\title{
Innate immunomodulation with MIS416: mechanism of action in experimental autoimmune encephalomyelitis
}

\author{
By \\ Madeleine White
}

A thesis submitted to the Victoria University of Wellington in fulfilment of the requirements for the degree of Doctor of Philosophy

Victoria University of Wellington

2015 


\begin{abstract}
Multiple sclerosis (MS) is an immune-mediated disease in which self-reacting T lymphocytes enter the central nervous system (CNS) and direct the damage of the myelin sheaths, which protect nerve axons. While there is no cure for MS currently, specific disease-modifying therapies are available that target the relapsing-remitting form of MS. However, these therapies are not effective in progressive forms of MS, which affect $\sim 50 \%$ of MS patients in New Zealand, and thus there is an urgent need for novel treatments to be developed to treat these patients. MIS416 is a microparticle, which targets phagocytic cells by activating cytosolic receptors NOD2 and Toll-like receptor 9, and has recently completed a phase $2 \mathrm{a}$ trial in the treatment of progressive MS with promising results. The aim of this thesis was to elucidate the mechanism(s) by which MIS416 modified autoimmune disease using an animal model of MS, experimental autoimmune encephalomyelitis (EAE).
\end{abstract}

We determined that weekly intravenous doses of MIS416 were effective at reducing disease in a chronic model of MS, EAE, and we used this dosing regimen to further understand mechanisms involved in MIS416-induced EAE protection. It was found that MIS416 treatment induced a number of peripheral immune changes which had the potential to alter a $\mathrm{T}$ cell-mediated autoimmune disease, including increased levels of serum NO and IFN- $\gamma$, increased numbers of Tregs and macrophages as well as increased expression of PD-L1, an anti-proliferative ligand. Additionally, we also determined that MIS416 treatment significantly reduced T cell proliferation in vitro and in vivo.

To investigate which of these peripheral changes were involved in MIS416-mediated protection from EAE, we assessed each change individually in our disease model. We found that the central immune-modulating factor responsible for the protective effects of MIS416 treatment was IFN- $\gamma$ (White, Webster, O'Sullivan, Stone, \& La Flamme, 2014) as IFN- $\gamma^{-1-}$ mice treated with MIS416 were not protected from EAE. This protective role of MIS416-induced IFN- $\gamma$ was likely to have been mediated through downstream effects given that in the absence of IFN- $\gamma$ many of these changes were not seen. In particular, the macrophage population did not expand in MIS416-treated IFN- $\gamma^{-/-}$mice, and the expression of the immunoregulatory ligand, PD-L1, was not enhanced suggesting that macrophage expressed PD-L1 may be one of the ways in which MIS416-induced IFN- $\gamma$ altered EAE. While NO was found to contribute to the MIS416-mediated suppression of T cell proliferation ex vivo and IFN- $\gamma^{-/}$mice did not have elevated levels of NO, the in vivo inhibition of iNOS by aminoguanidine did not ablate MIS416 protection suggesting that these pathways were not central to disease protection. Additionally, MIS416 treatment was shown to increase the number and function of Tregs, and in the absence of IFN- $\gamma$ this expansion was 
reduced. Altogether, this research indicated that IFN- $\gamma$ was key to MIS416-mediated protection possibly through an expansion of immunoregulatory macrophage populations, enhanced PD-L1 expression, and enhanced Treg numbers and function.

In addition to these peripheral immune effects, we found that MIS416 treatment also altered cellular trafficking to the CNS. MIS416-treated EAE mice had reduced CNS infiltration as measured by both flow cytometry and histology compared to untreated EAE mice, and MIS416 treatment also reduced the EAE-induced permeability of the blood brain barrier. Furthermore, our study determined that MIS416-induced trafficking of immune cells to the CNS in the absence of EAE, and this trafficking also occurred in an IFN- $\gamma$-dependent manner. Given the suppressive nature of cells found within the periphery of MIS416-treated mice, the cells which trafficked to the CNS in response to MIS416 treatment may have had beneficial roles in EAE by suppressing T cell responses from within the CNS.

In summary, the work outlined in this thesis revealed that MIS416 treatment induced an immunoregulatory state that was capable of suppressing $\mathrm{T}$ cell proliferation and reducing EAE disease. Moreover, this research highlighted that in the right context, a pro-inflammatory cytokine such as IFN- $\gamma$ may be protective in autoimmune disease. Finally, when these findings are applied more widely, they indicate that the immune modulations induced by MIS416 may have potential in other diseases mediated by immune dysregulation in addition to MS. 


\section{Acknowledgements}

Firstly, I would like to thank my supervisor A/Prof Anne La Flamme. Her guidance and support has been invaluable over the years and I could not have asked for a better supervisor. I would also like to thank my secondary supervisor Dr Gill Webster for her continual encouragement and steady flow of ideas for my $\mathrm{PhD}$ project.

Thank you to all the people in the LAF lab past and present: Marie, Degee, Dave, Danielle, Laura, Lisa, Jenni, Sarrabeth, Dahna, Lou, Andrew, Hajar, Vimal, Nikki and Pirooz. Together you have made the lab an enjoyable place to work and supplied plenty of caffeine breaks to ensure productivity!

I would like to particularly thank Sarrabeth, Marie, Nikki and Pirooz for helping me out during some of the more epic experiments, without you I would have never got through them all. To Pirooz for being an awesome editor and support person for the final few months, with many coffee breaks and science banter to keep me sane (or slightly insane?). To Nikki for helping section my spinal cords even though you had plenty of your own work to get through. Also, thanks to the Malaghan $\mathrm{PhD}$ club for some much needed science interaction and support.

I would also like to acknowledge the people who partially aided in the work presented in this thesis: David O'Sullivan for providing assistance in the early MIS416 EAE dosing experiments, Nikki Templeton and Pirooz Zareie for scoring the H\&E spinal cord sections, and Louise Rooney for assisting with the oral dosing of MIS416 and providing H\&E sections of the gut.

A big thank you to Rebecca Girvan, Vicky Pearson, Gill Webster and the rest of the team at Innate Immunotherapeutics for the constant supply of MIS416, sharing of ideas and financial support through my PhD. I would also like to thank various funding bodies who have supported my project including Victoria University Doctoral Award and the Great New Zealand Trek, among others.

I would also like to thank my family and close friends for being so understanding about my lack of spare time! A big thank you to my parents for helping out, especially looking after my dog while I was busy working late. And to my sister Charlotte, for always being willing to hang out - even if that means going on a big run with me.

Finally, big thanks to my support group at home. To my dog Max for always being happy to see me no matter what time I get home. And a special mention to my partner Sean, thank you for being so understanding and encouraging during the year, but most of all thank you for putting up with me. You helped me so much with all the little things, which made my PhD years manageable and I couldn't have done it without you (and Max). 


\section{Publications and conference presentations}

Below is a list of publications and conference presentations arising from the work in this thesis.

White, M., Webster, G., O'Sullivan, D., Stone, S., \& La Flamme, A. C. (2014). Targeting innate receptors with MIS416 reshapes Th responses and suppresses CNS disease in a mouse model of multiple sclerosis. PloS one, 9(1), e87712. doi: 10.1371/journal.pone.0087712

White, M., Webster, G., \& La Flamme, A. Innate cell modulator MIS416 suppresses CNS disease in EAE. Journal of Neuroimmunology, 275(1), 178. doi: 10.1016/j.jneuroim.2014.08.478 Conference proceeding 


\section{Table of Contents}

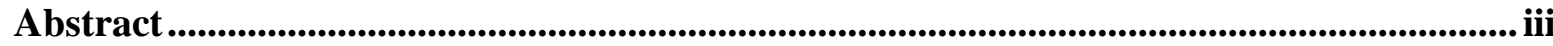

Acknowledgements ..............................................................................................................................

Publications and conference presentations ............................................................................................ vi

Table of Contents .............................................................................................................................................. vii

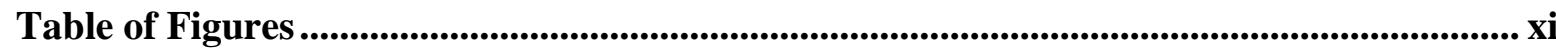

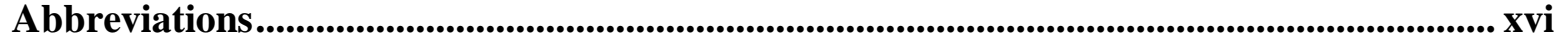

Chapter 1： Introduction ............................................................................................................. 1

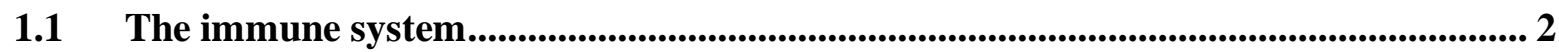

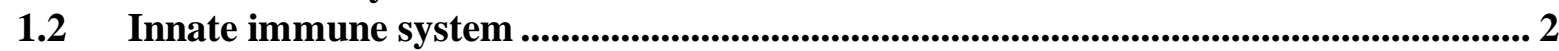

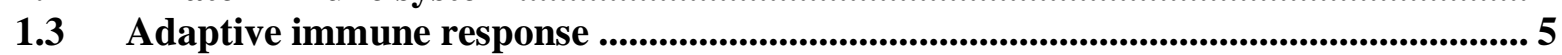

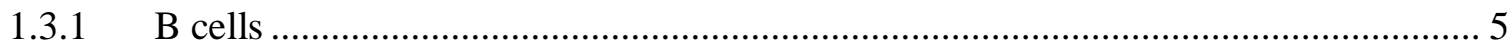

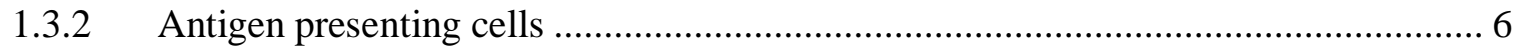

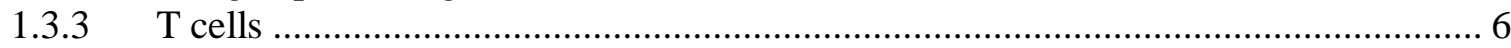

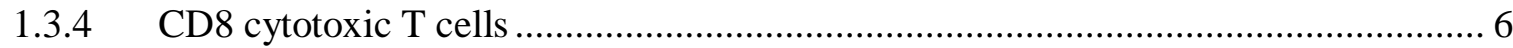

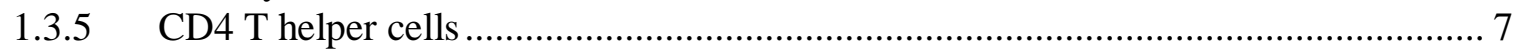

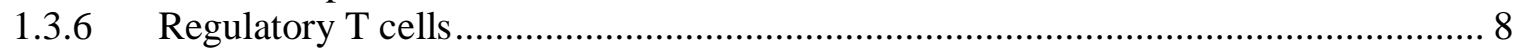

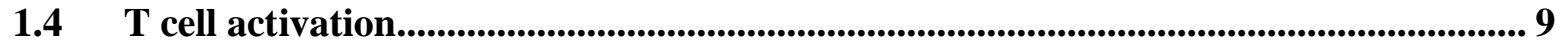

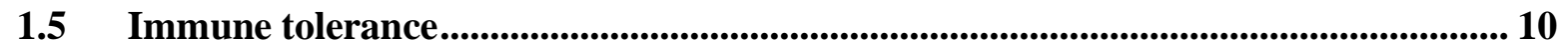

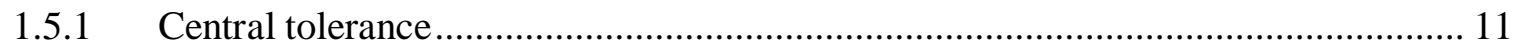

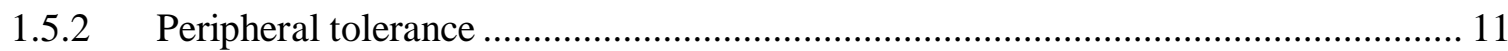

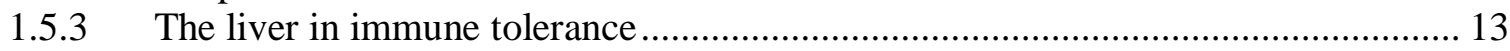

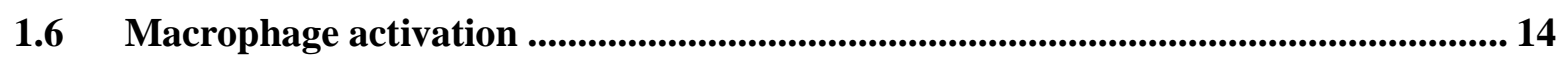

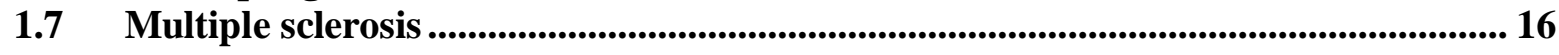

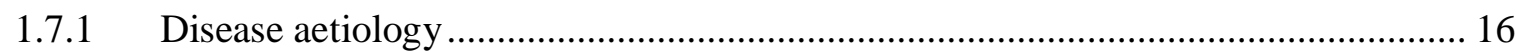

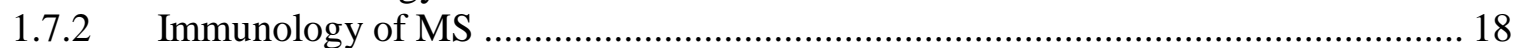

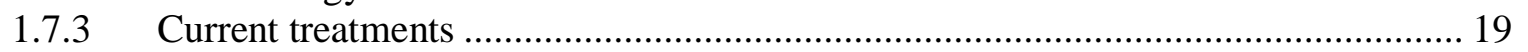

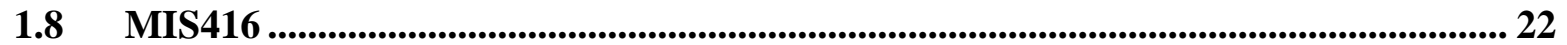

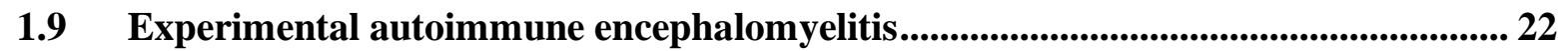

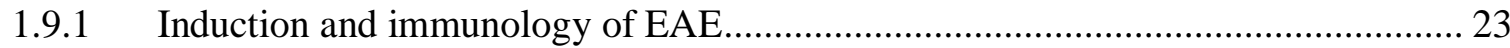

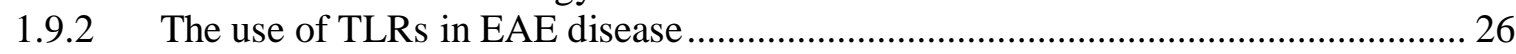

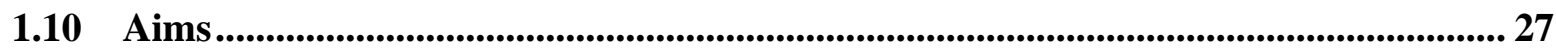

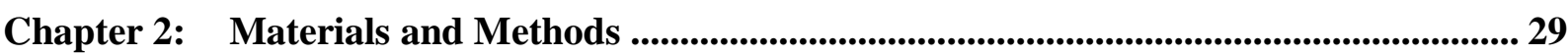

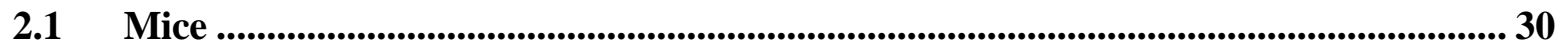

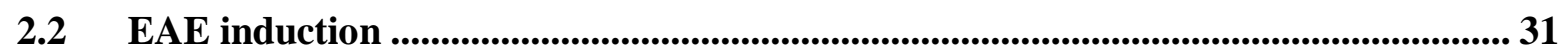

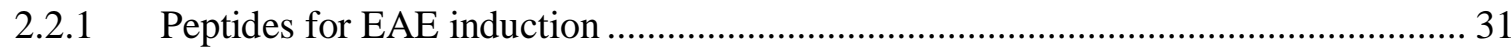

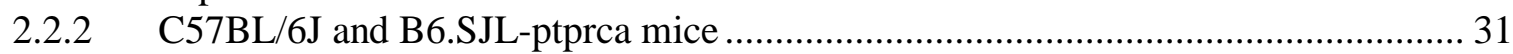

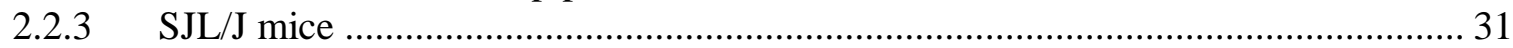

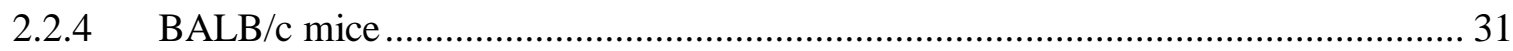

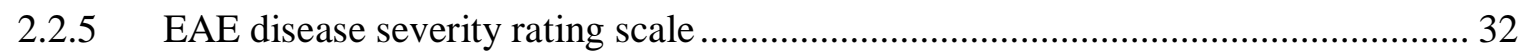

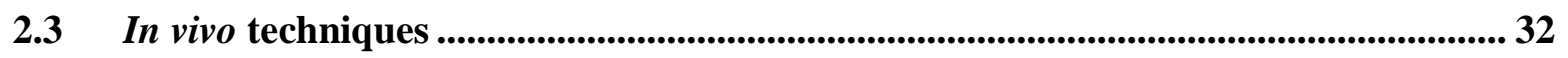

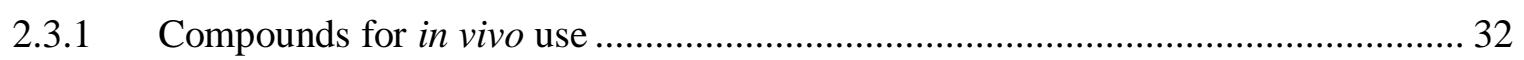

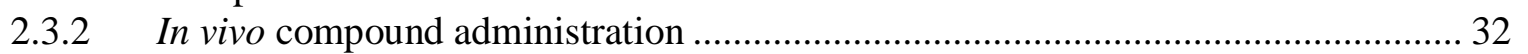

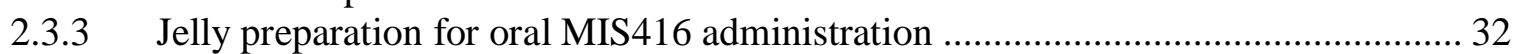

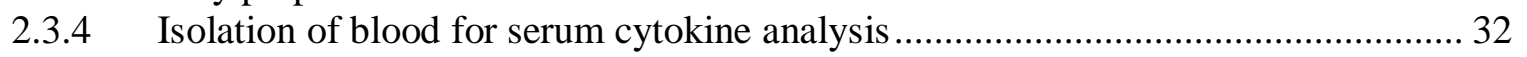

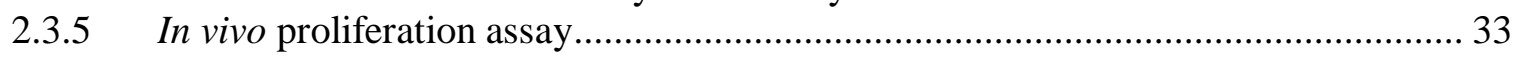




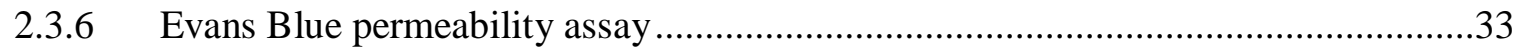

2.4 Ex vivo techniques ....................................................................................................................................33

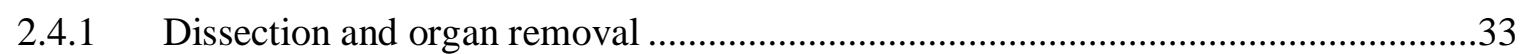

2.4.2 Preparation of a single cell suspension from the spleen ..........................................

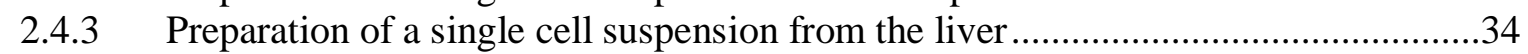

2.4.4 Preparation of a single cell suspension from the spinal cord ...................................35

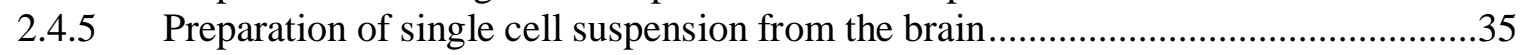

2.4.6 Preparation of whole blood for flow cytometry ......................................................

2.4.7 Preparation of a single cell suspension from the draining lymph node.....................36

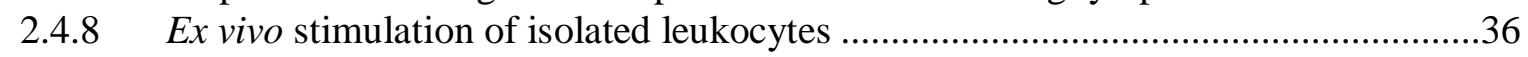

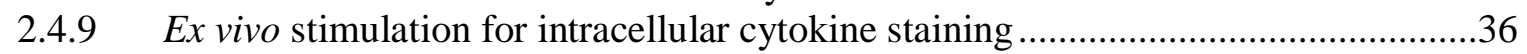

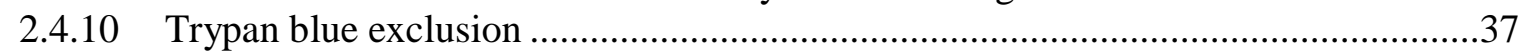

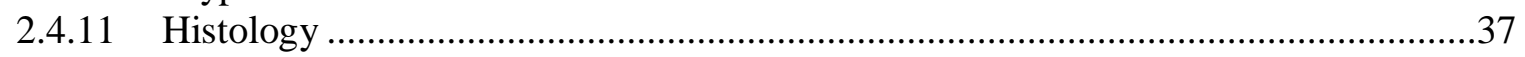

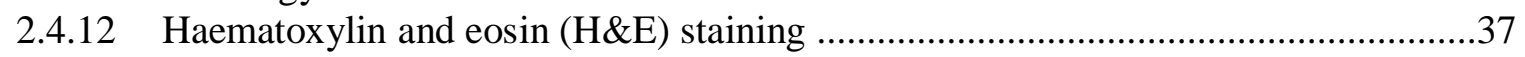

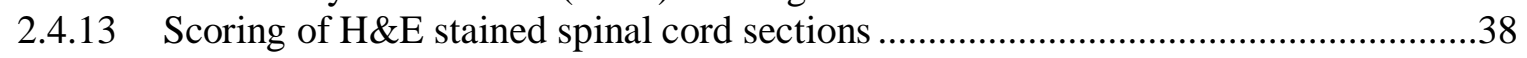

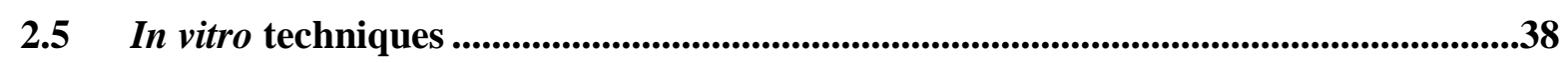

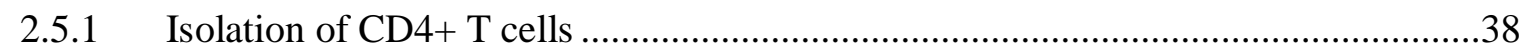

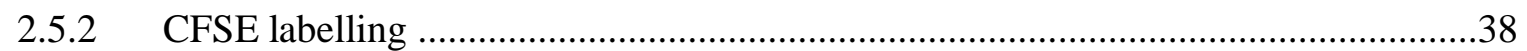

2.5.3 Preparation of bone marrow-derived macrophages ..................................................

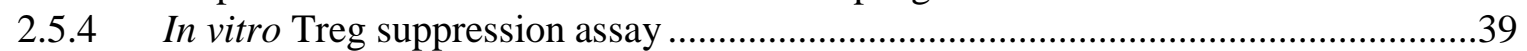

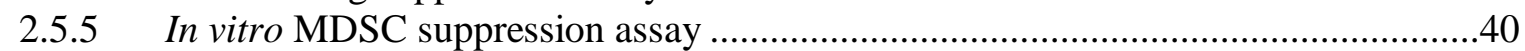

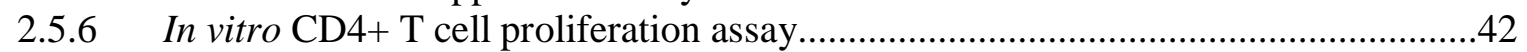

2.6 Enzyme linked immunosorbent assays (ELISA) ........................................................42

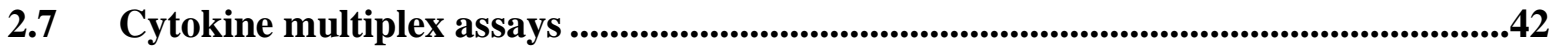

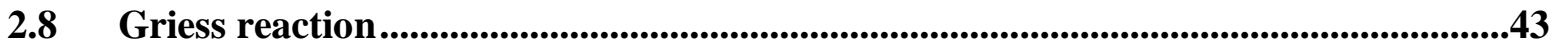

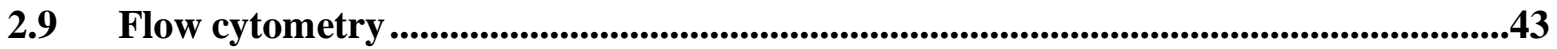

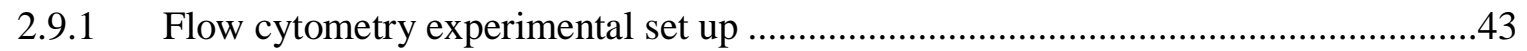

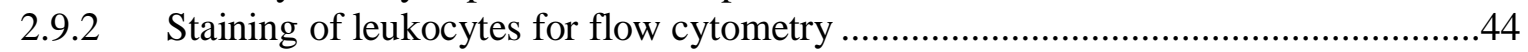

2.9.3 Cell cycle analysis using Vybrant ${ }^{\circledR}$ DyeCycle ${ }^{\mathrm{TM}}$ Green stain ................................44

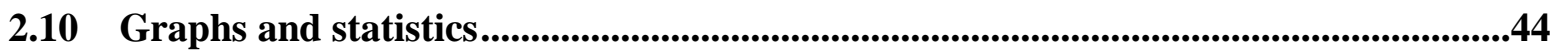

Chapter 3: Identifying an effective dose regime for MIS416 using experimental autoimmune encephalomyelitis .............................................................................................................................44

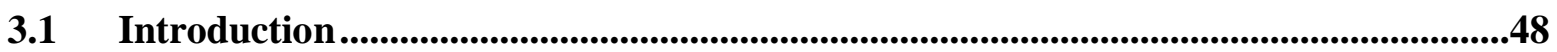

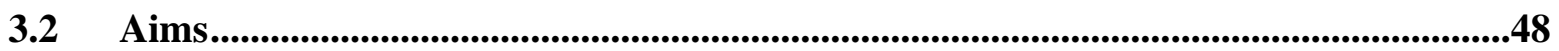

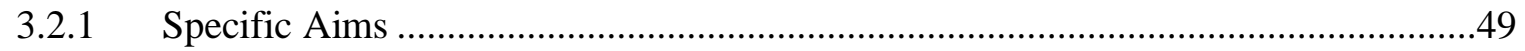

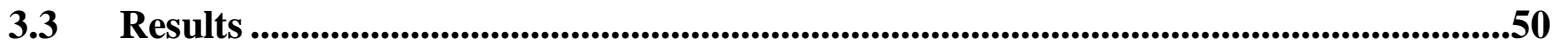

3.3.1 Dose response when MIS416 is given at day 0 in C57BL/6 mice with EAE ...........50

3.3.2 A $100 \mu \mathrm{g}$ weekly dose of MIS416 is the most effective dose in C57BL/6 EAE mice 53

3.3.3 Antigen specific responses in C57BL/6 EAE mice with weekly MIS416 doses ......55

3.3.4 MIS416 was ineffective at reducing EAE disease in BALB/c mice at either $100 \mu \mathrm{g}$

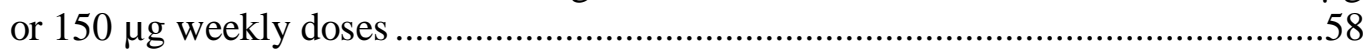

3.3.5 A $50 \mu \mathrm{g}$ dose was most effective at reducing severity of relapses in SJL/J EAE mice 60

3.3.6 Signalling through TLR9 is essential for MIS416 EAE disease protection ..............63

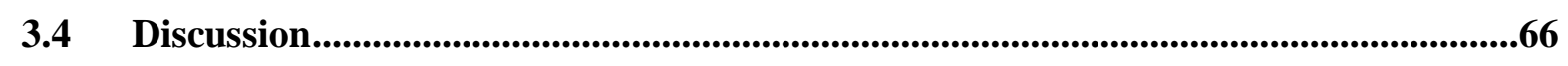

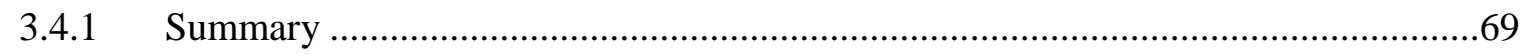


4.1 Introduction ....................................................................................................................................... 72

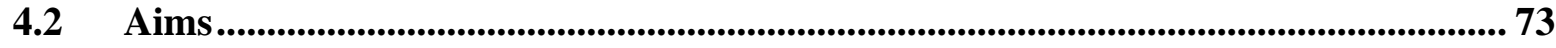

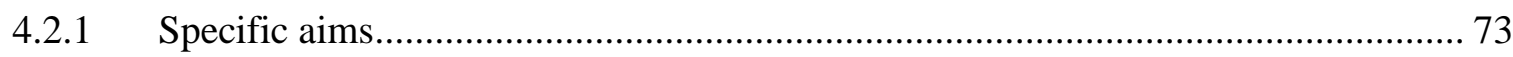

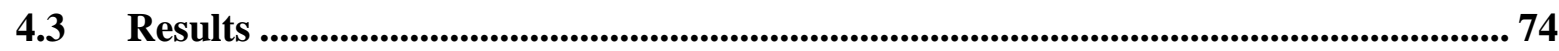

4.3.1 MIS416 treatment resulted in increased leukocyte count in the liver and spleen..... 74

4.3.2 MIS416 treatment induced cells of the spleen and liver to enter the cell cycle ....... 76

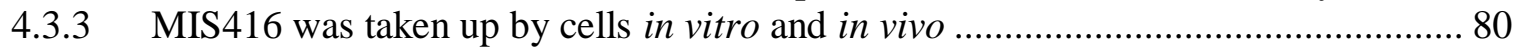

4.3.4 MIS416 treatment altered the myeloid populations in the spleen and liver .............. 83

4.3.5 MIS416 treatment altered T cell populations in the spleen and liver........................ 87

4.3.6 MIS416 treatment altered immune cell activation in the spleen and liver ................ 93

4.3.7 Spleen and liver cytokine profile was altered with in vivo MIS416 treatment ......... 95

4.3.8 Serum IFN- $\gamma$, IL-6 and NO were increased with MIS416 treatment ...................... 101

4.3.9 CD4 T cells and F4/80+ cells were the main producers of splenic IFN- $\gamma$.............. 103

4.3.10 Macrophages produced IL-12 in response to MIS416 ........................................... 108

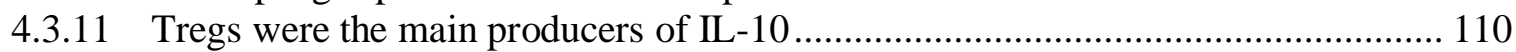

4.3.12 MIS416 reduced CD4 T cell proliferation in vitro and ex vivo ............................... 112

4.3.13 MIS416 induced effects were still apparent 6 days post injection ......................... 116

4.3.14 Using TLR-9-ligand-deficient MIS416 some immunomodulatory properties were

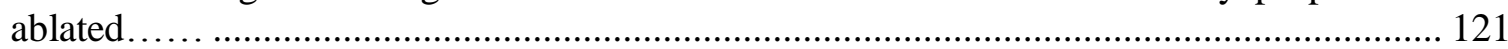

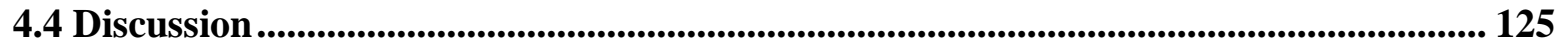

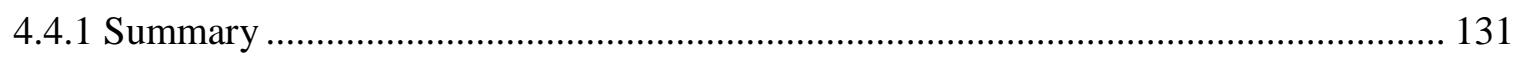

Chapter 5: Mechanisms of MIS416-mediated immunoregulation .................................... 133

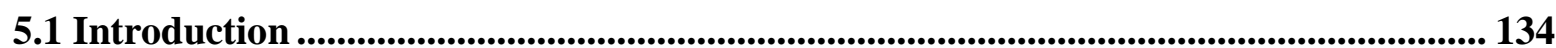

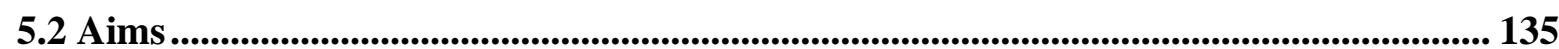

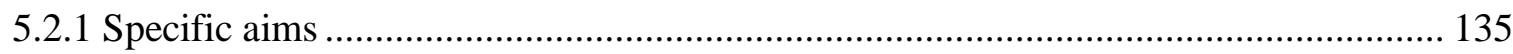

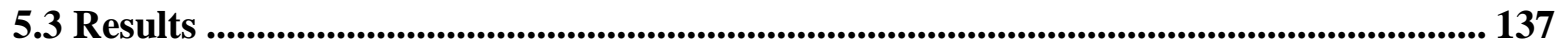

5.3.1 IFN- $\gamma$ was required for MIS416 protection in chronic EAE disease model ................ 137

5.3.2 MIS416-induced peripheral myeloid changes were abolished in IFN- $\gamma^{-/}$mice ........... 139

5.3.3 IFN- $\gamma$ was a key factor in MIS416-mediated suppression of T cell responses with ConA 145

5.3.4 Nitric oxide was a key factor in MIS416-mediated suppression of ConA-induced T cell proliferation

5.3.5 Nitric oxide inhibition during disease onset did not affect MIS416-mediated disease protection....

149

5.3.6 MIS416-mediated effects in non-EAE mice were preserved when nitric oxide

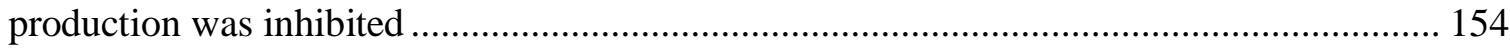

5.3.7 Tregs from MIS416-treated mice were more suppressive than Tregs from untreated mice 159

5.3.8 CD4+ T cells isolated from MIS416-treated mice responded similarly to CD3/CD28

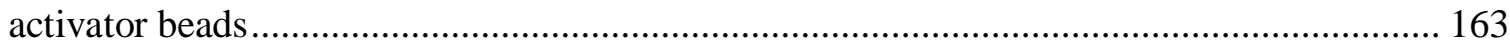

5.3.9 Myeloid-derived suppressor cells (MDSC) from MIS416-treated mice were more

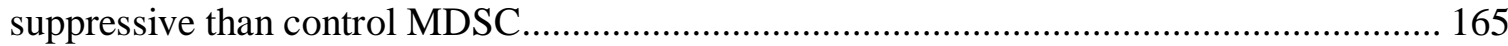

5.3.10 MIS416 treatment reduced in vivo proliferation of MOG-specific $\mathrm{T}$ cells in EAE

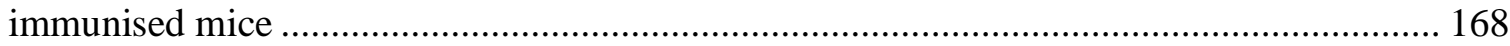

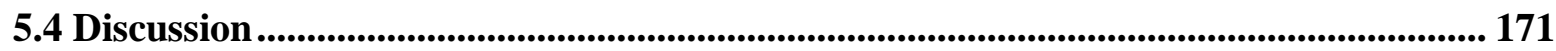

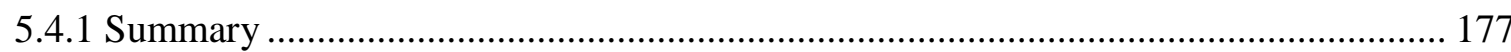


Chapter 6: MIS416-driven alterations in the CNS .................................................................179

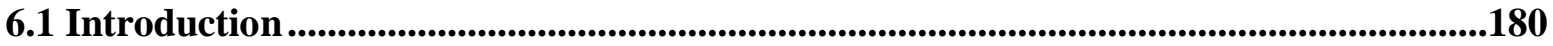

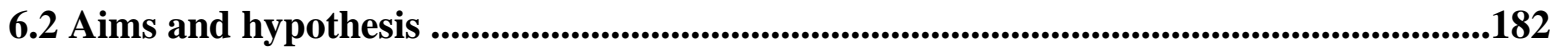

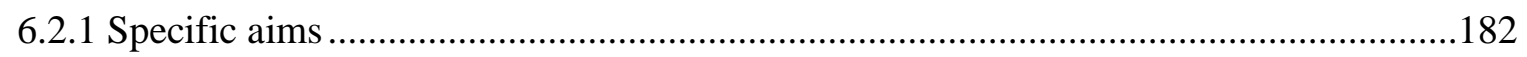

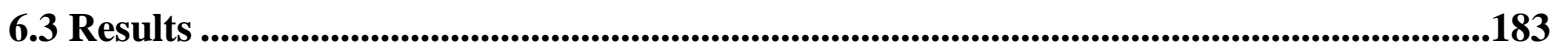

6.3.1 Trace levels of MIS416-AF488 were found within the brain and spinal cord..............183

6.3.2 MIS416 treatment reduced CNS-infiltrating inflammatory cells in EAE mice .............185

6.3.3 Heat-treated MIS416 was less effective at reducing CNS infiltration in EAE mice ....191

6.3.4 MIS416 increased myeloid and non-myeloid CNS infiltration without EAE ..............194

6.3.5 MIS416-treated EAE mice had reduced lesion size in the spinal cord at day 22 .........196

6.3.6 MIS416 treatment reduced EAE-induced BBB permeability .....................................198

6.3.7 IFN- $\gamma$ was important in the infiltration of cells into the CNS of MIS416-treated mice in

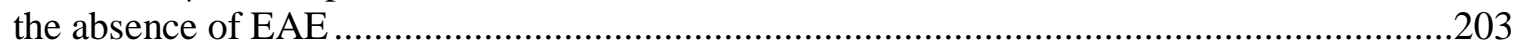

6.4 Discussion ..........................................................................................................................................206

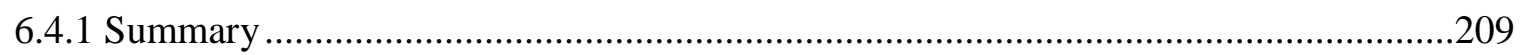

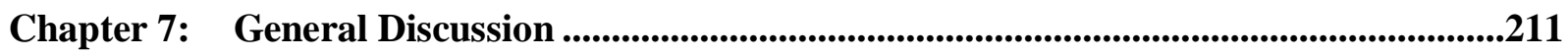

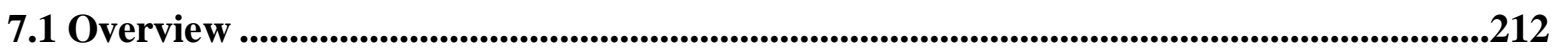

7.2 Comparisons of MIS416 treatment between mouse and human .........................................215

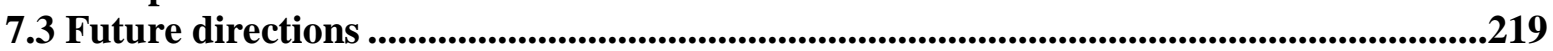

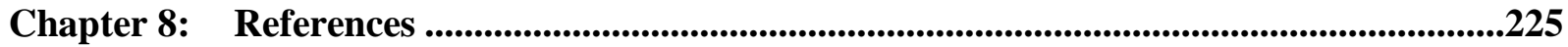

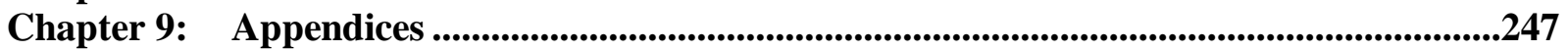

Appendix 1: Flow gating strategy and systemic cytokines ................................................247

Appendix 1.1 Flow gating strategies for identifying cell populations ................................247

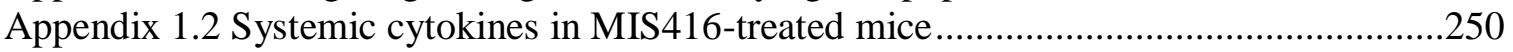

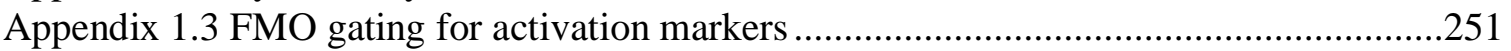

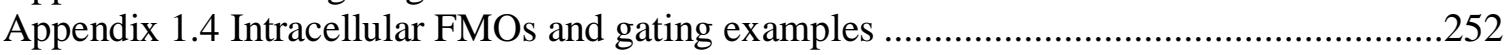

Appendix 2: Assessing CFSE labelling and concentration of CD3/CD28 activator beads256 Appendix 3: Ly6G+ and Gr-1dim MDSC have different expression profiles .....................257 Appendix 4: In vivo proliferation assay gating strategy ...............................................................258 Appendix 5 Oral dosing of MIS416 .........................................................................................................259

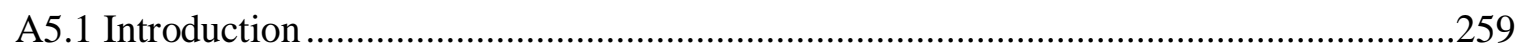

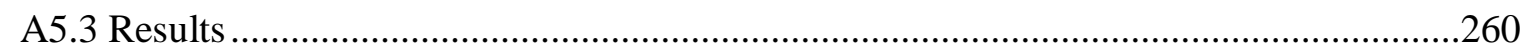

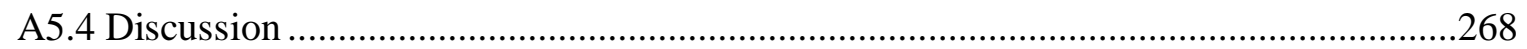

Appendix 6: Buffers and solutions...........................................................................................................270

Appendix 7: Antibodies and Proteins ................................................................................................272

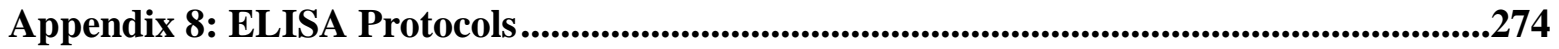

Appendix 9: Alterations of the MIS416 compound.........................................................................2275

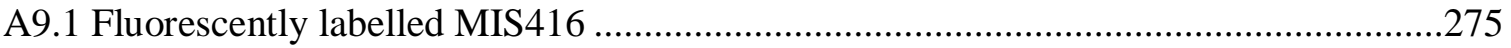

A9.2 Heat treatment of MIS416 removes TLR9 activity only .........................................2.

Appendix 10: Spinal cord sections ..............................................................................................................277 


\section{Table of Figures}

\section{Chapter 3}

Figure 3-1 $250 \mu \mathrm{g}$ dose of MIS416 was effective at reducing disease in C57BL/6 mice when given at day 0 .

Figure 3-2 $250 \mu \mathrm{g}$ dose of MIS416 reduced disease in C57BL/6 mice when given at day $12 \ldots . . .52$

Figure 3-3. Weekly doses of $100 \mu \mathrm{g}$ of MIS416 was the most effective treatment in C57BL/6 EAE mice

Figure 3-4 MIS416 treatment significantly altered splenocytes cytokine production in response to MOG at day 15 and 22. 56

Figure 3-5 MIS416 treatment significantly altered liver cells cytokine production in response to MOG at day 15 and 22 . 57

Figure 3-6 Weekly treatments of either $100 \mu \mathrm{g}$ or $150 \mu \mathrm{g}$ were ineffective in the BALB/c model of EAE 59

Figure 3-7 MIS416 was effective at reducing relapsing-remitting EAE disease in SJL/J mice when given $50 \mu \mathrm{g} / \mathrm{mouse}$ weekly doses beginning at day 21 .

Figure 3-8 MIS416 treatment in SJL/J mice significantly reduced the cytokine response to PLP at day 42 .

Figure 3-9 Heat-treated-MIS416 was not effective at reducing EAE disease in C57/B16 mice when used at the same dose and timing as regular MIS416.

Figure 3-10 Heat-treated MIS416 reduced the IL-17A response to MOG with no effect on MOG induced IFN- $\gamma$ or NO production in splenocytes. 65

\section{Chapter 4}

Figure 4-1 Spleen and liver cell counts were elevated at various time points after MIS416 treatment.

Figure 4-2 MIS416 given to splenocytes in vitro induced a small percentage of cells to undergo proliferation.

Figure 4-3 MIS416-treated mice i.v showed an increase in the number of proliferating cells in the spleen and liver.

Figure 4-4 MIS416 was taken up by isolated splenocytes in vitro............................................. 81

Figure 4-5 MIS416 was deposited primarily in the liver 24-hours after a $100 \mu \mathrm{g}$ dose i.v............ 82

Figure 4-6 Gating strategy for liver and spleen myeloid populations ......................................... 84 
Figure 4-7 The splenic myeloid compartment was significantly altered by MIS416 administration.

Figure 4-8 The liver myeloid compartment was significantly altered by MIS416 administration. 86 Figure 4-9 Administration of MIS416 increased the total number of Treg, CD4 and CD8 T cells in EAE mice. . .89

Figure 4-10 Administration of MIS416 increased the total number of Treg, CD4 and CD8 T cells in non-EAE mice. . .90

Figure 4-11 Administration of MIS416 increased the liver T cell populations . .91

Figure 4-12 Weekly MIS416 treatment increased FoxP3+ populations in spleen and liver using FoxP3+GFP mice. . .92

Figure 4-13 MIS416 activated myeloid populations .094

Figure 4-14 MIS416 enhanced MIS416-stimulated production of IFN- $\gamma$, NO and IL-10 but not IL17A by splenocytes from EAE mice.

Figure 4-15 MIS416 enhanced MIS416-stimulated production of IFN- $\gamma$, NO and IL-10 by splenocytes from non-EAE mice. . .98

Figure 4-16 MIS416 enhanced MIS416-stimulated production of IFN- $\gamma$, NO and IL-10 but not IL17A by liver leukocytes from EAE mice.

Figure 4-17 MIS416 enhanced MIS416-stimulated production of IFN- $\gamma$ and NO by liver leukocytes from non-EAE mice.

Figure 4-18 Serum cytokines increased with MIS416 treatment in vivo 102

Figure 4-19 IFN- $\gamma$ positive cells were found after 5-hour ex vivo stimulation 105

Figure 4-20 IFN- $\gamma$ positive cells were characterised after 48-hour ex vivo stimulation. 106

Figure 4-21 IFN- $\gamma$ positive cells were characterised after 48-hour ex vivo stimulation without PMA/ionomycin.

Figure 4-22 MIS416 stimulated splenocytes and macrophages to produce high levels of IL-12 .109 Figure 4-23 In vivo stimulation with MIS416 enhanced IL-10 production by Treg, macrophages and NK cells after 5-hour ex vivo re-stimulation

Figure 4-24 MIS416 suppressed MOG proliferation response in vitro. 114

Figure 4-25 MIS416 suppressed ConA proliferation and IFN- $\gamma$ response ex vivo. 115

Figure 4-26 MIS416-induced splenocyte alterations were still apparent 6-days post treatment...118 Figure 4-27 MIS416-induced lymphocyte alterations were still apparent 6-days post treatment.119 Figure 4-28 MIS416 treatment altered cytokine profile at day 13 120

Figure 4-29 Heat-treated MIS416 treatment resulted in minor peripheral immune changes. .......123

Figure 4-30 Heat-treated MIS416 altered the cytokine profile of EAE mice 124 
Figure 4-31 Observations of spleen and liver cultures with MIS416, or serum cytokines which occurred with MIS416 treatment in vivo.

\section{Chapter 5}

Figure 5-1 IFN- $\gamma^{-/-}$mice were not protected from EAE disease with MIS416 treatment............ 138

Figure 5-2 IFN- $\gamma^{-/-}$mice had increased spleen counts with MIS416 treatment ........................... 141

Figure 5-3 MIS416-treated IFN- $\gamma^{-1-}$ mice did not have the same myeloid alterations as WT mice.

Figure 5-4 MIS416-treated IFN- $\gamma^{-/-}$mice had altered responses to re-stimulation 143

Figure 5-5 MIS416-treated IFN- $\gamma^{-/}$mice had low levels of serum NO compared to MIS416-treated WT mice 144

Figure 5-6 Splenocytes from IFN- $\gamma^{-/-}$MIS416-treated mice were unable to suppress $\mathrm{T}$ cell proliferation response to ConA 146

Figure 5-7 Nitric oxide was a key factor for MIS416-induced suppression of CD4 T cell proliferation 148

Figure 5-8 Nitric oxide inhibition did not affect EAE disease protection with MIS416 treatment 151

Figure 5-9 NO inhibition did not significantly alter the cellular composition and activation of cells in MIS416-treated EAE mice. 152

Figure 5-10 Cytokine production in AG treated mice was not significantly altered compared to controls 153

Figure 5-11 AG treatment in non-EAE mice did not abolish MIS416-induced changes .... 156

Figure 5-12 AG treatment in non-EAE mice did not abolish MIS416-induced cytokines. 157

Figure 5-13 Serum NO levels were suppressed by aminoguanidine 158

Figure 5-14 Tregs isolated from MIS416-treated mice were more suppressive than Tregs from untreated mice. 161

Figure 5-15 PD-L1 and PD-1 expression was increased on T cell populations 162

Figure 5-16 CD4+ T cell proliferation was not significantly altered with MIS416 treatment ..... 164 Figure 5-17 MDSC isolated from MIS416-treated mice were more suppressive than control MDSC and this suppression was not mediated by NO. 167

Figure 5-18 in vivo proliferation assay 169

Figure 5-19 MIS416 treatment reduced CD4+ $\mathrm{T}$ cell proliferation in vivo in response to $\mathrm{EAE}$ immunisation. 170 


\section{Chapter 6}

Figure 6-1 Traces of MIS416 were found within the brain 48 hours after a weekly dose in nonEAE mice 184

Figure 6-2 MIS416 treatment reduced infiltrating myeloid cells during EAE to the level seen in non-EAE MIS416-treated mice at day 16.

Figure 6-3 MIS416 treatment maintained resting microglial state in brain and spinal cord of EAE mice at day 16.

Figure 6-4 MIS416 treatment protected mice against EAE-induced infiltrating lymphocytes in the brain and spinal cord at day 16 . 190

Figure 6-5 Weekly HT-MIS416 treatment did not significantly reduce leukocyte infiltration into the brain in EAE mice at day 22 192

Figure 6-6 Weekly HT-MIS416 treatment did not significantly reduce leukocyte infiltration into the spinal cord in EAE mice at day 22.

Figure 6-7 Weekly MIS416 treatment increased leukocyte infiltration into the CNS in non-EAE mice.

Figure 6-8 MIS416-treated EAE mice had reduced lesion size in the spinal cord.

Figure 6-9 Optimising Evans blue in BALB/c mice showed that $200 \mu 1$ of $2.5 \%$ in saline given i.p. was effective in EAE mice compared to non-EAE. 200

Figure 6-10 BBB permeability in EAE mice was reduced with MIS416 treatment. 201

Figure 6-11 BBB permeability in non-EAE mice was not significantly altered with MIS416 treatment. .202

Figure 6-12 MIS416 infiltration into the CNS in non-EAE mice required IFN- $\gamma$. .204

Figure 6-13 MIS416-induced trafficking into the brain was comprised of different cells compared to spinal cord in non-EAE mice. .205

\section{$\underline{\text { Appendices }}$}

Figure A1-1 Live cell gating strategy for liver and spleen. .247

Figure A1-2 Isolating live-single-CD45+ cells from the spleen and liver..... 248

Figure A1-3 Liver and spleen cell populations and differential Gr1+ populations 248

Figure A1-4 The splenic myeloid compartment was significantly altered by MIS416 administration and immunization (as published in White et al, 2014).

Figure A1-5 MIS416 treatment suppressed systemic antigen-specific responses (as published in White et al, 2014). .250 
Figure A1-6 Example of FMO gating for activation marker PD-L1. 251

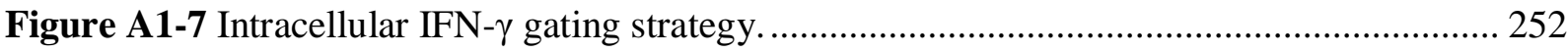

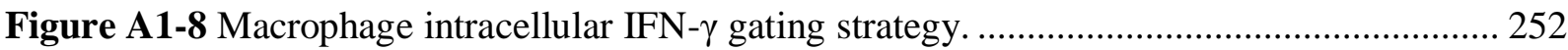

Figure A1-9 Lymphocytes intracellular IFN- $\gamma$ gating strategy. ............................................... 253

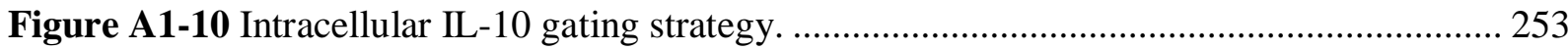

Figure A1-11 Lymphocytes intracellular IL-10 gating strategy.............................................. 254

Figure A1-12 Lymphocyte intracellular FoxP3 gating strategy.............................................. 255

Figure A2-1 CD3/CD28 activator beads in culture with isolated CD4+ T cells. ....................... 256

Figure A3-1 MDSC were either monoyctic or granulocytic and express different levels of Ly6C

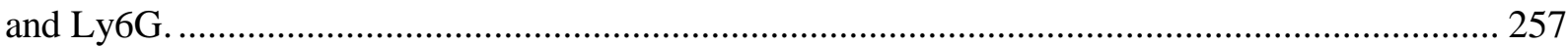

Figure A4-1 gating for in vivo proliferation experiments and examples of each treatment. ....... 258

Figure A5-1 MIS416 given in feed at 100 ug daily did not alter the peripheral immune changes

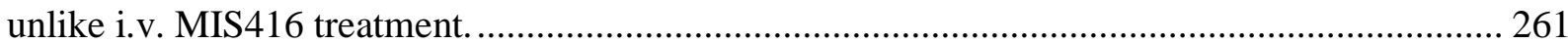

Figure A5-2 Cytokine re-call was not significantly enhanced in diet-treated MIS416 mice. ...... 262

Figure A5-3 Oral MIS416-treated mice had minor cellular changes ......................................... 264

Figure A5-4 Oral MIS416 administration altered activation state within the spleen ................. 264

Figure A5-5 Oral MIS416 administration did not alter crypt integrity ....................................... 265

Figure A5-6 Oral MIS416 administration did not induce gut immune infiltration ...................... 266

Figure A5-7 Oral MIS416 administration reduced macrophage infiltration in the small intestine 267

Figure A9-1 Heat treatment of MIS416 retains NOD2 cytokines and TLR9 cytokines are suppressed..... 276 
Abbreviations

\begin{tabular}{|l|l|}
\hline AEC & Animal ethics committee \\
\hline AG & aminoguanidine \\
\hline APC & antigen presenting cell \\
\hline Arg-1 & Arginase-1 \\
\hline AUC & Area under the curve \\
\hline BBB & blood brain barrier \\
\hline BCR & B cell receptor \\
\hline BCSFB & Blood cerebro-spinal fluid barrier \\
\hline BrdU & 5-bromo-2-deoxyuridine \\
\hline BRU & Biomedical research unit \\
\hline CCL5 & chemokine (C-C motif) ligand 5 \\
\hline CCR5 & chemokine (C-C motif) receptor 5 \\
\hline CD & cluster of differentiation \\
\hline CFA & complete Freund's adjuvant \\
\hline CFDA-SE & carboxyfluorescein diacetate N-succinimidyl ester \\
\hline CFSE & carboxyfluorescein succinimidyl ester \\
\hline c-FLIP & Cellular FLCE-inhibitory protein \\
\hline CLRs & C-type lectins receptors \\
\hline ConA & Concanavalin A \\
\hline CSF & Cerebro-spinal fluid \\
\hline CTCM & complete T cell media \\
\hline CTL & cytotoxic lymphocyte \\
\hline CTLA-4 & Cytotoxic lymphocyte associated protein-4 \\
\hline DC & dendritic cell \\
\hline dLN & inguinal lymphnode that drains the EAE immunisation site \\
\hline DMSO & dimethyl sulfoxide \\
\hline dPBS & Dulbecco's phosphate buffered saline \\
\hline EAE & experimental autoimmune encephalomyelitis \\
\hline ELISA & enzyme-linked immunosorbant assay \\
\hline FACS & fluorescent activated cell sorting \\
\hline FCS & foetal calf serum \\
\hline FDA & Food and Drug Administration \\
\hline Foxp3 & forkhead box p3 \\
\hline g & gravity \\
\hline GA & glatiramer acetate \\
\hline GATA3 & GATA-binding protein 3 \\
\hline GM-CSF & granulocyte-macrophage colony-stimulating factor \\
\hline GPCR & G protein-coupled receptors \\
\hline HLA & human leukocyte antigen \\
\hline HRP & horseradish peroxidase \\
\hline IBD & Irritable bowel disease \\
\hline I-CAM-1 & intracellular adhesion molecule-1 \\
\hline IDO & Indoleamine-pyrrole 2,3-dioxygenase \\
\hline IFN- & interferon \\
\hline IgG & Immunoglobulin G \\
\hline IL- & interleukin \\
\hline iNOS & Inducible nitric oxide synthase \\
\hline i.p. & intraveritonous \\
\hline i.v. & \\
\hline &
\end{tabular}




\begin{tabular}{|c|c|}
\hline JC virus & John Cunningham virus \\
\hline LFA-1 & lymphocyte function-associated antigen-1 \\
\hline LN & lymph node \\
\hline LPS & lipopolysaccharides \\
\hline LSEC & Liver sinusoidal endothelial cells \\
\hline M & moles \\
\hline MAPK & mitogen activated protein kinases \\
\hline MBP & myelin basic protein \\
\hline MDSC & myeloid-derived suppressor cells \\
\hline MHC & major histocompatability complex \\
\hline MMP-9 & Matrix metallopeptidase 9 \\
\hline MOG35-55 & myelin oligodendrocyte glycoprotein \\
\hline MS & multiple sclerosis \\
\hline MSDC & Myeloid derived suppressor cell \\
\hline MTB & Mycobacterium tuberculosis \\
\hline MyD88 & myeloid differentiation primary response gene 88 \\
\hline$N F-\kappa B$ & nuclear factor $-\kappa \mathrm{B}$ \\
\hline NLR & nucleotide oligomerization domain like receptor \\
\hline $\mathrm{nM}$ & nanomole \\
\hline $\mathrm{NO}$ & nitric oxide \\
\hline NOD & nucleotide oligomerization domain \\
\hline PAMPs & pathogen-associated molecular patterns \\
\hline PBS & phosphate buffered saline \\
\hline $\mathrm{PD}-\mathrm{L}(1$ or 2$)$ & Programmed death ligand-1 (also referred to CD274 in the literature) \\
\hline PD-1 & Programmed death-1 (also referred to CD279 in the literature) \\
\hline p.i. & post immunisation \\
\hline PLP & proteolipid protein \\
\hline PML & Progressive multifocal leukoencephalopathy \\
\hline PPMS & primary progressive multiple sclerosis \\
\hline PRR & pattern recognition receptor \\
\hline RA & Rheumatoid arthritis \\
\hline RANTES & regulated upon activation, normal T-cell expressed, and secreted \\
\hline RICK & Receptor-interacting serine/threonine kinase \\
\hline RLRs & RIG-I-like receptors \\
\hline ROR $\gamma \mathrm{t}$ & retinoic acid receptor-related orphan receptor- $\gamma \mathrm{t}$ \\
\hline RRMS & relapsing-remitting multiple sclerosis \\
\hline RT & room temperature \\
\hline SA & streptavidin \\
\hline S.c & subcutaneous \\
\hline SC & spinal cord \\
\hline SEM & standard error of the mean \\
\hline T-bet & T-box transcription factor expressed in T-cell \\
\hline TCR & T cell receptor \\
\hline TEC & Thymic epithelial cell \\
\hline Th & T helper cell \\
\hline TLR & Toll like receptor \\
\hline TNF & Tumor necrosis factor \\
\hline Treg & regulatory T cells \\
\hline$\mu \mathrm{M}$ & micro moles \\
\hline VCAM-1 & vascular adhesion molecule-1 \\
\hline
\end{tabular}




\begin{tabular}{|l|l|}
\hline VCF & Vertebrate containment facility \\
\hline VLA-4 & $\alpha 4 \beta 1$ integrin \\
\hline 2D2 & Transgenic mice that express a TCR for the $\mathrm{MOG}_{35-55-}$ \\
\hline
\end{tabular}


Chapter 1: Introduction 


\subsection{The immune system}

The main function of the immune system is to protect the body from potential harm and by doing so the immune system needs to recognise self from non-self. The immune system recognises a potential threat through a collection of cells which can be divided into two lineages, the innate immune system and the adaptive immune system. The innate immune response provides us with a rapid defence against foreign pathogens, and it is this part of the immune system with which we are born. However as it is non-specific, it has a limited response and does not develop immunological memory. On the other hand, the adaptive immune response is slow acting but is very specific and improves over time. Furthermore, the adaptive immune response is able to form immunological memory (i.e. recognises previously encountered pathogens). Together the adaptive and innate immune systems are effective in eliminating most pathogens.

Given that the role of the immune system is eliminating pathogens, it needs to be able to distinguish between self and non-self to avoid initiating an inappropriate immune response. The immune system has several ways to prevent this type of self-activation from occurring, which include central and peripheral tolerance; however, these mechanisms are not absolute, and failure can lead to autoimmunity where the immune system directs its response towards the body's own cells. Multiple sclerosis (MS) is thought to be one of many autoimmune diseases in which the immune system has become confused and directs an inappropriate immune response towards self. Like other autoimmune diseases, MS is difficult to treat and current treatment options are limited with an increasing demand for more effective treatment options to be developed. The current study uses an experimental model to determine the mechanisms of a novel treatment for MS, with the hope that further understanding of this compound will allow it to be an approved therapeutic for MS patients.

\subsection{Innate immune system}

The innate immune system is made up of several components which include physical barriers such as skin and mucosal membranes, as well as soluble proteins and specific immune cells which work together to defend against pathogens and assist the adaptive immune response (Janeway \& Medzhitov, 2002). The cells of the innate immune system include basophils, eosinophils, mast cells, natural killer cells and phagocytic cells such as dendritic cells, neutrophils and macrophages (Turvey \& Broide, 2010). These cell types can recognise substances as foreign because of their expression of pathogen-associated molecular patterns (PAMPs) which are conserved structures expressed on bacteria, viruses and yeast but are not expressed on mammalian cells, preventing the innate immune system from reacting to self (Mogensen, 2009). The innate cells recognise these PAMPs through pattern recognition receptors (PRR) which can be found either on the surface of 
the innate cells or within the cell cytoplasm (Medzhitov \& Janeway, 1997). The major classes of PRRs identified include transmembrane proteins such as Toll-like receptors (TLRs), cytoplasmic proteins such as Retinoic acid-inducible gene-I-like receptors (RLRs), C-type lectin receptors (CLRs) and NOD-like receptors (NLRs) (Takeuchi \& Akira, 2010). Together these PRRs cover a wide range of conserved motifs and activation of these receptors results in the activation of downstream signalling which in turn activate transcription factors such as NFאB to produce inflammatory, antimicrobial and antiviral responses accordingly (Shizuo Akira \& Takeda, 2004).

The TLRs are an important class of PRRs given that there are 10 and 12 functional TLRs identified in mouse and human respectively, and nine of these were conserved in both species (Kawai \& Akira, 2010). Furthermore, each TLR has a unique function in PAMP recognition and the signalling downstream of TLRs triggers the activation of the innate immune response, which in turn provides the signals to recruit the adaptive response (Shizuo Akira \& Takeda, 2004; Takeuchi \& Akira, 2010). Many different cell types, not just innate cells, are able to respond to PAMPs however, the innate cells which respond to PAMPs are able to recruit other cells and direct the destruction of invading pathogens.

Neutrophils are relatively short-lived, granulocytic, innate immune cells with a life span of only 68 hours in humans (Amulic, Cazalet, Hayes, Metzler, \& Zychlinsky, 2012). Despite their short lifespan, neutrophils are recruited early during an inflammatory response and given that they have the ability to engulf and kill pathogens as well as secrete granules containing antimicrobial factors, these cells are important for innate immunity (Appelberg, 2007). Additionally, neutrophils function to secrete chemokines which are able to recruit macrophages and dendritic cells to the site of infection (Silva, 2010).

Basophils, another type of granulocyte, are found at a relatively low abundance in the blood $(<1 \%)$ (Falcone, Haas, \& Gibbs, 2000). Although the low number of these cells has made it hard for them to be studied, basophils have been identified as important responders to a range infections including parasitic and bacterial infections (Karasuyama, Obata, Wada, Tsujimura, \& Mukai, 2011). Furthermore, basophils are thought to have a role in driving $\mathrm{T}$ cell immune responses associated with parasite infection (Min et al., 2004). Eosinophils are also granulocytic and have a role in immune responses to parasites by releasing inflammatory cytokines and cytotoxic granules and in addition, it has been shown that eosinophils play a pathogenic role in allergic disease (Shamri, Xenakis, \& Spencer, 2011).

Mast cells are granulocytes found within tissues and known to be the main effector cell in $\mathrm{T}$ helper type 2 (Th2) and IgE-mediated hypersensitivity response. Upon stimulation by PAMP, antibody 
cross-linking or $\mathrm{C} 3$ binding the complement receptor, mast cells degranulate and release mediators such as histamine and other chemokines, cytokines and proteases (Mekori \& Metcalfe, 2000). Mast cells can also enhance immune responses through the release of factors such as histamine which increases local vascular permeability and therefore enhances the recruitment of other innate and adaptive immune cells (Abraham \& St. John, 2010).

The natural killer (NK) cells are a unique cell type which has overlapping functions with $\mathrm{T}$ lymphocytes; however, their involvement in the early immune defence defines them as part of the innate immune system. The classical NK cells do not express the T cell receptor (TCR) and can mediate perforin-dependent cell lysis and activate other innate cells by secreting cytokines such as IFN- $\gamma$ and GM-CSF. NK cells function to control growth of tumours as well as limit microbial infections, indicating these cells can limit or exacerbate immune responses depending on the immune environment (Vivier, Tomasello, Baratin, Walzer, \& Ugolini, 2008).

NKT cells are a unique population of T cells that share NK characteristics and express the TCR, and are found at high frequencies in unchallenged livers (Biron, Nguyen, Pien, Cousens, \& Salazar-Mather, 1999). NKT cells express semi-invariant TCR which recognises CD1d expressing antigen-presenting cells (APCs) or $\alpha$-galactosylceramide and, upon stimulation, NKT cells are able to induce innate-like antimicrobial functions as well as secrete cytokines typical of $\mathrm{T}$ cell responses (Bendelac, Savage, \& Teyton, 2007). It has been shown that NKT cells are capable of rapidly producing high levels of immunoregulatory cytokines such as IL-4, IFN- $\gamma$ and TNF and therefore these cells have an extremely diverse range of actions including inducing autoimmunity as well as driving tumour rejection (Godfrey, Hammond, Poulton, Smyth, \& Baxter, 2000).

Monocytes and macrophages, which reside within the blood and other tissues respectively, are cells derived from bone marrow precursors of the myeloid lineage. These cells have two main functions, firstly they are involved in the innate immune response and secondly they also have a role in maintaining homeostasis by clearing debris. When macrophages are activated they are able to directly kill pathogens by phagocytosis and secrete inflammatory cytokines such as TNF and IL-12. Dendritic cells (DCs) also express PRR and like macrophages are able to phagocytose pathogens and secrete cytokines (Parihar, Eubank, \& Doseff, 2010). Furthermore, macrophages and dendritic cells share a key function of being able to present pathogen derived antigens to the cells of the adaptive immune system, and therefore are able to recruit the adaptive immune response (R. M. Steinman \& Hemmi, 2006). 


\subsection{Adaptive immune response}

The adaptive immune system is made up of $\mathrm{T}$ and $\mathrm{B}$ cells, and while this immune response is much slower than the innate immune response, it can specifically target invading pathogens. B or $\mathrm{T}$ cells which specifically recognise an antigen undergo rapid proliferation resulting in identical progeny which amplifies the immunological response; this response is known as the clonal selection theory. In addition, the adaptive immune response develops immunological memory, therefore when reencountering a particular pathogen the resulting response is faster and stronger than that seen in the initial infection with that pathogen. The adaptive immune responses can be divided into humoral and cell-mediated immunity, which are characterised by $\mathrm{B}$ and $\mathrm{T}$ cell involvement respectively.

\subsubsection{B cells}

B cells are bone marrow-derived cells which perform many different functions in the immune system however, they are mostly known for their role in the production of antibodies. Other functions of B cells include antigen presentation, production of cytokines and regulation of immune responses (Harris et al., 2000; Mauri \& Bosma, 2012). Newly formed B cells produce large amounts of the B cell receptor (BCR) which are inserted to the plasma membrane, and upon activation of the B cell they are secreted as antibodies. Each B cell produces one type of BCR which is specific for a particular antigen; although, within an individual there is a vast array of B cell receptors as a result of gene rearrangement and this allows the B cell population to recognise approximately $5 \times 10^{13}$ different antigens (Murphy, Travers, Walport, \& Janeway, 2012). Antigen engagement occurs via the BCR, which recognises either soluble or membrane-associated antigen, which allows naïve B cells to become activated and proliferate. Following antigen recognition, B cells are able to process and present antigen on MHCII which results in recruitment of specific CD4+ $\mathrm{T}$ cells that can aid in B cell proliferation and differentiation (Batista \& Harwood, 2009). Activated B cells can undergo somatic hypermutation and antibody class switching, together these processes increase the affinity for a particular antigen and thus produces a stronger immune response (Li, Woo, Iglesias-Ussel, Ronai, \& Scharff, 2004). In addition, the majority of B cells form plasma B cells, which produce large quantities of antibody but are short-lived, while a small amount become memory B cells (McHeyzer-Williams \& McHeyzer-Williams, 2005; Murphy et al., 2012). B cell-produced antibodies function in a number of ways including pathogen neutralisation, opsonisation or activation of the complement system and disruption of these cells can lead to a range of conditions including autoimmunity, allergy and reduced clearance of infection (Murphy et al., 2012). 


\subsubsection{Antigen presenting cells}

Unlike B cells which require BCR recognition of antigen to become activated and mount an immune response, $\mathrm{T}$ cells can only respond to antigen which is presented by antigen-presenting cells (APC). Therefore, APCs play an important role in the initiation of $\mathrm{T}$ cell responses although the APC involved may be a part of the innate (DC, macrophage), adaptive immune system (B cell), or a non-immune cell as well. In APCs, the antigen is presented on major histocompatibility complex (MHC) II molecules. There are 2 types of MHC; MHC class I (MHCI), which is expressed by all cell types, and MHC class II (MHCII), which expression is limited to professional APCs such DCs, macrophages and B cells (Murphy et al., 2012). Antigen presentation occurs through two main mechanisms, either endogenous antigens via MHCI molecules or exogenous via MHCII molecules. With MHCI, endogenous antigens are derived from either the host cells or intracellular pathogens (i.e viral proteins) and these antigens get digested into peptides in the cells proteasome after which they are processed and become expressed on MHCI molecules on the surface of cells (Vyas, Van der Veen, \& Ploegh, 2008). In contrast, exogenous antigens are derived from pathogens or debris which has been phagocytosed and digested in the lysosome, followed by presentation on MHCII molecules which get transported to the surface of APCs (Vyas et al., 2008). Interestingly these two pathways are not always distinct, and cross-presentation can occur where extracellular antigens are expressed on MHCI molecules (H. Shen et al., 2006).

\subsubsection{T cells}

$\mathrm{T}$ cells are produced in the bone marrow but are "educated" in the thymus where $\mathrm{T}$ cells which recognise self MHC are selected for as well as removal of self-reacting cells (Murphy et al., 2012). $\mathrm{T}$ cells are a key component in the cell-mediated arm of the adaptive immune response. There are two different lineages of T cells based on the type of TCR they express. The $\alpha \beta \mathrm{T}$ cells constitute the majority of the T cell population, while $\gamma \delta \mathrm{T}$ cells are only a small subset of $\mathrm{T}$ cells which have both innate and adaptive characteristics, and are mainly found within epithelial and mucosal tissues (Carding \& Egan, 2002). The $\alpha \beta$ T cells can be further divided based on the expression of the CD4 or CD8 co-receptor which recognise antigens presented on MHCII and MHCI respectively (Murphy et al., 2012). Similar the B cell receptor, the T cell receptor is generated by gene rearrangement resulting in $10 \times 10^{18}$ possible TCR rearrangements which recognise antigen presented by MHC molecules (Murphy et al., 2012).

\subsubsection{CD8 cytotoxic $T$ cells}

CD8+ $\mathrm{T}$ cells express the co-receptor (i.e. a surface receptor acting with other receptors to modulate cellular activation) CD8, a receptor which is also expressed on the surface of some innate cells, and like the TCR is able to bind MHC molecules but is specific to MHCI. CD8 + T 
cells are able to differentiate into cytotoxic lymphocytes (CTLs) upon activation (Gibbings \& Befus, 2009). CD8 T cells recognise endogenous antigens expressed on MHCI molecules on the surface of many cell types, however it has also been noted that cross presentation occasionally occurs where exogenous proteins are expressed on MHCI and therefore can be recognised by CD8 T cells (H. Shen et al., 2006). The main function of activated CD8 T cells is killing abnormal or virus infected cells by the release of cytotoxic factors (Murphy et al., 2012). CTLs mediate their effects through several pathways including the release of perforin and granzyme and induction of apoptosis through the activation of the FAS-FASL pathway (Barry \& Bleackley, 2002; ChavezGalan, Arenas-Del Angel, Zenteno, Chavez, \& Lascurain, 2009). Perforin is stored in secretory granules inside CTLs as well as NK cells, and upon activation these granules are released allowing pores to form on the surface of target cells, which allows serine proteases (granzymes) to enter the target cell and induce cell death possibly through the activation of the caspase pathway (Voskoboinik \& Trapani, 2006). Additionally, CTLs can mediate apoptosis indirectly through the secretion of cytokines such as TNF and INF- $\gamma$, which can activate apoptosis or necrosis in the target cell by promoting a burst of reactive oxygen species (Chavez-Galan et al., 2009; Harty, Tvinnereim, \& White, 2000). Given that CTLs have mechanisms which can induce apoptosis in target cells, it not surprising that these cells play a key role in suppressing viral infections.

\subsubsection{CD4 T helper cells}

Unlike CD8 $\mathrm{T}$ cells, the CD4 $\mathrm{T}$ helper cells function to activate or direct other immune cells to induce an appropriate immune response. The roles of a $\mathrm{CD} 4+\mathrm{T}$ cell include $\mathrm{B}$ cell activation, aiding in cytotoxic $\mathrm{T}$ cell activation and controlling macrophage responses (Murphy et al., 2012). T helper (Th) cells were originally thought to be activated into either Th1 or Th2 subtypes based on the profile expression of cytokines (Mosmann, Cherwinski, Bond, Giedlin, \& Coffman, 1986), however more recently the Th1/Th2 dichotomy has been reviewed and Th subsets have been defined even further into Th1, Th2, Th17 or regulatory T cell (Treg) responses (Nakayamada, Takahashi, Kanno, \& O'Shea, 2012). All of these subsets have been defined by their cytokine profiles, expression of certain transcription factors and their effect on other immune cells.

Th1 cells are capable of producing several cytokines including IL-2 and IFN- $\gamma$. Additionally, these cells are induced through the interaction with MHCII-antigen complexes on APC in conjunction with IL-12 (Hsieh et al., 1993). Th1 cells can be differentiated from other Th subsets by the expression of T-box transcription factor (T-bet), in addition to the production of IFN- $\gamma$ (Szabo et al., 2000). Th1 cells play an important role in defending against intracellular pathogens and are involved in cell-mediated immunity as well as inducing immunoglobulin isotype switching in B cells (Abbas, Murphy, \& Sher, 1996). Furthermore, Th1 cells alter macrophages by increasing 
their antimicrobial effects through IFN- $\gamma$, resulting in increased MHC expression, increased IL-12 production and nitric oxide (NO) production (Schroder, Hertzog, Ravasi, \& Hume, 2004). In addition to the host defence mechanisms, Th1 cells have also been implicated in autoimmune conditions such as MS.

Th2 cells are thought to play a key role in inducing humoral immune responses, although it has been shown that Th1 cells can provide help for antibody production. Th2 cells can be characterised by the expression of GATA-3 and produce cytokines such as IL-4, IL-5, IL-10 and IL-13 (Mosmann \& Sad, 1996; Swain, Weinberg, English, \& Huston, 1990). It has been shown that Th2 cells have important roles in defending against intracellular parasites, whereas inappropriate $\mathrm{Th} 2$ responses to otherwise innocuous antigens are involved in allergy and asthma; and are involved in the humoral immunity by induction of immunoglobulin class switching (Abbas et al., 1996).

A more recent addition to the T helper subset is the Th17 cells which support neutrophil-mediated inflammation (Z. Zhang et al., 2009) and have roles in protecting against extracellular bacteria and fungi. Th17 cells are induced by IL-6 and TGF- $\beta$, in conjunction with IL-23 which has a role in stabilising Th17 polarity (Bettelli, Korn, Oukka, \& Kuchroo, 2008). These Th17 cells primarily secrete IL-17, IL-21 and IL-22 cytokines (Curtis \& Way, 2009) and can be identified by the expression of ROR $\gamma \mathrm{t}$ (Ivanov, Zhou, \& Littman, 2007).

Th subsets are continually being redefined into further subtypes, other than the three types mentioned above. More recently the introduction of IL-9 producing Th9 cells and IL-22 secreting Th22 cells are being identified as unique subtypes (Schmitt, Klein, \& Bopp, 2014; N. Zhang, Pan, \& Ye, 2011). However, dividing CD4 T cells into subsets needs to be reviewed given there is evidence to suggest that these cells may have some plasticity and therefore functions between each cell type may not be discrete (Bluestone, Mackay, O'Shea, \& Stockinger, 2009)

\subsubsection{Regulatory $\mathbf{T}$ cells}

In contrast to the other CD4 T cells mentioned, Tregs are thought to regulate the immune response and maintain immune tolerance (Murphy et al., 2012). These cells are identifiable by their high expression of CD25, the IL-2 receptor. However, CD25 is also upregulated on activated CD4 T cells from other subtypes and therefore Tregs are further identified by the expression of the transcription factor Forkhead box P3 (FoxP3) (Banham, Powrie, \& Suri-Payer, 2006). FoxP3 is highly expressed by Tregs and is also thought to be a master regulator for the Treg lineage both within the thymus and periphery (Hori, Nomura, \& Sakaguchi, 2003). There are a number of different Treg types identified, however in vivo there are two main types of Tregs; natural Tregs which differentiate in the thymus and inducible Tregs which are induced in the periphery (i.e. 
outside of the thymus) (Campbell \& Koch, 2011). There has been evidence to suggest that induction of iTregs periphery rely on TGF- $\beta$, whereas nTregs in the thymus rely on TNF to become suppressive (Chen et al., 2003; Housley et al., 2011).

Tregs suppress $\mathrm{T}$ cell responses through FoxP3-mediated effects such as the secretion of regulatory cytokines including IL-10 (Sakaguchi, Wing, Onishi, Prieto-Martin, \& Yamaguchi, 2009). Additionally, $\mathrm{T}$ cell responses can be suppressed through cell-cell contact mechanisms including CTLA-4 expression which competes for CD28 co-stimulation on T cells, as well as Tregs sequestering IL-2, as this cytokine functions to promote $\mathrm{T}$ cell proliferation (Wing \& Sakaguchi, 2012). Importantly, it is the balance between regulatory and pathogenic Th subsets which determines whether immune tolerance or autoimmune inflammation occurs.

\section{$1.4 \mathrm{~T}$ cell activation}

TCR recognition of antigen presented on MHC molecules on the surface of APCs is not enough to constitute activation of naïve $\mathrm{T}$ cells. In addition, these cells require the stimulation of costimulatory pathways and soluble factors (i.e. cytokines) to become completely activated and drive T cell differentiation (Murphy et al., 2012). Each naïve T cell carries one type of TCR which is specific for a particular antigen, due to gene recombination in the thymus there are $10 \times 10^{18}$ possible TCR rearrangements (Murphy et al., 2012). MHC presented antigen is recognised by the variable regions of the TCR $\alpha \beta$ chains, a necessary process for antigen recognition (Murphy et al., 2012). In conjunction with TCR stimulation, T cells also express co-receptors, either CD4 or CD8, which recognise conserved regions of MHCII or MHCI respectively. While the CD4 and CD8 coreceptors contribute to antigen recognition by stabilising MHC:T cell binding, signalling through CD3, which is co-expressed with TCR $\alpha \beta$ chains, is required for T cell activation (Smith-Garvin, Koretzky, \& Jordan, 2009).

To successfully activate naïve T cells co-stimulation is also required in addition to TCR activation. There are several different types of co-stimulation, most of which are activating although some are negative regulators of $\mathrm{T}$ cell activation (Chambers \& Allison, 1997). In the absence of costimulation, TCR activation on naïve T cells can result in anergy (a state in which T cells become functionally inactivated) and lack of $\mathrm{T}$ cell responsiveness (Saibil, Deenick, \& Ohashi, 2007). The most well-known co-stimulatory molecules are CD80 and CD86 (previously referred to as B7.1 and B7.2 in the literature) expressed on the surface of APCs which interact with CD28 or cytotoxic lymphocyte associated protein-4 (CTLA-4, also referred to as CD152) on the surface of naïve T cells (Krummel \& Allison, 1995). Although the functional difference between CD80 and CD86 is unclear, it appears that these cells may have differential expression on the surface of APCs and T 
cells (Chambers \& Allison, 1997) and that binding of both CD80 and CD86 to CD28 results in T cell activation (Krummel \& Allison, 1995). In contrast, when CD80 and CD86 binds CTLA-4 T cell responses are suppressed; although CD80 and CD86 have a higher affinity for CTLA-4 than CD28, given that CTLA-4 is not constitutively expressed like CD28, the negative regulation of $\mathrm{T}$ cell responses is tightly controlled (Krummel \& Allison, 1995). The role of CTLA-4 expression is therefore to regulate $\mathrm{T}$ cell responses considering that Tregs highly express CTLA-4 (Sakaguchi et al., 2009), and effector T cells also upregulate expression of CTLA-4 as a negative feedback of T cell activation (Jain, Nguyen, Chambers, \& Kang, 2010).

There are a range of co-stimulatory molecules, not just CD80/CD86, which include CD40 and programmed death-ligand 1 (PD-L1) and programmed death-ligand 2 (PD-L2). CD40L (also referred to as CD154) expressed on the surface of T cells interacts with CD40 expressed by APCs and plays important roles in the both the T cell and the APC. As a result of TCR activation, CD40L expression is upregulated; in addition, CD40 binding to CD40L induces APCs to secrete cytokines such as IL-12 and TNF (Cella et al., 1996), which can drive T cell differentiation and upregulate expression of MHC molecules (Suttles \& Stout, 2009).

Programmed death-1 (PD-1) and its ligands PD-L1 and PD-L2 have an important role regulating T cell responses as it has been shown peripheral tolerance can be mediated through the PD-1 pathway (Keir, Francisco, \& Sharpe, 2007). PD-L1 and PD-L2 have very different expression profiles, with PD-L1 being constitutively expressed on a wide range of haematopoietic and nonhaematopoietic cell types, while PD-L2 expression is restricted to APCs, and PD-1 is expressed on T cells, B cells and activated macrophages (Keir et al., 2007). Interactions of PD-1 on T cells with PD-L1 on APCs can in some cases suppress $\mathrm{T}$ cell responses and in some cases $\mathrm{T}$ cell proliferation is enhanced (Keir et al., 2007; Latchman et al., 2004). Furthermore, it has been suggested that PD1/PD-L1 interactions have a role in negative feedback of T cell responses as seen in PD-L1 ${ }^{-/}$mice which develop more severe EAE disease (Carter et al., 2007); additionally, PD-L1 expression may not just suppress $\mathrm{T}$ cell responses directly, but expression of PD-L1 is able to induce Treg formation which may further suppress T cell proliferation (Francisco et al., 2009).

\subsection{Immune tolerance}

The immune system has the ability to respond to a wide range of pathogens due to the random rearrangements of genes which make up the TCR and BCR. However, during rearrangement of these receptors some may recognise self-antigens and therefore to prevent the body of attacking itself, these cells need to be deleted from the immune repertoire. There are several mechanisms by which self-reactive cells are removed and will be broadly divided into central and peripheral 
tolerance. Failure of these tolerance mechanisms can occur, resulting in autoimmune disease (Hanahan, 1998).

\subsubsection{Central tolerance}

Central tolerance occurs in the primary lymphoid organs during development and is the main mechanism by which self-recognising $\mathrm{T}$ cells are eliminated. In the thymus $\mathrm{T}$ cells undergo both positive and negative selection processes. $\mathrm{T}$ cells are positively selected for if they are able to interact with MHC molecules, and T cells which do not recognise MHC are not allowed to survive (Starr, Jameson, \& Hogquist, 2003). Therefore, functional T cells which are able recognise MHC presented antigen are positively selected. Additionally, $\mathrm{T}$ cells undergo negative selection, where $\mathrm{T}$ cells that recognise self-antigen are removed (Hogquist, Baldwin, \& Jameson, 2005). Thymic epithelial cells (TEC) and thymic DCs express many, but not all, self-antigens and T cells which strongly recognise a self-antigen are deleted (Palmer, 2003). T cells which weakly recognise selfantigen are not deleted however, they are induced to express FoxP3 and become a natural Treg (nTreg) (Murphy et al., 2012).

\subsubsection{Peripheral tolerance}

Despite the extensive mechanisms involved in central tolerance, a small number of self-reactive $\mathrm{T}$ cells escape into the periphery where they may become activated against self-proteins resulting in autoimmunity. To counter autoimmunity several mechanisms are in place to prevent inappropriate T cell activation, and these mechanisms are referred to as peripheral tolerance. Peripheral tolerance can occur through $\mathrm{T}$ cell intrinsic pathways such as $\mathrm{T}$ cell anergy, or $\mathrm{T}$ cell extrinsic pathways mediated through other cell populations such as Tregs or myeloid suppressor cells (Mueller, 2010; Walker \& Abbas, 2002).

To successfully activate naïve T cells, TCR stimulation as well as co-stimulation is required. In the absence of a strong co-stimulatory signal, often this is CD28-mediated, T cells undergo anergy and become permanently unresponsive (Appleman \& Boussiotis, 2003). Co-stimulatory molecules expressed on the surface of APCs are upregulated after PRR activation by microbial products (S. Akira, Takeda, \& Kaisho, 2001); therefore, in the absence of pathogen infection, the expression of self-antigens on APCs will lack sufficient co-stimulation to successfully activate T cells. This is likely to be the case for self-reactive $\mathrm{T}$ cells recognising self-antigen presented on APCs under steady-state conditions and therefore the lack of co-stimulation prevents autoimmunity.

Additionally, specific surface receptors are important in maintaining a balance between $\mathrm{T}$ cell activation, tolerance and immune-driven tissue damage. These receptors include, but are not limited to, CTLA-4 and PD-1 which when activated induce inhibitory pathways within T cells 
expressing these ligands (Mueller, 2010). T cell expression of CTLA-4, which has a higher affinity for CD80/CD86 co-stimulatory markers than CD28, induces T cell anergy when ligated (Xing \& Hogquist, 2012). PD-1 expressed on T cells binds to PD-L1 or PD-L2 resulting in suppressed T cell responses. PD-L1 is expressed constitutively across many haematopoietic and nonhaematopoietic cell types, while PD-L2 expression is more conserved and, upon activation the expression of these ligands are upregulated (Keir et al., 2007). Tolerogenic DCs express high levels of PD-L1 and PD-L2 ligands which provide insight into how these cells may suppress T cell activation and induce tolerance (Probst, McCoy, Okazaki, Honjo, \& van den Broek, 2005). Furthermore, there has been evidence to suggest that lack of PD-1 signalling results in an increased susceptibility to autoimmune disease in PD- $1^{-/}$animals (Francisco, Sage, \& Sharpe, 2010). Another mechanism by which PD-1/PD-L1 interaction may add to peripheral tolerance is through the formation of Tregs, as it has been shown that PD-L1-coated beads induce Tregs in vitro and that PD-L1 can sustain and enhance Treg function and FoxP3 expression (Francisco et al., 2009).

There are also particular cell types which have a role in peripheral tolerance. These cells include Tregs, myeloid-derived suppressor cells (MDSC) and some macrophage subsets (Gabrilovich \& Nagaraj, 2009; Munn et al., 1999; Walker \& Abbas, 2002). MDSCs are a heterogeneous population of myeloid progenitor cells which are prevented from fully differentiating (Gabrilovich \& Nagaraj, 2009); and are able to mediate T cell responses by upregulating enzymes involved in L-arginine metabolism, such as iNOS and Arg1 which increase nitric oxide (NO) and depletes arginine respectively (Bronte, Serafini, Mazzoni, Segal, \& Zanovello, 2003). The depletion of local arginine can induce $\mathrm{T}$ cells to undergo apoptosis and is therefore one method of MDSC regulation on T cells. Other mechanisms of MDSC-mediated suppression include production of reactive oxygen species, through cell-cell contacts mediated by receptor-ligand interactions such as PD-1/PD-L1 and by the induction of Tregs (Condamine \& Gabrilovich, 2011; Gabrilovich \& Nagaraj, 2009) suggesting that these cells may also play a role in maintaining peripheral tolerance. Tregs are well known suppressors of autoreactive T cells through FoxP3 mediated effects such as the secretion of regulatory cytokines including IL-10 (Sakaguchi et al., 2009), cell-cell contact mechanisms including CTLA-4 expression, and sequestering IL-2 (Wing \& Sakaguchi, 2012). Macrophages can also be activated into suppressive subtypes characterised by the cytokines and surface markers they express. It has been shown that macrophages can suppress $\mathrm{T}$ cells through the expression of tryptophan-depleting enzyme indoleamine 2,3-dioxygenase (IDO), which suppresses T cell proliferation by depriving tryptophan (Munn et al., 1999) or by down-regulating the expression of co-stimulatory markers (Tierney, Kharkrang, \& La Flamme, 2009). 
In conjunction with the peripheral tolerance mechanisms, there are a number of sites in the body which are thought be less immunologically reactive such as eyes, central nervous system (CNS) and testes (Niederkorn \& Stein-Streilein, 2010). While it was originally thought that these sites were "immune privileged" it now appears that these sites are less reactive due to a number of tolerance mechanisms, similar to those mentioned already (Benhar, London, \& Schwartz, 2012). The cells within these "immune privileged" tissues express certain factors such as inhibitors of macrophage migration and release of suppressive cytokines (Apte, Sinha, Mayhew, Wistow, \& Niederkorn, 1998; D'Orazio \& Niederkorn, 1998). The suppressive nature of cells within these tissues combined with the physical barriers in these tissues results in reduced immune responses within these sites.

In this introduction thus far we have highlighted some of the cells and mechanisms involved in immunological tolerance however if these mechanisms fail, immune cells can become reactive to self. If immune cells become autoreactive this can lead to autoimmune disease such as MS.

\subsubsection{The liver in immune tolerance}

While the balance of $\mathrm{T}$ effector cells and Tregs is important for peripheral immune tolerance, the liver has also been shown to have a role in aiding tolerance. The liver is a unique immunological site where blood rich in gut-derived antigens passes through a network of sinusoids allowing the surveillance of these antigens by liver-resident APCs and lymphocytes. Therefore, the liver has developed a number of tolerance mechanisms to prevent over-stimulation from occurring (Crispe et al., 2006).

The liver contains a number of different innate and adaptive immune cells, with the overall composition being quite different to other secondary lymphoid organs (e.g. spleen). The lymphocytes found within the liver include conventional B and T cell subsets however, unlike the spleen, the liver is a rich source of $\gamma \delta$ T cells and NK cells (Gao, Radaeva, \& Park, 2009; Vito Racanelli \& Barbara Rehermann, 2006). The liver also contains a number of resident APCs, three of which are thought to play a crucial role in maintaining tolerance. These cells include Kupffer cells which are liver resident macrophages and make up the largest group of macrophages within the body (V. Racanelli \& B. Rehermann, 2006). Kupffer cells are located within the sinusoidal vascular space where they function to clear endotoxins from the blood and phagocytose passing debris and microorganisms (V. Racanelli \& B. Rehermann, 2006). Other APCs are the liver sinusoidal endothelial cells (LSEC) and resident hepatic DCs.

The APCs within the liver favour immune tolerance through several different mechanisms. Liver DCs have reduced expression of co-stimulatory receptors, compared to other organs, and also 
preferentially express IL-10 over Th1 driving cytokines (Tiegs \& Lohse, 2010). Given that liver DCs lack strong co-stimulation and secrete IL-10, it is not surprising that these cells can induce the formation of Tregs and overall result in T cell hypo-responsiveness (Bamboat et al., 2009). Other mechanisms involved in liver-induced immune tolerance include reduced production of proinflammatory cytokines, high levels of PD-L1 expression and depletion of essential amino acids with arginase and IDO (Thomson \& Knolle, 2010; Tiegs \& Lohse, 2010).

Given that the liver favours immune tolerance over immune activation, it is not surprising that treating with orally ingested antigens has been tested as a therapeutic option for several diseases, especially considering that oral treatments are an attractive option clinically. Several animal models of autoimmune disease and inflammation have found orally dosing with disease peptides results in increased Tregs and reduced disease severity (Howard L. Weiner, da Cunha, Quintana, $\& \mathrm{Wu}, 2011)$. Additionally, one study has suggested that oral tolerance can be induced in an antigen non-specific manner using a monoclonal antibody to CD3 (anti-CD3), by showing that anti-CD3 can induce CD4+CD25-LAP+ Tregs which functionally suppressed T cell responses and reduced autoimmune disease (Ochi et al., 2006).

\subsection{Macrophage activation}

Macrophages are thought to be a key population in directing the adaptive immune response towards pro-inflammatory or anti-inflammatory pathways depending on the expression of particular cytokines and activation markers. These cells can be found throughout the body, in the blood they are monocytes, microglia in the brain and Kupffer cells within the liver. While previous literature has split macrophages into 2 different subsets reflecting Th nomenclature, proinflammatory (M1) and alternatively activated (M2), more recently it has been identified that macrophages show plasticity in their activation state and activation is now considered on a spectrum (Mosser \& Edwards, 2008). Considering the confusing nomenclature, the general introduction in this thesis will outline three broadly unique macrophage populations which are classified as classically activated macrophages, alternatively activated macrophages and type IIactivated macrophages, with the understanding that macrophages may not always easily fit into a defined group.

Classically activated macrophages are the most well-defined within the literature; these cells are thought to be activated by microbial products such as LPS (lipopolysaccharide) and direct CD4+ T cell responses to a Th1 phenotype. Classically activated macrophages are thought to require two signals for activation; IFN- $\gamma$, which primes macrophages for activation, in addition to TNFsignalling which can either be endogenous e.g. via TLR activation or exogenous TNF (Mosser, 
2003). These macrophages express high levels of co-stimulatory molecules and produce IL-12 which together favour Th1 activated CD4+ T cells (Desmedt, Rottiers, Dooms, Fiers, \& Grooten, 1998). Additionally, classically activated macrophages have an increased ability to kill intracellular pathogens as they produce high levels of NO and reactive oxygen species (Nathan \& Shiloh, 2000).

Alternatively activated macrophages are considered to be activated by IL-4 or IL-13 and are involved in allergy and Th2-type responses (Gordon, 2003). Alternatively activated macrophages upregulate expression of arginase and therefore produce very little NO in comparison to classically activated macrophages (Rutschman et al., 2001), which makes this subtype of macrophages inefficient in killing intracellular pathogens. These cells can also be characterised by reduced $\mathrm{Ag}$ presentation and produce higher levels of IL-10 than classically activated macrophages (Mosser, 2003). Unlike classically activated macrophages, alternatively activated macrophages may inhibit $\mathrm{T}$ cell proliferation and have reduced potential to differentiate Th cells.

More recently type II-activated macrophages have been recognised as a unique sub-population that is considered to be immunoregulatory. Like classically activated macrophages, type-II activated macrophage are thought to be induced by two signals; the ligation of the Fc $\gamma \mathrm{R}$ (the receptors for the Fc portion of immunoglobulin $\mathrm{G}$ which are expressed on a number of hematopoietic cells including macrophages) in addition to TLR-stimulation or CD40/CD44-stimulation drives the type-II phenotype (Anderson \& Mosser, 2002). Type-II activated macrophages are able to drive the production of Th2 cells (Anderson \& Mosser, 2002). The cytokine profile of type-II activated macrophages include low levels of IL-12 and high levels of IL-10; interestingly, these cells retain the ability to produce cytokines which are thought to characterise the classically activate macrophage subtype (Mosser, 2003).

Given that macrophages can be activated into a number of different subtypes, some of which are regulatory or suppress $\mathrm{T}$ cell responses, it is reasonable that modulation of macrophages is becoming a therapeutic target to treat a range of diseases. An example of this therapeutic approach is the induction of type-II macrophages in an animal model for MS, which reduced disease severity (Tierney et al., 2009). Additionally, glatiramer acetate (GA) also alters the activation state of monocytes in MS patients by promoting anti-inflammatory type II monocytes which are characterised by increased secretion of TGF- $\beta$ and IL-10 and decreased production of IL-12 (Rina Aharoni, 2014). 


\subsection{Multiple sclerosis}

MS is an autoimmune disease characterised by inflammation of the central nervous system (CNS). The usual age of disease onset is between 20-40 years of age, however MS can also be diagnosed within the first decade of life (Banwell, Ghezzi, Bar-Or, Mikaeloff, \& Tardieu, 2007) and it appears that women are more susceptible than men with a prevalence ratio of 2.5:1 (women to men) amongst relapsing-remitting patients (Keegan \& Noseworthy, 2002; Willer, Dyment, Risch, Sadovnick, \& Ebers, 2003). In the vast majority of patients (85\%), MS presents in a relapsing remitting form in which periods of exacerbation of symptoms are followed by a period of reduced symptoms (Greenstein, 2007), and these patients often go on to develop secondary progressive MS within 5-10 years of onset, whereas $10-15 \%$ of patients initially present with a primary progressive form of the disease.

MS disease is thought to be caused by an activation of self-reactive lymphocytes which enter the CNS and attack the myelin sheath which protects nerve axons, causing inflammatory lesions (Vinay Kumar \& Stanley Leonard Robbins, 2007). The symptoms of MS vary depending on which part of the brain is affected by these lesions and may include progressive paralysis, weakness in the limbs and visual disturbances (Vinay Kumar \& Stanley L. Robbins, 2007). There is much heterogeneity between patients in both the disease presentation and pathology as particular disease symptoms can be chronic or transient and range from mild to severe (Lucchinetti et al., 2000). The diversity in disease symptoms, clinical progression and the immune responses that underlie these, adds to the complexity of both diagnosing and treating MS.

\subsubsection{Disease aetiology}

The cause of MS is not completely understood however several risk factors have been identified for MS including genetics, smoking, infectious agents and geographical location (O'Gorman, Lucas, \& Taylor, 2012). The human leukocyte antigen (HLA; i.e. the human MHC molecule), has consistently been implicated as a risk factor for development of MS. It has been shown that the DRB1*1501 HLA allele and other associated alleles have a strong link to MS susceptibility in both European and non-European populations (Sawcer et al., 2011; Schmidt, Williamson, \& Ashley-Koch, 2007). Additionally, it has been determined that several genes outside of the HLADR loci have also been identified as risk factors for MS including cytokines and receptors, such as IL2RA and IL7RA (Hafler et al., 2007), as well as co-stimulatory molecules (De Jager et al., 2009). Studies on familial aggregation and twin pairs have determined that there is an increased risk to developing MS with increased relatedness to a family member with the disease (Dyment, Ebers, \& Dessa Sadovnick, 2004; O'Gorman et al., 2012). It was also found in adoptive studies that children raised from infancy in a family with a parent who has MS, are no more likely to 
develop MS than someone in the general population (Dyment et al., 2004) indicating that familial risk is genetic and not due to shared family environment. In saying that, the risk of MS for offspring of one parent with MS is $2.49 \%$ and this is increased to $30.5 \%$ when both parents have the disease (Dyment et al., 2004), therefore indicating that risk factors other than genetics play a strong role in the aetiology of MS.

Microorganisms have been identified as possible triggers for the development of MS disease, however the mechanisms behind these associations have not yet been shown (Gilden, 2005). There have been associations between bacterium such as Chlamydia pneumoniae and several viruses including human herpes virus 6, Epstein-Barr virus (EBV) and John Cunningham (JC) virus (Gilden, 2005). The most compelling research on the link between MS and viruses has focused on EBV and, in particular, its clinical presentation infectious mononucleosis. It was identified in a meta-analysis that infectious mononucleosis in adolescence or young adulthood was associated with a 2.3-fold risk in developing MS (Thacker, Mirzaei, \& Ascherio, 2006). Although this link appeared to be quite striking, it should be noted that EBV is thought to infect over $90 \%$ of the global population and within these infections $40 \%$ occur during adolescence or adults (A. Ascherio \& Munger, 2010; Macsween \& Crawford, 2003). Given that EBV is so wide spread it is likely that this virus is only one contributing risk factor among many.

A strong correlation has been observed between latitude and MS, with increasing risk of developing MS at the higher latitudes. This correlation was highlighted in a meta-analysis including 321 studies which concluded that there was a significant positive association linking MS prevalence and latitude; however, part of Italy and northern Scandinavia were an exception to this rule (Simpson, Blizzard, Otahal, Van der Mei, \& Taylor, 2011). Interestingly, the latitude association has also been found in New Zealand with an increasing MS prevalence from North $\left(35^{\circ} \mathrm{S}\right)$ to South $\left(48^{\circ} \mathrm{S}\right)$ of three-fold (Taylor et al., 2010). The link between latitude and MS prevalence is proposed to be associated with number of sunlight hours which may mediate its effects through vitamin D levels. It has been shown that MS patients living further away from the equator (i.e Nordic countries) have vitamin D insufficiency and furthermore, vitamin D levels can influence the rate of relapse and lesions within MS patients (Pierrot-Deseilligny \& Souberbielle, 2013). While it appears that the majority of vitamin D is synthesised from UVB radiation in sunlight, some foods can also provide a rich source and populations which consume large amounts of vitamin D rich foods could account for the lower than expected MS prevalence predicted by latitude (Alberto Ascherio, Munger, \& Simon, 2010). In addition to vitamin D, UVB radiation may be protective against autoimmunity through the induction of regulatory cells and secreted immunoregulatory factors such as IL-10 (Marsh-Wakefield \& Byrne, 2015). 
The number of factors which may contribute to MS disease highlight that it is likely no single set of factors causing the disease, and the factors involved may be different from individual to individual. Given this, MS is likely to be a difficult disease to prevent. However, understanding the immunology behind the disease will aid the development of therapeutic treatments.

\subsubsection{Immunology of MS}

MS is a disease mediated by the immune system, a hypothesis which is supported by several findings such as the efficacy of immunomodulatory and immune suppressing treatments. Disease is driven by $\mathrm{CD} 4+\mathrm{T}$ cells which specifically recognise peptides from the myelin protein such as myelin basic protein (MBP), myelin oligodendrocyte glycoprotein (MOG) and proteolipid protein (PLP) (L. Steinman, 1996). Interestingly, it has been found that T cells specific for these peptides are present in healthy individuals which indicates that the presence of autoreactive $\mathrm{T}$ cells is not sufficient to drive MS disease. The myelin-specific CD4+ T cells from MS patients are present at a higher number and are more reactive than in healthy individuals suggesting that in MS patients these cells are triggered to become activated, after which the cells undergo clonal expansion (Pender, Csurhes, Houghten, McCombe, \& Good, 1996; Pette et al., 1990). Although CD4+ T cells appear to be a key cell type activated during the early stages of disease, there are many different cell types involved in the pathogenesis of disease including macrophages, B cells and CD8+ T cells.

The initial event which triggers the activation of CD4+ T cells is still unknown, and it is likely that the factors involve vary from individual to individual. Bacterial and viral infections, such as EBV, have been proposed as an initial step in activating CD4+ T cells through molecular mimicry which occurs when the antigens from the invading pathogen resembles self-peptides (Libbey, McCoy, \& Fujinami, 2007); and therefore $\mathrm{T}$ cells which become activated towards the pathogen antigen are cross-reactive to self, resulting in autoimmune disease. Molecular mimicry could explain the variability in MS disease as various viruses may resemble different myelin peptides resulting in different disease presentations, an idea which is supported by several viruses being linked to the cause of MS (O'Gorman et al., 2012). Another theory is that the immune environment becomes pro-inflammatory, resulting in the bystander activation of autoreactive CD4+ T cells (Fujinami, von Herrath, Christen, \& Whitton, 2006). This could potentially occur through the upregulation of co-stimulatory molecules, cytokine production or other pro-inflammatory molecules which reflect the loss of peripheral tolerance (Sospedra \& Martin, 2005). Although bystander activation and molecular mimicry are attractive options for MS-triggers, neither has been proven and therefore the initial step remains elusive. 
After the activation of autoreactive CD4+ $\mathrm{T}$ cells these cells must migrate into the CNS to elicit disease, this is known as the "outside-in' theory of MS disease and is the most commonly accepted theory for MS disease progression (Peterson \& Fujinami, 2007). In MS patients the blood brain barrier (BBB) becomes more cell-permeable and this breakdown is evident in magnetic resonance imaging (MRI) scans (Bennett et al., 2010; Carson, Doose, Melchior, Schmid, \& Ploix, 2006). Although, it is unclear whether the BBB breakdown occurs before or after disease symptoms begin, it is clear that the movement of cells into the CNS is key for disease pathogenesis as blocking of these cells with natalizumab (see 1.7.3) reduces symptoms in relapsing-remitting MS patients (D. H. Miller et al., 2003). Once self-reacting CD4+ T cells are in the CNS they direct the damage of the myelin sheath, resulting in inflammatory lesions. This process also involves the recruitment of other cells both inside the CNS, such as microglia and astrocytes and also peripheral cells such as macrophages and CD8+ T cells (Friese \& Fugger, 2009; Sospedra \& Martin, 2005). After the initial CNS infiltration occurs the inflammation can begin to subside with time. This gives the CNS a chance to regenerate depending on the severity of lesions, with scars and irreversible axonal damage occurring in some patients (Frohman, Racke, \& Raine, 2006). The level of neuronal damage is reflected in disease severity, and patients who have resolution of lesions are likely to have a "remission" of symptoms as seen in relapsing-remitting MS disease.

There are obvious limitations for assessing the immune infiltrates into the CNS of MS patients, therefore the majority of knowledge gained thus far has been from post-mortem studies and animal models of MS (see 1.9).

\subsubsection{Current treatments}

MS disease is degenerative, and there is currently no cure; however, there are some drug therapies that may help patients manage the disease. These include treatment of the symptoms only, immunosuppressive therapies which dampen down the immune response and more recently disease modifying treatments have been developed. These disease modifiers can be broken down into two types: inhibitors of migration, such as natalizumab (Clifford et al., 2010) which blocks the entry of self-reactive lymphocytes in the CNS, and immune modifiers such as the cytokine interferon $\beta$ (IFN- $\beta$ ) and GA both of which alter the immune response in a way that suppresses autoimmune disease (Yong, 2002). Although there are a number of ways to treat MS, two of the frontline immune modifying treatments (i.e. IFN- $\beta$ and GA) for MS have a low efficacy with variable results among the literature suggesting that treatment results in only a $30 \%$ reduction in symptoms in relapsing and remitting MS patients (O. A. Khan et al., 2001). Some therapies have a higher efficacy however their use is limited by the potential for severe side effects, such as 
progressive multifocal leukoencephalopathy which is associated with the use of the inhibitor natalizumab (Clifford et al., 2010).

One of the first disease modifying MS drugs introduced to the market was Betaferon ${ }^{\circledR}$ in 1993, a recombinant interferon- $\beta$ cytokine. Recombinant IFN- $\beta$ is still on the market today, given as Avonex ${ }^{\circledR}$ and Rebif ${ }^{\circledR}$. IFN- $\beta$ has been shown to have immunomodulatory properties including promoting cell differentiation, antiviral and anti-proliferative effects; and although the full mechanism is not completely understood it is widely agreed that the major effects are reliant on the activation of the IFN transmembrane receptor leading to the up- or down-regulation of target genes (Yong, 2002). A study by Schrenier et al showed that IFN- $\beta$ treatment enhances monocyte and dendritic cell expression of PD-L1, an inhibitor of T cell activation, and when PD-L1 expression was neutralised the suppression of $\mathrm{T}$ cell proliferation and activation by IFN- $\beta$-treated APCs was relieved (Schreiner et al., 2004). IFN- $\beta$ treatment exerts its actions through a range of other mechanisms including inhibiting CNS infiltration by stabilising the BBB, T cell apoptosis, cytokine modulation and induction of Tregs (Dhib-Jalbut \& Marks, 2010). Administration of IFN$\beta$ is by intramuscular or subcutaneous injection 1-3 times per week and is well tolerated although some patients report flu-like symptoms (Limmroth, Putzki, \& Kachuck, 2011), which is to be expected with immunostimulatory agents. Unfortunately, the efficacy of IFN- $\beta$ diminishes over time as the body develops antibodies to the recombinant protein which renders it inactive (Bertolotto et al., 2002).

GA, clinically referred to as Copaxone ${ }^{\circledR}$, is another effective example of an immune modulator altering the immune environment in a way that reduces MS disease, particularly in patients with relapsing-remitting MS (Ge et al., 2000). GA is given subcutaneously every day in some patients and as little as three times weekly is effective at reducing relapsing-remitting MS, with adverse effects being limited to the injection site (O. Khan, Rieckmann, Boyko, Selmaj, \& Zivadinov, 2013). Interestingly, as GA is a mixture of polypeptides made up of four amino acids resembling myelin basic protein (MBP), which is thought to be one of the key proteins auto-reactive T cells recognise in MS, its mechanism is likely to occur in an antigen-specific manner unlike IFN- $\beta$ (Yong, 2002). There are a number of mechanisms by which GA may exert its effects which include the formation of $\mathrm{T}$ cell tolerance to MBP, competition between GA and the natural MBP proteins for binding to MHC molecules and therefore displacing MBP antigen presentation, and potentially the formation of GA dependent Th2-like regulatory cells which suppress normal $\mathrm{T}$ cell activation (Neuhaus, Farina, Wekerle, \& Hohlfeld, 2001). Although anti-GA antibodies do not have an impact on treatment efficacy, GA treatment has relatively low efficacy with only a $30 \%$ reduction in symptoms and many patients do not respond to treatment (Boster, Bartoszek, 
O’Connell, Pitt, \& Racke, 2011; O. A. Khan et al., 2001), creating a need for more effective treatments to be developed and approved.

Natalizumab, clinically referred to as Tysabri ${ }^{\circledR}$, is an antibody which selectively blocks $T$ lymphocytes from entering the CNS by binding to their $\alpha 4 \beta 1$-integrin proteins and preventing lymphocyte-vascular endothelium interaction. It is currently approved as an effective treatment for relapsing-remitting MS patients (D. H. Miller et al., 2003), and is administered intravenously (i.v.) monthly with side effects including fatigue, limb swelling and nausea (Pucci et al., 2011). However, while the early trials showed natalizumab was safe, it wasn't until larger cohorts of patients were treated that it was found that a small number $(0.01 \%)$ of treated patients developed a serious and often fatal CNS disease called progressive multifocal leukoencephalopathy (PML). It is believed that natalizumab blocks the lymphocyte immune surveillance in the CNS and therefore in MS patients who carry the John Cunningham (JC) virus, the virus reactivates and causes PML (Ransohoff, 2005). Despite the finding of PML in patients treated with natalizumab, it has been reapproved with strict monitoring guidelines, pre-treatment testing for the JC virus and patient awareness of the risk of developing PML (Yousry et al., 2006).

Fingolimod, clinically marketed as Gilenya ${ }^{\circledR}$, modulates the function of S1P receptor and is important for regulating the egress of lymphocytes from the lymph nodes into the circulation (Chun \& Hartung, 2010). Fingolimod has shown efficacy in relapsing-remitting MS when given daily doses orally, and this efficacy was found to be above what was found with IFN- $\beta$ therapy (Cohen et al., 2010). Patients treated with fingolimod have a significant reduction in circulating lymphocytes (60-80\% reduction) which is due to the inhibition of the S1P gradient which allows CD4+ T cells from egressing from the lymph node and treatment may also drive lymphocytes back into the lymph nodes, resulting in a reduction of CD4+ T cells able to enter the CNS and cause MS disease (Antel, 2014; Ingwersen et al., 2012). There is potential for fingolimod to have CNSspecific effects given that it can cross the BBB and may activate gliosis and endogenous repair mechanisms (Chun \& Hartung, 2010). Adverse effects with fingolimod included common cold, mild headaches, fatigue and gastrointestinal dysfunction which are similar to effects seen with other immune mediators such as IFN- $\beta$ (Ingwersen et al., 2012). However, in early 2015 there was a reported case of PML in an individual who received Gilenya (fingolimod) for more than 4 years for the treatment of relapsing-remitting MS, and had not previously received natalizumab treatment, suggesting there is some risk for PML in patients receiving fingolimod.

Current treatments for MS are aimed at altering the disease progression and none of the treatments are able to cure the disease. Many patients who begin taking immune modifying treatments for MS will remain on such drugs for the rest of their lives and, given that there is limited efficacy, the 
cost effectiveness of current treatments is very low (Noyes et al., 2011). In addition, current treatments are ineffective in patients with progressive forms of MS; therefore, there is a need for more effective MS therapeutics with good safety profiles to be developed.

\subsection{MIS416}

MIS416 is a novel microparticle derived from Propionibacterium acnes comprising a minimal cell wall skeleton that is rich in immunostimulatory muramyl dipeptide (MDP) cross-linked with bacterial single stranded DNA, which together signal through cytosolic receptors NOD2 and TLR9, respectively (Girvan et al., 2011). The MIS416 particle is about $0.2 \mu \mathrm{m}$ in size and is therefore thought to target its intracellular receptors in innate phagocytic cells such as monocyte and dendritic cells, as these cells have the ability to take up the particle (Girvan et al., 2011). While MIS416 was originally developed as a vaccine adjuvant, interest has grown around the potential use of MIS416 as a standalone immunomodulatory agent for treatment of inflammatory disorders when administered systemically. Recently, MIS416 was evaluated in a single centre, open-label, non-randomized, dose-escalation study in which the safety and tolerability of i.v. administered MIS416 weekly for 4 weeks in patients with chronic progressive multiple sclerosis. Although this study was not powered to look at disease outcomes, it was suggested that MIS416 was having a positive effect with the majority of patients opting to remain on MIS416 treatment under the compassionate use program (Gill Webster, Innate Immunotherapeutics Limited, Auckland, NZ).

\subsection{Experimental autoimmune encephalomyelitis}

MS research is greatly facilitated by the use of animal models of the disease with the most commonly used model being experimental autoimmune encephalomyelitis (EAE). EAE is a CD4+ $\mathrm{T}$ cell-mediated disease in which activated CD4+ $\mathrm{T}$ cells and mononuclear cells mediate demyelination of axonal tracks within the central nervous system. By using this model it is possible to study the immune regulation and pathogenesis of a CD4+ Th1/Th17 mediated disease which is considered an acceptable model for understanding human demyelinating diseases such as MS. EAE can be induced in animals by immunising with a myelin specific protein or peptide with an immune adjuvant (Kuchroo \& Weiner, 1998) or the disease can be established by adoptively transferring myelin specific T cells from an immunised animal into a healthy recipient, as these are the cells that are thought to be primarily responsible for mediating the disease (Kuchroo et al., 1992). 


\subsubsection{Induction and immunology of EAE}

Mice are often used in EAE experiments as they show strain specific differences in disease pathology and susceptibility to a particular antigens which make them useful models in understanding different aspects of the human disease (S. D. Miller \& Karpus, 2007). The SJL strain of mice is susceptible to PLP, MOG and MBP and often exhibits a relapsing-remitting form of the disease (Kono et al., 1988; S. D. Miller \& Karpus, 2007); which is therefore useful to study $\mathrm{T}$ cell-mediated demyelination as opposed to the resident macrophage driven damage that controls the later stages of chronic progressive disease. Furthermore, the relapsing-EAE shows similar histopathological and clinical similarities to human relapsing-remitting MS which makes it an effective model for studying the cellular and molecular basis of disease progression. Alternatively, the C57BL/6 strain of mice is often induced with MOG peptide plus adjuvant and develops a severe chronic-progressive form of EAE (Bernard et al., 1997). By taking advantage of this range of disease in the EAE model researchers are able to study different aspects of MS disease pathology.

Active EAE can be induced in mice by subcutaneous (s.c.) injections in the hind flanks with myelin peptide in the presence of complete Freund's adjuvant (CFA), which contains heatinactivated Mycobacterium tuberculosis (MTB), and intraperitoneal (i.p.) injections of pertussis toxin which are typically given on the day of injection and two days later (Stromnes \& Goverman, 2006). Together the s.c and i.p injections are often referred to as EAE immunisation. Freund's adjuvant is thought to play a number of roles during the induction of EAE which are due to both the MTB and the paraffin oil containing surfactant which together make up CFA. MTB is a strong inducer of IL-12 and in the presence of myelin peptides drives the production of a Th1-type immune response that drives the development of EAE. Furthermore, MTB has been shown to increase the permeability of the BBB which further drives EAE development (Billiau \& Matthys, 2001; C. Constantinescu \& Hilliard, 2005). In conjunction, the paraffin oil in CFA acts as a depot for the immunising antigen and has also shown to be immunologically active through the induction of inflammatory cytokines such as TNF (Billiau \& Matthys, 2001). The exact role of pertussis toxin in EAE is still unclear as yet however, it is clear that EAE immunisation with pertussis toxin is more reliable and reproducible than without. There is evidence to show that pertussis toxin is able to increase vascular permeability and drives the expansion of encephalogenic $\mathrm{T}$ cells through the activation of macrophages and DCs, and both of these effects appear to be mediated through the histamine $\mathrm{H}_{1}$ receptor (C. Constantinescu \& Hilliard, 2005).

Following EAE immunisation in the hind flanks, resident antigen presenting cells (i.e. DCs) pick up the myelin antigen and become activated then migrate to the draining (inguinal) lymph node 
where they induce the activation of naïve $\mathrm{T}$ cells. These autoreactive $\mathrm{T}$ cells are induced following exposure to myelin-based peptides presented on the surface of APCs along with the correct costimulatory molecules, such as CD80/CD86. Following $\mathrm{T}$ cell activation, these cells undergo maturation and clonal expansion which results in a large pool of myelin-specific CD4+ $\mathrm{T}$ cells in the periphery. As mentioned earlier, $\mathrm{T}$ cells can be activated into different subtypes (Th1/Th2/Th17) and these subtypes are determined by the cytokines present during activation and this process is often directed by APCs. Originally Th1 cells were believed to be the drivers of EAE disease however, it is now widely accepted that Th17 also play a key role and may even be the key drivers of disease (Komiyama et al., 2006; L. Steinman, 2007).

There is evidence to support the role of Th17 in driving EAE disease and suggest that full activation of EAE requires both Th1 and Th17 autoreactive T cells. It has been shown that IFN- $\gamma$ deficient and IFN- $\gamma$ receptor-deficient mice exhibit more severe EAE disease than WT mice (I. A. Ferber et al., 1996; Sabatino, Shires, Altman, Ford, \& Evavold, 2008), suggesting that inhibiting Th1 signalling does not seem to alter disease. Interestingly, given that IFN- $\gamma$ deficient mice develop severe EAE disease suggests that IFN- $\gamma$ may actually play a role in regulating the Th17mediated side of disease, an idea which is supported by increased number of Th17 cells present in IFN- $\gamma$ deficient mice (Sabatino et al., 2008). Additionally, mice which receive antibodies against IL-17 have attenuated disease severity indicating that IL-17 is a key driver in the pathogenesis of EAE disease (Hofstetter et al., 2005). Furthermore, it has been shown that mice lacking functional IL-23 (a cytokine involved in sustaining Th17 cells) are resistant to EAE, while mice lacking IL12 (involved in sustaining Th1 cells) are not EAE resistant (Cua et al., 2003). The accepted model is that full EAE induction involves both Th1 and Th17 autoreactive cells given that it has been shown that adoptive transfer of reactive Th17 only induces mild disease, and inhibition of IL-17A doesn't completely suppress EAE disease whereas the addition of reactive Th1 cells allows full EAE severity (R. A. O'Connor et al., 2008). Furthermore, it has been suggested that Th1 cells may allow Th17 cells entry into the CNS where they can exert their action (R. A. O'Connor et al., 2008).

A key part of EAE disease is the migration of autoreactive $\mathrm{T}$ cells from the periphery into the CNS. It has been shown that leukocyte migration into the CNS occurs in healthy individuals although the movement of cells is restricted by the BBB. In a steady state with normal immune surveillance, the cells are maintained in the cerebro-spinal fluid (CSF) drained vascular spaces where they can interact with strategically located antigen presenting cells in search for their specific antigen (Engelhardt \& Ransohoff, 2012). The migration of activated immune cells into the CNS first involves crossing the BBB or blood cerebro-spinal fluid barrier (BCSFB) and into the 
CSF, followed by crossing the glia limitans into the CNS parenchyma (Engelhardt, 2006). Furthermore, it has been noted that in EAE, only the entry of immune cells into the CNS parenchyma results in clinical disease as cells which are only found in the perivascular or leptomeningeal CSF-drained spaces do not contribute to disease symptoms (Toft-Hansen et al., 2006).

The expression of adhesion molecules on the surface on vascular endothelial cells is important for the infiltration of activated T cells to cross the BBB. In the EAE model, it has been found that vascular adhesion molecule-1 (VCAM-1) and intracellular adhesion molecule-1 (ICAM-1) are both upregulated (Steffen, Butcher, \& Engelhardt, 1994). These receptors interact with their ligands, lymphocyte function-associated antigen-1 (LFA-1) and $\alpha 4 \beta 1$ integrin (VLA-4), which are known to be expressed on the surface of trafficking encephalogenic $\mathrm{T}$ cells (Engelhardt \& Ransohoff, 2012). Additionally, the interaction between these receptors and ligands are shown to have an important role in driving EAE and MS disease, as inhibition of $\alpha 4 \beta 1$ integrin with natalizumab is an effective treatment (Pucci et al., 2011).

Once encephalogenic T cells are present within the CNS and recognise their target antigens, the inflammatory response is amplified through the recruitment of other cells. This recruitment occurs through the secretion of cytokines (e.g. IL-1 and TNF) and chemokines (e.g. CCL2 and CCL5) bringing in more effector CD4+ T cells, NK cells, CD8+ T cells and macrophages into the CNS which are all known to play a role in CNS inflammation during EAE, resulting in tissue damage and demyelination (Elhofy, Kennedy, Fife, \& Karpus, 2002; Karpus \& Ransohoff, 1998; McQualter \& Bernard, 2007). CD8+ T cells mediate cell apoptosis within the CNS through FasFasL interactions and the release of Granzyme B (Huseby et al., 2001; Johnson, Jirik, \& Fournier, 2010). Infiltrating macrophages and resident microglia play a role in EAE disease through secretion of NO and other reactive oxygen species as well as assisting the breakdown of the BBB by secreting MMPs and therefore aid the recruitment of additional cells which further amplify CNS inflammation (Kieseier et al., 1998; McQualter \& Bernard, 2007).

The inflammatory responses do not go unchecked in EAE disease, and there are a number of cells which are also recruited to regulate the immune response. It has been shown that Tregs play a key role in the disease resolution of EAE given that these cells are found at higher numbers during the later stages of disease and can also be found within the CNS to locally reduce $\mathrm{T}$ cell responses (Adam P. Kohm, Carpentier, Anger, \& Miller, 2002). It has also been suggested that PD-L1 is another mechanism which regulates EAE disease (Carter et al., 2007) by suppressing $\mathrm{T}$ cell responses and inducing immune tolerance or T cell anergy. Moreover, it has been found that high levels of cytokines including IFN- $\gamma$ and NO may have a regulatory role in the later stages of EAE 
disease by suppressing T cell responses (Espejo et al., 2002; van der Veen, Dietlin, \& Hofman, 2003).

Animal models of multiple sclerosis have been used for the development and validation of novel therapeutic treatments, although only a couple of treatments have been developed in the model several therapies have been secondarily assessed in EAE (Mix, Meyer-Rienecker, \& Zettl, 2008; L. Steinman \& Zamvil, 2005). Some aspects, such as spontaneity, cannot be accounted for in EAE, although considering many of the current front-line therapeutics for MS have utilized EAE at some stage during their development and all have shown efficacy within the model (L. Steinman \& Zamvil, 2005) EAE is still a useful tool for evaluating MS therapies.

\subsubsection{The use of TLRs in EAE disease}

The activation of innate PRRs, such as the TLRs, has been implicated in both the pathogenesis as well as the regulation of multiple sclerosis and its animal model EAE (Carty \& Bowie, 2011; Waldner, 2009). It has been shown that signalling through some TLRs, such as TLR2 and TLR9, is necessary for the development of EAE and deficiency for these receptors or the downstream TLR-adaptor myeloid differentiation primary response gene 88 (MyD88)-mediated signalling inhibits EAE induction (Miranda-Hernandez et al., 2011). However, the role of TLR9 in establishing EAE disease is controversial; it has been shown that administration of TLR9 ligands, such as CpG, exacerbates disease in some studies (Miranda-Hernandez et al., 2011; M. Prinz et al., 2006) while in another study there is evidence that indicates TLR9 may also have a protective function in EAE (Marta, Andersson, Isaksson, Kampe, \& Lobell, 2008).

In addition to the TLRs, another intracellular PRR known as the NOD family have also been implicated in the progression of EAE. Recent studies have suggested that EAE is dependent on NOD-2 and downstream receptor-interacting serine/threonine kinase (RICK) signalling as it has a key role in the activation of CNS-infiltrating DCs (Shaw et al., 2011). Although the role that these PRR may play in the induction or pathogenesis of MS is less clear (Marta et al., 2008), the association between bacterial infections and MS as well as the detection of antigen presenting cells containing peptidoglycan, a NOD2 ligand, in the brains of MS patients support a possible pathogenic role for these PRR in MS (Schrijver et al., 2001).

Although some studies identify PRR agonists as has having a pathogenic role in MS, there has been recent evidence which points to a potential therapeutic application of these agonists as immunomodulatory agents to treat MS. An example of this is the administration of TLR3 and TLR7 ligands in EAE animal models which have proven to have a protective effect on disease in a number of studies (Hayashi et al., 2009; O'Brien, Fitzgerald, Rostami, \& Gran, 2010; Touil, 
Fitzgerald, Zhang, Rostami, \& Gran, 2006). In particular, the suppression of EAE by TLR3 activation has been attributed directly to the induction of endogenous IFN- $\beta$ (Touil et al., 2006) and similar studies by O'Brien et al. found that the protective TLR7 agonist, imiquimod, also enhanced IFN- $\beta$ production during EAE (O'Brien et al., 2010). In contrast to this mechanism, recent work has found that multiple low doses of a TLR7 agonist induced a tolerogenic state that was able to suppress CNS inflammation during EAE (Hayashi et al., 2009). This finding suggests that repeated induction of low levels of inflammatory cytokine signalling may favour the enhancement of negative feedback immune mechanisms that effectively down regulate inflammatory T cell activity (Hayashi et al., 2009). Another study has shown that B cell regulation of EAE requires the stimulation of TLRs and the MyD88 pathway to produce IL-10 which suppresses inflammatory Th1 and Th17 cell responses and therefore drives EAE recovery (Lampropoulou et al., 2008).

\subsection{Aims}

The aim of my research was to determine whether the immunotherapeutic agent MIS416 protects against and reduces the severity of EAE, a model of neuroinflammation. Additionally, to achieve this key aim it was necessary first to identify a dosing regimen in which MIS416 was effective and use this model to elucidate the mechanism of MIS416 in an autoimmune disease setting. Understanding the exact mechanism of MIS416 immunotherapy will allow this compound to be used more effectively in patients with MS as well as other inflammatory diseases. I addressed this key aim through four complementary specific aims:

1. To develop an optimal MIS416 dosing regimen for further mechanistic studies.

2. To characterise the alterations in cells numbers, activation state and cytokine profile of MIS416-treated mice.

3. To elucidate which cell types and cytokines were involved in MIS416-mediated protection against EAE.

4. To determine if MIS416 altered CNS inflammation in EAE. 
Chapter 2: Materials and Methods 


\subsection{Mice}

Animals were housed at Victoria University of Wellington, New Zealand in the animal facility. The experimental protocols were pre-approved by the Victoria University of Wellington Animal Ethics Committee (AEC) under the licenses 2008R12 (September 23 ${ }^{\text {rd }}$, 2008) and 2011R21 (October $7^{\text {th }}, 2011$ ). The animals were maintained in a temperature-regulated environment with a 12-hour light/dark cycle and were kept in filter-top cages. Any handling of the animals was conducted in a laminar flow workspace.

C57BL/6J (H-2b) mice were bred at the biomedical research unit (BRU) at the Malaghan Institute of Medical Research (Wellington, NZ). Breeding stock was originally purchased from the Jackson Laboratory (Bar Harbour, ME, USA).

SJL/J mice were bred at the vertebrate containment facility (VCF), Victoria University of Wellington. The original breeding stock was purchased from Animal Resource Centre (Canning Vale, WA, Australia).

BALB/c mice were bred at the VCF, Victoria University of Wellington. The breeding stock was originally obtained from Frank Brombacher (University of Cape Town, Cape Town, South Africa).

B6.SJL-ptprca mice, which are congenic mice on the C57BL/6 background expressing CD45.1, were bred at the BRU at the Malaghan Institute of Medical Research. Breeding stock was originally purchased from Animal Resource Centre.

2D2 mice were bred at the VCF, Victoria University of Wellington. These mice express a transgenic TCR for the MOG35-55 peptide (Bettelli et al., 2003). The breeding stock was originally obtained from Prof. Vijay K. Kuchroo (Harvard Medical School, Boston, MA, USA) via A/Prof. Thomas Backstrom (Malaghan Institute of Medical Research).

FoxP3 GFP mice (B6x129(Foxp3 GFP-g2)) were bred at the BRU at the Malaghan Institute of Medical Research. Mice were used between 8-12 weeks of age and only male mice were used for these experiments.

C57BL/6-ifngtm1Ts IFN- $\gamma$-deficient mice (Dalton et al., 1993) were bred at the BRU at the Malaghan Institute of Medical Research. The breeding stock was originally obtained from the Walter and Eliza Hall Institute of Medical Research (Melbourne, VIC, Australia). 


\subsection{EAE induction}

\subsubsection{Peptides for EAE induction}

MOG $_{35-55}$ peptide (MEVGWYRSPFSRVVHLYRNGK), PLP $_{139-151}$ peptide (HSLGKWLGHPDKF), and PLP $180-199$ peptide (WTTCQSIAFPSKTSASIGSL) were synthesised by GenScript (Piscataway, NJ, USA).

\subsubsection{C57BL/6J and B6.SJL-ptprca mice}

Eight to twelve week old female mice were immunised by subcutaneous (s.c.) injection with an emulsion consisting of $50 \mu \mathrm{g} \mathrm{MOG}_{35-55}$ peptide (Genscript) and $500 \mu \mathrm{g}$ heat-inactivated Mycobacterium tuberculosis (Difco Laboratories, Detroit, USA) in incomplete Freund's adjuvant (Sigma, St. Louis, MO, USA). The components were emulsified by repeatedly passing through an 18 gauge needle attached to a glass syringe until a thick homogenised emulsion was created. The mice were injected s.c. in each hind flank with $100 \mu$ l of emulsion after which, each mouse was injected 200 ng pertussis toxin (Sapphire Bioscience, Redfern, NSW, Australia) in $200 \mu 1$ pertussis toxin buffer (see Appendix 6) intraperitoneally (i.p.) on days 0 and 2 post-immunisation (p.i.) as previously described (Crume et al., 2009).

\subsubsection{SJL/J mice}

Eight to sixteen week old female mice were immunised by s.c. injection with an emulsion consisting of $50 \mu \mathrm{g} \mathrm{PLP}_{139-151}$ peptide (Genscript) and $500 \mu \mathrm{g}$ heat-inactivated Mycobacterium tuberculosis (Difco Laboratories) in incomplete Freund's adjuvant (Sigma). The components were emulsified by repeatedly passing through an 18 gauge needle attached to a glass syringe until a thick homogenised emulsion was created. The mice were injected s.c. in each hind flank with 100 $\mu \mathrm{l}$ of emulsion after which, each mouse was injected $100 \mathrm{ng}$ pertussis toxin (Sapphire Bioscience) in $200 \mu \mathrm{l}$ pertussis toxin buffer i.p. on days 0 and 2 p.i as this dose has been optimised in our lab for this model.

\subsubsection{BALB/c mice}

Eight to sixteen week old female mice were immunised by s.c. injection with an emulsion consisting of $100 \mu \mathrm{g}$ PLP180-199 peptide (Genscript) and $500 \mu \mathrm{g}$ heat-inactivated Mycobacterium tuberculosis (Difco Laboratories) in incomplete Freund's adjuvant (Sigma). The components were emulsified by repeatedly passing through an 18 gauge needle attached to a glass syringe until a thick homogenised emulsion was created. The mice were injected s.c. in each hind flank with 100 $\mu \mathrm{l}$ of emulsion after which, each mouse was injected $400 \mathrm{ng}$ pertussis toxin (Sapphire Bioscience, Redfern, NSW, Australia) in $200 \mu$ pertussis toxin buffer i.p. on days 0 and 2 p.i, as this dose has been optimised in our lab for this model. 


\subsubsection{EAE disease severity rating scale}

After immunisation mice were weighed daily using a Mettler PB6000 balance (Mettler Tolodo; Columbus, $\mathrm{OH}, \mathrm{USA}$ ) and monitored for the duration of the experiment for signs of disease or unexpected illness. EAE disease was scored using a 0-5 disease severity scale as follows: $0=$ unaffected, $0.5=$ loss of tonicity in distal region of tail, $1=$ loss of tail tonicity or half-tail paralysis; 2 = full tail paralysis; $3=$ one hind limb paralysis or severe weakness in both hind limbs; 4 = full hind limb paralysis; and 5 = moribund (Keating et al., 2009; Tierney et al., 2009). Mice which reached a disease score of 5 (moribund) were euthanised.

\subsection{In vivo techniques}

\subsubsection{Compounds for in vivo use}

MIS416, heat-treated MIS416 and AF488 or FITC-labelled MIS416 were generously provided by Innate Immunotherapeutics (Auckland, NZ) (see Appendix 9).

\subsubsection{In vivo compound administration}

MIS416, heat-treated MIS416, fluorescently-labelled MIS416 or saline vehicle was injected at indicated amounts (between 100 and $250 \mu$ total volume) into the lateral tail vein using a 31 gauge needle.

\subsubsection{Jelly preparation for oral MIS416 administration}

Mice were orally treated with $2 \mathrm{mg}$ MIS416 daily in sugar-free strawberry jelly (Weight watchers). Jelly was prepared by dissolving 1 packet of jelly in $250 \mathrm{ml}$ of autoclaved tap water (a $2 \mathrm{x}$ concentrated jelly) and adding an additional $10 \mathrm{~g}$ of gelatine. This " $2 \mathrm{x}$ jelly solution" was aliquoted and stored at $-20^{\circ} \mathrm{C}$ for future use. In a 24 -well plate, $1000 \mu 1$ of the melted " $2 \mathrm{x}$ jelly solution" was added to $800 \mu \mathrm{l}$ of $10 \mathrm{mg} / \mathrm{ml}$ MIS416, and gently pipetted up and down to mix the MIS416 into the jelly. The jelly was left to set in the fridge and fresh jelly was made every 4 days. A control jelly was also made using sterile saline solution in place of MIS416. On the day of treatment each 24-well was divided into 4 pieces and each mouse received 1 piece of jelly containing $2 \mathrm{mg}$ of MIS416.

\subsubsection{Isolation of blood for serum cytokine analysis}

Mice were placed under a heat-lamp for a few minutes to encourage vasodilation then restrained within a mouse restraint. The distal tip of their tails nicked with sharp sterile scissors and drops of blood were collected in sterile microcentrifuge tubes. Following incubation at room temperature (RT) when the blood had coagulated the samples were centrifuged at 7,800 x g for 10 minutes, 
after which serum was removed from the surface and used immediately or stored at $-80^{\circ} \mathrm{C}$ until required.

\subsubsection{In vivo proliferation assay}

Cells were isolated from the spleen and lymph nodes of 2D2 mice which were age and sex matched to B6.SJL-ptprca mice. The cells processed into a single cell suspension as indicated by methods 2.4.2 and 2.4.7 and CFSE-labelled with $5 \mu \mathrm{M}$ CFSE in PBS using the methods discussed in 2.5.1. After which, the cells were resuspended at 50x $10^{6}$ cells $/ \mathrm{ml}$ in PBS and B6.SJL-ptprca mice were injected i.v. with $200 \mu \mathrm{l}$ of the CFSE-labelled 2D2 cells. The following day mice were given MIS416 treatment and EAE immunisations (see 2.2.2 and 2.3.2). Mice were culled 5 days after MIS416 and EAE injections and the spleen, blood and draining lymph node were processed and analysed by flow cytometry to assess $2 \mathrm{D} 2 \mathrm{CD} 4+\mathrm{T}$ cells proliferation.

\subsubsection{Evans Blue permeability assay}

Mice were immunised for EAE as per methods and weighed and scored daily. At disease onset, which is characterised by weight loss in the majority of untreated mice with some mice showing early disease symptoms (usually day 9-11), the mice were inject i.p. with $200 \mu 12.5 \%$ Evans blue (EB) in saline solution. After which the mice were scarified between 40-90 minutes using $\mathrm{CO}_{2}$ asphyxiation and cardiac perfused with $20 \mathrm{ml}$ of room temperature PBS. The brain and spinal cord were removed and placed on glass slides for photography then placed in $700 \mu 1$ and $500 \mu 1$ formamide solution for the brain and spinal cord respectively. The tissue samples were left for 3 days at room temperature in the dark to allow the EB dye to leech out of the tissue and into solution. Then $200 \mu \mathrm{l}$ of the formamide solution was removed and placed into a flat bottomed 96well plate and the absorbance was measured at $650 \mathrm{~nm}$ using an EnSpire 2300 multilabel plate reader (PerkinElmer, USA). A permeability index was calculated using the following calculation:

Permeability index $=($ absorbance of sample $) /($ absorbance of no evans blue control tissue $)$.

\subsection{Ex vivo techniques}

\subsubsection{Dissection and organ removal}

Mice were sacrificed by $\mathrm{CO}_{2}$ asphyxiation and washed with $100 \%$ ethanol. To remove the lymph nodes and spleen, the peritoneal cavity was opened with scissors sterilised in ethanol and using sterile tweezers the spleen and lymph nodes (mescenteric for oral dosing experiments and inguinal for EAE experiments) were isolated and placed in $1 \mathrm{ml}$ of wash buffer (see appendix 6). Bone marrow was taken following blunt dissociation from connective tissues; after skeletal tissue was 
removed the tibias and femurs were cut at both ends and flushed with dPBS (Invitrogen, Waltham, Massachusetts, USA) with 1\% PenStrep (Invitrogen) using a 23 gauge needle (BD Biosciences, Franklin Lakes, New Jersey, USA). Gastrointestinal tract was removed by cutting the small intestine, just below the stomach, and at the end of the colon. Blood was harvested by cutting the diaphragm and exposing the heart followed by a cardiac puncture in the right ventricle with a 27 gauge needle attached to a $1 \mathrm{ml}$ syringe (BD Biosciences). For the removal of brains, spinal cord and liver, the mice were first perfused with PBS (see appendix 6) to remove contaminating blood cells from the tissues, which was done by first severing the portal vein. A 23 gauge needle attached to a $20 \mathrm{ml}$ syringe (BD Biosciences) containing $20 \mathrm{ml}$ PBS was inserted in to the left ventricle of the heart and the mouse was slowly perfused. Following perfusion, the liver was removed using sterile tweezers and scissors and placed into $5 \mathrm{ml}$ of PBS. Mice were decapitated and spinal cords were removed by severing the spinal column at the lower back and inserting a 19 gauge needle into this lumbar region of the vertebral column and exerting positive pressure onto the spinal cord via a syringe containing PBS. Brains were isolated by removing the upper half of the skull with small dissection scissors and using sterile tweezers to remove the brain from the lower part of the skull, then placing the brain in $1 \mathrm{ml}$ of PBS.

\subsubsection{Preparation of a single cell suspension from the spleen}

Following isolation, spleens were placed in $1 \mathrm{ml}$ of wash buffer (see appendix 6) for re-stimulation assays (see 2.4.8) or isolation buffer (see appendix 6) for $\mathrm{T}$ cell isolation (see 2.5.1). A single cell suspension was made by dissociating the tissue through a $70 \mu \mathrm{m}$ cell strainer (BD Biosciences) and the remaining cells in the strainer were washed with either wash buffer or isolation buffer into a $50 \mathrm{ml}$ conical tube. The cells were centrifuged at $730 \mathrm{x}$ g for 5 minutes and the red blood cells were lysed by resuspending the cells in $2 \mathrm{ml}$ of red blood cell lysis buffer (Sigma) for 3 minutes at room temperature. The splenocytes were washed by adding $8 \mathrm{ml}$ of wash buffer followed by centrifugation at $730 \mathrm{x} \mathrm{g}$ for 5 minutes. Splenocytes were resuspended in $10 \mathrm{ml}$ of fresh wash buffer or isolation buffer and live cell numbers were determined using trypan blue exclusion (see 2.4.10).

\subsubsection{Preparation of a single cell suspension from the liver}

Livers were perfused until light brown in colour and placed into $5 \mathrm{ml}$ of PBS (see appendix 6). A single cell suspension was made by dissociating the tissue using the rubber end of a $10 \mathrm{ml}$ syringe plunger and passing the cells through a $70 \mu \mathrm{m}$ cell strainer (BD Biosciences). The cells were washed through the strainer at regular intervals using $1 \mathrm{ml}$ of PBS until the whole liver had passed through the strainer into a $50 \mathrm{ml}$ conical tube. The tubes were filled to $40 \mathrm{ml}$ with PBS and spun at $730 \mathrm{x} g$ for 5 minutes to pellet the cells of interest and remove unwanted debris. The pellet was 
resuspended in 33\% percoll solution in PBS containing 2\% Alsevers solution (Sigma) (see appendix 6) and centrifuged at $770 \mathrm{x} g$ for 30 minutes with no brake. The supernatant was removed and the cells were washed in $15 \mathrm{ml}$ PBS by centrifugation at $730 \mathrm{x}$ g for 5 minutes. The red blood cells were lysed by resuspending the cells in $5 \mathrm{ml}$ red blood cell lysis buffer (Sigma) for 5 minutes at RT. The liver cells were washed by adding $10 \mathrm{ml}$ of PBS, and the red blood cell lysis was repeated if the pellet contained red blood cells. The splenocytes were washed again by centrifugation at $730 \mathrm{x}$ g for 5 minutes and resuspended in either fresh culture media (see appendix 6) for culturing of cells or FACs buffer (see appendix 6) for flow cytometry.

\subsubsection{Preparation of a single cell suspension from the spinal cord}

Using a common process spinal cords were processed into a single cell suspension (Sedgwick et al., 1991; Weir et al., 2006). Spinal cords were minced in a Petri dish using a surgical blade until only very small pieces remained. The small cord pieces were resuspended in $5 \mathrm{ml}$ of $2.4 \mathrm{mg} / \mathrm{ml}$ collagenase type II (see appendix 6) in a $50 \mathrm{ml}$ conical tube and incubated for 30 minutes at $37^{\circ} \mathrm{C}$ in a shaking incubator. After incubation, a p1000 pipette was used to break up clumps by rapidly pipetting the solution up and down for 1-2 minutes and left for a further 10 minutes at $37^{\circ} \mathrm{C}$. Following incubation the cells were passed through a $70 \mu \mathrm{m}$ cell strainer and pelleted by spinning at $760 \mathrm{x}$ g for 5 minutes. After discarding the supernatant the cells were resuspended in $10 \mathrm{ml}$ of $37 \%$ Percoll (see appendix) and spun for 30 minutes at $760 \mathrm{x}$ g without the break on. Following centrifugation the myelin layer and supernatant was carefully removed and the cells were resuspended in $5 \mathrm{ml}$ of PBS. The cells were pelleted again by centrifuging at $760 \mathrm{xg}$ for 5 minutes then resuspended in a suitable volume of FACs buffer (see appendix 6).

\subsubsection{Preparation of single cell suspension from the brain}

Single cell suspensions from the brain were made by mashing the brain through a $70 \mu \mathrm{m}$ cell strainer and adding PBS in $1 \mathrm{ml}$ lots until all the tissue had passed through the strainer. The cells were pelleted by centrifugation at $760 \mathrm{x} g$ for 5 minutes and discarding the supernatant. The cells were resuspended in $10 \mathrm{ml}$ of $37 \%$ Percoll solution (see appendix 6) and centrifuged at $760 \mathrm{x} \mathrm{g}$ with low acceleration and no break for 30 minutes. The myelin layer and supernatant were removed and the cells were washed in $5 \mathrm{ml}$ PBS by centrifugation at $760 \mathrm{xg}$ for 5 minutes. After discarding the supernatant the cells were resuspended in a suitable volume of FACs buffer (see appendix 6).

\subsubsection{Preparation of whole blood for flow cytometry}

$100 \mu \mathrm{l}$ of whole blood was mixed into $500 \mu 1$ of 1 xPBS containing $0.5 \mu \mathrm{M}$ EDTA and left on ice until prepared. The blood was washed by centrifugation at $400 \mathrm{x} g$ for 5 minutes and the supernatant was discarded. The red blood cells were lysed by adding $1 \mathrm{ml}$ of red blood cell lysis 
buffer (Sigma) and incubating at RT for 5 minutes. The blood solution was transferred to a $15 \mathrm{ml}$ tube and the cells were washed by adding $5 \mathrm{ml}$ of PBS followed by centrifugation at $730 \mathrm{x} \mathrm{g}$ for 5 minutes. The supernatant was discarded and the red blood cell lysis was repeated until the pellet was no longer red and the cells were resuspended in FACs buffer for further analysis.

\subsubsection{Preparation of a single cell suspension from the draining lymph node}

Inguinal or mesenteric lymph nodes (LN) were isolated with minimal fatty tissue and were placed in either $1 \mathrm{ml}$ of wash (see appendix 6) buffer or $1 \mathrm{ml}$ of PBS depending on application. A single cell suspension was made by dissociating the tissue through a $70 \mu \mathrm{m}$ cell strainer (BD Biosciences) and washing the remaining cells in the strainer into a $50 \mathrm{ml}$ centrifuge tube using wash buffer. The cells were pelleted by centrifugation at $730 \mathrm{x}$ g for 5 minutes and resuspended in either PBS or FACs buffer. Live cell numbers were determined using trypan blue exclusion and were processed further for either FACs staining (see 2.10.1) or CFSE labelling (see 2.5.2) depending on the experiment.

\subsubsection{Ex vivo stimulation of isolated leukocytes}

Splenocytes and liver cells were plated at $1 \times 10^{6}$ and $2 \times 10^{5}$ cells per well respectively, in culture medium (see appendix 6) in 96-well flat bottom plates. Ex vivo stimuli were added to the cells at varying concentrations where MIS416 was given between 5 and $20 \mu \mathrm{g} / \mathrm{ml}$, MOG at either $9 \mu \mathrm{g} / \mathrm{ml}$ or $27 \mu \mathrm{g} / \mathrm{ml}$, LPS at $200 \mathrm{ng} / \mathrm{ml}$ or ConA between 0.3 and $1 \mu \mathrm{g} / \mathrm{ml}$. Cells were incubated $\left(5 \% \mathrm{CO}_{2}\right.$ at $37^{\circ} \mathrm{C}$ ) for 24,48 or 72 -hours as indicated. The supernatant was collected for ELISA by centrifuging the plates at $400 \mathrm{x}$ g and $160 \mu \mathrm{l}$ of supernatant was transferred into a clean 96-well Ubottom plate. Supernatants were frozen at $-20^{\circ} \mathrm{C}$ for long term storage or placed in the fridge for up to 5 days.

\subsubsection{Ex vivo stimulation for intracellular cytokine staining}

Freshly isolated or in vitro stimulated (see 2.4.8) cells were re-stimulated in a 96-well plate with $50 \mathrm{ng} / \mathrm{ml}$ of PMA (phorbol myristate acetate), $500 \mathrm{ng} / \mathrm{ml}$ ionomycin and $1 \mu \mathrm{g}$ of GolgiStop (BD Biosciences) per $1 \times 10^{6}$ cells with or without additional stimulation of MIS416 at $20 \mu \mathrm{g} / \mathrm{ml}$ or ConA at $1 \mu \mathrm{g} / \mathrm{ml}$, and samples were left to incubate at $37^{\circ} \mathrm{C}$ with $5 \% \mathrm{CO}_{2}$ for 5-hours. The cells were washed by centrifugation at $400 \mathrm{x} g$ for 5 minutes and the supernatant discarded followed by resuspension in FACs buffer (see appendix 6). The cells were washed again and resuspended in 50 $\mu 1$ FACs buffer containing Fc receptor block and extracellular antibodies and left for 25 minutes on ice in the dark. Following extracellular staining the cells were fixed and permeabilised using Transcription Factor Buffer (BD Biosciences) following a scaled down version of the manufacturer's instructions for staining in a 96-well plate. In brief, $120 \mu 1$ of fix/perm buffer was added to cells and incubated on ice for 40 minutes. The cells were washed twice with perm/wash 
solution, where an excess of perm/wash solution was added and the 96-well plate was spun at 400 $\mathrm{x} g$ and supernatant discarded. The cells were then incubated with $50 \mu \mathrm{l}$ of perm/wash solution containing intracellular antibodies on ice for 40 minutes in the dark. The cells were then washed twice in perm/wash buffer and resuspended in FACs buffer, followed by analysis using BD FACs Canto II flow cytometer (BD Biosciences).

\subsubsection{Trypan blue exclusion}

To count live splenocytes, the cells were resuspended in wash buffer and counted using $0.4 \%$ trypan blue (Sigma) in a 1:10 dilution. $10 \mu \mathrm{l}$ of the cell and dye mix was placed onto a Neubauer haemocytometer (Hawksley, Lancing, UK) and counted using a compound microscope (CX41: Olympus, PA, USA).

Liver cells were also counted as above, however the cell:dye ratio was 1:1 as there were significantly fewer leukocytes found within the liver.

\subsubsection{Histology}

Spinal cords from EAE mice were fixed in 4\% paraformaldehyde solution (see appendix 6) for 2448 hours post-extraction. The spinal cords were then washed in PBS and put into 30\% sucrose solution (see appendix 6) and left at $4{ }^{\circ} \mathrm{C}$, the sucrose solution was replaced a few days later and left at $4{ }^{\circ} \mathrm{C}$ for long term storage. One day before the spinal cords were sectioned the sucrose was replaced with $70 \%$ ethanol and the samples were placed in labelled plastic cassettes. The samples were successively dehydrated and put into paraffin using a tissue processor (Leica TP1020 automatic tissue processor, Lecia Biosystems, Nussloch, Germany). After this, the samples were embedded into paraffin wax in the correct orientation for sectioning with the cervical section of the spinal cord on the surface of the paraffin block (Leica EG1160 Tissue Embedding Station, Lecia Biosystems). Using a microtome $10 \mu \mathrm{m}$ cross-section spinal cord slices were taken and placed on poly L-lysine slides (Thermo Fisher, Waltham, Massachusetts, USA) and left to dry at room temperature overnight.

\subsubsection{Haematoxylin and eosin (H\&E) staining}

Slides were de-paraffinised in xylene (Sigma) for at least 20 minutes or until the majority of wax was removed. The slides were then rehydrated using successive incubation of ethanol starting at $100 \%$, 95\% then $75 \%$ with 5 minute incubations at each stage. Slides were rinsed briefly with $\mathrm{ddH}_{2} \mathrm{O}$ and incubated in haematoxylin solution (Sigma) for 30 seconds, followed by another rinse in $\mathrm{ddH}_{2}$. Slides were left to incubate in $75 \%$ ethanol for 5 minutes followed by eosin (Sigma) for 10 seconds and quickly place in acid alcohol (see appendix 6) for 20 seconds. The slides were then placed in $0.01 \%$ lithium carbonate (see appendix 6) for 30 seconds followed by dehydration with 5 
minutes of $95 \%$ ethanol and 5 minutes of $100 \%$ ethanol. Slides were then placed in xylene (Sigma) for a further 45 minutes before mounting with Depex mounting medium (DBH laboratory supplies, Poole, England) and a coverslip placed over the sections. The slides were left to dry overnight before microscope work took place.

\subsubsection{Scoring of H\&E stained spinal cord sections}

Sections were analysed on an Olympus IX51 inverted microscope (Olympus) and photos were taken using CellA software (Olympus). The severity of CNS infiltration was assessed by independent investigators who scored the spinal cord sections based on a scale of 1 to 4 , in a blinded fashion using photographs of the sections. Spinal cord sections which appeared to have no infiltration were scored as a 1 and spinal cords with maximum infiltration were given a score of 4 (as based on a comparison to pre-prepared example photos). Given that the scoring process was subjective, sections were scored independently by three different individuals.

\subsection{In vitro techniques}

\subsubsection{Isolation of CD4+ $\mathrm{T}$ cells}

This was done using the Dynabeads Untouched mouse CD4 Kit (Life Technologies, Carlsbad, CA, USA) and following the recommended protocol. In brief, the cells from lymphoid organs were processed into single cell suspension (see 2.4.2 and 2.4.7) and resuspended in isolation buffer containing $2 \%$ FCS and 2 mM EDTA in dPBS (see appendix 6). For example, $5 \times 10^{7}$ cells were resuspended in $500 \mu \mathrm{l}$ in isolation buffer and $100 \mu \mathrm{l}$ heat-inactivated FCS was added followed by $100 \mu \mathrm{l}$ of antibody mix and after mixing thoroughly left for 20 minutes in the fridge. The cells were washed by adding $10 \mathrm{ml}$ isolation buffer and spun at $350 \mathrm{x}$ g for 8 minutes and supernatant discard. The cells were resuspended in $4 \mathrm{ml}$ isolation buffer and $1 \mathrm{ml}$ of pre-washed mouse depletion beads was added and left to incubate with gentle rotation at RT for 15 minutes. Followed by the addition of $5 \mathrm{ml}$ of isolation buffer and the cells were resuspended by pipetting up and down using a P1000 pipette. The tube was then placed onto a DynaMag magnet (Invitrogen) for 2 minutes and the supernatant was removed and placed into a new tube. The supernatant contained the untouched CD4+ T cell fraction, and these cells were washed, counted using trypan blue (see 2.4.10) and resuspended in PBS or culture media depending on the application. Note that with a total starting population of 50 million cells, a yield of around 7 million is normal.

\subsubsection{CFSE labelling}

Cells were prepared from the desired organ as indicated earlier, and resuspended in $1 \mathrm{ml}$ of PBS (not complete tissue culture media, CTCM, as this contains FCS which binds CFSE resulting in inconsistent CFSE labelling (Quah \& Parish, 2010)). For up to $1 \times 10^{7}$ cells a clean $15 \mathrm{ml}$ falcon 
tube was used and $1 \mathrm{ml}$ of cells at $1 \times 10^{7} / \mathrm{ml}$ were added to the bottom. The tube was laid on its side and $110 \mu \mathrm{l}$ PBS added to the top of the tube followed by $1.1 \mu 1$ of $5 \mathrm{mM}$ CFSE mixed into the $110 \mu 1$ of PBS. The cap was carefully replaced and the tube was vortexed on its lid briefly to combine the cells with the CFSE dye. The cells were left to incubate at RT in the dark for 5 minutes. The reaction was quenched with the addition of $2 \mathrm{ml}$ of heat-inactivated FCS to the cells and left for another 3 minutes at room temperature. The cells were then washed by adding $7 \mathrm{ml}$ of PBS and centrifuged at $730 \times \mathrm{g}$ for 5 minutes and discarding the supernatant. The cells were washed again using $10 \mathrm{ml}$ of either PBS or wash buffer (see appendix 6) and resuspended in the appropriate volume of culture media for the experiment. For staining 10-50x $10^{6}$ cells at one time, $1 \mathrm{ml}$ of $10 \mu \mathrm{M}$ CFSE was added to $1 \mathrm{ml}$ of cells (creating a final concentration of $5 \mu \mathrm{M}$ CFSE), mixed thoroughly and left at room temperature for 5 minutes in the dark and the same quenching and washing steps were used as that used in CFSE-labelling $1 \times 10^{7}$ cells.

\subsubsection{Preparation of bone marrow-derived macrophages}

Bone marrow progenitor cells were harvested by flushing the femurs of C57BL/6 mice, between the ages of 8-12 weeks, with sterile PBS containing 1\% PenStrep. Bone marrow cells were homogenised by pipetting the cells up and down using a $10 \mathrm{ml}$ serological pipette. Once the cells were collected the red blood cells were lysed with $2 \mathrm{ml}$ of red blood cell lysis buffer (Sigma) for 2 minutes at RT. The cells were washed with $8 \mathrm{ml}$ of wash buffer (see appendix 6) and resuspended at $1 \times 10^{6}$ cells $/ \mathrm{ml}$ in culture media (see appendix 6), and left overnight at $37^{\circ} \mathrm{C}\left(5 \% \mathrm{CO}_{2}\right)$ in a $\mathrm{T} 75$ $\mathrm{mm}^{2}$ tissue culture flask (BD Biosciences). The next day non-adherent cells were isolated from the flask and transferred into $90 \mathrm{~mm}$ petri dishes (Techno Plas, St Marys, Australia). The cells were then cultured for a total of 9 days in the presence of IL-3 and GM-CSF (both at $5 \mathrm{ng} / \mathrm{ml}$, Peprotech, New Jersey, USA) at $37^{\circ} \mathrm{C}\left(5 \% \mathrm{CO}_{2}\right)$. On the $5^{\text {th }}$ day additional media containing GMCSF and IL-3 were added to the petri dishes at a final concentration of $2.5 \mathrm{ng} / \mathrm{ml}$ for both factors. At the end of the culture period bone marrow derived macrophages (BMM $\varnothing$ ) were removed from the petri dishes by blasting off the cells with ice cold dPBS (Invitrogen). For experiments the BMM $\varnothing$ were cultured in culture media at $1 \times 10^{5}$ cells/well in a 96 -well flat-bottomed plate (BD Biosciences) and cultured overnight with IFN- $\gamma(20 \mathrm{U} / \mathrm{ml}$; Sigma, USA). Following overnight culture the cells were incubated for 24 hours with or without lipopolysaccharide (LPS $200 \mathrm{ng} / \mathrm{ml}$; Sigma) and experimental drugs were added as indicated.

\subsubsection{In vitro Treg suppression assay}

Tregs were isolated from MIS416-treated to untreated non-EAE mice using MACs CD4+CD25+ mouse regulatory $\mathrm{T}$ cell isolation kit (Miltenyi Biotec, Cologne, Germany) following the protocol to the manufacturer's instructions. Briefly, cells were processed into a single cell suspension from 
the spleen in isolation buffer (see appendix 6) using methods from 2.4.2, for each Treg assay the spleens of 2 mice were combined and $125 \times 10^{6}$ splenocytes were used at the beginning of the isolation. The cells were resuspended in $500 \mu \mathrm{l}$ isolation buffer and $125 \mu \mathrm{l}$ of antibody cocktail was added and the sample was refrigerated for 10 minutes. An additional $375 \mu 1$ of isolation buffer was added to the cell mixture followed by $250 \mu \mathrm{l}$ of anti-biotin microbeads and $125 \mu \mathrm{l}$ of anti$\mathrm{CD} 25-\mathrm{PE}$ and left to refrigerate in the dark for 15 minutes. After incubation, the sample was then washed with the addition of $10 \mathrm{ml}$ isolation buffer and centrifugation at $350 \mathrm{x}$ for 8 minutes. The supernatant was discarded and cells were resuspended in $500 \mu 1$ isolation buffer followed by magnetic separation. Sample was run through a wetted LD column using midiMACs magnet (Miltenyi Biotec) and the total effluent was collected as the unlabelled CD4+ T cell fraction. The collected CD4+ cells were washed in $10 \mathrm{ml}$ of isolation buffer and resuspended in $1125 \mu \mathrm{l}$ isolation buffer plus $125 \mu \mathrm{l}$ of anti-PE microbeads and refrigerated in the dark for 15 minutes. The cells were then washed in $10 \mathrm{ml}$ isolation buffer by centrifugation at $350 \mathrm{x}$ g for 8 minutes and resuspended in $500 \mu 1$ isolation buffer. The sample was then passed through a wetted MS column using a miniMACs magnet (Miltenyi Biotec, Germany) followed by 3x $500 \mu$ l isolation buffer washes. The MS column was removed from the magnet and $1 \mathrm{ml}$ of isolation buffer was added and plunged through the column, the CD4+CD25+ cells were collected and this process was repeated over a second MS column to increase Treg purity. The Tregs were then counted and resuspended in culture media (see appendix 6) at $4 \times 10^{6}$ cells $/ \mathrm{ml}, 50 \mu \mathrm{l}$ of Tregs were added to a U-bottom 96well plate at a 2:1 Treg:T activator ratio for the top concentration and serial 2-fold dilutions were performed to create a range of Treg:T activator cell ratios. CFSE labelled CD4+ cells were isolated as per section 2.5.1 and used as the T activator cells in this assay. Each well contained $1 \times 10^{5} \mathrm{~T}$ activator cells and these cells were stimulated with $2.5 \times 10^{4} \mathrm{CD} 3 / \mathrm{CD} 28$ beads (added into the assay in $50 \mu \mathrm{l}$ of culture media). The Treg suppression assay was left at $37{ }^{\circ} \mathrm{C}$ for 72 -hours and CD4+ T cell proliferation was measured using flow cytometry and FlowJo proliferation platform software (Tree Star, Ashland, OR, USA).

\subsubsection{In vitro MDSC suppression assay}

The MDSCs were isolated using MACs Myeloid-Derived Suppressor Cell Isolation kit (Miltenyi Biotec) following the manufacturer's instructions, as described briefly here using 100 million cells as the starting number. A single-cell suspension was prepared from the spleen using standard methods (2.4.2) and resuspended in isolation buffer (see appendix 6). The cells were counted passed through a cell strainer to ensure that there were no clumps followed by centrifugation at $300 \mathrm{x}$ g for 10 minutes discarding the supernatant. The cells were resuspended in $350 \mu 1$ isolation buffer per 100 million cells and $50 \mu \mathrm{l}$ of FcR blocking reagent was added, mixed well and left to incubate in the refrigerator for 10 minutes. After incubation $100 \mu \mathrm{l}$ of anti-Ly6G biotin was added 
and mixed well, followed by a further 10 minute incubation in the refrigerator. The cells were washed in $10 \mathrm{ml}$ of isolation buffer and centrifuged at $300 \mathrm{x} \mathrm{g}$ for 10 minutes and aspirated completely, after which, samples were resuspended in $800 \mu 1$ of isolation buffer and $200 \mu 1$ of antibiotin microbeads. The samples were mixed well and left to incubate in the refrigerator for 15 minutes and washed in $10 \mathrm{ml}$ of isolation buffer and centrifuged at $300 \mathrm{x} \mathrm{g}$ for 10 minutes. The supernatant was aspirated completely and samples were resuspended in $500 \mu \mathrm{l}$ of isolation buffer followed by magnetic separation. Before the sample was processed through the LS column it was prepared by rinsing with $3 \mathrm{ml}$ of isolation buffer. The sample was pipetted onto the column (on the magnet) and the flow through was collected as unlabelled cells (Ly6G-) after which the column was rinsed 3 times with $3 \mathrm{ml}$ of isolation buffer. The column was then removed from the magnet and placed on a second collection tube, $5 \mathrm{ml}$ of isolation buffer was pipetted onto the column and using the column plunger the Ly6G+ cells were flushed out of the column. This process was completed over a second LS column which increased the purity of the Ly6G+ cells.

Following isolation of Ly6G+ cells the Ly6G- fraction from above was purified to isolate the Gr1dim population. This was done by first washing the Gr1dim cells by centrifugation at $300 \mathrm{x} \mathrm{g}$ for 10 minutes and aspirating the supernatant completely. The sample was then resuspended in 400 $\mu \mathrm{l}$ of isolation buffer (per 100 million starting cells) and $100 \mu \mathrm{l}$ of anti-Gr1 biotin was added and mixed then left to incubate in the refrigerator for 10 minutes. The sample was then washed with 10 $\mathrm{ml}$ of isolation buffer and spun at $300 \mathrm{x} \mathrm{g}$ for 10 minutes and the supernatant was aspirated, this was followed by resuspension in $900 \mu \mathrm{l}$ isolation buffer and $100 \mu \mathrm{l}$ of streptavidin microbeads, the sample was then mixed well and left to incubate in the refrigerator for 15 minutes. After the incubation the sample was washed in $10 \mathrm{ml}$ of isolation buffer and centrifuged at $300 \mathrm{x} \mathrm{g}$ for 10 minutes, the supernatant was removed and the sample was resuspended in $1 \mathrm{ml}$ of isolation before proceeding to magnetic separation of Gr1dim cells. An MS column was placed on the magnetic field and prepared by rinsing with $3 \times 500 \mu 1$ of isolation buffer. The sample was then placed on the column and the unbound cells were collected. The column was then removed from the magnet and placed on a collection tube. To collect the Gr1dim cells from the column $1 \mathrm{ml}$ of isolation buffer was added followed by applying the plunger which pushed the Grldim cells from the column. This process was repeated using a fresh MS column to increase the purity of the Gr1dim population. After these two populations of MDSCs were isolated, the purity and populations were confirmed using flow cytometry. The Ly6G+ cells were confirmed as CD11b+Gr $1{ }^{\text {high }} \mathrm{Ly} 6 \mathrm{G}+$ cells (granular - see appendix 3) and the Gr1dim cells were confirmed as CD11b+Gr1 ${ }^{\text {int/dim } L y 6 G-~}$ Ly6C+ cells (monocytic - see appendix 3). 


\subsubsection{In vitro $\mathrm{CD} 4+\mathrm{T}$ cell proliferation assay}

Splenocytes or CD4+ T cells were stained with CFSE following 2.5.1 and plated into a 96-well plate at either $1 \times 10^{6}$ or $1 \times 10^{5}$ respectively. Splenocytes were cultured with $1 \mu \mathrm{g} / \mathrm{ml}$ of ConA for 48-hours and CD4 $\mathrm{T}$ cells were stimulated with $2.5 \times 10^{4} \mathrm{CD} 3 / \mathrm{CD} 28$ activator beads for $48-72$ hours. After the incubation period plates were spun at $400 \mathrm{x}$ g for 5 minutes and samples were resuspended in extracellular stain in FACs buffer and following a staining period of 25 minutes on ice the samples were centrifuged at $400 \mathrm{x} g$ and resuspended in FACs buffer ready for analysis by flow cytometry.

\subsection{Enzyme linked immunosorbent assays (ELISA)}

ELISAs were conducted as per the manufacturer's instructions (see Appendix 8; BD Bioscience). In short, 96 well ELISA plates (BD Biosciences or Thermo Fisher) were coated overnight with 50 $\mu 1$ of a primary purified "capture" antibody per well diluted in a sodium phosphate buffer (see appendix 6), either $\mathrm{pH} 6$ or 9 , and incubated at $4^{\circ} \mathrm{C}$. The next day, the capture antibody was removed and the plates were coated in excess blocking buffer containing FCS either 5 or 10\% in PBS for 2 hours at room temperature. After the 2 hour incubation the blocking buffer was removed and the plates were washed 4 times in a PBS wash buffer containing 0.05\% Tween 20 (Sigma). After the plates had been washed the standards and samples were added in a $50 \mu \mathrm{l}$ volume and left for 2 hours at room temperature or overnight at $4^{\circ} \mathrm{C}$, all dilutions for the standards and samples were done in blocking buffer. After the 2 hour standards and samples incubation or the following day, the plates were washed another 4 times in wash buffer and a biotinylated detection antibody was added in blocking buffer and incubated at room temperature for a further 1 hour. Following the 1 hour incubation, the detection antibody was removed and the plate was washed a further 6 times before streptavidin conjugated horse-radish peroxidase was added and left in the dark at room temperature for another 1 hour incubation. Finally, the streptavidin was removed and the plates were washed a further 8 times before tetramethyl benzidine (TMB) reagents A and B (BD Bioscience) were mixed in equal volumes and $100 \mu \mathrm{l}$ was added to each well. This was followed by $100 \mu \mathrm{l}$ of stop solution ( $0.18 \mathrm{M}$ sulphuric acid; see appendix 6) once sufficient colour had developed. Absorbance values were measured at $450 \mathrm{nM}$ using an EnSpire 2300 multilabel plate reader (PerkinElmer). A standard curve was constructed from the absorbance values of the standards and cytokine concentrations were calculated using the Enspire Software (PerkinElmer). A complete list of ELISA antibody combinations see appendix 8.

\subsection{Cytokine multiplex assays}

Murine cytokines were assessed in the serum by cytokine bead assay using a using a custom cytokine bead assay matrix (Becton Dickinson CBA Flex Sets TM). Protocol was conducted as per 
manufacturer's instructions and data was collected by Rebecca Girvan at Innate Immunotherapeutics (Auckland, NZ). The detection limit for cytokines assessed ranged from 2-20 $\mathrm{pg} / \mathrm{ml}$ as was indicated in the instruction booklet.

\subsection{Griess reaction}

The measurement of nitric oxide (NO) is difficult to detect as it is unstable and rapidly degrades to nitrate and nitrites, therefore measuring these breakdown products is a relative measure of NO production. Using a Griess assay we are able to measure nitrite and this is commonly used as an indicator of NO production within cell culture supernatant (Sun, Zhang, Broderick, \& Fein, 2003). This was done by combining Griess reagents A and B (see appendix 6) in equal volume followed by $50 \mu 1$ of this mixture being added to $50 \mu 1$ of culture supernatant in a flat bottom 96-well plate (BD Bioscience). The standard for this assay was serial dilutions of sodium nitrite performed in duplicate. Absorbance was measured at $570 \mathrm{nM}$ using an EnSpire 2300 multilabel plate reader (PerkinElmer) and a standard curve of nitrite standards was produced which allowed the determination of the unknown sample nitrite concentrations using the Enspire Software (PerkinElmer). The detection limit of this assay was $2-4 \mu \mathrm{M}$.

\subsection{Flow cytometry}

\subsubsection{Flow cytometry experimental set up}

FACs Canto II (BD Biosciences) flow cytometer was maintained at the Victoria University of Wellington flow cytometry suite and quality control measures were in place to ensure the ongoing reliability of the machine. Daily calibration was performed using cytometer set up and tracking (CST) beads. Experiments were set up using the Diva software (BD Biosciences), including the optimisation of PMT voltages to ensure that for each fluorophore unstained cells sat at $2.5 \mathrm{x}$ RSD of the electronic noise, to ensure that dim populations can be correctly identified. Each flow cytometry experiment included a set of single stained cells for compensation and these cells were treated in the same manner as the samples (i.e. fix/permeabilised controls in intracellular experiments). Manual compensation was performed using the Diva software and single stained samples were recorded for future compensation in Flowjo (Tree Star).

Antibodies were titrated prior to use to identify the concentration which had the best signal with minimal background staining (i.e. the highest signal to noise ratio). The titrated antibodies were then tested in the desired mixed antibody panel to ensure the optimum detection of each cell population was achieved. Furthermore, given that particular antigens have variable expression, such as PD-L1 and MHC, and intracellular antibodies have a high level of background, fluorescence minus one (FMO) controls plus isotype antibodies were used for each sample for 
gating accuracy. As shown in Appendix 1 the geometric mean of PD-L1 was determined by subtracting the geometric mean of the isotype control from the PD-L1 geometric mean, which accounts for any differences in background fluorescence in that channel. Similarly, FoxP3 and IFN- $\gamma$ positive cells were gated on using an FMO for these markers to ensure these events represented true positives and not background straining (Appendix 1).

\subsubsection{Staining of leukocytes for flow cytometry}

Cells for flow cytometry were plated at $1 \times 10^{6}$ cells/well in a U-bottomed 96-well plate. The cells were washed in FACs buffer (see appendix 6) and centrifuged at $400 \mathrm{x}$ g for 5 minutes. The supernatant was flicked out and plates were vortexed to resuspend cells in residual FACs buffer. The samples were stained with primary antibodies in $50 \mu 1$ of FACS buffer and left on ice for 25 minutes in the dark. All the antibodies used were titrated prior to experimentation which minimised background fluorescence and maximised separate between cell populations. The cells were washed by adding $150 \mu \mathrm{l}$ FACs buffer and centrifuged at $400 \mathrm{x}$ g for 5 minutes followed by streptavidin conjugated fluorophores if secondary antibodies were required which were left on ice in the dark for another 25 minutes. The cells were washed again in excess FACs buffer and centrifuged at $400 \mathrm{x}$ g and resuspended in $200 \mu 1$ FACs buffer. The samples were filtered through mesh into a clean 96-well plate or FACs tubes and analysed on a FACs CantoII (BD Biosciences). Data was analysed using FlowJo software version 7 or X (Tree Star). See appendix 7 for a complete list of antibodies used.

\subsubsection{Cell cycle analysis using Vybrant ${ }^{\circledR}$ DyeCycle ${ }^{\mathrm{TM}}$ Green stain}

The cells were placed in $500 \mu 1$ of PBS (see appendix) and $1 \mu 1$ of DyeCycle green (Thermo Fisher) was added, left to incubate at room temperature in the dark for 1 hour. If multivariate analysis was performed the cells were stained in PBS and washed first.

\subsection{Graphs and statistics}

All graphs and statistics were generated using GraphPad Prism 5 (GraphPad Software Inc., La Jolla, CA, USA). Many of the figures presented in this thesis were generated and statistically analysed by combining experimental replicates (i.e mouse disease scores from independent experiments were graphed together if the experimental conditions were identical) which accounts for the day to day variations and increases statistical power. If the sample size was large enough a Shapiro-Wilk normality test was used to determine if the values formed a Gaussian distribution. To compare two groups an unpaired Student's t-test was used. For experiments in which more than two groups were compared a 1-way ANOVA with a Bonferroni post-test was used if the groups has equal sample sizes or a Tukey's post-test if group sizes were unequal. For samples which were 
non-normally distributed a non-parametric a Kruskal-Wallis test with Dunn's post-test was used in place of a 1-way ANOVA. For the comparison of 2 variables (e.g. treatment and time), a 2-way ANOVA with Bonferonni's post-test was used. Differences which gave $\mathrm{p}$ values of $<0.05$ were deemed statistically significant. 


\section{Chapter 3: Identifying an effective dose regime for MIS416 using experimental autoimmune encephalomyelitis}




\subsection{Introduction}

MIS416, which was originally developed as a vaccine adjuvant for cancer therapy, has been identified as a potential immunomodulatory agent for the treatment of chronic inflammatory disorders, such as multiple sclerosis (MS) and Crohn's disease. MIS416 has been approved for compassionate use (under section 25 and 29 of NZ medicines Act 1989, which allows the use of an unapproved experimental medicine when prescribed by a medical practitioner) and a preliminary cohort of 8 patients suffering progressive forms of MS were administered with MIS416 intravenously once a week for at least 12 weeks. These patients tolerated MIS416 treatment and self-reported improvements in their disease symptoms (personal communication; Dr Gill Webster, Innate Immunotherapeutics, Auckland NZ). The preliminary results from these patients led Innate Immunotherapeutics to run an open label, dose-escalation phase 2a trial of MIS416 in patients with progressive MS. This trial demonstrated that MIS416 was safe and well tolerated in these patients and there was evidence of disease improvements. Furthermore, many of these patients wished to continue treatment with MIS416 after the trial had ended suggests that MIS416 could be an effective novel treatment for progressive MS and immune modulating effects should be assessed further. However, although we know that MIS416 activates TLR-9 and NOD-2 pathways, its full effects on the immune system are not well understood. Therefore, this chapter is aimed at identifying an effective dose and dosing regimen in the animal model of MS, experimental autoimmune encephalomyelitis (EAE).

EAE is a widely accepted model of MS. Although some aspects, such as spontaneity, cannot be accounted for in EAE, it is an effective way to assess the efficacy of a treatment as many of the current front-line therapeutics for MS have utilized EAE at some stage during their development and all have shown efficacy within the model (L. Steinman \& Zamvil, 2005). Because different mouse strains model different aspects of MS disease, such as the relapsing-remitting form in the SJL strain, it is possible to assess the effect a novel treatment may have on a certain type of disease, making the EAE model a useful tool for MS studies. By using the EAE model in mice we hope to be able to find a MIS416 treatment regime that shows consistent efficacy, and use this regime to understand further how MIS416 is able to alter the immune system in a way that reduces EAE disease. Furthermore, as anecdotal evidence from the compassionate use patients suggests that MIS416 appears to be effective in MS patients, the focus in these studies is not to show efficacy to justify translation into humans but to dissect MIS416 immunomodulatory effects.

\subsection{Aims}

This chapter explores the use of the microparticle, MIS416, at varying concentrations and timing in different strains of mice, which model distinct aspects of MS. These results will allow us to 
determine the optimal treatment regime that provides consistent and long term protection against the development of EAE in mice. Using this treatment regime, we can then begin to determine how MIS416 provides disease protection in EAE. Furthermore, we will start to understand how the innate immune system can be modified by MIS416 in a way that can reduce autoimmune disease.

\subsubsection{Specific Aims}

1. To determine the minimal dose of MIS416 that is effective at reducing disease in C57BL/6 mice with EAE.

2. To evaluate whether multiple dosing is more effective than a single dose at day 0 in C57BL/6 mice with EAE.

3. To evaluate the effect of MIS416 on disease progression in different mouse strains which model different aspects of MS disease.

4. To determine whether the TLR9 activity is essential for MIS416 disease protection. 


\subsection{Results}

\subsubsection{Dose response when MIS416 is given at day 0 in C57BL/6 mice with EAE}

Previous research using MIS416 in the EAE model indicated that a single high dose of MIS416 $(250 \mu \mathrm{g})$ administered on the day of EAE immunisation but not 3 days before or 7 days after was effective at reducing disease (White et al., 2014). Thus, it was important to determine if this high dose was required for the efficacy of MIS416 in the C57BL/6 model or if a lower single dose was still effective when administered on the day of immunisation. In this strain of mice, immunisation with EAE develops as a consistent progressive paralysis between days 8-14 post-immunisation in $90-100 \%$ of the immunised mice. Initially, the doses of MIS416 used were $50 \mu \mathrm{g}, 100 \mu \mathrm{g}$ and 250 $\mu \mathrm{g}$ as these had been shown to be safe in early toxicity studies completed in mice and rabbits.

As shown previously, mice which received a high dose of $250 \mu \mathrm{g}$ of MIS416 intravenously (i.v) on the day of EAE immunisation (i.e day 0) showed a reduction and slight delay in EAE disease onset however, due to the small sample size in this experiment the difference did not reach significance unlike published data in which a larger sample size was used (White et al., 2014). In comparison, the treatment with the lower doses of MIS416 followed a similar disease course to untreated mice (Figure 3-1). This result indicates that when given as a single bolus, MIS416 was only effective at the highest dose (i.e $250 \mu \mathrm{g}$ ). Furthermore, as these early preliminary studies showed that control mice receiving saline i.v had the same disease progression as untreated mice (O'Sullivan, 2012), we did not include a saline injected control group in further studies to reduce unnecessary pain and manipulation of the mice.

For MS, it is important to show efficacy in a therapeutic setting as the treatments need to be able to alter on-going disease progression, particularly in patients with the progressive form of MS. To test whether MIS416 had efficacy when given in a therapeutic setting (i.e after disease onset) we treated mice with a single bolus of MIS416 on day 12 post immunisation (p.i), which was disease onset for the majority of the EAE mice. As seen when administered at day 0 , mice which received the $250 \mu \mathrm{g}$ dose of MIS416 at day 12 showed a reduction in disease score compared to the control EAE mice (Figure 3-2) again, due to the small sample size in this experiment the difference did not reach significance unlike published data in which a larger sample size was used (White et al., 2014). It also appeared that the lower doses of 50 and $100 \mu \mathrm{g}$ showed some efficacy at reducing disease when given at day 12. However, the higher dose of $250 \mu \mathrm{g}$ showed a more consistent reduction in disease score over a longer period of time, up to day 38 p.i (Figure 3-2). Together, these results show that MIS416 treatment was effective in the EAE model when a single high dose $(250 \mu \mathrm{g})$ is given prophylactically on day 0 or therapeutically on day 12 . 
A
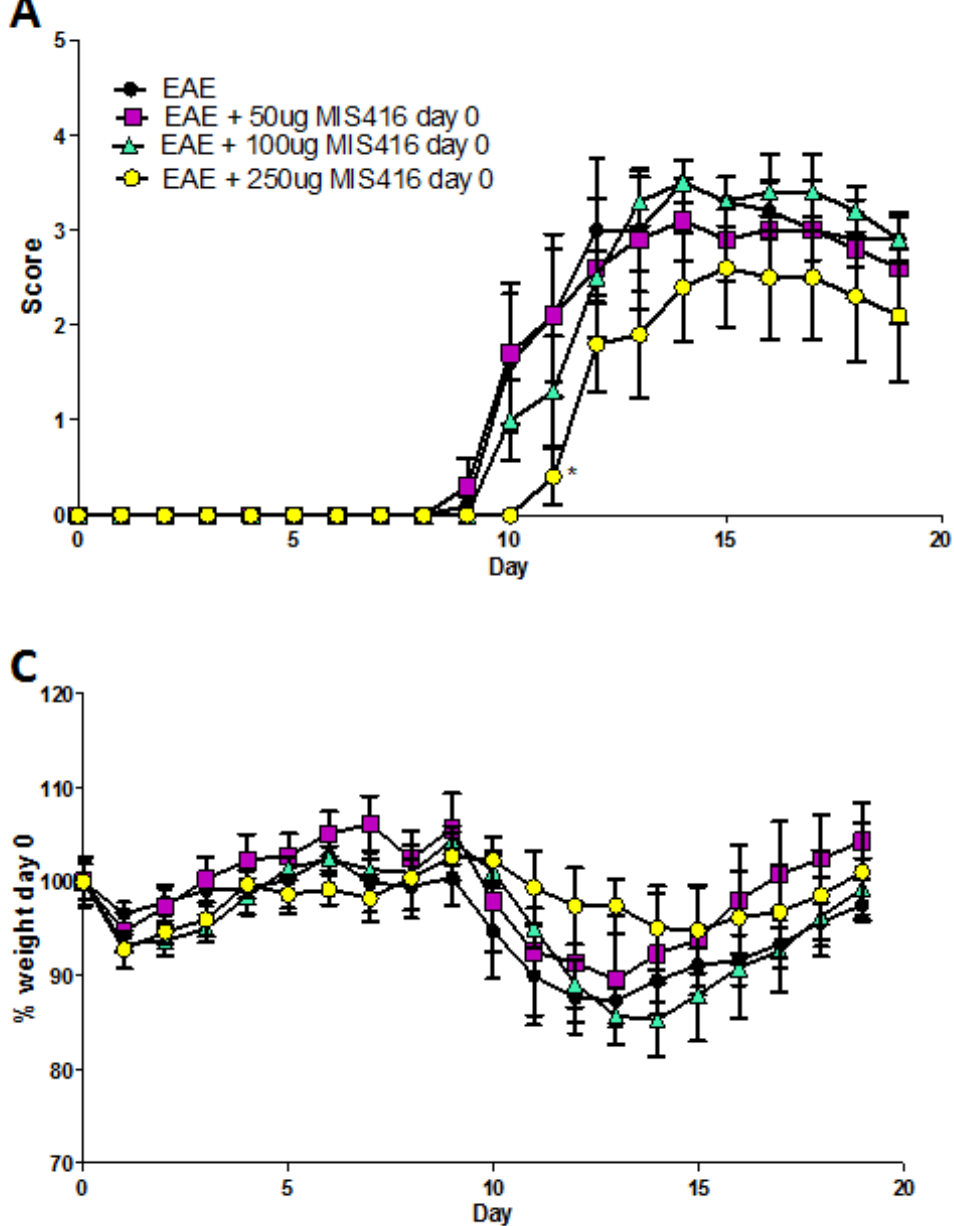

B

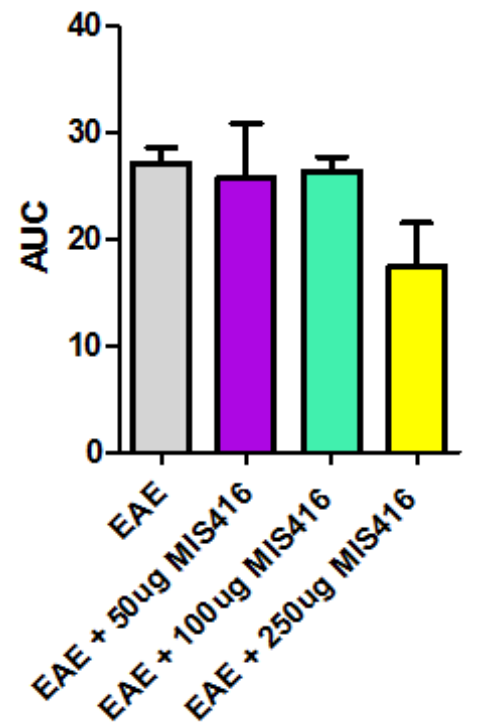

Figure 3-1 $250 \mu \mathrm{g}$ dose of MIS416 was effective at reducing disease in C57BL/6 mice when given at day 0 .

C57BL/6 mice were immunised for EAE and treated with either 50, 100 or $250 \mu \mathrm{g}$ MIS416 i.v on day 0. Mice were weighed and scored daily. A) Disease score of all mice, day 11 showed a significant reduction in disease between untreated and $250 \mu \mathrm{g}$ MIS416 using a 2-way repeated measures ANOVA with a Bonferronis multiple comparisons post-test, the overall effect of treatment between these two groups was also not significant $(\mathrm{p}=0.0582)$. B) Cumulative disease burden of mice; measured by the area under the curve. NS using 1-way ANOVA with Bonferroni post-test to compare EAE to treated groups. C) Percentage weight change; calculated as $\%$ of baseline weight where $100 \%$ was weight at day 0 . Shown are the means \pm SEM of one experiment $(n=5$ mice per group). 

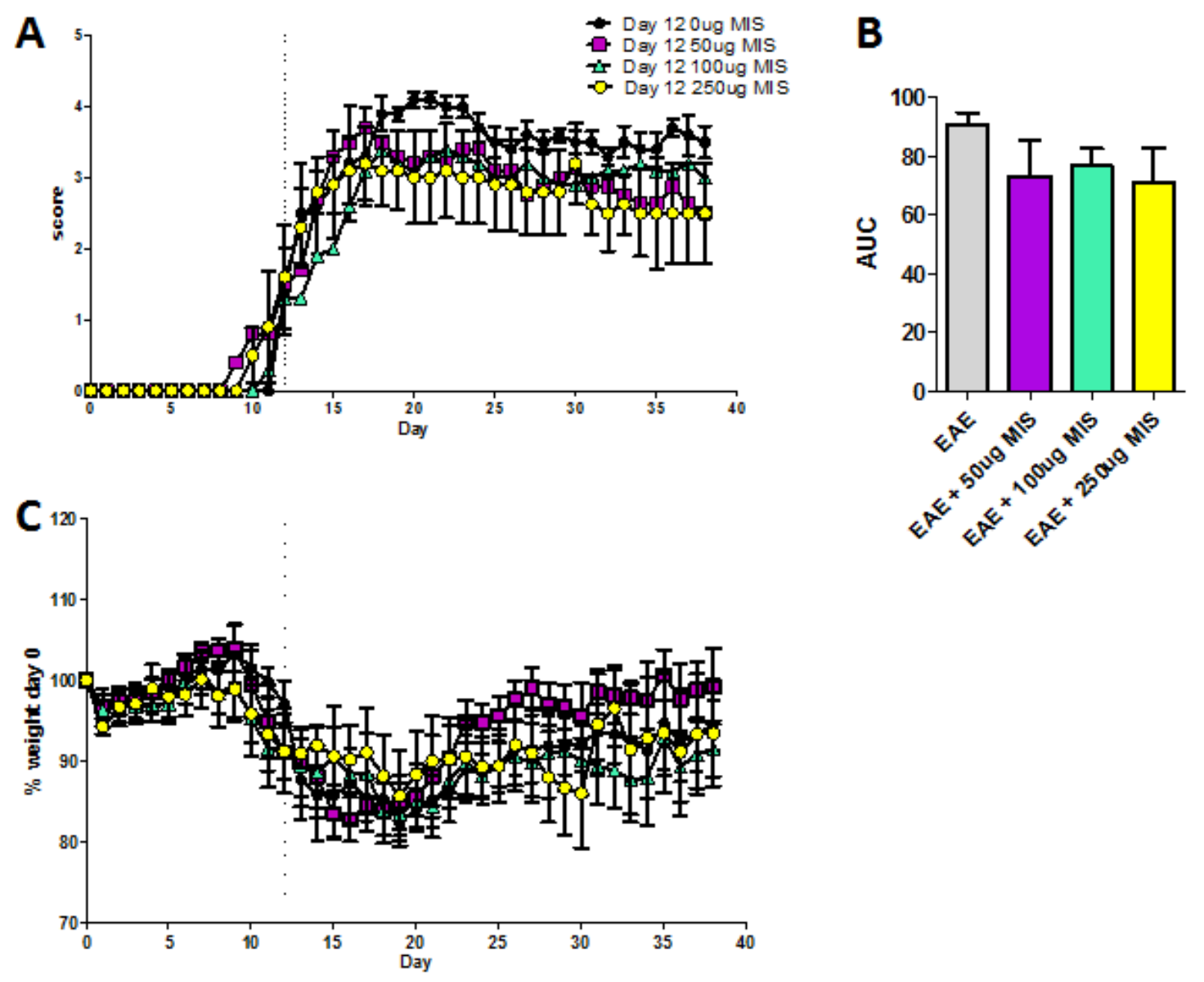

Figure 3-2 $250 \mu \mathrm{g}$ dose of MIS416 reduced disease in C57BL/6 mice when given at day 12.

C57BL/6 mice were immunised for EAE and treated with either 50, 100 or $250 \mu \mathrm{g}$ MIS416 i.v on day 12, as indicated by the dotted line. Mice were weighed and scored daily. A) Disease score of all mice, NS by 2-way repeated measures ANOVA with Bonferronis multiple comparisons post-test. B) Cumulative disease burden of mice; measured by the area under the curve. NS using 1-way ANOVA with Bonferroni post-test to compare EAE to treated groups. C) Percentage weight change; calculated as $\%$ of baseline weight where $100 \%$ was weight at day 0 . Shown are the means \pm SEM of one experiment $(n=5$ mice per group). 


\subsubsection{A $100 \mu \mathrm{g}$ weekly dose of MIS416 is the most effective dose in C57BL/6 EAE mice}

Although a single dose of MIS416 administered on day 0 significantly reduced disease in the EAE model, over time the protective effect of MIS416 appeared to wane (White et al., 2014). Therefore to determine if repeated administration of MIS416 would provide lasting efficacy, and to mimic the treatment regime that is used in secondary progressive patients in the compassionate use program, a single $250 \mu \mathrm{g}$ dose was compared to a weekly $250 \mu \mathrm{g}$ or $100 \mu \mathrm{g}$ dose. While it appeared that the high weekly dose was more effective than a single high dose with no mice showing signs of disease by the end of the experimental time at day 22 , this regimen was fatal for about $80 \%$ of the mice tested, and only became evident within 24 hours after the $3^{\text {rd }}$ dose (i.e day 15) (Figure 3-3A\&B). This finding suggests that with the higher dose, there are cumulative immune effects that become lethal only after multiple high doses.

In contrast to the weekly high dose, mice which receive the weekly low dose had a slight delay in onset of disease symptoms, but did not develop as severe disease as that seen in untreated mice. Moreover, even after the $3^{\text {rd }}$ dose, the mice consistently appeared to recover slightly and no lethality was seen, as evidenced over 3 independent experiments (Figure 3-3C). These results suggest that MIS416 treatment in the C57BL/6 EAE model at a weekly dose of $100 \mu \mathrm{g}$ is sufficient to reduce the disease burden significantly and consistently. Furthermore, these results also indicate the MIS416 treatment may drive disease resolution as there was a reduction in disease score even after established disease. 

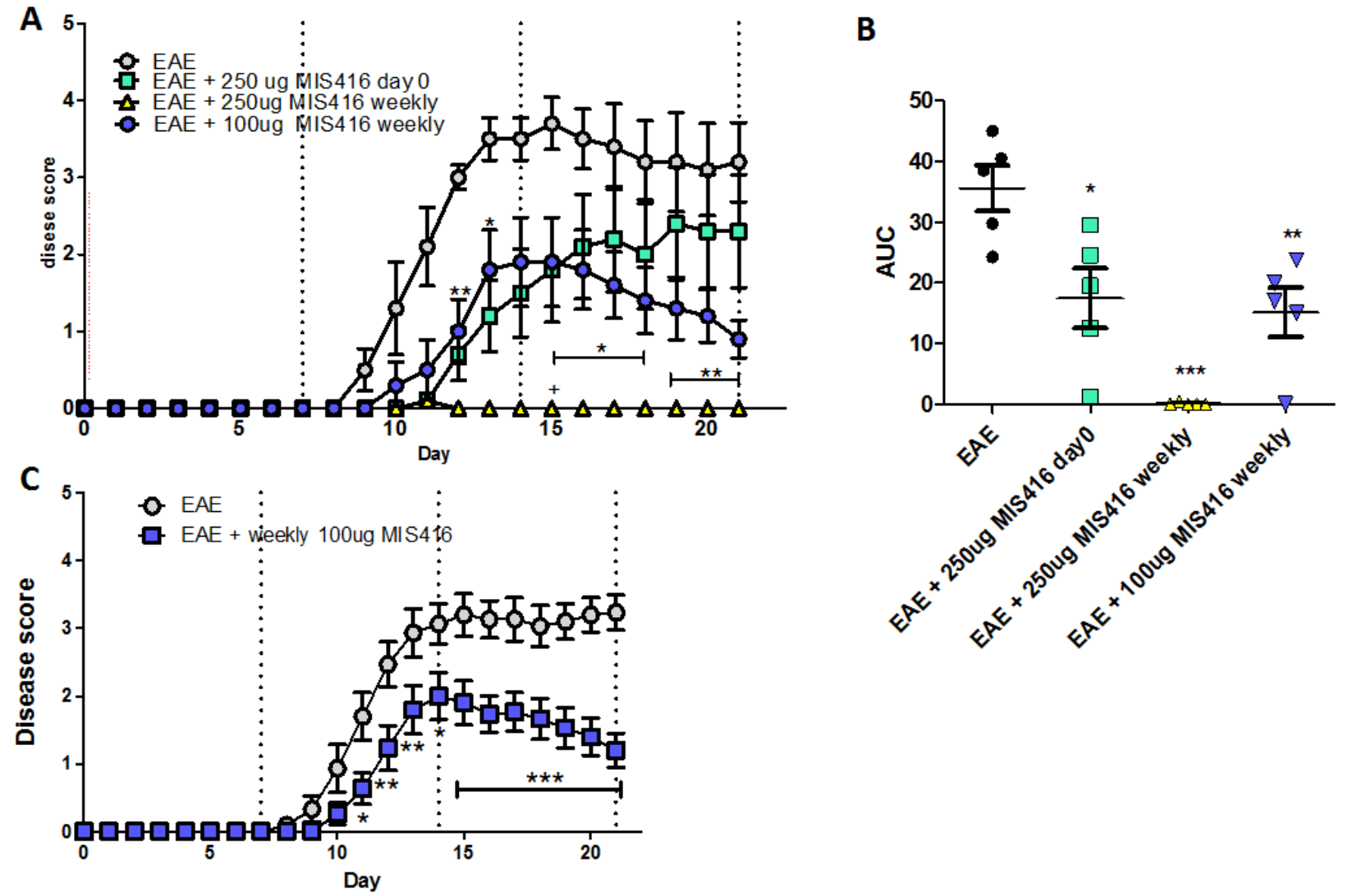

Figure 3-3. Weekly doses of $100 \mu \mathrm{g}$ of MIS416 was the most effective treatment in C57BL/6 EAE mice.

C57BL/6 mice were induced with EAE at day 0 and given either a single dose or weekly doses of MIS416 beginning at day 0 and repeat doses were performed on days indicated by vertical dotted lines. Mice were weighed and scored daily. A) Disease scores of all mice; + represents where lethality occurred in the $250 \mu \mathrm{g}$ weekly treated mice. B) Cumulative disease burden of mice; measured by the area under the curve (AUC). C) Disease scores of EAE and EAE + $100 \mu \mathrm{g}$ MIS416 weekly. Shown are the disease scores (A\&C) or area under the curve (B) \pm SEM. Data represents a single experiment ( $n=5$ mice per treatment group) for A and B and 3 independent experiments for $\mathrm{C}\left(\mathrm{n}=15\right.$ mice/group). ${ }^{*} \mathrm{p}<0.05, * * \mathrm{p}<0.01$ and $* * * \mathrm{p}<0.001$ Untreated EAE mice compared to MIS416-treated EAE mice by 2-way repeated measures ANOVA with Bonferronis multiple comparisons post-test (A\&C) and a 1-way ANOVA with Bonferroni post-test was used to compare EAE to MIS416-treated groups (B). 


\subsubsection{Antigen specific responses in C57BL/6 EAE mice with weekly MIS416 doses}

As part of this chapter is aimed at setting up a model in which the mechanism of MIS416 can be assessed, the effect MIS416 treatment has on the antigen-specific cytokine response was an important factor to support the reduced disease burden seen in these mice. Therefore, using the weekly low dose of MIS416 (i.e $100 \mu \mathrm{g}$ /mouse), the systemic antigen-specific response was assessed at various time points post-immunisation to identify whether MIS416 treatment also altered this measurement of disease. This was done by restimulating splenocytes and liver leukocytes with the EAE immunising antigen, MOG, and assessing the level of cytokines within the culture supernatant at the time points indicated.

In mice which received MIS416 treatments weekly (i.e $100 \mu \mathrm{g}$ ) there was a significant reduction in the MOG-specific IFN- $\gamma$ production seen in the MIS416-treated mice compared to control EAE mice at both the day 15 (peak disease) and day 22 (chronic disease) time points (Figure 3-4A). A similar reduction was seen in the IL-17A MOG-specific response as well however, the IL-17A response appeared to peak at the day 15 time point with much less IL-17A being produced by both groups of mice in response to MOG at the day 22 time point (Figure 3-4B). We also assessed the nitric oxide (NO) production in response to MOG using a Griess assay. Interestingly, in response to MOG, mice which received MIS416 treatment in vivo had significantly lower NO production compared to control mice at day 15, however, by day 22 these were reversed (Figure 3-4C). Together the reduced IFN- $\gamma$ and IL-17A response in MIS416-treated EAE mice support the reduced disease scores seen in these mice and therefore further indicate that this weekly dose is a good model for assessing the mechanism of MIS416 in EAE.

Early toxicology studies have indicated the liver as an important site for MIS416 deposit and therefore we also compared the MOG response within the liver of MIS416-treated EAE mice to EAE control mice. Similar to our findings in the spleen, there was a significant reduction in IFN- $\gamma$ production in response to MOG in the MIS416-treated mice at both the day 15 and day 22 time points (Figure 3-5A). Likewise with IL-17A, there were also significant reductions with MIS416 treatment (Figure 3-5B). In contrast to IFN- $\gamma$ and IL-17A, we found that immune cells from the livers of MIS416-treated mice produced much more NO particularly at the disease onset time point (day 15) (Figure 3-5C). These results show that while the liver and spleen may constitute different immune environments, they both have the ability to develop an antigen-specific response to the EAE antigen MOG, and have similar cytokine but not nitric oxide responses to MOG with MIS416 treatment. 
A

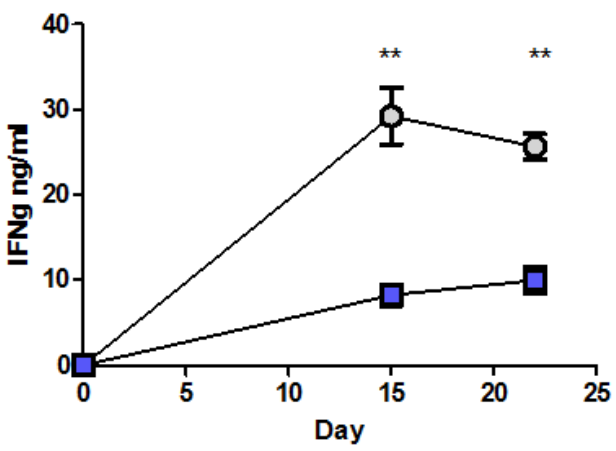

C

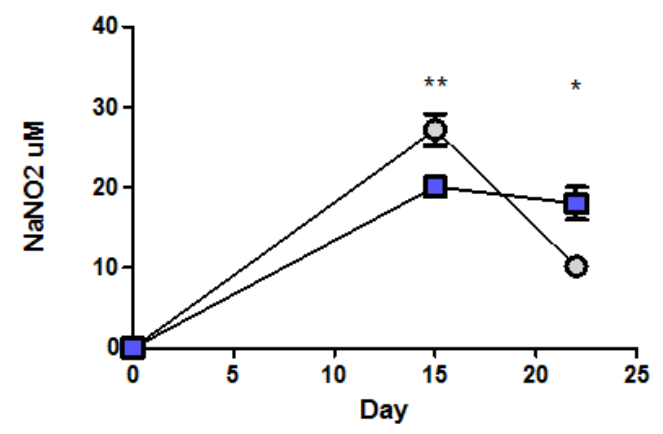

B

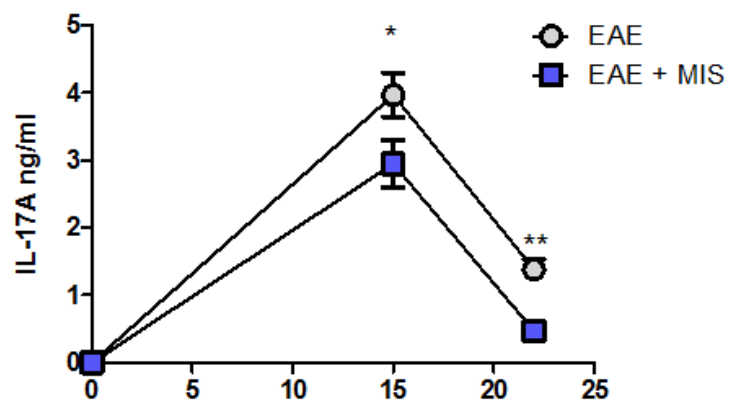

Figure 3-4 MIS416 treatment significantly altered splenocytes cytokine production in response to MOG at day 15 and 22.

EAE was induced in C57BL/6 mice and MIS416 treatment began at day 0, one day after a MIS416 dose splenocytes were harvested and cultured at $1 \times 10^{6}$ cells/well and stimulated with $27 \mu \mathrm{g} / \mathrm{ml} \mathrm{MOG}$ and supernatant was analysed 72 hours later A) IFN-g levels from ELISA, B) IL-17A levels from ELISA, C) NO (nitrate) production as measured by Griess reaction. Results are from between 1 and 3 experiments per time point. Shown are the means \pm SEM $(n=5$ day $15, n=10-15$ day 22 , in triplicate). $* \mathrm{p}<0.05$ and $* * * p<0.001$ EAE was compared to MIS416-treated EAE by 2-way ANOVA with Bonferroni's multiple comparison post-test. 
A

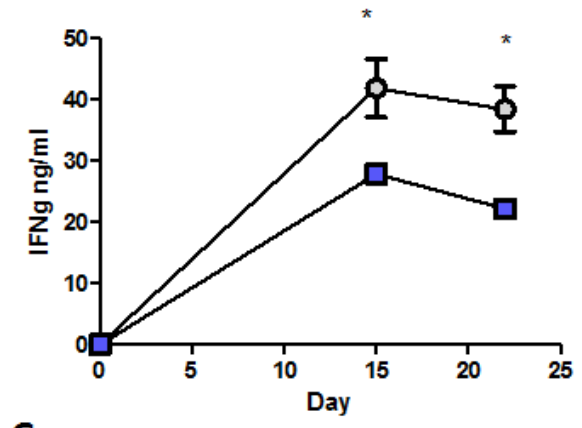

C

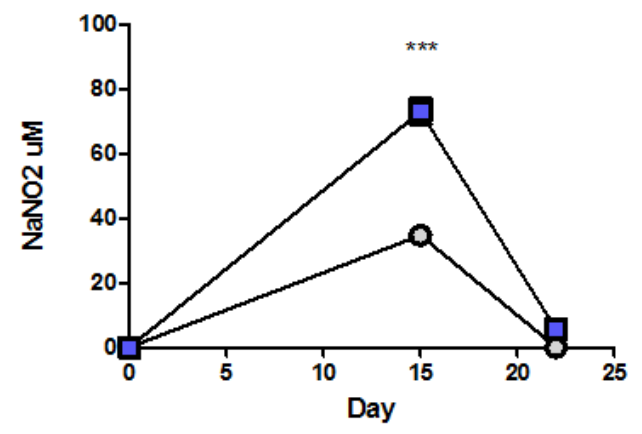

B

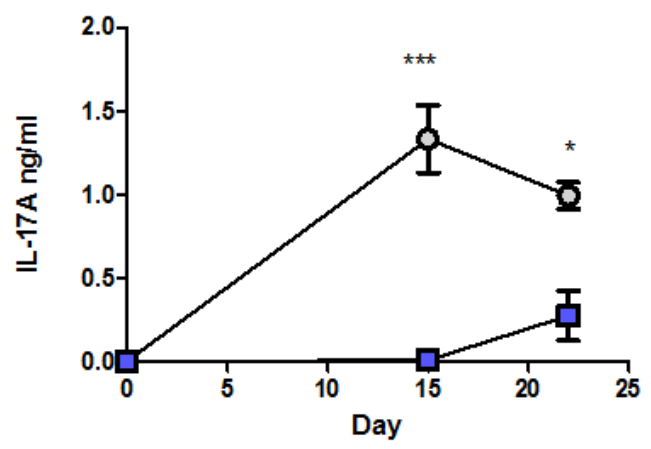

Figure 3-5 MIS416 treatment significantly altered liver cells cytokine production in response to MOG at day 15 and 22.

EAE was induced in C57BL/6 mice and MIS416 treatment began at day 0, one day after a MIS416 dose liver cells were harvested and cultured at $2 \times 10^{5}$ cells/well and stimulated with $27 \mu \mathrm{g} / \mathrm{ml}$ MOG and supernatant was analysed 72 hours later A) IFN-g levels from ELISA, B) IL-17A levels from ELISA, C) NO (nitrate) production as measured by Griess reaction. Results are from one experiment for each time point. Shown are the means \pm SEM ( $n=3) * p<0.05$ and $* * * p<0.001$ EAE was compared to MIS416-treated EAE by 2-way ANOVA with Bonferroni's multiple comparison post-test. 


\subsubsection{MIS416 was ineffective at reducing EAE disease in BALB/c mice at either $100 \mu \mathrm{g}$ or $150 \mu \mathrm{g}$ weekly doses}

The BALB/c stain of mice develop a single episode form of EAE in which most of the CNS lesions are found within the brain and few in the spinal cord in comparison to the C57BL/6 model, where the mice develop a consistent ascending paralysis with the lesions predominately being found within the spinal cord (Orian et al., 2015). As we have shown that weekly treatments were effective at reducing EAE disease in the C57BL/6 model, we decided to determine whether MIS416 treatment was also effective in the BALB/c model using a similar dosing regimen.

Using the $100 \mu \mathrm{g}$ weekly dose and a higher dose of $150 \mu \mathrm{g}$, we found that MIS416 treatment was not protective in the BALB/c model (Figure 3-6A). However, comparing the mouse weights instead of the disease scores, the MIS416-treated mice at the $100 \mu \mathrm{g}$ dose did not lose as much weight as control mice during the disease onset suggesting that these mice might not be as sick as the disease scores imply however, this difference did not reach statistical significance (Figure 3-6B). In our lab we have found the EAE mice from the BALB/c stain difficult to score as they do not develop the classic ascending paralysis seen in the C57BL/6 model and therefore by looking at the disease scores alone may not be indicating the potential protection MIS416 may be having (Orian et al., 2015). Furthermore, studies in our lab have shown that weight is a better indicator of disease with decreasing weight and increasing lesions in the brain (Orian et al., 2015). From these results it appears that the C57BL/6 model is a better model of EAE to use in our pursuit of understanding the mechanism of action for MIS416 in the protection against EAE and MS. 


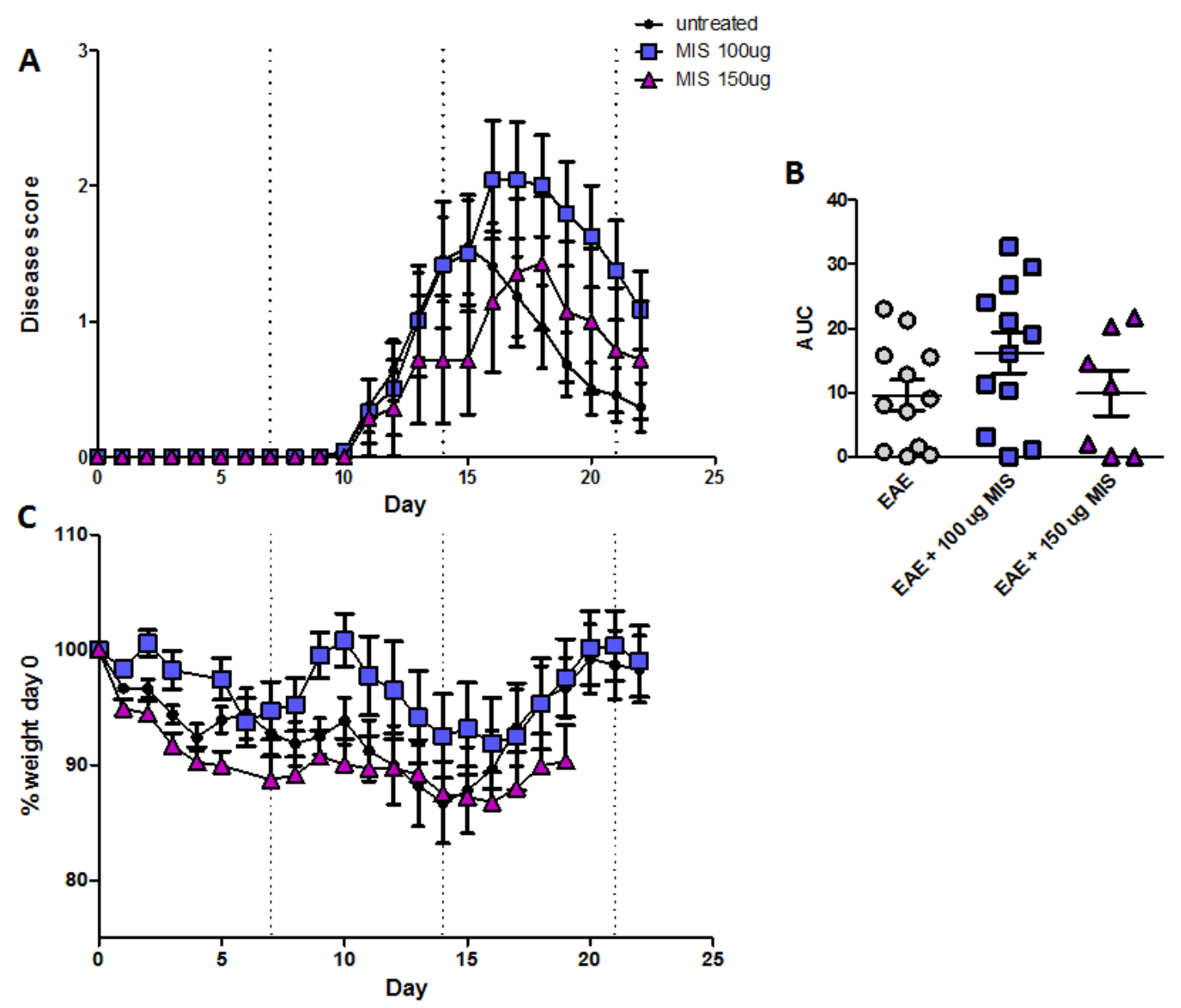

Figure 3-6 Weekly treatments of either $100 \mu \mathrm{g}$ or $150 \mu \mathrm{g}$ were ineffective in the BALB/c model of EAE

$\mathrm{BALB} / \mathrm{c}$ mice were immunised with EAE and given either 100 or $150 \mu \mathrm{g}$ dose of MIS416 weekly beginning on the day of disease induction, doses are indicated by the dotted lines. Mice were weighed and scored daily. A) Disease scores of all immunised mice. B) Cumulative disease burden as indicated by the area under the curve; NS using 1-way ANOVA with Tukey post-test to compare EAE to treated groups. C) Weight as a percentage of baseline for each treatment group. Shown are the disease score means \pm SEM of 1, $\mathrm{n}=7$ /group (150 $\mu \mathrm{g}$ dose) or 2 $\mathrm{n}=13$ /group $(100 \mu \mathrm{g}$ dose $)$ independent experiments. Data for disease scores and weight change NS using 2-way repeated measures ANOVA. 


\subsubsection{A $50 \mu$ g dose was most effective at reducing severity of relapses in SJL/J EAE mice}

EAE induced in SJL/J mice using PLP peptide (residues 139-151) results in a relapsing-remitting form of the disease which is characterised by an initial peak of disease symptoms followed by a recovery phase, after which most of the mice will suffer severe relapses. Preliminary experiments were performed with the SJL/J strain to optimise the immunisation protocol to achieve sufficient EAE disease in this model as the concentrations of adjuvant and antigen used to induce EAE in these mice vary substantially across lab groups. In the following experiments $100 \%$ of mice developed EAE disease, in which the first peak of disease all mice had a disease score of at least 3.5 .

MIS416 treatment began during the first remission stage of disease, at day 21. As SJL/J mice have previously been shown to be over sensitive to a $100 \mu \mathrm{g}$ weekly dose of MIS416 (data not shown), we chose a lower dose of $50 \mu \mathrm{g}$ MIS416/mouse weekly, however we did maintain the same injection volume of $100 \mu \mathrm{l}$ with this lower dose. SJL/J mice which received $50 \mu \mathrm{g}$ of MIS416 on day 21 had a delay in relapses and a reduced severity of these relapses compared to untreated control mice as seen by the disease scores over time (Figure 3-7A). Furthermore, MIS416 treatment significantly reduced the disease burden, as indicated by the area under the curve. These results were significantly different when comparing both the disease burden for the duration of the experiment (data not shown) and the disease burden as calculated from the start of weekly MIS416 treatment (Figure 3-7C). Additionally, MIS416 treatment significantly reduced the peak disease seen after the first remission, and first MIS416 treatment (Figure 3-7B), which suggests that MIS416 was able to reduce the severity of relapses in this model. Together, these results indicate that MIS416 is effective at reducing and altering disease in a relapsing-remitting model of MS.

The MOG-specific response was significantly reduced in the C57BL/6 model with MIS416 treatment and therefore we determined whether MIS416 treatment in the SJL model also reduced the antigen-specific cytokine response. The supernatants of splenocytes re-stimulated with PLP were analysed, and similar to the C57BL/6 model, we found significantly lower levels of antigenspecific IFN- $\gamma$ and IL-17A being produced in the mice that received MIS416 treatment in vivo compared to control mice (Figure 3-8A \& B). There was also an increase in the amount of NO and IL-10 produced by PLP-stimulated splenocytes from MIS416-treated mice compared to controls; however, this increase did not reach statistical significance (Figure 3-8C \& D). Together with the disease scores, these results show that MIS416 treatment was effective at reducing immune parameters associated with disease in the SJL relapsing-remitting model using a dose of $50 \mu \mathrm{g}$ weekly. These findings are important for the development of MIS416 as a therapeutic treatment as 
we have shown that MIS416 treatment was effective at reducing disease and antigen-specific cytokine response using weekly doses in two different mouse models of MS.

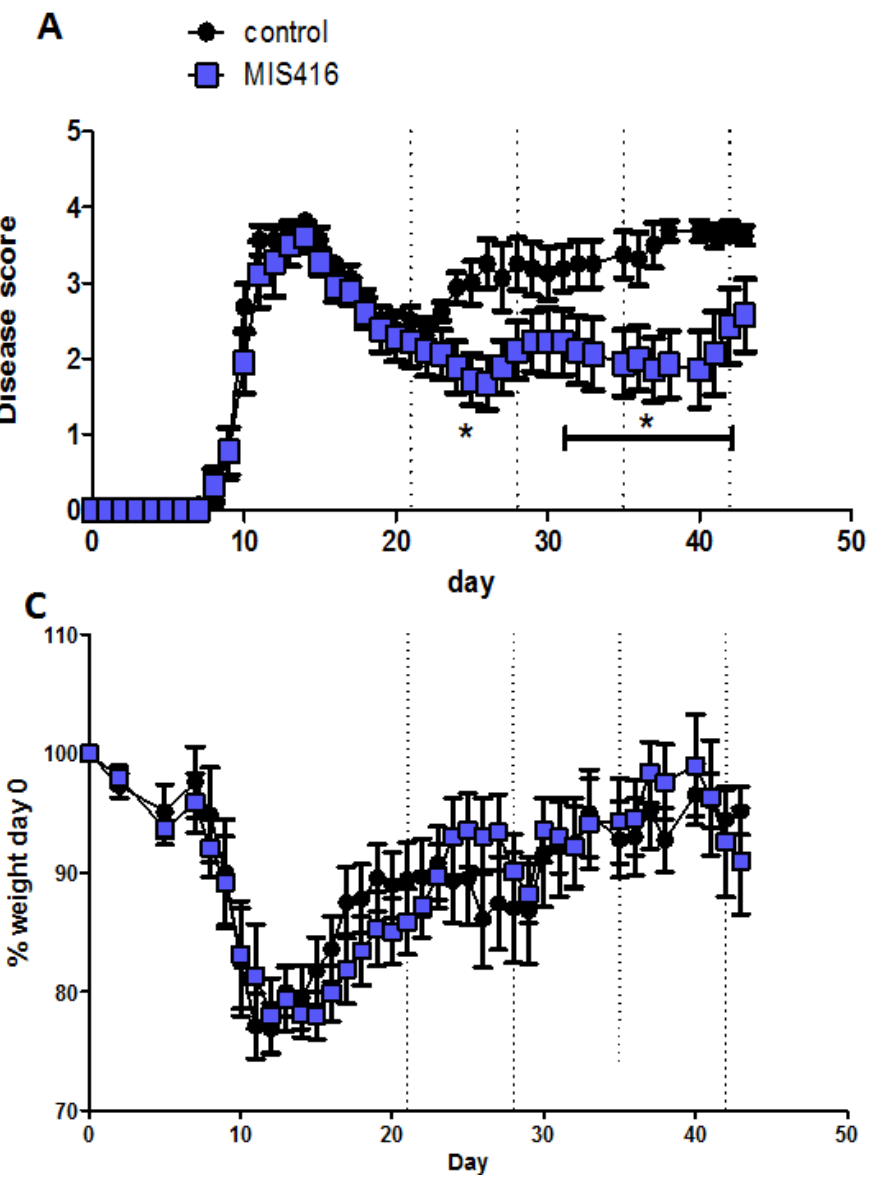

B

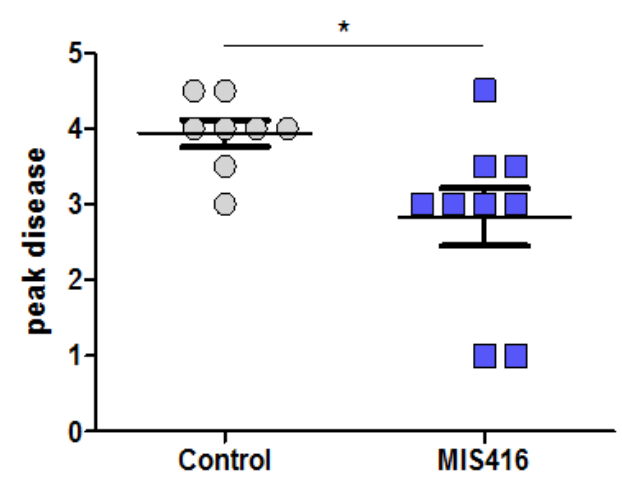

D

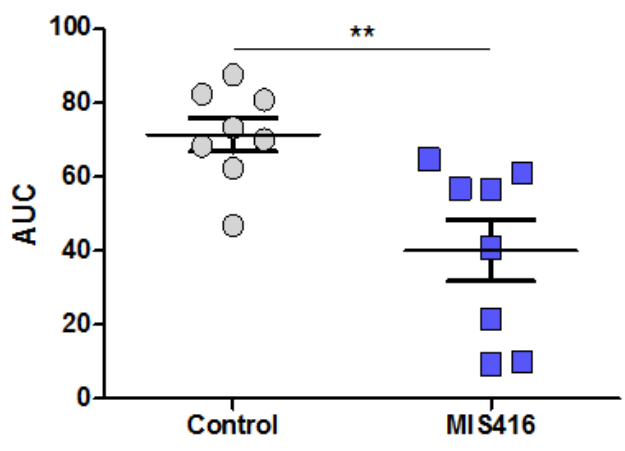

Figure 3-7 MIS416 was effective at reducing relapsing-remitting EAE disease in SJL/J mice when given 50 $\mu \mathrm{g} /$ mouse weekly doses beginning at day 21 .

$\mathrm{SJL} / \mathrm{J}$ mice were induced for EAE at day 0 and scored daily until they reached their first remission state at day 21 , then weekly $50 \mu \mathrm{g} /$ mouse MIS416 treatments were administered i.v. as indicated by the vertical dotted lines. A) Disease scores of all immunised mice with MIS416 treatment beginning at day 21 B) peak disease after MIS416 treatment began at day $21 \mathrm{C}$ ) weight as a percentage of baseline for each treatment group D) area under the curve of disease scores after MIS416 treatment. Shown are the means \pm SEM, $n=8-9 /$ group, 2 independent experiments. ${ }^{*} \mathrm{p}<0.05$ and $* * \mathrm{p}<0.01$ by 2 -way repeated measures ANOVA with Bonferronis multiple comparison post-test (A) or an unpaired Students t-test was used to compare control mice to MIS416-treated EAE mice (B\&D with welches correction for unequal variances in B). 
A

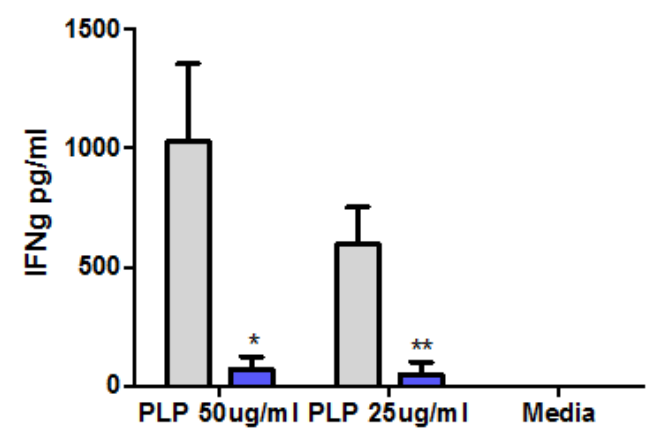

C

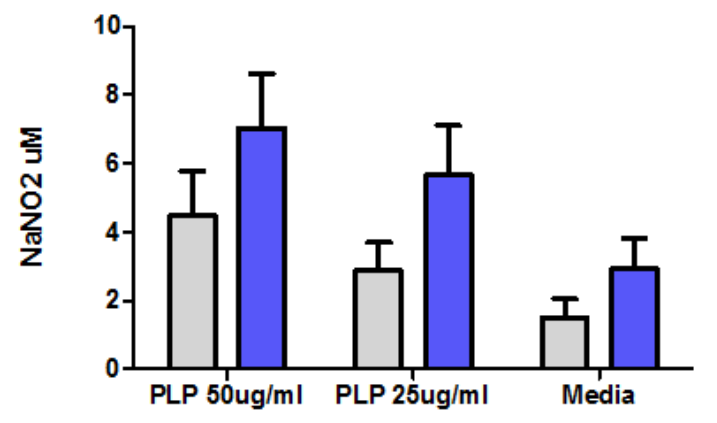

B
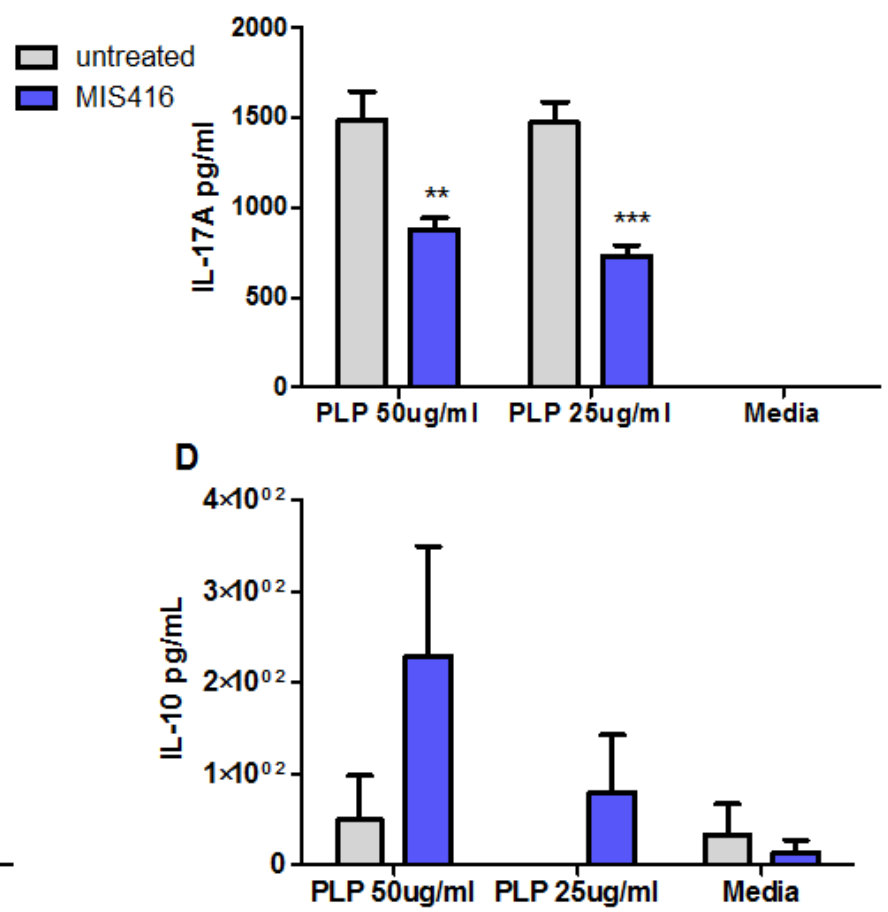

Figure 3-8 MIS416 treatment in SJL/J mice significantly reduced the cytokine response to PLP at day 42. Mice were immunised for EAE at day 0 and weekly MIS416 treatments began at day 21 during the first remission state. Splenocytes were harvested at day 42 which coincided with 1-day post MIS416 treatment, and splenocytes were cultured with either 50 or $25 \mu \mathrm{g} / \mathrm{ml}$ PLP for 72 hours and supernatants were analysed for cytokines. A) IFN$\gamma$ levels from ELISA, B) IL-17A levels from ELISA, C) NO (nitrate) production as measured by Griess reaction, D) IL-10 levels from ELISA. Results are from 1 experiment. Shown are the means \pm SEM ( $n=4-5$, in triplicate) ${ }^{*} \mathrm{p}<0.05, * * \mathrm{p}<0.01$ and $* * * \mathrm{p}<0.001$ EAE compared to MIS EAE using Mann-Whitney test. 


\subsubsection{Signalling through TLR9 is essential for MIS416 EAE disease protection}

In this chapter we successfully identified a treatment regime which showed MIS416 is effective at reducing the disease burden within the C57BL/6 EAE model. However we also want to show that manipulation of either the immune system or its signalling pathways will led to altered protection with MIS416. To this end, we compared regular MIS416 with a heat-treated version of MIS416 (HT-MIS416), which renders the bacterial DNA (i.e. the TLR-9 agonist) inactive and thus allowing us to evaluate the effect of the NOD-2 activity only. When the HT-MIS416 was given at the same concentration and dosing as the regular MIS416 it did not provide any disease protection as the mice followed a similar disease course to control mice (Figure 3-9). Comparing the disease scores of the EAE mice, we saw that the control and HT-MIS416-treated mice developed EAE at a similar time and had peak disease at about day 16, whereas the MIS416-treated mice showed a reduced disease score by the day 14 dose and this reduction continued until the experiment was completed (Figure 3-9A). Furthermore, the weight change in these mice also reflected a similar pattern as the disease scores where MIS416 treatment protected the mice from the major weight loss associated with disease onset in the both untreated and heat-treated MIS416 mice (Figure 3-9C).

These results using the HT-MIS416 indicated that the MIS416 protection in EAE requires the TLR-9 ligand, at least when administered at the same weekly $100 \mu \mathrm{g}$ concentration as MIS416. Therefore, NOD-2 stimulation alone at this concentration was not sufficient to have disease altering effects in EAE and it is possible that both the NOD-2 and TLR-9 pathways are required to produce the disease protective effect in these mice.

Comparing the cytokine response to MOG, there was a similar trend to that found with the disease scores, where the mice which received HT-MIS416 did not show the same reduced IFN- $\gamma$ response to MOG as seen in the MIS416-treated EAE mice (Figure 3-10A). In contrast, with the MOGspecific IL-17A response, there was a slight reduction in the HT-MIS416 mice but not the same level of reduction as seen with MIS416 treatment (Figure 3-10B). Furthermore, the nitric oxide concentration in these cultures showed that HT-MIS416 did not drive a strong nitric oxide production in contrast to MIS416 which had a strong NO response to MOG at day 22 in these experiments (Figure 3-10C).

Together the disease scores and antigen-specific cytokine production in these experiments further validate that not only is the TLR-9 signalling important for MIS416 mechanism of action, but that the treatment regimen in this model can be manipulated to allow us to determine key aspects of the mechanism. 
A

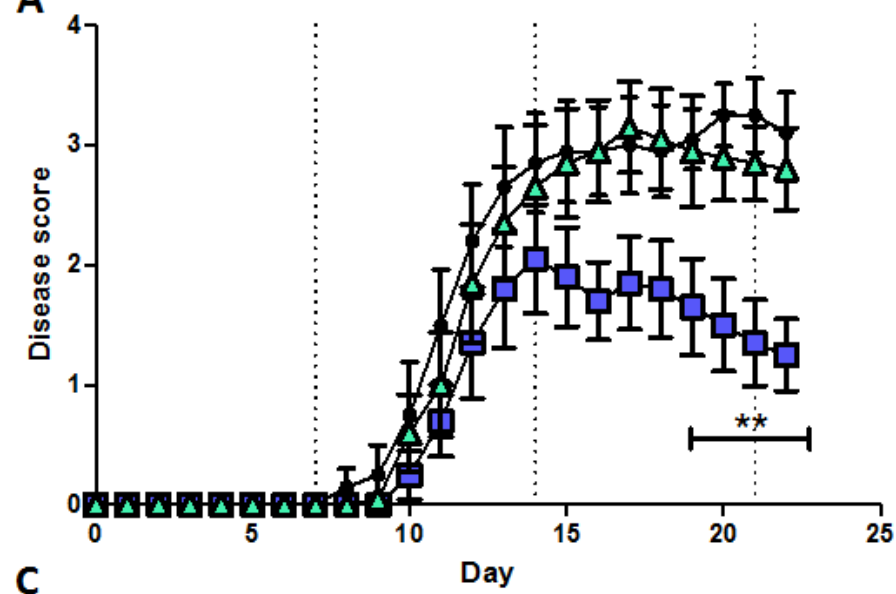

C

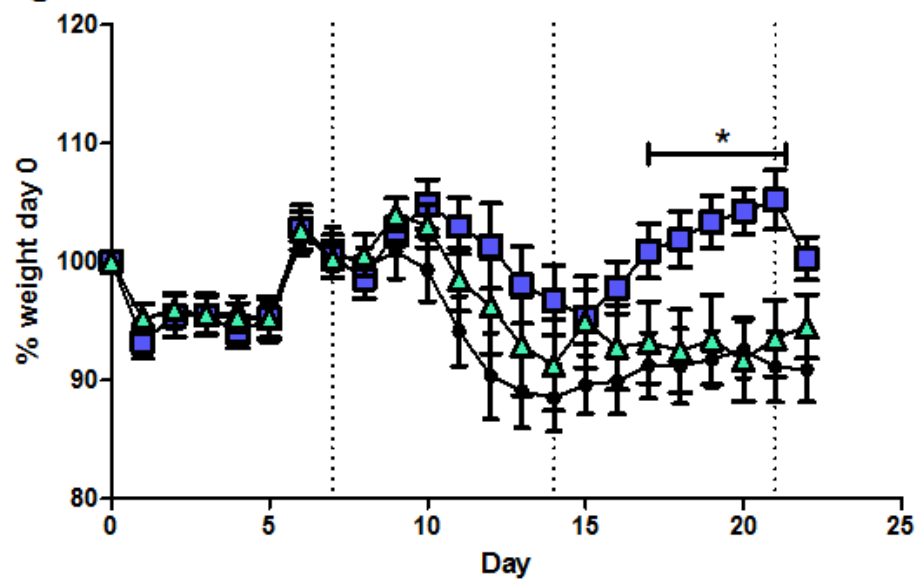

B

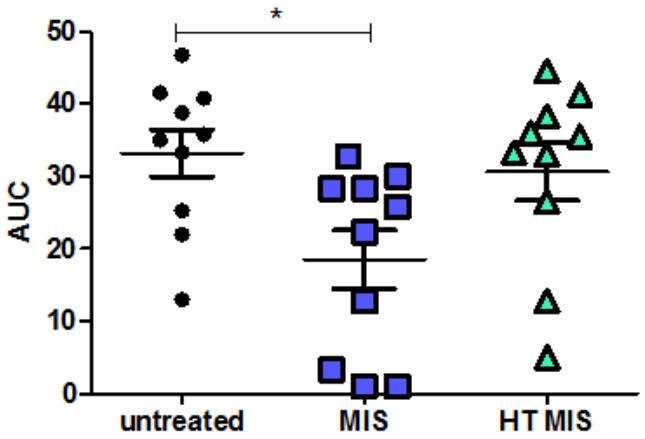

Figure 3-9 Heat-treated-MIS416 was not effective at reducing EAE disease in C57/B16 mice when used at the same dose and timing as regular MIS416.

C57BL/6 mice were immunised for EAE at day 0 and received $100 \mu \mathrm{g}$ weekly doses of either MIS416 or a heattreated version of MIS416 and weighed and scored daily. A) Disease scores of all immunised mice with MIS416 treatment. B) Area under the curve as a measure of disease burden. C) Weight as a percentage of baseline for each treatment group. Shown are the means \pm SEM, $n=9-10$ per treatment group of 2 independent experiments. * $\mathrm{p}<0.05$ and ${ }^{*} \mathrm{p}<0.01$ by 2 -way repeated measures ANOVA with Bonferronis multiple comparisons test was used (A\&C) or a Kruskal-Wallis test with Dunns post-test analysis (B) comparing MIS416-treated or HT-MIS416treated to control. 
A
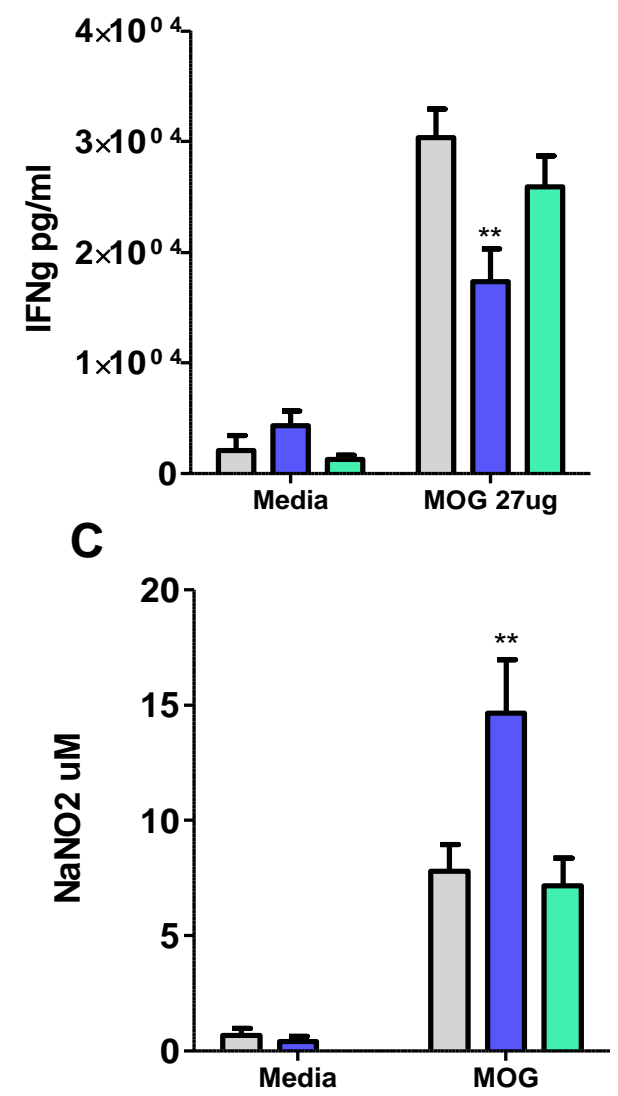

B

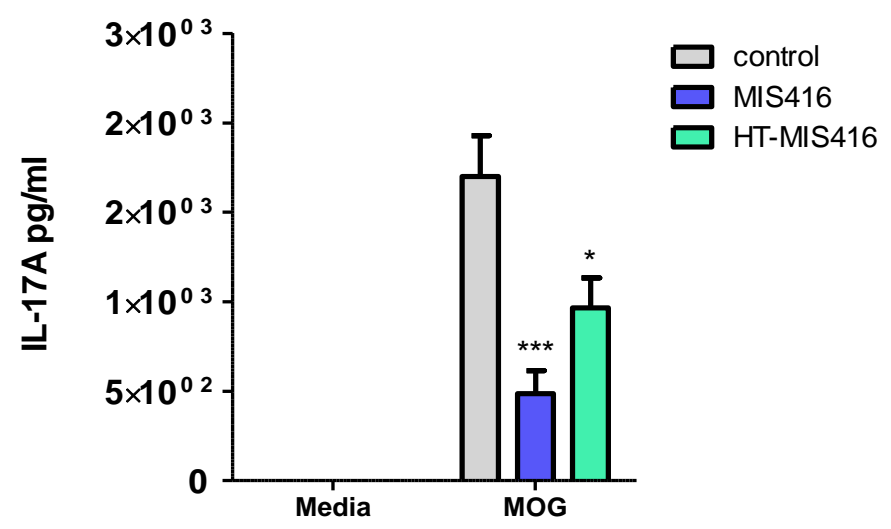

Figure 3-10 Heat-treated MIS416 reduced the IL-17A response to MOG with no effect on MOG induced IFN- $\gamma$ or NO production in splenocytes.

C57BL/6 mice were immunised for EAE at day 0 and received $100 \mu \mathrm{g}$ weekly doses of either MIS416 or a heattreated version of MIS416. Mice were culled at day 22 and splenocytes were harvested and cultured with 27 $\mu \mathrm{g} / \mathrm{ml}$ MOG for 72 hours then supernatants were collected. A) IFN-g levels from ELISA, B) IL-17A levels from ELISA, C) NO (nitrate) production as measured by Griess reaction. Shown are the means \pm SEM $(n=9-10)$ from 2-independent experiments, ${ }^{*} \mathrm{p}<0.05, \mathrm{p}<0.01,{ }^{*} * \mathrm{p}<0.001$ comparing control mice to either MIS416-treated or HT-MIS416 treated by 1-way ANOVA with Tukey's post-test. 


\subsection{Discussion}

This chapter explores the use of MIS416, which is composed of bacterial nucleic acid and muramyl dipeptide, TLR-9 and NOD-2 ligands respectively, as a treatment for MS. The key outcome for this chapter was to determine an effective and consistent treatment regimen which could be used to assess the immune-mediated mechanism of action of MIS416. Using various EAE strains of mice, we have identified two mouse strains in which MIS416 is beneficial (C57BL/6 and SJL) against the development of EAE and one type in which MIS416 is not beneficial in $(\mathrm{BALB} / \mathrm{c})$.

Also, we used different dosing and timing of MIS416 with the aim to identify a suitable dose that reduces EAE disease scores without causing detrimental effects. We showed that using varying concentrations of MIS416 at day 0 or day 12 is safe and tolerable in these mice, and furthermore that the highest dose of $250 \mu \mathrm{g}$ leads to significant reduction in disease compared to the control mice (Figure 3-1 \& Figure 3-2). These first experiments were part of the initial finding that this compound, which specifically activates innate cell TLR-9 and NOD-2, is able to alter the immune environment in a protective way in the EAE model and we have recently published these findings (White et al., 2014).

Before our work began however, other studies had shown that the role of PRR stimulation in the EAE model can in some cases be detrimental and in others beneficial. For example the role of TLR9 signalling was thought to be harmful in EAE, as EAE induced in a TLR9 deficient mice had significantly later disease onset and reduced severity compared to wild type mice. Furthermore, this study also showed that mice deficient in the downstream signalling molecule for TLR9, MyD88, were completely EAE resistant (M. Prinz et al., 2006). Another study also suggested that the use of bacterial DNA, a TLR9 ligand, in both the viral-induced and autoimmune EAE exacerbates disease (Tsunoda et al., 1999). In contrast to this finding another group showed that $\mathrm{TLR}^{-{ }^{--}}$and TLR4 ${ }^{-/-}$mice suffered surprisingly more severe EAE compared to control mice and therefore suggesting that signalling through TLR9 and TLR4 can regulate disease severity in some EAE models (Marta et al., 2008). A key concept to keep in mind for these studies is that induction of EAE relies on PRR signalling, such as through MyD88 (Marta et al., 2008), and therefore the lack of disease in a knockout mice could be due to an inability to develop disease and not due to an alteration of disease processes.

Similar to the role of TLR9 in disease, the role which NOD2 signalling may have in EAE is unclear. However, there are reports that antigen presenting cells containing peptidoglycan, another NOD2 ligand, can be found within the brains of MS patients suggesting a detrimental role for this 
PRR signal in MS (Schrijver et al., 2001). Other studies have demonstrated that administration of TLR3 and TLR7 ligands can have a protective effect in EAE (Hayashi et al., 2009; O'Brien et al., 2010; Touil et al., 2006). It is studies such as these mentioned which indicate that while there is potential for PRR to be either beneficial or detrimental, it is likely that the response depends on the dose and localisation of the signal which can alter the outcome of an immune response. It is also possible that the effect it is not the activation of the TLR itself, but the standby effects by cytokines such as endogenous IFN- $\beta$ which is the key driver in the disease protection seen by O'Brien et al and his study with the TLR7 agonist, imiquimod. Despite what has been found with other TLR-9 and NOD2 agonists, the treatment of EAE mice with MIS416 shows disease protective effects and further analysis into how this immune modifier is acting is of great interest.

We have shown that MIS416 treatment is effective at altering EAE disease in 2 strains of mice (C57BL/6 and SJL), therefore we then determined if both the TLR9 and NOD2 ligands were required for this effect. To do this we used a heat-treated version of the compound which renders the TLR9 signalling inactive and allows signalling only through the NOD2 pathway (personal communication, Gill Webster, IIT, see Appendix 9.2). Using weekly doses in the C57 model we identified that the HT-MIS416 was not effective at altering EAE disease when used at the same concentration as MIS416 (Figure 3-9). Together with the cytokine profile from the HT-MIS416 experiments, we have shown that the TLR9 ligand is essential for the mechanism of MIS416, and it is also likely that both ligands are acting in a synergistic manner to alter the EAE disease. However, as the muramyl dipeptide (MDP) is a major component of the microparticle, giving it unique size properties that render MIS416 microparticle unique to other TLR agonist treatments, it would be difficult to treat mice with the bacterial DNA component in the same way without MDP. Furthermore, as NOD2 deficient mice are somewhat resistant to EAE (Shaw et al., 2011) it would be difficult for us to evaluate the role of muramyl dipeptide is having in the disease protection seen with MIS416.

As MIS416 is a relatively large particle, and has been shown to be preferentially taken up by phagocytic cells (Girvan et al., 2011), it is likely that the way it activates the immune system is completely unique compared to the majority of ligands previously used in EAE. Also, another major difference in our study is the route of administration (i.e. i.v) and therefore the cell types which come into contact with MIS416 first are likely to be different to those when administered intraperitoneally or subcutaneously. Interestingly, one other study has also looked into the effect of immune-modifying microparticles and determined that negatively charged carboxylated polystyrene microparticles are able to alter inflammatory monocyte responses (Getts et al., 2014). Furthermore, Ghetts et al showed that with the microparticle monocytes did not traffic to sites of 
inflammation and instead migrated to the spleen where they were degraded via induction of apoptosis and cell clearance mechanisms. Like MIS416, these immune modifying microparticles were intravenously injected and were found to have disease protective effects within the EAE model (as well as other models of inflammation) (Getts et al., 2014). However, the study by Ghetts et al suggest it is solely the microparticle structure of MIS416 which is inducing the disease protective effects in EAE; although considering we have determined that TLR9-deficient MIS416 is not capable of inducing disease protection, and has maintained its microparticle structure, the disease protective effects with MIS416 are unlikely to be purely due to the structure of the compound.

Another aim for this chapter was to explore if MIS416 treatment was effective in different models of MS by inducing EAE in different strains of mice. The purposes of these experiments were to show that MIS416 was not only effective in one model of EAE but was more widely effective. While the C57BL/6 model is the most widely used EAE mouse model, the BALB/c and the SJL strains develop EAE quite differently to the C57BL/6 strain, are becoming more widely used as well. The BALB/c mice develop a progressive form of EAE that presents as mild to moderate paralysis, and these mice often have lesions within the brain as opposed to the spinal cord as seen in the C57 model. The nature of these lesions make these mice difficult to score and many in the field often use weight loss as an indicator of disease followed by lesion analysis (Orian et al., 2015). When the BALB/c mice were EAE immunised and treated with MIS416 weekly using the same dose as what we see as protective in the C57 model, we did not see any disease protection compared to control mice (Figure 3-6) however, in the peak disease stage it appeared that MIS416-treated mice had a reduced weight loss compared to control mice. Although, it is possible that MIS416 was having a protective effect in these mice, because there was no effect on disease scores it is unlikely. On the other hand BALB/c mice have a very different immune predisposition compared to $\mathrm{C} 57$ mice. For example, BALB/c mice are more prone to mounting a Th2-type response whereas C57 mice are more prone to Th1 (Mills, Kincaid, Alt, Heilman, \& Hill, 2000), and these differences could explain the differences in MIS416-induced effects. Therefore, further assessment of the mechanisms behind the protective effect of MIS416 in the C57BL/6 model may allow us to understand whether it is this immune predisposition that underlies the difference between the two models.

In contrast to the BALB/c model, we found that the SJL relapsing-remitting mice responded well to MIS416 treatment, and weekly doses of MIS416 reduced the severity and duration of relapses (Figure 3-8). It was exciting to demonstrate that MIS416 was effective in a relapsing-remitting model when administered after the first wave of disease and thus provided further evidence that 
MIS416 was effective in altering already established disease. Furthermore, as we know that between $80-90 \%$ of MS patients present initially with the relapsing-remitting form of the disease (Hemmer, Archelos, \& Hartung, 2002), these results in an animal model support the use of this novel compound in the treatment of this form of MS.

By analysing the cytokine profile of splenocytes and liver cells of treated mice re-stimulated with MOG ex vivo we discovered that MIS416 treatment significantly reduced the antigen-specific proinflammatory IFN- $\gamma$ and IL-17A cytokine production (Figure 3-4 \& Figure 3-5). These cytokines have been implicated as evidence of the Th1 and Th17 antigen specific response which occurs in the EAE model (Rostami \& Ciric, 2013), and are consistent with the reduced disease scores in these mice. Therefore, the IFN- $\gamma$ and IL-17A data provide further evidence of the immune modulating effect MIS416 has in EAE indicating that the treatment regimen used is an effective model for further studying the immunological events. Of note, the NO response to MOG appeared to be increased by MIS416 treatment. As NO is acts in an anti-proliferative manner (Kawabe, Isobe, Hasegawa, Nakashima, \& Shimokata, 1992), this could contribute to the reduced antigenspecific cytokine response in vitro and possibly in vivo as well. Further investigation into these immune mediating effects with MIS416 will help us understand beneficial effects of the compound.

\subsubsection{Summary}

Altogether the findings for this chapter have identified a weekly dosing regimen that was effective in the C57BL/6 progressive model of MS. While it is not clear why MIS416 was effective at reducing EAE in C57BL/6 and SJL mice but not BALB/c mice, we determined an effective method for analysing the immunomodulatory effects of MIS416 further. The peripheral and CNSspecific immune alterations will be assessed in the upcoming chapters. 


\section{Chapter 4: MIS416-induced alterations on the peripheral immune system}




\subsection{Introduction}

The previous chapter successfully identified a dosing method for MIS416 that shows a consistent reduction in disease burden in the C57BL/6 EAE model. Using this dosing regimen and EAE model, the upcoming chapters are aimed at identifying how systemic administration of MIS416, which is believed to target phagocytic cells, is able to alter the immune environment in EAE such that disease is reduced. The current chapter focuses on the changes that occur in the peripheral immune system following weekly MIS416 treatment and is therefore mostly observational whereas the subsequent chapter (Chapter 5) is aimed at determining which of these changes is important for the MIS416-induced effects during EAE.

Within the peripheral immune compartment there are a number of lymphoid organs that could be of importance to MIS416 treatment, including blood, spleen, liver and draining lymph nodes. As MIS416 is delivered i.v, it is first encountered by cells within the circulating blood and it is likely to be picked up by phagocytic cells there, including monocytes and dendritic cells. Studies by Garvin et al have shown that monocytes, myeloid dendritic cells (mDC) and plasmacytoid dendritic cells (pDC) isolated from human blood were able to internalise MIS416, with monocytes and mDCs being better at picking up MIS416 than pDC (Girvan et al., 2011). Therefore, in mice treated with MIS416, we expect that similar phagocytic cells within the blood will pick up the microparticle. Furthermore, we know from early toxicology studies that MIS416 deposits within the liver and to some extent the spleen (personal communication, Gill Webster, IIL); therefore, we will include these immune compartments in our investigations in this observational chapter.

Immune modulation for the treatment of MS is an already established therapeutic avenue; we know that it is possible to manipulate the peripheral immune system to promote beneficial diseasealtering effects. For example, IFN- $\beta$ is a cytokine that is produced by many different cell types, including fibroblasts, and when given as a recombinant protein subcutaneously to patients it is an effective therapeutic treatment in relapsing-remitting MS (Duquette et al., 1993). Glatiramer acetate (GA) is another effective example of an immune modulator which alters the immune environment in a way that effectively reduces MS disease, particularly in patients with the relapsing-remitting form (Ge et al., 2000). Interestingly, as GA is a mixture of polypeptides made up of four amino acids that resemble myelin basic protein (MBP), which is thought to be one of the key proteins auto-reactive $\mathrm{T}$ cells recognise in MS, its mechanism is likely to occur in an antigen-specific manner unlike IFN- $\beta$ (Yong, 2002).

These treatments highlight the idea that with the right kind of immunological manipulation it is possible to alter the disease course of a pro-inflammatory autoimmune disease, such as MS, 
by changing the peripheral immune environment. To determine the mechanism by which MIS416 is beneficial in reducing inflammation, the first step was to observe what immunological changes occur with MIS416 treatment in the animal model of MS. We hypothesise that MIS416 is an effective immune modulator which is able to recruit different cell types and produce specific cytokines that allows it to reduce disease in EAE mice and furthermore, its mechanism of action is likely to be quite unique compared to current immune modulating treatments such as IFN- $\beta$ and GA.

\subsection{Aims}

The aim of this chapter is to identify changes in the peripheral immune system including alterations in cell populations and their activation states induced by MIS416 treatment. By identifying the ways in which MIS416 alters the immune system, we can then investigate if and how these immunological changes are beneficial in EAE, and ultimately MS patients.

\subsubsection{Specific aims}

1. To identify the key cell types responding directly to MIS416.

2. To identify the cytokines produced in response to MIS416 and their cellular source.

3. To determine the effect of MIS416 treatment on antigen-specific and antigen nonspecific stimulation.

4. To determine whether heat-treated MIS416, which lacks the TLR9 agonist activity, induces the same immunological changes as non-heat treated MIS416. 


\subsection{Results}

\subsubsection{MIS416 treatment resulted in increased leukocyte count in the liver and spleen}

One of the first observations made with weekly $100 \mu \mathrm{g}$ doses of MIS416 was that mice had enlarged spleens and to some extent enlarged livers as well. In Figure 4-1A the spleens from untreated control mice were much smaller than those from MIS416-treated mice at day 15, and the spleens also appeared darker.

Additionally, the MIS416-treated mice had significantly more leukocytes in the spleen than untreated mice at most of the time points analysed (Figure 4-1B). This increase was also seen in the EAE mice although the difference was not as great as that observed in the non-EAE mice (Figure 4-1C). However, as it is known that EAE immunisation alone transiently increases the neutrophil population in the spleen around the time of disease onset (White et al., 2014), this finding may explain why the total number of cells in the control and MIS416-treated mice did not differ significantly at the day 15 time point in EAE mice (Figure 4-1D).

Strikingly, there was also a significant increase in the leukocyte count in the liver of MIS416treated non-EAE (Figure 4-1E) and EAE mice (Figure 4-1F). However, as observed in the spleen, it appeared that immunisation alone increased the liver leukocyte count compared to non-EAE mice at the day 15 time point, although this increase in EAE mice did not alter the significant difference found between the MIS416-treated and untreated groups. 

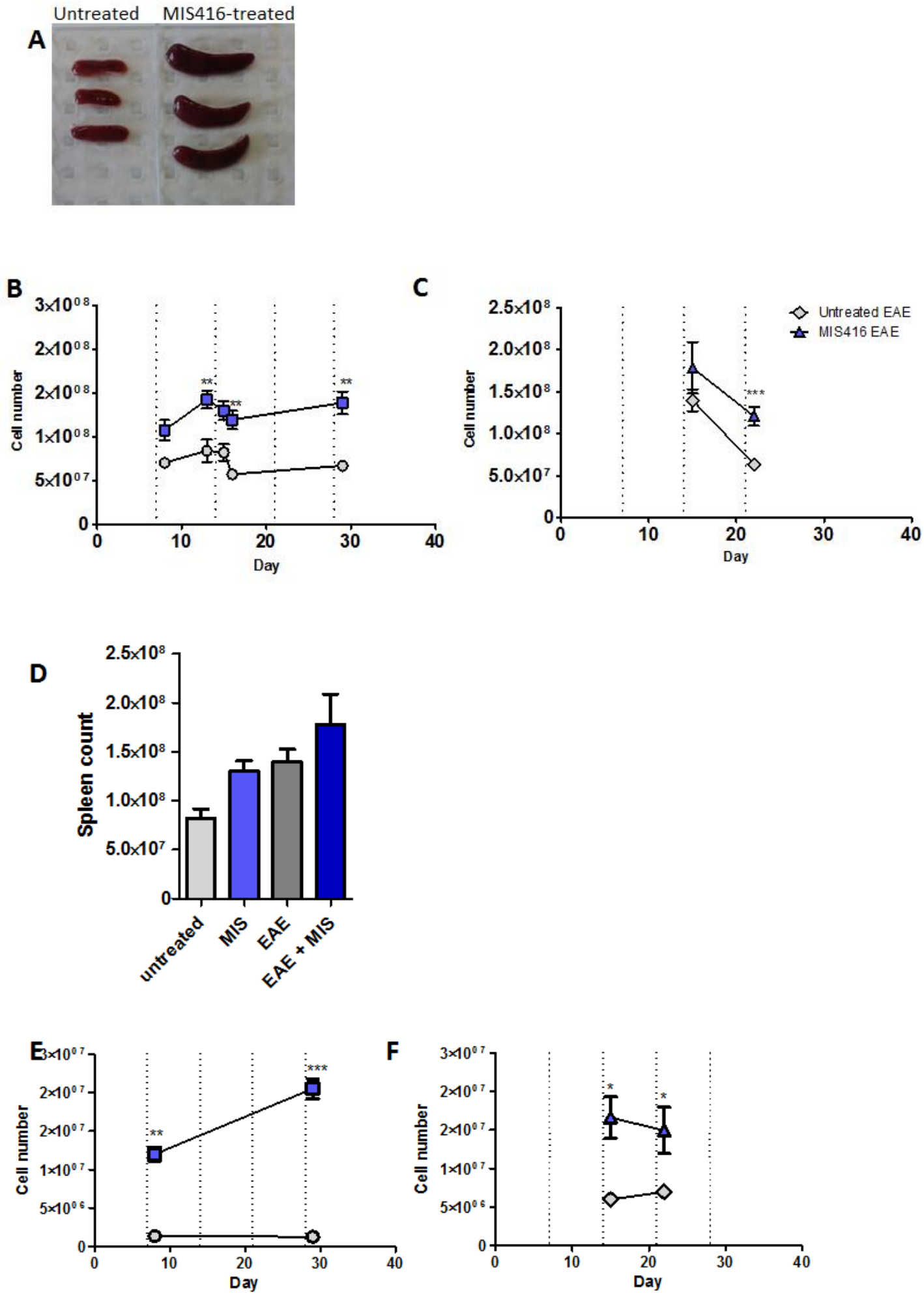

Figure 4-1 Spleen and liver cell counts were elevated at various time points after MIS416 treatment.

Spleen and liver cells were isolated and counted on the days indicated. A) Picture representing how spleens become enlarged with MIS416 treatment at day 15. B) Spleen counts without EAE immunisation. C) Spleen counts in EAE mice. D) Comparison of spleen counts at day 15. E) Liver counts without EAE immunisation. F) Liver counts in EAE mice. Shown are the means \pm SEM of counts from individual mice from 1-3 experiments at each time point with $\mathrm{n}=3-15 .{ }^{*} \mathrm{p}<0.05, * * \mathrm{p}<0.01,{ }^{*} * \mathrm{p}<0.001$, B-F untreated compared to MIS416-treated at each time point by unpaired t-test with Welches Correction. 


\subsubsection{MIS416 treatment induced cells of the spleen and liver to enter the cell cycle}

Because we observed an increase in the liver and spleen counts, we investigated whether the increase was due to a migration of cells into these organs or if a local progenitor population underwent proliferation in response to MIS416 treatment. To do this we used a cell cycle dye (Vybrant DyeCycle Green Stain) which is cell-permeant and binds to the DNA in live cells and provides information about which stage of the cell cycle each cell is at. Firstly, we looked at how MIS416 altered the cell cycle compared to known proliferative agents such as ConA (polyclonal) and MOG (antigen-specific). By using splenocytes from 2D2 mice, which express a MOG-specific TCR, the proliferation of naïve antigen-specific T cells could be assessed. After 48 hours of stimulation, a profound increase in the number of cells in S/G2 phase was observed in the ConA culture (Figure 4-2A). This finding was expected as it is known that ConA acts as a mitogen in spleen cultures by causing both the CD4+ and CD8+ T cell populations to proliferate (Kruisbeek, Shevach, \& Thornton, 2004). We also saw an increase in the percentage of proliferating cells in the MOG culture and to a lesser extent the MIS416 stimulation compared to controls (Figure 4-2A\&B). These results indicate that MIS416 may be able to induce proliferation in a cell population within the spleen; however, the specific cell type proliferating in response to MIS416 is, as yet, unknown.

To determine if MIS416 treatment in vivo altered cellular proliferation, we also analysed the cell cycle of liver and spleen leukocytes isolated from MIS416-treated mice at days 15 and 29. MIS416 treatment significantly increased the percentage of splenocytes that underwent cell division compared to control mice at day 15 (Figure 4-3A), and in the pooled liver leukocytes from the same experiment, a similar increase in the percentage of cells which were dividing was found (Figure 4-3B). Comparison of the two time points tested (day 15 and 29) showed that there was a stronger effect, with more dividing cells, at day 15 compared to day 29, and overall there were significantly more dividing cells in splenocytes from MIS416-treated mice compared to control mice (Figure 4-3C). To see if there was a positive correlation between spleen count and proliferation, the spleen count was plotted against the percentage of cells undergoing division (in the G2/S phase) and we found a significant linear relationship, where the mice that had higher spleen counts also had a higher percentage of cells dividing (Figure 4-3D). This finding suggested that the better MIS416 responders had more splenocytes, and this increase was related to more proliferation within the spleen (Figure 4-3D).

These studies investigating the relationship between cell division were the first to show that MIS416 is able to alter the peripheral immune compartment within the spleen and liver by 
inducing cellular proliferation. While we cannot rule out that MIS416 treatment also induced migration of cells into the spleen and liver, we know that there was proliferation of leukocytes within these organs and that this cell division was directly linked to the total cell count. 
A

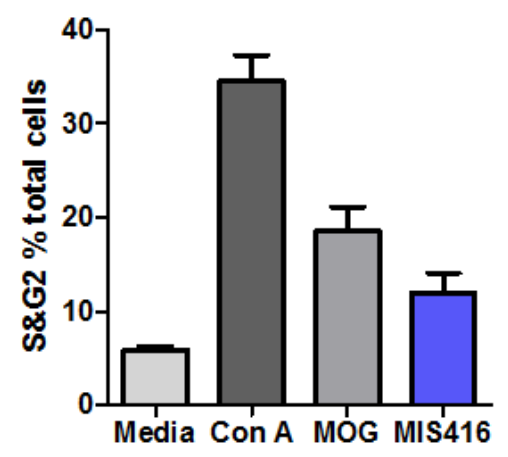

B
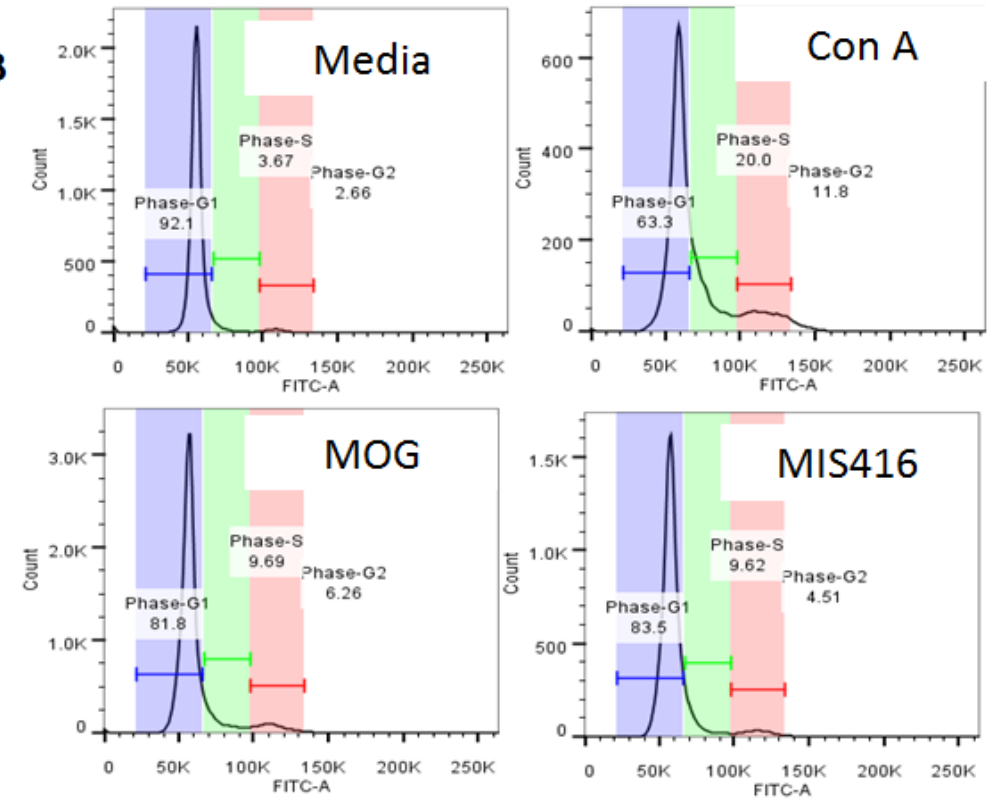

Figure 4-2 MIS416 given to splenocytes in vitro induced a small percentage of cells to undergo proliferation.

Splenocytes were isolated from C57BL/6 mice and cultured for 48 hours with ConA ( $3 \mu \mathrm{g} / \mathrm{ml})$, MOG $(27 \mu \mathrm{g} / \mathrm{ml})$ or MIS416 $(20 \mu \mathrm{g} / \mathrm{ml})$. A) Percentage of splenocytes in the G2/S phase of the cell cycle. B) A representation of the cell cycle platform with each stimulation type. Shown in $\mathbf{A}$ are the mean \pm SEM of duplicate wells from 1 experiment. 

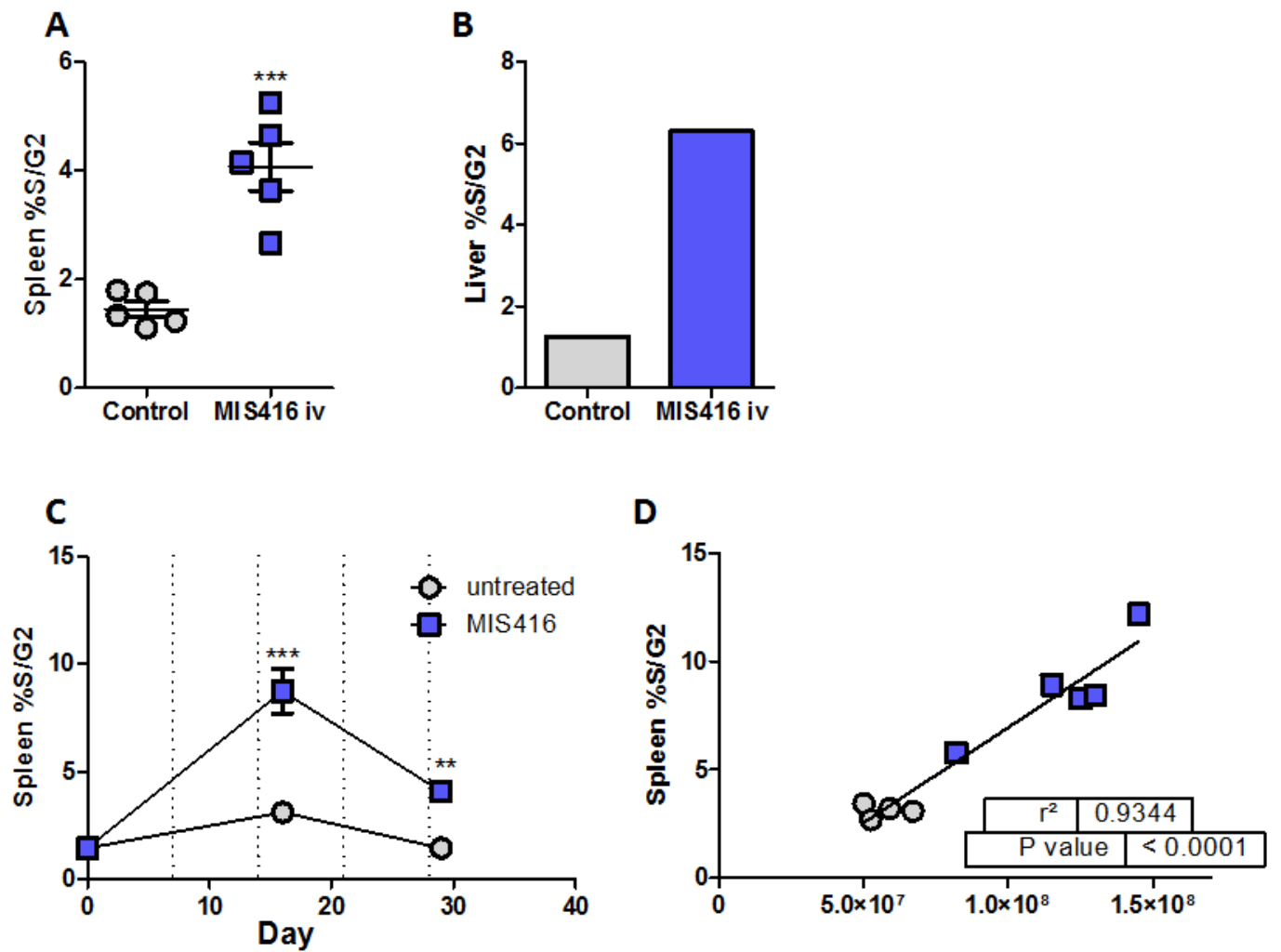

Figure 4-3 MIS416-treated mice i.v showed an increase in the number of proliferating cells in the spleen and liver.

C57BL/6 mice were treated with $100 \mu \mathrm{g}$ MIS416 weekly for 15 days and splenocytes and liver cells were isolated and analysed for cell cycle stages (this occurred 1 day p.i). A) Spleen cells show a significant increase in the percentage of cells in the G2/S phase of the cell cycle. B) Liver cells have an increase in the percentage of cells in the G2/S phase. C) Over time the percentage of G2/S cells is increased by day15 after first weekly MIS416 dose and maintained difference at day 29. D) Positive correlation between elevated spleen count and percentage of cells in G2/S phase of the cell cycle. Shown are the mean \pm SEM of 1-2 experiments with $n=3-5$ per time point, ** $\mathrm{p}<0.01,{ }^{* * *} \mathrm{p}<0.001$ untreated compared to MIS416-treated by students t-test with Welches Correction for unequal variances $(\mathrm{A} \& \mathrm{C})$ and linear regression in $\mathrm{D}$. 


\subsubsection{MIS416 was taken up by cells in vitro and in vivo}

MIS416 has been shown to be taken up by phagocytic cell types in the blood such as monocytes and dendritic cells (Girvan et al., 2011). To determine which populations took up MIS416 in the mouse, we investigated the uptake of fluorescently labelled-MIS416 in in vitro splenocyte cultures. Using the gating strategy shown in Figure 4-4A, we determined that a variety of cells became MIS416 positive after incubation with labelled-MIS416 including myeloid cells such as macrophages $\left(\mathrm{CD} 11 \mathrm{~b}+\mathrm{Gr} 1^{\text {low }}\right)$, granulocytes, some $\mathrm{CD} 4+$ and $\mathrm{CD} 8+$ cells and other non-CD11b cells, which could include red-pulp macrophages (Figure 4-4B). Furthermore, MIS416 was taken up fairly slowly in these splenocytes cultures (Figure 4-4C), with the percentage of MIS416 positive cells increasing over time. The greatest difference between the percentage of positive cells was observed at 4 hours, the latest time point assessed. If MIS416 were picked up quickly, we would expect the percentage of MIS416-positive cells to plateau between 1-4 hours which did not occur; therefore we believe that these cells are slow to phagocytose MIS416. As expected, when we incubated splenocytes with increasing concentrations of MIS416, the fluorescence of the MIS416-positive cells increased (Figure 4-4C).

To determine if MIS416 accumulated in cells within the blood, liver or spleen, mice were treated i.v. with $100 \mu \mathrm{g}$ fluorescently labelled-MIS416, and cells isolated from the liver, spleen, and blood 24-hours later. We found that the majority of MIS416 remaining after 24 hours was found within the liver and only small amounts were still located in cells circulating in the blood (Figure 4-5A). After characterising these cells further, the majority of cells that were MIS416-positive in the liver were F4/80+, which were likely to be the resident macrophages of the liver (i.e. kupffer cells). The other cell types that were MIS416-positive comprised of granulocytes, dendritic cells and NK1.1+ cells (Figure 4-5B). It is interesting to find that the NK1.1+ cells appeared to be able to phagocytose MIS416 as this marker is usually on NK cells, which are not phagocytic; however, it is possible that these cells were simply very closely associated with the MIS416 (e.g. bound to the cell surface) and hence appear to have taken up MIS416 by flow cytometry.

These results provide information about the types of cells that first come into contact with MIS416 when it is given i.v. in the mouse, and may indicate not only which cells are the first responders to MIS416 but also which key cell types are involved in the mechanism of action of MIS416 in EAE. 

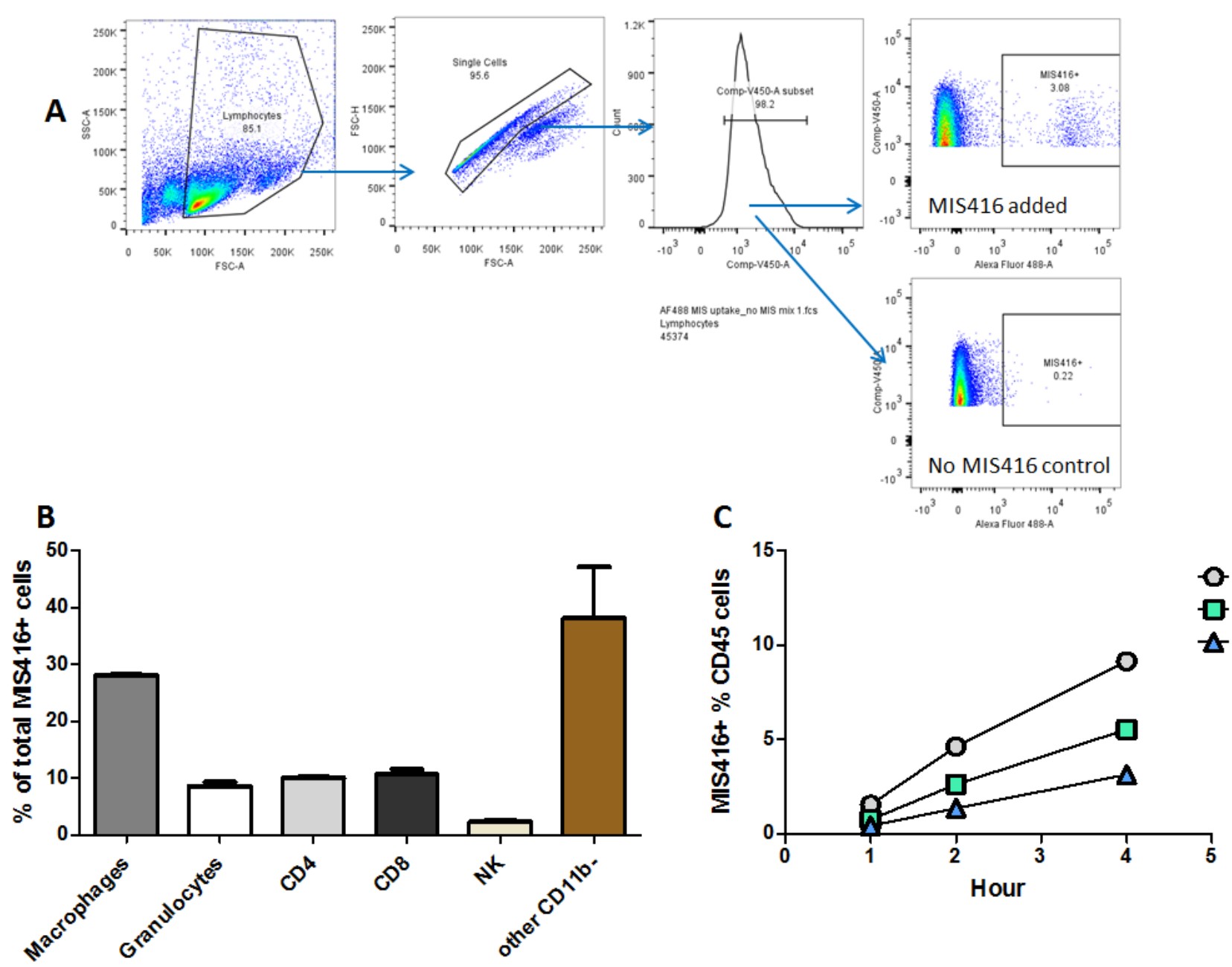

$20 \mathrm{ug} / \mathrm{ml}$

ㅁ. $10 \mathrm{ug} / \mathrm{ml}$

$\Delta \mathrm{ug} / \mathrm{ml}$

Figure 4-4 MIS416 was taken up by isolated splenocytes in vitro.

Splenocytes were isolated and cultured at $1 \times 10^{6}$ cells per well in a flat bottom 96 well plate, with the indicated amount of MIS416 per ml given. A) Using flow cytometry we are able to gate on live, single cells that were CD45+ and determine the percentage of MIS416+ cells, shown is a gating example B) Immunophenotyping the cells that were MIS416+ after 4 hours of culture with $5 \mu \mathrm{g} / \mathrm{ml}$, macrophages (F4/80+), granulocytes (CD11b+Gr1+), CD4 T cells (CD4+CD11b-), CD8 T cells (CD8+CD11b-), NK (NK1.1+), other CD11b+ (CD45+CD11b-). C) The uptake of MIS416 over 4 hours using various concentrations of MIS416. Shown are the means \pm SEM of 1 experiment in duplicate. 

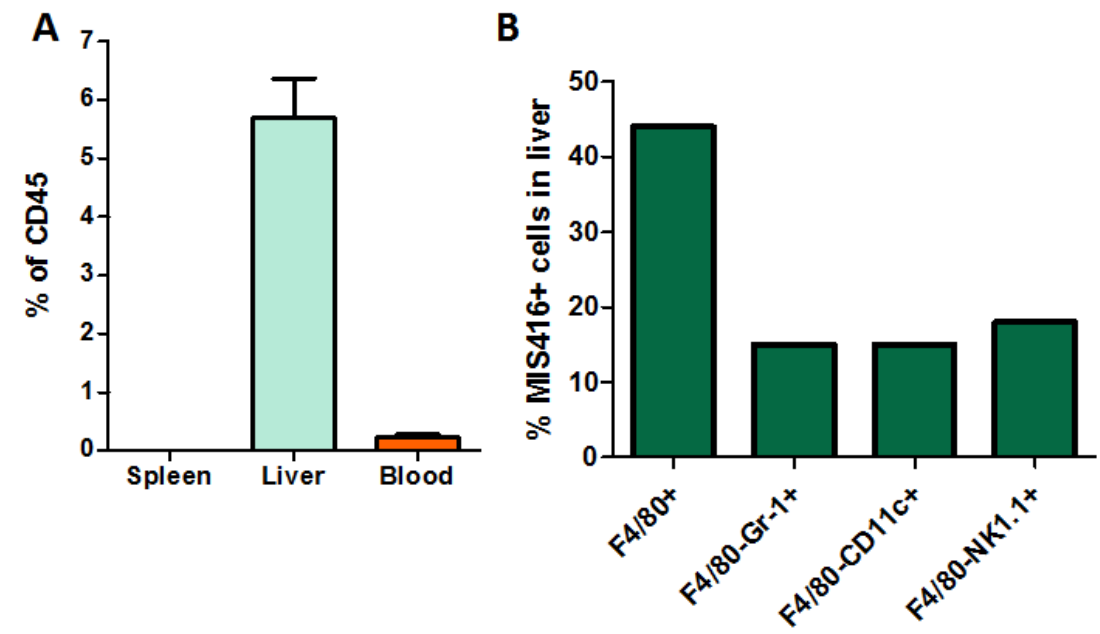

Figure 4-5 MIS416 was deposited primarily in the liver 24-hours after a $100 \mu \mathrm{g}$ dose i.v.

FITC or AF488 labelled MIS416 was injected i.v 24-hours prior to euthanisation and the percentage of MIS416+ cells as a proportion of CD45+ cells in each spleen, liver and blood was calculated. A) Percentage of MIS416+ cells within the total leukocyte population of the spleen, liver and blood. B) Immunophenotyping showing which cell populations took up the labelled MIS416. Shown are the mean \pm SEM of 3 individual mice from 1 experiment (A) and an average breakdown of cell populations which were MIS416+ shown in B. 


\subsubsection{MIS416 treatment altered the myeloid populations in the spleen and liver}

As a low dose of MIS416 administered i.v. accumulated within the liver, and previous toxicology studies using higher doses of MIS416 found depositions within the spleen also, we assessed if weekly MIS416 treatment altered the myeloid composition of splenocytes and liver leukocytes both within the context of EAE and without. As shown in appendix 1.1, we developed a gating strategy that first removed dead cells followed by gating on single cells (linear population in FSCA/FSC-H) and CD45+ cells to identify leukocytes found within the liver and spleen (appendix 1.2). We analysed splenocytes for the surface markers Gr-1, CD11b, CD11c, and F4/80 and using these markers we created a gating strategy to allow us to identify red pulp macrophages, dendritic cells, granulocytes and monocytic Gr-1dim cells which will be referred to as monocytic myeloidderived suppressor (mMDSC) cells although the functionality of this population has not yet been assessed (Figure 4-6).

With MIS416 treatment, there were significant effects on the myeloid compartment, mainly within the red pulp macrophage population, which was CD11b- and F4/80+ (Figure 4-7A \&B). As shown in the previous section, the red pulp macrophages were key cells to pick-up and respond to MIS416 through their receptors NOD-2 and TLR9. Within the spleen of EAE immunised mice, there were significant increases in the proportion of red pulp macrophages (as a percentage of total cells) and due to an increase in total cell number, there was also a significant increase in the total number of red pulp macrophages in the spleens from MIS416-treated animals (Figure 4-7A \&B). A similar response was seen in non-EAE mice although, in the absence of immunisation the changes in other myeloid populations such as the dendritic cells and granulocytes also reached statistical significance (Figure 4-7C \&D).

Within the liver a similar effect to that in the spleen was observed. There was no significant difference in the proportion of any myeloid subtype between MIS416-treated and untreated mice, regardless of EAE immunisation (Figure 4-8A \&C). However, as the total cell count of the liver was significantly increased with MIS416 treatment, a significant increase in the macrophage (F4/80+), dendritic cell and Gr $1^{\text {low }}$ populations was found within the liver of MIS416-treated nonEAE mice (Figure 4-8D). Although increased, the differences between the total myeloid populations of MIS416-treated and untreated EAE mice did not reach significance, likely because EAE immunisation alone increases cell counts which confounds the MIS416-induced changes. 
B
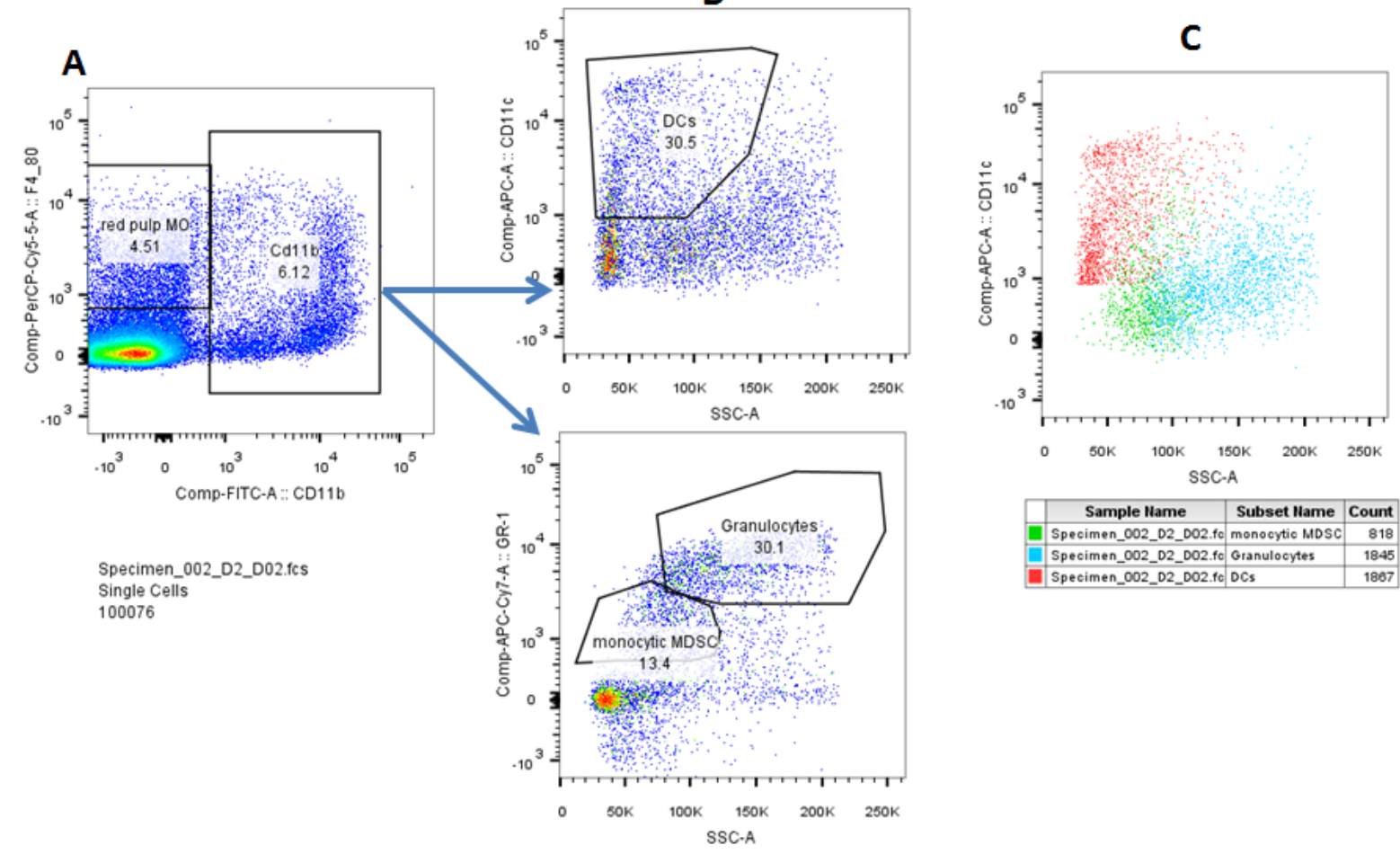

Figure 4-6 Gating strategy for liver and spleen myeloid populations

Gating strategy shown on splenocytes. A) Red pulp macrophages were gated as CD11b-F4/80+ cells and $\mathrm{CD} 11 \mathrm{~b}+$ cells were isolated and gated further. B) Within the CD11b+ population the dendritic cells (DCs) were pulled out as $\mathrm{CD} 11 \mathrm{c}+\mathrm{SSC} \mathrm{C}^{\text {low }}$, granulocytes were $\mathrm{Gr} 1^{\text {high }} \mathrm{SSC}$ variable which are likely to include neutrophils and $\mathrm{Gr} 1^{\text {low }} \mathrm{SSC}^{\text {low }}$ were identified as monocytic myeloid-derived suppressor cells. Differential break down of Gr1 populations (see appendix 1.3) show that $\mathrm{Gr} 1^{\text {high }}$ are $\mathrm{Ly} 6 \mathrm{G}+\mathrm{Ly} 6 \mathrm{C}^{\text {low }}$ and $\mathrm{Gr} 1^{\text {low }}$ populations are Ly6G-Ly6C $\mathrm{C}^{\text {high }}$. C) DCs as shown in red have very little overlap with mMDSC using CD11c to gate the cells, indicating this population is mostly exclusive using this gating strategy. 
A
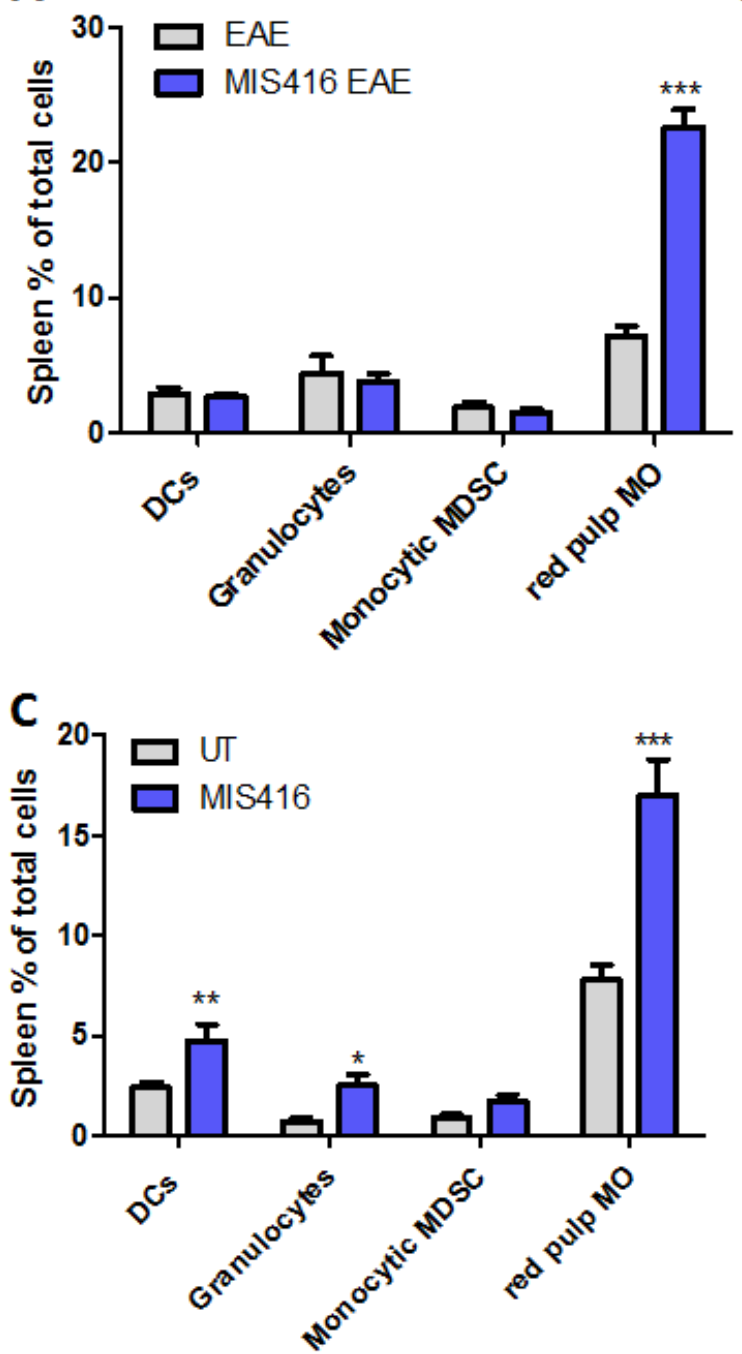

B

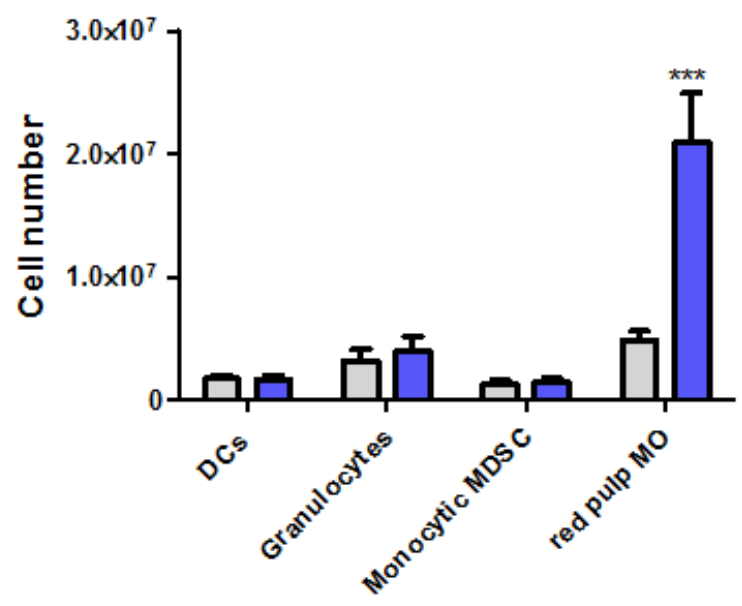

D

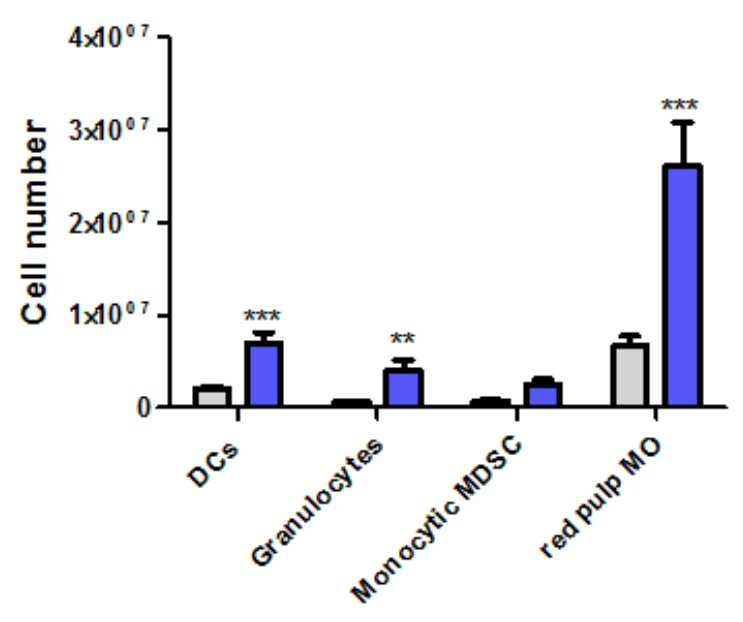

Figure 4-7 The splenic myeloid compartment was significantly altered by MIS416 administration.

C57BL/6 mice were either immunised for EAE or non-immunised and treated weekly by i.v. administration with $100 \mu \mathrm{g} /$ mouse MIS416 starting on the day of immunization (day 0), and splenocytes were isolated at day 22 for EAE experiments and day 15 for non-EAE experiments, and assessed by flow cytometric analysis. A) MIS416 treatment in EAE mice resulted in an increase in the proportion of macrophages (red pulp MØ) and no changes in other myeloid populations. B) MIS416 treatment in EAE mice resulted in an expansion of the macrophage population while other myeloid subsets remained unchanged. C) MIS416-teatment without EAE expanded the proportion of macrophages and non-significant increases in other myeloid populations. D) MIS416 treatment resulted in an expansion of the total macrophage population while other myeloid subsets showed moderate nonsignificant increases. Shown are the means \pm SEM of values from individual mice ( $\mathrm{n}=10$ per group) from 2 experiments. ${ }^{* * *} \mathrm{p}<0.001$ by students t-test (A\&B) and Mann-whitney test $(\mathrm{C} \& \mathrm{D})$. 
A
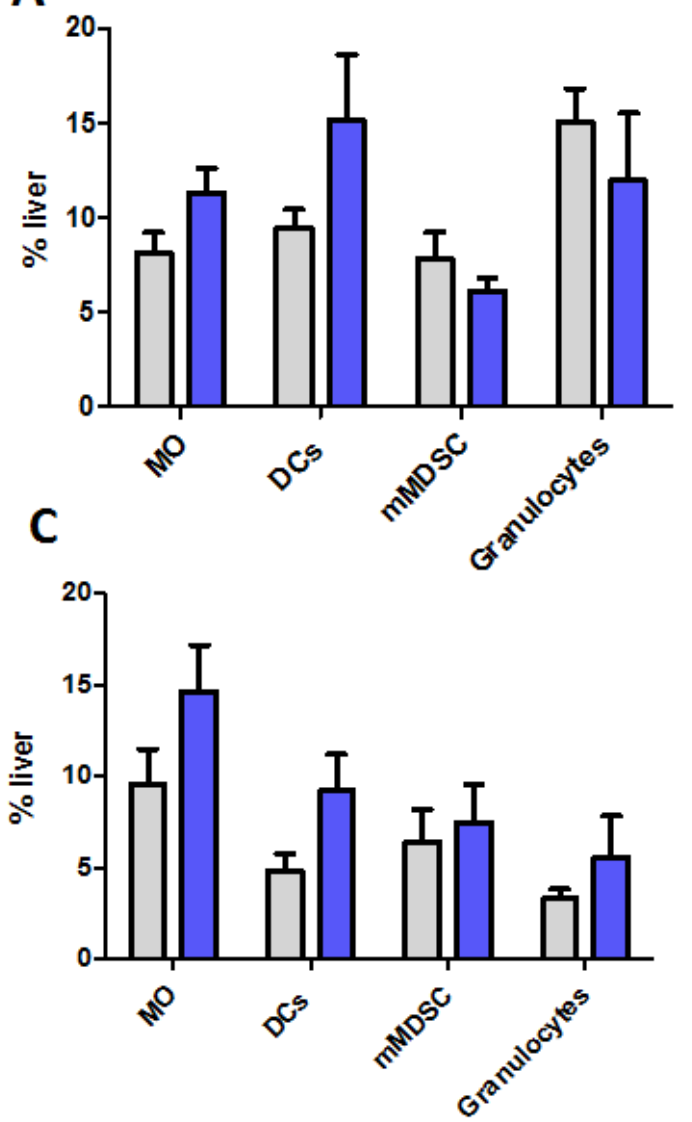

B

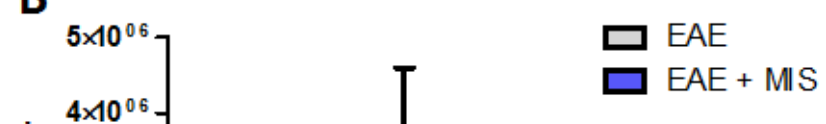

Figure 4-8 The liver myeloid compartment was significantly altered by MIS416 administration.

C57BL/6 mice were either immunised for EAE or non-immunised and treated weekly by i.v. administration with $100 \mu \mathrm{g} /$ mouse MIS416 starting on the day of immunization (day 0), and liver cells were isolated at day 22 for EAE experiments and day 15 for non-EAE experiments, and assessed by flow cytometric analysis. A) MIS416 treatment in EAE mice resulted in no changes in the proportion of myeloid populations. B) MIS416 treatment in EAE mice appeared to non-significantly increase most myeloid populations analysed. C) MIS416-teatment without EAE non-significantly expanded the proportion myeloid populations. D) MIS416 treatment resulted in an expansion of the total macrophage, dendritic cells and monocytic MDSC populations while the neutrophils showed moderate non-significant increase. Shown are the means \pm SEM of values from individual mice $(n=3-$ 10/group) from 1-2 experiments. ${ }^{*} \mathrm{p}<0.05, * * \mathrm{p}<0.01$ unpaired t-test with Welches correction for unequal variances. 


\subsubsection{MIS416 treatment altered $T$ cell populations in the spleen and liver}

MIS416 treatment had the ability to alter the myeloid populations within the spleen and liver; however in the context of EAE, it was also important to identify MIS416-induced changes to the T cell populations. In particular the CD4+ T cells, which are thought to be the drivers of EAE disease, and the Treg population, which is a regulatory population that may be involved in the regulation or reduction of disease symptoms in the later stages of EAE disease, (A. P. Kohm, Carpentier, \& Miller, 2003; Paust \& Cantor, 2005) were of importance in our study. EAE mice treated with MIS416 had no significant alterations in the splenic proportion of $\mathrm{T}$ cell populations including CD4, CD8, NK and Tregs, however the total number of CD4+ and CD8+ T cells were significantly increased with MIS416 treatment at day 22 post immunisation (Figure 4-9B \&C). Interestingly, the total number of Tregs (as indicated by CD4+CD25 $5^{\text {high }}$ ) in the spleen of MIS416treated EAE mice was significantly increased at the day 22 time point (Figure 4-9C) which indicated that MIS416 treatment in EAE mice may drive a more regulatory phenotype.

This analysis was the first to show that MIS416 treatment altered not only the splenic myeloid compartment within EAE mice, but also the $\mathrm{T}$ cell populations as well. To establish if the changes in $\mathrm{T}$ cell populations induced by MIS416 were EAE disease specific or non-specific, we determined if these changes were also occurring in non-EAE MIS416-treated mice. We found there were similar alterations of $\mathrm{T}$ cell populations in MIS416-treated non-EAE mice after three MIS416 treatments (i.e. at day 15) as those observed in the EAE mice after four MIS416 treatments (i.e. at day 22). Again, the proportions of $\mathrm{T}$ cell populations were not altered whereas the total number of splenic CD4+ and CD8+ T cells were significantly increased with MIS416 treatment (Figure 4-10A \&B). The Treg population was also significantly altered in MIS416treated mice without EAE (Figure 4-10C), but the difference was not as great nor as consistent as that seen during EAE (Figure 4-10D). This result could suggest that MIS416 treatment in EAE mice may enhance the Treg induction pathway in EAE, which is present in later stages of disease to naturally limit the disease progression, and may also explain why we saw a more consistent and higher number of Tregs in MIS416-treated EAE mice compared to non-EAE mice given that this pathway would already be activated in the EAE mice compared to the healthy controls.

As the liver is an organ of particular interest in MIS416 treatment and we know that the liver is a naturally tolerogenic organ (Tiegs \& Lohse, 2010), we investigated whether similar T cell alterations as observed in the spleen also occurred in the liver. We found that were significant increases in both the percentage and total number of CD4+ T cells in the livers of MIS416-treated EAE mice but no significant alterations in the CD8+ T cells and NK cells were seen (Figure 
4-11A \&B). In contrast, while there were significant decreases in proportions of the CD4 $\mathrm{T}$ cells and NK cells in the MIS416-treated non-EAE mice, there were between 2 to 4-fold increase in the total number of $\mathrm{CD} 4, \mathrm{CD} 8$ and NK cells compared to the control mice, which reflected the large increases in the total number of leukocytes found within the liver after MIS416 treatment (Figure 4-11C \&D).

Initially Tregs were identified as CD4+CD25+; however CD25, the receptor for IL-2, is also upregulated on activated $\mathrm{CD} 4+\mathrm{T}$ cells and therefore under inflammatory conditions not all CD4+CD25+ cells may be Tregs. Instead the transcription factor FoxP3 can be used as a more definitive marker for Tregs although, being a transcription factor it is more difficult to detect by flow cytometry. As an accurate way of assessing FoxP3 expression, we used mice that expressed GFP-labelled FoxP3 and using flow cytometry gated on CD4+CD25+FoxP3GFP+ cells to quantify the Treg population. Using these mice, the same trend in MIS416-induced increases in liver and splenocyte counts was observed, although due to the small sample size this experiment was not subject to statistical analysis (Figure 4-12A $\mathbf{\& B}$ ). While the percentage of CD4 cells that were CD25+FoxP3+ increased in the livers but not the spleens of MIS416-treated mice (Figure 4-12C), the total number of Treg cells increased in both the livers and spleens of MIS416-treated (Figure 4-12E). These findings are consistent with the studies performed without FoxP3 expression (Figure 4-9C). Furthermore, previous studies have described another Treg population which is FoxP3+ but CD25-, and this population appears to be overexpressed in the liver of MIS416-treated mice compared to control mice (Figure 4-12D).

Taken together, these results show that MIS416, an innate cell modifier, affected the $\mathrm{T}$ cell compartment within the spleen and liver and in particular, increased in the number of Tregs which are known to have a role in regulating EAE. 

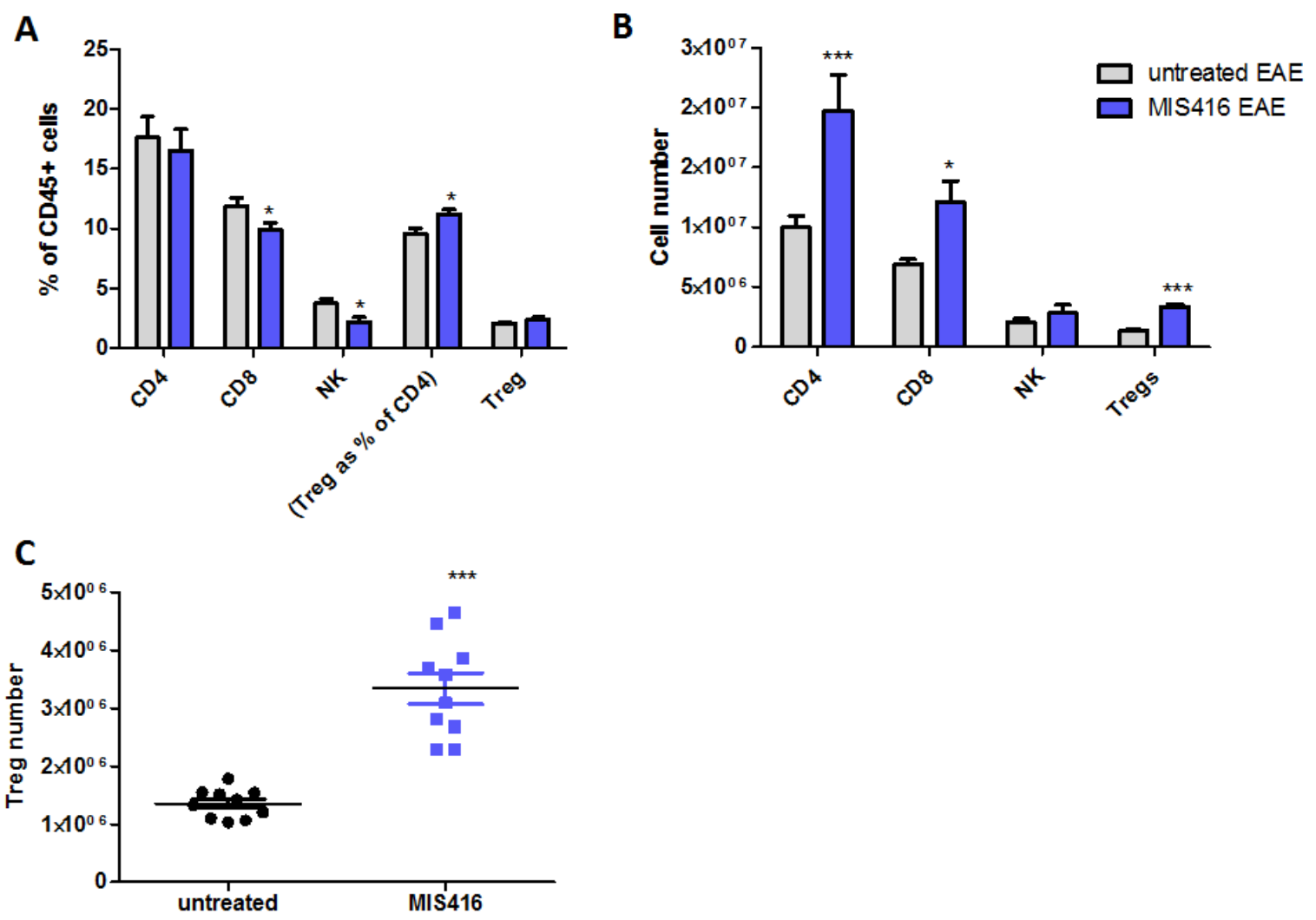

Figure 4-9 Administration of MIS416 increased the total number of Treg, CD4 and CD8 T cells in EAE mice.

C57BL/6 mice were either immunised for EAE or non-immunised and treated weekly by i.v. administration with $100 \mu \mathrm{g} /$ mouse MIS416 starting on the day of immunization (day 0). On day 22 splenocytes were isolated from MIS416-treated or untreated mice and run on the flow cytometer with T cell markers. A) MIS416 treatment significantly altered $\mathrm{T}$ cell populations as a proportion of CD45+ (except Treg \% CD4 shows the proportion of CD4+ T cells which were Tregs). B) Total cell numbers of $\mathrm{T}$ cell populations were also altered by MIS416 treatment. C) Treg numbers were significantly increased in MIS416-treated EAE mice compared to controls. Shown are the means \pm SEM of values from individual mice from 2 ( $n=5$-10/group) experiments. ${ }^{*} p<0.05$ and *** $p<0.001$ MIS416-treated compared to control mice by unpaired t-test with Welch's correction. 
A
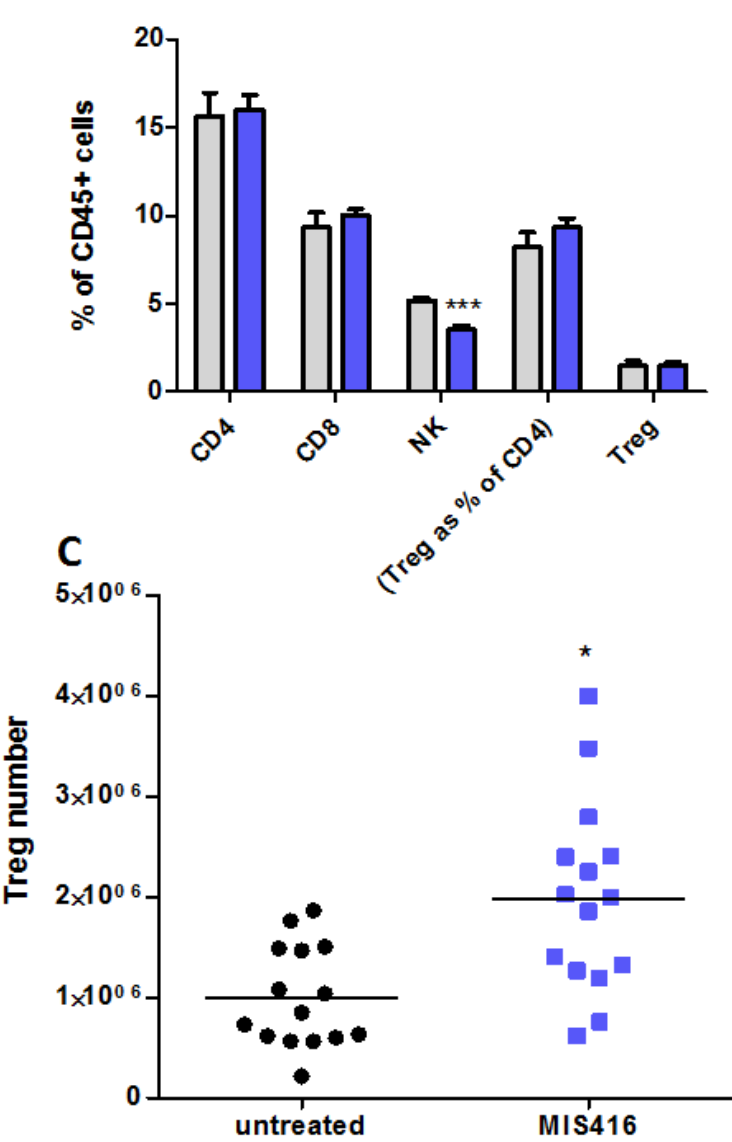

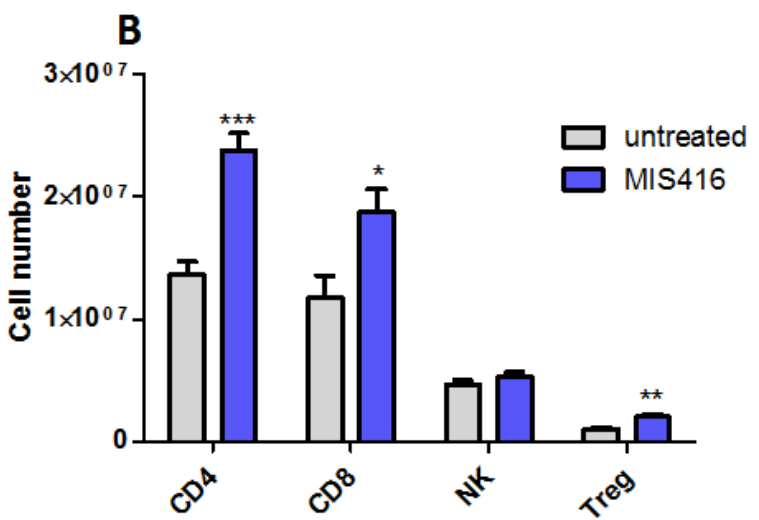

D

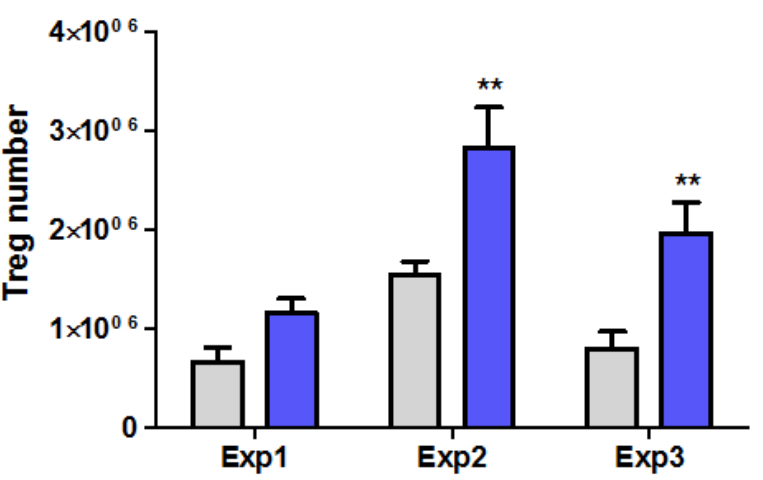

Figure 4-10 Administration of MIS416 increased the total number of Treg, CD4 and CD8 T cells in nonEAE mice.

C57BL/6 mice were treated weekly by i.v. administration of $100 \mu \mathrm{g} /$ mouse MIS416. On day 15 splenocytes were isolated from MIS416-treated or untreated mice and run on the flow cytometer with T cell markers. A) MIS416 significantly reduced NK cells as a proportion of CD45+, note (Treg \% CD4) shows the proportion of CD4+ T cells which were Tregs. B) MIS416 treatment significantly increased the number of CD4, CD8 and Treg cells). C) Treg number was significantly increased in MIS416-treated non-EAE mice. D) Variable levels of Treg number across experiments however a trend of increased Tregs with MIS416 is seen across all 3 experiments. Shown are the means \pm SEM of values from individual mice from 3 ( $n=15 /$ group) experiments unless indicated otherwise $* \mathrm{p}<0.05, * * \mathrm{p}<0.01$ and $* * \mathrm{p}<0.001$ MIS416-treated compared to untreated mice by unpaired t-test with Welch's correction for unequal variances. 

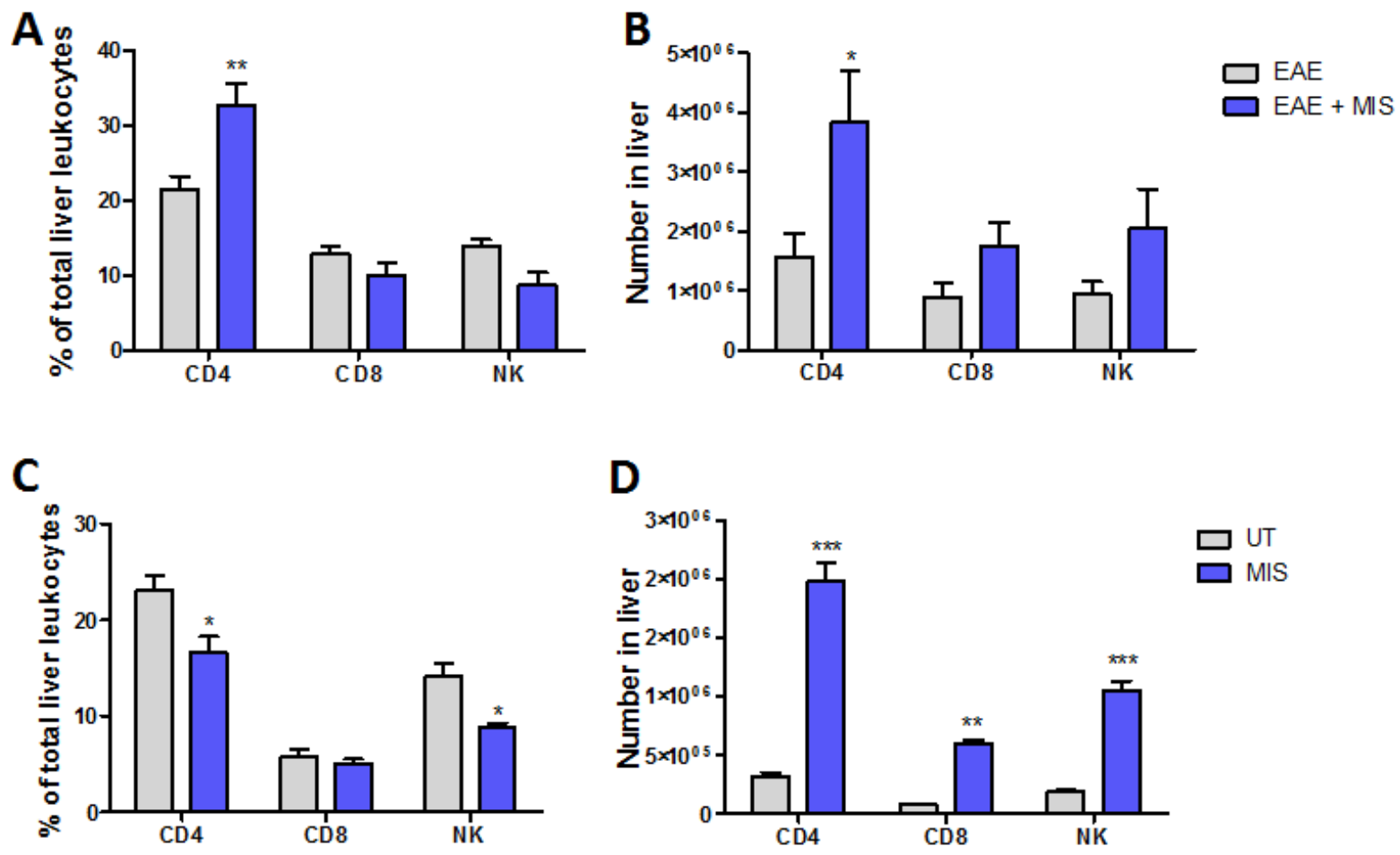

Figure 4-11 Administration of MIS416 increased the liver T cell populations

C57BL/6 mice were either immunised for EAE or non-immunised and treated weekly by i.v. administration with $100 \mu \mathrm{g} /$ mouse MIS416 starting on the day of immunization (day 0), and liver cells were isolated at day 22 for EAE experiments and day 15 for non-EAE experiments, and assessed by flow cytometric analysis. A) MIS416 treatment in EAE resulted in increased percentage of CD4+ cells. B) MIS416 treatment in EAE mice significantly increased total CD4+ cells C) MIS416-teatment without EAE non-significantly expanded the proportion CD4+ cells and decreased the proportion of NK cells. D) MIS416 treatment resulted in an expansion of the total CD4+, CD8+ and NK cells within the liver. Shown are the means \pm SEM of values from individual mice $(n=3-$ 10 /group) from 1-2 experiments. ${ }^{*} \mathrm{p}<0.05,{ }^{*} \mathrm{p}<0.01$ and ${ }^{* * *} \mathrm{p}<0.001$ comparing MIS416-treated to untreated mice by an unpaired $t$-test. 
A

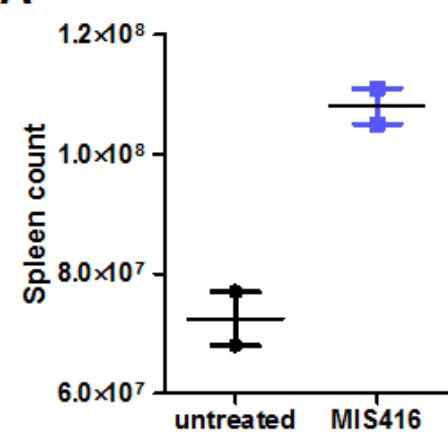

C

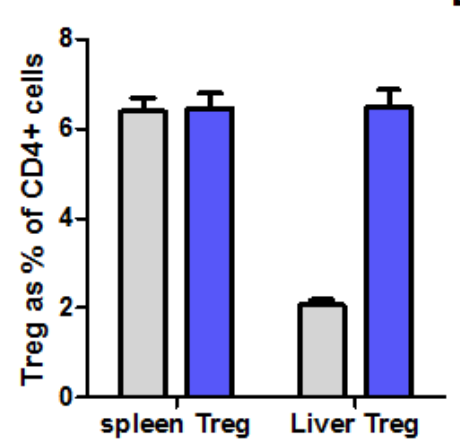

B

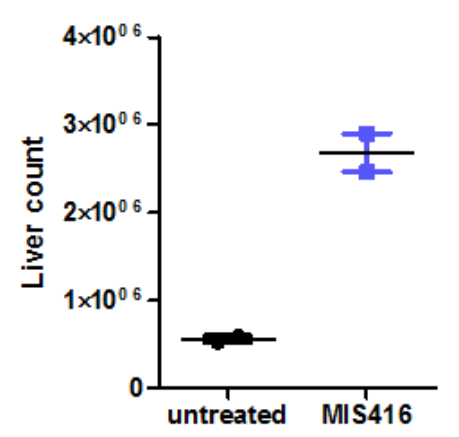

D

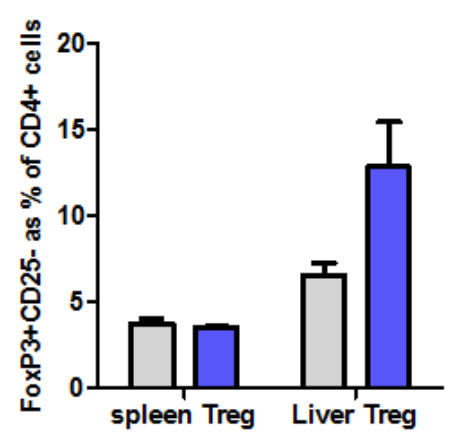

$\mathbf{E}$

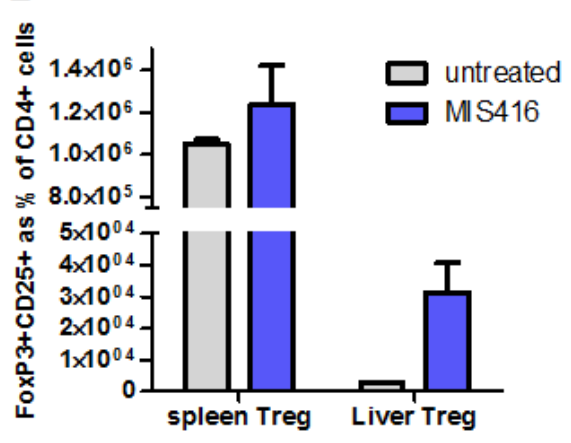

Figure 4-12 Weekly MIS416 treatment increased FoxP3+ populations in spleen and liver using FoxP3+GFP mice.

FoxP3-GFP mice were treated weekly by i.v. administration of $100 \mu \mathrm{g} / \mathrm{mouse}$ MIS416. On day 15 splenocytes and liver leukocytes were isolated from MIS416-treated or untreated mice and analysed with flow cytometry for regulatory T cell markers. A) Spleen count appeared to increase with MIS416 treatment. B) Liver count appeared to increase with MIS416 treatment. C) Treg cells as percentage of CD4+ cells. D) FoxP3+CD25- cells as percentage of CD4+ cells. E) FoxP3+CD25- cells as a percentage of CD4+ cells. Shown are the means \pm SEM of values from individual mice from 1 experiment $n=2 /$ group, given the small sample size no statistical tests were performed. 


\subsubsection{MIS416 treatment altered immune cell activation in the spleen and liver}

Myeloid cells such as macrophages and dendritic cells appeared to be the key cell types that could pick up and respond to MIS416 and, as we showed that MIS416 had major effects on these myeloid populations in the spleen and liver, we characterised the activation state of these cells. The assessment of MHCII (major histocompatibility class II) and PD-L1 (programmed death ligand 1) expression was determined because it is known these ligands are able to interact with receptors on T cells (the TCR and PD-1, respectively), and therefore have the ability to alter an adaptive immune response (Corse, Gottschalk, \& Allison, 2011; Yamazaki et al., 2002).

Within the spleen of control mice we found that different myeloid populations expressed different levels of PD-L1 and MHCII and that with MIS416 treatment there were significant increases in both PD-L1 and MHCII expression across all myeloid populations assessed (Figure 4-13 A \&B). The same trend was found within the liver in the macrophage (single-positive gated F4/80+ cells) and general myeloid (single-positive gated CD11b+ cells) populations, with PD-L1 and MHCII expression significantly up-regulated after MIS416 treatment (Figure 4-13C \& D). We also assessed the expression of CD14 on liver macrophages since CD14 is part of the TLR4 complex and it is known to be up regulated in response to macrophage stimulation (G. L. Su, 2002). Similar to PD-L1 and MHC-II expression the expression of CD14 was also significantly upregulated on liver macrophages from MIS416-treated mice compared to controls (Figure 4-13E).

These results suggest that MIS416 treatment had the ability to alter the activation state of all myeloid populations within the spleen and liver and these alterations may have led to downstream alterations in $\mathrm{T}$ cell activation. 

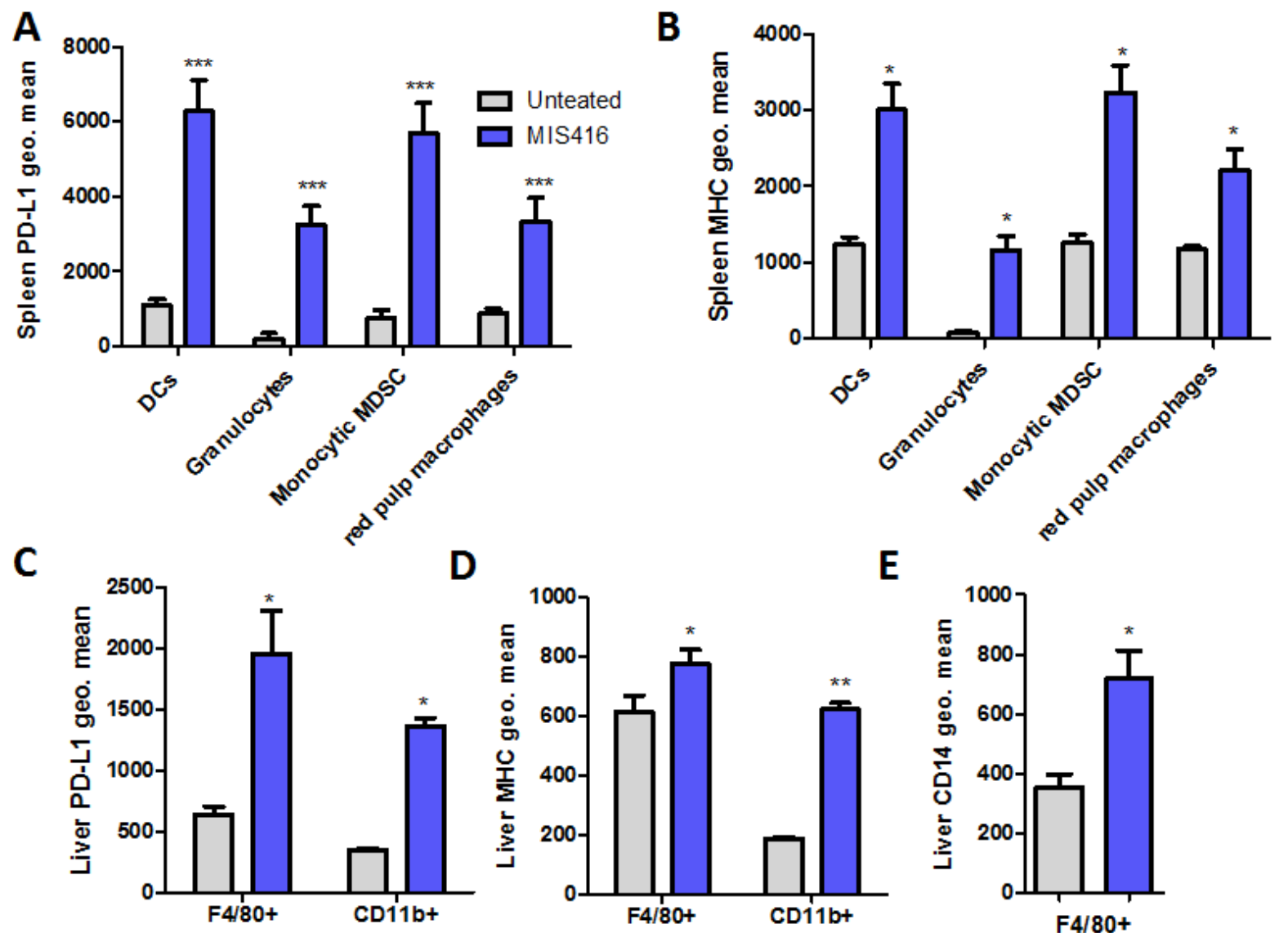

E

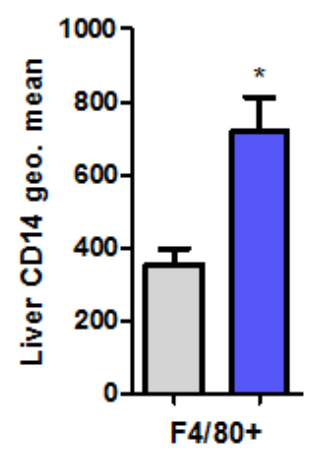

\section{Figure 4-13 MIS416 activated myeloid populations}

C57BL/6 mice were treated weekly by i.v. administration of $100 \mu \mathrm{g} /$ mouse MIS416. On day 15 splenocytes and liver leukocytes were isolated stained for myeloid activation markers. A) PD-L1 expression on splenic myeloid populations. B) MHC II expression on splenic myeloid populations. C) PD-L1 expression on liver myeloid populations. D) MHC II expression on liver myeloid populations. E) CD14 expression on liver macrophages (F4/80+ cells). Shown are the means \pm SEM of values from individual mice from 1-2 experiments $(n=4-$ $15 /$ group $) * \mathrm{p}<0.05,{ }^{* *} \mathrm{p}<0.01$ and $* * * \mathrm{p}<0.001$ MIS416-treated compared to control mice by Mann-Whitney Utest. 


\subsubsection{Spleen and liver cytokine profile was altered with in vivo MIS416 treatment}

Activated cells not only increase their expression of surface markers but also produce cytokines in response to a stimulus (Janeway \& Medzhitov, 2002). Therefore, to determine the cytokines produced in MIS416-treated mice, we assessed the production of IFN- $\gamma$, IL-17A, IL-10 and nitric oxide (NO) after re-stimulation with various compounds including MIS416 in splenocyte and liver leukocyte cultures. At day 22 post-immunisation in vivo MIS416 treatment significantly reduced the MOG-specific production of IFN- $\gamma$ and IL-17A by splenocytes, while there was no significant difference in MOG-induced NO and IL-10 production with MIS416 treatment (Figure 4-14). These results were consistent with the disease protection seen in Chapter 3 as we would expect that with the reduced disease seen in MIS416 treated mice we would also see reduced antigenspecific cytokine production. Additionally, splenocytes from MIS416-treated mice re-stimulated in vitro with MIS416 produced significantly higher levels of IFN- $\gamma$, nitric oxide and IL-10 compared to untreated mice (Figure 4-14A, C\& D). Furthermore, while there was very little IL-17A produced in response to MIS416, it appears that untreated mice produced more IL-17A than MIS416-treated mice (Figure 4-14B).

In non-EAE mice, a similar increase in IFN- $\gamma$, NO and IL-10 production in response to MIS416 by splenocytes from MIS416-treated mice compared to control was observed at day 15, while no IL17A was detected (data not shown). The response to LPS was similar to that found with the MIS416 re-stimulation, and there were significant increases in IFN- $\gamma$ and NO production. IL-10 production was increased in response to LPS; however this increase was not of the same magnitude as the MIS416 response indicating that MIS416 is a better inducer of IL-10 under these conditions than LPS. Using ConA (concanavalin A) as an antigen non-specific T cells stimulus, we found IFN- $\gamma$ levels were not affected by MIS416 treatment whereas significantly more NO and IL-10 were produced in response to ConA in MIS416-treated mice compared to control mice (Figure 4-15).

In liver leukocytes isolated on day 15 from EAE mice and re-stimulated with MOG, MIS416 and LPS it was also found that MIS416-induced specific changes in cytokines productions. The MOGspecific response by liver leukocytes was characterised by significant reductions in IFN- $\gamma$ and IL17A and a significant increase in NO in mice receiving MIS416 treatment in vivo (Figure 4-16A,B \&C). In response to MIS416 re-stimulation ex vivo high levels of IFN- $\gamma$, NO and IL-10 were produced by mice which received MIS416 treatment in vivo producing significantly more than liver leukocytes from mice which were untreated. This response was also similar in the LPS cultures where MIS416-treated mice produce higher levels of IFN- $\gamma$, NO and IL-10 than liver 
leukocytes from mice which were untreated (Figure 4-16). In the liver of MIS416-treated nonEAE mice there was significant production of IFN- $\gamma$ and NO in cultures stimulated with media, MIS416 and LPS, whereas untreated non-EAE mice had no detectable IFN- $\gamma$ and NO when restimulated with the same factors (Figure 4-17).

Although only a small number of cytokines have been shown here, we have also assessed the production of various other cytokines in MIS416-treated mice including IL-6, IL-4, IL-13 and IL22 (see appendix 1.5; (White et al., 2014)) and found these ones to be of most interest in the model. Taken together these results show that not only did MIS416 alter cell number and activation states in treated mice, but also the liver leukocytes and splenocytes were primed to respond differently to immunological stimuli (i.e. had a different cytokine profile) than untreated mice. 


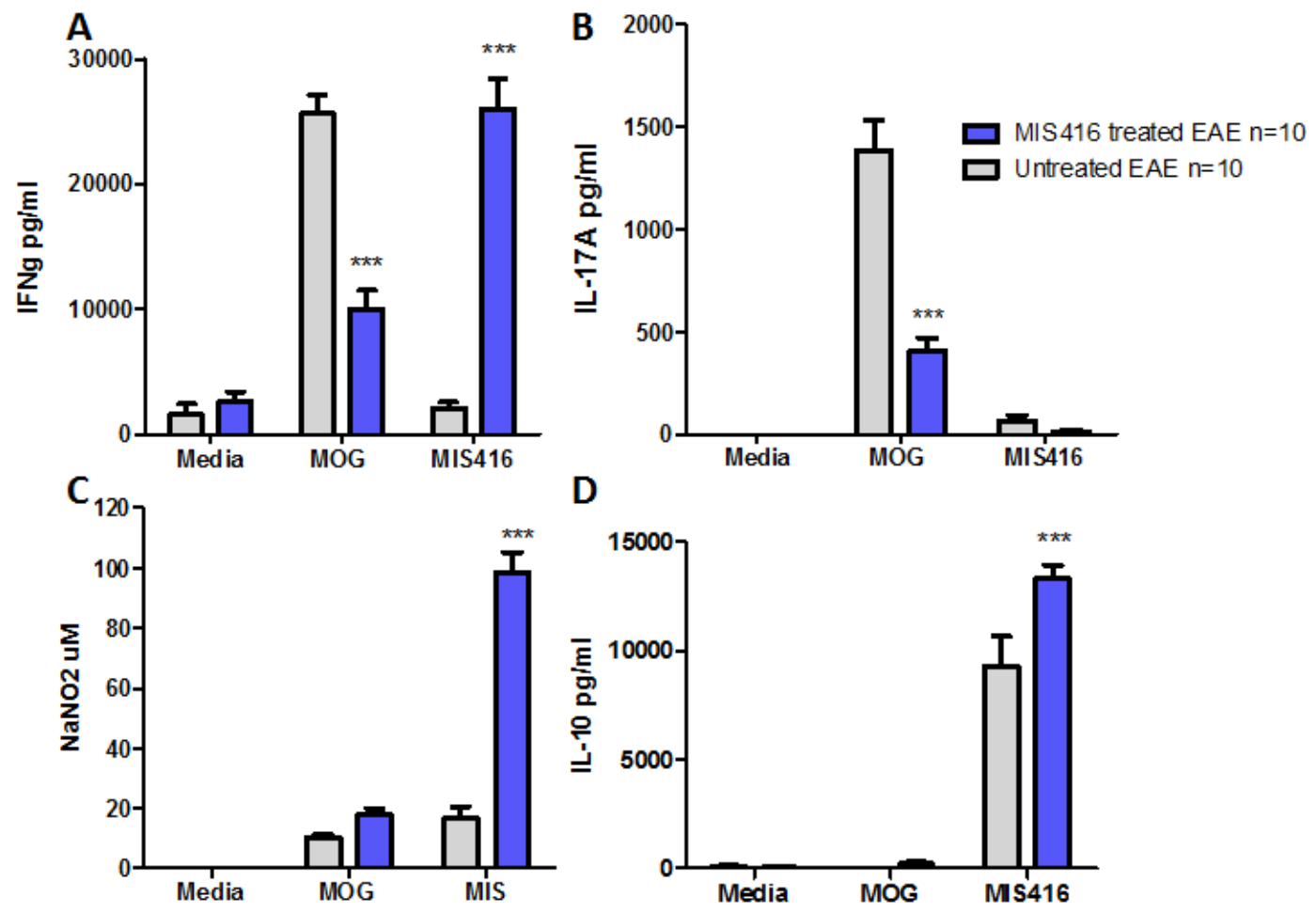

Figure 4-14 MIS416 enhanced MIS416-stimulated production of IFN- $\gamma$, NO and IL-10 but not IL-17A by splenocytes from EAE mice.

C57BL/6 mice were immunised to induce EAE and treated weekly by i.v. administration with $100 \mu \mathrm{g} / \mathrm{mouse}$ MIS416 starting on the day of EAE induction. On day 22 splenocytes were isolated from MIS416-treated or untreated EAE mice and cultured $\left(10^{6}\right.$ cells/well) with media alone, MOG peptide $(27 \mu \mathrm{g} / \mathrm{ml})$ or MIS416 (20 $\mu \mathrm{g} / \mathrm{ml}$ ) for 72 hours. Cytokines in the culture supernatants were assayed by ELISA (A, B \&D) or Griess (C). A) IFN- $\gamma$ production by splenocytes. B) IL-17A production. C) NO production. D) IL-10 production. Shown are the means \pm SEM of values from individual mice from 3 ( $n=15 /$ group, a,c,d) or 2 ( $n=10 /$ group; b) experiments. * $\mathrm{p}<0.05, * * \mathrm{p}<0.01$, and $* * * \mathrm{p}<0.001$ EAE compared to MIS EAE by 2 -way ANOVA with Bonferronis post-test. 
A

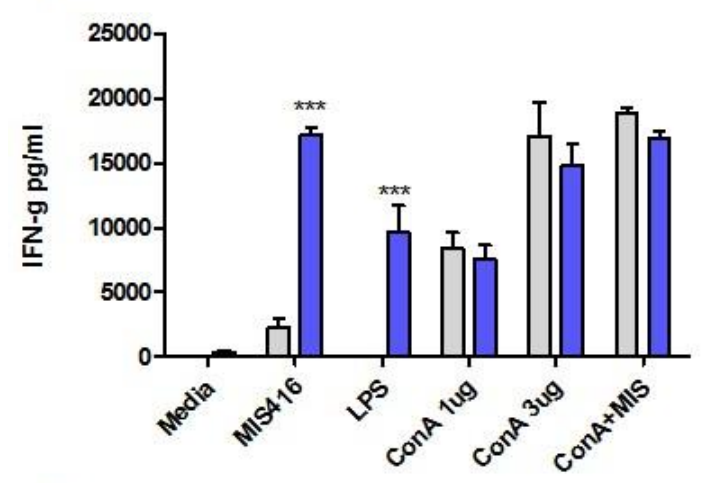

C

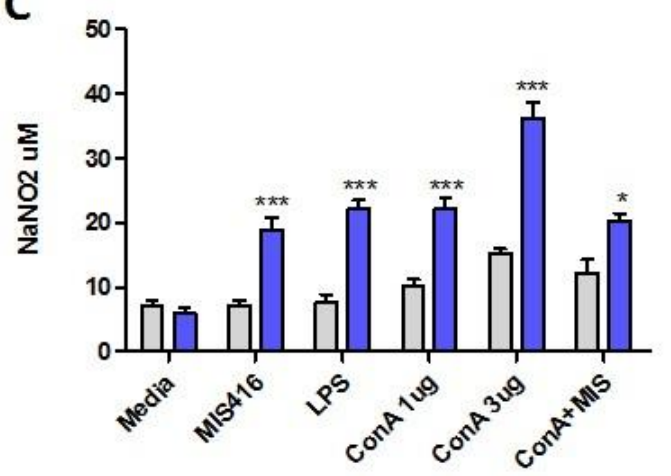

B

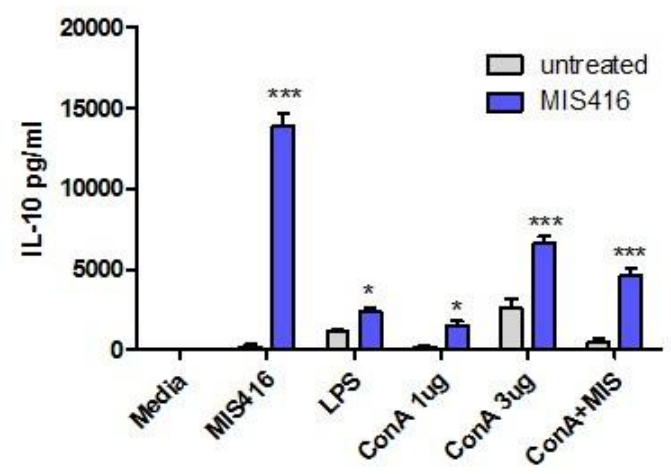

Figure 4-15 MIS416 enhanced MIS416-stimulated production of IFN- $\gamma$, NO and IL-10 by splenocytes from non-EAE mice.

C57BL/6 mice were treated weekly by i.v. administration of $100 \mu \mathrm{g} /$ mouse MIS416. On day 15 splenocytes were isolated from MIS416-treated or untreated mice and cultured $\left(10^{6}\right.$ cells/well) with media alone, MIS416 (20 $\mu \mathrm{g} / \mathrm{ml}$ ), LPS (200 ng/ml) ConA (1 or $3 \mu \mathrm{g} / \mathrm{ml}$ ) or ConA and MIS416 ( $1 \mu \mathrm{g} / \mathrm{ml}$ and $20 \mu \mathrm{g} / \mathrm{ml} \mathrm{respectively)} \mathrm{for} 48$ hours. Cytokines in the culture supernatants were assayed by ELISA (A \&B) or Griess (C). A) IFN-ץ production. B) IL-10 production. C) NO production. Shown are the means \pm SEM of values from individual mice from 1-3 (n=5-15/group) experiments. $* \mathrm{p}<0.05, * * \mathrm{p}<0.01$, and ${ }^{* * *} \mathrm{p}<0.001$ MIS416-treated compared to untreated mice by 2-way ANOVA with Bonferronis post-test. 
A

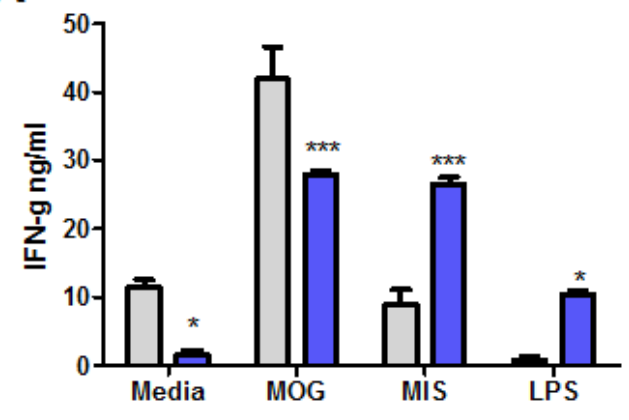

C

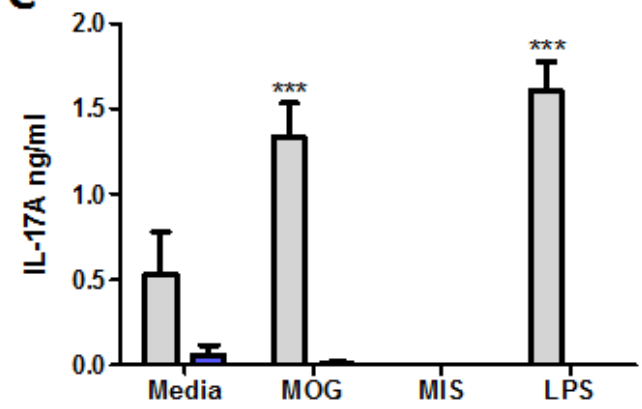

B

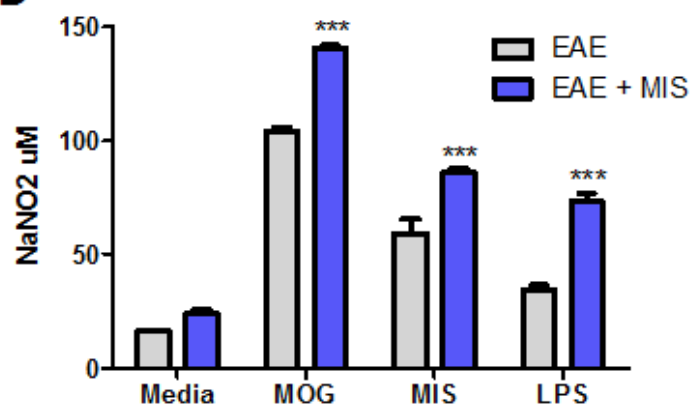

D

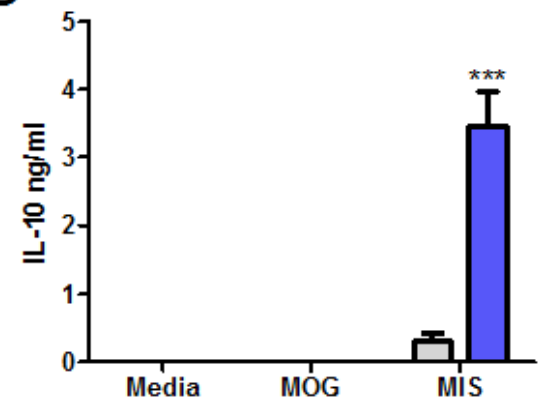

Figure 4-16 MIS416 enhanced MIS416-stimulated production of IFN- $\gamma$, NO and IL-10 but not IL-17A by liver leukocytes from EAE mice.

C57BL/6 mice were immunised to induce EAE and treated weekly by i.v. administration of $100 \mu \mathrm{g} / \mathrm{mouse}$ MIS416 starting on the day of EAE induction. On day 15 leukocytes were isolated from MIS416-treated or untreated EAE mice and cultured $\left(2.5 \times 10^{5}\right.$ cells/well $)$ with media alone, MOG peptide $(27 \mu \mathrm{g} / \mathrm{ml})$, MIS416 (20 $\mu \mathrm{g} / \mathrm{ml})$ or LPS $(200 \mathrm{ng} / \mathrm{ml})$ for 72 hours. Cytokines in the culture supernatants were assayed by ELISA (A, C\&D) or Griess (B). A) IFN- $\gamma$ production. B) NO production. C) IL-17A production. D) IL-10 production. Shown are the replicate means \pm SEM of values from pooled liver cells from 1 experiment, $n=5$ originally. $* p<0.05$, and *** $\mathrm{p}<0.001$ MIS416-treated compared to untreated mice by 2-way ANOVA with Bonferronis post-test. 
A

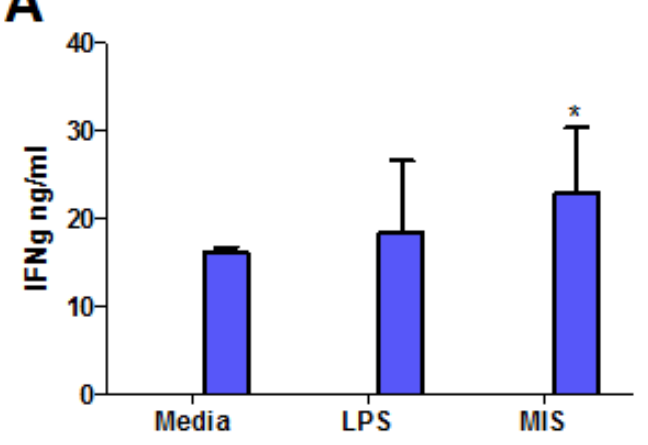

B

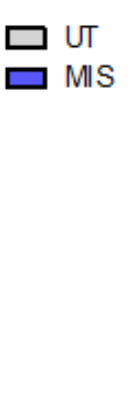

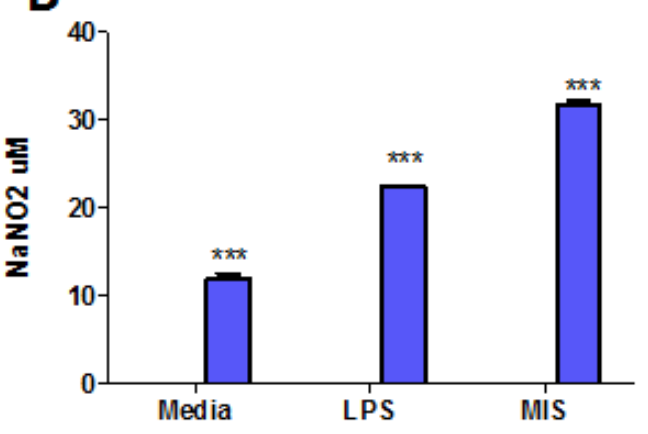

Figure 4-17 MIS416 enhanced MIS416-stimulated production of IFN- $\gamma$ and NO by liver leukocytes from non-EAE mice.

C57BL/6 mice were treated weekly by i.v. administration of $100 \mu \mathrm{g} / \mathrm{mouse}$ MIS416. On day 15 liver cells were isolated from MIS416-treated or untreated mice and cultured $\left(2.5 \times 10^{5}\right.$ cells/well) with media alone, MIS416 (20 $\mu \mathrm{g} / \mathrm{ml}$ ), LPS (200 $\mathrm{ng} / \mathrm{ml}$ ) for 48 hours. Cytokines in the culture supernatants were assayed by ELISA (A) or Griess (B). A) IFN- $\gamma$ production. B) NO production. Shown are the replicate means \pm SEM of values from pooled liver cells from 1 experiment, $\mathrm{n}=5$ originally. MIS416-treated compared to untreated mice by 2-way ANOVA with Bonferronis post-test. * $\mathrm{p}<0.05$, and $* * * \mathrm{p}<0.001$ 


\subsubsection{Serum IFN- $\gamma$, IL-6 and NO were increased with MIS416 treatment}

The cytokine response in stimulated splenocytes and liver cells showed that MIS416 treatment can alter the cytokine profile, however to determine if any of these cytokines were being secreted systemically at physiologically relevant concentrations, we assessed the serum cytokine levels. In the MIS416-treated mice, there was significant production of IFN- $\gamma$, which peaked at day 15 and could be found in the serum of both EAE and non-EAE mice. In contrast, IL-6 was consistently produced at all time-points assessed in MIS416-treated mice compared to untreated mice and was also consistent in both EAE and non-EAE mice (Figure 4-18A \&B). Because we found significantly elevated NO production induced by MIS416 in the in vitro cultures, we assessed the concentration of serum NO in MIS416-treated mice. Similar to the in vitro culture results, we found that MIS416-treated mice also had significantly increased serum NO both with and without EAE when compared to untreated mice (Figure 4-18C). Given that the increased serum NO and IFN- $\gamma$ concentrations induced by MIS416 were consistent with results from the spleen and liver cultures, one possible explanation is that these cytokines significantly contribute to the mechanism of action of MIS416. Interestingly, we also observed that independent of MIS416, EAE mice had significantly higher levels of serum NO than non-EAE mice at day 22, indicating that NO may have a role in EAE disease resolution or pathogenesis independently of MIS416 treatment (Figure 4-18D). 
A

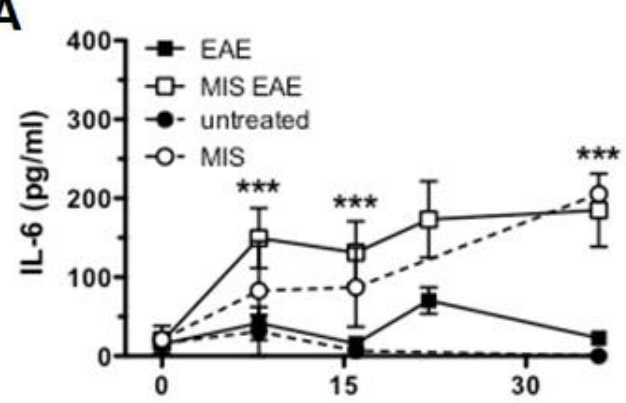

B

day after immunization

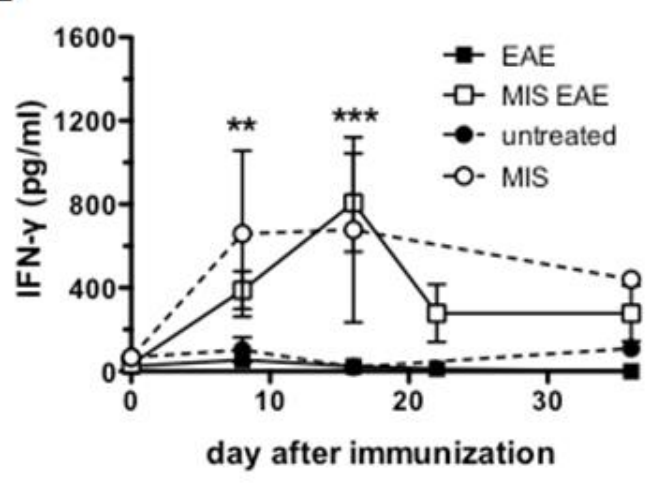

C

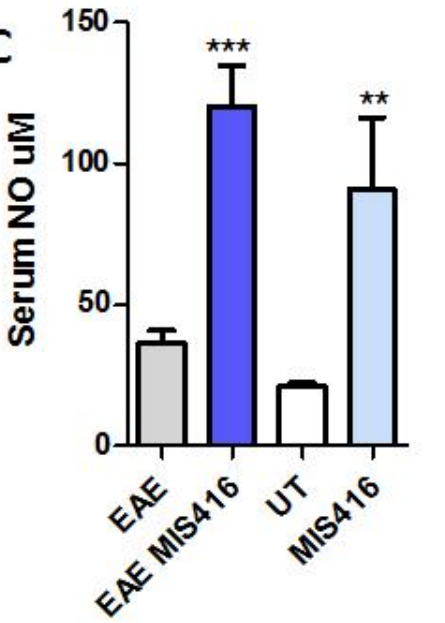

D

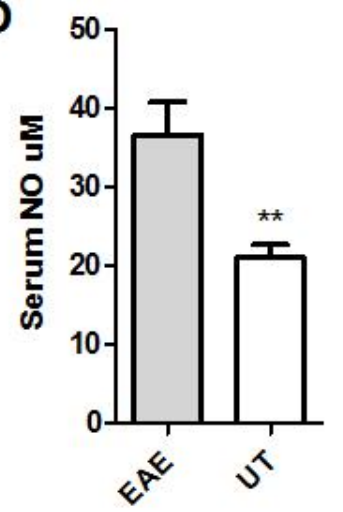

Figure 4-18 Serum cytokines increased with MIS416 treatment in vivo

C57BL/6 mice were either immunised for EAE on day 0 or left untreated, and some received weekly i.v. administration of $100 \mu \mathrm{g} /$ mouse of MIS416 also beginning on day 0 . Serum was collected as per methods at each time point, and a cytokine bead assay or Enzo nitric oxide detection kit was used to assess serum IFN- $\gamma$, IL- 6 and NO. A) Serum IFN- $\gamma$ over time in both non-EAE and EAE mice. B) IL-6 production over time in both non-EAE and EAE mice. C) Total NO at day 22 in EAE and day 15 in non-EAE mice with and without MIS416 treatment. D) Total serum NO in untreated versus EAE mice at day 22. Shown are the means \pm SEM of values from 1-5 experiments $(\mathrm{n}=3-26)$. $* * \mathrm{p}<0.01$ and $* * * \mathrm{p}<0.001$ MIS416-treated compared to untreated mice, 2-way ANOVA with Bonferronis Multiple Comparison post-test (A) \& (B), 1-way ANOVA with Kruskal-Wallis post-test (C) or Mann-Whitney U-test (D). 


\subsubsection{CD4 $\mathrm{T}$ cells and F4/80+ cells were the main producers of splenic IFN- $\gamma$}

Considering that IFN- $\gamma$ was consistently produced in response to MIS416, it was important to determine what cell types were producing this cytokine. Additionally, as IFN- $\gamma$ was produced at similar levels in both EAE and non-EAE mice, we investigated the cellular source of IFN- $\gamma$ in the absence of confounding effects induced by EAE immunisation. Because splenocytes that have been in culture for 48 hours are likely to become IFN- $\gamma$ positive during the culture time, we initially assessed IFN- $\gamma$-positive splenocytes after a 5-hour re-stimulation as a more accurate measure of cells that were producing IFN- $\gamma$ in vivo. When we assessed the total number of IFN- $\gamma$ positive cells in these short-term cultures, we found that the MIS416-treated mouse splenocytes had more IFN- $\gamma$-positive cells than control mouse splenocytes under both the media and MIS416 re-stimulation conditions (Figure 4-19A). Further characterisation of the IFN- $\gamma$-positive cell populations showed that the percentage of CD4+ cells (likely to be CD4+ T cells) which were IFN- $\gamma$ positive was significantly increased in cultures from MIS416-treated mice stimulated with either media or MIS416 (Figure 4-19B) whereas there was no change in the NK or macrophage populations (Figure 4-19C \&D).

To determine the cell types which produced the high levels of IFN- $\gamma$ within the cell culture period in response to MIS416, we assessed intracellular IFN- $\gamma$ staining at 48 hour post re-stimulation. The positive cells within the media culture were not significantly altered by in vivo MIS416 treatment; however, there was a significant decrease in the percentage of IFN- $\gamma$ positive cells within the ConA cultures of MIS416-treated mice compared to untreated mice (Figure 4-20A). As expected, the percentage of IFN- $\gamma$-positive cells after MIS416 re-stimulation of cells from MIS416-treated mice was significantly increased (Figure 4-20A). Many different cell populations were IFN- $\gamma$ positive in the ConA cultures including a large proportion of the CD4+, CD8+, NK, NKT and B cells, and while most populations were similar in the MIS416-treated and untreated mice, there was a significant reduction in IFN- $\gamma$-positive CD4+ cells in ConA cultures from mice which were MIS416-treated in vivo. These results show that MIS416 treatment affected the ability of CD4 T cells to produce IFN- $\gamma$ upon ConA stimulation while in contrast, there were significant increases in IFN- $\gamma$-positive CD4+ T cells and the NKT cells (NK1.1+CD3+) upon MIS416 re-stimulation ex vivo (Figure 4-20).

While these results were attained with the addition of PMA/ionomycin to the cultures, when PMA and ionomycin were absent from the cultures, we found a similar trend in the percentage of total IFN- $\gamma$ positive cells with media, ConA and MIS416 (Figure 4-21A). Furthermore, it was found that the majority of positive cells with MIS416 treatment either in vivo or ex vivo, were made up of 
CD4 $\mathrm{T}$ cells and F4/80+ macrophages. Therefore, we believe these are the key cell populations in producing IFN- $\gamma$ in response to MIS416 treatment and thus, could be the key cell types responsible for disease protection seen in MIS416-treated EAE mice. 


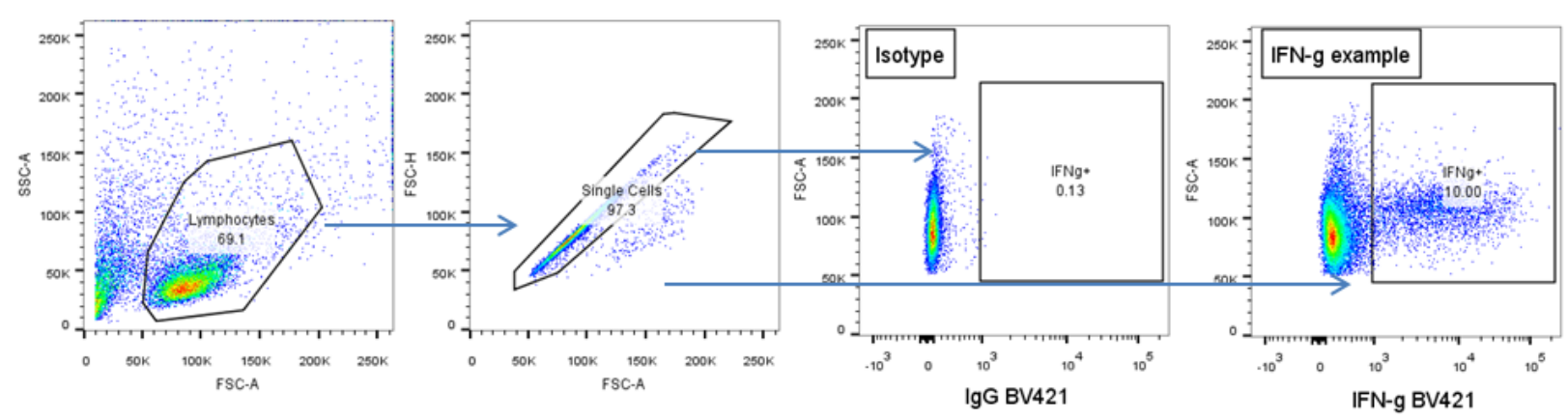

A

B
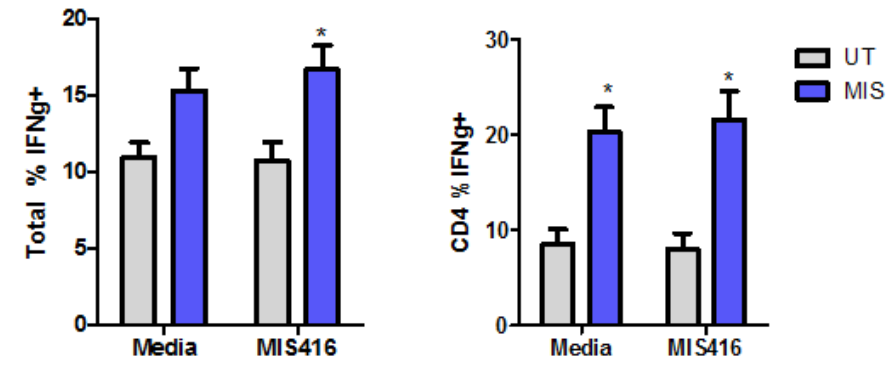

C

D
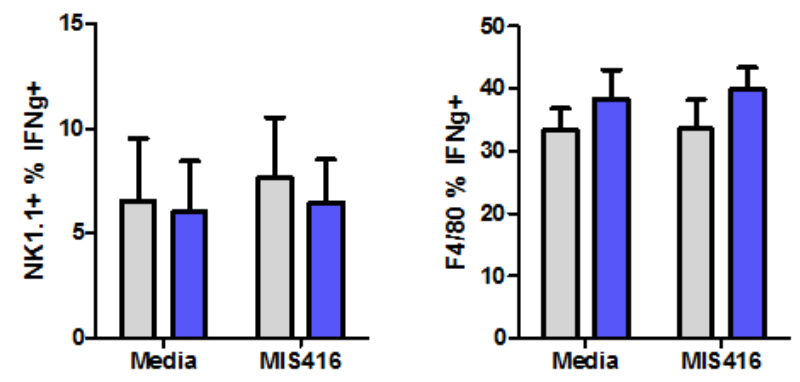

Figure 4-19 IFN- $\gamma$ positive cells were found after 5-hour ex vivo stimulation.

Splenocytes were isolated from untreated and MIS416-treated mice at day 15, and cultured with PMA/ionomycin/golgi stop for 5 hours and analysed for intracellular IFN- $\gamma$, as per methods. A) Total IFN- $\gamma$ positive cells were increased in MIS416-treated mice. B) CD4+ IFN- $\gamma$ positive cells were increased in MIS416treated mice. C) NK1.1+ IFN- $\gamma$ positive cells showed no difference between treatments. D) F4/80+ IFN- $\gamma$ positive cells showed no difference between treatments. Shown are the means \pm SEM of values from 2 experiments $(n=4) * p<0.05$ MIS416-treated compared to untreated mice by Mann-Whitney U-test. 
A
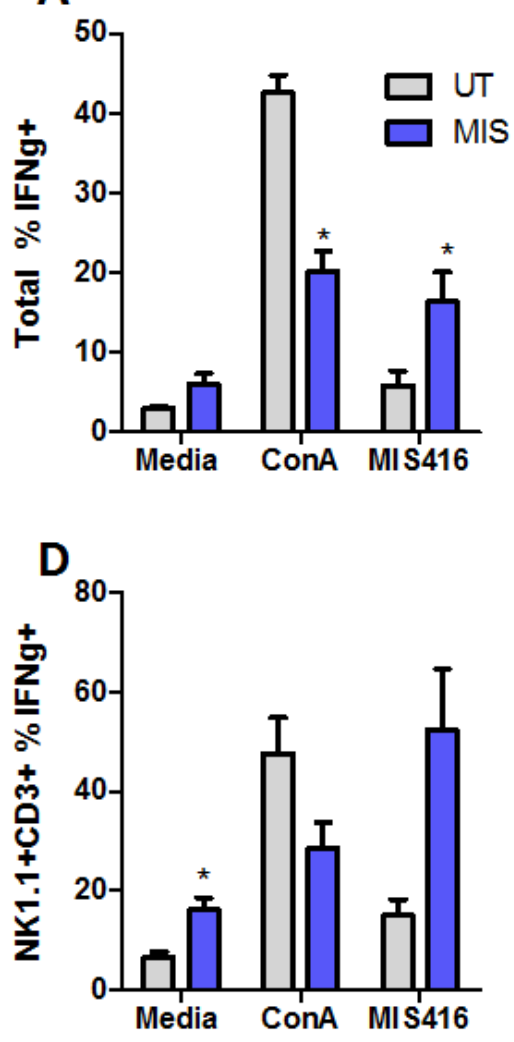

B
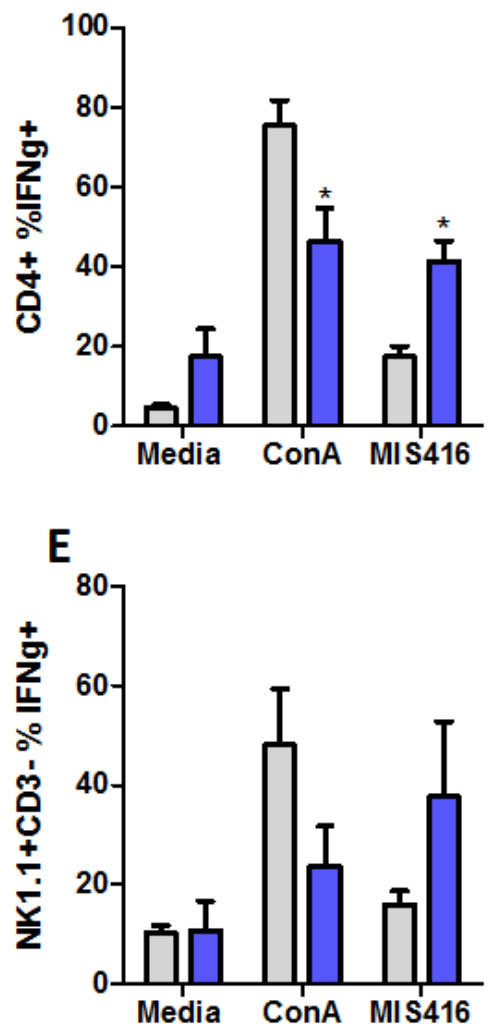

C

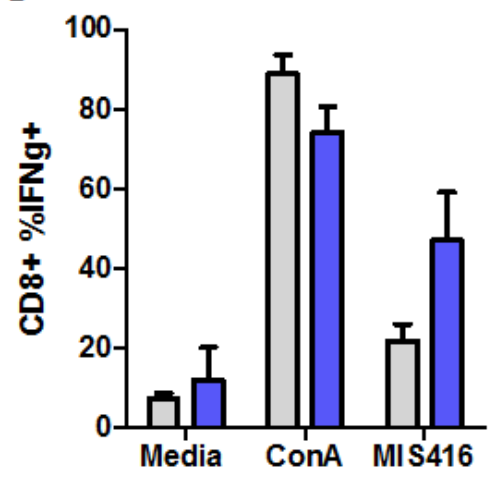

$\mathbf{F}$

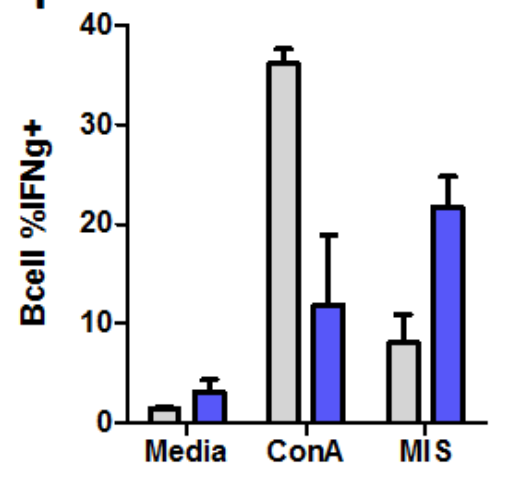

Figure 4-20 IFN- $\gamma$ positive cells were characterised after 48-hour $e x$ vivo stimulation.

Splenocytes were isolated from untreated and MIS416-treated mice at day 15, and cultured with media, ConA (1 $\mu / \mathrm{ml})$ or MIS416 $(20 \mu \mathrm{g} / \mathrm{ml})$ for 48 hours with PMA/ionomycin/golgi stop for the last 5 hours and analysed for intracellular IFN- $\gamma$, as per methods. See appendix 1.3 for further gating examples. A) Total IFN- $\gamma$ positive cells comparing ex vivo treatment. B) CD4+ IFN- $\gamma$ positive cells comparing ex vivo treatment. C) CD8+ IFN- $\gamma$ positive cells comparing ex vivo treatment. D) NKT cell IFN- $\gamma$ positive comparing ex vivo treatment. E) NK cell IFN- $\gamma$ positive comparing ex vivo treatment. F) B cell (B220+) IFN- $\gamma$ positive comparing ex vivo treatment. Shown are the means \pm SEM of values from 2 experiments $(n=4) * p<0.05$ MIS416-treated compared to untreated mice by Mann-Whitney U-test. 

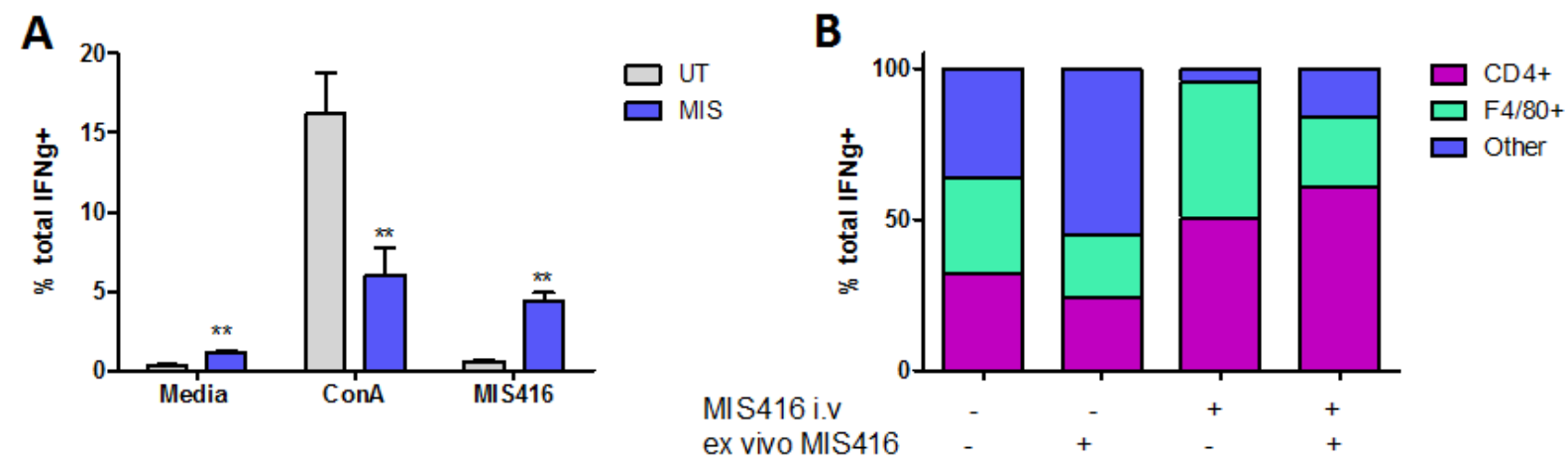

Figure 4-21 IFN- $\gamma$ positive cells were characterised after 48-hour ex vivo stimulation without PMA/ionomycin.

Splenocytes were isolated from untreated and MIS416-treated mice at day 15, and cultured with media, ConA (1 $\mu / \mathrm{ml})$ or MIS416 $(20 \mu \mathrm{g} / \mathrm{ml})$ for 48 hours with golgi stop for the last 5 hours and analysed for intracellular IFN- $\gamma$, as per methods. A) Total IFN- $\gamma$ positive cells comparing ex vivo treatment. B) Comparing percentage of cells that make up the IFN- $\gamma$ positive population. Shown are the means \pm SEM of values from 2 experiments $(n=4)$ ** $p<0.01$ MIS416-treated compared to untreated mice by Mann-Whitney U-test (A). 


\subsubsection{Macrophages produced IL-12 in response to MIS416}

Our results indicated that IFN- $\gamma$ was produced by CD4+ T cells, NK cells and macrophages in response to MIS416 and previous studies suggest that IL-12 production by macrophages drives these cells (esp. CD4+ T cells and NK cells) to produce IFN- $\gamma$ (Lieberman \& Hunter, 2002; Puddu et al., 1997). Therefore, we determined whether macrophages produced IL-12 in response to MIS416 treatment. As shown in Figure 4-22A splenocytes cultured with MIS416 produce high levels of IL-12 and these levels peaked at $2 \mu \mathrm{g} / \mathrm{ml}$, and dropped off at $20 \mu \mathrm{g} / \mathrm{ml}$. This reduction of IL-12 at the highest dose could be due to cellular death or negative feedback regulation induced by over-cytokine production. To confirm that the IL-12 produced in the splenocyte cultures could have been macrophage-derived, IFN- $\gamma$-primed BMMØ were stimulated with MIS416, and it was found that IL-12 was secreted in a concentration-dependent manner (Figure 4-22B). Together, these results provide evidence that the macrophage population was indeed likely to be a key producer of IL-12 in response to MIS416, and in turn this IL-12 may have initiated the production of other cytokine such as IFN- $\gamma$ and IL-10 by other macrophages, NK cells and CD4+ T cells. 
A

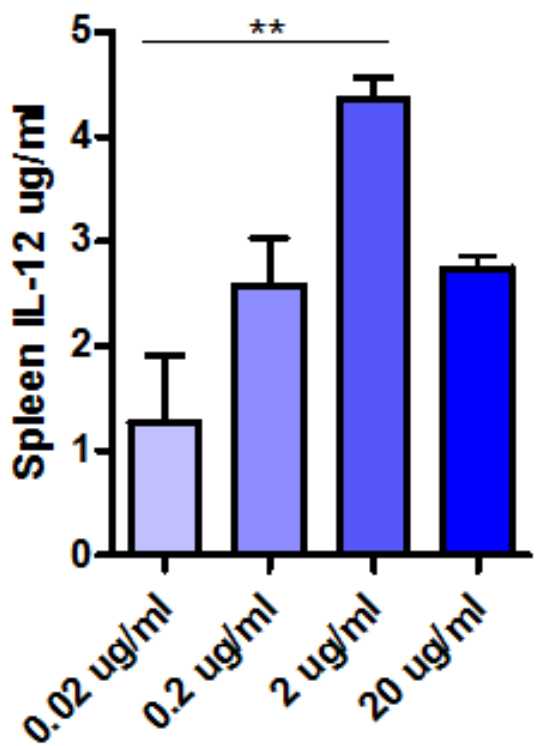

B

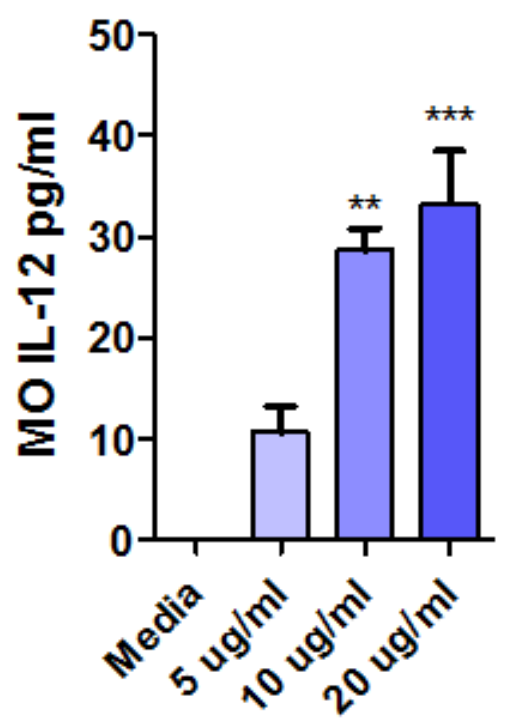

Figure 4-22 MIS416 stimulated splenocytes and macrophages to produce high levels of IL-12

Splenocytes or IFN- $\gamma$ primed bone marrow macrophages (BMMØ) were stimulated with MIS416 at various concentrations $(0.02-20 \mu \mathrm{g} / \mathrm{ml})$ and supernatants taken at 24 hour post-stimulation were assessed for IL-12p40 by ELISA. A) Spleen IL-12 production with various MIS416 concentrations. B) BMM $\varnothing$ IL-12 production with various MIS416 concentrations. Shown are the means \pm SEM of 1 experiment done in triplicate, 1-way ANOVA with Tukey's multiple comparisons test to compare $0.02 \mu \mathrm{g} / \mathrm{ml}$ (A) or media (B) to treated cultures, where ** indicate $\mathrm{p}<0.01$ and $* * * \mathrm{p}<0.001$. 


\subsubsection{Tregs were the main producers of IL-10}

In the culture supernatants with MIS416 re-stimulation there were very high levels of IL-10 produced by splenocytes from untreated mice, and even more IL-10 in cultures from mice which received MIS416 treatment in vivo. Therefore, to characterise which cells were induced to produce IL-10 in vivo, we characterised the IL-10-positive cells by flow cytometry after a 5 hour culture. It was found that while there was no significant difference between the total percentage of cells which were IL-10 positive (data not shown), some populations of cells from MIS416-treated mice produced much higher levels of IL-10 compared to controls as indicated by the increase in the geometric mean fluorescence of IL-10 (Figure 4-23). Interestingly, the Treg population from MIS416-treated mice produced much higher levels of IL-10 compared to untreated mice, and this increase was observed cultures in which media or MIS416 was present during the 5 hour stimulation (Figure 4-23). Although, the Treg population was a key producer of IL-10 in response to MIS416 in these cultures, we also found significantly more IL-10 being produced by the NK cells and macrophages from MIS416-treated mice compared to controls (Figure 4-23). Therefore, it was likely that several cellular populations including the Tregs, NK cells and macrophages contributed to the IL-10 being produced in both in vivo and in the ex vivo cultures. 

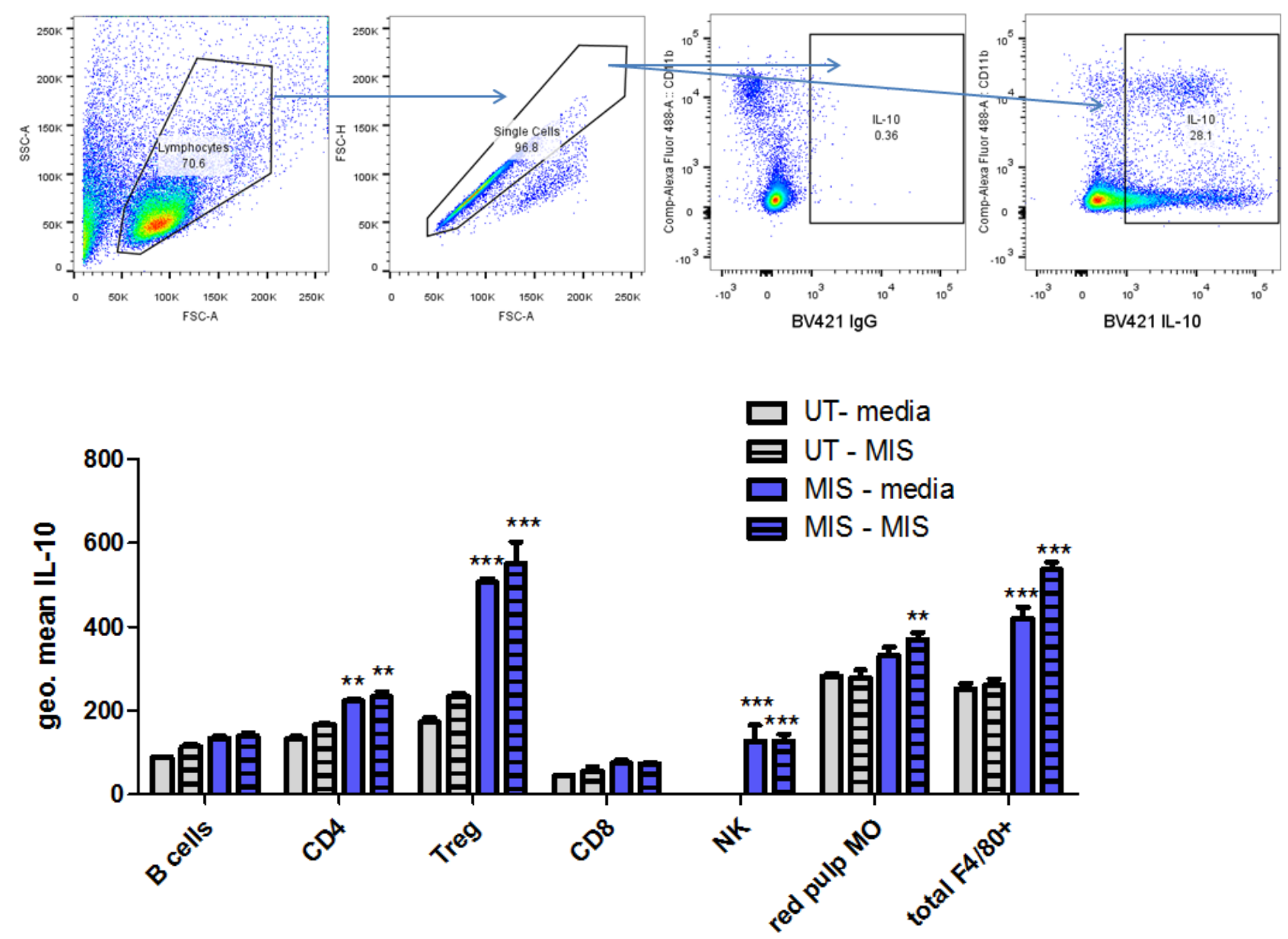

Figure 4-23 In vivo stimulation with MIS416 enhanced IL-10 production by Treg, macrophages and NK cells after 5-hour ex vivo re-stimulation

Splenocytes were isolated from untreated and MIS416-treated mice at day 15, and cultured with media, or MIS416 $(20 \mu \mathrm{g} / \mathrm{ml})$ for 5 hours with golgi stop for the last 5 hours and analysed for intracellular IL-10, as per methods. MIS416-treated compared to untreated mice, results from 1 experiment $(n=2), 2$-way ANOVA with Bonferronis post-test, ${ }^{* *} \mathrm{p}<0.01$ and $* * * \mathrm{p}<0.001$. 


\subsubsection{MIS416 reduced CD4 $\mathrm{T}$ cell proliferation in vitro and ex vivo}

Given that MIS416 treatment induced immunoregulatory factors such as NO, PD-L1 and IL-10, we investigated whether MIS416 treatment had the ability to reduce CD4 T cell proliferation. Firstly, we assessed the function of MIS416 in an in vitro setting where splenocytes from EAE mice at day 7 post-immunisation were isolated, CFSE-labelled and re-stimulated with MOG, MOG + MIS416 and MIS416 alone. As expected with an antigen-specific response, we found that in MOG re-stimulated cultures from untreated EAE mice, the CD4 T cells proliferated more than the media control where the media had a proliferation index of 1. However, when MIS416 was added with the MOG antigen in vitro, there was a significant reduction in the proliferative response to MOG. Furthermore, the same level of proliferation was also seen in the MIS416 only culture (Figure 4-24). These results suggest that MIS416 has the ability to alter the immune environment in a way that could reduce CD4 $\mathrm{T}$ cells from responding to an antigen, and furthermore, this effect did not require MIS416 exposure in vivo.

To assess if in vivo MIS416 treatment altered the ability of CD4 T cells to proliferate in culture, mice were culled 1 day after the $3^{\text {rd }}$ weekly MIS416 treatment (i.e. day 15) and CD4 T cell proliferation in response to ConA was compared to untreated mice. The proliferation index of untreated mice in response to ConA was very high, nearly 40 times the media control, however the ConA response in MIS416-treated mice was much lower ( $\sim 10$ times the media control) (Figure 4-25A), which suggested the in vivo immune environment of MIS416-treated mice significantly altered the ability of $\mathrm{T}$ cells to respond in vitro. In comparison to the reduced proliferation response seen with MOG with the addition of MIS416 into the culture ex vivo (Figure 4-24), the same effect was not observed with ConA and MIS416 which had a proliferation level similar to ConA alone (Figure 4-25). This effect could have been due to ConA being such a strong mitogen in the splenocyte culture whereas MOG caused a weaker proliferative response, and the addition of MIS416 with ConA was unable to exert any additional suppressive effect. The proliferative response to MIS416 was similar to the media control and was not significantly different in mice that were untreated or received MIS416 in vivo.

Because IFN- $\gamma$ is a key cytokine produced in response to ConA (Makarova et al., 2005), we correlated IFN- $\gamma$ production to the ConA-induced proliferative response after MIS416 treatment. Interestingly, with MIS416 treatment alone there was an increase in IFN- $\gamma$ production by CD4 T cells however, in the ConA-stimulated cultures from MIS416-treated mice, there was a significant reduction in the percentage of IFN- $\gamma$ positive CD4 T cells (Figure 4-25B). These results suggest that while MIS416 treatment alone drove a strong CD4 $\mathrm{T}$ cell IFN- $\gamma$ response, when ConA, a 
strong non-specific mitogen, was given to splenocytes from MIS416-treated mice, proliferation and cytokine production was suppressed compared to control mice. Again, these results indicate that MIS416 treatment was inducing a suppressive or regulatory immune environment that may be important in its ability to reduce disease in EAE. 


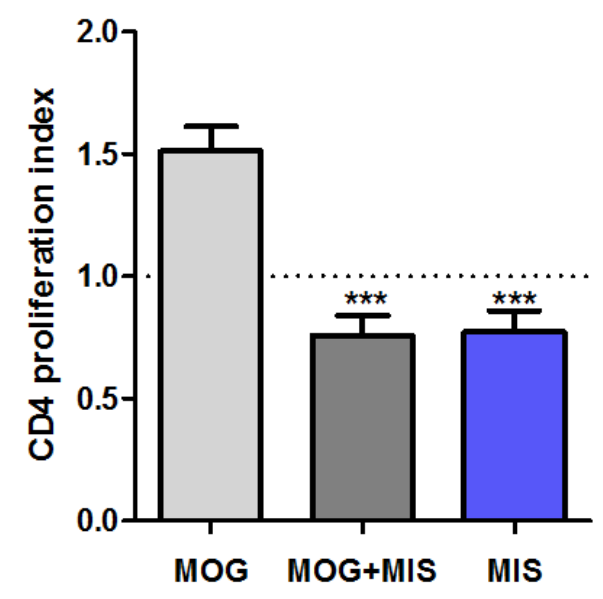

Figure 4-24 MIS416 suppressed MOG proliferation response in vitro.

Splenocytes were isolated from EAE-immunised mice at day 7 and re-stimulated with MOG (27 $\mu \mathrm{g} / \mathrm{ml})$, MOG + MIS or MIS416 (20 $\mu \mathrm{g} / \mathrm{ml})$ only and cultured for 72 hours followed by flow cytometry. Dotted-line represents proliferation in media alone. Shown are the means \pm SEM from 1 experiment, $n=4$. *** $p<0.001$ MOG + MIS and MIS compared to MOG, 1-way ANOVA with Bonferroni's post-test. 

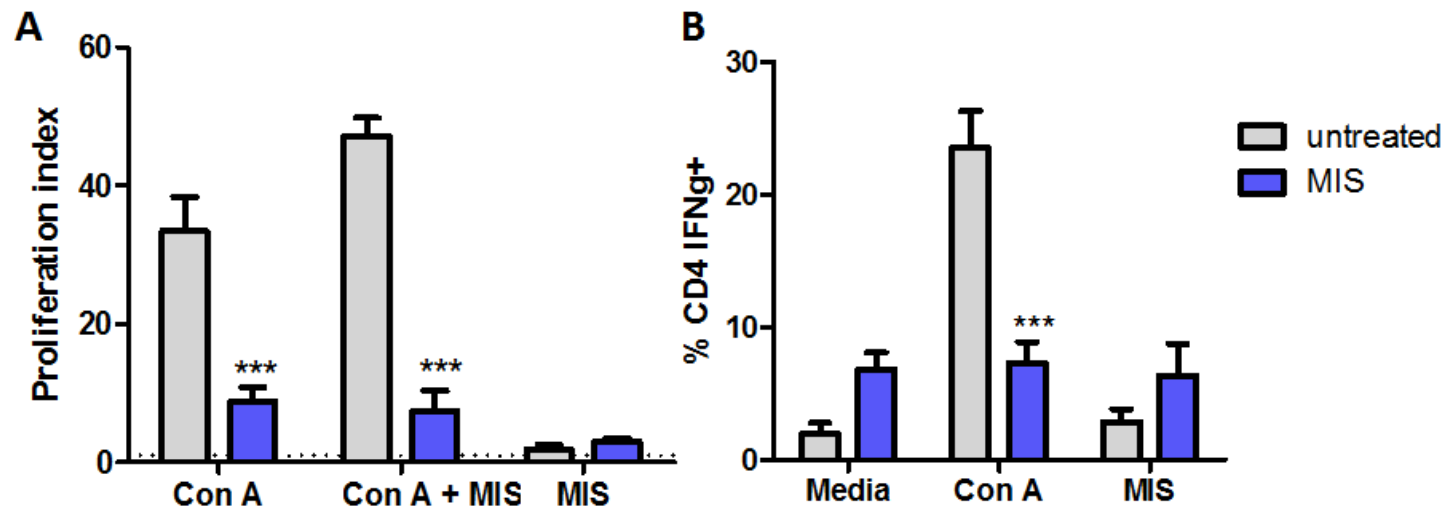

Figure 4-25 MIS416 suppressed ConA proliferation and IFN- $\gamma$ response ex vivo.

Splenocytes were isolated from untreated or MIS416-treated mice at day 15 and re-stimulated with media, ConA, ConA + MIS or MIS- only and cultured for 48 hours followed by flow cytometry. A) MIS416 treatment in vivo significantly reduces proliferation response to ConA ex vivo, the dotted-line represents proliferation in media alone. B) MIS416-treated mice also produce significantly less IFN- $\gamma$ in response to ConA stimulation. Shown are the means \pm SEM from 3 experiments ( $n=6-8 /$ group). $* * * p<0.001$ Untreated compared to MIS-treated by 2-way ANOVA with Bonferroni's post-test. 


\subsubsection{MIS416 induced effects were still apparent 6 days post injection}

The previous results assessing peripheral MIS416-induced effects were obtained 1 day postMIS416 treatment. To test the longevity of these effects, we assessed peripheral immune changes at day 13 (6 days post-MIS416 treatment), after which mice had received 2 weekly doses of MIS416. Similar to the day 15 and 22 time-points (which are 1 day post-treatment) we determined that MIS416-treated mice had a significantly higher spleen count compared to control mice (Figure 4-26A). There was also a significant increase in F4/80+ cells (the macrophage population), as represented by the percentage of total cells, and a significant increase in the expression of PD-L1 on myeloid cells and specifically macrophages (Figure 4-26B \&C) in mice which received MIS416 treatment compared to untreated mice. These results showed that the alterations within the splenic myeloid populations were apparent after only 2 MIS416 injections and furthermore, that the myeloid population changes with MIS416 were maintained within the spleen 6 days after MIS416 treatment.

Similar to the peripheral immune effects measured 1 day post-treatment, at day 13 (6 days posttreatment) there were no significant alterations in the percentage of CD4, CD8 and regulatory T cell populations with MIS416 treatment (Figure 4-27A\&C). However, in contrast to the day 15 time point, the MIS416-treated mice at day 13 did not have significantly more regulatory $\mathrm{T}$ cells within the spleen although, there was a slight increase in the total Treg number this increase did not reach statistical significance (Figure 4-27B). Taken together the lymphocyte results within the spleen and liver showed that while some of the alterations were similar to the day 15 time point, the Treg number was not significantly higher than control mice at this time. This finding suggests that either more MIS416 treatments would be required to induce high Treg numbers or that the Treg population was not sustained in the spleen up to 6 days-post MIS416 treatment.

We also assessed the cytokine production of MIS416-treated splenocytes in response to restimulation with MIS416, LPS and ConA at day 13 post-MIS416 treatment. We found similar increases in IFN- $\gamma$, IL-10 and NO production when re-stimulated with MIS416 or LPS, and similar to the day 15 time-point, MIS416-treated mice produced significantly higher levels of these cytokines than untreated mice (Figure 4-28A,B\&D). In contrast to the day 15 results, there were much higher levels of IL-17A found in cultures re-stimulated with MIS416 and ConA in the MIS416-treated mice at day 13 compared to untreated mice (Figure 4-28C). This was an unexpected result as within splenocyte culture from non-EAE mice at day 15 very little IL-17A can be detected within culture supernatants; and although IL-17A is produced by MOG-stimulated splenocytes from EAE immunised mice, MIS416-treated mice had a reduced production of IL-17A when compared to untreated mice. These results indicate that MIS416 drove a delayed IL-17 
response, although the role this cytokine may play is unknown. The assessment of serum NO levels showed that MIS416-treated mice at day 15 had significantly high levels of serum NO compared to untreated mice, and at day 13 there were elevated levels of NO, although they were much lower than at day 15 (1 day post-MIS416 treatment) and did not reach statistical significance (Figure 4-28E). Even though the serum NO levels 6 days post MIS416 treatment (day 13) were not significantly higher than untreated mice the elevated levels may still have biological relevance.

These cytokine results suggest that some of the cytokine responses seen ex vivo were consistent whether we assessed splenocytes 1 day post injection or 6 days post injection and these may have been the key cytokines involved in the mechanism of action of MIS416. However, while MIS416 treatment significantly reduced the Th17 type response in EAE, it appeared that this cytokine may have had a role in the later phases of MIS416 treatment. The precise role this cytokine had in MIS416 treatment is unclear at this point. 

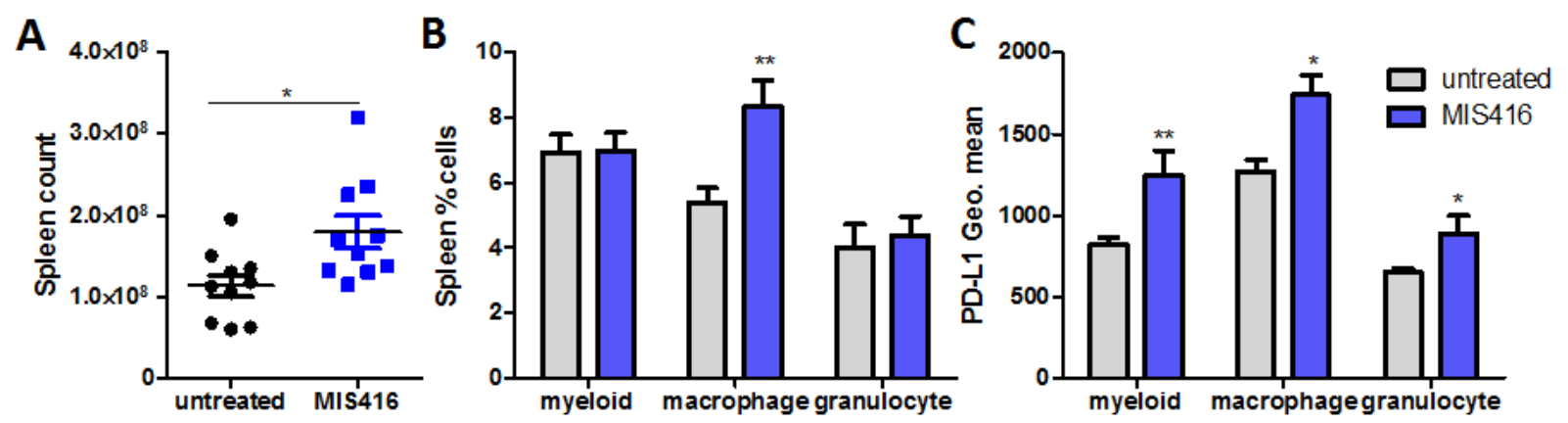

Figure 4-26 MIS416-induced splenocyte alterations were still apparent 6-days post treatment

C57BL/6 mice were treated weekly by i.v. administration with $100 \mu \mathrm{g} /$ mouse of MIS416 and culled at day 13 . Splenocytes were isolated and assessed by flow cytometry. A) Spleen count of MIS416-treated mice was higher than untreated. B) Myeloid populations as a percentage of total spleen, (single positive staining where myeloid = $\mathrm{CD} 11 \mathrm{~b}+$, macrophage $=\mathrm{F} 4 / 80+$, granulocyte $=\mathrm{Gr}-1+)$. C) Myeloid PD-L1 expression. Shown are the means \pm SEM of values from individual mice ( $n=10$ /group) from 2 experiments for $A$ and $B$, and 1 experiment in $C(n=5)$ $* \mathrm{p}<0.05$ and $*$ * $\mathrm{p}<0.01$ MIS-treated compared to untreated by Mann-Whitney U-test. 
A

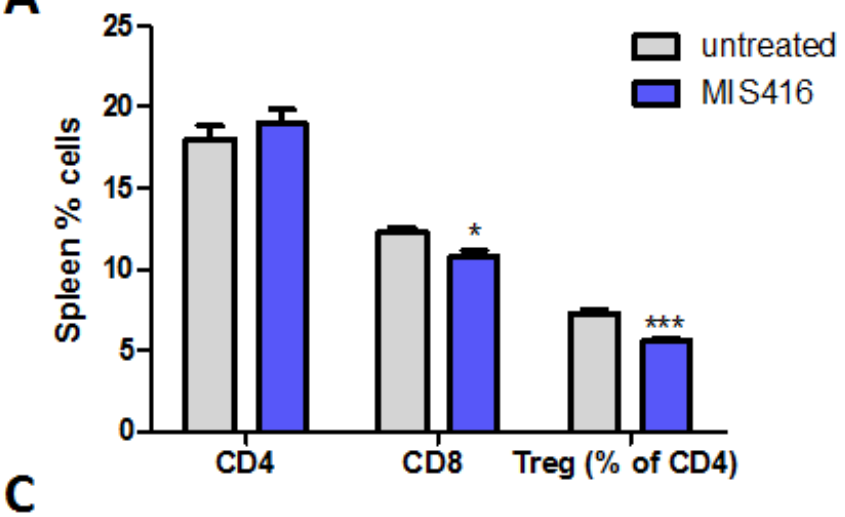

C

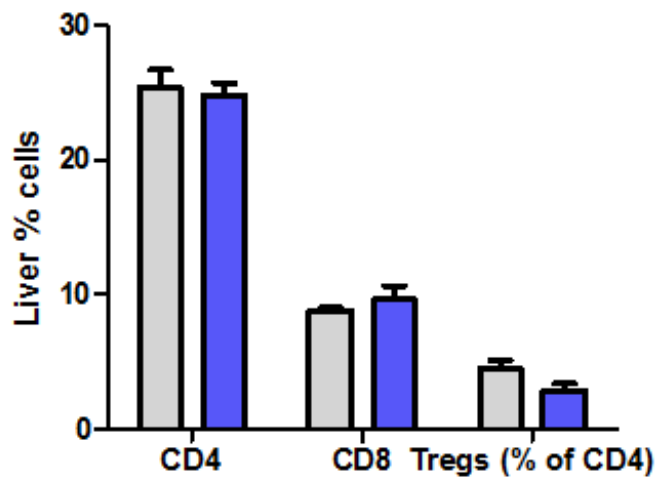

B

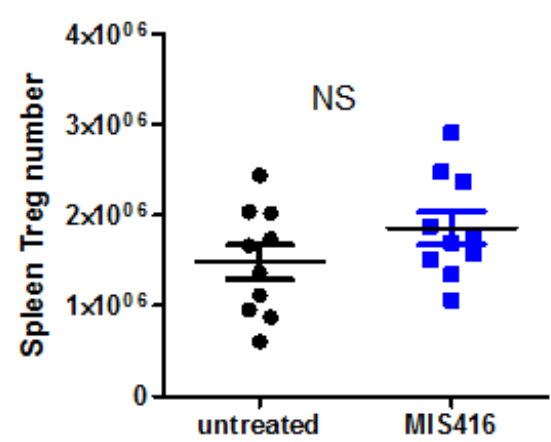

Figure 4-27 MIS416-induced lymphocyte alterations were still apparent 6-days post treatment

C57BL/6 mice were treated weekly by i.v. administration with $100 \mu \mathrm{g} / \mathrm{mouse}$ of MIS416 and culled at day 13. Splenocytes and liver cells were isolated and assessed by flow cytometry. A) T cell populations as a percentage of total spleen. B) Total number of Tregs within the spleen. C) $\mathrm{T}$ cell populations as a percentage of total liver. Shown are the means \pm SEM of values from individual mice $(n=5-10 /$ group $)$ from $1-2$ experiments. * $p<0.05$ and $* * * \mathrm{p}<0.001$ or NS (not significant) MIS416-treated compared to untreated by Mann-Whitney U-test. 

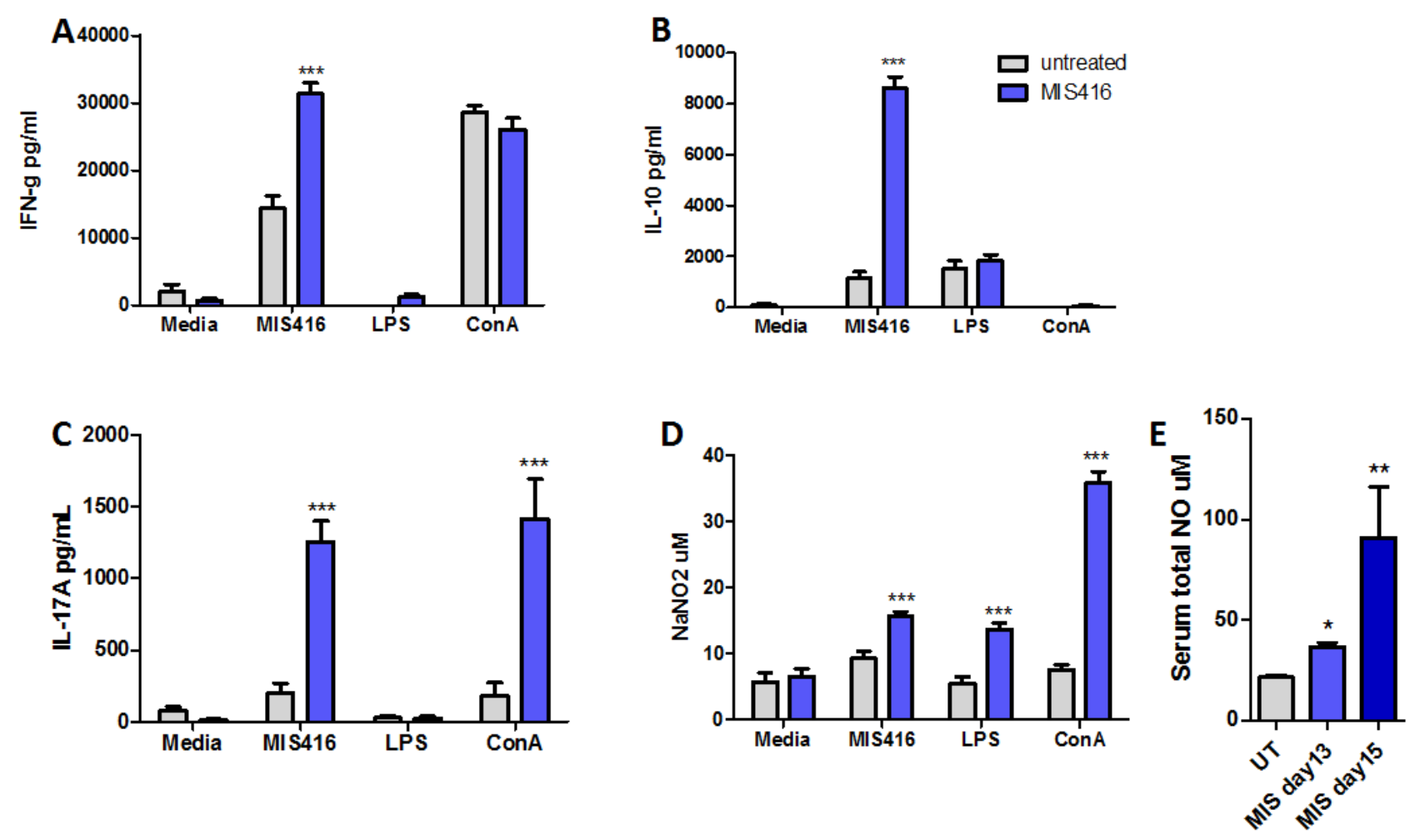

Figure 4-28 MIS416 treatment altered cytokine profile at day 13

C57BL/6 mice were treated weekly by i.v. administration with $100 \mu \mathrm{g} /$ mouse of MIS416 and culled at day 13 . Splenocytes were isolated and cultured $\left(10^{6}\right.$ cells/well) with media alone, MIS416 $(20 \mu \mathrm{g} / \mathrm{ml})$, LPS $(200 \mathrm{ng} / \mathrm{ml})$ or ConA ( 1 or $3 \mu \mathrm{g} / \mathrm{ml}$ ) for 48 hours (A-D) Serum was also assessed for nitric oxide (E). A) IFN- $\gamma$ production. B) IL-10 production. C) IL-17A production. D) Nitric oxide production. E) Serum NO. Shown are the means \pm SEM of values from individual mice ( $\mathrm{n}=5-10$ /group) from 2 experiments. $* \mathrm{p}<0.05, * * \mathrm{p}<0.001$ and ${ }^{* * *} \mathrm{p}<0.001$ Untreated compared to MIS-treated by 2-way ANOVA with Bonferroni's post-test (A-D) and 1-way ANOVA with Kruskal-Wallis post-test. 


\subsubsection{Using TLR-9-ligand-deficient MIS416 some immunomodulatory properties were ablated}

The MIS416 microparticle is made up of both TLR9 agonist (bacterial ssDNA) and NOD2 agonist (muramyl dipeptide), and therefore to determine if the TLR9 is essential for the disease protection, we used a heat-treated version of MIS416, in which the bacterial DNA is denatured and no TLR9 signalling occurs (personal communication, Gill Webster, IIT, see Appendix 9.2). As found in Chapter 3, we did not see disease protection when $100 \mu \mathrm{g}$ of heat-treated MIS416 (i.e. the same concentration used with non-heat-treated MIS416) was used, however some of the peripheral immune effects some were still apparent albeit to a lesser extent than with MIS416, and some effects were not seen all together.

Firstly, we found that heat-treated MIS416 (HT-MIS416) mice had an increase in total spleen count compared to untreated mice; however, the spleen count was not increased to the extent as MIS416-treated mice (Figure 4-29A). Assessment of T cell populations in HT-MIS416-treated mice showed that while total CD4 and CD8 T cells were increased to the same extent as MIS416treated mice, only MIS416-treated mice had a significant increase in the total number of Tregs within the spleen and HT-MIS416 mice did not have an expansion of Tregs (Figure 4-29B \&C). In the myeloid compartment (using the gating strategy shown in appendix 1.4) there was a significant 3-fold increase in the percentage and number of macrophages (F4/80+) with MIS416 treatment, but HT-MIS416 mice had a similar percentage and number of macrophages as untreated mice (Figure 4-29D\&E). These results showed that because HT-MIS416 treatment did not result in disease protection in EAE mice, the peripheral immune effects retained in HT-MIS416-treated mice were not responsible for the mechanism of protection induced by MIS416 in EAE.

To understand more fully the unique effects that MIS416 had on altering the immune environment, splenocytes from HT-MIS416-treated mice were stimulated ex vivo and cytokine production was compared to that of splenocytes from MIS416-treated mice. We found that HT-MIS416-treated EAE mice produced a similar level of MOG-specific IFN- $\gamma$ production to untreated EAE mice, whereas MIS416-treated mice had a significant reduction compared to untreated mice (Figure 4-30A). The unaffected cytokine recall response to MOG was not surprising considering HTMIS416-treated mice also did not have the disease protection seen with MIS416 treatment. Interestingly, the IL-17A response to MOG in HT-MIS416-treated mice was significantly reduced compared to control EAE mice although, not reduced to the same extent seen in MIS416-treated mice (Figure 4-30D). The MOG-specific IL-17A response in HT-MIS416-treated mice indicated that with HT-MIS416 this pathway was reduced to some extent but did not appear to have a significant effect on the disease score of these mice. 
The re-stimulation of splenocytes with MIS416 ex vivo produced very high levels of IFN- $\gamma$, IL-10 and NO, and MIS416-treated mice produced even greater levels of these cytokines compared to untreated mice. Therefore, the assessment of these cytokines in splenocytes from HT-MIS416treated mice were compared to MIS416-treated and untreated mice. It was found that while HTMIS416-treated mice produced much higher levels of IFN- $\gamma$, IL-10 and NO compared to untreated mice, the levels were not as high as those seen in MIS416-treated mice (Figure 4-30A,B\&C). We also assessed the levels of serum NO, and found that HT-MIS416-treated mice had similar levels of NO to untreated mice whereas the MIS416-treated mice had significantly higher levels of NO compared to both the untreated and HT-MIS416-treated mice (Figure 4-30E). The peripheral immune effects which were identified as different between MIS416-treated and HT-MIS416treated mice help us to understand which peripheral immune changes identified with MIS416 treatment may have a key involvement in the mechanism of action of MIS416. 
A
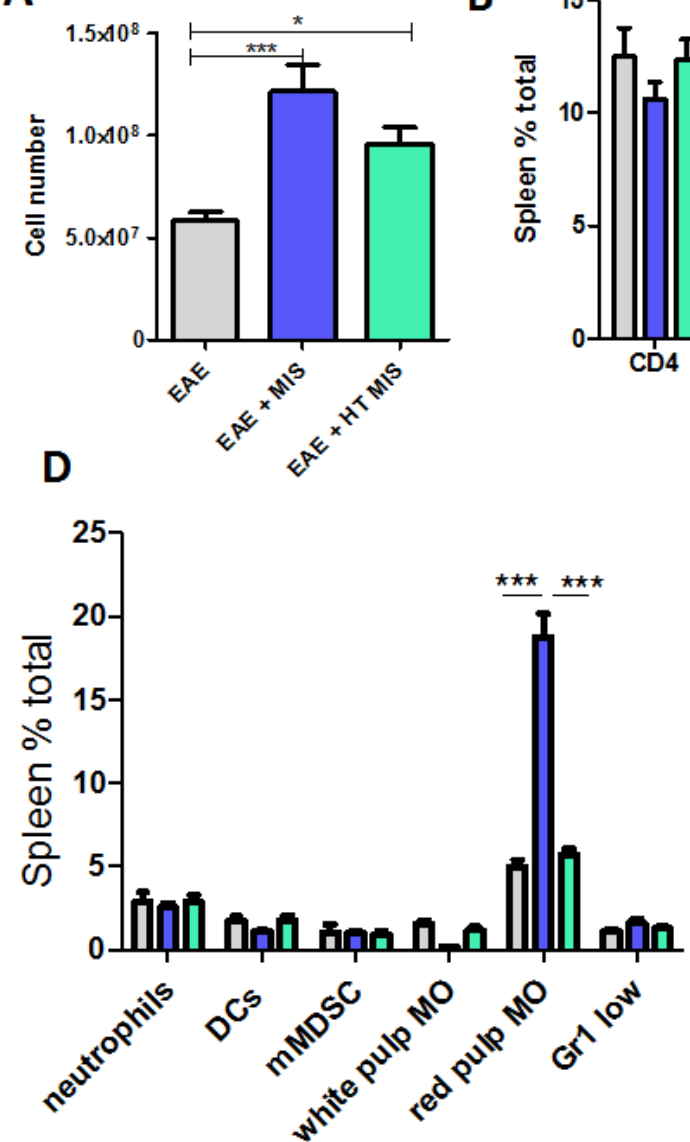

B

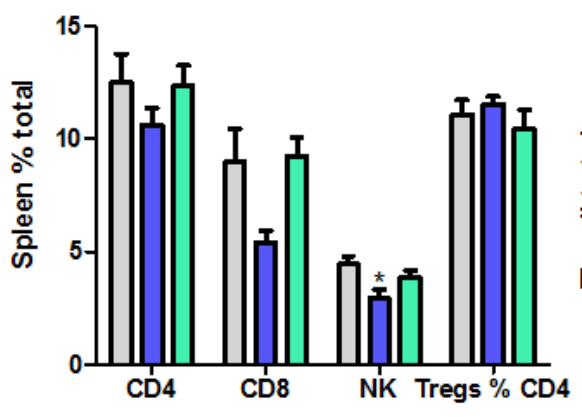

$\mathbf{E}$
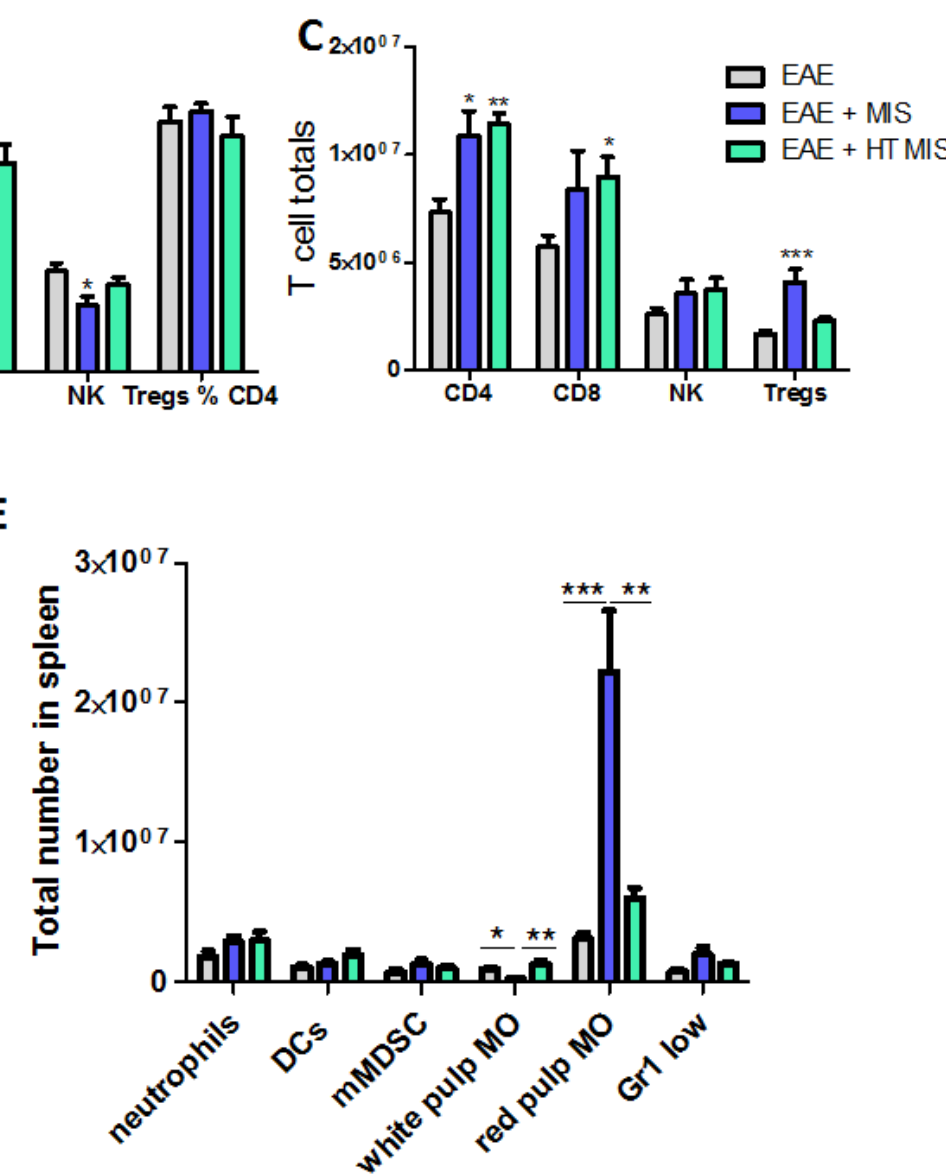

Figure 4-29 Heat-treated MIS416 treatment resulted in minor peripheral immune changes.

C57BL/6 mice were either immunised for EAE and treated weekly by i.v. administration with either 100 $\mu \mathrm{g} /$ mouse of MIS416 or $100 \mu \mathrm{g} /$ mouse of heat-treated MIS416 starting on the day of immunization (day 0), and splenocytes were isolated at day 22, and assessed by flow cytometric analysis using gating strategy from White $e t$ $a l, 2014$ (see Appendix 1.4). A) HT-MIS416 treatment significantly increases spleen count but not to the same extent as regular MIS416. B) Spleen proportion of lymphocytes C) Total number of lymphocytes shows heattreated MIS416 increases total CD4+ and CD8+. D) Heat-treated MIS416 did not cause an increase in red pulp macrophages whereas regular MIS416 did. E) Heat-treated MIS416 did not cause an increase in total red pulp macrophages. Shown are the means \pm SEM of values from individual mice $(n=5-10$ /group $)$ from 1-2 experiments. MIS416-treated compared to untreated and HT-MIS416* ${ }^{*}<0.05$ and $* * * p<0.001$ by 1-way ANOVA with Bonferonni's post-test. 
A

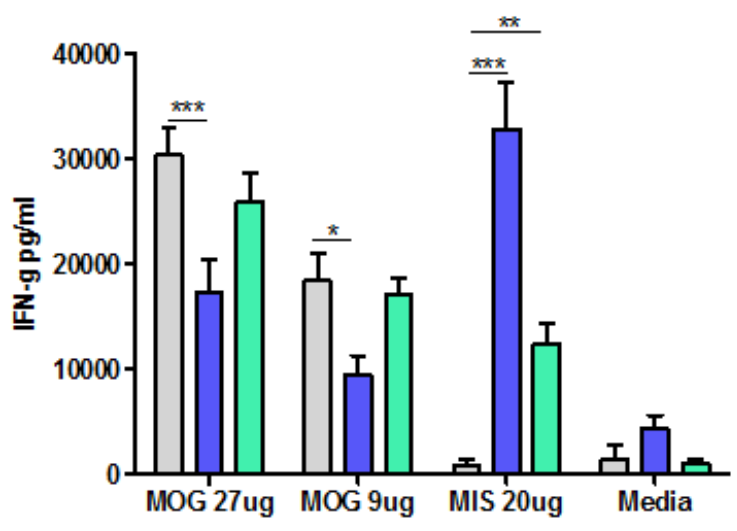

B

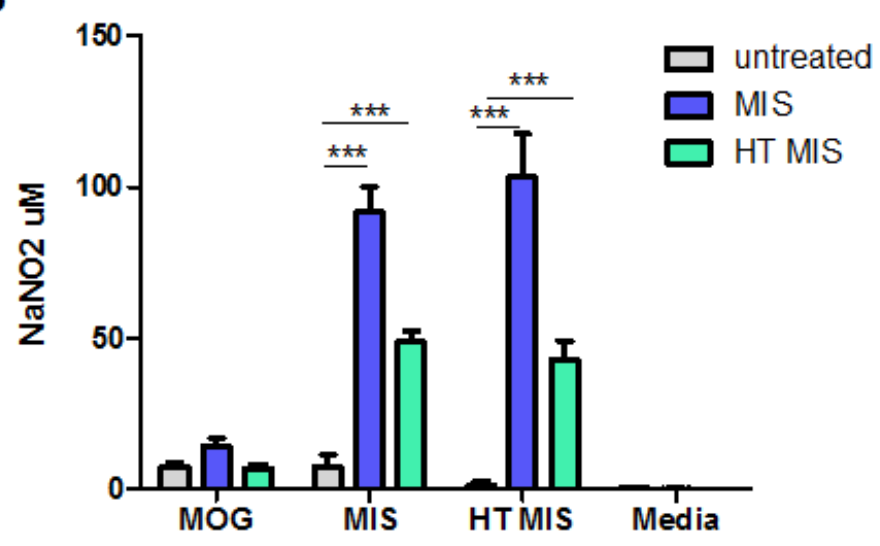

C

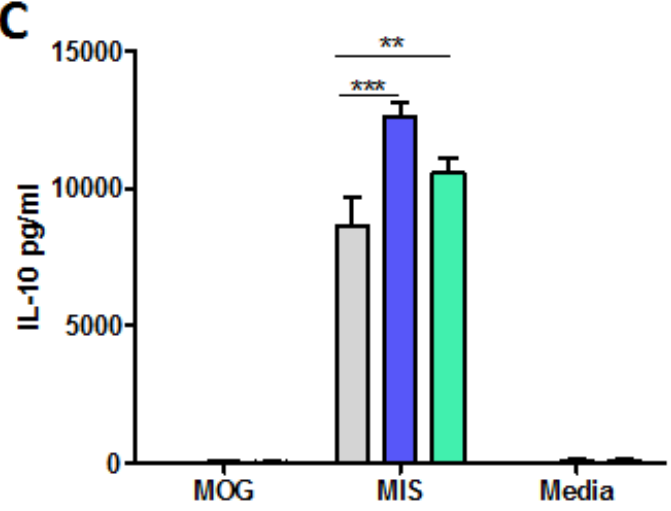

D

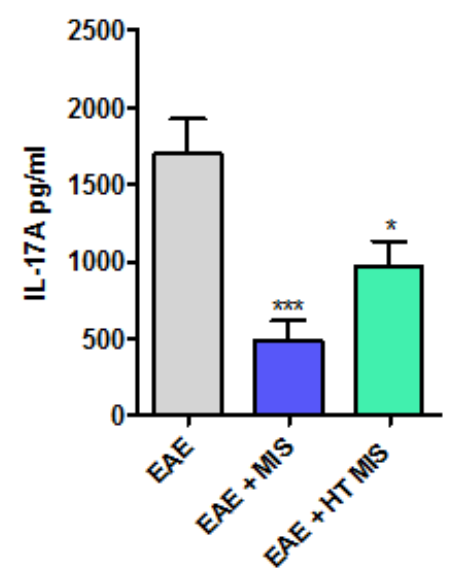

$\mathbf{E}$

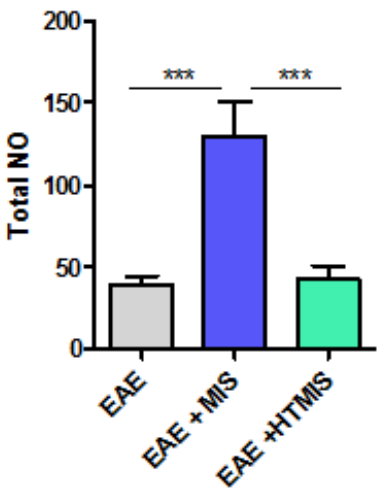

Figure 4-30 Heat-treated MIS416 altered the cytokine profile of EAE mice

C57BL/6 mice were either immunised for EAE and treated weekly by i.v. administration with either 100 $\mu \mathrm{g} /$ mouse of MIS416 or $100 \mu \mathrm{g} /$ mouse of heat-treated MIS416 starting on the day of immunization (day 0). Splenocytes were isolated at day 22 cultured with MOG (27 and $9 \mu \mathrm{g} / \mathrm{ml})$, MIS416 $(20 \mu \mathrm{g} / \mathrm{ml})$ or heat-treated MIS416 $(20 \mu \mathrm{g} / \mathrm{ml})$ for 72 hours. Serum was also taken as per methods. A) MOG-specific and MIS416-induced splenic IFN- $\gamma$ responses. B) MOG-specific, MIS416 and heat-treated MIS416-induced splenic nitric oxide responses. C) MOG-specific and MIS416 splenic IL-10 production. D) MOG-specific splenic IL-17A production is reduced in heat-treated MIS416 mice. E) Serum nitric oxide levels at euthanasia (day 22). Shown are the means \pm SEM of values from individual mice ( $\mathrm{n}=5-10$ /group) from 1-2 experiments. * $\mathrm{p}<0.05$, ** $\mathrm{p}<0.01$ and $* * * \mathrm{p}<$ 0.001 by 1 or 2-way ANOVA with Bonferroni's post-test. 


\subsection{Discussion}

Chapter 4 aimed to determine the MIS416-induced peripheral immune alterations using the weekly dosing regimen identified in Chapter 3. The initial work identified that MIS416-treated mice had enlarged spleen and livers as a result of MIS416 treatment, which prompted us to determine whether the cells within the spleen and liver were altered by MIS416 treatment in a way that may have contributed to the mechanism of action. Unsurprisingly, the enlarged spleen and livers were associated with significantly increased spleen and liver leukocyte counts, and furthermore some of these new cells were a result of MIS416-induced proliferation of local progenitor cells, as indicated by cell cycle analysis. MIS416 treatment also resulted in a shift of cell populations within the spleen and liver, as indicated by significant increases in myeloid populations (i.e dendritic cells, red pulp macrophages and granulocytes) as well as increased number of Tregs. The increased macrophage population was of particular interest as this population has been shown to pick up MIS416 in the liver. Additionally, the myeloid cells within the spleen and liver of MIS416-treated mice became activated and expressed higher levels of PD-L1, MHC-II and CD14 compared to cells from untreated mice. Finally, splenocyte cultures and serum analysis studies revealed that MIS416-treated mice produced high levels of IFN- $\gamma$, NO and IL-10 in response to restimulation to MIS416 while in contrast, produced lower levels of MOG-specific IFN- $\gamma$ and IL17A. Further analysis identified CD4+ T cells, macrophages and NK cells as key producers of IFN- $\gamma$ and IL-10, indicating that these cells could be central to the peripheral mechanism of MIS416.

Phagocytic cells were found to be key responders to MIS416 and were able to take up large amounts of labelled-MIS416, both in vitro and in vivo, which resulted in the activation of intracellular receptors NOD2 and TLR9 (Kaisho \& Akira, 2006; Strober, Murray, Kitani, \& Watanabe, 2006). The macrophage is of particular interest in MIS416-treated mice as these cells are known to be very plastic and like dendritic cells, direct adaptive immune responses (Stout \& Suttles, 2004). Results from this chapter have shown that MIS416-treated mice had at least a 3fold expansion of their splenic red pulp macrophages both in EAE mice and non-EAE mice. The red pulp macrophages have a major role in the phagocytosis and degradation of aged erythrocytes and the recycling of iron within the spleen (den Haan \& Kraal, 2012); however, the role these cells may play in the mechanism of MIS416 is unclear. It is possible these cells have other functions, and as reported in one study splenic $\mathrm{F} 4 / 80+\mathrm{CD} 11 \mathrm{~b}^{\text {low }}$ (red pulp macrophages) cells express different surface markers to $\mathrm{F} 4 / 80+\mathrm{CD} 11 \mathrm{~b}^{\text {high }}$ cells, are able to regulate $\mathrm{CD} 4+\mathrm{T}$ cell responses through the secretion of cytokines and can also induce the transformation of naïve CD4+ T cells into FoxP3+ Tregs (Kurotaki et al., 2011). Therefore, the increase in red pulp macrophages seen in 
MIS416-treated mice may have been responsible for the increase in Treg numbers in the spleen, and may also have produced the high levels of IL-10 found in the splenocyte cultures from MIS416-treated mice.

The liver-resident macrophage population or Kupffer cells $\left(\mathrm{F} 4 / 80^{+}\right)$, may also be an important phagocytic cell in MIS416 treatment. Kupffer cells were shown to pick up large amounts of MIS416 after intravenous MIS416 injection and, similar to the spleen red pulp macrophages, the liver Kupffer cell population was significantly increased post-MIS416 treatment. During homeostasis the Kupffer cells are involved in the clearance of aged erythrocytes however, under inflammatory conditions these cells are known to have roles in host-defence mechanisms, immune tolerance and liver regeneration (Bilzer, Roggel, \& Gerbes, 2006). The MOG-specific responses within the liver were significantly lower with MIS416 treatment (Chapter 3) indicating that the immune responses within the liver were altered with MIS416 treatment. The liver is known to direct the induction of immune tolerance, as opposed to inflammation, due to the constant exposure to gut-derived antigens; therefore the reduced MOG-specific responses in the liver could have been due to the induction of immune tolerance mediated directly through MIS416 containing Kupffer cells. A study using isolated kupffer cells showed that in vitro $\mathrm{T}$ cell proliferation was reduced in Kupffer cell:T cell cultures compared to peritoneal macrophage:T cell cultures and furthermore, addition of Kupffer cells into an in vitro dendritic cell:CD4+ proliferation assay showed suppression of proliferation which was likely mediated through soluble factors (You, Cheng, Kedl, \& Ju, 2008). This study suggests that an increase in the number of resident macrophages in the liver (i.e. Kupffer cells) may be reducing $\mathrm{T}$ cell responses in EAE mice, and therefore may explain how MIS416 treatment is working in EAE.

As macrophages are able to act very differently under different conditions, we characterised several activation markers expressed during MIS416 treatment. It was found that cells isolated from the spleen and livers of MIS416-treated mice expressed higher levels of PD-L1, MHCII and CD14 compared to untreated mice. Interestingly, as different macrophage activation states can alter the course of EAE disease, these cells may contribute to the mechanism of action of MIS416, particularly as with MIS416 treatment there is an abundant increase in this cell type. There are many faces of macrophage activation such as type-II activation, alternatively activated and classically activated macrophages, which are all associated with unique immune functions and may have opposing roles in EAE. Macrophages which are classically or alternatively activated are typically thought to be associated with Th1- and Th2-type responses respectively, and therefore activating macrophages to these responses will have either inflammatory (Th1) or wound healing (Th2) type immune response (Fairweather \& Cihakova, 2009). Another study identified type-II 
activated macrophages, which can be induced with the addition of immune complexes such as antibody opsonised sheep red blood cells, (SRBC:Ig), and these macrophages were shown to express lower levels of co-stimulatory markers CD40 and CD80 (Tierney et al., 2009). Interestingly, type-II activated macrophages have down regulated expression of PD-L1, unlike macrophages from MIS416-treated mice, yet EAE mice treated with type-II activated macrophages have significantly reduced disease (Tierney et al., 2009). Macrophages have also been shown to be a central cell type for the immunomodulatory effects of GA although acting differently to other types of macrophage activators. GA is a small peptide which contains similar residues to myelin and when GA is bound to MHCII on macrophages, the resulting immune response is an antigen-specific Th2/Th3-type which is able to reduce disease in EAE and MS (Rina Aharoni, 2014; Yong, 2002). The Th2 cells produced in the presence of GA are characterised by secretion of IL- 4 and IL-10 whilst Th3 cells produce TGF- $\beta$, together these cells are capable of down-regulating autoimmunity (A. Miller et al., 1998). While it seems unlikely we induced a similar state of activation to those listed above, we potentially activated macrophages into a unique suppressive state, although the full characterisation of these cells was not completed in this study.

The expression of anti-proliferative ligands, such as PD-L1, on the surface of antigen-presenting cells such as macrophages and DCs can alter the outcome of a T cell response (Pen et al., 2014). Of particular interest is PD-L1 as MIS416-treated mice were shown to have a significant increase in surface expression of this ligand on myeloid populations, including macrophages and DCs. PDL1 expressed on the surface of APCs interacts with its receptor, PD-1, on T cells and suppresses effector T cell responses, although the role of PD-L1 expression on non-APCs is less clear (Blank \& Mackensen, 2007). Therefore, as CD4+ $\mathrm{T}$ cell responses were significantly reduced in splenocyte cultures from MIS416-treated mice compared to untreated mice, and as the macrophage and other myeloid cells within MIS416-treated splenocyte cultures were expressing PD-L1, these differences could be the reason why $\mathrm{T}$ cell proliferation was reduced. Additionally, PD-L1 expression has been shown to be important in naturally limiting EAE disease, as work by Latchman et al showed that PD-L1 ${ }^{-/}$mice have enhanced $\mathrm{T}$ cell responses in vitro and develop more severe EAE than wild type mice (Latchman et al., 2004). It is possible that the up regulation of PD-L1 expression in both the antigen presenting and non-antigen presenting myeloid populations in MIS416-treated mice enhanced the natural feedback loop in EAE mice and thus could have been one of the key mechanisms in MIS416-reduced disease.

MIS416 treatment also resulted in an increased expansion of Tregs (CD4+CD25+FoxP3+) in both the spleen and the liver, and this trend was enhanced in EAE mice compared to non-EAE mice. It 
has been shown that the expansion of Tregs occurs in EAE mice as a normal regulatory control of disease, and this expansion is most apparent in the CNS after peak disease (Richard A. O'Connor \& Anderton, 2008). Furthermore, the depletion of Tregs in mice before EAE immunisation results in more severe disease symptoms and delayed disease resolution, indicating Tregs exert their action in the later stages of EAE disease (McGeachy, Stephens, \& Anderton, 2005). MIS416 treatment induced a further expansion of Tregs at peak disease (day 15) indicating that these cells could play a role in MIS416 mechanism of disease resolution in EAE. Additionally, natural and induced Tregs are able to suppress $\mathrm{T}$ cell function, including proliferation and cytokine production, and it has been shown that the addition of Tregs into EAE mice reduces disease burden (Adam P. Kohm et al., 2002). Therefore, we believe that MIS416-induced Treg expansion is another pathway in MIS416 treatment responsible for the reduced disease burden in EAE mice.

MOG-specific CD4+ T cells, which are driven towards either Th1 or Th17, are induced early-on in EAE, and in response to re-stimulation with MOG, these cells produce high levels of IFN- $\gamma$ and IL-17A (El-behi, Rostami, \& Ciric, 2010). MIS416 treatment significantly reduced the production of MOG-specific IFN- $\gamma$ and IL-17A, both within the spleen and liver of MIS416-treated mice EAE compared to untreated EAE mice. These results suggest that MIS416 treatment acted to reduce EAE-specific $\mathrm{T}$ cell responses in the periphery, a mechanism which is used by other immune modulating MS treatment. For example GA, which shifts antigen-specific T cell responses away from the disease-driving Th1-type and promotes a more Th2/Th3-type response, results in lower levels of MOG-specific pro-inflammatory cytokines (Rina Aharoni, 2014; Yong, 2002). Similarly, IFN- $\beta$ treatment alters $\mathrm{T}$ cell responses, and when used in the EAE model, also results in reduced production of MOG-specific IFN- $\gamma$ and IL-17A. IFN- $\beta$ treatment alters antigen presentation leading to suppressed $\mathrm{T}$ cell responses (Yong, 2002), and interestingly, one way this suppression occurs is through the expression of PD-L1 (B7-H1) on the surface of monocytes and DCs (Schreiner et al., 2004). Therefore, reduced MOG-specific responses seen in MIS416-treated mice suggest peripheral immune modulation is occurring, and as MIS416-treated mice express high levels of PD-L1 on APCs, there may be some similarities between MIS416 treatment and IFN- $\beta$ treatment.

While ConA-induced IFN- $\gamma$ production by splenocytes from MIS416-treated mice were not significantly reduced compared to untreated mice, there was a significant reduction in the CD4+ T cell proliferative response to ConA which was also associated with a reduced number of IFN- $\gamma$ positive cells in the ConA cultures. These results suggest that MIS416 treatment may alter the peripheral immune environment in an antigen non-specific manner, which is similar to the IFN- $\beta$ induced PD-L1 expression mechanism which has also been shown to be antigen non-specific 
(Schreiner et al., 2004); but unlike the GA peripheral mechanisms which are thought to be antigenspecific (Ziemssen \& Schrempf, 2007).

One of our major findings was the significant induction of IFN- $\gamma$ by CD4+ cells, macrophages and NK cells in response to MIS416 in vitro and in vivo. Interestingly, a pilot study conducted in the 1980s in relapsing-remitting MS patients identified that i.v. IFN- $\gamma$ treatment can lead to disease exacerbations (Panitch, Hirsch, Schindler, \& Johnson, 1987). However, later on it was suggested by Sriram et al that the apparent worsening of disease was due to pseudo-relapses induced by IFN$\gamma$-enhancing the pre-existing MS-associated inflammation in these patients. This conclusion was supported by the lack of new clinical activity 6-12 months after this treatment as measured using neurological disability scoring (Panitch et al., 1987; Sriram \& Steiner, 2005). Unlike the relapsingremitting IFN- $\gamma$ studies, systemic release of IFN- $\gamma$ may be more effective in progressive forms of MS disease in which the $\mathrm{T}$ cell responses are no longer involved in driving disease. Instead, the innate cells (i.e. macrophages and microglia) are believed to mediate the CNS inflammation in progressive MS disease (H. L. Weiner, 2008). The effectiveness of IFN- $\gamma$ may also depend on its location and context within the immune system, and since MIS416-induced IFN- $\gamma$ is innate cellderived, the effect of secreted IFN- $\gamma$ may be beneficial unlike cytokine therapy.

High levels of MIS416-induced IFN- $\gamma$ may be able to alter EAE disease through the release of immunosuppressive factors such as NO and IDO (indoleamine-pyrrole 2,3-dioxygenase). Firstly, it was found that MIS416-treated mice produced significant amounts of NO in response to MIS416 in vitro and in vivo, and therefore, this factor is likely to play a major role in MIS416 treatment. NO has a variety of immunological functions through both intra- and inter-cellular signalling, although the immunomodulatory role NO has in EAE disease is relatively unclear. A review published by Willenborg et al in 1999 noted that 27 papers suggested a negative role for NO in EAE while 15 papers show that NO has a protective role, and several studies showed no alteration (Willenborg, Staykova, \& Cowden, 1999). Even now, some 15 years later the role of NO in EAE disease is still unclear; however, it is likely that the context and timing of this particular secreted factor is important.

By using aminoguanidine (AG), a selective iNOS inhibitor, one group found that when NO was depleted during early stages of disease there was a significant delay in disease progression; however, when AG was used in the later stages of disease, after onset, EAE disease was exacerbated with enhanced clinical severity and mortality (Okuda, Sakoda, Fujimura, \& Yanagihara, 1998). Their results suggest that during early stages of disease, NO may have a detrimental role whereas during later stages of disease NO may be disease protective. Another 
study has also linked the production of IFN- $\gamma$ and NO by suggesting that NO production by APCs, particularly in the CNS, requires T cell-derived IFN- $\gamma$; and furthermore, the production of NO inhibits other $\mathrm{T}$ cells from becoming activated and proliferating (van der Veen et al., 2003). Furthermore, several studies have shown that NO can inhibit $\mathrm{T}$ cell proliferation in vitro (Albina, Abate, \& Henry, 1991; van der Veen, Dietlin, Pen, Gray, \& Hofman, 2000), and given that splenocytes from MIS416-treated mice produced high levels of NO, this could be the mechanism by which MIS416-treated CD4+ T cell responses to ConA were significantly reduced compared to untreated samples. These studies provide evidence that high levels of IFN- $\gamma$ may be protective in EAE through the induction of iNOS, and our results showed that MIS416 treatment may rely on NO to induce its disease protective effects.

The production of IDO by activated cells may be another way in which IFN- $\gamma$ exerts disease protective effects. It has been shown that splenic DCs which were isolated and treated with IFN- $\gamma$ for 48 hours, before being transferred to EAE mice or Lewis rats day 5-7 post immunisation, induced CD4+ $\mathrm{T}$ cell apoptosis and reduced the migration of inflammatory cells into the CNS of EAE animals (Xiao et al., 2004). Further analysis showed that the IFN- $\gamma$-treated DCs upregulate their expression of IDO, which may result in $\mathrm{CD} 4+\mathrm{T}$ cell apoptosis by reducing the availability of tryptophan metabolites (Xiao et al., 2004). The results from this study provide evidence that IFN- $\gamma$ may mediate its disease protective effects through a multitude of pathways that are not all dependent on NO.

MIS416 treatment induced high levels of splenic IL-10, produced by Tregs and macrophages, suggesting that this regulatory cytokine may also play a role in the mechanism of MIS416. IL-10 has been implicated as a suppressive factor in EAE, as mice which are deficient for IL-10 develop more severe EAE and exhibit stronger Ag-specific proliferation than wild-type mice (Bettelli et al., 1998). Furthermore, IL-10 production by Tregs in EAE mice acts to naturally limit the disease, since Tregs from IL-10 deficient mice are not able to suppress EAE disease (X. Zhang et al., 2004). Therefore IL-10 was a key cytokine which was produced by Tregs in response to MIS416, and given that the number of Tregs also increased with MIS416 treatment these cells may have a role in the disease protective mechanism of MIS416 treatment.

The longevity of the MIS416-induced peripheral changes were assessed at 6 days post-treatment and most of the cellular and cytokine changes were maintained, including slight elevations in serum NO at day 13. Together, these results suggest that the MIS416-induced effects were long lasting and therefore may have maintained disease suppression with MIS416 treatment. In contrast to day 15 (1 day post-MIS416 treatment) in which no detectable IL-17A was produced, at day 13 
splenocytes from MIS416-treated mice produced elevated levels of IL-17A in response to MIS416 re-stimulation when compared to splenocytes from untreated mice. This result was interesting since MIS416-treated EAE mice had a reduced MOG-specific IL-17A response compared to untreated EAE mice. Initially IL-17A has been characterised as a pro-inflammatory cytokine but may have dual roles in an immunological response. Although in the early stages of EAE disease this cytokine is thought to have detrimental effects and be associated with a Th17 response (Aranami \& Yamamura, 2008), there is also evidence to suggest that under certain circumstances IL-17A can be regulatory through the recruitment of specific cells such as neutrophils (Fujie et al., 2012).

Results from the HT-MIS416 experiments indicated that TLR9 stimulation was required to reduce EAE disease. Interestingly, the literature suggests the role of TLR9 in EAE disease is controversial with some studies suggesting TLR9 ligands exacerbate disease and are involved in EAE disease induction (Marco Prinz et al., 2006; Tsunoda et al., 1999). In contrast another study has shown more severe disease using TLR9 deficient mice compared to WT mice (Marta et al., 2008). The difference between the protective role of TLR9 in our study could be that MIS416 has a longer half-life compared to soluble CpG motifs (Palma \& Cho, 2007; White et al., 2014) or that TLR9 stimulation was protective only when given in the presence of a NOD2 agonist. In support of the latter idea a study by Castellaneta et al reported that the in vivo administration of MDP (NOD2 agonist) alters the response of liver pDCs to TLR9 resulting in reduced TLR9-induced IFN- $\alpha$, IL-6 and TNF and indicating that a more tolerogenic state was achieved (Castellaneta, Sumpter, Chen, Tokita, \& Thomson, 2009). Although disease protection was not seen in the HT-MIS416-treated mice, some of the peripheral immune effects were altered, such as a small increase in spleen count which mostly consisted of CD4 and CD8+ T cells and no increase in red pulp macrophages was seen. Therefore, these results indicate that NOD2 was involved in some of the peripheral immune effects of MIS416 but the NOD2-induced changes were not sufficient to reduce EAE disease.

\subsubsection{Summary}

There were a number of key observations which could be contributing to the mechanism of MIS416-induced disease protection in EAE mice. As outlined in the summary figure (Figure 4-31) it was observed that there were increased numbers of macrophages and Tregs within both the spleen and liver of MIS416-treated mice. These cell types have been identified as potential modifiers of EAE disease depending on which factors they express and how they interact with an activated $\mathrm{T}$ cell population. Furthermore, we assessed the activation markers including expression of anti-proliferative ligand PD-L1 and suppressive factors such as IL-10 and NO which were also 
elevated within the spleen and liver. Together with the observation that MIS416 treatment reduced proliferation of splenic CD4 $\mathrm{T}$ cells in response to ConA, these results suggest that MIS416 was using these mechanisms to alter the immune environment in a way that induced an immunoregulatory phenotype. It is likely that these factors were not independent of each other, however which factors contribute to the mechanism of MIS416 in EAE mice will be assessed further in the next chapter.

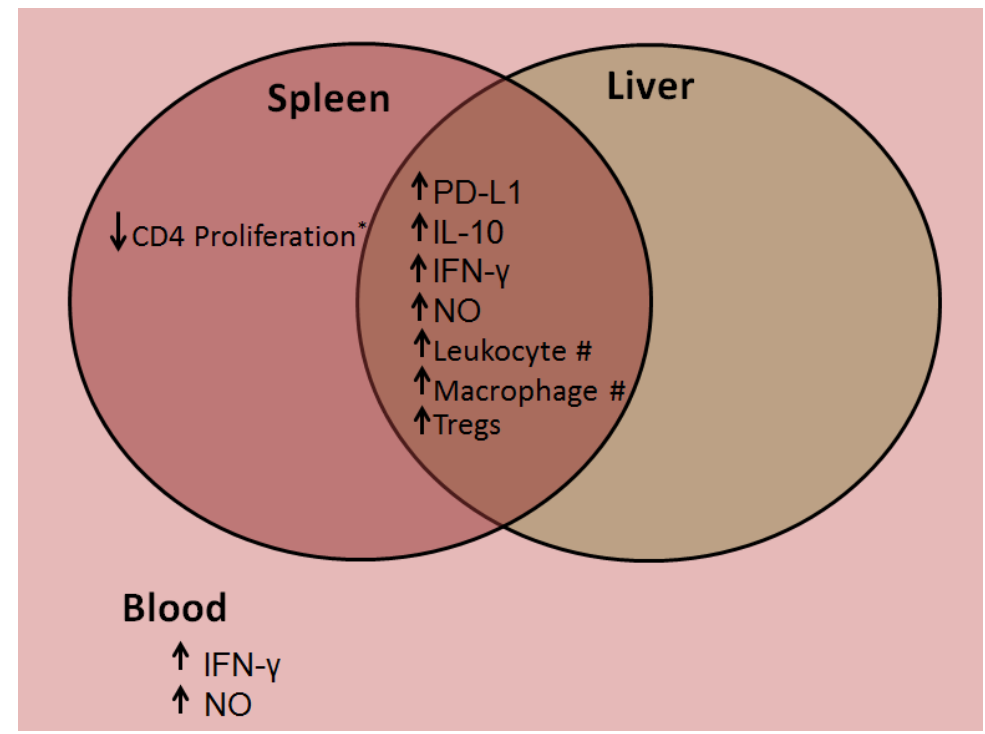

Figure 4-31 Observations of spleen and liver cultures with MIS416, or serum cytokines which occurred with MIS416 treatment in vivo.

* not assessed in liver. 


\section{Chapter 5: Mechanisms of MIS416- mediated immunoregulation}




\subsection{Introduction}

The previous chapter (Chapter 4) provided insight into the peripheral immune changes which occurred during MIS416 treatment, and because some of these changes have known immunosuppressive effects, the current chapter is aimed at assessing if these changes account for the disease suppressing effects of MIS416 during EAE. Specifically, we found that MIS416treated mice had significantly increased serum IFN- $\gamma$ and NO, as well as Tregs and MDSC within the spleen and liver. Therefore, this chapter investigated if these immune cells and factors mediated functional immunoregulation to determine mechanism of action of MIS416 in EAE.

Serum IFN- $\gamma$ levels in MIS416-treated EAE mice peaked at onset of disease, indicating that this cytokine could be involved in the mechanism of MIS416, particularly in the early stages of disease. However, the role IFN- $\gamma$ may have in MIS416's mechanism of action is counter-intuitive given this cytokine is typically considered to be pro-inflammatory. Furthermore, EAE disease has long been considered a Th1-driven disease characterised by the expansion of antigen-specific IFN$\gamma+\mathrm{CD} 4 \mathrm{~T}$ cells, indicating that high levels of IFN- $\gamma$ could drive disease pathology. Interestingly, it has been identified that IFN- $\gamma^{-/-}$mice actually develop more severe EAE than WT controls (I A Ferber et al., 1996) which suggests that IFN- $\gamma$ may have a negative feedback control on EAE severity, and therefore it is possible that MIS416 treatment is enhancing the negative feedback pathway of EAE.

A potential way in which MIS416-induced IFN- $\gamma$ may be exerting its immunoregulatory effects is through the secretion of NO. Our results from the previous chapter (Chapter 4) showed that MIS416-treated mice produced high levels of NO, and this NO was found within splenocyte and liver leukocyte cultures and also within the serum of MIS416-treated mice, regardless of EAE immunisation. Similar to IFN- $\gamma$, the role NO has in EAE disease is controversial in the literature and it is likely to have dual roles in disease depending on the timing and location. One study has suggested that selectively inhibiting iNOS at the early stages of disease was protective, whereas in later stages of disease NO appeared to be disease limiting (Okuda et al., 1998). Therefore, NO could also be another factor which, at high levels, reduces EAE disease.

In addition to the secreted factors produced in response to MIS416, there were also cellular changes which could participate in the immunoregulatory mechanism. MIS416 treatment significantly increased the number of Tregs within the spleen and liver, and this increase was apparent in both EAE and non-EAE mice. It has previously been shown that EAE mice have an expansion of Tregs during the later stages of disease, and therefore Tregs are thought to be a natural regulatory mechanism of limiting EAE disease (Richard A. O'Connor \& Anderton, 2008). 
Additionally, it has been identified that the addition of Tregs into EAE mice reduces disease burden (Adam P. Kohm et al., 2002); therefore we believe that increased Treg number in MIS416treated mice may contribute to reduced EAE disease seen in MIS416-treated EAE mice. As the results from Chapter 4 have identified Tregs as CD4+CD25+FoxP3+ cells, this chapter assessed the functionality of this population, an important consideration in determining whether these cells contribute to the mechanism of MIS416 in EAE.

Another cell population increased by MIS416 treatment was MDSC (myeloid derived suppressor cell). These cells are a relatively newly identified population which can be isolated by fairly broad markers, CD11b+ and Ly6G+ or Ly6C+ and are likely to include a wide range of cells not just MDSC. This cell population was initially identified as suppressors of anti-tumour responses in tumour models, as increasing levels of MDSC cells are associated with a worse prognosis within a tumour setting (Youn, Nagaraj, Collazo, \& Gabrilovich, 2008). In contrast to the tumour response, treatment of EAE mice with MDSC has shown to reduce disease burden (Gabrilovich \& Nagaraj, 2009). As we have seen an increase in a cell population which express the markers of MDSCs with MIS416 treatment, this chapter also focused on assessing whether MDSC function is increased, and if these cells could have a role in MIS416 mechanism.

While the previous chapter highlighted several key ways in which MIS416 treatment could be altering the immune system such that EAE disease is reduced, it is also likely that these factors are not acting independently of each other. Therefore, in this chapter we tested whether some of these factors are acting independently or not and the possible cell types involved. These experiments will take us one step closer to identifying the mechanism and key cell types involved in MIS416induced EAE suppression.

\subsection{Aims}

The aim of this chapter is to identify which peripheral immune changes are key parts of MIS416 mechanism of action in EAE disease, with the idea that not all the immunological changes seen in the previous chapter are equally important for the suppression of EAE. By understanding which parts of the immune system are crucial for MIS416 we may be able to identify how MIS416 can be used more effectively in MS patients.

\subsubsection{Specific aims}

1. To elucidate the role of IFN- $\gamma$ in MIS416-mediated immunoregulation.

2. To identify the role of NO in MIS416-mediated immunoregulation. 
3. To determine whether Tregs and MDSC from MIS416-treated mice can suppress CD4+ T cell proliferation in vitro.

4. To determine if MIS416 treatment reduces CD4 T cell proliferation in vivo. 


\subsection{Results}

\subsubsection{IFN- $\gamma$ was required for MIS416 protection in chronic EAE disease model}

The assessment of cytokine responses in MIS416-treated mice have identified IFN- $\gamma$ as a key cytokine produced post-MIS416 treatment with high levels detected in culture supernatants and serum even in the absence of EAE. Therefore, by determining the involvement of IFN- $\gamma$ in MIS416-treated EAE mice, we can identify whether this cytokine is important in MIS416's mechanism of action in EAE. To this end, we compared the disease scores of IFN- $\gamma$-deficient (hence forth IFN- $\gamma^{-1}$ ) MIS416-treated EAE mice to that of WT, and it was found that MIS416treated IFN- $\gamma^{-1}$ EAE mice suffered a severe disease which followed a similar course to IFN- $\gamma^{-1-}$ untreated EAE mice. In contrast, the MIS416-treated WT EAE mice showed significant disease protection (Figure 5-1A). Given the severity of EAE disease developed by IFN- $\gamma^{-/-}$mice, these mice were culled earlier than WT mice. The weights were also recorded daily as increased EAE disease severity is correlated to increased weight loss. We found a similar lack of protection against weight loss in MIS416-treated IFN- $\gamma^{-/-}$EAE mice whereas the MIS416-treated WT EAE mice had minimal weight loss (Figure 5-1B). These results suggest that IFN- $\gamma$ was involved in MIS416-induced disease suppression since without IFN- $\gamma$, MIS416-treated mice were not protected from EAE disease. 
A

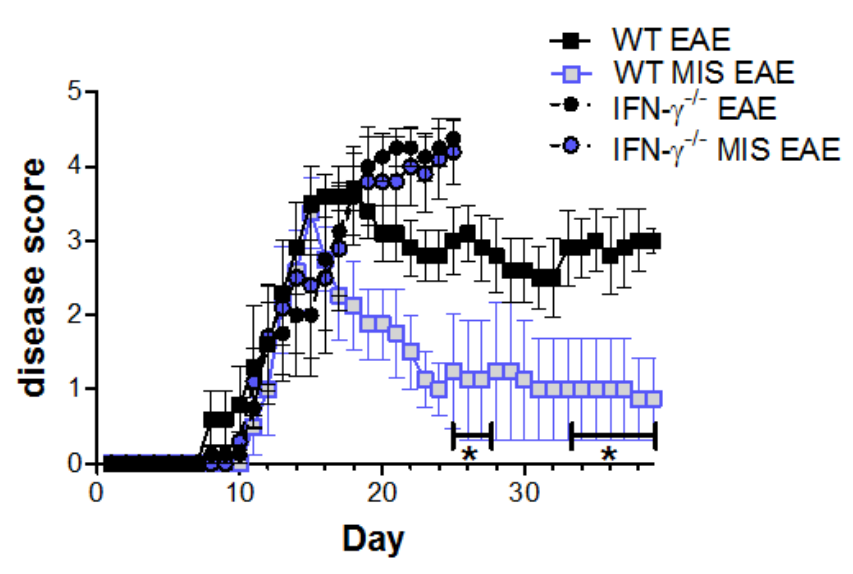

B

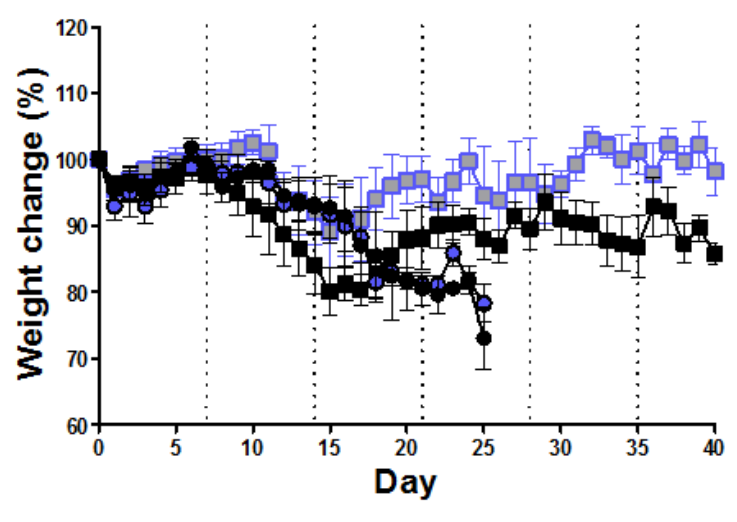

Figure 5-1 IFN- $\gamma^{-/-}$mice were not protected from EAE disease with MIS416 treatment

Wild type (C57BL/6) or IFN- $\gamma^{-1-}$ mice were immunised to induce EAE and treated with a weekly dose of MIS416 (100 $\mu \mathrm{g} / \mathrm{mouse})$, and weighed and scored daily. A) MIS416 treatment did not protect IFN- $\gamma^{-/ 2}$ mice from EAE. B) MIS416 lack of disease protection was evident in weight loss seen over time with EAE disease, although differences were not significant. Shown are the means \pm SEM of disease scores from 2 independent experiments ( $\mathrm{n}=10$ /group) ${ }^{*} \mathrm{p}<0.05$ WT MIS EAE compared to WT EAE by 2-way ANOVA with Bonferroni's multiple comparison post-test. 


\subsubsection{MIS416-induced peripheral myeloid changes were abolished in IFN- $\gamma^{-/-}$mice}

Given that IFN- $\gamma^{-/-}$EAE mice did not respond to MIS416 treatment, the next step was to observe the peripheral immune effects of MIS416 treatment in IFN- $\gamma^{-1-}$ mice in the absence of EAE. In the previous chapter (Chapter 4), we discovered that MIS416 treatment increased the number of splenic Tregs and total spleen counts. In IFN- $\gamma^{-/-}$mice MIS416 treatment also induced an increase in the total spleen count (Figure 5-2A), and although Tregs were increased in number, this increase was not significant (Figure 5-2B\&C). Interestingly, this difference could be due to a higher baseline number of Tregs in IFN- $\gamma^{-/-}$mice compared to WT mice and although the baseline differences were not statistically significant, it could have influenced the lack of increased Treg number in MIS416-treated IFN- $\gamma^{-/-}$mice compared to untreated IFN- $\gamma^{-/}$mice. Given the less substantive increase of Tregs in MIS416-treated IFN- $\gamma^{-/-}$mice, it is possible that IFN- $\gamma$ contributes to the expansion of the Treg population by MIS416.

The assessment of other $\mathrm{T}$ cell and NK populations in IFN- $\gamma^{-/-}$MIS416-treated mice indicated a similar trend to WT MIS416-treated mice, where relatively consistent frequencies of CD4, CD8, NK cells and Tregs with and without MIS416 treatment were observed (Figure 5-3A). There was a significant decrease in the percentage NK cells with MIS416 treatment, which was seen in both the WT and IFN- $\gamma^{-/}$(Figure 5-3A). Together with the Treg numbers, these data suggest that the observed MIS416-mediated changes to the lymphoid populations in the spleen were not abolished in the absence of IFN- $\gamma$.

F4/80+ cells, which represent the red pulp macrophages within the spleen, were consistently up regulated within the spleens of MIS416-treated WT mice (Chapter 4). In contrast, IFN- $\gamma^{-1-}$ MIS416-treated mice did not have an increased number or percentage of red pulp macrophages within the spleen compared to untreated WT or IFN $-\gamma^{-/-}$mice (Figure 5-3B). The percentage of Gr-1+ (e.g. granulocytes) cells were not significantly altered with MIS416 treatment in either WT or IFN- $\gamma^{-/-}$mice (Figure 5-3B). Together, these results indicated that IFN- $\gamma$ directly or indirectly contributes to the expansion of the red pulp macrophage populations in the spleen of MIS416treated mice. Interestingly, although there was a significant increase in total spleen counts in IFN$\gamma^{-/-}$MIS416-treated mice, we did not identify a single population which was significantly upregulated in the spleen. One possible explanation is that there was an increase in all cell populations within the spleen.

The expression of activation markers on myeloid cells, both macrophages and Gr-1+ cells, has been shown to be increased with MIS416 treatment. Therefore, we investigated whether IFN- $\gamma$ was involved in the up-regulation of these activation markers with MIS416 treatment and found that unlike the WT controls, there was no significant increase in the expression of MHCII on 
macrophages or Gr-1+ cells from IFN- $\gamma^{-/-}$MIS416-treated mice (Figure 5-3C). Similarly, the expression of PD-L1 was also not significantly up-regulated on macrophages and Gr-1+ from IFN$\gamma^{-1-}$ MIS416-treated mice compared to either untreated IFN- $\gamma^{-/-}$or untreated WT mice (Figure 5-3D). These data suggest that IFN- $\gamma$ is involved in the up-regulation of these markers by MIS416.

The re-stimulation response to MIS416 and ConA was compared between MIS416-treated and untreated IFN- $\gamma^{-1-}$ mice, as well as the WT controls. Splenocytes from MIS416-treated IFN- $\gamma^{-1-}$ mice produced significant levels of IL-10 compared to splenocytes from untreated IFN- $\gamma^{-/}$and WT mice, which had no detectable IL-10 in the supernatant cultures; although MIS416-treated WT mice produced significantly more IL-10 than MIS416-treated IFN- $\gamma^{-/-}$mice (Figure 5-4A). In response to ConA, only low levels of IL-10 were detectable in splenocyte culture supernatants from WT or IFN- $\gamma^{-/-}$mice, regardless of MIS416 treatment. Similar to the re-stimulation response with MIS416 alone, the ConA + MIS416 re-stimulation produced strong IL-10 responses by splenocytes from MIS416-treated WT and IFN- $\gamma^{-/-}$mice compared to their respective controls, although it appeared that with the addition of ConA the MIS416-induced IL-10 production was lower than MIS416 alone (Figure 5-4A). Together these results indicate that IFN- $\gamma$ is not required for the production of IL-10 in response to MIS416, although without IFN- $\gamma$ less IL-10 was produced.

In contrast to the IL-10 production, the level of NO in splenocyte culture supernatants from IFN- $\gamma^{-}$ I- mice was significantly lower across all the stimulations tested when compared to the WT control (Figure 5-4B). For example, both MIS416 and ConA re-stimulation cultures from WT mice had significantly higher levels of NO, particularly in the mice which received MIS416 treatment in vivo, whereas cultures from IFN- $\gamma^{-/}$mice had barely detectable levels of NO regardless of MIS416 treatment. Thus, the NO results from IFN $-\gamma^{-/}$mice indicate that NO production in splenocyte cultures may be dependent on IFN- $\gamma$.

To determine if a similar effect was found in vivo, NO levels in the serum were assessed, and we found that in the absence of IFN- $\gamma$ (i.e. IFN- $\gamma^{-/-}$mice), there was no increase in serum NO by MIS416 treatment (Figure 5-5). This finding is in contrast to MIS416 treatment in WT mice in which serum NO was significantly increased compared to untreated mice (Figure 5-5). Altogether these results using IFN- $\gamma^{--}$mice implicate IFN- $\gamma$ as major contributor to MIS416-induced disease protection. Additionally, these data reveal a potential role for NO in disease protection which merits further investigation. 
A

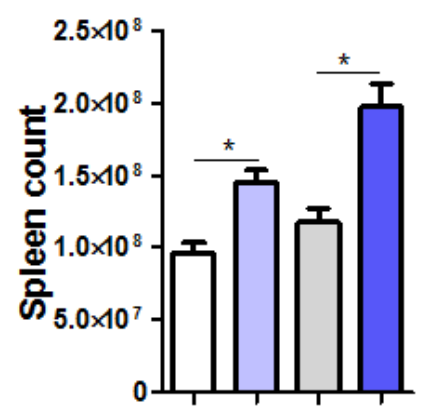

B

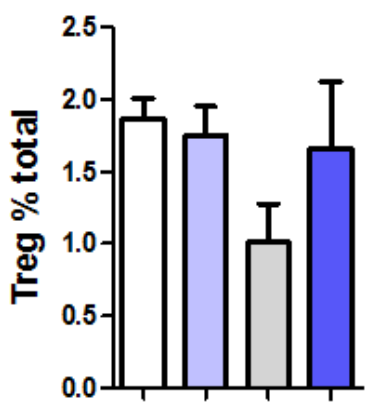

C

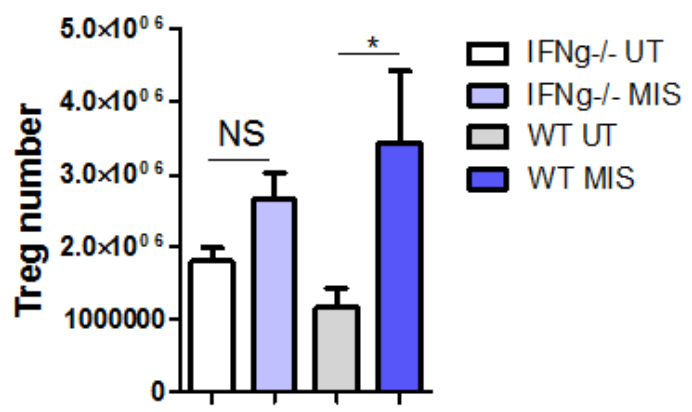

Figure 5-2 IFN- $\gamma^{-1-}$ mice had increased spleen counts with MIS416 treatment

Wild type (WT - C57BL/6) or IFN- $\gamma^{-1-}$ mice were treated with a weekly dose of MIS416 (100 $\left.\mu \mathrm{g} / \mathrm{mouse}\right)$ and at day 15, 1 day post MIS416 treatment, mice were culled and splenocytes were isolated and assessed using flow cytometry. A) MIS416 treatment increased spleen count in both WT and IFN- $\gamma^{-1-}$ mice. B) MIS416 treatment did not alter the percentage of Tregs in the spleen. C) MIS416 treatment significantly increased the number of Tregs in the spleen of WT mice. Shown are the means \pm SEM from 3 independent experiments ( $=7-12 /$ group) *p $<0.05$ by MIS416-treated compared to control mice 1-way ANOVA with Bonferroni's post-test. 
A
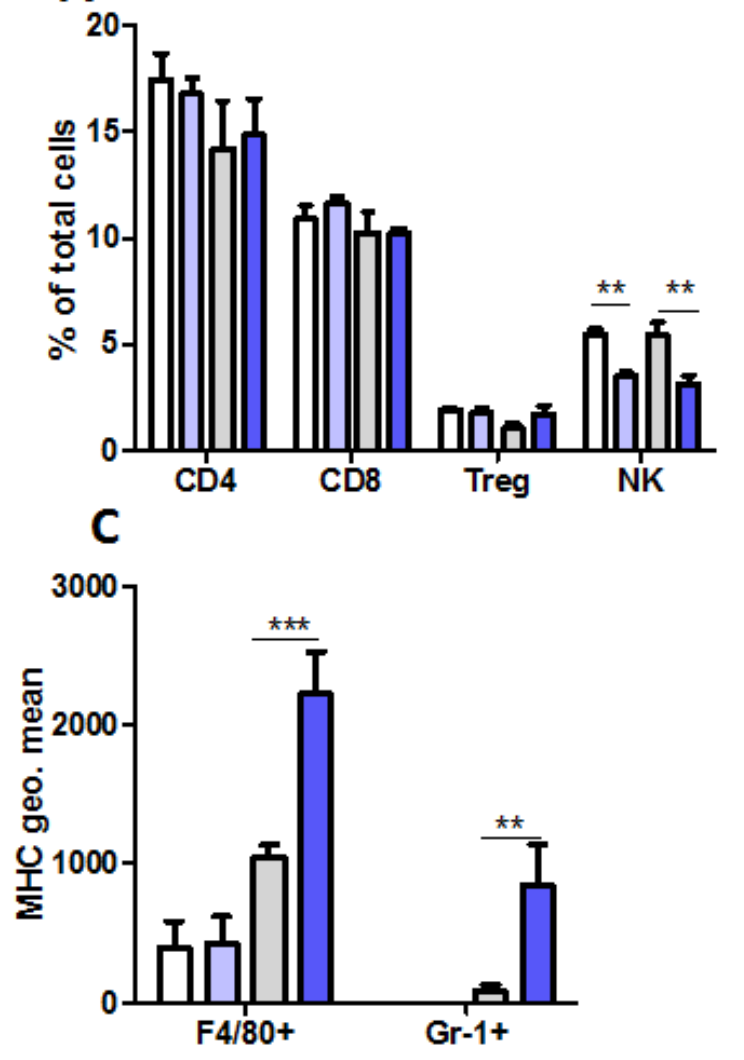

B
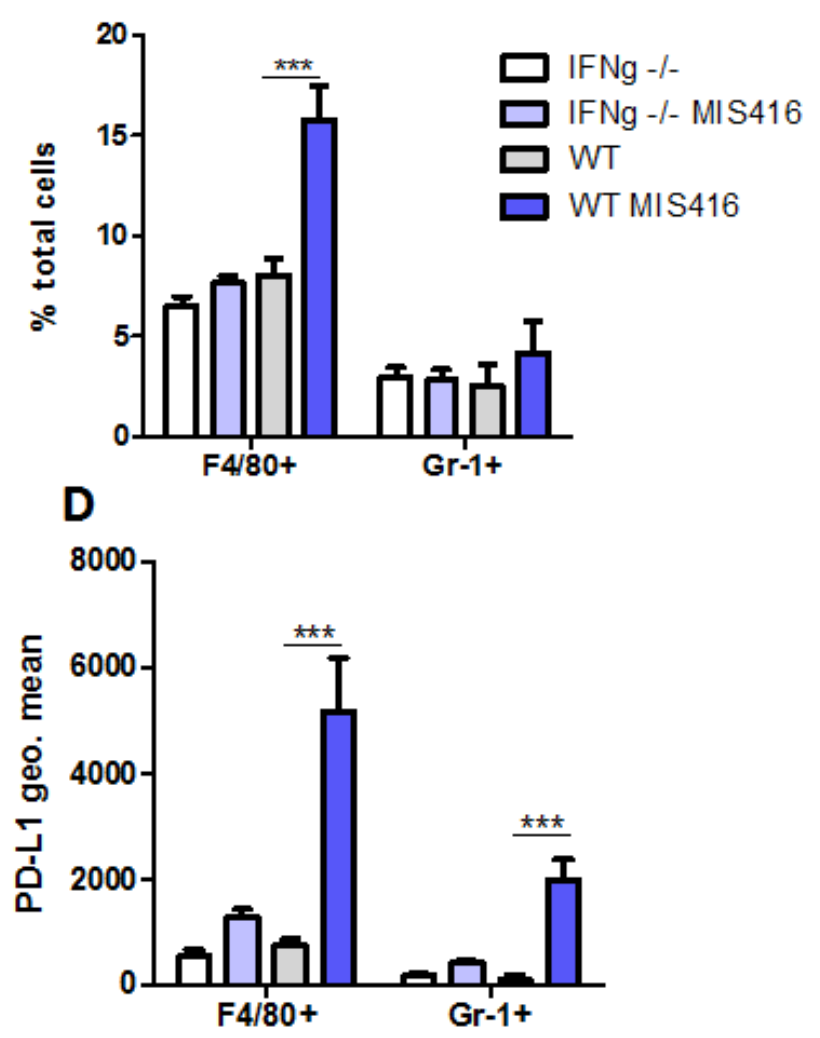

Figure 5-3 MIS416-treated IFN- $\gamma^{-1 /}$ mice did not have the same myeloid alterations as WT mice.

Wild type (WT - C57BL/6) or IFN- $\gamma^{-1 /}$ mice were treated with a weekly dose of MIS416 (100 $\left.\mu \mathrm{g} / \mathrm{mouse}\right)$ and at day 15, 1 day post MIS416 treatment, mice were culled and splenocytes were isolated and assessed using flow cytometry, for the myeloid populations single positive gating was used. A) Spleen lymphocyte and NK populations within IFN- $\gamma^{-1-}$ mice were not altered with MIS416 treatment. B) MIS416 treatment in IFN- $\gamma^{-1-}$ mice did not result in increased myeloid cells. C) MIS416-treated IFN- $\gamma^{-/-}$mouse myeloid cells did not have increased MHC expression. D) MIS416-treated IFN- $\gamma^{-/-}$mouse myeloid cells did not have increased PD-L1 expression. Shown are the means \pm SEM from 2 independent experiments ( $\mathrm{n}=7-12 /$ group) $* * \mathrm{p}<0.01$ and $* * * \mathrm{p}<0.001$ MIS416-treated mice compared to untreated mice by 1-way ANOVA with Bonferroni’s post-test. 
A

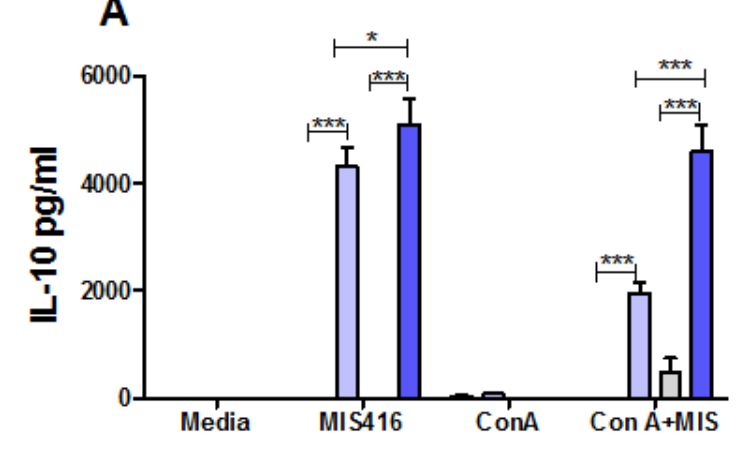

B

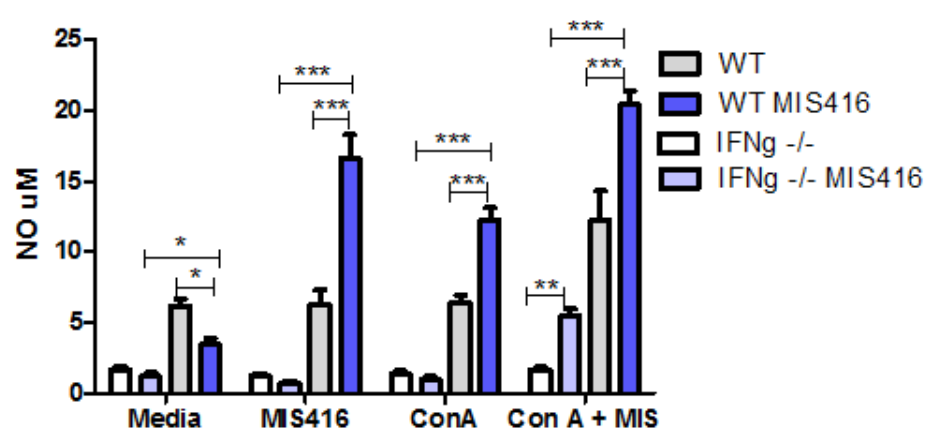

Figure 5-4 MIS416-treated IFN- $\gamma^{-/}$mice had altered responses to re-stimulation

Wild type (WT - C57BL/6) or IFN- $\gamma^{-1-}$ mice were treated with a weekly dose of MIS416 (100 $\left.\mu \mathrm{g} / \mathrm{mouse}\right)$ and at day 15, 1 day post MIS416 treatment, mice were culled and splenocytes were cultured for 48-hours with media, ConA $(1 \mu \mathrm{g} / \mathrm{ml})$, MIS416 $(20 \mu \mathrm{g} / \mathrm{ml})$ or ConA + MIS416 and culture supernatants were assessed at 48-hours for IL-10 by ELISA (A) and NO by Griess (B). Shown are the means \pm SEM from 2 independent experiments (n=8/group) $* \mathrm{p}<0.05, * * \mathrm{p}<0.01$ and $* * * \mathrm{p}<0.001$ MIS416 compared to respective control mice by 2-way ANOVA with Bonferroni's post-test. 


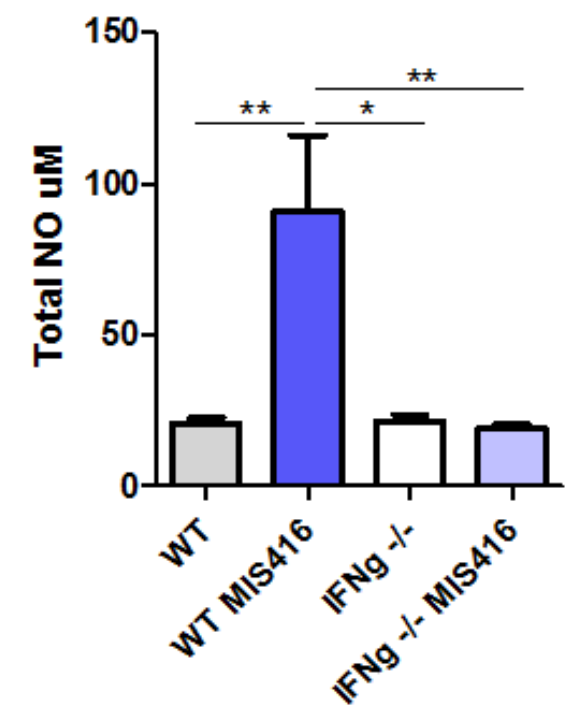

Figure 5-5 MIS416-treated IFN- $\gamma^{-1 /}$ mice had low levels of serum NO compared to MIS416-treated WT mice

Wild type (WT - C57BL/6) or IFN- $\gamma^{-1-}$ mice were treated with a weekly dose of MIS416 (100 $\left.\mu \mathrm{g} / \mathrm{mouse}\right)$ and at day 15, 1 day post MIS416 treatment, mice were culled and blood was taken via cardiac puncture for serum. Shown are the means \pm SEM from 2 independent experiments $(n=8-10) * \mathrm{p}<0.05$ and $* * \mathrm{p}<0.01$ MIS416 compared to control mice by 1-way Kruskal-Wallis ANOVA with Dunns post-test. 


\subsubsection{IFN- $\gamma$ was a key factor in MIS416-mediated suppression of $\mathrm{T}$ cell responses with ConA}

The previous chapter (Chapter 4) revealed that MIS416-treated mice had reduced CD4+ T cell proliferative responses to ConA. Therefore, using CD4+ T cells from MIS416-treated IFN- $\gamma^{-1-}$ mice, we investigated whether a reduced proliferative response could occur in the absence of IFN$\gamma$. We found that, in contrast to MIS416-treated WT mice, there was no significant effect on the proliferation of CD4+ T cells from MIS416-treated IFN- $\gamma^{-/-}$mice compared to untreated IFN- $\gamma^{-/-}$ mice (Figure 5-6). Furthermore, this effect was consistent across all proliferation measurements, which included the percentage of cells which divided, the division index of all cells and the proliferation index of only cells which divided using the proliferation platform in Flowjo (Tree Star). The proliferation index in the FlowJo platform is calculated by the total number of divisions divided by the number of cells which underwent division, whereas the division index is the average number of cell divisions which occur by a cell in the original population including cells which never divided, therefore all measurements of proliferation are relevant to the suppression of proliferation induced by MIS416 treatment. These results suggest that MIS416 treatment did not induce an anti-proliferative immune environment in the spleens of IFN- $\gamma^{-1-}$ mice whereas in MIS416-treated WT mice, the expected anti-proliferative effect was similar to previous experiments. These findings indicate that MIS416-induced IFN- $\gamma$ directly, or indirectly through a down-stream mediator, which significantly reduced polyclonal $\mathrm{T}$ cell proliferation in vitro. 

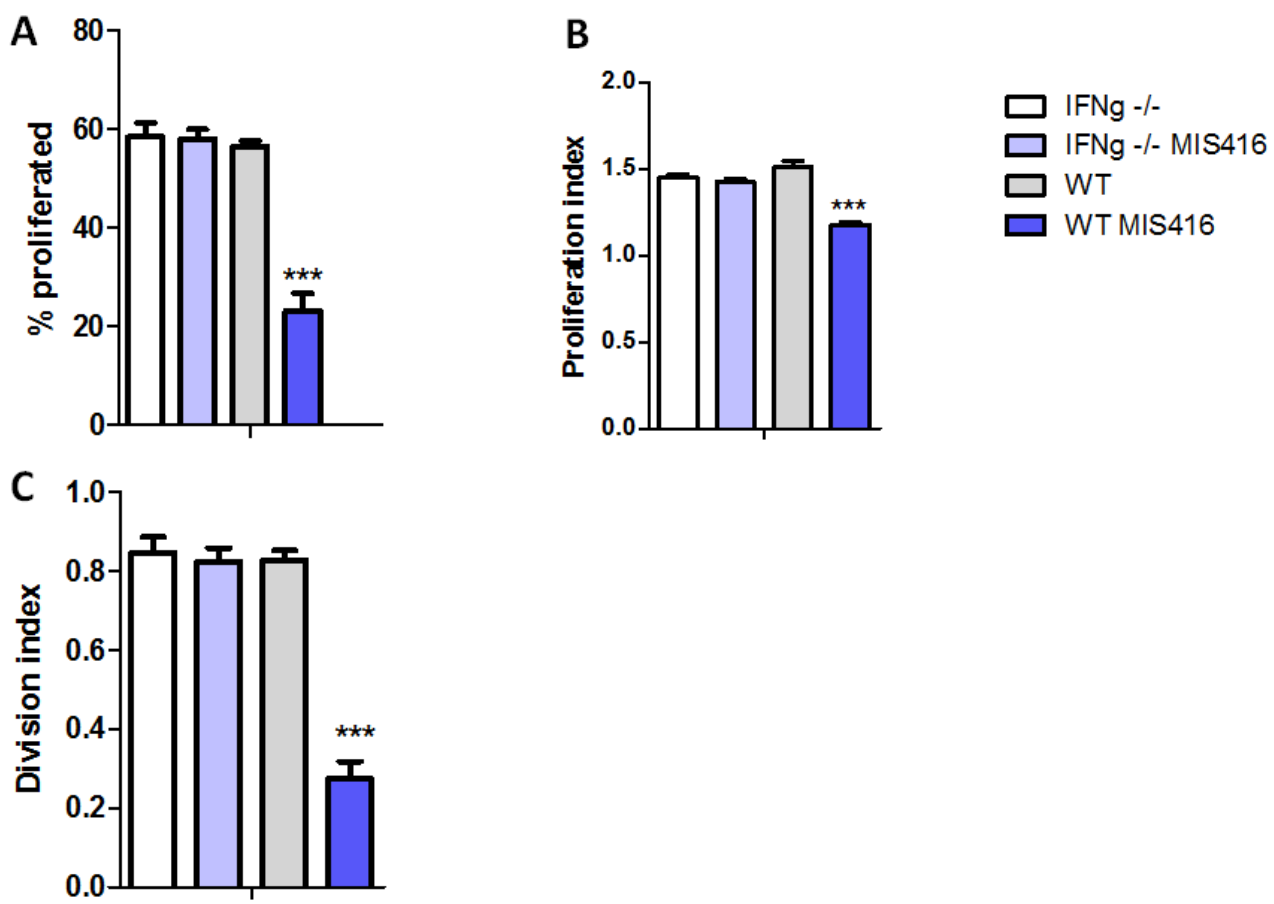

Figure 5-6 Splenocytes from IFN- $\gamma^{-/-}$MIS416-treated mice were unable to suppress $T$ cell proliferation response to ConA

Wild type (WT - C57BL/6) or IFN- $\gamma^{-1-}$ mice were treated with a weekly dose of MIS416 (100 $\mu \mathrm{g} / \mathrm{mouse}$ ) and at day 15, 1 day post MIS416 treatment, mice were culled and splenocytes were isolated and CFSE labelled then cultured with media or ConA $(1 \mu \mathrm{g} / \mathrm{ml})$. A) Percentage of CD4+ cells which proliferated. B) Proliferation index of CD4+ cells in splenocyte culture C) Division index of CD4+ cells in splenocyte cultures Shown are the means \pm SEM from 3 independent experiments ( $\mathrm{n}=8$-12/group) $* * * \mathrm{p}<0.001$ MIS416 compared to control mice by 1 -way ANOVA with Bonferroni's post-test 


\subsubsection{Nitric oxide was a key factor in MIS416-mediated suppression of ConA-induced $T$ cell proliferation}

Because previous IFN- $\gamma^{-/}$mouse experiments showed that without IFN- $\gamma$ very little NO was produced in response to MIS416 (Figure 5-4B) and NO is known to have anti-proliferative effects on CD4+ T cells (Albina et al., 1991), we determined whether NO produced in response to MIS416 treatment may be one of the ways systemic IFN- $\gamma$ provides protection against EAE. Therefore, we assessed the effect of inhibiting NO production on the proliferative response of CD4+ $\mathrm{T}$ cells to ConA. To do this, aminoguanidine (AG) a known inhibitor of nitric oxide synthase (iNOS) that inactivates both the citrulline and NADPH oxidase-forming properties of iNOS enzyme (Wolff \& Lubeskie, 1995), was added to splenocyte cultures. A concentration of $200 \mathrm{mM}$ AG within splenocyte cultures has been shown to deplete almost all of the NO produced in response to MIS416 (data not shown), and therefore by using AG in these cultures it was possible to determine whether NO production contributed to the suppression of $\mathrm{T}$ cell proliferation in response to ConA.

ConA-stimulated splenocytes from untreated mice had a proliferation index of 1.5 and $60 \%$ of all cells were proliferating, whereas splenocytes from MIS416-treated mice had a significantly lower proliferation index (less than 1.2; Figure 5-7). However, in the presence of AG, MIS416-treated mouse splenocytes stimulated with ConA had a partial restoration of the proliferation index (i.e. higher proliferation in the presence of AG than without AG) (Figure 5-7A\&D). These results indicate that the high levels of NO produced in ConA re-stimulated splenocyte cultures from MIS416-treated mice had a suppressive effect on CD4+ T cell proliferation capacity, given that the proliferation index only measures cells which have begun to divide and this effect was restored with iNOS inhibition. Interestingly, although the ConA re-stimulated cultures had high levels of NO present, the addition of MIS416 to these cultures elevated the levels of NO further but did not enhance the suppressive effect, suggesting the suppressive effect of NO could not be further enhanced (Figure 5-7D).

The division index and percent divided were not reversed completely with the addition of NO indicating that when iNOS is blocked, splenocytes from MIS416-treated mice were still able to prevent some CD4+ T cells from undergoing proliferation. These results suggest that NO may not be the only mechanism by which MIS416 treatment suppressed T cell responses and that there may be other contributing factors (Figure 5-7B\&C). Altogether these results provide evidence that MIS416 treatment mediated some of its immunosuppressive effects on T cell proliferation through $\mathrm{NO}$, and furthermore NO appeared to be IFN- $\gamma$-dependent suggesting a potential mechanism by which IFN- $\gamma$ production may regulate disease in EAE. 
A
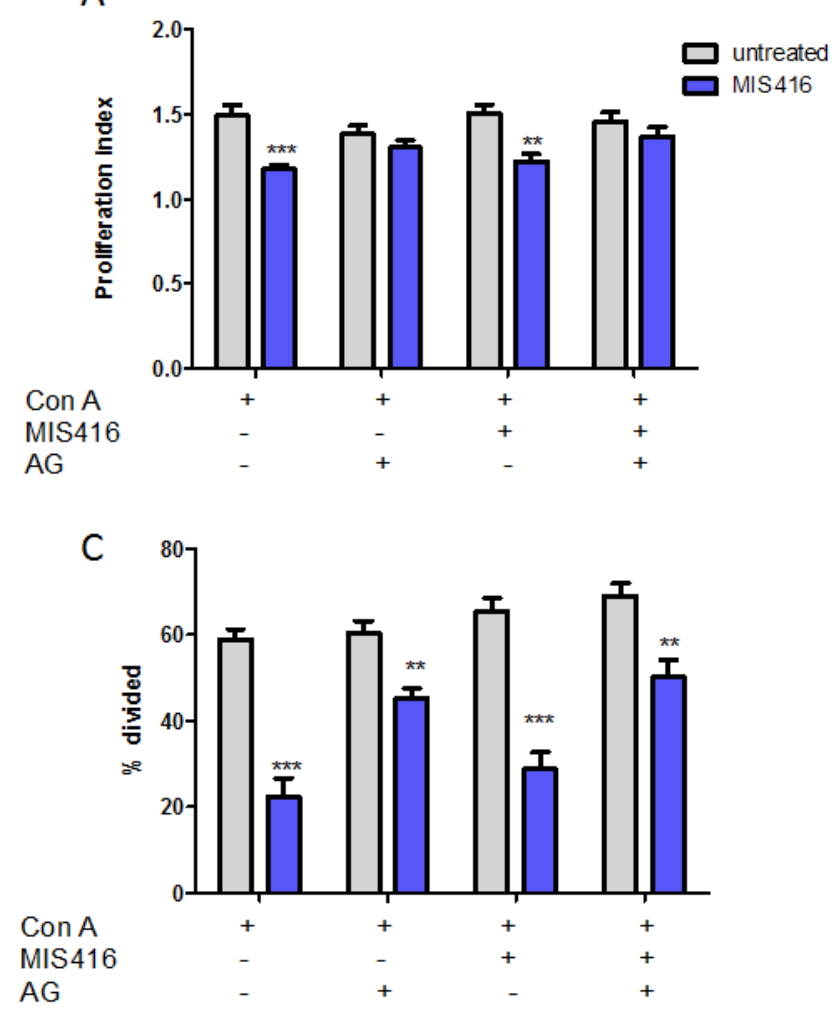

B
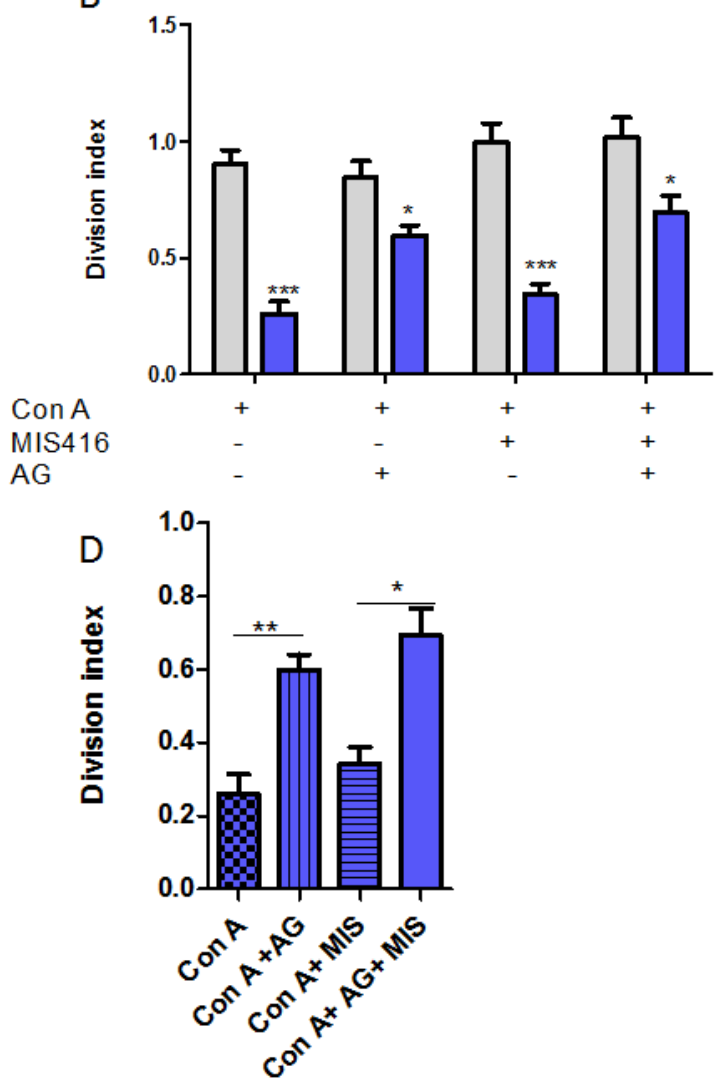

Figure 5-7 Nitric oxide was a key factor for MIS416-induced suppression of CD4 T cell proliferation

C57BL/6 mice were treated with a weekly dose of MIS416 (100 $\mu \mathrm{g} /$ mouse) and at day 15, 1 day post MIS416 treatment, mice were culled and splenocytes were isolated and CFSE labelled then cultured with media, ConA (1 $\mu \mathrm{g} / \mathrm{ml})$, ConA + MIS416 (1 $\mu \mathrm{g} / \mathrm{ml}$ and $20 \mu \mathrm{g} / \mathrm{ml}$ respectively), ConA + AG (1 $\mu \mathrm{g} / \mathrm{ml}$ and $200 \mathrm{mM}$ respectively $)$ or ConA + MIS416 + AG (1 $\mu \mathrm{g} / \mathrm{ml}, 20 \mu \mathrm{g} / \mathrm{ml}$, and $200 \mathrm{mM})$. A) Proliferation index of CD4+ cells in splenocyte culture. B) Division index of CD4+ cells in splenocyte cultures. C) Percentage of CD4+ T cells which divided. D) Division index of CD4+ T cells from MIS416-treated splenocytes cultures. Shown are the means \pm SEM from 3 independent experiments ( $\mathrm{n}=8$-12/group) $* \mathrm{p}<0.05$, $* * \mathrm{p}<0.01$ and $* * * \mathrm{p}<0.001$ MIS416-treated mice were compared to control mice by Mann-Whitney t-test (A-C) or 1-way Kruskal-Wallis ANOVA (D) with Dunn's post-test. 


\subsubsection{Nitric oxide inhibition during disease onset did not affect MIS416-mediated disease protection}

As the previous results showed that nitric oxide was a key factor contributing to the reduced CD4 $\mathrm{T}$ cell proliferative response seen within ex vivo splenocytes cultures from MIS416-treated mice, we next assessed whether suppressing NO production in vivo would inhibit MIS416-induced EAE disease suppression and MIS416-induced peripheral immunological changes. To do this, the function of iNOS was inhibited using AG in the drinking water at $100 \mathrm{mM}$, the same concentration our lab has previously demonstrated to inhibit NO production in EAE mice (Keating et al., 2009). However, since suppressing iNOS activity has previously been shown to affect the induction of EAE (Okuda et al., 1998), we chose to use AG after EAE onset by treating mice with AG from day 12 onwards. Mice that received AG treatment developed EAE at the same time as control EAE mice and with a similar disease severity, indicating that inhibiting iNOS at this stage in disease did not affect the progression of EAE (Figure 5-8A). Mice that received both MIS416 and AG treatment had significantly reduced disease symptoms compared to AG-treated EAE mice (Figure 5-8B). These results suggest that NO may not play a significant role in MIS416-mediated disease given that MIS416 treatment was still effective at reducing disease burden in the absence of NO. However, as AG treatment was not given until day 12, we can only conclude that NO was not a key part of MIS416 mechanism of action in EAE after disease onset. Additionally, it is possible that MIS416-induced NO may play a role in disease reduction in the earlier stages of EAE, particularly since T cell proliferation in EAE mice is thought to occur earlier than day 12, and our work has shown that in vitro MIS416-induced NO acts as a suppressor of $\mathrm{T}$ cell proliferation (Figure 5-7).

In addition to disease parameters, the peripheral immune changes in AG-treated EAE mice were also assessed. AG treatment appeared to have minimal effect on the increased spleen and liver counts with MIS416 treatment (Figure 5-8C\&D) although it did appear that spleen and liver counts of MIS416-treated AG-treated mice were not increased to the same extent as MIS416treated mice without AG treatment, suggesting that NO may play a small role in increased cell number with MIS416 treatment. NO inhibition by administration of AG in the drinking water was confirmed by assessing serum NO levels, and it was found that AG-treated mice had very low levels of NO regardless of MIS416 treatment, proving that the lack of observed disease protection was not due to ineffective or inadequate-AG treatment (Figure 5-8E).

The assessment of liver and splenic cellular populations showed that AG treatment did not affect the trends seen with MIS416 treatment. The proportion of macrophages within the spleen was increased with MIS416 treatment in both AG and non-AG treated EAE mice and this trend was 
consistent in both the spleen and liver. Similarly, the increased percentage of Tregs in the liver but not spleen was apparent in MIS416-treated mice regardless of AG treatment (Figure 5-9A\&B). This was also the case with the activation markers PD-L1 and CD14, which were assessed on myeloid cells within the spleen and liver, and the same trend of increased cellular activation was seen with MIS416 treatment, regardless of whether these mice received AG treatment (Figure 5-9C\&D).

The culture supernatants of splenocytes re-stimulated with MOG and MIS416 with and without additional AG in the culture were also assessed. NO production by splenocytes re-stimulated with MOG was very low, and the addition of AG in MOG cultures diminished any NO produced. Surprisingly, the addition of AG in the MIS416 re-stimulation culture did not completely abolish the NO production by MIS416-treated splenocytes, although the NO levels were much lower than without the addition of AG (Figure 5-10A). Supernatant NO levels from this experiment show that AG treatment in vivo did not significantly alter the ability of splenocytes to produce NO ex vivo in response to a strong NO-inducing stimulus like MIS416, and also that giving AG in vitro did not completely suppress NO production by splenocytes re-stimulated with MIS416 when the splenocytes were from MIS416-treated mice.

In contrast to the lack of disease suppression seen when EAE mice were treated with AG at day 12 , in vivo AG treatment reduced the ability of splenocytes to produce IL-17A in response to MOG at day 22, indicating a reduced EAE-specific Th17 response (Figure 5-10B). Furthermore, as previously seen in Chapter 4, splenocytes from MIS416-treated mice also had a significantly reduced MOG-specific IL-17A production compared to untreated EAE mice, and this effect was observed regardless of AG treatment (Figure 5-10B).

In contrast to IL-17A and NO, IL-10 and IFN- $\gamma$ production by splenocytes in response to MIS416 and MOG re-stimulation were very similar to the responses seen without AG treatment (Figure 5-10C\&D), suggesting that depleting NO either in vivo or in vitro did not affect these MIS416induced cytokine changes. Taken together, these findings suggest that in the absence of NO, the Th17 but not the Th1 or IL-10 responses were reduced. Moreover, the altered MOG-specific IL$17 \mathrm{~A}, \mathrm{IFN}-\gamma$, and IL-10 production in MIS416-treated mice was not mediated by NO.

Altogether, the results with the AG-treated EAE mice suggest that most of the MIS416-induced immune alterations occurred whether AG was administered or not, and MIS416-induced disease protection was also not altered. Finally, it is important to consider that when AG is given at day 12, the MIS416-induced changes may have already taken place and may not be subject to reversal by suppressing NO production at this later time point. 
A
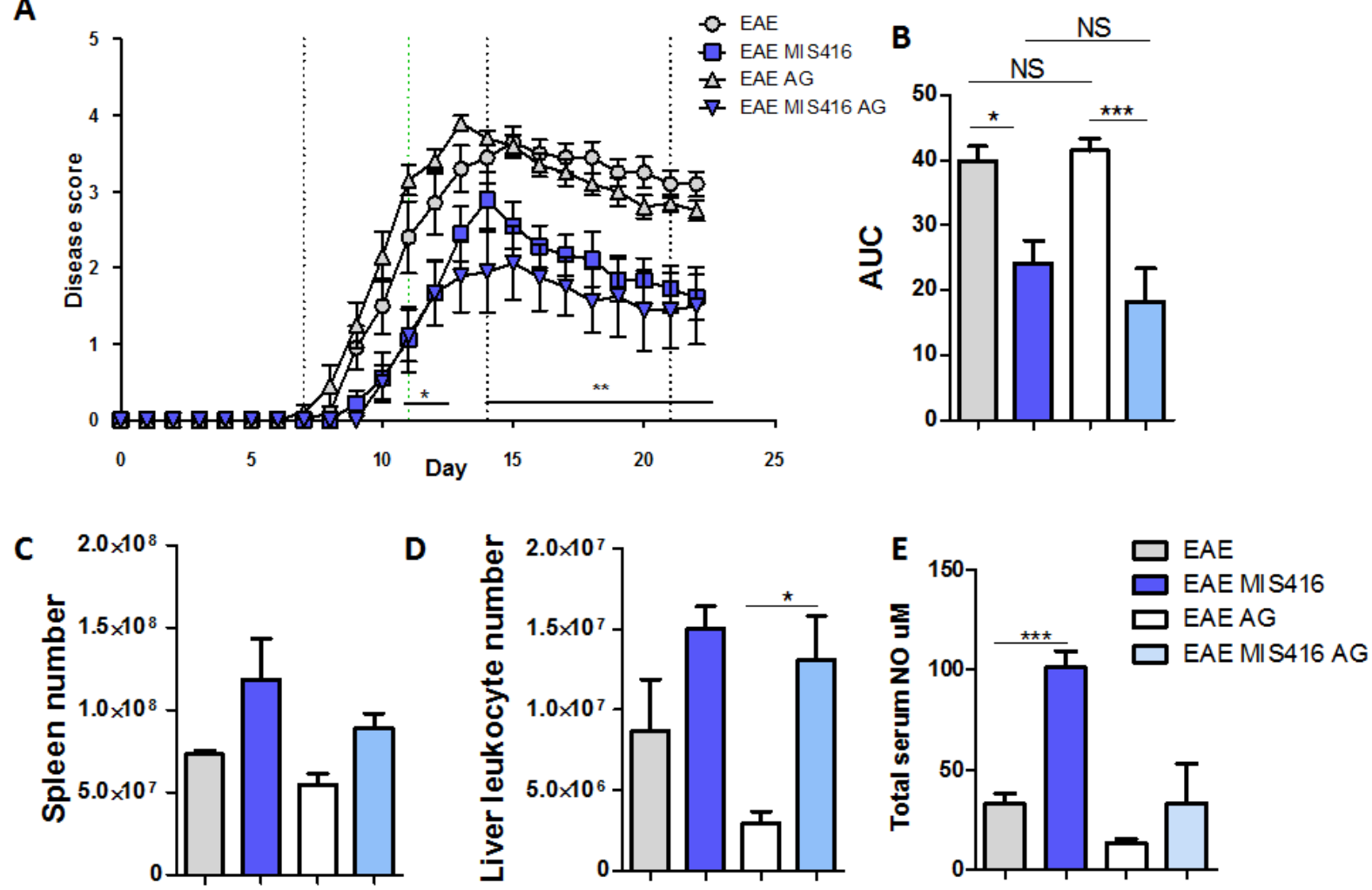

Figure 5-8 Nitric oxide inhibition did not affect EAE disease protection with MIS416 treatment

C57BL/6 mice were immunised to induce EAE and treated with a weekly dose of MIS416 (100 $\mu \mathrm{g} / \mathrm{mouse})$, with half of the mice receiving aminoguanidine (AG) treatment in the drinking water $(100 \mathrm{mM})$ at day 12 , and mice were weighed and scored daily. Serum, spleen and liver were taken at euthanisation (day 22) and serum NO was detected using ENZO life science kit. A) MIS416-treated mice that received aminoguanidine treatment still showed protection from EAE. B) Area under the curve of disease score showed MIS416 treatment was still effective with NO inhibition. C) MIS416-treated mice that received aminoguanidine treatment had a reduced increase in spleen count compared to controls. D) MIS416-treated mice which received aminoguanidine treatment had similar increase in liver count compared to controls. E) Serum NO concentration. Shown are the means \pm SEM of disease scores from 2 independent experiments ( $n=10$ /group), and spleen and liver counts from 1 experiment $(\mathrm{n}=5)$, and serum NO levels from 1 experiment $(\mathrm{n}=4-5) * \mathrm{p}<0.05, * * \mathrm{p}<0.01$ and $* * * \mathrm{p}<0.001$ MIS EAE mice compared to EAE and EAE AG mice compared to EAE MIS416 AG by 2-way ANOVA with Bonferroni's multiple comparison post-test (A) or 1-way ANOVA with Tukey's post-test (B-F). 

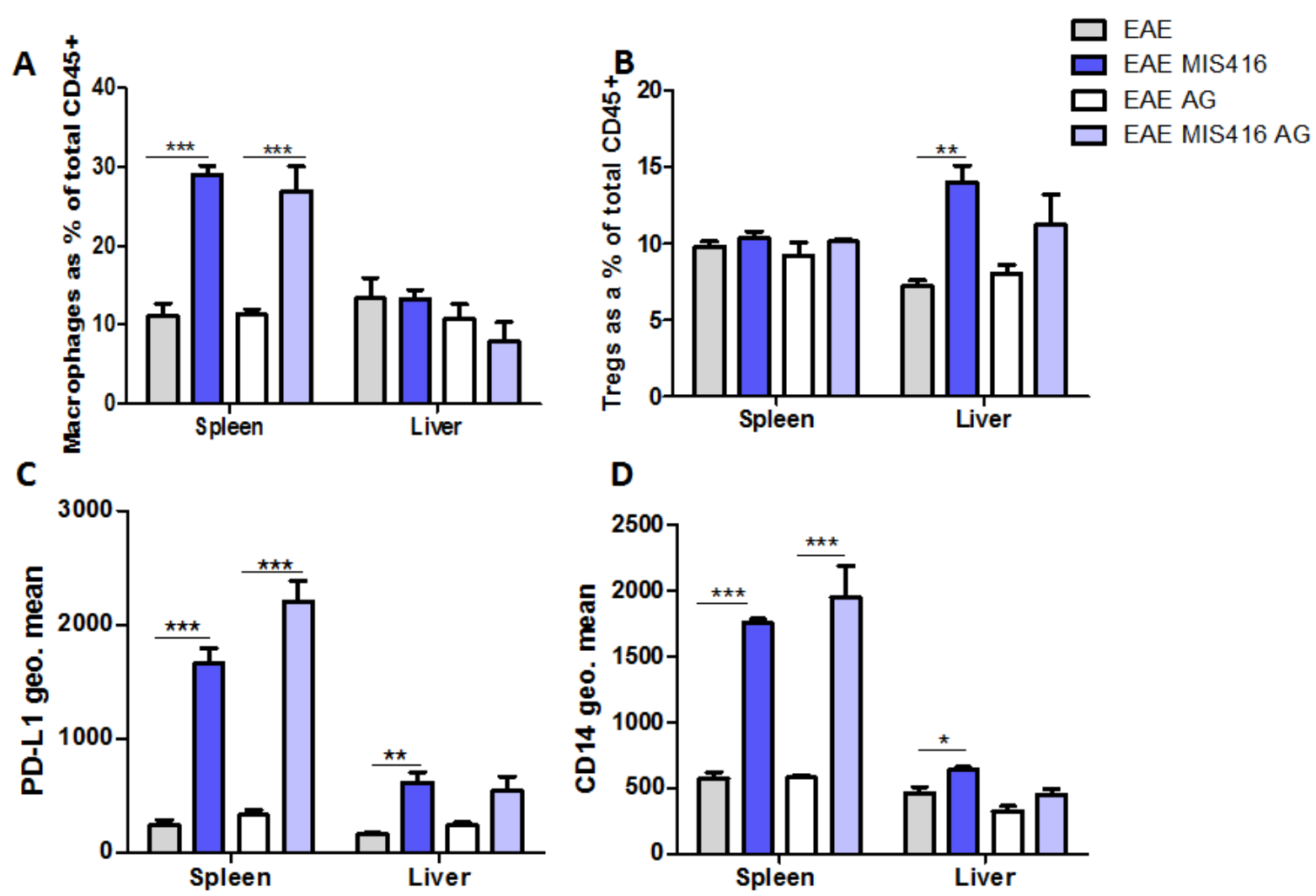

Figure 5-9 NO inhibition did not significantly alter the cellular composition and activation of cells in MIS416-treated EAE mice.

C57BL/6 mice were immunised to induce EAE and treated with a weekly dose of MIS416 (100 $\mu \mathrm{g} / \mathrm{mouse}$ ), with half of the mice receiving aminoguanidine treatment in the drinking water $(100 \mathrm{mM})$ from day 12 onwards. Splenocyte and liver cells were isolated on day 22 and immunophenotyped using flow cytometry. A) Splenic macrophages (F4/80+) were increased as a percentage whilst liver macrophages remained unchanged. B) Liver but not splenic Treg proportions were increased by MIS416 treatment. C) Myeloid (CD11b+) PD-L1 expression was up-regulated in MIS416 treated mice. D) Myeloid (CD11b+) CD14 expression was up-regulated in MIS416treated mice. Shown are the means \pm SEM from 1 experiment (n=5/group), ${ }^{*} \mathrm{p}<0.05, * * \mathrm{p}<0.01$ and $* * * \mathrm{p}<0.001$ MIS EAE mice were compared to EAE and EAE AG mice were compared to EAE MIS416 AG by 1-way ANOVA with Bonferroni's post-test. 
A

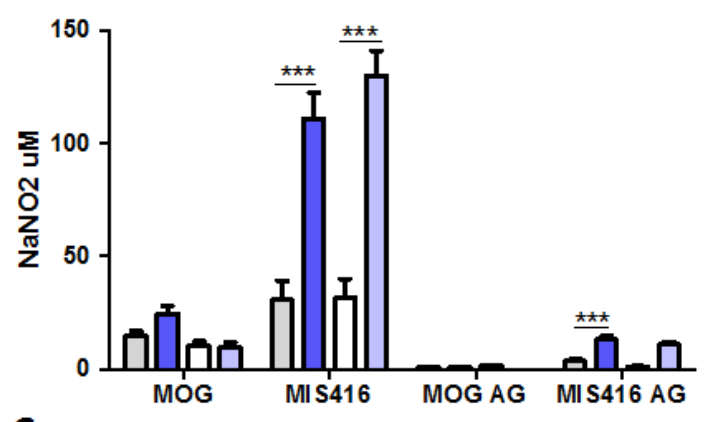

C

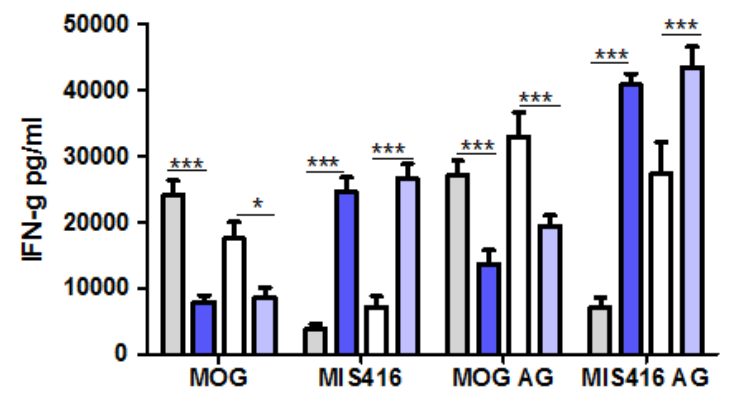

B
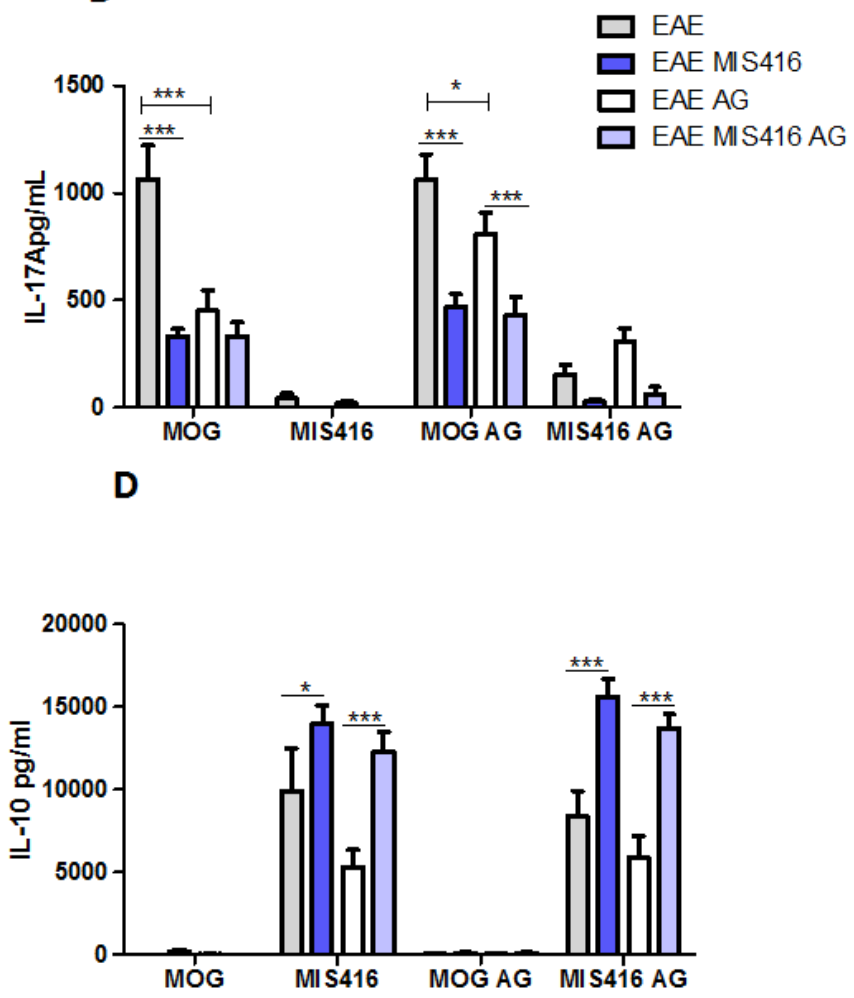

Figure 5-10 Cytokine production in AG treated mice was not significantly altered compared to controls C57BL/6 mice were immunised to induce EAE and treated with a weekly dose of MIS416 (100 $\mu \mathrm{g} / \mathrm{mouse})$, with half of the mice receiving aminoguanidine treatment in the drinking water $(100 \mathrm{mM})$ from day 12 onwards. Splenocytes were isolated and cultured with MOG $(27 \mu \mathrm{g} / \mathrm{ml})$, MIS416 $(20 \mu \mathrm{g} / \mathrm{ml})$ with and without the addition of AG (200 mM) for 72 hours and supernatants analysed by Griess (A) or ELISA (B-D). A) NO production. B) IL-17A production. C) IFN- $\gamma$ production. D) IL-10 production. Shown are the means \pm SEM from 1 experiment ( $\mathrm{n}=5$ /group), $* \mathrm{p}<0.05$ and $* * * \mathrm{p}<0.001$ MIS EAE mice were compared to EAE and EAE AG mice were compared to EAE MIS416 AG, unless indicated otherwise by 2-way ANOVA with Bonferroni's post-test. 


\subsubsection{MIS416-mediated effects in non-EAE mice were preserved when nitric oxide production was inhibited}

As AG treatment did not alter the disease protection provided by MIS416, we aimed to determine what effect the MIS416-induced NO had on the peripheral immune system in the absence of EAE. By treating mice with AG 1 day before weekly MIS416 treatment began, we determined the effect of NO on leukocyte populations and cytokine production. MIS416-treated AG-treated mice showed an increase in total spleen cell number and Treg count although not to the same extent as MIS416-treated mice without AG (Figure 5-11A\&B). These results suggest that MIS416-induced NO production may be involved in the increased number of cells within the spleens of MIS416treated mice. Also, AG treatment had no effect on the proportion $\mathrm{T}$ cell populations within the spleens of MIS416-treated mice; however, MIS416-treated mice receiving AG had an increase the proportion of myeloid cells (i.e. macrophages and dendritic cells) and these levels were elevated above those in MIS416-treated mice without AG treatment. Interestingly, the proportion of NK cells was decreased with MIS416 treatment and although not significant this trend was also present with both AG and MIS416 treatment. Furthermore, given that the macrophage proportion in MIS416-treated mice receiving AG was elevated above that seen with MIS416 alone, it was surprising we did not identify a population which was significantly decreased with AG treatment (Figure 5-11D\&E). Despite the increase in proportion, AG treatment did not affect the activation state of myeloid cells with MIS416 treatment, for there were similar levels of PD-L1 expression on both MIS416-treated mice regardless of AG treatment (Figure 5-11C). While this experiment had low numbers, these results are a representation of 2 experiments showing the same result therefore indicating that by inhibiting the production of NO with AG treatment did not abolish MIS416induced increased spleen count, elevated macrophage number or increased expression of PD-L1.

The pattern of cytokines produced by re-stimulated splenocytes from non-EAE mice that received AG treatment in vivo were very similar to untreated controls, and similar to the response seen in the EAE AG-treated experiments (Figure 5-10\&Figure 5-12). For example, splenocytes from MIS416-treated mice produced high levels of IFN- $\gamma$ in response to MIS416 re-stimulation regardless of whether they were AG-treated or not indicating in vivo AG-treatment did not affect this recall response. Similarly, IL-10 and NO produced in response to MIS416 and ConA restimulation of splenocytes from AG-treated mice had a similar profile to untreated mice. However, in contrast the ConA-induced IL-17A response was enhanced with in vivo AG treatment in untreated mice but not the MIS416-treated mice. While this difference may indicate the IL-17A response was dominant when $\mathrm{NO}$ was not present in vivo, it is difficult to explain, in light of the fact that we saw the opposite effect in MOG stimulated splenocytes from AG-treated EAE mice compared to untreated mice. 
Finally, since we observed only modest effects on the MIS416-induced peripheral changes by AG, we assessed whether the dose of AG was able to completely inhibit NO production induced by MIS416 in vivo. Therefore we measured the total serum NO levels in mice treated with $100 \mathrm{mM}$ AG and $2.5 \%$ AG (which is just over $200 \mathrm{mM}$ ) with and without MIS416 (Figure 5-13). We found only minimal levels of serum NO with both the high and low dose of AG regardless of whether the mice were MIS416-treated or not. Additionally, using this higher dose of AG led to similar immune alterations as the lower dose (data not shown). To conclude, NO was not found to mediate the peripheral immune changes induced by MIS416. 

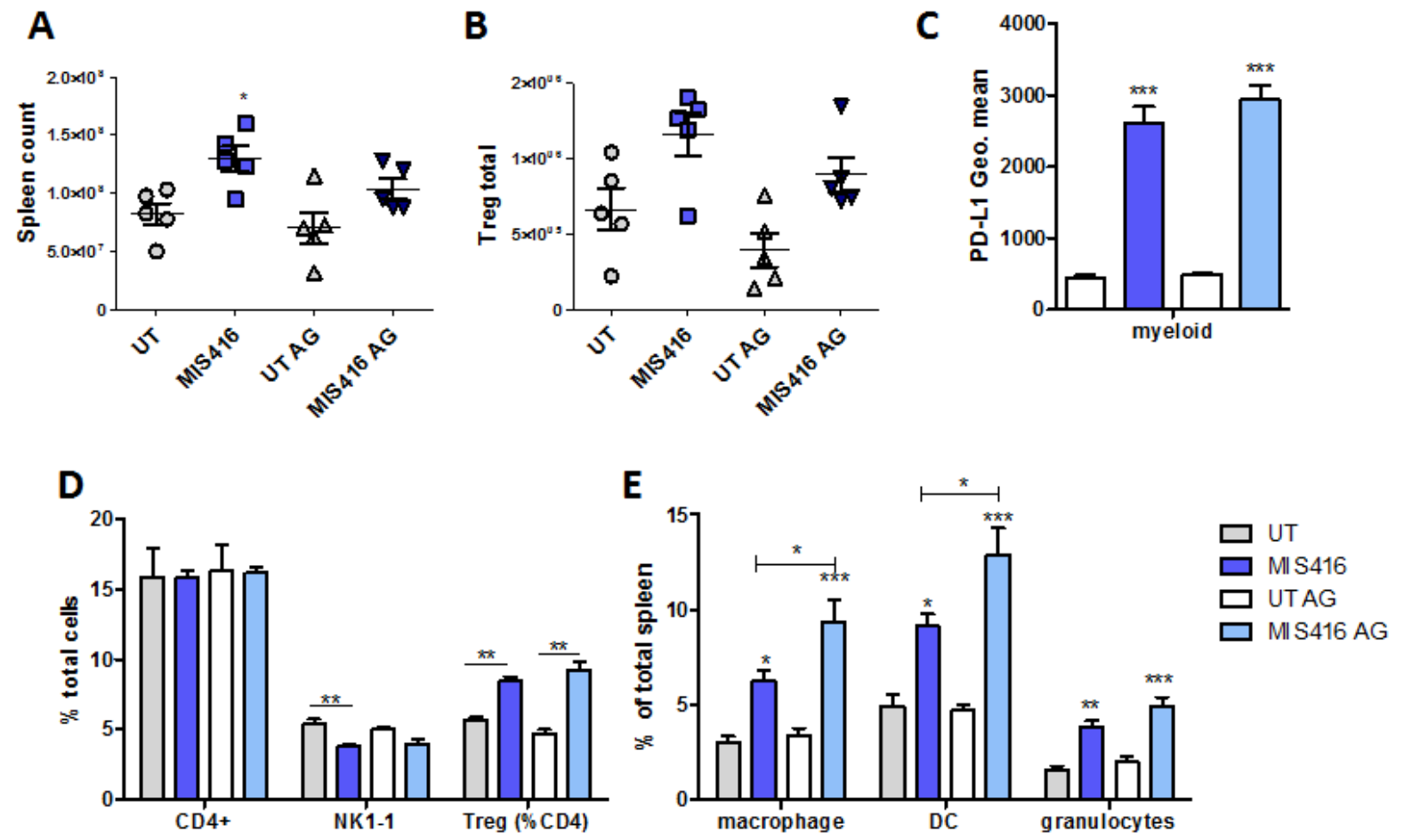

Figure 5-11 AG treatment in non-EAE mice did not abolish MIS416-induced changes

C57BL/6 mice were treated with a weekly dose of MIS416 (100 $\mu \mathrm{g} / \mathrm{mouse})$ and with half of the mice receiving aminoguanidine in the drinking water $(100 \mathrm{mM})$ from day -1 onwards. Splenocytes were isolated and analysed using flow cytometry. A) Spleen count. B) Treg count within the spleen. C) Percentage of CD4+, NK and Tregs within the spleen. D) Percentage of macrophages (CD11b+F4/80+), DCs (single positive CD11C-expressing cells may include an activated macrophage population) and granulocytes (CD11b+GR-1+) within the spleen. E) PDL1 expression on myeloid cells (CD11b+). This is a representative of two experiments, shown are the means \pm SEM of one experiment (n=5/group), ${ }^{*} \mathrm{p}<0.05, * * \mathrm{p}<0.01$ and $* * * \mathrm{p}<0.001$ MIS416 compared to UT and UT AG compared to MIS416 AG, unless indicated otherwise, by 1-way ANOVA with Bonferroni’s post-test. 
A

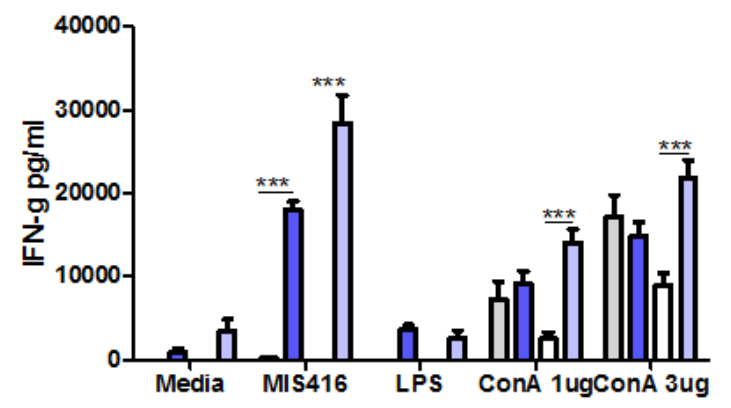

C

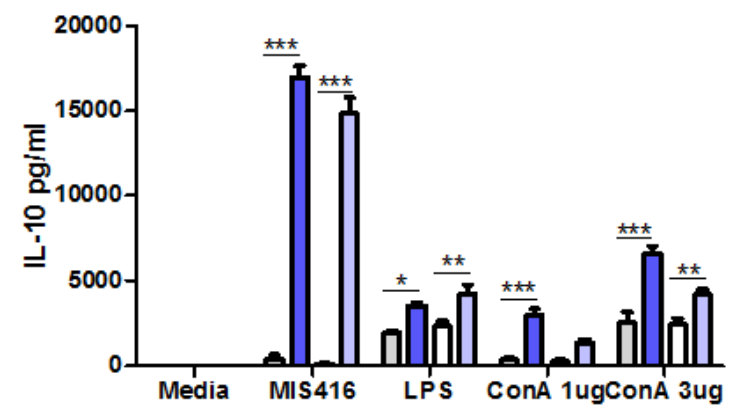

B

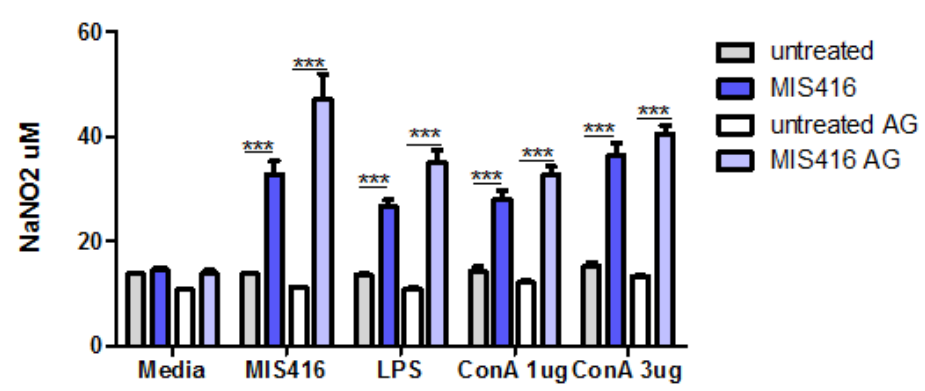

D

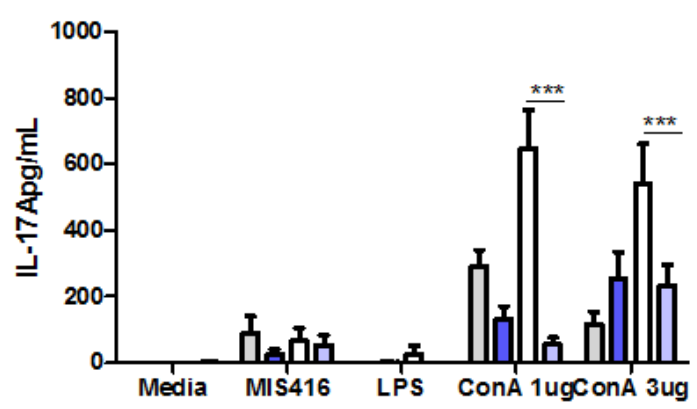

Figure 5-12 AG treatment in non-EAE mice did not abolish MIS416-induced cytokines.

C57BL/6 mice treated with a weekly dose of MIS416 (100 $\mu \mathrm{g} /$ mouse) and half of the mice receiving aminoguanidine in the drinking water $(100 \mathrm{mM})$ from day -1 onwards. Splenocytes were isolated and cultured with LPS $(200 \mathrm{ng} / \mathrm{ml})$, ConA (1 or $3 \mu \mathrm{g} / \mathrm{ml})$ or MIS416 $(20 \mu \mathrm{g} / \mathrm{ml})$ with and without the addition of AG (200 $\mathrm{mM}$ ) for 48 hours and supernatants analysed for cytokines using ELISA (A,C \& D) or Griess (B). A) IFN- $\gamma$ production. B) NO production. C) IL-10 production. D) IL-17A production. This is a representative of two experiments, shown are the means \pm SEM of one experiment. Shown are the means \pm SEM from 1 experiment ( $\mathrm{n}=5$ /group), $* \mathrm{p}<0.05$ and $* * * \mathrm{p}<0.001$ MIS416 mice were compared to UT and AG mice were compared to MIS416 AG by 2-way ANOVA with Bonferroni’s post-test. 


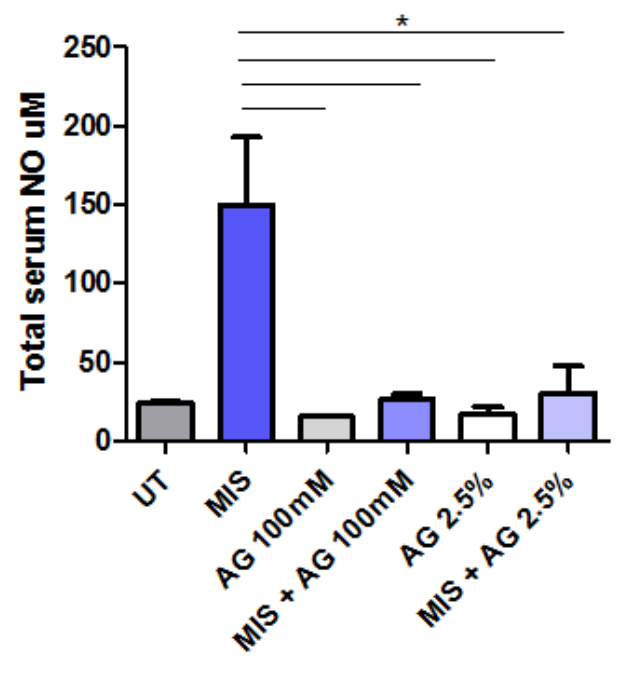

Figure 5-13 Serum NO levels were suppressed by aminoguanidine

C57BL/6 mice treated with a weekly dose of MIS416 (100 $\mu \mathrm{g} / \mathrm{mouse})$ and aminoguanidine in the drinking water $(100 \mathrm{mM}$ or $2.5 \%$, which is the equivalent of just over $200 \mathrm{mM})$ from day -1 onwards. Serum was analysed for total NO. Shown are the means \pm SEM of 1 experiment, $n=2-3 /$ treatment, ${ }^{*} \mathrm{p}<0.051$-way ANOVA with Tukey's post-test. 


\subsubsection{Tregs from MIS416-treated mice were more suppressive than Tregs from untreated} mice

Because the previous chapter (Chapter 4) demonstrated that MIS416 treatment increased the number of Tregs present in both the liver and spleen, it was important to determine whether this expanded Treg population was functional and thus able to contribute to MIS416-mediated protection in EAE. To do this, splenic Tregs were isolated from MIS416-treated mice using a MACs CD4+CD25+ mouse regulatory isolation kit (Miltenyi Biotec) as per methods, and the purity of the Treg population was determined to be 96-98\% using flow cytometry. Although the FoxP3 expression was not confirmed the suppressive nature of these cells were confirmed by the functional assay. Tregs were co-cultured in the presence of CFSE labelled CD4+ cells from untreated mice (Dynabeads Untouched mouse CD4 Kit; Life Technologies, USA) and CD3/CD28 stimulator beads (see Appendix 2). Because functional Tregs are able to suppress CD4+ T cell proliferation in a dose dependent manner (Kruisbeek et al., 2004), we assessed whether Tregs isolated from MIS416-treated mice could dose-dependently suppress CD4+ T cell responses.

Interestingly, the Tregs isolated from MIS416-treated mice were more suppressive than the Tregs isolated from untreated mice (Figure 5-14). Proliferation was determined using several different measurements and the Tregs from MIS416-treated mice showed the greatest effect on the proliferation index (Figure 5-14B) compared to the division index and percentage divided (Figure 5-14A\&C). The proliferation index in the FlowJo platform is calculated by the total number of divisions divided by the number of cells which underwent division, whereas the division index is the average number of cell divisions which occur by a cell in the original population including cells which never divided. Therefore, these results suggest that MIS416 treatment induced Tregs to be more suppressive overall and suppress or slow down the proliferation of cells which had already begun dividing.

Since MIS416-induced NO production was shown to be a contributing factor to the suppression of $\mathrm{T}$ cell proliferation in splenocyte cultures of MIS416-treated mice, the assessment of NO in the Treg assay was used to determine if NO contributed to Treg-mediated suppression. However, no detectable NO was found within in the Treg cultures indicating that the suppression observed in the Treg assay was not mediated by NO (data not shown). We also assessed the PD-L1 expression on cells isolated from MIS416-treated mice and found higher levels of PD-L1 expression on Tregs, as well as CD4+ and CD8+ cells, compared to untreated mice (Figure 5-15A). In addition, the assessment of PD-1, the receptor for PD-L1, was also assessed to ensure that high levels of the ligand could result in interaction with its receptor on activated CD4+ T cells. It was found that PD1 expression was slightly but not significantly increased by MIS416 treatment (Figure 5-15B), 
suggesting that the PD-L1 ligand was expressed and able to interact with its receptor to promote suppression. Unlike the results from freshly isolated Tregs, the PD-L1 expression on Tregs at the end of the suppression assay was similar between both MIS416-treated Tregs and untreated Tregs and was much higher than on freshly isolated Tregs (data not shown). Together these results show that MIS416-treated Tregs were functionally suppressive in vitro, and this suppression was not NO-dependent but may have involved PD-L1. 

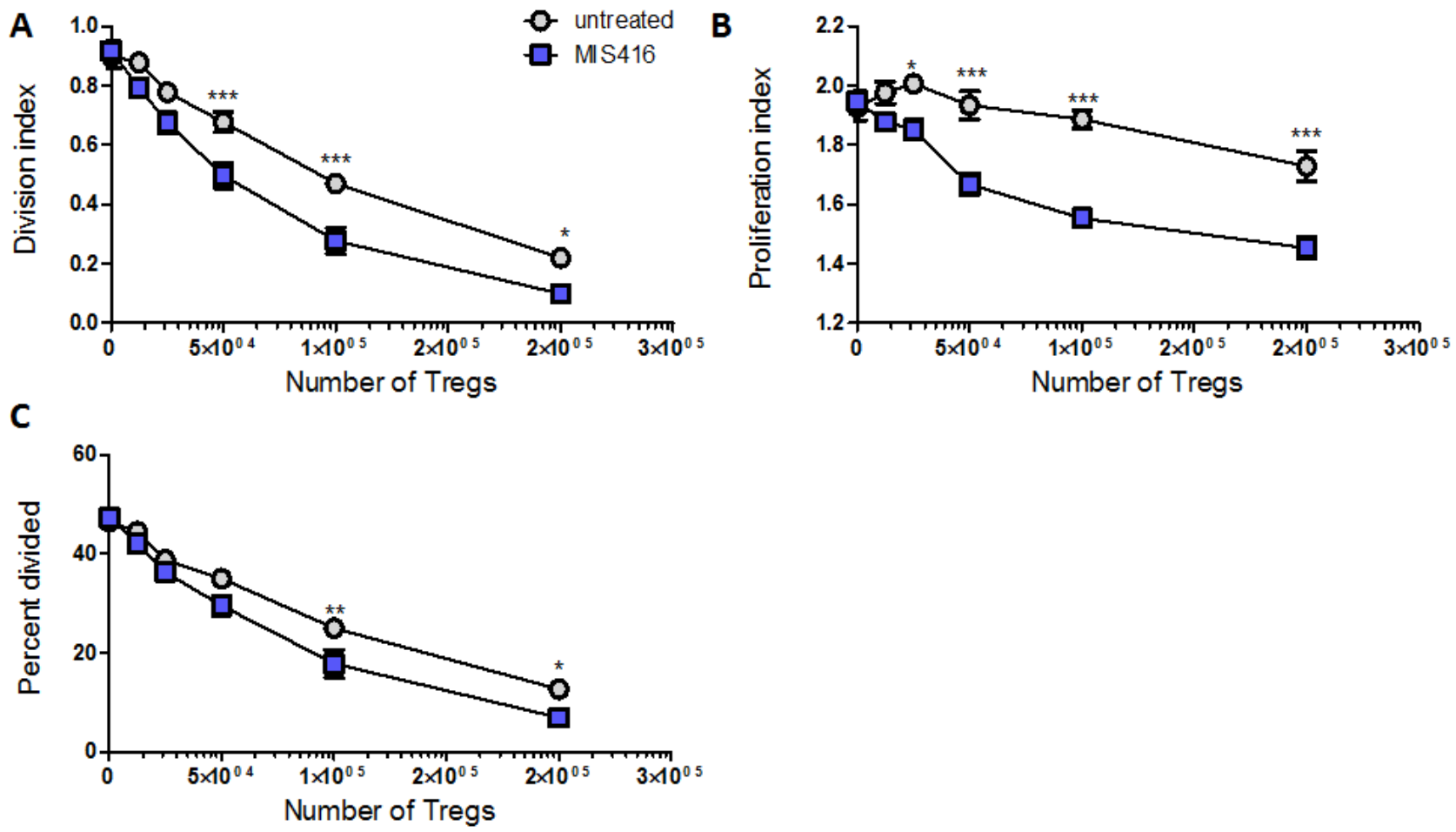

Figure 5-14 Tregs isolated from MIS416-treated mice were more suppressive than Tregs from untreated mice.

C57BL/6 mice were treated with a weekly dose of MIS416 (100 $\mu \mathrm{g} / \mathrm{mouse})$. Splenic Tregs were isolated at day 15, 1 day post MIS416 treatment and co-cultured at increasing concentrations with $1 \times 10^{5}$ CFSE-labelled CD4+ T cells isolated from untreated mice, and stimulated with $2.5 \times 10^{4} \mathrm{CD} 3 / \mathrm{CD} 28$ beads. A) Division index. B) Proliferation index. C) Percentage of CD4+ T cell which divided. Shown are the replicate means \pm SEM of 2 experiments in which spleens from 2 mice were pooled for each experiment $(\mathrm{n}=2) * \mathrm{p}<0.05,{ }^{*} \mathrm{p}<0.01$ and $* * * p<0.001$ by 2 -way ANOVA with Bonferroni’s multiple comparison post-test. 

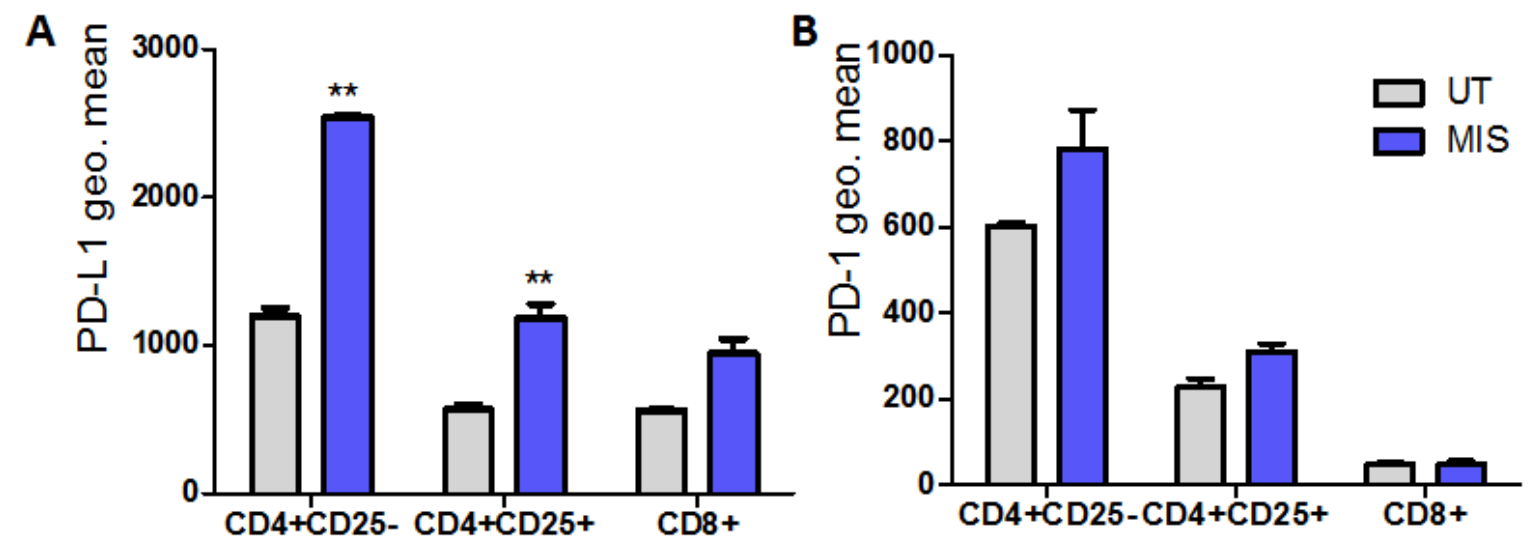

Figure 5-15 PD-L1 and PD-1 expression was increased on T cell populations

C57BL/6 mice were treated with a weekly dose of MIS416 (100 $\mu \mathrm{g} / \mathrm{mouse})$ and splenocytes were harvested at day 15, 1 day post MIS416 treatment. T cells were assessed for surface expression of PD-L1 and PD-1 using flow cytometry. A) PD-L1 expression level on various T cell populations. B) PD-1 expression on various T cell populations. Shown are the means \pm SEM of 1 experiment $(n=2-3) . * * p<0.01$ by unpaired students t-test. 
5.3.8 CD4+ T cells isolated from MIS416-treated mice responded similarly to CD3/CD28 activator beads

Based upon the results from the Treg suppression assay, the proliferation of the whole CD4+ population (i.e. without the removal of CD4+CD25+ Tregs) was assessed to determine if MIS416 treatment altered the overall CD4+ T cell environment. Similar to the Treg assay, the proliferation index was significantly lower in CD4+ T cells from MIS416-treated mice compared to untreated mice (Figure 5-16A). However, the division index and percentage of divided cells were significantly higher in CD4 $\mathrm{T}$ cells from MIS416-treated compared to CD4+ $\mathrm{T}$ cells from untreated mice (Figure 5-16B\&C). These results suggested that while MIS416 treatment reduced the rate of proliferation in these $\mathrm{CD} 4 \mathrm{~T}$ cells as indicated by the proliferation index, it also increased the number of cells which underwent division as indicated by the division index and percentage of divided cells.

Even though the CD4+ T cells isolated from MIS416-treated mice will contain a Treg population which we defined as more suppressive than Tregs isolated from untreated mice (Figure 5-14), it is likely the proportion of Tregs was small too small to have an effect in this particular assay. Therefore, from these mixed results we believe that CD4+ T cells isolated from MIS416-treated mice had the ability to proliferate at the same capacity as CD4+ T cells from untreated mice, and while MIS416-treated T cells had a reduced rate of proliferation, the ability to proliferate was not reduced. 
A

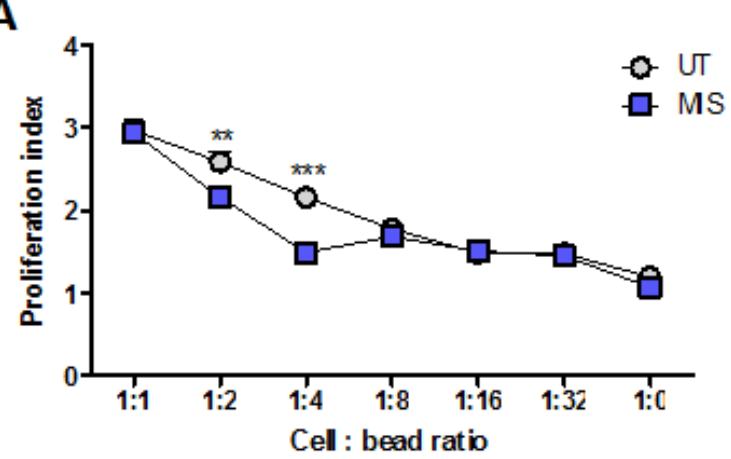

C

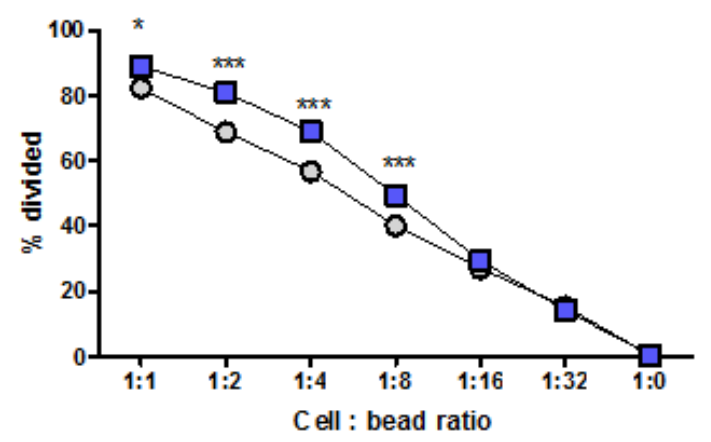

B

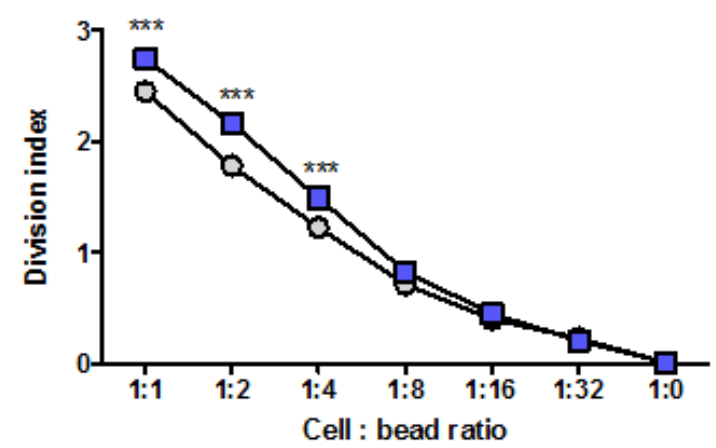

Figure 5-16 CD4+ T cell proliferation was not significantly altered with MIS416 treatment

C57BL/6 mice were treated with a weekly dose of MIS416 (100 $\mu \mathrm{g} /$ mouse $)$ and splenocytes were harvested at day 15,1 day post MIS416 treatment. 1 x10 $0^{5}$ CFSE-labelled CD4+ T cells were isolated and stimulated with 2.5 $\mathrm{x} 10^{4}$ CD3/CD28 beads. A) Proliferation index was higher in untreated mice. B) Division index was higher in MIS416-treated mice. C) The percentage of dividing cells was higher in MIS416-treated mice. Shown are the means \pm SEM of 1 experiment $(n=2-3) . * p<0.05, * * \mathrm{p}<0.01$ and $* * * \mathrm{p}<0.001$ by 2 -way ANOVA with Bonferroni post-test. 


\subsubsection{Myeloid-derived suppressor cells (MDSC) from MIS416-treated mice were more suppressive than control MDSC}

The peripheral changes assessed in Chapter 4 showed that MIS416-treated mice had increased myeloid cell number within the spleen and liver, and some of these myeloid cells expressed markers of MDSC. Therefore, we determined whether the MDSC from MIS416-treated mice had the ability to suppress $\mathrm{T}$ cell responses, and if so, how the level of $\mathrm{T}$ cell suppression compared to MDSC isolated from untreated mice. To do this, the MDSC were isolated using the MACs isolation kit which separates two populations of MDSCs, either granular (Gr-1hi) or monocytic (Gr-1dim) and these populations are characterised by different levels of the MDSC markers Ly6G and Ly6C as shown in Appendix 3. MDSC were added at increasing concentrations to a $\mathrm{T}$ cell proliferation assay, which was similar to that used to assess Treg suppression.

In cultures with the highest concentration of Ly6G+ MDSC (granular), an almost complete suppression of T cell proliferation with either MDSC from MIS416-treated or untreated mice was observed. However, with decreasing concentrations of MDSCs, the cells from MIS416-treated mice appeared to maintain a stronger suppression of $\mathrm{T}$ cell proliferation compared to the MDSC isolated from untreated mice (Figure 5-17A-C). This difference was also observed in the suppression assay using the Gr-1dim (monocytic) MDSC in which the MDSC isolated from MIS416-treated mice were more suppressive than the MDSC from untreated mice (Figure 5-17E\&F). However, the results from the proliferation index indicate that MIS416-treated monocytic MDSC were effective at suppressing T cell responses only at higher concentrations and untreated monocytic MDSCs were not suppressive at any concentrations as indicated by the proliferation index (Figure 5-17D). These results are interesting as the division index and percentage divided parameters indicated a high level of $\mathrm{T}$ cell suppression at all concentrations assessed; however as already mentioned in this chapter, the proliferation index only takes into account the cells which have divided and how much they divided. Therefore, it is possible to have higher levels of proliferation but lower numbers of cells actually dividing.

NO has been suggested as a method by which MDSC suppress T cell responses (Gabrilovich \& Nagaraj, 2009), and thus AG was added to these cultures to suppress iNOS and inhibit NO production. Interestingly, it appeared that suppressing iNOS activity did not alter either monocytic or granulocytic MDSC-mediated T cell suppression by cells isolated from MIS416-treated mice or untreated mice given that the suppression of proliferation remained unchanged with the addition of AG (Figure 5-17).

Altogether, the results from these MDSC suppression assays showed that both monocytic and granulocytic MDSC cultured with CD3/CD28-stimulated CD4+ T cells were able to suppress $\mathrm{T}$ 
cell proliferation in a concentration-dependent manner. Furthermore, MDSCs isolated from MIS416-treated mice were even more suppressive than MDSCs from untreated mice since MDSCs from MIS416-treated mice suppressed $\mathrm{T}$ cell responses at much lower concentrations. We have also shown that the enhanced suppression by MDSCs from MIS416-treated mice was not mediated by NO production. Furthermore, these results indicate that MDSCs may be another mechanism by which MIS416 suppressed $\mathrm{T}$ cell proliferation thus reduced the $\mathrm{T}$ cell-mediated autoimmune disease, EAE. That said, given that these results were only from a single experiment, further repetition is needed to confirm these conclusions. 

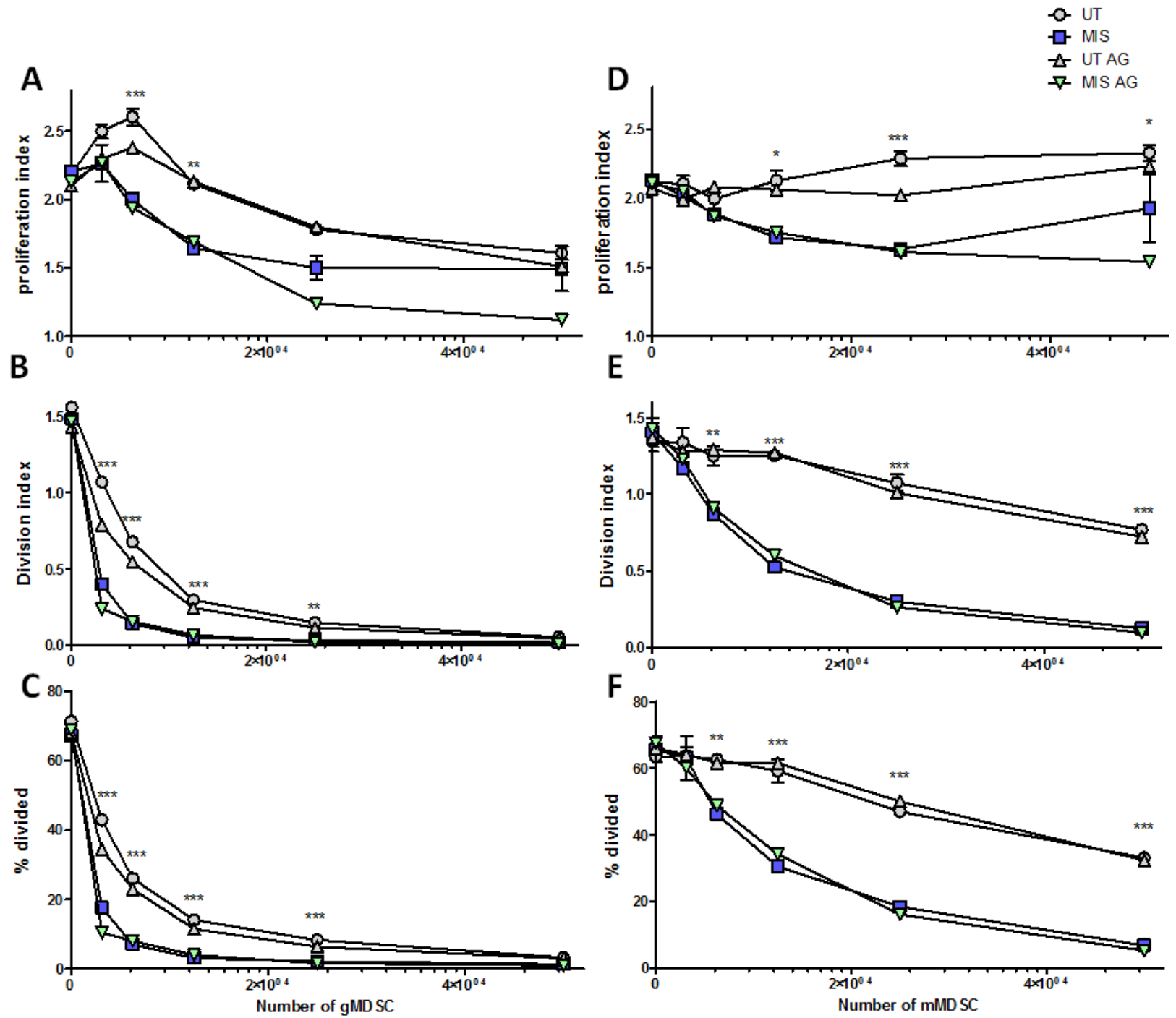

Figure 5-17 MDSC isolated from MIS416-treated mice were more suppressive than control MDSC and this suppression was not mediated by NO

C57BL/6 mice were treated with a weekly dose of MIS416 (100 $\mu \mathrm{g} /$ mouse) and splenocytes were harvested at day 15,1 day post MIS416 treatment. MDSC were isolated from splenocytes and co-cultured at various concentrations with $1 \times 10^{5}$ CFSE-labelled CD4+ T cells, and stimulated with $2.5 \times 10^{4} \mathrm{CD} 3 / \mathrm{CD} 28$ beads, with or without the addition of $200 \mathrm{mM}$ aminoguanidine. The proliferation levels of T cells were assessed in the presence of granular Ly6G+ MDSC (A-C) or monocytic Gr-1dim/Ly6C+ MDSC (D-F). A) Proliferation index with Ly6G+ MDSC. B) Division index with Ly6G+ MDSC. C) Percentage divided with Ly6G+ MDSC. D) Proliferation index with Gr-1dim MDSC. E) Division index with Gr-1dim MDSC. F) Percentage divided with Gr-1dim MDSC. Shown are the duplicate means of experimental replicates \pm SEM of 1 experiment $(n=2$ pooled splenocytes). $* \mathrm{p}<0.05, * * \mathrm{p}<0.01$ and $* * * \mathrm{p}<0.001$ MIS416-treated compared to untreated MDSC by 2-way ANOVA with Bonferroni post-test. 


\subsubsection{MIS416 treatment reduced in vivo proliferation of MOG-specific $T$ cells in EAE immunised mice}

The parameters assessed in this chapter so far have focused on isolating cells which were altered by MIS416 treatment, and analysing the ability of these cell populations to suppress polyclonal CD4+ T cell proliferation in vitro. However, a key part of understanding how MIS416 works is assessing the ability of this treatment to affect $\mathrm{T}$ cell proliferation in vivo. To do this, a model of in vivo proliferation was used (Crume, O'Sullivan, Miller, Northcote, \& La Flamme, 2009). Firstly, as shown in Figure 5-18 the splenocytes and LN cells of 2D2 mice, which express a transgenic MOG-specific TCR (Bettelli et al., 2003), were isolated and transferred to congenic mice as the T cells in this population will expand rapidly in response to EAE immunisation. The cells were assessed for CD4 expression (21\%), and V $\beta 11 / \mathrm{V} \alpha 3.2$ (20.7\%) confirming that these cells had the MOG-specific TCR as expected. After isolation, the cells were labelled with CFSE and transferred into B6.SJL-ptprca mice (i.e. CD45.1+) the day before EAE immunisation and MIS416 treatment. Five days after EAE immunisation, mice were culled and LN, spleen and blood were harvested to assess CD4 proliferation by flow cytometry.

For this analysis both the percentage of cells which proliferated (see Appendix 4) and the number of proliferation events was assessed. In MIS416-treated EAE mice there was a significant reduction in the percentage of cells proliferating in response to EAE immunisation compared to untreated EAE mice and this reduction was found in all organs (Figure 5-19). Additionally, the number of proliferation events was also much lower in MIS416-treated compared to untreated mice. In mice that did not receive EAE immunisation, there was very little proliferation of the transferred CD4+ $\mathrm{T}$ cells indicating that the proliferation was antigen-dependent and was consistent between MIS416-treated and untreated controls (Figure 5-19).

These results show that MIS416 treatment suppressed CD4+ T cell proliferation in vivo in an antigen-specific manner. Taken together, these results indicate that MIS416 suppressed antigenspecific T cell proliferation during the early stages of EAE (i.e. up to day 5), which may suggest a potential mechanism for MIS416-mediated EAE suppression. Interestingly, because the number of proliferation events was also much lower in MIS416-treated mice, as indicated by less 2D2 cells present at day 5, suggests that MIS416 treatment was able to eliminate activated cells through an unidentified mechanism. 

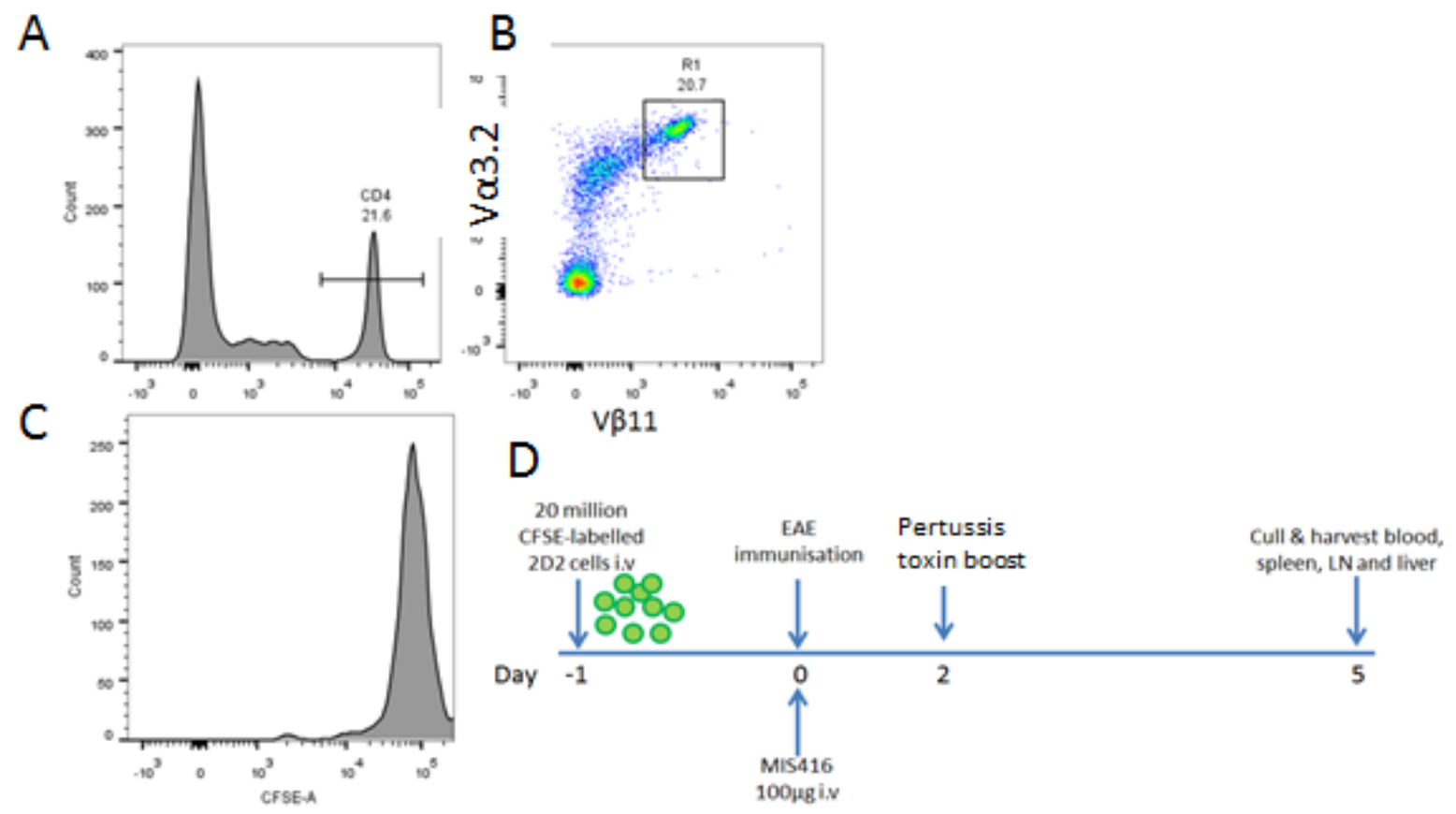

Figure 5-18 in vivo proliferation assay

Splenocytes and LNs were harvested from 2D2 mice and injected i.v. into B6.SJL-ptprca mice. A) Total splenocyte pool contained $21 \%$ of CD4 T cells. B) $20 \%$ of splenocytes from 2D2 mice were expressing both V $\alpha 3.2$ and V $\beta 11$, indicating they were MOG-specific CD4+ T cells. C) Splenocytes were stained for CFSE. D) Protocol for experiment where $20 \times 10^{6} 2 \mathrm{D} 2$ cells were injected i.v into B6.SJL-ptprca at day -1 after which mice received EAE immunisation on day 0 and were treated with $100 \mu \mathrm{g}$ MIS416. Mice were culled at day 5 and proliferation of CFSE labelled 2D2 cells was assessed by flow cytometry. 
A

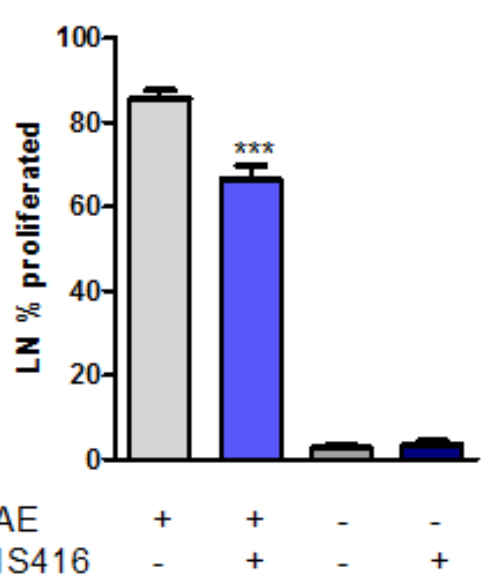

C

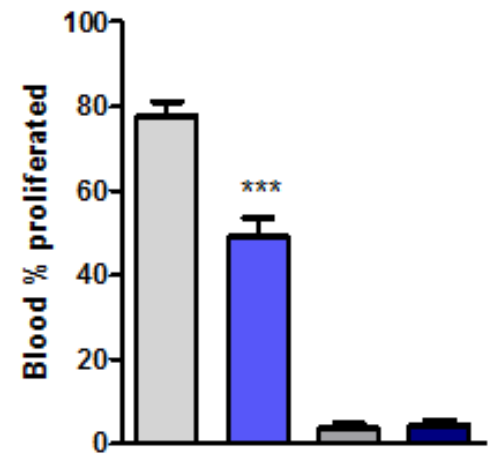

EAE MIS416

$\mathbf{E}$

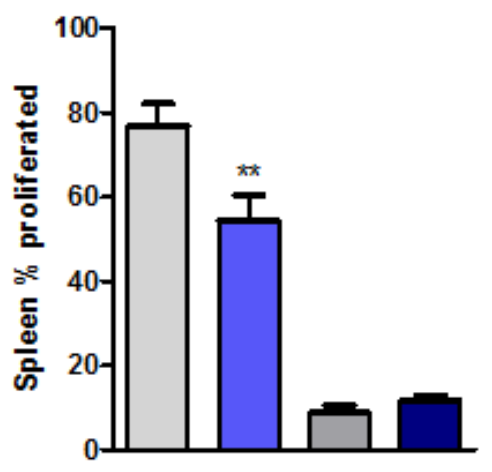

EAE

MIS416
B

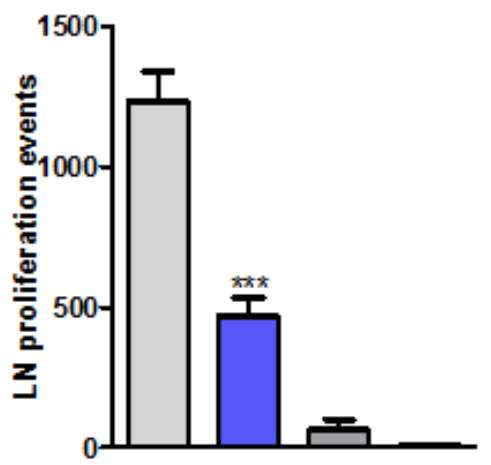

EAE

MIS416

D

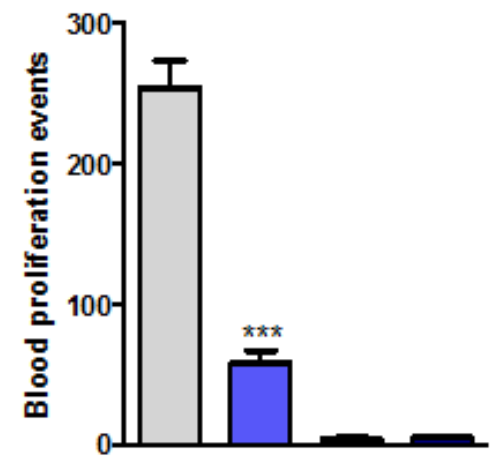

EAE

MIS416 - $+\quad+$

$\mathbf{F}$

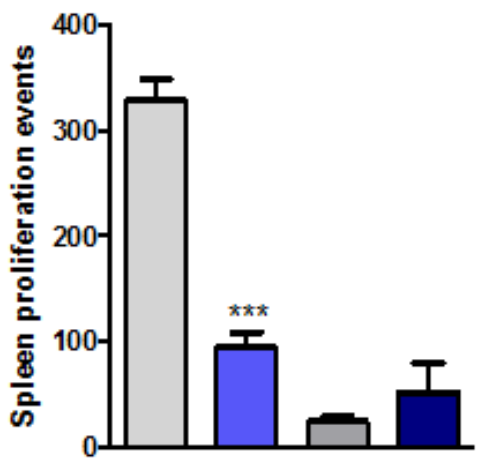

EAE

MIS416

Figure 5-19 MIS416 treatment reduced CD4+ T cell proliferation in vivo in response to EAE immunisation. B6.SJL-ptprca were injected i.v. with $20 \times 10^{6}$ 2D2 cells at day -1 after which mice received EAE immunisation on day 0 and were treated with $100 \mu \mathrm{g}$ MIS416. Mice were culled at day 5 and proliferation of CFSE labelled 2D2 cells was assessed using CD45.2 to distinguish injected cells (see Appendix 4). A) Percentage of proliferating CD4+ cells in the inguinal draining lymph node (dLN). B) Proliferation events in dLN. C) Percentage of proliferating CD4+ cells in the blood. D) Proliferation events in blood. E) Percentage of proliferating CD4+ cells in the spleen. F) Proliferation events in the spleen. Shown are the means \pm SEM of 2 independent experiments ( $\mathrm{n}=8-10$ /group). $\mathrm{p}<0.01$ and $* * * \mathrm{p}<0.001$ MIS416-treated compared to untreated by 1way ANOVA with Tukey's post-test. 


\subsection{Discussion}

The aim of this chapter was to determine if any of the specific immunological changes identified in Chapter 4 were important for MIS416 to exert its disease protective effects in EAE. Because elevations in both NO and IFN- $\gamma$ occurred with MIS416 treatment and these factors have known immunosuppressive activity, we assessed their role by depleting or reducing the level of these factors in MIS416-treated mice. Using IFN- $\gamma^{-/-}$mice, we discovered that MIS416-induced IFN- $\gamma$ was involved in the disease protection provided by MIS416 as in the absence of IFN- $\gamma$, no disease protection was observed. Furthermore, these experiments highlighted the possibility that IFN- $\gamma$ may have driven the production of NO since MIS416-treated IFN- $\gamma^{-/-}$mice produced very little NO, both within the splenocyte cultures and the serum levels. Thus, we demonstrated that the production of NO was dependent on IFN- $\gamma$; however, the inhibition of NO in vivo had very little effect on MIS416-induced EAE disease protection and peripheral immune alterations indicating that $\mathrm{NO}$ was not involved in disease protection. We also determined that splenic Treg and MDSC populations, which were expanded by MIS416 treatment, were able to suppress T cell responses ex vivo. Finally, we also found that MIS416 treatment was able to suppress Ag-specific T cell proliferation in vivo indicating that MIS416 acted as an immune modulator to suppress T cell responses in the periphery, which may contribute to its disease protection in EAE.

Our results clearly implicate IFN- $\gamma$ as a major contributor to MIS416-induced disease protection in EAE, therefore assessing the MIS416-induced immune alterations in the absence of IFN- $\gamma$ helped identify changes that may be central to the mechanism in EAE. The number of Tregs was increased in MIS416-treated IFN- $\gamma^{-/-}$mice, but not significantly, and not to the extent seen in the MIS416-treated WT mice. Therefore, the enhancement of IFN- $\gamma$ production by MIS416 may be contributing to either Treg formation or the induction of CD4 $\mathrm{T}$ cells to Tregs, and therefore in IFN- $\gamma^{-/-}$mice, MIS416 treatment had a reduced capacity to induce the expansion of Tregs. One study suggested that the lack of IFN- $\gamma$-induced Tregs may be one of the reasons why IFN- $\gamma^{-/-}$mice suffer more severe EAE disease (Wang et al., 2006) and therefore the production of IFN- $\gamma$ can have disease limiting effects. In this study by Wang et al they showed that in IFN- $\gamma$ deficient mice Tregs were reduced in frequency and function and were characterised by a decreased FoxP3 expression compared to WT mice. Furthermore, they also determined that the in vitro treatment of CD4+CD25- cells from IFN- $\gamma$ deficient mice with IFN- $\gamma$, allowed the conversion of these cells to functional Tregs as shown by reduced disease when transferred into EAE mice (Wang et al., 2006). It is also possible that IFN- $\gamma$ indirectly drives the formation of Tregs through the increased expression of PD-L1 and NO (Francisco et al., 2009; Niedbala et al., 2007) both of which were increased in MIS416-treated WT mice and not MIS416-treated IFN- $\gamma^{-/-}$mice. Considering that 
MIS416-treated IFN- $\gamma^{-/-}$mice had reduced expansion of Tregs, it is possible that MIS416-induced IFN- $\gamma$ is immunoregulatory through the induction of Tregs, which suppress disease in MIS416treated EAE mice. Moreover, it may be that the in the absence of IFN- $\gamma$ MIS416-induced Treg function may be impaired and therefore functional assessment of Tregs from MIS416-treated IFN$\gamma^{-/-}$mice would be an interesting follow up experiment for the role of IFN- $\gamma$ in Treg formation.

Although the percentages of other lymphocyte populations such as the NK and CD8 cells in MIS416-treated IFN- $\gamma^{-/-}$mice were similar to those in control mice, the substantial increase in F4/80+ macrophages by MIS416 treatment was not seen in MIS416-treated IFN- $\gamma^{-/-}$mice indicating that IFN- $\gamma$ is important in either the proliferation or migration of these cells into the spleen after MIS416 treatment. Furthermore, we found that the F4/80+ cell population was directly correlated to the total spleen number (data not shown) and that this population was either an important event in the mechanism or a strong indicator of the immune changes seen with MIS416 treatment. The role that these F4/80+ macrophages may have in reducing EAE disease is unclear; however, it is possible that these cells are the major producers of NO (Hibbs Jr, Taintor, Vavrin, \& Rachlin, 1988). Therefore, the reduced production of NO in MIS416-treated IFN- $\gamma^{-1-}$ mice may be caused by the lack of expansion of this F4/80+ macrophage population in the absence of IFN- $\gamma$. Furthermore, macrophages are also able to regulate $\mathrm{T}$ cell responses and under the right conditions can induce the transformation of CD4+ T cells into Tregs (Kurotaki et al., 2011), which together suggest that the lower Treg numbers in MIS416-treated IFN- $\gamma^{-/}$mice may be due to a lack of IFN$\gamma$-induced expansion of macrophages. Additionally, since the activation state of the macrophages is able to shape an immune response, as seen in the treatment of EAE mice with type II-activated macrophages (Tierney et al., 2009), it may be that the macrophage population contributed significantly to MIS416's mechanism of action in EAE and without IFN- $\gamma$, this population was not primed to provide this protection.

In addition to the lack of expansion of the macrophage population in the absence of IFN- $\gamma$, the myeloid population (including macrophages) from MIS416-treated IFN- $\gamma^{-/-}$mice did not express high levels of PD-L1 and MHC-II like MIS416-treated WT mice suggesting that IFN- $\gamma$ is involved in upregulating these markers. These results are not surprising given that the expression of PD-L1 has been shown to be upregulated by IFN- $\gamma$ (Loke \& Allison, 2003), and MIS416 treatment has been shown to induce high levels of serum IFN- $\gamma$ (White et al., 2014). As mentioned previously, the expression of PD-L1 on the surface of antigen presenting cells such as macrophages and DCs occurs naturally to limit EAE (Carter et al., 2007; Latchman et al., 2004); and PD-L1 binding to the PD-1 receptor on $\mathrm{T}$ cells also suppresses CD4 $\mathrm{T}$ cell proliferation (Shi, Chen, Yang, \& Li, 2013). Therefore the up-regulation of these markers in MIS416-treated mice may be a way in 
which MIS416 exerts its disease protective effects by suppressing antigen-specific $\mathrm{T}$ cell responses.

Another pathway by which PD-L1 expression may be protective in EAE is through the induction of Tregs. Several studies have shown that the expression of PD-L1 on myeloid cells induces the formation of Tregs as well as maintains the suppressive nature of the Treg population (Francisco et al., 2009; Shi et al., 2013). In agreement with these findings, we found that myeloid cells from MIS416-treated mice expressed high levels of PD-L1 and these mice had increased Treg numbers. Moreover, in the absence of IFN- $\gamma$ there was very little PD-L1 expression and Treg expansion was reduced. Therefore, these experiments provide an explanation for how the Treg population may be expanded in MIS416 treatment. Together the results comparing WT and IFN- $\gamma^{-1-}$ mice have highlighted the idea that many of the peripheral immune changes seen with MIS416 treatment are linked together and indicate that IFN- $\gamma$ may be a key contributor to the mechanism of action of MIS416.

As mentioned previously, MIS416 treatment induced high levels of NO within splenocyte cultures treated with MIS416 and also high levels of NO within the serum even days after MIS416 treatment (Chapter 4). However, IFN- $\gamma^{-/-}$mice produced only low levels of NO in response to MIS416 treatment in vitro and in vivo suggesting that IFN- $\gamma$-induced NO could be another way in which IFN- $\gamma$ is reducing disease in MIS416-treated EAE mice. A study by van der Veen et al showed that $\mathrm{T}$ cell-derived IFN- $\gamma$ is required for the expression of iNOS in both the periphery and CNS during EAE, and the production of NO inhibits activated T cells from proliferating (van der Veen et al., 2003). Similar to the response seen by van der Veen et al, we found that the ConAinduced proliferative response in MIS416-treated $\mathrm{IFN}^{-\gamma^{--}}$mice was not significantly altered compared to control IFN- $\gamma^{--}$mice, unlike the reduced proliferation seen in WT MIS416-treated mice compared to WT untreated mice. These results were not surprising given that many of the immunoregulatory changes did not occur without IFN- $\gamma$ present including reduced NO production and reduced expression of PD-L1, both of which are able to suppress T cell responses (Albina et al., 1991; Shi et al., 2013).

Given that MIS416-induced disease suppression was not found in IFN- $\gamma^{-/-}$mice and these mice also produced very little NO compared to MIS416-treated WT mice, the possible contribution of NO to reduced $\mathrm{T}$ cell proliferation and disease protection was directly demonstrated by inhibiting $\mathrm{NO}$ production in vivo and in vitro with $\mathrm{AG}$. We found that while CD4+ T cells within whole splenocyte culture from MIS416-treated mice had a reduced proliferation response to ConA, in the presence of AG the proliferation was partially restored. This finding is consistent with studies which have shown that NO suppresses T cell responses (Albina et al., 1991) and is consistent with 
the high levels of ConA-induced NO produced by splenocytes from MIS416-treated mice compared to untreated mice. We also determined that NO was not responsible for reducing disease burden in MIS416-treated EAE mice by depleting NO in vivo using AG. The AG treatment was started on day 12 post-immunisation, because EAE disease is not able to be consistently induced in the absence of NO (Okuda et al., 1998), and did not alter MIS416-induced disease reduction. However, when AG was used at day -1 in non-EAE mice, it was found that NO may have a role in MIS416-induced migration or proliferation of cells since the effect was reduced by AG treatment.

These results were partly supported by a study in which mice were immunised with Salmonella typhimurium, a bacterium which is likely to stimulate PRR receptors similar to MIS416, which showed markedly suppressed splenocyte proliferation response to mitogens. The investigators concluded that this suppressed proliferation was mediated by activated splenic macrophages and their production of NO (MacFarlane, Schwacha, \& Eisenstein, 1999). They also noted that the spleen of S. typhimurium immunised mice had an influx of macrophage and polymorphonuclear leukocytes and that the depletion of NO in these mice significantly reduced the migration of these cells into the spleen (MacFarlane et al., 1999). This study suggests that NO produced in response to MIS416 treatment may be involved in the recruitment of cells into the spleen, resulting in the enlarged size and increased spleen counts following MIS416 treatment; although AG treatment did not completely abolish the increased cell count in MIS416-treated mice, suggesting another pathway contributes to increased spleen counts in these mice. Furthermore, similar to MacFarlane et al we have established that NO is part of the mechanism by which MIS416 suppressed T cell responses ex vivo; however, as $\mathrm{T}$ cell proliferation occurs during the early stages of EAE disease (i.e. we have shown significant proliferation by day 5), the depletion of NO at an earlier time point in EAE mice would determine if MIS416-induced NO has similar effects on T cell proliferation in vivo.

Our studies in Chapter 4 revealed an expansion of the Treg population with MIS416 treatment. In these early studies the assessment of Tregs was solely done on the expression of CD4+CD25+ and later the expansion of the Treg population was found to include both FoxP3+ and FoxP3 ${ }^{\text {low/- }}$ cells. Within the literature there has been evidence to suggest that both CD4+CD25-FoxP3+ and CD4+CD25+FoxP3- cells are important suppressive cell populations which function similarly to "classic" CD4+CD25+FoxP3+ Tregs. Thus it was important to assess their ability to suppress T cell responses to truly characterise them as regulatory T cells (Vieira et al., 2004). Our studies in this chapter identified that the Tregs (isolated as CD4+CD25+ cells) expanded by MIS416 treatment were able to suppress $\mathrm{T}$ cell proliferation to a higher extent than Tregs isolated from untreated mice. The assessment of PD-L1 expression on the Treg population confirmed that cells 
isolated from MIS416-treated mice had a higher level of this anti-proliferative ligand; and since it has been shown that Tregs can suppress T cells responses through PD-L1 expression (Dilek et al., 2013), this increase in PD-L1 may explain why Tregs isolated from MIS416-treated mice were somehow primed during in vivo MIS416 treatment to express higher levels of PD-L1 and to be more suppressive ex vivo. Further work is required to confirm that this enhanced suppression is indeed mediated by PD-L1.

A second population which was increased in the spleens of MIS416-treated mice was the myeloidderived suppressor cells (MDSC) and in particular, the monocytic MDSC (White et al., 2014). MDSC are thought to have immunosuppressive functions by reducing $\mathrm{T}$ cell responses and were initially identified as a detrimental cell type in cancer immunity by suppressing anti-tumour responses (Talmadge \& Gabrilovich, 2013). More recently, these cells have been targeted as a potential treatment for autoimmune diseases and several studies have shown that adoptive transfer of MDSCs reduces disease severity (Gabrilovich \& Nagaraj, 2009; Slaney, Toker, La Flamme, Backstrom, \& Harper, 2011). For example, CD11b+Ly6C ${ }^{\text {high }}$ myeloid cells which were adoptively transferred into EAE mice showed significantly reduced disease scores compared to control mice (B. Zhu et al., 2007). It has also been shown that an expansion of the MDSC is a natural feedback loop in autoimmune disease and an expansion of this population can be found in the peripheral blood of MS patients (Ioannou et al., 2012).

MDSC within the mouse have been divided into two different types, the granulocytic Ly6G+ cells and the monocytic Ly6C+ cells, both of which are able to suppress $\mathrm{T}$ cell responses, although the exact protective mechanisms of these populations have not been fully identified (Gabrilovich \& Nagaraj, 2009). In this chapter we determined that both Ly6C+ and Ly6G+ MDSC isolated from MIS416-treated mice were able to suppress $\mathrm{T}$ cell responses to a much greater extent than MDSC isolated from untreated mice. Interestingly, we found that the increased suppression in MIS416treated MDSC was not due to NO despite these cells being linked to expressing high levels this factor and MIS416 treatment being an inducer of NO production. Particularly since other groups have shown the Ly6C+ MDSC suppress T cell responses through the iNOS pathway (Raber et al., 2014), it was surprising that the depletion of NO did not affect T cell suppression in these assays. The suppressive response from these cells could be due to the expression of PD-L1 as work in other labs has shown that MDSC can suppress T cell responses through this ligand (Noman et al., 2014) and we know that with MIS416 treatment there is a overexpression of this ligand in myeloid populations.

The MDSC from MIS416-treated mice could also be acting through other known suppressive mechanisms such as increased activity of arginase, increased production of reactive oxygen 
species and peroxynitrite (Gabrilovich \& Nagaraj, 2009). Therefore, future experiments could include the depletion of these factors alongside NO and PD-L1 to further determine the mechanisms responsible for the enhanced $\mathrm{T}$ cell suppression by MDSC from MIS416-treated mice. Finally, another link to how MDSC expansion by MIS416 in vivo may be protective is through the induction of Tregs, as one group has shown that MDSC are able to expand the Treg population (Huang et al., 2006). Moreover, this group showed that in their tumour model MDSC induced the expansion of Tregs through the production of IL-10 and IFN- $\gamma$ (Huang et al., 2006), and we know that both of these cytokines were also upregulated in MIS416 treatment. Therefore, the results from such studies show that many of the mechanisms involved in MIS416 may be interlinked and highlight that IFN- $\gamma$ is likely to be a key player.

Most of the mechanistic studies in this chapter focused on determining whether the cellular changes resulted in functional immunosuppression, but we also wanted to confirm whether MIS416 treatment reduced proliferation of MOG-specific CD4+ T cells in vivo. We found that compared to control mice there was a significant reduction in both the number and percentage of proliferative events in MIS416-treated mice compared to controls. This finding was the first evidence to show that MIS416 treatment was able to directly reduce the antigen-specific proliferation response in vivo, and indicated that MIS416 induced a peripherally acting immunoregulatory state. The exact mechanism behind this immunoregulatory state has not yet been elucidated and as there was both a reduced percentage of proliferation with MIS416 and a reduced number of proliferation events, it is possible that MIS416 induced T cell apoptosis.

Two of the current first line immune-modifying treatments for MS (IFN- $\beta$ and GA) have well characterised peripheral immune changes which act quite differently from each other and our studies suggest that MIS416 has immune modifying actions, some of which are also different from each of them. One of these immune-modifying treatments is IFN- $\beta$, which has a variety of actions including decreased expression IFN- $\gamma$ and molecules necessary for antigen presentation, resulting in a reduced expansion of antigen-specific T cell clones (Dhib-Jalbut \& Marks, 2010; Hussien, Sanna, Söderström, Link, \& Huang, 2001). IFN- $\beta$ has also been shown to increase T cell apoptosis by inhibiting the anti-apoptotic protein cellular FLICE-inhibitory protein (c-FLIP) (Sharief, Semra, Seidi, \& Zoukos, 2001). In contrast to IFN- $\beta$, GA, a random polymer that mimics myelin basic protein (MBP) and has a high affinity for MHC, and when expressed on APC the resulting $\mathrm{T}$ cell activation is Th2/Th3 biased (Rina Aharoni, 2014; Rizvi, Kim, \& Moodie, 2006). It has been noted that Th2/3 differentiated cells produce high levels of IL-10, IL-4 and TGF- $\beta$ which do not drive EAE/MS and may suppress the disease through promoting wound repair (Rina Aharoni, 2014; Yong, 2002). Additionally it has been shown that GA treatment induces type-II activated 
monocytes and promotes Treg formation, both of which are able to suppress EAE disease (Jee et al., 2007b; Weber et al., 2007). MIS416 on the other hand, appears to up-regulate the expression of IFN- $\gamma$ and mediate its affects through this cytokine and like GA, expands suppressive cell population's including Tregs and MDSCs. Therefore, MIS416 appears to exert a unique set of immune-modifying effects indicating its potential as a novel treatment for MS.

\subsubsection{Summary}

The results from this chapter clearly support that IFN- $\gamma$ is a key factor involved in the mechanism of action of MIS416. While there has been evidence to suggest that IFN- $\gamma$ is detrimental in both EAE and MS, it is likely that the context and location of this cytokine is central to its therapeutic effect. Furthermore, we identified a number of ways in which IFN- $\gamma$ may be exerting its effects such as enhancing the number and functionality of Tregs, inducing myeloid cells to produce NO and upregulating suppressive surface ligands such as PD-L1. Further assessment of the disease stage during which these factors are important in EAE disease would be helpful to further understand how MIS416 is exerting its action and ultimately, how MIS416 may be used more effectively. 


\section{Chapter 6: MIS416-driven alterations in the CNS}




\subsection{Introduction}

Multiple Sclerosis (MS) is a disease which is characterised by the breakdown of the blood brain barrier (BBB) allowing the entry of self-reactive CD4+ T cells, which then direct the damage of the myelin sheath surrounding the nerve axons. The continual damage of the BBB contributes to the sustained inflammatory pathology seen in MS, such as the demyelination, edema formation and axonal loss (Martin, Sturzebecher, \& McFarland, 2001). Similar to the outside-in theory of MS, the EAE model is also characterised by the generation of myelin-specific T cells followed by their migration and infiltration into the CNS, leading to subsequent demyelination and inflammatory lesions (C. S. Constantinescu, Farooqi, O'Brien, \& Gran, 2011). Therefore, the EAE model will be useful to help us understand if, and how, an immune modulator can alter trafficking and infiltration into the CNS. Given that MIS416-treated EAE mice had profound changes within the peripheral immune response resulting in reduced EAE disease burden, it was important to also investigate the role MIS416 treatment may have on CNS infiltration and inflammation in EAE.

The CNS has often been considered an immunologically and pharmacologically privileged site as few immune cells or therapeutic compounds are thought to be able to pass through an intact BBB. However, in recent years immune surveillance of the CNS has proven to be very important. For example, natalizumab, an antibody which selectively blocks T lymphocytes from entering the CNS by binding to their $\alpha 4 \beta 1$-integrin proteins and preventing lymphocyte-vascular endothelium interaction, is an effective treatment for relapsing-remitting MS patients (D. H. Miller et al., 2003). However, while the early trials showed natalizumab was safe, it wasn't until larger cohorts of patients were treated it was found that a small number $(0.01 \%)$ of treated patients developed a serious and often fatal CNS disease called progressive multifocal leukoencephalopathy (PML). It is believed that natalizumab blocks the lymphocyte immune surveillance in the CNS and therefore in MS patients who carry the John Cunningham (JC) virus, the virus reactivates and causes PML (Ransohoff, 2005). As we now know that immune surveillance of the CNS is essential, the understanding of the natural structure of the BBB and the migration of cells into the CNS both in a normal and disease setting is key to identifying how a potential treatment for MS is exerting its action.

The barrier to the CNS is made up of the BBB and the blood-cerebrospinal fluid barrier (BCSFB) which are located in the CNS microvessels and the choroid plexus respectively. The migration of activated immune cells into the CNS first involves crossing the BBB or BCSFB and into the cerebro-spinal fluid (CSF), followed by crossing the glia limitans into the CNS parenchyma (Engelhardt, 2006). In a steady state with normal immune surveillance, the cells are maintained in the CSF drained vascular spaces where they can interact with strategically located antigen 
presenting cells in search for their specific antigen (Engelhardt \& Ransohoff, 2012). Furthermore, it has been noted that in EAE, only the entry of immune cells into the CNS parenchyma results in clinical disease as cells which are only found in the perivascular or leptomeningeal CSF-drained spaces do not contribute to disease symptoms (Toft-Hansen et al., 2006). Results from these studies suggest that while the assessment of immune infiltration into the CNS using flow cytometry is a helpful tool, the accompanied histological sections of the brains and spinal cords should also be taken in to consideration as infiltration doesn't always correspond to disease.

Current immune modifying treatments, GA and IFN- $\beta$, are mostly thought to exert their actions in the periphery; however, they do have some suppressive effects at the level of the BBB and CNS. Firstly, IFN- $\beta$ is able to alter some of processes required for immune cell entry into the CNS, including the decreased level of adhesion molecule expression. One study has shown that IFN- $\beta$ can convert VCAM-1 into its soluble form, resulting in activated $\mathrm{T}$ cells interacting with this soluble form of VCAM-1 and therefore reducing their interaction with endothelial cells (Calabresi, Pelfrey, Tranquill, Maloni, \& McFarland, 1997). Other studies have shown that IFN- $\beta$ also decreases the level of the CCR5 receptor and several chemokines (Zang et al., 2001) as well as decreasing the level of MMP-9 produced by activated T cells (Stuve et al., 1996) which together, result in reduced cellular recruitment into the CNS.

Unlike IFN- $\beta$, the effects of GA treatment on the CNS are unclear and it appears that the majority of its immunological effects occur in the periphery (i.e at injection site and draining LN). These peripheral alterations include reduced monocyte reactivity (Weber et al., 2004) and the increased frequency and function of Tregs (Jee et al., 2007a) which ultimately suppresses disease by reducing CNS infiltration. Interestingly, mouse work in the early 2000s reported that GA treatment was able to polarise lymphocyte responses in the periphery, and after disease-induced cellular trafficking there was in an increased number of GA-specific Th2/Th3 cells within the CNS (R. Aharoni et al., 2000). It is believed that upon activation in the CNS, these GA-specific Th2/Th3 cells underwent proliferation and released anti-inflammatory cytokines IL-10 and TGF- $\beta$ (Rina Aharoni, Kayhan, Eilam, Sela, \& Arnon, 2003; Yong, 2002), resulting in bystander suppression within the CNS and therefore reducing EAE disease. More recently, GA has been shown to reduce the infiltration of pro-inflammatory Th17 cells and also induced the expansion or infiltration of Tregs in the CNS of EAE mice at later stages of disease (Aharoni et al., 2010). Together the results from these studies show that GA has immune-mediating effects in the periphery and some which act directly on the CNS to reduce EAE and possibly MS disease.

Given that both GA and IFN- $\beta$ have mechanisms within the periphery and also act directly in the CNS, the evaluation of CNS-specific mechanisms involved in MIS416 treatment is of great 
significance. Previous results in our study have shown that MIS416 results in a wide range of effects on both the innate and adaptive cells (Chapter 4\&5) including increased secreted factors such as NO and IFN- $\gamma$ which, like the effects seen with IFN- $\beta$ treatment, may alter the BBB and receptors involved in cellular trafficking. Interestingly, we have also shown that MIS416 treatment results in an increased number and function of Tregs in the periphery (Chapter $4 \& 5$ ) and, as shown with GA treatment, these Tregs may migrate to the CNS and directly suppress EAE disease (Aharoni et al., 2010). Similar to Tregs, we have shown that MIS416 treatment alters myeloid frequency and activation in the periphery; therefore these cells also have the potential to migrate to the CNS and act in a disease suppressive manner. In conjunction with myeloid activation, it has been shown that MIS416 is taken up by phagocytes (Chapter 4), such as macrophages and dendritic cells, and therefore the migration of MIS416-positves cells (or MIS416 itself) may have direct effects on the CNS.

\subsection{Aims and hypothesis}

Given that MIS416 is injected i.v. and was present in the blood up to 24-hours post-treatment, there is potential for MIS416 to enter and have direct alterations in the CNS, such as being phagocytosed by resident macrophages (microglia) or for MIS416-positive cells to migrate to the CNS. Furthermore, as it has been shown that MIS416 treatment significantly reduced the disease burden of EAE mice (Chapter 3), we hypothesise this reduction of disease also correlates with reduced CNS inflammation and reduced BBB breakdown compared to untreated EAE mice.

\subsubsection{Specific aims}

1. To determine whether MIS416 is able to enter the CNS.

2. To identify whether MIS416-treated EAE mice have a reduced number of immune cells infiltrating into the CNS.

3. To identify if heat-treated MIS416 EAE mice have a similar level of CNS infiltration as regular MIS416-treated mice.

4. To investigate whether MIS416-treated mice have CNS alterations in the absence of EAE.

5. To determine if MIS416 treatment reduces BBB permeability. 


\subsection{Results}

\subsubsection{Trace levels of MIS416-AF488 were found within the brain and spinal cord}

MIS416 is a large particle which cannot freely diffuse across cellular membranes and when injected intravenously deposits can be found within in the blood, liver and to some extent the spleen up to 24 hours post-treatment. Therefore, we investigated whether MIS416-positive cells were present in the CNS. To this end, AF488-labelled MIS416 was injected intravenously into non-EAE mice at a weekly dose of $100 \mu \mathrm{g}$ for 3 doses and the mice were then culled 48 hours after the last dose, at day 16. The gating strategy shown in Figure 6-1A identified cells which are CD45hiCD11b+ and were CNS infiltrating myeloid cells which had the ability to pick up the microparticle. At 48 hours post-treatment it appeared that there were only a few MIS416-positive cells detected in the brain and the spinal cord, although some mice appeared to have more trafficking of these MIS416-positive cells than others as indicated by the yellow stars (Figure 6-1B\&C). The number of MIS416-positve cells entering the CNS appeared to be very low, therefore it is unlikely that the microparticle is able to have a significant direct effect on the CNS resident cells. 


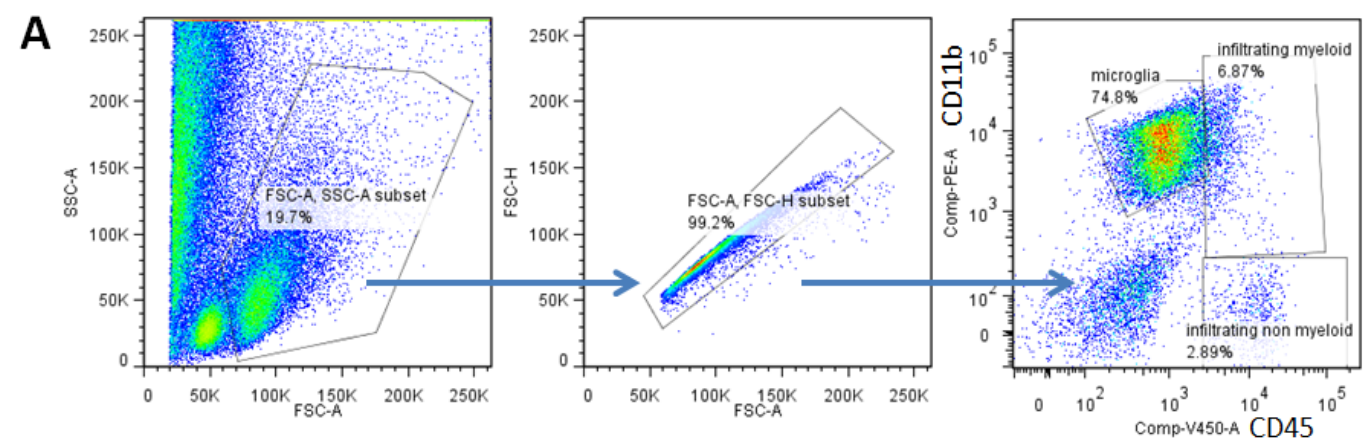

B
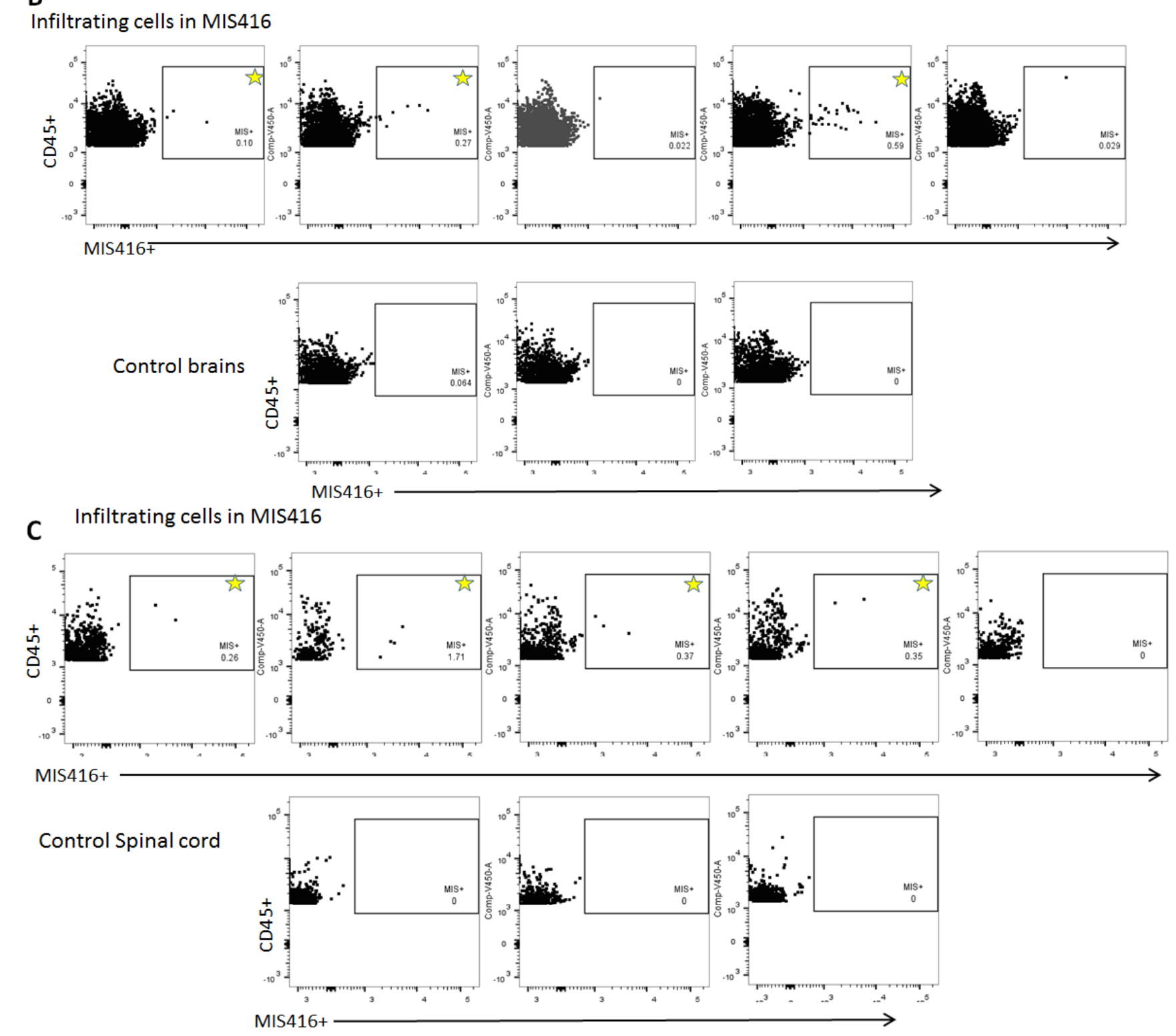

Figure 6-1 Traces of MIS416 were found within the brain 48 hours after a weekly dose in non-EAE mice

Mice receiving weekly MIS416 treatments were culled on day 16, 48 hours after the last MIS416 dose and brains and spinal cord lysate were analysed using flow cytometry. A) Grating strategy used in brain and spinal cord, using CD45hiCD11b+ cells as infiltrating myeloid cells in which B and C were gated on. B) Small amounts of MIS416+ cells were found within the brain as indicated by a star, whereas none in the control brains. C) Small amounts of MIS416+ cells found within the spinal cord as indicated by a star, whereas none in the control spinal cords. Shown are the FACs plots from an individual representative experiment ( 2 experiments done total). 


\subsubsection{MIS416 treatment reduced CNS-infiltrating inflammatory cells in EAE mice}

The EAE model in C57BL/6 mice is characterised by the infiltration of inflammatory monocytes and effector CD4 $\mathrm{T}$ cells into the CNS, and in particular the spinal cord, and to assess how MIS416 treatment affected the infiltration of these cells in EAE, flow cytometry was utilized. To gain a full understanding of the CNS alterations several gating strategies were used. Firstly, in Figure 6-2A the live single cells were gated, followed by the macrophage population $\left(\mathrm{CD} 45^{\text {hi }} \mathrm{CD} 11 \mathrm{~b}+\right)$, non-myeloid infiltrating cells $\left(\mathrm{CD} 45^{\mathrm{hi}} \mathrm{CD} 11 \mathrm{~b}-\right)$, resident microglia $\left(\mathrm{CD} 45^{\text {int }} \mathrm{CD} 11 \mathrm{~b}+\right)$ and granulocyte infiltrating cells $(\mathrm{CD} 45+\mathrm{CD} 11 \mathrm{~b}+\mathrm{Gr}-1)$. Using this gating strategy we found that MIS416 treatment significantly reduced the proportion of infiltrating macrophages in EAE mice as indicated by the ratio of infiltrating macrophages to resident microglia, and this trend was found in both the spinal cord and the brain (Figure 6-2B\&C). It was also determined that the spinal cord had many more infiltrating macrophages than the brain, which is not surprising in this model as the majority of lesions are found within the spinal cord in the C57BL/6 strain, and very little activity in the brain occurs at this early time point (day 16). We saw a similar trend in the Gr-1+ population which was also reduced in MIS416-treated EAE mice compared to untreated EAE mice, and this reduction was found in both the spinal cord and brain (Figure 6-2D\&E). The percentage of non-CD11b+ cells in the brain and spinal cord was elevated in EAE mice compared to non-EAE mice and with MIS416 treatment the percentage of these cells were also reduced, although only significantly in the brain (Figure 6-2F\&G). Interestingly, the infiltrating populations in the brain and spinal cord of MIS416-treated mice in the absence of EAE were also altered compared to untreated mice, although in this particular experiment the change did not reach statistical significance.

As we have identified a number of changes to myeloid cells which occurred in the CNS of both EAE and non-EAE mice with MIS416 treatment, we wanted to assess these changes further using a gating strategy focused on the activation of microglia. As shown in Figure 6-3A cells were gated based off their CD11b/CD45 expression where resting microglia were $\mathrm{CD} 11 \mathrm{~b}+\mathrm{CD} 45^{\text {low }}$, active microglia have been shown to upregulate their CD45 expression (Sedgwick et al., 1991) and therefore are $\mathrm{CD} 11 \mathrm{~b}+\mathrm{CD} 45^{\text {int }}$ and infiltrating myeloid cells are CD11b+CD45 $5^{\text {high }}$ and non-myeloid were similar to the other gating strategy and express no CD11b but are CD45 ${ }^{\text {high }}$. Using this strategy we assessed not only the percentage of positive cells but also using a gate over time we were able to effectively count the number of cells. By using a gate set over 20 seconds of running flow cytometry time we can effectively count the cells given that samples were running with a fixed flow rate (using high-through put, HTS, system on the FACs Canto II flow cytometer) and as all samples were processed in the same way and resuspended in the same volume, therefore giving us a relative count of each cell population. Although cell counting over time may not the most 
accurate counting method, the trends were consistent with the data presented as a percentage of total CD45+ cells.

Unsurprisingly, it was determined that spinal cords in MIS416-treated EAE mice had a significantly higher proportion of resting microglia and a lower proportion of activated microglia compared to untreated EAE mice (Figure 6-3B) and this trend was more evident in the relative cell counts (Figure 6-3C). There were also similar trends within the brains of MIS416-treated EAE mice although the number of infiltrating cells was much lower (Figure 6-3D\&E). These results using the cell count over time gave us a good indication of the number of cells making up each population, since previous gating strategies have looked only at the cells as a proportion of total events, which may not reflect the true difference in the number of infiltrating cells. For example, this method has showed that there were high numbers of cells within the spinal cord of EAE mice and that MIS416 treatment significantly reduced the number of these cells. The alterations in the brain were minimal compared to effects seen in the spinal cord which was unsurprising given that unpublished data from our lab has shown there is little brain involvement this early on in disease. The lack of infiltration was also reflected in the previous gating strategy (Figure 6-2A); however using the gating strategy in Figure 6-3 it was determined that EAE mice had a significant reduction in the proportion of non-myeloid infiltrating cells with MIS416 treatment and this trend was seen in the cell counts as well (Figure 6-3D\&E).

An interesting finding in this study was that with EAE immunisation there appeared to be an increase in the number of resting microglia $\left(\mathrm{CD} 45^{\text {low }} \mathrm{CD} 11 \mathrm{~b}+\right)$ compared to unimmunised and MIS416-treated mice. This effect could be due to several reasons such as an increase in resting microglia as one study has shown that during early stages of EAE there is an increased proliferation of microglia (Mendel, Kerlero de Rosbo, \& Ben-Nun, 1995); or could be due to the down-regulation of CD45 expression on infiltrating macrophages which then sit in the gate for "resting microglia", which may occur if infiltrating macrophages were replacing the microglia population (Mildner et al., 2007; Streit, Graeber, \& Kreutzberg, 1988). A more simple explanation could be the gating strategy used considering that while the populations were clear in the unimmunised mice, after EAE immunisation the populations were no longer distinct and due to the high number of cells collected some cells crossed into a different gate; given this we still believe the two gating strategies used in Figure 6-2A \& Figure 6-3A were effective for assessing CNS infiltration. While this slight elevation of resting cells will have an effect on the ratio used for the gating strategy in Figure 6-2 (i.e. number of infiltrating macrophages/number of microglia), due to the high number of infiltrating cells in the immunised mice the increase in untreated EAE mice is still significantly higher than non-immunised mice. 
Because EAE is characterised not only by infiltrating myeloid cells but also CD4+ T cells, we assessed the percentage and number of infiltrating cells into the brains and spinal cords of MIS416-treated mice with and without EAE. It was found that in the brains of EAE mice at day 16 (same experiment as the myeloid analysis; Figure 6-2) there was a significant reduction in the percentage and number of both CD4+ and CD8+ T cells in MIS416-treated compared to untreated animals (Figure 6-4A\&B). Surprisingly, there was an increase in the proportion and number of CD4+ $\mathrm{T}$ cells in the brain of MIS416-treated non-EAE mice compared to untreated mice indicating that MIS416 treatment alone significantly increased trafficking of this population into the brains of "healthy" mice (Figure 6-4A).

The lymphoid changes were also assessed in the spinal cord; however, the spinal cord expression of CD4 was very low in this particular experiment. Therefore, as surrogate markers of effector CD4 T cells, we used the expression of CD25 and lack of CD8 (CD8-CD25+). Given that within the effector $\mathrm{T}$ cell population (i.e. CD25+ cells), once the CD8+ $\mathrm{T}$ cells have been removed the remaining cells are likely to include a large population of CD4+ cells; and as a similar trend in the spinal cord as was found in the brain using this gating strategy, we conclude that there was a reduction in "CD4" $\mathrm{T}$ cell infiltration with in MIS416-treated mice (Figure 6-4C\&D). The inability to detect CD4 staining in the spinal cord has been attributed to the type of clone our lab had switched to, and the epitope for this antibody was likely altered during the enzymatic dissociation (with collagenase) of the spinal cord tissue and the antibody could no longer recognised the epitope. This idea was confirmed in the brain as the staining was not altered with the new clone and the brain tissue was not processed using collagenase. 
A
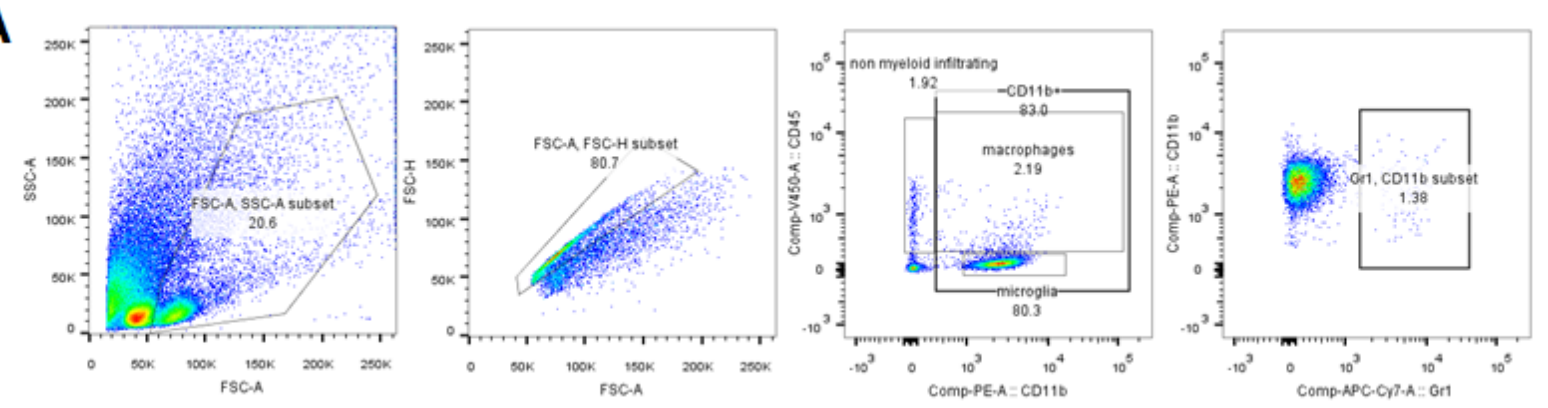

B
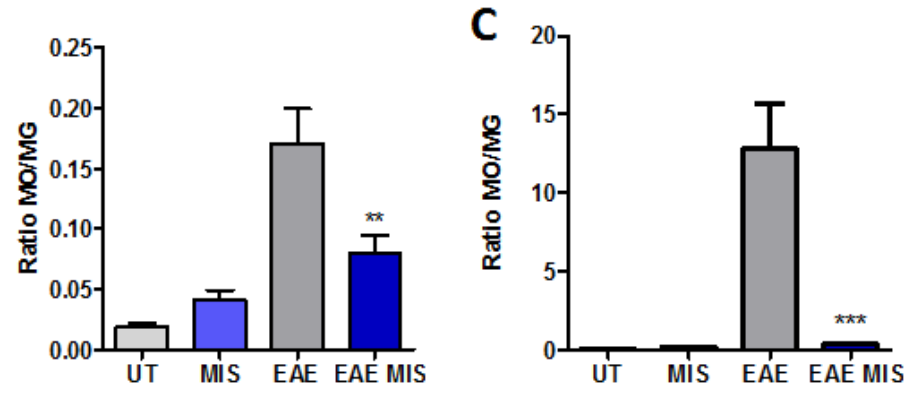

D

\section{E}
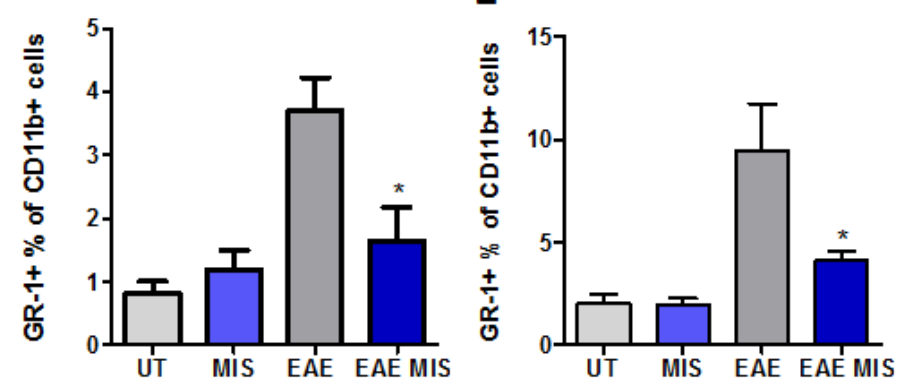

$\mathbf{F}$
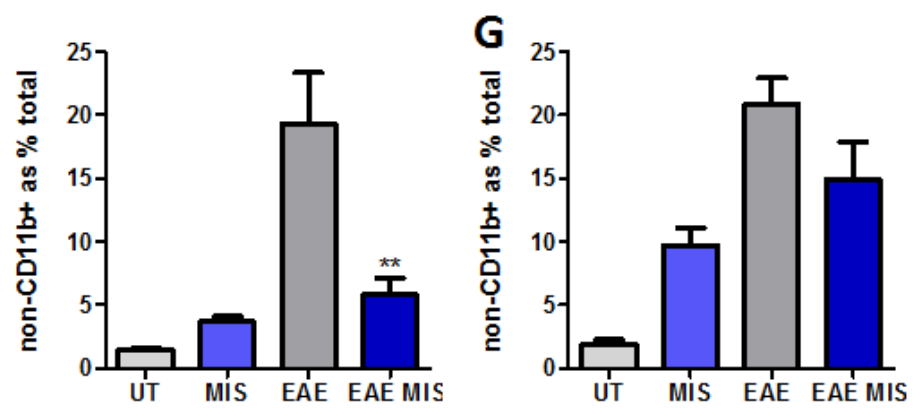

Figure 6-2 MIS416 treatment reduced infiltrating myeloid cells during EAE to the level seen in non-EAE MIS416-treated mice at day 16.

Mice were either immunised with EAE on day 0 or left untreated and were either untreated or treated weekly with MIS416 beginning at day 0. Mice were culled on day 16, 48 hours post MIS416 treatment, and CNS cell populations were analysed using flow cytometry. The cell populations were analysed as shown above (A), firstly the leukocyte population was gated on using FSC and SSC, then single cells (FSC-A vs FSC-H), then $\mathrm{CD} 45+\mathrm{CD} 11 \mathrm{~b}$ - cells are non-myeloid, CD45+CD11b+ are macrophages, and $\mathrm{CD} 45^{\text {low }} \mathrm{CD} 11 \mathrm{~b}+$ are microglia, then gating on all $\mathrm{CD} 11 \mathrm{~b}+$ cells, Gr-1+CD11b+ cells were assessed. Ratio of infiltrating macrophages (MO) to resident microglia (MG) was used as a measure of CNS infiltration. B), D) and F) show myeloid infiltrates into the brain. C), E) and G) show myeloid infiltrates into the spinal cord. Shown are the means \pm SEM from 1 experiment, $\mathrm{n}=5$ /treatment group. ${ }^{*} \mathrm{p}<0.05,{ }^{*} \mathrm{p}<0.01, \mathrm{p} * * *<0.001$ MIS416-treated compared to untreated mice unless indicated otherwise by 1-way ANOVA with Bonferroni's post-test. 
A
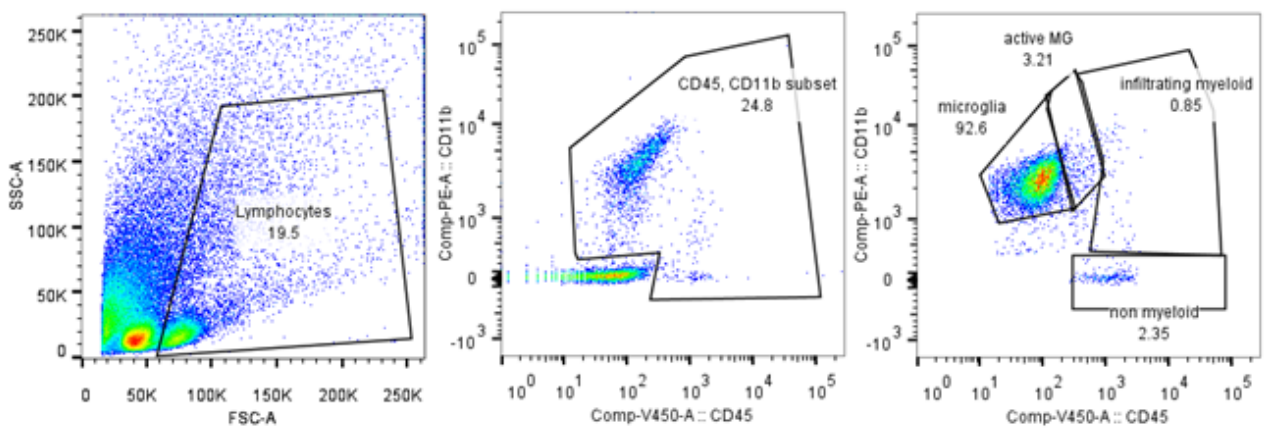

B
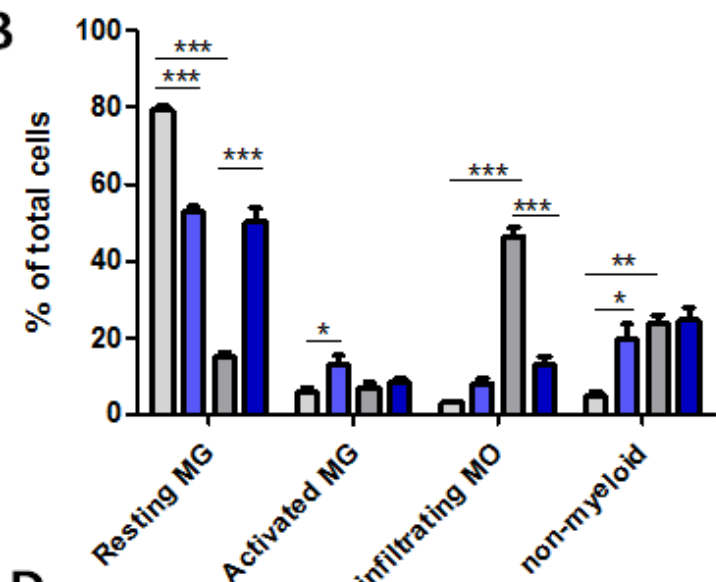

D

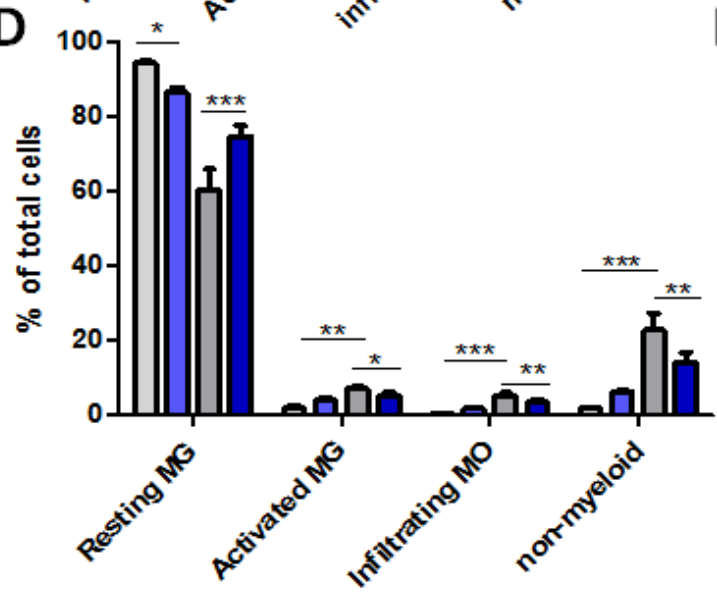

C
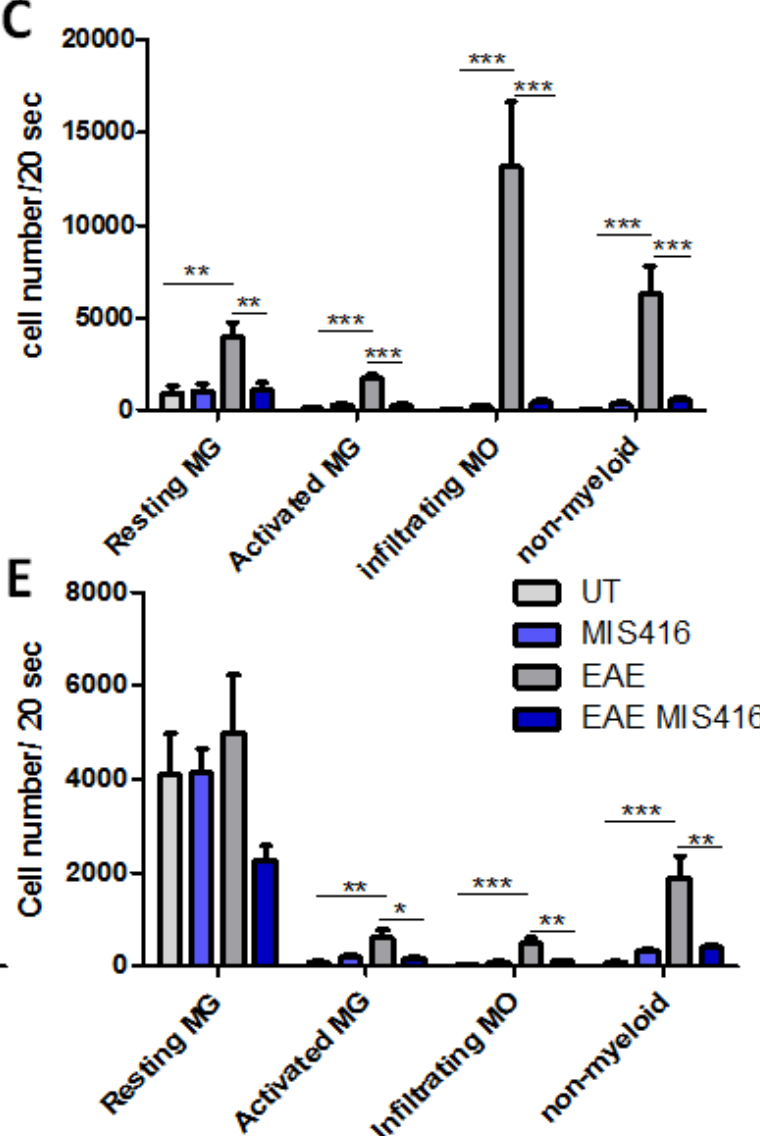

Figure 6-3 MIS416 treatment maintained resting microglial state in brain and spinal cord of EAE mice at day 16.

Mice were either immunised or non-immunised for EAE on day 0 and either left untreated or MIS416-treated with $100 \mu \mathrm{g}$ weekly. Brains and spinal cord were harvested on day 16, 48-hours post MIS416 injection. A) Gating strategy used to identify cell populations. B) Percentage of cell populations within the spinal cord C) Count of cell populations in the spinal cord in 20 seconds of sample run-time on the flow cytometer. D) Percentage of cell populations in the brain. E) Count of cell populations in the brain in 20 seconds of sample runtime on the flow cytometer. Shown are the means \pm SEM from 1 experiment, where $n=5 /$ treatment. ${ }^{*} p<0.05$, $* * \mathrm{p}<0.01$ and ${ }^{* * *} \mathrm{p}<0.001$ MIS416-treated compared to untreated unless indicated otherwise by 1-way ANOVA with Bonferroni’s post-test. 


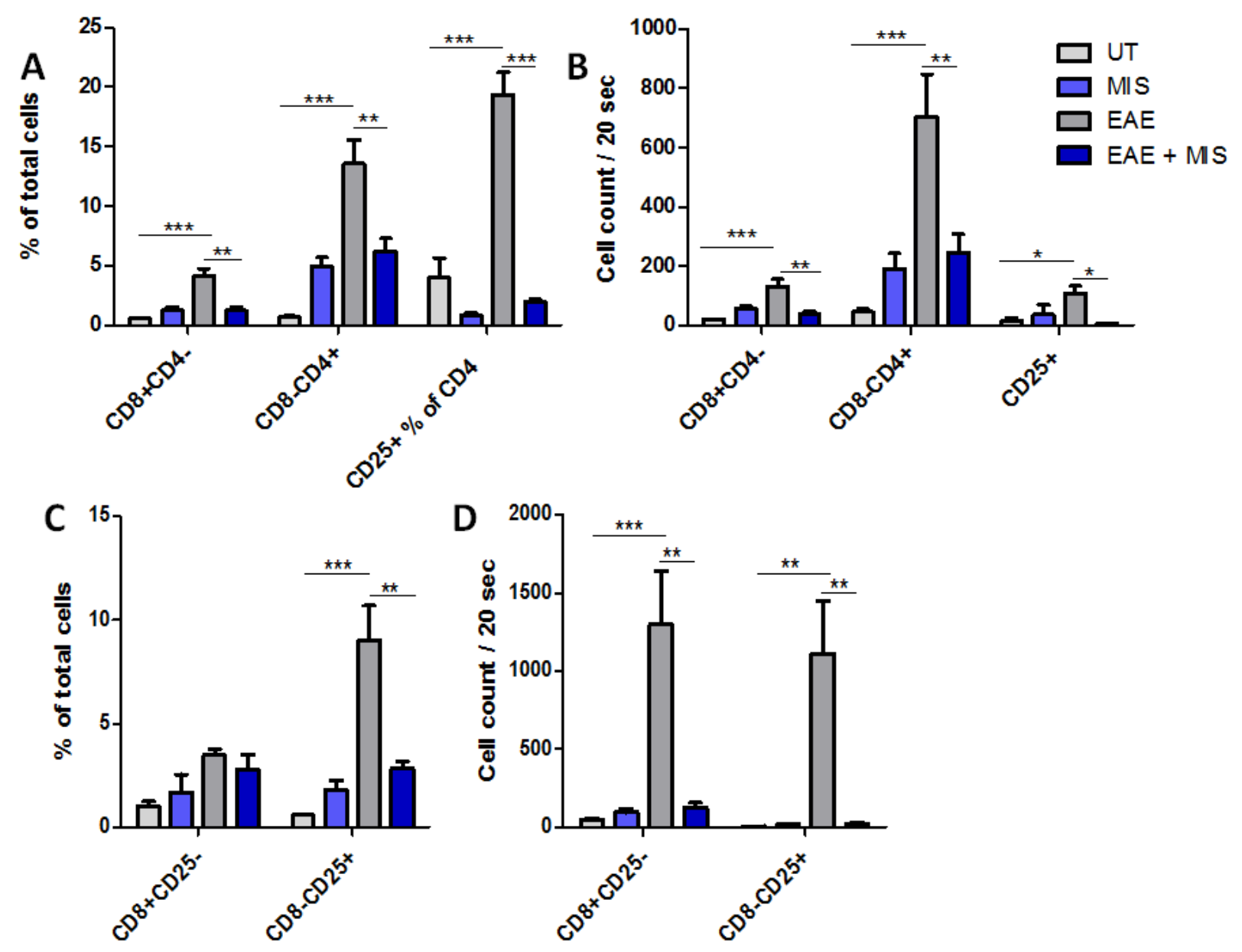

Figure 6-4 MIS416 treatment protected mice against EAE-induced infiltrating lymphocytes in the brain and spinal cord at day 16.

Mice were either immunised or non-immunised for EAE on day 0 and either left untreated or MIS416-treated with $100 \mu \mathrm{g}$ weekly. Brains and spinal cord were harvested on day 16, 48-hours post MIS416 injection. A) Percentage of cell populations within the brain. B) Cell count within the brain in 20 seconds of sample run-time on the flow cytometer. C) Percentage of cell populations within the spinal cord. D) Cell count within the spinal cord in 20 seconds of sample run-time on the flow cytometer. Shown are the means \pm SEM of 1 experiment, where $\mathrm{n}=5 /$ treatment. $* \mathrm{p}<0.05, * * \mathrm{p}<0.01$ and $* * * \mathrm{p}<0.001$ MIS416-treated compared to untreated and EAE compared to non-EAE by 1-way ANOVA with Bonferroni's post-test. 


\subsubsection{Heat-treated MIS416 was less effective at reducing CNS infiltration in EAE mice}

It was demonstrated in Chapter 3 that the heat-treated version of MIS416, which has no TLR9 activity, was not able to protect mice from EAE disease when given at the same dose and timing as regular MIS416. Therefore, in this chapter we evaluated the effect of heat-treated MIS416 on EAE-induced CNS changes. Using similar gating strategies to above, there was no significant reduction in the proportion of infiltrating macrophages in the brains MIS416-treated or HTMIS416-treated mice compared to untreated EAE mice in this experiment at day 22. Furthermore, neither treatment significantly affected the percentage of infiltrating CD4+ and CD8+ $\mathrm{T}$ cells within the brain (Figure 6-5). In contrast, we found a significant reduction in infiltrating macrophages in the spinal cords with MIS416 treatment but not with the HT-MIS416 treatment (Figure 6-6A). Although HT-MIS416 appeared to cause a reduction in infiltrating macrophages, this reduction did not reach significance and was not apparent when represented as a proportion of CD11b+ cells (Figure 6-6D). These results indicate that with HT-MIS416 treatment there may be some decrease in infiltrating macrophages despite there being no alteration in the disease scores of these mice compared to untreated EAE mice. Similarly, there was a significant reduction in the percentage of infiltrating CD8+ T cells in the spinal cord with MIS416 treatment but not with HTMIS416 (Figure 6-6B). Additionally, in this experiment spinal cord infiltrating CD4+ T cells were not assessed due to the lack of expression in the spinal cord, an effect that was also seen in the spinal cord at day 16 (Figure 6-4D).

Using the gating strategy shown in Figure 6-6C, which is the same microglia activation gating used previously, we established that MIS416-treated EAE mice had a more resting microglia state in the spinal cord compared to untreated EAE mice was consistent with data from EAE mice at day 16 as shown in Figure 6-3A (Figure 6-6D). Similar to the activation state in untreated EAE mice, the HT-MIS416-treated EAE mice also had a higher proportion of activated microglia and infiltrating macrophages with a reduction in the proportion of resting microglia (Figure 6-6D). Altogether these results indicated that the HT-MIS416-treated EAE mice had similar levels of microglial activation and CNS infiltration as untreated EAE mice indicating that TLR9 activity is required for the MIS416-induced CNS alterations in EAE. 
A
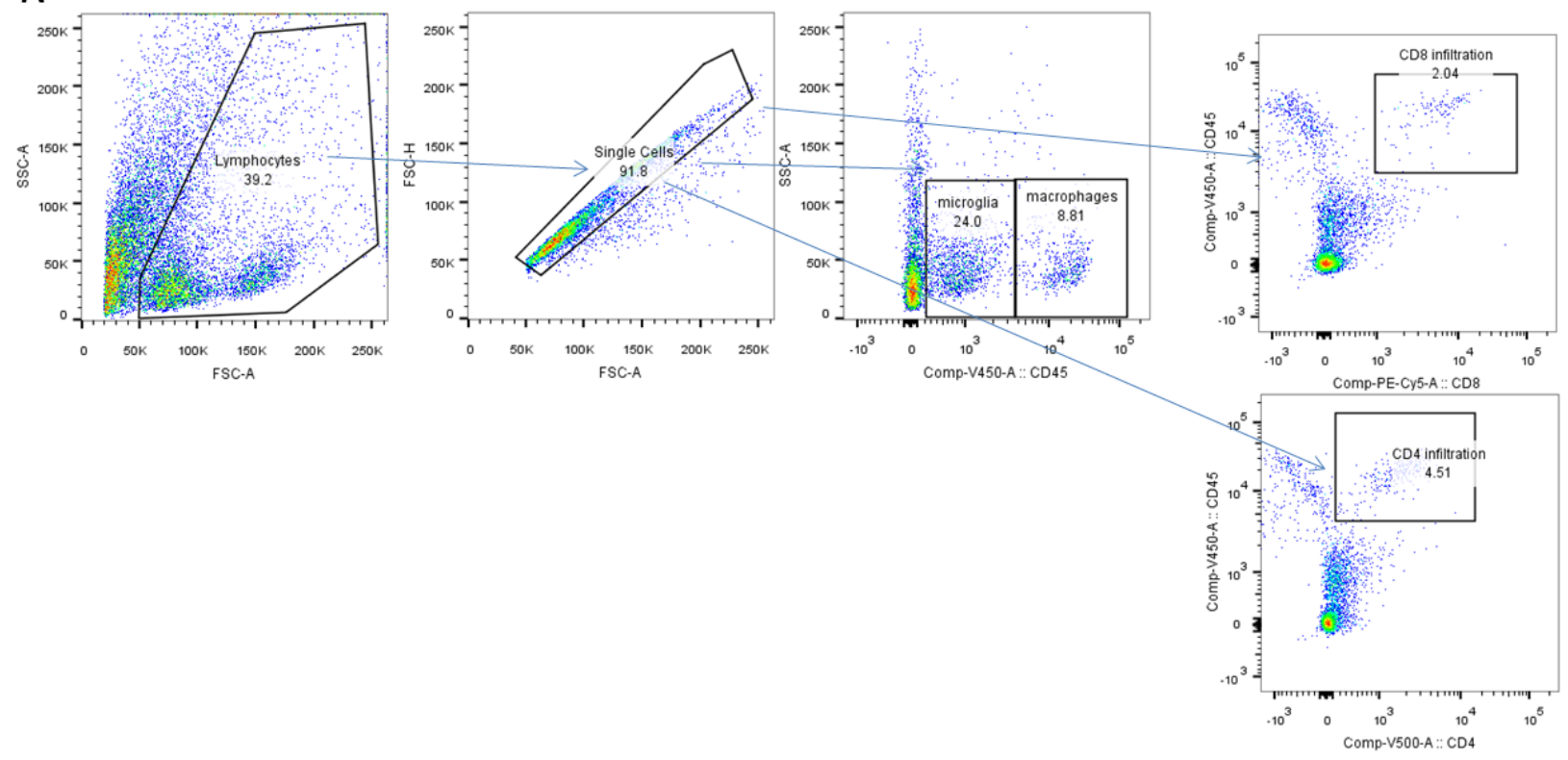

B

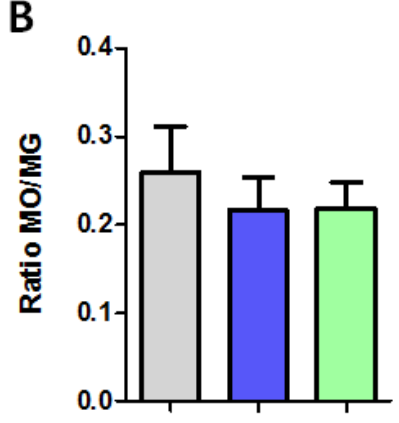

C

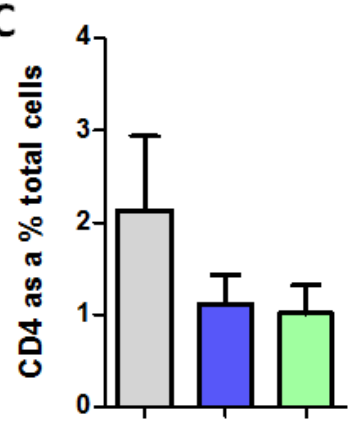

D

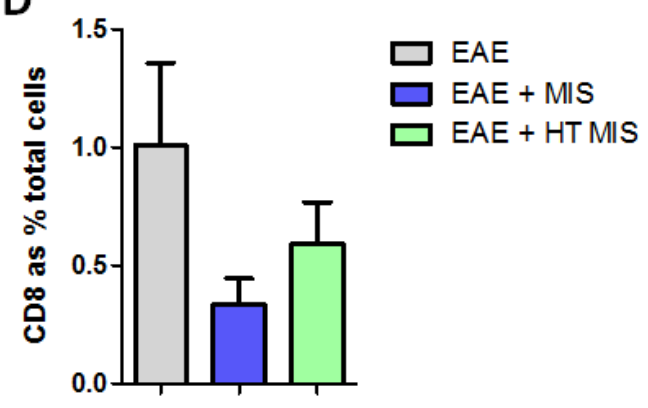

Figure 6-5 Weekly HT-MIS416 treatment did not significantly reduce leukocyte infiltration into the brain in EAE mice at day 22.

Mice were immunised with EAE on day 0 and received weekly treatments of $100 \mu \mathrm{g}$ of either MIS416 or heattreated MIS416. A) Gating strategy to show how macrophages, microglia, CD4 and CD8 T cells were defined. B) Ratio of infiltrating macrophages, MO, to microglia, MG, as calculated by number of macrophages divided by the number of resident microglia. C) CD4 T cells as a percentage of the total leukocyte gate as seen in the FCS/SSC graph. D) CD8 T cells as a percentage of the total leukocyte also. Shown are the means \pm SEM of 1 experiment ( $\mathrm{n}=5 /$ treatment group). NS using 1-way ANOVA with Bonferonni's post-test. 

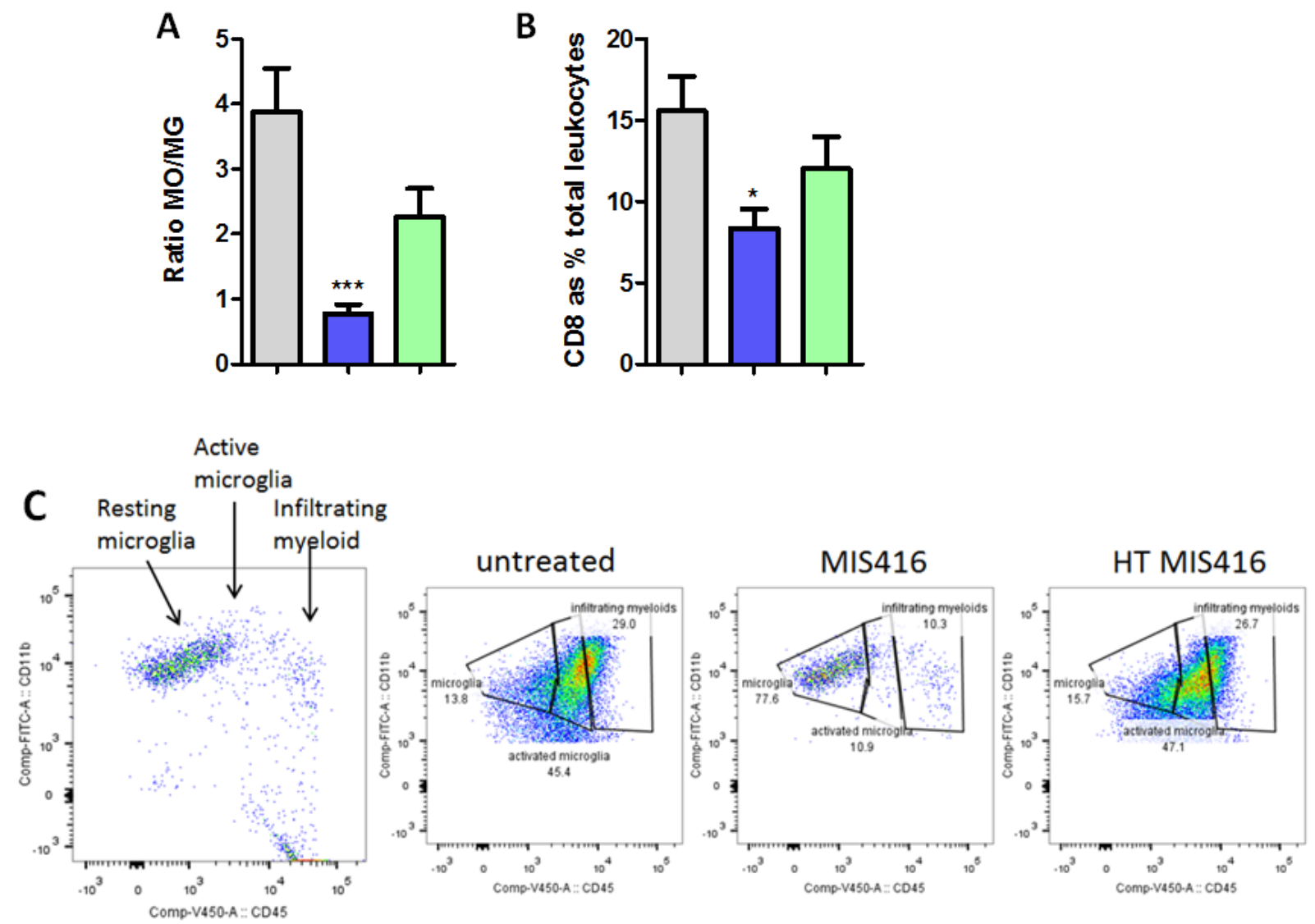

\section{Basic gating on \\ non-EAE brain}

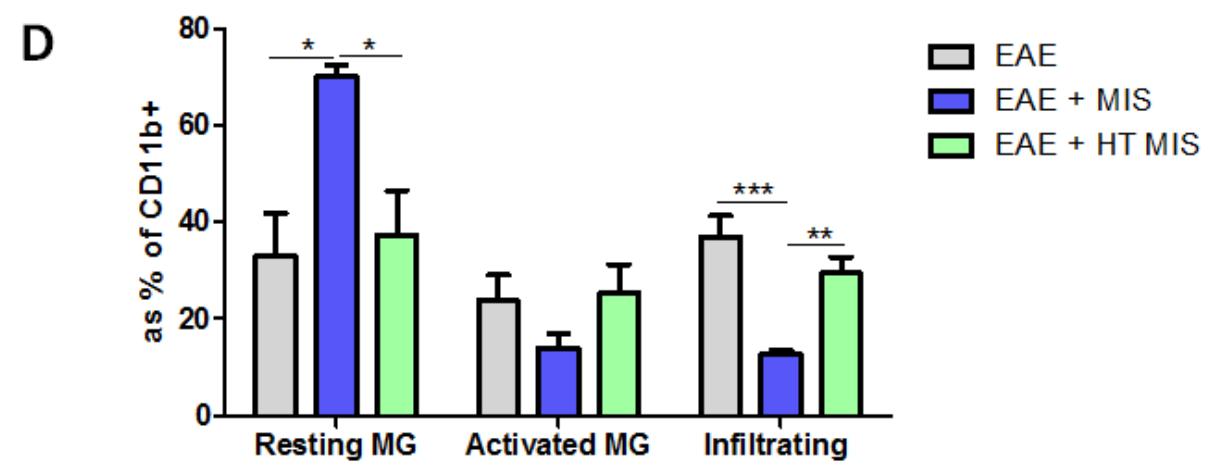

Figure 6-6 Weekly HT-MIS416 treatment did not significantly reduce leukocyte infiltration into the spinal cord in EAE mice at day 22.

Mice were immunised with EAE on day 0 and received weekly treatments of $100 \mu \mathrm{g}$ of either MIS416 or heattreated MIS416 and culled at day 22. Gating strategy to show how macrophages, microglia, CD4 and CD8 T cells were defined. A) Ratio of infiltrating macrophages, MO, to microglia, MG, as calculated by number of macrophages divided by the number of resident microglia. B) CD8 T cells as a percentage of the total leukocyte gate as seen in the FCS/SSC graph. C) Example of gating strategy for treated samples, using a non-EAE brain to gate on microglia $\left(\mathrm{CD} 45^{\text {low }} \mathrm{CD} 11 \mathrm{~b}+\right)$, active microglia $\left(\mathrm{CD} 45^{\text {int }} \mathrm{CD} 11 \mathrm{~b}+\right)$ and infiltrating myeloid cells $\left(\mathrm{CD} 45^{\mathrm{hi}} \mathrm{CD} 11 \mathrm{~b}+\right)$. D) Comparing CD11b+ populations based on CD45 expression as shown in the gating strategy shown in C. Shown above are the means \pm SEM of 1-2 independent experiments $(n=10 /$ treatment group (A\&B) and $\mathrm{n}=5 /$ treatment group (D)). ${ }^{*} \mathrm{p}<0.05, * * \mathrm{p}<0.01, \mathrm{p} * * *<0.001$ MIS416-treated compared to untreated and HTMIS416-treated mice by 1-way ANOVA with Bonferonni's post-test. 


\subsubsection{MIS416 increased myeloid and non-myeloid CNS infiltration without EAE}

Preliminary studies investigating CNS infiltration in EAE and non-EAE mice culled at day 16 identified that in the absence of EAE, MIS416 enhanced leukocyte trafficking. Therefore, we more specifically assessed the cell types which traffic into the CNS with MIS416 treatment in the absence of EAE. Firstly, we assessed the ratio of infiltrating macrophages to microglia using the gating strategy in Figure 6-7B and found that in spinal cords and brains of MIS416-treated mice, there was significant trafficking of myeloid cells $\left(\mathrm{CD} 11 \mathrm{~b}+\mathrm{CD} 45^{\mathrm{hi}}\right)$ compared to untreated controls (Figure 6-7A). Further assessment of these myeloid cells identified a subpopulation which was Gr-1+ (i.e. neutrophils), and although this increase did not reach significance, it appeared that granulocytes trafficked more to the brain in MIS416-treated mice. Interestingly, also using the gating strategy in Figure 6-7B for non-myeloid cells, it was found that MIS416 treatment significantly induced trafficking of non-myeloid cells into the spinal cord and brain (Figure 6-7C). Altogether these results suggested that MIS416 treatment can induce trafficking of both nonmyeloid and myeloid cells into the brain and spinal cord in the absence of EAE. While we have shown alterations in CNS trafficking with MIS416 treatment, further assessment into the role of the BBB as well as the location and activation state of cells which enter the CNS are of interest to understanding fully the impact of MIS416 treatment on the CNS. 

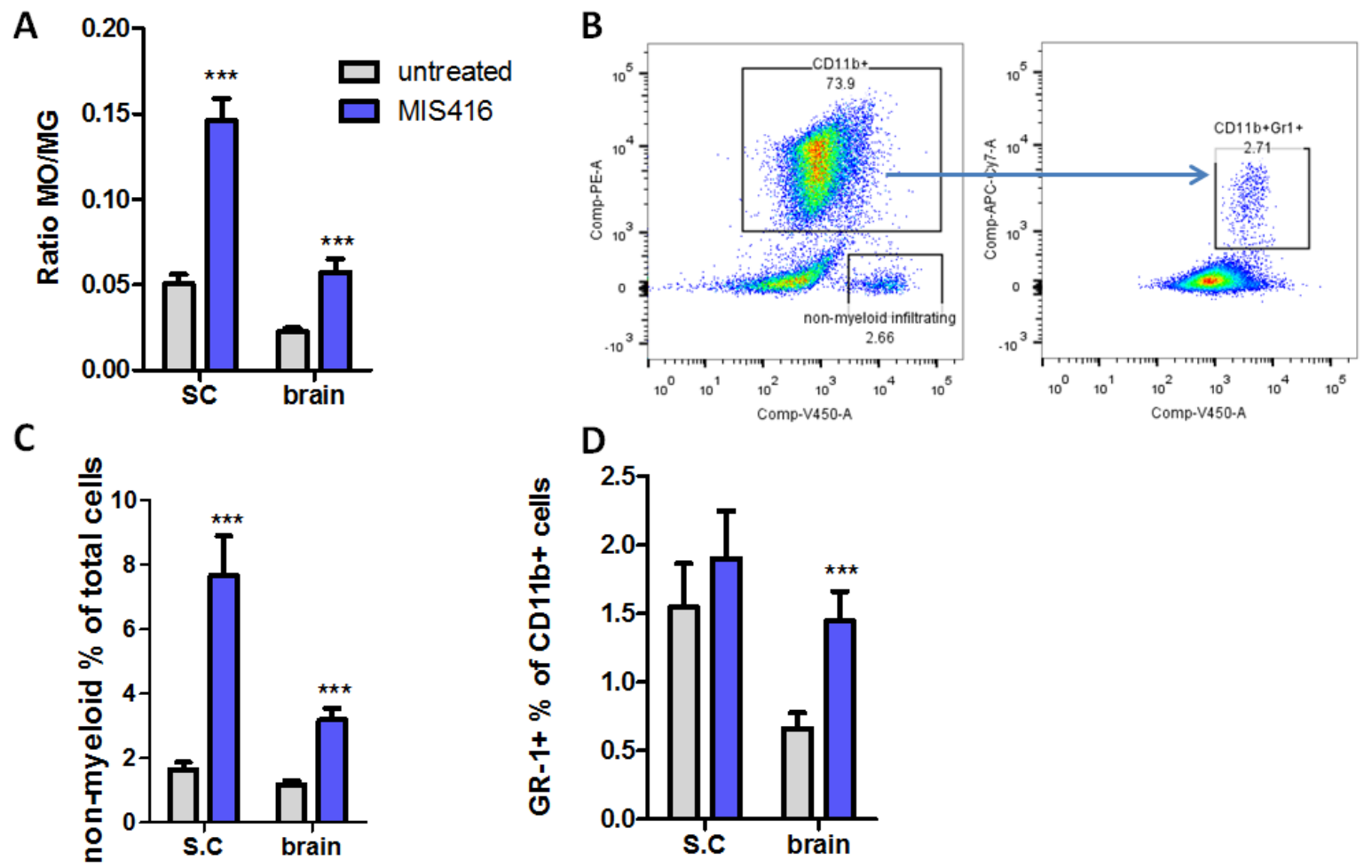

\section{D}

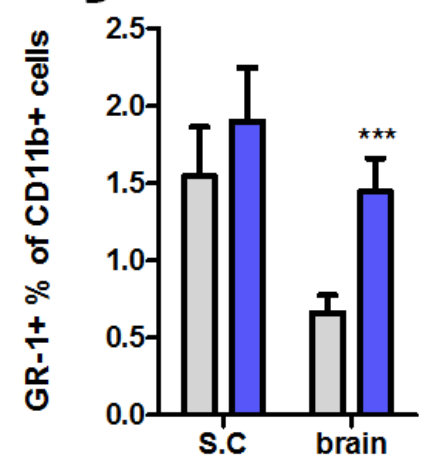

Figure 6-7 Weekly MIS416 treatment increased leukocyte infiltration into the CNS in non-EAE mice.

Mice receiving weekly MIS416 treatments were culled on day 16, 48 hours after the last MIS416 dose and brains and spinal cord lysate were analysed using flow cytometry. A) The ratio of MO, macrophages to MG, microglia. B) Gating strategy for C and D after FSC/SCC and single cells were gated on. C) Percentage of total single cells that were non-myeloid D) Percentage of CD11b+ cells that were also Gr-1+. Shown above are the means \pm SEM from 2 experiments, $\mathrm{n}=10 /$ treatment. $* * * \mathrm{p}<0.001$ MIS416-treated compared to untreated by unpaired Student's $\mathrm{t}$ test with Welches correction. 


\subsubsection{MIS416-treated EAE mice had reduced lesion size in the spinal cord at day 22}

The histological assessment of lesions within the brain and spinal cord of EAE mice is another method to assess infiltration in the CNS (esp. the spinal cord). Therefore, we assessed the lesion severity in haematoxylin and eosin $(\mathrm{H} \& \mathrm{E})$ stained spinal cord sections from untreated and MIS416-treated EAE mice at day 22 to determine whether MIS416 treatment reduced the lesion severity. The sections were scored by 3 independent assessors on a scale of 1-4, and we found that MIS416 reduced lesion severity compared to untreated EAE mice (Figure 6-8A). There was a non-significant linear correlation between lesion score and disease score, suggesting that with more replicates the lower disease score will correlate to lower lesion score; and considering MIS416-treated mice had lower disease scores, the lesion severity was also likely to be lower which was not surprising (Figure 6-8B). 
A

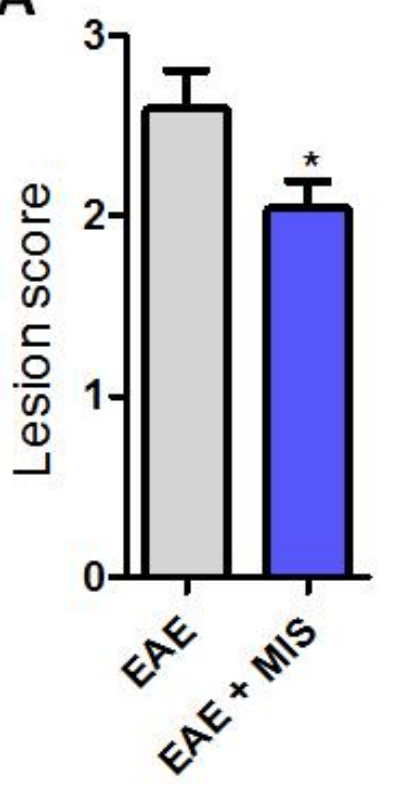

C

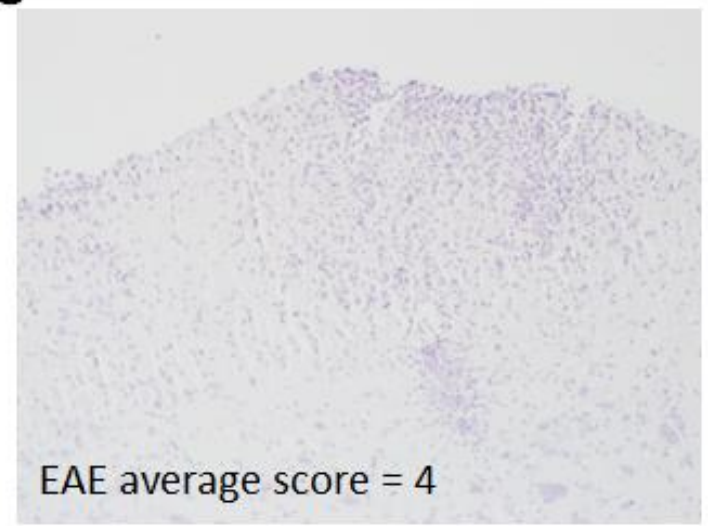

B

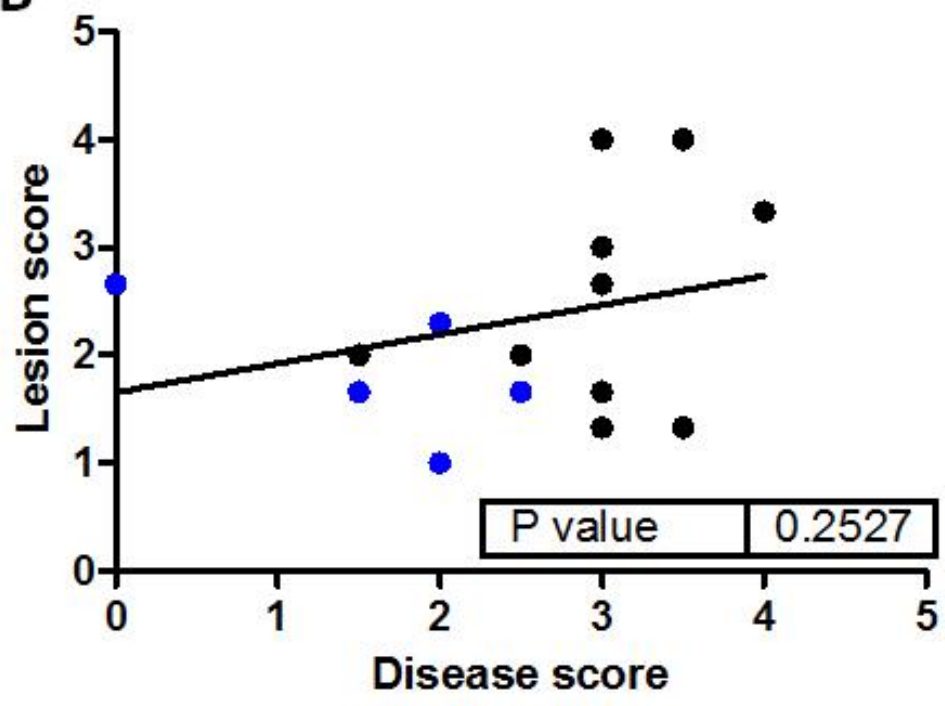

D

MIS416 average score $=1$

Figure 6-8 MIS416-treated EAE mice had reduced lesion size in the spinal cord

Mice were immunised with EAE on day 0 and received weekly treatments of $100 \mu \mathrm{g}$ MIS416. Mice were culled at day 22 and spinal cords were sectioned, stained with H\&E and the level of infiltration was scored on a scale of 1 (non-infiltration) and 4 (maximum infiltration), using 3 independent assessors. A) MIS416-treated mice show a significantly reduced level of spinal cord infiltration. B) A linear relationship between increased lesion score and increased disease score, not significant $\mathrm{p}=0.2572$. C) Example of average lesion score in EAE mouse. D) Example of average lesion score in MIS416-treated EAE mouse. Shown are the results of 2 experiments (n=7$10 /$ treatment group, some points on the plot are on top of each other). ${ }^{*} p<0.05$ MIS416-treated compared to untreated by an unpaired Student's t-test. 


\subsubsection{MIS416 treatment reduced EAE-induced BBB permeability}

The BBB is disrupted during EAE, resulting in increased vascular permeability and allowing the movement of auto-reactive $\mathrm{T}$ cells and inflammatory macrophages into the CNS and a similar change is thought to occur during MS (Bennett et al., 2010). Therefore, part of our CNS studies was to analyse the effect of MIS416 treatment on the integrity of the BBB in EAE mice. The integrity (i.e. ability to exclude the passage of albumin into the CNS) was assessed using Evans Blue (EB) dye. When this dye is injected into a mouse, it binds albumin proteins in the serum (Radu \& Chernoff, 2013), and if the integrity of the BBB is compromised, EB dye-bound albumin enters the CNS. To optimise the delivery of EB for our MIS416 experiments, we assessed whether the EB dye was more effective when injected intravenously (i.v) or intraperitoneally (i.p). We found that EAE mice at day 12, during early onset of EAE symptoms, showed an increase in the amount of EB dye visible in the brain and spinal cord with either i.v or i.p as shown by the black circles in Figure 6-9A compared to non-EAE mice injected with EB dye.

The amount of EB dye in the brain and spinal cord can be quantified using formamide to leech the dye from the tissue and the absorbance measured as per methods. A permeability index was created using the optical density (OD) of the sample divided by the OD of a non-EB control. Using this EB permeability index, the amount of BBB leakage can be quantified and compared. We found that i.v administration of EB had a higher permeability index in the brain compared to i.p (Figure 6-9B) whereas the i.p administration had a higher permeability index than i.v in the spinal cord (Figure 6-9C). Both routes of administration much higher than control samples, and therefore for ease and consistency, we chose to use i.p injection of EB-dye for future experiments.

Using this optimised protocol for EB dye staining, we determined that MIS416 did not significantly alter the increased permeability of the BBB in EAE mice however, there was a trend towards a reduction in BBB breakdown and with more replicates this trend may have reached significance. As shown in Figure 6-10A the brains and spinal cords of MIS416-treated EAE mice macroscopically appeared to have less blue staining compared to untreated EAE mice, although there was a lot of variation between the individual mice. However, using the permeability index, we found that the reduction with MIS416 treatment did not reach statistical significance although a trend was seen in both the brains and spinal cords of EAE mice receiving MIS416 treatment compared to untreated EAE mice (Figure 6-10B\&D). Because these mice had not yet developed disease symptoms, we assessed the severity of disease by the percentage of weight change and correlated this measurement to the permeability index. Using this analysis, EAE mice showed greater weight loss compared to MIS416-treated EAE mice which is consistent with MIS416treated mice developing less severe EAE disease. Furthermore, the permeability index correlated 
significantly with weight loss with EAE mice at day 10 showing the greatest weight loss and increase in permeability index (Figure 6-10C\&E).

Given that MIS416 also altered trafficking of cells into the CNS in the absence of EAE immunisation, the assessment of BBB leakage in MIS416-treated non-EAE mice was also investigated. Using the EB dye method, we found no significant difference between untreated and MIS416-treated mice in the BBB permeability in either the spinal cords or brains (Figure 6-11). Altogether, these results suggest that MIS416 itself does not induce a significant change in the $\mathrm{BBB}$ integrity although during $\mathrm{EAE}$ when the $\mathrm{BBB}$ is compromised, there was a trend towards a reduced $\mathrm{BBB}$ breakdown compared to untreated EAE mice. 


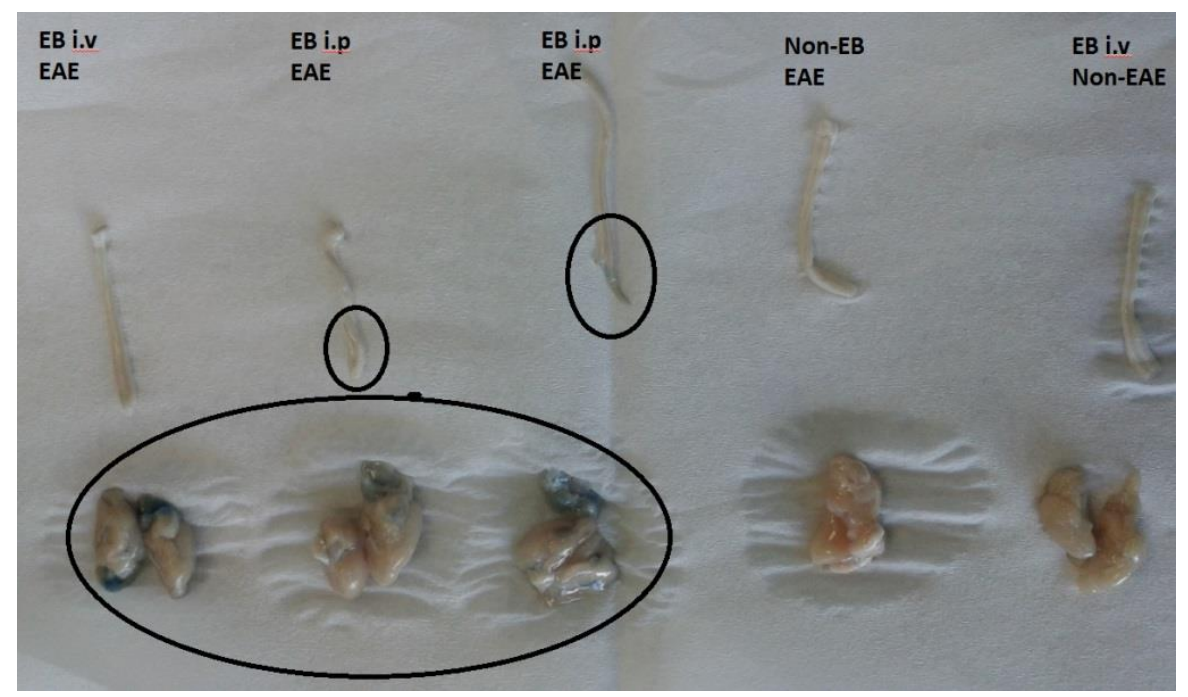

B

\section{Brain}

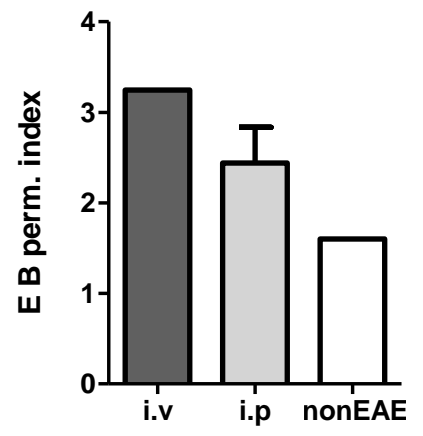

C

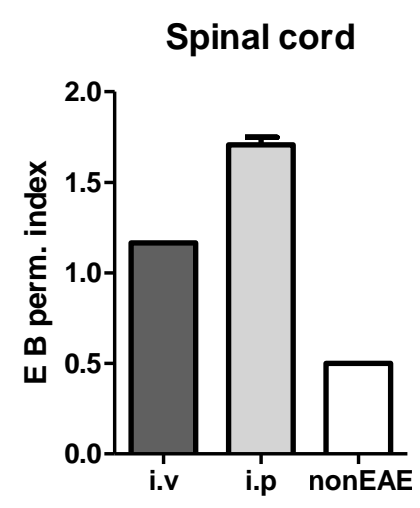

Figure 6-9 Optimising Evans blue in BALB/c mice showed that $200 \mu \mathrm{l}$ of $2.5 \%$ in saline given i.p. was effective in EAE mice compared to non-EAE.

Mice were either immunised or non-immunised for EAE on day 0 and evans blue dye was given either i.v or i.p on day 12. Mice were sacrificed 90 minutes later and perfused via the heart with $20 \mathrm{ml}$ PBS, then the brains and spinal cords were removed for examination A) Brains isolated from mice showed significant blue staining in the cerebellum and small spots of staining in the spinal cord and no evidence of blue staining in the non-EAE mice. B) Brain permeability index, where perm. Index = (OD sample/OD no evans blue brain). C) Spinal cord permeability index, where perm. Index $=(\mathrm{OD}$ sample/OD no evans blue spinal cord $)$. These results represent a single experiment, $\mathrm{n}=1-2$ per treatment. 
A Untreated

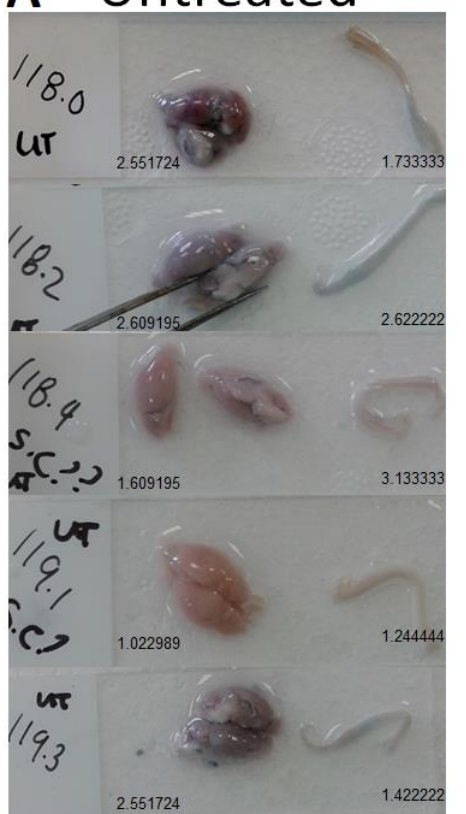

MIS416

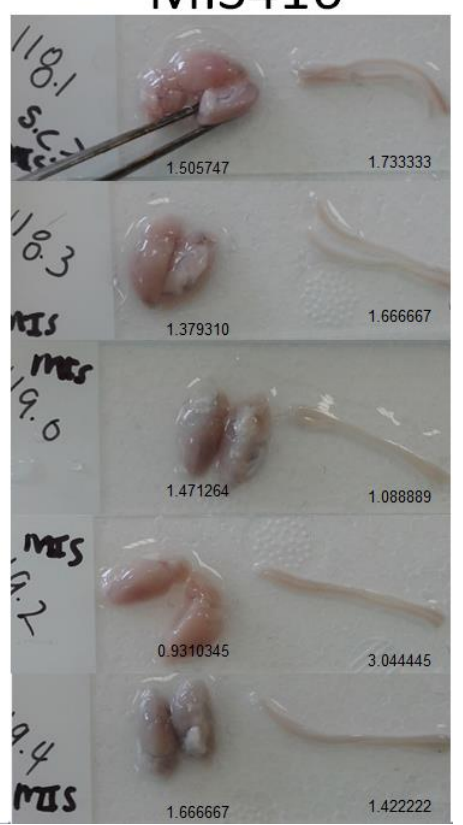

C

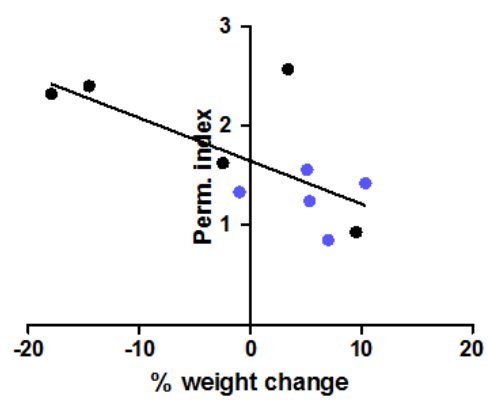

4

E

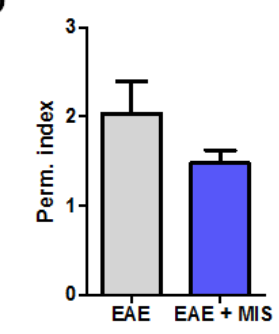

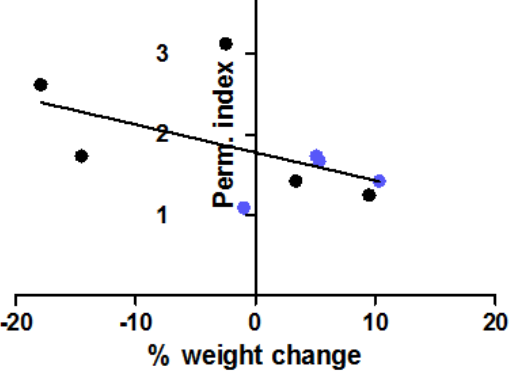

Figure 6-10 BBB permeability in EAE mice was reduced with MIS416 treatment.

Mice were immunised for EAE on day 0 and EB dye was given i.p on day 10. Mice were sacrificed between 4060 minutes later and perfused via the heart with $20 \mathrm{ml}$ PBS, then the brains and spinal cords were removed for examination and permeability index was created using (OD sample/OD no-Evans Blue control brain or spinal cord). A) Brains and spinal cords isolated from mice B) Brain permeability index ( $\mathrm{p}=0.0952)$. C) Brain correlation of percentage weight change at day 10 to permeability index. C) Spinal cord permeability index $(\mathrm{p}=0.387)$. E) Spinal cord correlation of percentage weight change at day 10 to permeability index. Shown are the means \pm SEM from 1 experiment, where $n=4-5 /$ treatment. Data not significant by unpaired Students t-test. 

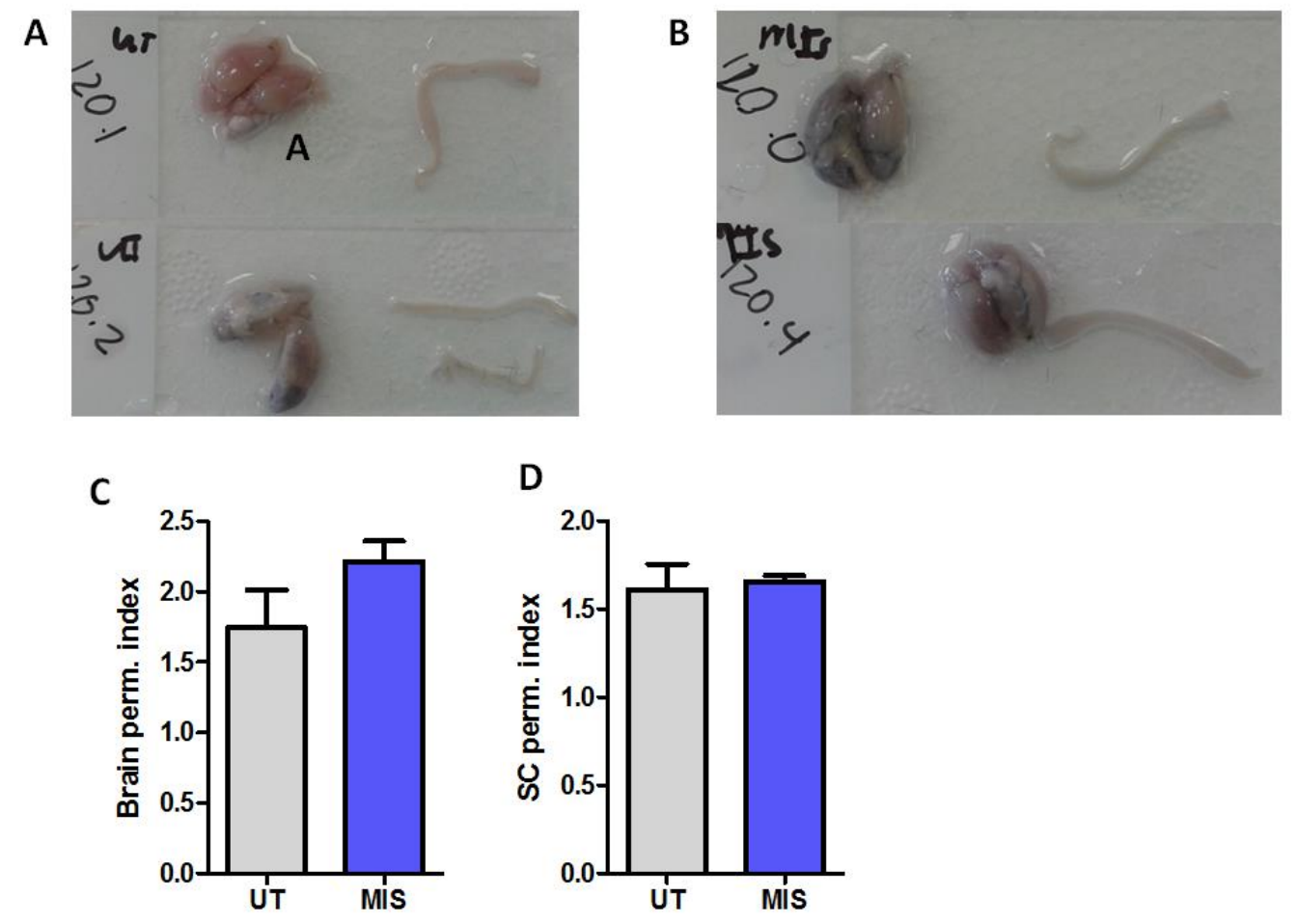

Figure 6-11 BBB permeability in non-EAE mice was not significantly altered with MIS416 treatment.

C57BL/6 mice were either untreated or MIS416-treated with $100 \mu \mathrm{g}$ weekly and were given Evans Blue dye at $2.5 \%$ in $200 \mu \mathrm{l}$ i.p on day 15 (one day post MIS416 treatment). Mice were sacrificed between 80-100 minutes later and perfused via the heart with $20 \mathrm{ml}$ PBS, then the brains and spinal cords were removed for examination and permeability index was created using (OD sample/OD no-Evans Blue control brain or spinal cord). A) Brains and spinal cords isolated from untreated mice B) Brains and spinal cords isolated from MIS416 mice C) Brain permeability index. D) Spinal cord permeability index. Shown are the means \pm SEM from 1 experiment, where $\mathrm{n}=2 /$ treatment. Data was not significant by unpaired Students t-test. 


\subsubsection{IFN- $\gamma$ was important in the infiltration of cells into the CNS of MIS416-treated mice in} the absence of EAE

A study by Kunis et al identified IFN- $\gamma$ as a key molecule involved in the trafficking of leukocytes into the CNS via the choroid plexus (Kunis et al., 2013). Given that MIS416-treated mice had high levels of circulating IFN- $\gamma$, we hypothesised that increased trafficking into the CNS with MIS416 treatment would be IFN- $\gamma$ dependent. Therefore to test this theory, we assessed trafficking of leukocytes in MIS416-treated IFN- $\gamma^{-/-}$mice in the absence of EAE. As hypothesised, there was no increase in the ratio of infiltrating macrophage to microglia in either the brain or spinal cord of IFN- $\gamma^{-/-}$mice by MIS416 treatment, in contrast to wild type MIS416-treated mice in which there was a significant increase (Figure 6-12). Further assessment of these cells determined that while MIS416 induced trafficking of CD4+ T cells, macrophages and other myeloid cells in the presence of IFN- $\gamma$ signalling, there was no difference in any of the leukocyte populations in the absence of IFN- $\gamma$ signalling with MIS416 treatment compared to untreated mice (Figure 6-13). Interestingly, the data from this experiment further highlight that the brain and spinal cord have different leukocyte trafficking in response to MIS416 treatment in the presence of IFN- $\gamma$ signalling, given that within the brain infiltrating cells were mostly macrophages (CD11b+F4/80+) and spinal cord infiltrating cells were CD4+ T cells (Figure 6-13). 

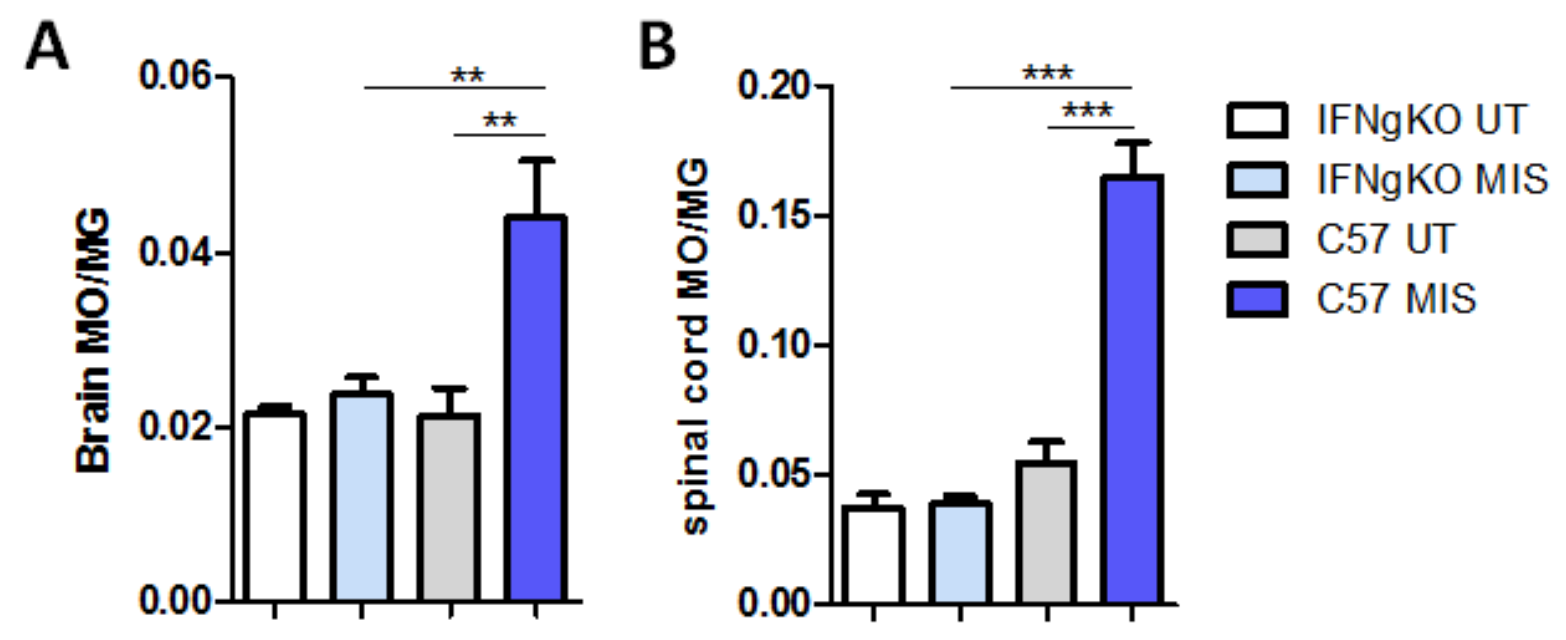

Figure 6-12 MIS416 infiltration into the CNS in non-EAE mice required IFN- $\gamma$.

Mice receiving weekly MIS416 treatments were culled on day 15, 24 hours after the last MIS416 dose and brains and spinal cord lysate were analysed using flow cytometry. A) The ratio of macrophages (MO) to microglia (MG) in the brain. B) The ratio of macrophages (MO) to microglia (MG) in the spinal cord. Shown is the means \pm SEM from 2 experiments, $\mathrm{n}=7 /$ treatment. ${ }^{* *} \mathrm{p}<0.01, * * * \mathrm{p}<0.001$ MIS416-treated compared to untreated controls and MIS416-treated WT compared to MIS416-treated IFN- $\gamma^{-1-}$ mice by 1-way ANOVA with Bonferroni's post-test. 
A

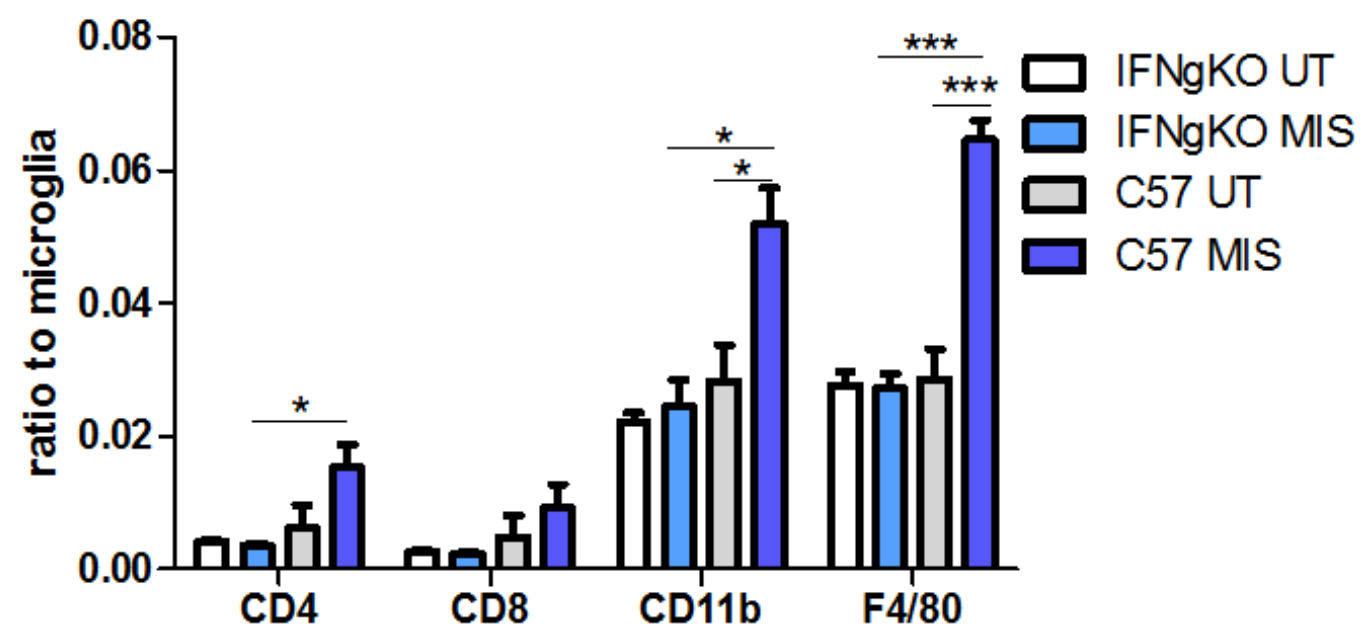

B

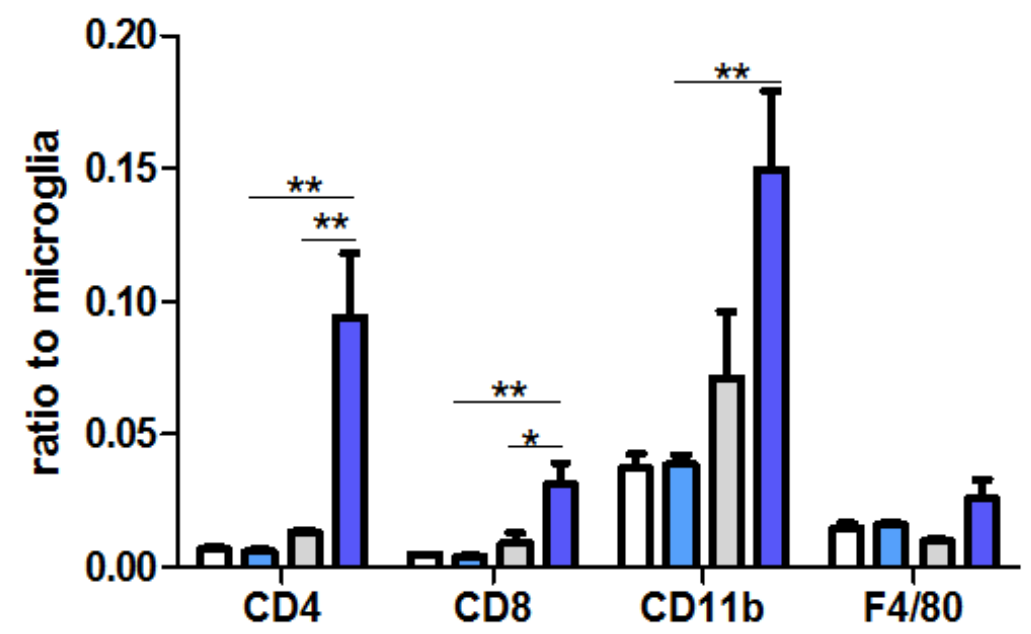

Figure 6-13 MIS416-induced trafficking into the brain was comprised of different cells compared to spinal cord in non-EAE mice.

Mice receiving weekly MIS416 treatments were culled on day 15, 24 hours after the last MIS416 dose and brains and spinal cord lysate were analysed using flow cytometry. A) The ratio of different cell populations to microglia in the brain. B) The ratio of different cell populations to microglia in the spinal cord. Shown is the means \pm SEM from 1 experiment, $\mathrm{n}=3 /$ treatment. $* \mathrm{p}<0.05$, $* * \mathrm{p}<0.01$, $* * * \mathrm{p}<0.001$ MIS416-treated compared to untreated controls and MIS416-treated WT compared to MIS416-treated IFN- $\gamma^{-/-}$mice by 1-way ANOVA with Tukey's post-test. 


\subsection{Discussion}

This chapter was aimed at identifying the effects of MIS416 on the CNS, either directly or indirectly, and determining how these effects are related to the disease protection seen in EAE mice. We found that weekly MIS416 treatment resulted a few MIS416-positive cells in the brain and spinal cord and, while the MIS416 particle is too large to freely pass through cellular membranes, it is possible that the transfer of MIS416 into the CNS is through cells which take up the particle; if so, the movement of MIS416 would be limited to the trafficking of specific cellular populations into the CNS. Additionally, MIS416 treatment reduced BBB breakdown, thereby suppressing the infiltration of macrophages and T cells into the CNS of EAE mice. Consequently, MIS416 treatment led to reduced inflammatory lesions in the spinal cord during EAE. Together these results were consistent with reduced disease scores in MIS416-treated mice. Finally, in contrast to EAE, in non-EAE mice, MIS416 enhanced trafficking of macrophages and lymphocytes (potentially CD4+ T) into the spinal cord and this trafficking was IFN- $\gamma$-dependent.

The amount of MIS416 present in the CNS was quite low using the $100 \mu \mathrm{g}$ weekly dose however, recent unpublished work by collaborators also suggests that at higher doses, more of the MIS416 can be found within the brain by histological sections as well as flow cytometry (personal communication, Gill Webster, IIT). While the amount of MIS416 found within the CNS of nonEAE mice was low and appeared to be mostly associated with trafficking myeloid cells, under EAE conditions where the BBB breaks down, the amount of MIS416 found within the CNS is likely to be increased. Therefore, the MIS416 which does enter the CNS may be taken up by resident APC, such as microglia (Fu, Shen, $\mathrm{Xu}, \mathrm{Luo}, \&$ Tang, 2014) resulting in TLR9 and NOD2 signalling within the CNS. Because the activation of microglia with TLR9 and NOD2 ligands has shown to be detrimental for CNS inflammation (Butchi, Woods, Du, Morgan, \& Peterson, 2011; Chauhan, Sterka, Furr, Young, \& Marriott, 2009), the entry of MIS416 into the CNS could be detrimental for EAE. Alternatively, it is possible that microglia are induced into a more tolerogenic state when both NOD2 and TLR9 are stimulated together as shown by previous studies investigating peripheral APCs by Castellaneta et al (Castellaneta et al., 2009).

The migration of macrophages and activated T cells into the CNS is a critical part of disease pathology in EAE and MS (Bar-Or, Oliveira, Anderson, \& Hafler, 1999). MIS416-treated mice have reduced entry of inflammatory macrophages and CD4+ T cells into both the brain and spinal cord during EAE; furthermore HT-MIS416-treated mice, which showed reduced peripheral effects and no disease protection, had similar levels of CNS infiltration to that found in untreated EAE mice. The reduced infiltration in MIS416-treated mice is likely a consequence of reduced T cell proliferation in the periphery accompanied by reduced migration of disease-driving cells into the 
CNS. Other immune modulating treatments have similar effects on infiltration; for instance, IFN$\beta$-treated mice have reduced leukocyte trafficking into the CNS, which is thought to be due to decreased expression of adhesion molecules and an inhibition of chemokine expression (Yong, 2002). In contrast to IFN- $\beta$, GA treatment does not appear to alter the trafficking of cells into the CNS and in contrast, the cells which enter the CNS are able to suppress immune responses from within (Yong, 2002). Although there was reduced infiltration into the CNS of MIS416-treated EAE mice, MIS416 treatment resulted in cellular trafficking to the CNS in healthy mice. It is possible that these cells which entered the CNS had anti-inflammatory effects and therefore could act similarly to GA.

During disease onset in EAE, the BBB has increased permeability which allows the infiltration of inflammatory cells and further drives disease progression. With MIS416 treatment there was a reduction in $\mathrm{BBB}$ breakdown in $\mathrm{EAE}$ mice, and in non-EAE mice the BBB remained unchanged despite enhanced cellular infiltration seen with MIS416. These results were not surprising considering other treatments which reduce disease symptoms and lesion size also reduce the BBB break-down. For example, IFN- $\beta$ treatment is thought to have direct action by stabilising the BBB and therefore reducing CNS entry and inflammation (Kraus \& Oschmann, 2006). Some of the mechanisms suggested to be involved in IFN- $\beta$ BBB stabilisation include distribution of tight junction proteins, ZO-1 and claudin, to stabilise the endothelial tight junctions and BBB permeability (Kraus \& Oschmann, 2006). This could be a potential mechanism by which MIS416 treatment could also stabilise the BBB to reduce EAE disease.

MIS416-treated EAE mice have reduced infiltrating macrophages and CD4+ T cells however, MIS416 treatment in non-EAE mice results in increased trafficking of these cells compared to untreated mice. Moreover, the cells traffic differently into the brain than the spinal cord, and the trafficking appeared to be IFN- $\gamma$-dependent. Studies from Engelhardt et al have described the entrance of CD4+ $\mathrm{T}$ cells across the BBB requiring several key molecules which are expressed at only low levels under steady-state conditions. To enter the CNS, the leukocytes express ligands which bind to receptors on the surface of the BBB, such as $\alpha 4 \beta 1$ integrin/V-CAM and LFA-1/ICAM; and under inflammatory conditions the expression of these molecules is much higher, allowing CNS infiltration to occur (Engelhardt, 2006). As CNS trafficking appeared to be reduced in MIS416-treated IFN- $\gamma^{-/-}$mice, we believe that MIS416-induced IFN- $\gamma$ is involved in the increased trafficking in the absence of EAE. A study conducted by Kunis et al described the alteration of the choroid plexus by IFN- $\gamma$ and found that in response to IFN- $\gamma$, the choroid plexus upregulated a range of trafficking molecules including I-CAM1, V-CAM as well as chemokines (Kunis et al., 2013). Furthermore, Kunis et al determined that in the absence of IFN- $\gamma$ signalling 
there were reduced CD4+ $\mathrm{T}$ cells in the choroid plexus and after spinal cord injury there was no up-regulation of trafficking molecules and therefore reduced recovery compared to WT mice (Kunis et al., 2013). As we found MIS416 treatment increased leukocyte trafficking into the CNS, one possibility is that the recruited cells are able to act in a suppressive manner to reduce disease symptoms and therefore may contribute to the mechanism of MIS416.

The migration of CD11b+ cells was significantly increased with MIS416 treatment and in an IFN$\gamma$ dependent manner. It was also found that in the brain these cells were made up of $\mathrm{CD} 11 \mathrm{~b}+\mathrm{F} 4 / 80+$ cells whereas in the spinal cord these cells were mainly CD11b+F4/80-. Although we did not characterise these cells further, it has been suggested that IFN- $\gamma$ upregulates the expression of CCL2 on the choroid plexus epithelium (Kunis et al., 2013), which is a chemokine that is thought to be involved in the recruitment of monocytes into the CNS through the expression of CCR2 (Szmydynger-Chodobska et al., 2012). Given that MIS416 treatment alters the activation state of cells within the periphery, it is possible that the CNS infiltrating myeloid cells in MIS416treated mice could have a protective role in EAE. Previous work has shown that the expansion of CD11b+Ly6C ${ }^{\text {high }}$ cells (monocytic-MDSC), which suppress $\mathrm{T}$ cell responses through NO and induce $\mathrm{T}$ cell apoptosis, are at a high frequency in the CNS of EAE mice indicating they may have a suppressive function (Bing Zhu et al., 2007). Another study which has assessed Gr-1+ cells from the CNS of EAE mice, found that these cells suppressed myelin-specific T cell responses in a NOdependent manner (Zehntner et al., 2005). The results from these studies suggest that MIS416 induced trafficking of myeloid-suppressor cells which could suppress $\mathrm{T}$ cell responses from within the CNS and may have a role in the mechanism of MIS416.

Like the CD11b+ cells, increased numbers of CD4+ T cells trafficked to the spinal cord in an IFN$\gamma$-dependent manner in MIS416-treated mice. However, as the functionality of these cells was not assessed, it is possible that these cells may be regulatory. As we found that there was a significant expansion of Tregs in the spleens of MIS416-treated mice (White et al., 2014), it is possible that the increased trafficking of CD4+ T cells includes a Treg population. Furthermore, we have shown in Chapter 5 that Tregs from MIS416-treated mice are more suppressive than those from untreated mice; therefore these cells may act to suppress responses within the CNS. A study assessing the accumulation of IL-10-producing CD4+CD25+ T cells within the CNS confirmed that these cells are FoxP3+ and supress $\mathrm{T}$ cell responses ex vivo; furthermore these Tregs appear to be increased during the recovery phase in EAE (McGeachy et al., 2005). Together with the macrophage trafficking data in non-EAE mice, it appears that MIS416 treatment may enhance the infiltration of cells which may suppress inflammatory responses within the CNS, and trafficking of these cells is likely to occur in EAE mice albeit the effect is masked by EAE-driven CNS infiltration. 
Another potential mechanism by which MIS416 reduces EAE disease severity in mice is through the activation of CNS repair mechanisms. One study has shown that administration of TLR and NOD2 ligands are able to induce proliferation of spinal cord neuronal progenitor cells, some of which were of microglial origin (Y. Su, Zhang, Trautmann, Xu, \& Schluesener, 2005). This finding was partially supported by our study as indicated by the increased number of resting microglia within the spinal cord of MIS416-treated non-EAE mice compared to untreated controls. Interestingly, in the study by $\mathrm{Su}$ et al it was reported that the administration of $\mathrm{CpG}$, a TLR9 agonist, did not induce proliferation, while TLR3, TLR4, TLR7/8 and NOD2 agonists temporarily increased the number of BrdU+ cells within the spinal cord (Y. Su et al., 2005). This study could indicate a way in which NOD2 but not TLR9 stimulation is protective within the CNS by promoting repair and disease recovery. However, while the study by Su et al suggests that TLR9 signalling may not be neuroprotective, it did appear that this agonist had a significant role in the mechanism of MIS416 within the periphery given the lack of disease protection in the HTMIS416-treated EAE. Furthermore, studies have also shown that low levels, but not high levels, of IFN- $\gamma$ can induce microglia into a neuroprotective-state (Butovsky, Talpalar, Ben-Yaakov, \& Schwartz, 2005), which may occur in MIS416-treated mice due to the elevated serum IFN- $\gamma$. Together, these studies show the potential for MIS416 to have CNS-specific neuroprotective effects.

\subsubsection{Summary}

In this chapter we have shown that MIS416 treatment significantly reduced CNS inflammation compared to that seen in untreated EAE mice. This reduction was evidenced by the significant reduction of infiltrating CD4+ cells and macrophages into the CNS, the reduced breakdown of the $\mathrm{BBB}$ and the decrease in severity of inflammatory lesions in MIS416-treated EAE mice compared to untreated mice. An unexpected finding was that small amounts of the MIS416 microparticle were found within the CNS, indicating the potential for TLR9 and NOD2 signalling to occur from within the CNS. Furthermore, MIS416 treatment induced IFN- $\gamma$-dependent CNS trafficking of macrophages and $\mathrm{CD} 4+\mathrm{T}$ cells in the absence of $\mathrm{EAE}$, and it is possible that these cells may play a regulatory role within the CNS and contribute to the mechanism of MIS416. 
Chapter 7: General Discussion 


\subsection{Overview}

MS is thought be an autoimmune disease of the CNS in which self-reactive lymphocytes cross the $\mathrm{BBB}$ and direct damage to the myelin sheath, creating inflammatory lesions and causing the symptoms seen in MS (Vinay Kumar \& Stanley Leonard Robbins, 2007). There are a range of approaches used to treat MS including treatment of the individual disease symptoms, immunosuppressive therapies, and disease-modifying treatments, which target specific immune pathways driving disease. The disease modifiers include inhibitors of migration, such as natalizumab, which blocks the entry of lymphocytes into the CNS (Clifford et al., 2010), and also immune modifiers such as GA and IFN- $\beta$ which alter the immune environment to reduce MS disease pathology (O. A. Khan et al., 2001).

The innate immune system is thought to play a pivotal role in MS pathogenesis, particularly in progressive disease, and therefore targeting innate cell receptors has recently been recognised as a potential therapeutic strategy (Mayo, Quintana, \& Weiner, 2012). MIS416 is a microparticle which targets myeloid cells by activating cytosolic receptors TLR9 and NOD2, and has completed a phase $1 \mathrm{~b} / 2 \mathrm{a}$ trial for the treatment of secondary progressive MS with positive outcomes (White et al., 2014). Using EAE, a model of MS, we investigated the pathways in which activating TLR9 and NOD2 in myeloid cells altered the innate immune environment and how this alteration was able to reduce $\mathrm{T}$ cell-mediated autoimmune responses.

Previous studies had shown that PRR receptors, such as TLR9 and NOD2, can play both detrimental and beneficial roles in the EAE model (Marta et al., 2008; Schrijver et al., 2001; Tsunoda et al., 1999). In this study, we found that targeting TLR9 and NOD2 with MIS16 in weekly doses was effective in C57BL/6 and SJL mice induced with EAE as indicated by reduced CNS lesions and disease scores. The disease protection induced in EAE mice relied on TLR9 signalling for treatment with the HT-MIS416 (i.e. only stimulates NOD2 pathways) did not suppress EAE disease. Moreover, studies from Castellaneta et al have shown that dual TLR9 and NOD2 signalling induces a more tolerogenic state than stimulation with either NOD2 or TLR9 alone, and therefore it is possible that MIS416 relies on activation of both pathways to exert its actions in EAE. However, in this study we did not assess disease protective effects in the absence of NOD2 signalling as this is difficult to do given the MDP (i.e NOD2 agonist) is a component in the structure of the microparticle therefore, we cannot conclude whether NOD2 signalling is essential for MIS416-induced disease suppression.

In the current study it was found that MIS416 treatment induced a number of peripheral immune changes which had the potential to alter a $\mathrm{T}$ cell-mediated autoimmune disease. The central factor 
identified was IFN- $\gamma$ (White et al., 2014). High levels of IFN- $\gamma$ were found in the supernatants of splenocyte and liver leukocyte cultures after stimulation with MIS416 and high levels were also found in the serum of MIS416-treated mice (Chapter 4); it was determined that CD4+ T cells, NK cells and macrophages were producers of IFN- $\gamma$ in response to MIS416 (Chapter 4). The importance of MIS416-induced IFN- $\gamma$ was determined in IFN- $\gamma^{-/-}$mice which, when immunised for EAE and treated with MIS416, did not show reduced disease unlike WT controls (Chapter 5). The disease protective role of IFN- $\gamma$ was surprising given that IFN- $\gamma$ monotherapy in relapsingremitting MS patients appeared to have detrimental effects (Panitch et al., 1987); although it was later suggested by Sriram et al that the apparent worsening in these patients could have been due to pseudo-relapses induced by IFN- $\gamma$-enhancing the pre-existing MS-associated inflammation in these patients (Panitch et al., 1987; Sriram \& Steiner, 2005). In contrast to the human monotherapy study, IFN- $\gamma$ can play a protective role in the EAE model given that IFN- $\gamma^{-/}$mice suffer more severe disease symptoms than WT mice (I A Ferber et al., 1996), suggesting IFN- $\gamma$ may be required for disease resolution.

The protective role of MIS416-induced IFN- $\gamma$ may be mediated through downstream effects since our study has shown that MIS416 treatment induced many peripheral immune changes, and in the absence of IFN- $\gamma$ some of these changes were not seen. MIS416 treatment induced the expansion of Tregs (White et al., 2014) and these Tregs were able to suppress T cell proliferation to a higher extent than Tregs from untreated mice (Chapter 5). There is evidence in the literature which suggests that IFN- $\gamma$ can drive the production of Tregs (Wang et al., 2006), and this idea was supported by a reduced expansion of Tregs in MIS416-treated IFN- $\gamma^{-/-}$mice compared to MIS416treated WT mice (Chapter 5). IFN- $\gamma$-induced Tregs may have a key role in the mechanism of MIS416 in EAE since EAE mice treated with additional Tregs had reduced disease severity (Adam P. Kohm et al., 2002).

IFN- $\gamma$-induced expression of PD-L1 may be another way in which MIS416 treatment reduces EAE. We have shown that IFN- $\gamma^{-/}$mice did not upregulate PD-L1 expression in response to MIS416 treatment, unlike MIS416-treated WT mice which had increased expression of PD-L1 on myeloid cells and Tregs (Chapter 4 and 5). A study by Latchman et al has shown that in the EAE model PD-L1 expression is a suppressive pathway for regulating disease severity (Latchman et al., 2004). In conjunction with PD-L1 expression our work has also identified that MIS416 treatment expanded a population of myeloid cells expressing markers of MDSCs (White et al., 2014), and that this population of MDSCs was able to suppress $\mathrm{T}$ cell proliferation to a higher extent than MDSCs from untreated mice (Chapter 5). Although this suppression was shown to be independent 
of NO, it is possible that PD-L1 was involved considering these cells expressed higher levels than MDSCs from untreated mice (Chapter 4).

This study also revealed a potential role for macrophages in mediating the protective effects of MIS416 and in an IFN- $\gamma$-dependent fashion given that IFN- $\gamma^{-/-}$mice treated with MIS416 did not have the expansion of macrophages in the spleen and liver, unlike MIS416-treated WT mice (Chapter 5). Given that macrophages are known to be very plastic and, like dendritic cells, can direct adaptive immune responses (Stout \& Suttles, 2004), the increased number of these cells could be part of the mechanism by which MIS416 treatment protects against EAE. Furthermore, macrophages are prime producers of $\mathrm{NO}$ and given that IFN- $\gamma^{-{ }_{-}^{-}}$mice did not have increased levels of NO with MIS416 treatment, the lack of macrophage expansion could explain this finding. Another explanation for low NO levels in IFN- $\gamma^{-/-}$mice could be that the release of NO is IFN- $\gamma-$ mediated (Albina et al., 1991; Ding, Nathan, \& Stuehr, 1988), and therefore without IFN- $\gamma$, minimal NO was produced. Additionally, in our study we have shown that both NO and IFN- $\gamma$ play a role in the MIS416-induced suppression of $\mathrm{T}$ cell proliferation ex vivo (Chapter 5), indicating these secreted factors may also play a role in vivo by suppressing $\mathrm{T}$ cell proliferation during EAE; and suppressed $\mathrm{T}$ cell proliferation in vivo could reduce EAE disease severity by limiting the number of auto-reactive lymphocytes available to enter the CNS and direct myelin damage. Interestingly, we have shown that depleting NO during the later stages of disease (i.e. after day 12) did not affect MIS416-induced disease protection (Chapter 5). However, as we have also shown that auto-reactive $\mathrm{T}$ cell proliferation occurs during early stages of EAE disease (i.e day 0-5; Chapter 5), it is possible that MIS416-induced NO does have a role in disease protection at earlier stages, and this was not detected due to NO depletion being assessed only during the later stages of disease. Overall our results suggested that IFN- $\gamma$-dependent NO release may play a part in the MIS416 mechanism in EAE during the early T cell proliferation stages, and may not play an important role during the later stages of disease. Furthermore, given that we have shown that when MIS416 is administered after disease onset a reduction in disease is observed (Chapter 3), MIS416 treatment may rely on more than one mechanism to exert its action in EAE.

MIS416 treatment not only altered the peripheral immune system but also altered trafficking into the CNS. It was found that MIS416-treated EAE mice had reduced CNS infiltration as measured by both flow cytometry and histology; furthermore, there was a reduction in the EAE-induced permeability of the BBB in MIS416-treated mice (Chapter 6). These results were consistent with the reduction in EAE disease scores in MIS416-treated mice and were similar to effects seen with IFN- $\beta$, another immune modulator approved for use in MS (Yong, 2002). Interestingly, in nonEAE mice MIS416 treatment induced the trafficking of CD11b ${ }^{+}$(i.e myeloid) and CD4+ T cells 
into the CNS, albeit to a lesser extent than the cellular trafficking seen with EAE immunisation (Chapter 6). It was also determined that the BBB was not degraded due to MIS416 treatment alone, indicating MIS416 treatment enhances the natural immune surveillance in the brain and allows cells to enter in the absence of inflammation (Ousman \& Kubes, 2012). Our study also determined that MIS416-induced CNS trafficking occurred in an IFN- $\gamma$-dependent manner and, given that IFN- $\gamma$ can upregulate the expression of trafficking molecules in the choroid plexus (Kunis et al., 2013), this pathway may be one of the ways MIS416 treatment enhanced cellular trafficking. Furthermore, we believe the cells which enter the CNS may have beneficial roles in EAE, given our findings in the peripheral immune system, as these cells may also be able to suppress $\mathrm{T}$ cell responses from within the CNS.

The work outlined in the current study have identified that MIS416 is successfully taken up by phagocytic cells (i.e macrophages) and these MIS416-positive cells were found within the blood, liver and to some extent the CNS 24-hours after an i.v. dose. Furthermore, MIS416 treatment resulted in alterations of cellular composition and activation state in the liver and spleen which ultimately induced an immunoregulatory state capable of suppressing $\mathrm{T}$ cell proliferation and EAE disease. Surprisingly, it was found that IFN- $\gamma$ appeared to be a key secreted factor behind many of the MIS416-induced changes including increased Treg and macrophage numbers, nitric oxide production and non-EAE CNS trafficking. Overall the results from this study have highlighted some of the immune modulations induced by MIS416 which have not only identified potential mechanisms for MIS416 treatment in MS patients, but also indicated that in the right context, a pro-inflammatory cytokine such as IFN- $\gamma$ may be protective in an autoimmune disease.

\subsection{Comparisons of MIS416 treatment between mouse and human}

EAE is a commonly used model for assessing potential MS treatments; however, some aspects, such as spontaneity are not accounted for. EAE is a CD4+ T cell-mediated disease which can be induced in animals by immunising with a myelin specific protein or peptide with an immune adjuvant (Kuchroo \& Weiner, 1998) or by adoptively transferring myelin specific T cells from an immunised animal into a healthy recipient (Kuchroo et al., 1992). These forms of EAE follow the outside-in theory of MS and therefore rely on the disease being initially driven by T cells, which is thought to occur in a subset of MS patients (H. L. Weiner, 2009). Mice are often used in EAE experiments as they show strain-specific differences in disease pathology and susceptibility to particular antigens, which make them useful models in understanding different aspects of the human disease (S. D. Miller \& Karpus, 2007). For example the SJL strain of mice are susceptible to PLP, MOG and MBP and often exhibit a relapsing-remitting form of the disease (Kono et al., 1988; S. D. Miller \& Karpus, 2007); which is therefore useful to study T cell-mediated 
demyelination. Alternatively, the C57BL/6 strain of mice are often induced with MOG peptide plus adjuvant and develop a severe chronic-progressive form of EAE (Bernard et al., 1997), making this strain useful to model progressive forms of MS. By taking advantage of this range of disease manifestations in the EAE model, researchers are able to study different aspects of MS disease pathology.

Although EAE is not a perfect model of MS, many of the current front-line therapeutics for MS have utilized EAE at some stage during their development, and all have shown efficacy within the model (L. Steinman \& Zamvil, 2005). In the current study we used the EAE model to determine efficacy of MIS416 in two different strains of EAE mice (i.e. SJL and C57BL/6), and it was found that MIS416 treatment reduced disease when given either therapeutically or prophylactically. Considering that MIS416 treatment was already in phase 1b/2a trial when our study began, the aim was not just to show efficacy in the EAE model but to determine an effective dosing regimen for MIS416 which would help us identify the immune mechanisms involved in this novel disease modifying treatment and how these mechanisms relate to effects seen in MIS416-treated secondary-progressive MS patients.

Our studies determined that MIS416-induced IFN- $\gamma$ was a key factor responsible for the immunemediated disease reduction in EAE mice and it has also been shown that MS patients treated with MIS416 had dose-dependently elevated IFN- $\gamma$ levels (White et al., 2014). In this study, secondaryprogressive MS patients were enrolled for a phase $1 \mathrm{~b} / 2 \mathrm{a}$ dose confirmation trial (see White et al for study design details), and their serum proteins were compared to pre-MIS416 treatment levels. In these MS patients MIS416 was reported to be well tolerated with minor adverse symptoms such as headache, fatigue and myalgia; these symptoms were only transient and to be expected considering the immunostimulatory nature of MIS416. Adverse symptoms are harder to identify in the mouse, however we did not see any food aversion, behavioural changes, or excessive weight loss after treatment, indicating MIS416 treatments were well tolerated. Consistent with what we have found in the mouse studies, IFN- $\gamma$ was increased at 24-hours post-treatment, and patients who received a higher dose of MIS416 produced more IFN- $\gamma$ than those who received a lower dose (White et al., 2014). IP-10 and CD54 (IFN- $\gamma$-associated proteins) were also upregulated while nonassociated proteins were unaffected (White et al., 2014). These findings demonstrate that in both mice and humans MIS416 induced elevated levels of IFN- $\gamma$ and associated proteins, which may be central to the mechanism of disease reduction.

As already mentioned, exogenous IFN- $\gamma$ treatment in relapsing-remitting patients was found to exacerbate disease symptoms (Panitch et al., 1987) and although it was later suggested by Sriram et al that the apparent worsening in these patients could have been due to pseudo-relapses (Panitch 
et al., 1987; Sriram \& Steiner, 2005), it is possible that the location and context of IFN- $\gamma$ is equally important. In contrast to the IFN- $\gamma$ therapy study, the MS patients assessed in the MIS416 phase $1 \mathrm{~b} / 2 \mathrm{a}$ study were secondary progressive, and therefore in these patients the disease is likely to no longer be $\mathrm{T}$ cell dependent but innate cell driven (H. L. Weiner, 2008), which may affect the outcome of additional IFN- $\gamma$. Another difference between the two studies could be the context and location of the cytokine, given that systemic cytokine monotherapy will result in the lack of cellular targeting and potential for "off target" effects, unlike MIS416 which targets PRR receptors within phagocytic cells which may result in endogenous as well as exogenous cytokine changes. Additionally, it was found in the mouse studies that CD4+ T cells, macrophages and NK were the main source of IFN- $\gamma$ after splenocyte re-stimulation with MIS416 (Chapter 4) and since MIS416 was found to deposit within the liver (Chapter 3), a rich source of NK cells, it is likely that systemic IFN- $\gamma$ comes from these cells suggesting that the context is very different to IFN- $\gamma$ monotherapy.

MIS416-treated mice had significantly increased trafficking of both myeloid and $\mathrm{T}$ cells into the CNS in the absence of EAE, and this migration was shown to be IFN- $\gamma$-dependent. Interestingly, the assessment of adhesion molecules on peripheral blood mononuclear cells (PBMCs) from MIS416-treated patients showed that 24-hours after treatment the expression of CX3CL1, CXCL10, V-CAM-1 and I-CAM-1 were all upregulated (personal communication, Gill Webster, IIT). Although the upregulation of these adhesion molecules was not maintained 7-days posttreatment, this finding shows that cellular trafficking can occur in these patients at least 1-day post-treatment and the cells, which are trafficking to the CNS, could potentially have MS diseasemodifying effects. These results were consistent with MIS416-induced CNS trafficking in our mouse study.

It has also been shown that in humans MIS416 treatment deviates monocyte activation away from a pro-inflammatory state into a more M2-like state (personal communication, Gill Webster, IIT), and while monocytes were not studied in the mice, we did see alterations in the macrophage population. For example, macrophages in the spleens of MIS416-treated mice upregulated PD-L1 and MHC-II expression and were thought to be producers of NO, IL-10 and IFN- $\gamma$ in our study. The regulatory nature of macrophages/monocytes were not directly assessed in either the mouse or human studies however, these cells have the potential to reduce EAE/MS disease through the suppression of $\mathrm{T}$ cell responses or possibly through the promotion of remyelination and repair (Ravid Shechter \& Schwartz, 2013; B. Zhu et al., 2007).

The MIS416 clinical trials were on secondary-progressive MS patients, and there has been evidence to suggest that the immunology behind secondary-progressive MS is quite different from 
relapsing-remitting disease (H. L. Weiner, 2008). For example, relapsing-remitting MS is thought to be due to the reactivation of $\mathrm{T}$ cells, either in the CNS or periphery, which then recruit macrophages and other innate cells to direct damage and cause disease (L. Steinman, 2001). On the other hand, secondary-progressive MS is thought to be mostly innate cell driven $(\mathrm{H}$. L. Weiner, 2008). This difference may explain why current therapies, which mostly target peripheral $\mathrm{T}$ cell responses or migration, are ineffective in secondary progressive patients, creating a need for novel therapeutics, such as MIS416, to treat progressive MS. As our studies have shown that MIS416 is able to induce CNS-specific alterations such as trafficking and as MIS416 targets innate cells, these factors may explain why MIS416 appears to be effective in the progressive stages of the disease. Our studies in the mouse have shown that MIS416 may be effective in both the relapsingremitting and progressive forms of the disease; however, as very little therapeutic treatments are available for secondary-progressive patients, it was important to determine the mechanisms of MIS416 which may be relevant for the progressive forms of MS disease.

The method of MIS416 treatment (i.e. weekly infusions i.v.) is a potential downfall for a novel MS therapeutic due to the lack of mobility in these patients which may make hospital visits difficult. Therefore, we determined if MIS416 treatment was able to alter the local and/or the systemic immune profile of mice when given orally (see Appendix 5). The rationale behind this work was that MIS416 deposited within the liver of mice after an i.v. dose, and also appeared to alter the immunological response of cells from the liver which may have contributed to the disease-altering effects seen with MIS416 treatment. As the liver is a known tolerogenic organ in which gutderived antigens are often expressed (Lunz, Specht, Murase, Isse, \& Demetris, 2007; Tiegs \& Lohse, 2010), there was potential for oral doses of MIS416 to induce a disease protective effect similar to that seen with i.v. MIS416. Our preliminary results outlined in a small results chapter in Appendix 5 determined that using an oral dose of $100 \mu \mathrm{g}$ of MIS416 daily did not have any effect on the spleen and liver cell counts or activation state, unlike what has been shown with i.v MIS416 treatment (Appendix 5). However, evidence suggests that the dosage required orally must be much higher than i.v. considering that some of the product will be degraded and pass through the gut without travelling via the portal vein to the liver (H. L. Weiner, 2000). When we assessed oral MIS416 at much higher doses of $2 \mathrm{mg}$ daily, it was found that there were slight changes in cellular proportions within the local and systemic organs. For example the spleen had a slight reduction in the percentage of B220+ cells compared to untreated mice, and in the MLN (i.e. the mesenteric lymph node, draining LN for the gut) and blood there was a significant reduction in the proportion of CD4+ T cells compared to untreated mice. Furthermore, this trend was also found in the liver although it did not reach statistical significance (see Appendix 5). 
There were also slight increases in PD-L1 expression on myeloid populations from the spleen of oral-treated MIS416 mice which, although they did not reach statistical significance, indicated a similar immunological state was created with oral MIS416 as seen with i.v. MIS416 administration (see Appendix 5). Interestingly, the NO and IFN- $\gamma$ production in response to MIS416 and ConA was also altered with oral MIS416 administration. However, in contrast to i.v. administration, there was a reduction in both NO and IFN- $\gamma$ with MIS416 and ConA stimulation compared to untreated mice. The splenocyte cytokine data together with the cellular alteration results indicate that oral administration of MIS416 is able to alter systemic responses although the alterations were not fully consistent with i.v. MIS416-treated mice. Therefore, whether these alterations are disease protective are unclear as yet. Evidence in the literature suggests that alterations in the gut microbiota, i.e. altered PRR signalling, can alter EAE disease (Bhargava \& Mowry, 2014; Ochoa-Repáraz, Mielcarz, Begum- Haque, \& Kasper, 2011); therefore, given that we are stimulating PRRs with a bacterial-derived microparticle, it is possible that oral MIS416 administration may harness a similar pathway to alter EAE disease and potentially MS.

\subsection{Future directions}

The work in this thesis investigated the mechanisms involved in MIS416-induced disease reduction in EAE mice with the goal of understanding how MIS416 treatment is an effective treatment in secondary-progressive MS. Results from this study identified that IFN- $\gamma$ and IFN- $\gamma$ dependent effects may have a central role in the reduced disease severity of MIS416-treated EAE mice. The idea that IFN- $\gamma$ may also be central to the mechanism of MIS416 in human patients was highlighted by the elevated levels of IFN- $\gamma$, as well as IFN- $\gamma$ associated proteins, after treatment in secondary-progressive MS patients (White et al., 2014). While we identified several key ways in which MIS416-induced IFN- $\gamma$ may be important in EAE, there are several questions which need addressing to further validate some of the conclusions this study generated.

It has been shown that MIS416 treatment induced a number of peripheral immune effects, which may be important in suppressing MOG-specific T cell responses in the periphery and ultimately may suppress EAE disease. MIS416-treated mice had an increased frequency and function of Tregs compared to untreated mice and the expansion of these cells appeared to involve IFN- $\gamma$. Considering that Tregs have been shown to be able to suppress EAE (Richard A. O'Connor \& Anderton, 2008), the determination of whether MIS416 treatment is able to suppress disease in a Treg-independent manner (i.e. after Treg depletion) would identify if Tregs are a key player in the mechanism of MIS416. It would also be interesting to show that MIS416 induction of Tregs was IFN- $\gamma$ dependent as suggested by Wang et al, which showed that IFN- $\gamma$ treatment can induce the transition of CD4+ cells into functional Tregs (Wang et al., 2006). This study could be done by 
isolating CD4+CD25-FoxP3- T cells from FoxP3GFP mice and injecting them into either IFN- $\gamma^{-/-}$ or WT mice and treating with MIS416. If MIS416 induced Treg expansion (as indicated by FoxP3GFP+) in WT and not IFN- $\gamma^{-/}$mice then we could conclude that Treg expansion in MIS416treated mice is not only IFN- $\gamma$-dependent, but also relies on the conversion of naive CD4+ T cells into Tregs.

The current thesis identified that NO and PD-L1, which were shown to be IFN- $\gamma$-dependently upregulated in MIS416-treated mice, may have a role in the suppression of $\mathrm{T}$ cell responses in the periphery. Firstly, NO was shown to suppress $\mathrm{T}$ cell responses ex vivo but when depleted in vivo during disease onset there was no effect on MIS416-induced disease suppression. However, it is possible that NO could be a key part of the mechanism of MIS416 during early stages of EAE disease, as we have shown that a lot of peripheral $\mathrm{T}$ cell proliferation occurs at this time (Chapter 5), therefore it would be interesting to deplete NO production earlier on to determine if MIS416induced suppression of $\mathrm{T}$ cell proliferation is ablated. Additionally, NO depletion could be assessed in EAE mice which receive MIS416 treatment after disease onset (i.e. day 12) which would indicate whether MIS416-induced NO plays a role in disease reduction at the later stages of disease. PD-L1 is an anti-proliferative ligand which can suppress $\mathrm{T}$ cell responses and is involved in the regulation of EAE severity (Carter et al., 2007; Shi et al., 2013). The role of PD-L1 upregulation in MIS416 treatment needs to be assessed further, since it has been shown that PD-L1 expression is not only upregulated on myeloid cells but on Tregs as well. This experiment could be done by inhibiting PD-L1 both in vivo and ex vivo using antibodies specific for either PD-L1 or PD-1, which will block the signalling of this anti-proliferative pathway, and determine whether MIS416 treatment is able to suppress T cell proliferation in the absence of PD-1 signalling.

Given that our studies have indicated that MIS416 treatment induces the expression of antiproliferative factors like NO and PD-L1, the effect these factors have on disease may be due to the active immunisation used to induce EAE. Therefore, further studies using adoptive transfer of encephalogenic T cells to induce EAE will eliminate the need for antigen presentation to occur (C. S. Constantinescu et al., 2011), and may help identify whether treatment with MIS416 relies on APCs presenting myelin antigen to $\mathrm{T}$ cells. Additionally as it has been shown that Tregs are expanded with MIS416 treatment and while it was unclear whether Tregs in MIS416-treated EAE mice were MOG-specific, by using adoptively transferred EAE, the potential for inducing MOGspecific Tregs during Ag presentation would be eliminated.

An interesting finding in this thesis was the increased trafficking of myeloid and CD4+ T cells into the CNS in the absence of disease, which was not associated with an increased permeability of the BBB. Therefore, further characterisation of the types of cells which traffic to the CNS after 
MIS416 treatment will help us to understand CNS-specific mechanisms in both EAE and MS. In the current study it was determined that with MIS416 treatment there was an expansion of immunoregulatory-type cells, such as the MDSCs and Tregs (White et al., 2014), and if these were the cells entering the CNS they may act to suppress inflammation from within, a mechanism which has been postulated for GA treatment (Aharoni et al., 2010). These experiments would be crucial to understand how MIS416 may be effective in an innate-driven form of MS given that other immune modifying treatments which target $T$ cells are ineffective against this type of disease (D. H. Miller \& Leary, 2007). To do this, we could assess the expression of migratory markers, such as CCR2 and CX3CR1 (Prinz \& Priller, 2010), on the surface of potential suppressor populations in the blood of MIS416-treated mice. Additionally it would be important to isolate and characterise the functional nature of cells which enter the CNS after MIS416 treatment by using flow cytometry and transcriptome analysis to determine the markers expressed by these cells.

The EAE model was used to determine MIS416 mechanisms of action; however, in this model the disease is driven by the peripheral immune system and requires the breakdown of the $\mathrm{BBB}$ to cause disease (Bennett et al., 2010). Given that the mechanisms between relapsing-remitting and progressive disease are likely to be different (i.e. progressive disease is innate-driven from within the CNS) (H. L. Weiner, 2009), it would be interesting to assess the effect of MIS416 treatment on demyelination induced without BBB breakdown or peripheral immune involvement. This could be done using cuprizone, a copper chelator given in mouse chow (Matsushima \& Morell, 2001). Cuprizone induces a toxic model of demyelination which acts independently of MHC expression (Hiremath, Chen, Suzuki, Ting, \& Matsushima, 2008) allowing the process of demyelination to occur without the involvement of the adaptive immune system unlike the EAE model. After demyelination occurs and the cuprizone has been removed from the diet, spontaneous remyelination occurs (Matsushima \& Morell, 2001). Therefore, it would be possible to assess whether MIS416 treatment reduces demyelination and/or promotes remyelination in this model. Therapies which can promote remyelination would be beneficial to complement current disease modifying treatments used in MS and may be useful in other diseases of the CNS.

Spinal cord injury (SCI) is another type of CNS-specific inflammation in which MIS416 treatment may be beneficial. After SCI there is an influx of immune cells, including neutrophils, macrophages and $\mathrm{T}$ cells, and these cells are thought to be the determinants of the injury and repair processes within the damaged site (Trivedi, Olivas, \& Noble-Haeusslein, 2006). Previously, SCIs were treated with corticosteroids, such as methylprednisolone, with the hope that by suppressing the initial inflammatory response of the SCI the damage will be lessened (Kwon, Tetzlaff, Grauer, Beiner, \& Vaccaro, 2004). Recently the idea of using immune modifying treatments in SCI has 
started to emerge, with the idea of giving immune based vaccines to speed up the injury repair mechanisms becoming a widely accepted concept (Hauben \& Schwartz, 2003). It was shown by Shechter et al that monocyte-derived macrophages enter the CNS after SCI in the mouse, where they contribute to recovery by mediating immune responses through the release of IL-10 (Ravid Shechter et al., 2009). More recently, it was shown that these infiltrating macrophages were of the M2 phenotype $\left(\right.$ Ly $6 C^{\text {low }} \mathrm{CX} 3 \mathrm{CR} 1^{\text {high }}$ ), which migrated through the choroid-plexus, and when the migration of these cells was inhibited in SCI, there was impaired motor-function recovery (R. Shechter et al., 2013). Given that we have shown MIS416 treatment induced CNS trafficking and promoted a more regulatory phenotype in peripheral macrophages (i.e. shown to produce IL-10 and express PD-L1), there may be potential for MIS416 treatment to be therapeutic in SCI and should be investigated further.

MIS416 treatment may also be beneficial in other disease settings given that the weekly doses of MIS416 induced a range of peripheral alterations capable of altering $\mathrm{T}$ cell responses. There are several autoimmune conditions in which systemic administration of MIS416 may be beneficial such as type-1 diabetes, systemic lupus erythematosus (SLE) or rheumatoid arthritis (RA). Given that these conditions also have limited therapeutic treatments available, it would be beneficial to test the efficacy of MIS416 in these diseases. Considering that MIS416 treatment appears to be effective in MS, the use of one treatment for several autoimmune diseases is not uncommon. For example it has been shown in about two thirds of patients with RA, Crohn's disease and psoriasis that the blockade of tumour necrosis factor (TNF) is an effective treatment; likewise, anti-IL-12 therapy has shown potential in RA, Crohn's disease and psoriasis as well (L. Steinman, Merrill, McInnes, \& Peakman, 2012).

In this study we have also provided evidence to suggest that MIS416 may be effective in Crohn's disease and other forms of irritable bowel disease (IBD). It was shown that oral dosing of MIS416 was able to induce systemic effects and slight changes were found locally within the gut (see appendix 5). As shown in appendix 5 oral dosing of MIS416 did not induce local inflammation or cellular infiltration into the gut (small intestine and colon were examined), however there were slight changes in the mesenteric LN and earlier studies indicated an increased number and size of Peyers patches in oral MIS416-treated mice (data not shown). Considering that we have shown MIS416 can induce a tolerogenic immune state and also that the gut microbiome and PRR signalling can have a major effect on IBD (Reiff \& Kelly, 2010), it is possible that with either oral or systemic MIS416 treatment, the severity of IBD may be reduced. A future direction for broad MIS416 immunotherapy should therefore include the use of the dextran sulfate sodium (DSS) 
model of colitis (Low, Nguyen, \& Mizoguchi, 2013) with oral MIS416 treatment to determine if MIS416 could be a potential therapeutic for IBD.

In conclusion, the results from this thesis have clearly outlined potential mechanisms by which MIS416, a dual agonist targeting TLR9 and NOD2, can protect against EAE. In particular, IFN- $\gamma$ appeared to be a key immune modulating factor which induced alterations of both innate cells and $\mathrm{T}$ cells within the periphery, and these alterations favoured an immunoregulatory phenotype. Although MIS416 treatment reduced CNS infiltration and BBB permeability in EAE mice, there was increased cellular trafficking into the CNS in the absence of EAE, which may be due to the migration of immunoregulatory cells into the CNS. Further work is needed to confirm this possibility. Finally, the findings from this thesis show that MIS416 treatment may have potential in other diseases in addition to MS and also indicate that in the right context, a pro-inflammatory cytokine such as IFN- $\gamma$ may be protective in autoimmune disease. 



\section{Chapter 8: References}

Abbas, A. K., Murphy, K. M., \& Sher, A. (1996). Functional diversity of helper T lymphocytes. Nature, 383(6603), 787-793.

Abraham, S. N., \& St. John, A. L. (2010). Mast cell-orchestrated immunity to pathogens. Nat Rev Immunol, 10(6), 440-452.

Aharoni, Eilam, Stock, Vainshtein, Shezen, Gal, . . Arnon. (2010). Glatiramer acetate reduces Th-17 inflammation and induces regulatory T-cells in the CNS of mice with relapsingremitting or chronic EAE. J Neuroimmunol, 225(1-2), 100-111. doi: 10.1016/j.jneuroim.2010.04.022

Aharoni, R. (2014). Immunomodulation neuroprotection and remyelination - The fundamental therapeutic effects of glatiramer acetate: A critical review. J Autoimmun, 54(0), 81-92. doi: http://dx.doi.org/10.1016/j.jaut.2014.05.005

Aharoni, R., Kayhan, B., Eilam, R., Sela, M., \& Arnon, R. (2003). Glatiramer acetate-specific $\mathrm{T}$ cells in the brain express $\mathrm{T}$ helper $2 / 3$ cytokines and brain-derived neurotrophic factor in situ. Proceedings of the National Academy of Sciences of the United States of America, 100(24), 14157-14162. doi: 10.1073/pnas.2336171100

Aharoni, R., Teitelbaum, D., Leitner, O., Meshorer, A., Sela, M., \& Arnon, R. (2000). Specific Th2 cells accumulate in the central nervous system of mice protected against experimental autoimmune encephalomyelitis by copolymer 1. Proceedings of the National Academy of Sciences of the United States of America, 97(21), 11472-11477. doi: 10.1073/pnas.97.21.11472

Akira, S., \& Takeda, K. (2004). Toll-like receptor signalling. Nat Rev Immunol, 4(7), 499-511.

Akira, S., Takeda, K., \& Kaisho, T. (2001). Toll-like receptors: critical proteins linking innate and acquired immunity. Nat Immunol, 2(8), 675-680. doi: 10.1038/90609

Albina, J. E., Abate, J. A., \& Henry, W. L. (1991). Nitric oxide production is required for murine resident peritoneal macrophages to suppress mitogen-stimulated $\mathrm{T}$ cell proliferation. Role of IFN-gamma in the induction of the nitric oxide-synthesizing pathway. The Journal of Immunology, 147(1), 144-148.

Amulic, B., Cazalet, C., Hayes, G. L., Metzler, K. D., \& Zychlinsky, A. (2012). Neutrophil function: from mechanisms to disease. Annu Rev Immunol, 30, 459-489. doi: 10.1146/annurev-immunol-020711-074942

Anderson, C. F., \& Mosser, D. M. (2002). A novel phenotype for an activated macrophage: the type 2 activated macrophage. Journal of leukocyte biology, 72(1), 101-106.

Antel, J. (2014). Mechanisms of action of fingolimod in multiple sclerosis. Clinical and Experimental Neuroimmunology, 5(1), 49-54. doi: 10.1111/cen3.12079

Appelberg, R. (2007). Neutrophils and intracellular pathogens: beyond phagocytosis and killing. Trends in Microbiology, 15(2), 87-92. doi: http://dx.doi.org/10.1016/j.tim.2006.11.009

Appleman, L. J., \& Boussiotis, V. A. (2003). T cell anergy and costimulation. Immunol Rev, 192, 161-180.

Apte, R. S., Sinha, D., Mayhew, E., Wistow, G. J., \& Niederkorn, J. Y. (1998). Cutting edge: role of macrophage migration inhibitory factor in inhibiting NK cell activity and preserving immune privilege. J Immunol, 160(12), 5693-5696.

Aranami, T., \& Yamamura, T. (2008). Th17 Cells and autoimmune encephalomyelitis (EAE/MS). Allergol Int, 57(2), 115-120. doi: 10.2332/allergolint.R-07-159

Ascherio, A., \& Munger, K. L. (2010). Epstein-barr virus infection and multiple sclerosis: a review. J Neuroimmune Pharmacol, 5(3), 271-277. doi: 10.1007/s11481-010-9201-3 
Ascherio, A., Munger, K. L., \& Simon, K. C. (2010). Vitamin D and multiple sclerosis. The Lancet Neurology, 9(6), 599-612. doi: http://dx.doi.org/10.1016/S14744422(10)70086-7

Bamboat, Z. M., Stableford, J. A., Plitas, G., Burt, B. M., Nguyen, H. M., Welles, A. P., ... DeMatteo, R. P. (2009). Human liver dendritic cells promote $T$ cell hyporesponsiveness. J Immunol, 182(4), 1901-1911. doi: 10.4049/jimmunol.0803404

Banham, A. H., Powrie, F. M., \& Suri-Payer, E. (2006). FOXP3+ regulatory T cells: Current controversies and future perspectives. European journal of immunology, 36(11), 28322836. doi: 10.1002/eji.200636459

Banwell, B., Ghezzi, A., Bar-Or, A., Mikaeloff, Y., \& Tardieu, M. (2007). Multiple sclerosis in children: clinical diagnosis, therapeutic strategies, and future directions. Lancet Neurol, 6(10), 887-902. doi: 10.1016/S1474-4422(07)70242-9

Bar-Or, A., Oliveira, E. M. L., Anderson, D. E., \& Hafler, D. A. (1999). Molecular pathogenesis of multiple sclerosis. Journal of Neuroimmunology, 100(1-2), 252-259. doi: http://dx.doi.org/10.1016/S0165-5728(99)00193-9

Barry, M., \& Bleackley, R. C. (2002). Cytotoxic T lymphocytes: all roads lead to death. Nat Rev Immunol, 2(6), 401-409.

Batista, F. D., \& Harwood, N. E. (2009). The who, how and where of antigen presentation to B cells. Nat Rev Immunol, 9(1), 15-27.

Bendelac, A., Savage, P. B., \& Teyton, L. (2007). The biology of NKT cells. Annu Rev Immunol, 25, 297-336. doi: 10.1146/annurev.immunol.25.022106.141711

Benhar, I., London, A., \& Schwartz, M. (2012). The privileged immunity of immune privileged organs: the case of the eye. Frontiers in Immunology, 3, 296. doi: 10.3389/fimmu.2012.00296

Bennett, J., Basivireddy, J., Kollar, A., Biron, K. E., Reickmann, P., Jefferies, W. A., \& McQuaid, S. (2010). Blood-brain barrier disruption and enhanced vascular permeability in the multiple sclerosis model EAE. Journal of Neuroimmunology, 229(1-2), 180-191. doi: http://dx.doi.org/10.1016/j.jneuroim.2010.08.011

Bernard, C. C., Johns, T. G., Slavin, A., Ichikawa, M., Ewing, C., Liu, J., \& Bettadapura, J. (1997). Myelin oligodendrocyte glycoprotein: a novel candidate autoantigen in multiple sclerosis. J Mol Med (Berl), 75(2), 77-88.

Bertolotto, A., Malucchi, S., Sala, A., Orefice, G., Carrieri, P., Capobianco, M., . . . Giordana, M. (2002). Differential effects of three interferon betas on neutralising antibodies in patients with multiple sclerosis: a follow up study in an independent laboratory. Journal of neurology, neurosurgery, and psychiatry, 73(2), 148-153. doi: 10.1136/jnnp.73.2.148

Bettelli, E., Korn, T., Oukka, M., \& Kuchroo, V. K. (2008). Induction and effector functions of TH17 cells. Nature, 453(7198), 1051-1057.

Bettelli, E., Pagany, M., Weiner, H. L., Linington, C., Sobel, R. A., \& Kuchroo, V. K. (2003). Myelin Oligodendrocyte Glycoprotein-specific T Cell Receptor Transgenic Mice Develop Spontaneous Autoimmune Optic Neuritis. The Journal of experimental medicine, 197(9), 1073-1081. doi: 10.1084/jem.20021603

Bettelli, E., Prabhu Das, M., Howard, E. D., Weiner, H. L., Sobel, R. A., \& Kuchroo, V. K. (1998). IL-10 Is Critical in the Regulation of Autoimmune Encephalomyelitis as Demonstrated by Studies of IL-10- and IL-4-Deficient and Transgenic Mice. The Journal of Immunology, 161(7), 3299-3306.

Bhargava, P., \& Mowry, E. (2014). Gut Microbiome and Multiple Sclerosis. Current neurology and neuroscience reports, 14(10), 1-8. doi: 10.1007/s11910-014-0492-2

Billiau, A., \& Matthys, P. (2001). Modes of action of Freund's adjuvants in experimental models of autoimmune diseases. Journal of leukocyte biology, 70(6), 849-860. 
Bilzer, M., Roggel, F., \& Gerbes, A. L. (2006). Role of Kupffer cells in host defense and liver disease. Liver International, 26(10), 1175-1186. doi: 10.1111/j.14783231.2006.01342.x

Biron, C. A., Nguyen, K. B., Pien, G. C., Cousens, L. P., \& Salazar-Mather, T. P. (1999). Natural killer cells in antiviral defense: function and regulation by innate cytokines. Annu Rev Immunol, 17, 189-220. doi: 10.1146/annurev.immunol.17.1.189

Blank, C., \& Mackensen, A. (2007). Contribution of the PD-L1/PD-1 pathway to T-cell exhaustion: an update on implications for chronic infections and tumor evasion. Cancer immunology, immunotherapy : CII, 56(5), 739-745. doi: 10.1007/s00262-0060272-1

Bluestone, J. A., Mackay, C. R., O'Shea, J. J., \& Stockinger, B. (2009). The functional plasticity of T cell subsets. Nat Rev Immunol, 9(11), 811-816.

Boster, A., Bartoszek, M. P., O’Connell, C., Pitt, D., \& Racke, M. (2011). Efficacy, safety, and cost-effectiveness of glatiramer acetate in the treatment of relapsing-remitting multiple sclerosis. Therapeutic Advances in Neurological Disorders, 4(5), 319-332. doi: $10.1177 / 1756285611422108$

Bronte, V., Serafini, P., Mazzoni, A., Segal, D. M., \& Zanovello, P. (2003). L-arginine metabolism in myeloid cells controls T-lymphocyte functions. Trends Immunol, 24(6), 301-305. doi: http://dx.doi.org/10.1016/S1471-4906(03)00132-7

Butchi, N. B., Woods, T., Du, M., Morgan, T. W., \& Peterson, K. E. (2011). TLR7 and TLR9 Trigger Distinct Neuroinflammatory Responses in the CNS. The American journal of pathology, 179(2), 783-794. doi: 10.1016/j.ajpath.2011.04.011

Butovsky, O., Talpalar, A. E., Ben-Yaakov, K., \& Schwartz, M. (2005). Activation of microglia by aggregated $\beta$-amyloid or lipopolysaccharide impairs MHC-II expression and renders them cytotoxic whereas IFN- $\gamma$ and IL-4 render them protective. Molecular and Cellular Neuroscience, 29(3), 381-393. doi: http://dx.doi.org/10.1016/j.mcn.2005.03.005

Calabresi, P. A., Pelfrey, C. M., Tranquill, L. R., Maloni, H., \& McFarland, H. F. (1997). VLA-4 expression on peripheral blood lymphocytes is downregulated after treatment of multiple sclerosis with interferon beta. Neurology, 49(4), 1111-1116.

Campbell, D. J., \& Koch, M. A. (2011). Phenotypical and functional specialization of FOXP3+ regulatory T cells. Nat Rev Immunol, 11(2), 119-130.

Carding, S. R., \& Egan, P. J. (2002). [gamma][delta] T cells: functional plasticity and heterogeneity. Nat Rev Immunol, 2(5), 336-345.

Carson, M. J., Doose, J. M., Melchior, B., Schmid, C. D., \& Ploix, C. C. (2006). CNS immune privilege: hiding in plain sight. Immunol Rev, 213, 48-65. doi: 10.1111/j.1600065X.2006.00441.X

Carter, L. L., Leach, M. W., Azoitei, M. L., Cui, J., Pelker, J. W., Jussif, J., . . Marusic, S. (2007). PD-1/PD-L1, but not PD-1/PD-L2, interactions regulate the severity of experimental autoimmune encephalomyelitis. Journal of Neuroimmunology, 182(1-2), 124-134. doi: http://dx.doi.org/10.1016/j.jneuroim.2006.10.006

Carty, M., \& Bowie, A. G. (2011). Evaluating the role of Toll-like receptors in diseases of the central nervous system. Biochem Pharmacol, 81(7), 825-837. doi: 10.1016/j.bcp.2011.01.003

Castellaneta, A., Sumpter, T. L., Chen, L., Tokita, D., \& Thomson, A. W. (2009). NOD2 ligation subverts IFN-alpha production by liver plasmacytoid dendritic cells and inhibits their $\mathrm{T}$ cell allostimulatory activity via B7-H1 up-regulation. J Immunol, 183(11), 6922-6932. doi: 10.4049/jimmunol.0900582

Cella, M., Scheidegger, D., Palmer-Lehmann, K., Lane, P., Lanzavecchia, A., \& Alber, G. (1996). Ligation of CD40 on dendritic cells triggers production of high levels of 
interleukin-12 and enhances T cell stimulatory capacity: T-T help via APC activation. The Journal of experimental medicine, 184(2), 747-752. doi: 10.1084/jem.184.2.747

Chambers, C. A., \& Allison, J. P. (1997). Co-stimulation in T cell responses. Curr Opin Immunol, 9(3), 396-404. doi: http://dx.doi.org/10.1016/S0952-7915(97)80087-8

Chauhan, V. S., Sterka, D. G., Furr, S. R., Young, A. B., \& Marriott, I. (2009). NOD2 plays an important role in the inflammatory responses of microglia and astrocytes to bacterial CNS pathogens. Glia, 57(4), 414-423. doi: 10.1002/glia.20770

Chavez-Galan, L., Arenas-Del Angel, M. C., Zenteno, E., Chavez, R., \& Lascurain, R. (2009). Cell Death Mechanisms Induced by Cytotoxic Lymphocytes. Cell Mol Immunol, 6(1), 15-25.

Chen, W., Jin, W., Hardegen, N., Lei, K.-j., Li, L., Marinos, N., . . . Wahl, S. M. (2003). Conversion of Peripheral CD4+CD25- Naive T Cells to CD4+CD25+ Regulatory T Cells by TGF- $\beta$ Induction of Transcription Factor Foxp3. The Journal of experimental medicine, 198(12), 1875-1886. doi: 10.1084/jem.20030152

Chun, J., \& Hartung, H.-P. (2010). Mechanism of Action of Oral Fingolimod (FTY720) in Multiple Sclerosis. Clinical neuropharmacology, 33(2), 91-101. doi: 10.1097/WNF.0b013e3181cbf825

Clifford, D. B., DeLuca, A., Simpson, D. M., Arendt, G., Giovannoni, G., \& Nath, A. (2010). Natalizumab-associated progressive multifocal leukoencephalopathy in patients with multiple sclerosis: lessons from 28 cases. The Lancet Neurology, 9(4), 438-446. doi: 10.1016/s1474-4422(10)70028-4

Cohen, J. A., Barkhof, F., Comi, G., Hartung, H.-P., Khatri, B. O., Montalban, X., . . Kappos, L. (2010). Oral Fingolimod or Intramuscular Interferon for Relapsing Multiple Sclerosis. New England Journal of Medicine, 362(5), 402-415. doi: doi:10.1056/NEJMoa0907839

Condamine, T., \& Gabrilovich, D. I. (2011). Molecular mechanisms regulating myeloidderived suppressor cell differentiation and function. Trends Immunol, 32(1), 19-25. doi: 10.1016/j.it.2010.10.002

Constantinescu, C., \& Hilliard, B. (2005). Adjuvants in EAE. In E. Lavi \& C. Constantinescu (Eds.), Experimental Models of Multiple Sclerosis (pp. 73-84): Springer US.

Constantinescu, C. S., Farooqi, N., O'Brien, K., \& Gran, B. (2011). Experimental autoimmune encephalomyelitis (EAE) as a model for multiple sclerosis (MS). Br J Pharmacol, 164(4), 1079-1106. doi: 10.1111/j.1476-5381.2011.01302.x

Corse, E., Gottschalk, R. A., \& Allison, J. P. (2011). Strength of TCR-peptide/MHC interactions and in vivo $\mathrm{T}$ cell responses. J Immunol, 186(9), 5039-5045. doi: 10.4049/jimmunol.1003650

Crispe, I. N., Giannandrea, M., Klein, I., John, B., Sampson, B., \& Wuensch, S. (2006). Cellular and molecular mechanisms of liver tolerance. Immunol Rev, 213, 101-118. doi: 10.1111/j.1600-065X.2006.00435.X

Crume, K. P., O'Sullivan, D., Miller, J. H., Northcote, P. T., \& La Flamme, A. C. (2009). Delaying the onset of experimental autoimmune encephalomyelitis with the microtubule-stabilizing compounds, paclitaxel and Peloruside A. Journal of leukocyte biology, 86(4), 949-958. doi: 10.1189/jlb.0908541

Cua, D. J., Sherlock, J., Chen, Y., Murphy, C. A., Joyce, B., Seymour, B., . . Sedgwick, J. D. (2003). Interleukin-23 rather than interleukin-12 is the critical cytokine for autoimmune inflammation of the brain. Nature, 421(6924), 744-748. doi: http://www.nature.com/nature/journal/v421/n6924/suppinfo/nature01355_S1.html

Curtis, M. M., \& Way, S. S. (2009). Interleukin-17 in host defence against bacterial, mycobacterial and fungal pathogens. Immunology, 126(2), 177-185. doi: 10.1111/j.1365-2567.2008.03017.x 
D'Orazio, T. J., \& Niederkorn, J. Y. (1998). A novel role for TGF-beta and IL-10 in the induction of immune privilege. J Immunol, 160(5), 2089-2098.

De Jager, P. L., Baecher-Allan, C., Maier, L. M., Arthur, A. T., Ottoboni, L., Barcellos, L., . . . Hafler, D. A. (2009). The role of the CD58 locus in multiple sclerosis. Proceedings of the National Academy of Sciences of the United States of America, 106(13), 52645269. doi: 10.1073/pnas.0813310106

den Haan, J. M. M., \& Kraal, G. (2012). Innate Immune Functions of Macrophage Subpopulations in the Spleen. J Innate Immun, 4(5-6), 437-445.

Desmedt, M., Rottiers, P., Dooms, H., Fiers, W., \& Grooten, J. (1998). Macrophages Induce Cellular Immunity by Activating Th1 Cell Responses and Suppressing Th2 Cell Responses. The Journal of Immunology, 160(11), 5300-5308.

Dhib-Jalbut, S., \& Marks, S. (2010). Interferon-beta mechanisms of action in multiple sclerosis. Neurology, 74 Suppl 1, S17-24. doi: 10.1212/WNL.0b013e3181c97d99

Dilek, N., Poirier, N., Hulin, P., Coulon, F., Mary, C., Ville, S., . . . Vanhove, B. (2013). Targeting CD28, CTLA-4 and PD-L1 Costimulation Differentially Controls Immune Synapses and Function of Human Regulatory and Conventional T-Cells. PloS one, 8(12), e83139. doi: 10.1371/journal.pone.0083139

Ding, A. H., Nathan, C. F., \& Stuehr, D. J. (1988). Release of reactive nitrogen intermediates and reactive oxygen intermediates from mouse peritoneal macrophages. Comparison of activating cytokines and evidence for independent production. The Journal of Immunology, 141(7), 2407-2412.

Duquette, P., Girard, M., Despault, L., Dubois, R., Knobler, R. L., Lublin, F. D., . . Taylor, W. (1993). Interferon Beta-1b Is Effective in Relapsing-Remitting Multiple-Sclerosis Clinical-Results of a Multicenter, Randomized, Double-Blind, Placebo-Controlled Trial. Neurology, 43(4), 655-661.

Dyment, D. A., Ebers, G. C., \& Dessa Sadovnick, A. (2004). Genetics of multiple sclerosis. The Lancet Neurology, 3(2), 104-110. doi: http://dx.doi.org/10.1016/S14744422(03)00663-X

El-behi, M., Rostami, A., \& Ciric, B. (2010). Current views on the roles of Th1 and Th17 cells in experimental autoimmune encephalomyelitis. J Neuroimmune Pharmacol, 5(2), 189-197. doi: 10.1007/s11481-009-9188-9

Elhofy, A., Kennedy, K. J., Fife, B. T., \& Karpus, W. J. (2002). Regulation of experimental autoimmune encephalomyelitis by chemokines and chemokine receptors. Immunol Res, 25(2), 167-175. doi: 10.1385/IR:25:2:167

Engelhardt, B. (2006). Molecular mechanisms involved in T cell migration across the bloodbrain barrier. J Neural Transm, 113(4), 477-485. doi: 10.1007/s00702-005-0409-y

Engelhardt, B., \& Ransohoff, R. M. (2012). Capture, crawl, cross: the T cell code to breach the blood-brain barriers. Trends Immunol, 33(12), 579-589. doi: 10.1016/j.it.2012.07.004

Espejo, C., Penkowa, M., Saez-Torres, I., Hidalgo, J., Garcia, A., Montalban, X., \& MartinezCaceres, E. M. (2002). Interferon-gamma regulates oxidative stress during experimental autoimmune encephalomyelitis. Experimental neurology, 177(1), 21-31.

Fairweather, D., \& Cihakova, D. (2009). Alternatively activated macrophages in infection and autoimmunity. $J$ Autoimmun, 33(3-4), 222-230. doi: http://dx.doi.org/10.1016/j.jaut.2009.09.012

Falcone, F. H., Haas, H., \& Gibbs, B. F. (2000). The human basophil: a new appreciation of its role in immune responses (Vol. 96).

Ferber, I. A., Brocke, S., Taylor-Edwards, C., Ridgway, W., Dinisco, C., Steinman, L., . . . Fathman, C. G. (1996). Mice with a disrupted IFN-gamma gene are susceptible to the induction of experimental autoimmune encephalomyelitis (EAE). The Journal of Immunology, 156(1), 5-7. 
Ferber, I. A., Brocke, S., Taylor-Edwards, C., Ridgway, W., Dinisco, C., Steinman, L., . . . Fathman, C. G. (1996). Mice with a disrupted IFN-gamma gene are susceptible to the induction of experimental autoimmune encephalomyelitis (EAE). J Immunol, 156(1), $5-7$.

Francisco, L. M., Sage, P. T., \& Sharpe, A. H. (2010). The PD-1 Pathway in Tolerance and Autoimmunity. Immunol Rev, 236, 219-242. doi: 10.1111/j.1600-065X.2010.00923.x

Francisco, L. M., Salinas, V. H., Brown, K. E., Vanguri, V. K., Freeman, G. J., Kuchroo, V. K., \& Sharpe, A. H. (2009). PD-L1 regulates the development, maintenance, and function of induced regulatory T cells. The Journal of experimental medicine, 206(13), 3015-3029. doi: 10.1084/jem.20090847

Friese, M. A., \& Fugger, L. (2009). Pathogenic CD8(+) T cells in multiple sclerosis. Ann Neurol, 66(2), 132-141. doi: 10.1002/ana.21744

Frohman, E. M., Racke, M. K., \& Raine, C. S. (2006). Multiple Sclerosis - The Plaque and Its Pathogenesis. New England Journal of Medicine, 354(9), 942-955. doi: doi:10.1056/NEJMra052130

Fu, R., Shen, Q., Xu, P., Luo, J., \& Tang, Y. (2014). Phagocytosis of Microglia in the Central Nervous System Diseases. Molecular Neurobiology, 49(3), 1422-1434. doi: 10.1007/s12035-013-8620-6

Fujie, H., Niu, K., Ohba, M., Tomioka, Y., Kitazawa, H., Nagashima, K., . . Numasaki, M. (2012). A distinct regulatory role of Th17 cytokines IL-17A and IL-17F in chemokine secretion from lung microvascular endothelial cells. Inflammation, 35(3), 1119-1131. doi: $10.1007 / \mathrm{s} 10753-011-9419-0$

Fujinami, R. S., von Herrath, M. G., Christen, U., \& Whitton, J. L. (2006). Molecular Mimicry, Bystander Activation, or Viral Persistence: Infections and Autoimmune Disease. Clinical Microbiology Reviews, 19(1), 80-94. doi: 10.1128/CMR.19.1.8094.2006

Gabrilovich, D. I., \& Nagaraj, S. (2009). Myeloid-derived suppressor cells as regulators of the immune system. Nat Rev Immunol, 9(3), 162-174. doi: 10.1038/nri2506

Gao, B., Radaeva, S., \& Park, O. (2009). Liver natural killer and natural killer T cells: immunobiology and emerging roles in liver diseases. Journal of leukocyte biology, 86(3), 513-528. doi: 10.1189/jlb.0309135

Ge, Y., Grossman, R. I., Udupa, J. K., Fulton, J., Constantinescu, C. S., Gonzales-Scarano, F., . . . Cohen, J. A. (2000). Glatiramer acetate (Copaxone) treatment in relapsingremitting MS: Quantitative MR assessment. Neurology, 54(4), 813-817. doi: 10.1212/wnl.54.4.813

Getts, D. R., Terry, R. L., Getts, M. T., Deffrasnes, C., Müller, M., van Vreden, C., . . King, N. J. C. (2014). Therapeutic Inflammatory Monocyte Modulation Using ImmuneModifying Microparticles. Science Translational Medicine, 6(219), 219ra217. doi: 10.1126/scitranslmed.3007563

Gibbings, D., \& Befus, A. D. (2009). CD4 and CD8: an inside-out coreceptor model for innate immune cells. Journal of leukocyte biology, 86(2), 251-259. doi: 10.1189/jlb.0109040

Gilden, D. H. (2005). Infectious causes of multiple sclerosis. The Lancet Neurology, 4(3), 195202. doi: http://dx.doi.org/10.1016/S1474-4422(05)01017-3

Girvan, R. C., Knight, D. A., O'Loughlin, C. J., Hayman, C. M., Hermans, I. F., \& Webster, G. A. (2011). MIS416, a non-toxic microparticle adjuvant derived from Propionibacterium acnes comprising immunostimulatory muramyl dipeptide and bacterial DNA promotes cross-priming and Th1 immunity. Vaccine, 29(3), 545-557. doi: $10.1016 /$ j.vaccine.2010.10.040 
Godfrey, D. I., Hammond, K. J. L., Poulton, L. D., Smyth, M. J., \& Baxter, A. G. (2000). NKT cells: facts, functions and fallacies. Immunol Today, 21(11), 573-583. doi: http://dx.doi.org/10.1016/S0167-5699(00)01735-7

Gordon, S. (2003). Alternative activation of macrophages. Nat Rev Immunol, 3(1), 23-35.

Greenstein, J. I. (2007). Current concepts of the cellular and molecular pathophysiology of multiple sclerosis. Developmental Neurobiology, 67(9), 1248-1265. doi: 10.1002/dneu.20387

Hafler, D. A., Compston, A., Sawcer, S., Lander, E. S., Daly, M. J., De Jager, P. L., . . . Hauser, S. L. (2007). Risk alleles for multiple sclerosis identified by a genomewide study. The New England journal of medicine, 357(9), 851-862. doi: 10.1056/NEJMoa073493

Hanahan, D. (1998). Peripheral-antigen-expressing cells in thymic medulla: factors in selftolerance and autoimmunity. Curr Opin Immunol, 10(6), 656-662.

Harris, D. P., Haynes, L., Sayles, P. C., Duso, D. K., Eaton, S. M., Lepak, N. M., . . Lund, F. E. (2000). Reciprocal regulation of polarized cytokine production by effector B and T cells. Nat Immunol, 1(6), 475-482. doi: http://www.nature.com/ni/journal/v1/n6/suppinfo/ni1200_475_S1.html

Harty, J. T., Tvinnereim, A. R., \& White, D. W. (2000). CD8+ T cell effector mechanisms in resistance to infection. Annu Rev Immunol, 18, 275-308. doi: 10.1146/annurev.immunol.18.1.275

Hauben, E., \& Schwartz, M. (2003). Therapeutic vaccination for spinal cord injury: helping the body to cure itself. Trends in Pharmacological Sciences, 24(1), 7-12. doi: Pii S0165-6147(02)00013-5

Doi 10.1016/S0165-6147(02)00013-5

Hayashi, T., Gray, C. S., Chan, M., Tawatao, R. I., Ronacher, L., McGargill, M. A., . . Corr, M. (2009). Prevention of autoimmune disease by induction of tolerance to Toll-like receptor 7. Proceedings of the National Academy of Sciences of the United States of America, 106(8), 2764-2769. doi: 10.1073/pnas.0813037106

Hemmer, B., Archelos, J. J., \& Hartung, H. P. (2002). New concepts in the immunopathogenesis of multiple sclerosis. Nat Rev Neurosci, 3(4), 291-301. doi: $10.1038 / \mathrm{nrn} 784$

Hibbs Jr, J. B., Taintor, R. R., Vavrin, Z., \& Rachlin, E. M. (1988). Nitric oxide: A cytotoxic activated macrophage effector molecule. Biochemical and Biophysical Research Communications, 157(1), 87-94. doi: http://dx.doi.org/10.1016/S0006-291X(88)800159

Hiremath, M. M., Chen, V. S., Suzuki, K., Ting, J. P. Y., \& Matsushima, G. K. (2008). MHC class II exacerbates demyelination in vivo independently of $\mathrm{T}$ cells. Journal of Neuroimmunology, 203(1), 23-32. doi: 10.1016/j.jneuroim.2008.06.034

Hofstetter, H. H., Ibrahim, S. M., Koczan, D., Kruse, N., Weishaupt, A., Toyka, K. V., \& Gold, R. (2005). Therapeutic efficacy of IL-17 neutralization in murine experimental autoimmune encephalomyelitis. Cell Immunol, 237(2), 123-130. doi: 10.1016/j.cellimm.2005.11.002

Hogquist, K. A., Baldwin, T. A., \& Jameson, S. C. (2005). Central tolerance: learning selfcontrol in the thymus. Nat Rev Immunol, 5(10), 772-782.

Hori, S., Nomura, T., \& Sakaguchi, S. (2003). Control of Regulatory T Cell Development by the Transcription Factor Foxp3. Science, 299(5609), 1057-1061. doi: 10.1126/science. 1079490

Housley, W. J., Adams, C. O., Nichols, F. C., Puddington, L., Lingenheld, E. G., Zhu, L., . . . Clark, R. B. (2011). Natural but Not Inducible Regulatory T Cells Require TNF- $\alpha$ 
Signaling for In Vivo Function. The Journal of Immunology, 186(12), 6779-6787. doi: 10.4049/jimmunol.1003868

Hsieh, C. S., Macatonia, S. E., Tripp, C. S., Wolf, S. F., O'Garra, A., \& Murphy, K. M. (1993). Development of TH1 CD4+ T cells through IL-12 produced by Listeria-induced macrophages. Science, 260(5107), 547-549.

Huang, B., Pan, P.-Y., Li, Q., Sato, A. I., Levy, D. E., Bromberg, J., . . Chen, S.-H. (2006). Gr-1+CD115+ Immature Myeloid Suppressor Cells Mediate the Development of Tumor-Induced T Regulatory Cells and T-Cell Anergy in Tumor-Bearing Host. Cancer Research, 66(2), 1123-1131. doi: 10.1158/0008-5472.can-05-1299

Huseby, E. S., Liggitt, D., Brabb, T., Schnabel, B., Öhlén, C., \& Goverman, J. (2001). A Pathogenic Role for Myelin-Specific Cd8+ T Cells in a Model for Multiple Sclerosis. The Journal of experimental medicine, 194(5), 669-676. doi: 10.1084/jem.194.5.669

Hussien, Y., Sanna, A., Söderström, M., Link, H., \& Huang, Y.-M. (2001). Glatiramer acetate and IFN- $\beta$ act on dendritic cells in multiple sclerosis. Journal of Neuroimmunology, 121(1-2), 102-110. doi: http://dx.doi.org/10.1016/S0165-5728(01)00432-5

Ingwersen, J., Aktas, O., Kuery, P., Kieseier, B., Boyko, A., \& Hartung, H.-P. (2012). Fingolimod in multiple sclerosis: Mechanisms of action and clinical efficacy. Clinical Immunology, 142(1), 15-24. doi: http://dx.doi.org/10.1016/j.clim.2011.05.005

Ioannou, M., Alissafi, T., Lazaridis, I., Deraos, G., Matsoukas, J., Gravanis, A., . . Verginis, P. (2012). Crucial role of granulocytic myeloid-derived suppressor cells in the regulation of central nervous system autoimmune disease. J Immunol, 188(3), 11361146. doi: 10.4049/jimmunol.1101816

Ivanov, I. I., Zhou, L., \& Littman, D. R. (2007). Transcriptional Regulation of Th17 Cell Differentiation. Seminars in Immunology, 19(6), 409-417. doi: 10.1016/j.smim.2007.10.011

Jain, N., Nguyen, H., Chambers, C., \& Kang, J. (2010). Dual function of CTLA-4 in regulatory $\mathrm{T}$ cells and conventional $\mathrm{T}$ cells to prevent multiorgan autoimmunity. Proceedings of the National Academy of Sciences of the United States of America, 107(4), 1524-1528. doi: 10.1073/pnas.0910341107

Janeway, C. A., Jr., \& Medzhitov, R. (2002). Innate immune recognition. Annu Rev Immunol, 20, 197-216. doi: 10.1146/annurev.immunol.20.083001.084359

Jee, Y., Piao, W. H., Liu, R., Bai, X. F., Rhodes, S., Rodebaugh, R., . . . Vollmer, T. L. (2007a). CD4(+)CD25(+) regulatory $\mathrm{T}$ cells contribute to the therapeutic effects of glatiramer acetate in experimental autoimmune encephalomyelitis. Clin Immunol, 125(1), 34-42. doi: 10.1016/j.clim.2007.05.020

Jee, Y., Piao, W. H., Liu, R., Bai, X. F., Rhodes, S., Rodebaugh, R., . . . Vollmer, T. L. (2007b). CD4+CD25+ regulatory $T$ cells contribute to the therapeutic effects of glatiramer acetate in experimental autoimmune encephalomyelitis. Clinical Immunology, 125(1), 34-42. doi: http://dx.doi.org/10.1016/j.clim.2007.05.020

Johnson, T., Jirik, F., \& Fournier, S. (2010). Exploring the roles of CD8+ T lymphocytes in the pathogenesis of autoimmune demyelination. Seminars in Immunopathology, 32(2), 197-209. doi: 10.1007/s00281-010-0199-7

Kaisho, T., \& Akira, S. (2006). Toll-like receptor function and signaling. J Allergy Clin Immunol, 117(5), 979-987; quiz 988. doi: 10.1016/j.jaci.2006.02.023

Karasuyama, H., Obata, K., Wada, T., Tsujimura, Y., \& Mukai, K. (2011). Newly appreciated roles for basophils in allergy and protective immunity. Allergy, 66(9), 1133-1141. doi: 10.1111/j.1398-9995.2011.02613.x

Karpus, W. J., \& Ransohoff, R. M. (1998). Chemokine regulation of experimental autoimmune encephalomyelitis: temporal and spatial expression patterns govern disease pathogenesis. J Immunol, 161(6), 2667-2671. 
Kawabe, T., Isobe, K. I., Hasegawa, Y., Nakashima, I., \& Shimokata, K. (1992). Immunosuppressive activity induced by nitric oxide in culture supernatant of activated rat alveolar macrophages. Immunology, 76(1), 72-78.

Kawai, T., \& Akira, S. (2010). The role of pattern-recognition receptors in innate immunity: update on Toll-like receptors. Nat Immunol, 11(5), 373-384.

Keating, P., O'Sullivan, D., Tierney, J. B., Kenwright, D., Miromoeini, S., Mawasse, L., . . . La Flamme, A. C. (2009). Protection from EAE by IL-4Ralpha(-/-) macrophages depends upon T regulatory cell involvement. Immunol Cell Biol, 87(7), 534-545. doi: 10.1038/icb.2009.37

Keegan, B. M., \& Noseworthy, J. H. (2002). Multiple sclerosis. Annu Rev Med, 53, 285-302. doi: 10.1146/annurev.med.53.082901.103909

Keir, M. E., Francisco, L. M., \& Sharpe, A. H. (2007). PD-1 and its ligands in T-cell immunity. Curr Opin Immunol, 19(3), 309-314. doi: http://dx.doi.org/10.1016/j.coi.2007.04.012

Khan, O., Rieckmann, P., Boyko, A., Selmaj, K., \& Zivadinov, R. (2013). Three times weekly glatiramer acetate in relapsing-remitting multiple sclerosis. Ann Neurol, 73(6), 705713. doi: 10.1002/ana.23938

Khan, O. A., Tselis, A. C., Kamholz, J. A., Garbern, J. Y., Lewis, R. A., \& Lisak, R. P. (2001). A prospective, open-label treatment trial to compare the effect of IFN beta-1a (Avonex), IFNbeta-1b (Betaseron), and glatiramer acetate (Copaxone) on the relapse rate in relapsing-remitting multiple sclerosis. European journal of neurology : the official journal of the European Federation of Neurological Societies, 8(2), 141-148.

Kieseier, B. C., Kiefer, R., Clements, J. M., Miller, K., Wells, G. M., Schweitzer, T., . . . Hartung, H. P. (1998). Matrix metalloproteinase-9 and -7 are regulated in experimental autoimmune encephalomyelitis (Vol. 121).

Kohm, A. P., Carpentier, P. A., Anger, H. A., \& Miller, S. D. (2002). Cutting Edge: CD4+CD25+ Regulatory T Cells Suppress Antigen-Specific Autoreactive Immune Responses and Central Nervous System Inflammation During Active Experimental Autoimmune Encephalomyelitis. The Journal of Immunology, 169(9), 4712-4716. doi: 10.4049/jimmunol.169.9.4712

Kohm, A. P., Carpentier, P. A., \& Miller, S. D. (2003). Regulation of experimental autoimmune encephalomyelitis (EAE) by CD4+CD25+ regulatory $\mathrm{T}$ cells. Novartis Found Symp, 252, 45-52; discussion 52-44, 106-114.

Komiyama, Y., Nakae, S., Matsuki, T., Nambu, A., Ishigame, H., Kakuta, S., . . Iwakura, Y. (2006). IL-17 Plays an Important Role in the Development of Experimental Autoimmune Encephalomyelitis. The Journal of Immunology, 177(1), 566-573. doi: 10.4049/jimmunol.177.1.566

Kono, D. H., Urban, J. L., Horvath, S. J., Ando, D. G., Saavedra, R. A., \& Hood, L. (1988). Two minor determinants of myelin basic protein induce experimental allergic encephalomyelitis in SJL/J mice. The Journal of experimental medicine, 168(1), 213227. doi: 10.1084 jem.168.1.213

Kraus, J., \& Oschmann, P. (2006). The impact of interferon- $\beta$ treatment on the blood-brain barrier. Drug Discovery Today, 11(15-16), 755-762. doi: http://dx.doi.org/10.1016/j.drudis.2006.06.008

Kruisbeek, A. M., Shevach, E., \& Thornton, A. M. (2004). Proliferative assays for T cell function. Curr Protoc Immunol, Chapter 3, Unit 3 12. doi: 10.1002/0471142735.im0312s60

Krummel, M. F., \& Allison, J. P. (1995). CD28 and CTLA-4 have opposing effects on the response of $\mathrm{T}$ cells to stimulation. The Journal of experimental medicine, 182(2), 459465. 
Kuchroo, V. K., Sobel, R. A., Laning, J. C., Martin, C. A., Greenfield, E., Dorf, M. E., \& Lees, M. B. (1992). Experimental allergic encephalomyelitis mediated by cloned T cells specific for a synthetic peptide of myelin proteolipid protein. Fine specificity and $\mathrm{T}$ cell receptor V usage. Journal of Immunology, 148, 3776-3782.

Kuchroo, V. K., \& Weiner, H. L. (1998). Antigen-driven regulation of experimental autoimmune encephalomyelitis Research Immunology, 149, 759-771.

Kumar, V., \& Robbins, S. L. (2007). Robbins basic pathology (8th ed.). Philadelphia, PA: Saunders/Elsevier.

Kumar, V., \& Robbins, S. L. (2007). Robbins basic pathology (Vol. 8). Philadelphia: Saunders/Elsevier.

Kunis, G., Baruch, K., Rosenzweig, N., Kertser, A., Miller, O., Berkutzki, T., \& Schwartz, M. (2013). IFN-gamma-dependent activation of the brain's choroid plexus for CNS immune surveillance and repair. Brain, 136(Pt 11), 3427-3440. doi: 10.1093/brain/awt259

Kurotaki, D., Kon, S., Bae, K., Ito, K., Matsui, Y., Nakayama, Y., . . Morimoto, J. (2011). CSF-1-dependent red pulp macrophages regulate CD4 $\mathrm{T}$ cell responses. J Immunol, 186(4), 2229-2237. doi: 10.4049/jimmunol.1001345

Kwon, B. K., Tetzlaff, W., Grauer, J. N., Beiner, J., \& Vaccaro, A. R. (2004). Pathophysiology and pharmacologic treatment of acute spinal cord injury. The Spine Journal, 4(4), 451464. doi: http://dx.doi.org/10.1016/j.spinee.2003.07.007

Lampropoulou, V., Hoehlig, K., Roch, T., Neves, P., Gómez, E. C., Sweenie, C. H., . . . Fillatreau, S. (2008). TLR-Activated B Cells Suppress T Cell-Mediated Autoimmunity. The Journal of Immunology, 180(7), 4763-4773. doi: 10.4049/jimmunol.180.7.4763

Latchman, Y. E., Liang, S. C., Wu, Y., Chernova, T., Sobel, R. A., Klemm, M., . . Sharpe, A. H. (2004). PD-L1-deficient mice show that PD-L1 on T cells, antigen-presenting cells, and host tissues negatively regulates T cells. Proceedings of the National Academy of Sciences of the United States of America, 101(29), 10691-10696. doi: 10.1073/pnas.0307252101

Lee, Y. K., Menezes, J. S., Umesaki, Y., \& Mazmanian, S. K. (2011). Proinflammatory T-cell responses to gut microbiota promote experimental autoimmune encephalomyelitis. Proceedings of the National Academy of Sciences, 108(Supplement 1), 4615-4622. doi: 10.1073/pnas.1000082107

Li, Z., Woo, C. J., Iglesias-Ussel, M. D., Ronai, D., \& Scharff, M. D. (2004). The generation of antibody diversity through somatic hypermutation and class switch recombination. Genes \& Development, 18(1), 1-11. doi: 10.1101/gad.1161904

Libbey, J. E., McCoy, L. L., \& Fujinami, R. S. (2007). Molecular Mimicry in Multiple Sclerosis. In M. Alireza (Ed.), International Review of Neurobiology (Vol. Volume 79, pp. 127-147): Academic Press.

Lieberman, L. A., \& Hunter, C. A. (2002). Regulatory pathways involved in the infectioninduced production of IFN- $\gamma$ by NK cells. Microbes and Infection, 4(15), 1531-1538. doi: http://dx.doi.org/10.1016/S1286-4579(02)00036-9

Limmroth, V., Putzki, N., \& Kachuck, N. J. (2011). The interferon beta therapies for treatment of relapsing-remitting multiple sclerosis: are they equally efficacious? A comparative review of open-label studies evaluating the efficacy, safety, or dosing of different interferon beta formulations alone or in combination. Therapeutic Advances in Neurological Disorders, 4(5), 281-296. doi: 10.1177/1756285611413825

Loke, P. n., \& Allison, J. P. (2003). PD-L1 and PD-L2 are differentially regulated by Th1 and Th2 cells. Proceedings of the National Academy of Sciences of the United States of America, 100(9), 5336-5341. doi: 10.1073/pnas.0931259100 
Low, D., Nguyen, D. D., \& Mizoguchi, E. (2013). Animal models of ulcerative colitis and their application in drug research. Drug Design, Development and Therapy, 7, 13411357. doi: 10.2147/DDDT.S40107

Lucchinetti, C., Bruck, W., Parisi, J., Scheithauer, B., Rodriguez, M., \& Lassmann, H. (2000). Heterogeneity of multiple sclerosis lesions: implications for the pathogenesis of demyelination. Ann Neurol, 47(6), 707-717.

Lunz, J. G., 3rd, Specht, S. M., Murase, N., Isse, K., \& Demetris, A. J. (2007). Gut-derived commensal bacterial products inhibit liver dendritic cell maturation by stimulating hepatic interleukin-6/signal transducer and activator of transcription 3 activity. Hepatology, 46(6), 1946-1959. doi: 10.1002/hep.21906

MacFarlane, A. S., Schwacha, M. G., \& Eisenstein, T. K. (1999). In vivo blockage of nitric oxide with aminoguanidine inhibits immunosuppression induced by an attenuated strain of Salmonella typhimurium, potentiates Salmonella infection, and inhibits macrophage and polymorphonuclear leukocyte influx into the spleen. Infect Immun, 67(2), 891-898.

Macsween, K. F., \& Crawford, D. H. (2003). Epstein-Barr virus-recent advances. The Lancet Infectious Diseases, 3(3), 131-140. doi: http://dx.doi.org/10.1016/S14733099(03)00543-7

Makarova, O. V., Trunova, G. V., Diatroptov, M. E., Serebryakov, S. N., Kondashevskaya, M. V., \& Malaitsev, V. V. (2005). Comparative Characterization of Cytokine Production by Concanavalin A-Activated Splenocytes from BALB/c and C57B1/6 Mice after Cold Exposure. Bulletin of Experimental Biology and Medicine, 139(2), 220-222. doi: 10.1007/s10517-005-0253-y

Marsh-Wakefield, F., \& Byrne, S. N. (2015). Photoimmunology and Multiple Sclerosis. Curr Top Behav Neurosci. doi: 10.1007/7854_2014_359

Marta, M., Andersson, A., Isaksson, M., Kampe, O., \& Lobell, A. (2008). Unexpected regulatory roles of TLR4 and TLR9 in experimental autoimmune encephalomyelitis. European journal of immunology, 38(2), 565-575. doi: 10.1002/eji.200737187

Martin, R., Sturzebecher, C. S., \& McFarland, H. F. (2001). Immunotherapy of multiple sclerosis: where are we? Where should we go? Nat Immunol, 2(9), 785-788. doi: 10.1038/ni0901-785

Matsushima, G. K., \& Morell, P. (2001). The Neurotoxicant, Cuprizone, as a Model to Study Demyelination and Remyelination in the Central Nervous System. Brain Pathology, 11(1), 107-116. doi: 10.1111/j.1750-3639.2001.tb00385.x

Matteoli, G., Mazzini, E., Iliev, I. D., Mileti, E., Fallarino, F., Puccetti, P., . . . Rescigno, M. (2010). Gut CD103+ dendritic cells express indoleamine 2,3-dioxygenase which influences T regulatory/T effector cell balance and oral tolerance induction. Gut, 59(5), 595-604. doi: 10.1136/gut.2009.185108

Mauri, C., \& Bosma, A. (2012). Immune regulatory function of B cells. Annu Rev Immunol, 30, 221-241. doi: 10.1146/annurev-immunol-020711-074934

Mayo, L., Quintana, F. J., \& Weiner, H. L. (2012). The Innate Immune System in Demyelinating Disease. Immunol Rev, 248(1), 170-187. doi: 10.1111/j.1600065X.2012.01135.X

McGeachy, M. J., Stephens, L. A., \& Anderton, S. M. (2005). Natural Recovery and Protection from Autoimmune Encephalomyelitis: Contribution of CD4+CD25+ Regulatory Cells within the Central Nervous System. The Journal of Immunology, 175(5), 3025-3032. doi: 10.4049/jimmunol.175.5.3025

McHeyzer-Williams, L. J., \& McHeyzer-Williams, M. G. (2005). Antigen-specific memory B cell development. Annu Rev Immunol, 23, 487-513. doi: 10.1146/annurev.immunol.23.021704.115732 
McQualter, J. L., \& Bernard, C. C. (2007). Multiple sclerosis: a battle between destruction and repair. J Neurochem, 100(2), 295-306. doi: 10.1111/j.1471-4159.2006.04232.x

Medzhitov, R., \& Janeway, C. A., Jr. (1997). Innate immunity: the virtues of a nonclonal system of recognition. Cell, 91(3), 295-298.

Mekori, Y. A., \& Metcalfe, D. D. (2000). Mast cells in innate immunity. Immunol Rev, 173(1), 131-140. doi: 10.1034/j.1600-065X.2000.917305.x

Mendel, I., Kerlero de Rosbo, N., \& Ben-Nun, A. (1995). A myelin oligodendrocyte glycoprotein peptide induces typical chronic experimental autoimmune encephalomyelitis in $\mathrm{H}-2 \mathrm{~b}$ mice: fine specificity and $\mathrm{T}$ cell receptor $\mathrm{V}$ beta expression of encephalitogenic T cells. European journal of immunology, 25(7), 1951-1959. doi: 10.1002/eji.1830250723

Mildner, A., Schmidt, H., Nitsche, M., Merkler, D., Hanisch, U. K., Mack, M., . . Prinz, M. (2007). Microglia in the adult brain arise from Ly-6ChiCCR2+ monocytes only under defined host conditions. Nat Neurosci, 10(12), 1544-1553. doi: 10.1038/nn2015

Miller, A., Shapiro, S., Gershtein, R., Kinarty, A., Rawashdeh, H., Honigman, S., \& Lahat, N. (1998). Treatment of multiple sclerosis with Copolymer-1 (Copaxone®): implicating mechanisms of Th1 to Th2/Th3 immune-deviation. Journal of Neuroimmunology, 92(1-2), 113-121. doi: http://dx.doi.org/10.1016/S0165-5728(98)00191-X

Miller, D. H., Khan, O. A., Sheremata, W. A., Blumhardt, L. D., Rice, G. P. A., Libonati, M. A., . . . O'Connor, P. W. (2003). A Controlled Trial of Natalizumab for Relapsing Multiple Sclerosis. New England Journal of Medicine, 348(1), 15-23. doi: doi:10.1056/NEJMoa020696

Miller, D. H., \& Leary, S. M. (2007). Primary-progressive multiple sclerosis. The Lancet Neurology, 6(10), 903-912. doi: http://dx.doi.org/10.1016/S1474-4422(07)70243-0

Miller, S. D., \& Karpus, W. J. (2007). Experimental autoimmune encephalomyelitis in the mouse. Curr Protoc Immunol, Chapter 15, Unit 1511 . doi: 10.1002/0471142735.im1501s77

Mills, C. D., Kincaid, K., Alt, J. M., Heilman, M. J., \& Hill, A. M. (2000). M-1/M-2 macrophages and the Th1/Th2 paradigm. J Immunol, 164(12), 6166-6173.

Min, B., Prout, M., Hu-Li, J., Zhu, J., Jankovic, D., Morgan, E. S., . . Paul, W. E. (2004). Basophils produce IL-4 and accumulate in tissues after infection with a Th2-inducing parasite. The Journal of experimental medicine, 200(4), 507-517. doi: 10.1084/jem.20040590

Miranda-Hernandez, S., Gerlach, N., Fletcher, J. M., Biros, E., Mack, M., Korner, H., \& Baxter, A. G. (2011). Role for MyD88, TLR2 and TLR9 but not TLR1, TLR4 or TLR6 in experimental autoimmune encephalomyelitis. J Immunol, 187(2), 791-804. doi: 10.4049/jimmunol.1001992

Mix, E., Meyer-Rienecker, H., \& Zettl, U. K. (2008). Animal models of multiple sclerosis for the development and validation of novel therapies - potential and limitations. Journal of neurology, 255 Suppl 6, 7-14. doi: 10.1007/s00415-008-6003-0

Mogensen, T. H. (2009). Pathogen Recognition and Inflammatory Signaling in Innate Immune Defenses. Clinical Microbiology Reviews, 22(2), 240-273. doi: 10.1128/CMR.0004608

Mosmann, T. R., Cherwinski, H., Bond, M. W., Giedlin, M. A., \& Coffman, R. L. (1986). Two types of murine helper $\mathrm{T}$ cell clone. I. Definition according to profiles of lymphokine activities and secreted proteins. J Immunol, 136(7), 2348-2357.

Mosmann, T. R., \& Sad, S. (1996). The expanding universe of T-cell subsets: Th1, Th2 and more. Immunol Today, 17(3), 138-146.

Mosser, D. M. (2003). The many faces of macrophage activation. Journal of leukocyte biology, 73(2), 209-212. doi: 10.1189/jlb.0602325 
Mosser, D. M., \& Edwards, J. P. (2008). Exploring the full spectrum of macrophage activation. Nat Rev Immunol, 8(12), 958-969.

Mueller, D. L. (2010). Mechanisms maintaining peripheral tolerance. Nat Immunol, 11(1), 2127.

Munn, D. H., Shafizadeh, E., Attwood, J. T., Bondarev, I., Pashine, A., \& Mellor, A. L. (1999). Inhibition of T Cell Proliferation by Macrophage Tryptophan Catabolism. The Journal of experimental medicine, 189(9), 1363-1372. doi: 10.1084/jem.189.9.1363

Murphy, K., Travers, P., Walport, M., \& Janeway, C. (2012). Janeway's immunobiology (8th ed.). New York: Garland Science.

Nagler-Anderson, C. (2001). Man the barrier! strategic defences in the intestinal mucosa. Nat Rev Immunol, 1(1), 59-67.

Nakayamada, S., Takahashi, H., Kanno, Y., \& O'Shea, J. J. (2012). Helper T cell diversity and plasticity. Curr Opin Immunol, 24(3), 297-302. doi: http://dx.doi.org/10.1016/j.coi.2012.01.014

Nathan, C., \& Shiloh, M. U. (2000). Reactive oxygen and nitrogen intermediates in the relationship between mammalian hosts and microbial pathogens. Proceedings of the National Academy of Sciences of the United States of America, 97(16), 8841-8848.

Neuhaus, O., Farina, C., Wekerle, H., \& Hohlfeld, R. (2001). Mechanisms of action of glatiramer acetate in multiple sclerosis. Neurology, 56(6), 702-708.

Niedbala, W., Cai, B., Liu, H., Pitman, N., Chang, L., \& Liew, F. Y. (2007). Nitric oxide induces CD4+CD25+ Foxp3 regulatory T cells from CD4+CD25 T cells via p53, IL-2, and OX40. Proceedings of the National Academy of Sciences of the United States of America, 104(39), 15478-15483. doi: 10.1073/pnas.0703725104

Niederkorn, J. Y., \& Stein-Streilein, J. (2010). History and Physiology of Immune Privilege. Ocular Immunology \& Inflammation, 18(1), 19-23. doi: doi:10.3109/09273940903564766

Noman, M. Z., Desantis, G., Janji, B., Hasmim, M., Karray, S., Dessen, P., . . . Chouaib, S. (2014). PD-L1 is a novel direct target of HIF-1 $\alpha$, and its blockade under hypoxia enhanced MDSC-mediated T cell activation. The Journal of experimental medicine, 211(5), 781-790. doi: 10.1084/jem.20131916

Noyes, K., Bajorska, A., Chappel, A., Schwid, S. R., Mehta, L. R., Weinstock-Guttman, B., . . . Dick, A. W. (2011). Cost-effectiveness of disease-modifying therapy for multiple sclerosis: a population-based study. Neurology, 77(4), 355-363. doi: 10.1212/WNL.0b013e3182270402

O'Brien, K., Fitzgerald, D., Rostami, A., \& Gran, B. (2010). The TLR7 agonist, imiquimod, increases IFN-beta production and reduces the severity of experimental autoimmune encephalomyelitis. J Neuroimmunol, 221(1-2), 107-111. doi: S0165-5728(10)00011-1 [pii]10.1016/j.jneuroim.2010.01.006

O'Connor, R. A., \& Anderton, S. M. (2008). Foxp3+ regulatory T cells in the control of experimental CNS autoimmune disease. Journal of Neuroimmunology, 193(1-2), 1-11. doi: http://dx.doi.org/10.1016/j.jneuroim.2007.11.016

O'Connor, R. A., Prendergast, C. T., Sabatos, C. A., Lau, C. W., Leech, M. D., Wraith, D. C., \& Anderton, S. M. (2008). Cutting edge: Th1 cells facilitate the entry of Th17 cells to the central nervous system during experimental autoimmune encephalomyelitis. $J$ Immunol, 181(6), 3750-3754.

O'Sullivan, D. (2012). Using Experimental Autoimmune Encephalomyelitis to Identify Prospective Treatments for Multiple Sclerosis. (Doctor of Philosophy ), Victoria University of Wellington, Wellington. Retrieved from http://hdl.handle.net/10063/2126 
O’Gorman, C., Lucas, R., \& Taylor, B. (2012). Environmental Risk Factors for Multiple Sclerosis: A Review with a Focus on Molecular Mechanisms. International Journal of Molecular Sciences, 13(9), 11718-11752. doi: 10.3390/ijms130911718

Ochi, H., Abraham, M., Ishikawa, H., Frenkel, D., Yang, K., Basso, A. S., . . Weiner, H. L. (2006). Oral CD3-specific antibody suppresses autoimmune encephalomyelitis by inducing $\mathrm{CD} 4+\mathrm{CD} 25-\mathrm{LAP}+\mathrm{T}$ cells. Nature medicine, 12(6), 627-635. doi: http://www.nature.com/nm/journal/v12/n6/suppinfo/nm1408 S1.html

Ochoa-Repáraz, J., Mielcarz, D. W., Begum- Haque, S., \& Kasper, L. H. (2011). Gut, bugs, and brain: Role of commensal bacteria in the control of central nervous system disease. Ann Neurol, 69(2), 240-247. doi: 10.1002/ana.22344

Okuda, Y., Sakoda, S., Fujimura, H., \& Yanagihara, T. (1998). Aminoguanidine, a selective inhibitor of the inducible nitric oxide synthase, has different effects on experimental allergic encephalomyelitis in the induction and progression phase. J Neuroimmunol, 81(1-2), 201-210.

Orian, J. M., Keating, P., Downs, L. L., Hale, M. W., Jiang, X., Pham, H., \& LaFlamme, A. C. (2015). Deletion of IL-4R $\alpha$ in the BALB/c mouse is associated with altered lesion topography and susceptibility to experimental autoimmune encephalomyelitis. Autoimmunity, 48(4), 208-221. doi: doi:10.3109/08916934.2014.987344

Ousman, S. S., \& Kubes, P. (2012). Immune surveillance in the central nervous system. Nat Neurosci, 15(8), 1096-1101. doi: 10.1038/nn.3161

Palma, E., \& Cho, M. J. (2007). Improved systemic pharmacokinetics, biodistribution, and antitumor activity of $\mathrm{CpG}$ oligodeoxynucleotides complexed to endogenous antibodies in vivo. Journal of Controlled Release, 120(1-2), 95-103. doi: http://dx.doi.org/10.1016/j.jconrel.2007.03.016

Palmer, E. (2003). Negative selection--clearing out the bad apples from the T-cell repertoire. Nat Rev Immunol, 3(5), 383-391. doi: 10.1038/nri1085

Panitch, H. S., Hirsch, R. L., Schindler, J., \& Johnson, K. P. (1987). Treatment of multiple sclerosis with gamma interferon: exacerbations associated with activation of the immune system. Neurology, 37(7), 1097-1102.

Parihar, A., Eubank, T. D., \& Doseff, A. I. (2010). Monocytes and macrophages regulate immunity through dynamic networks of survival and cell death. J Innate Immun, 2(3), 204-215. doi: 10.1159/000296507

Paust, S., \& Cantor, H. (2005). Regulatory T cells and autoimmune disease. Immunol Rev, 204, 195-207. doi: 10.1111/j.0105-2896.2005.00247.x

Pen, J. J., Keersmaecker, B. D., Heirman, C., Corthals, J., Liechtenstein, T., Escors, D., . . . Breckpot, K. (2014). Interference with PD-L1/PD-1 co-stimulation during antigen presentation enhances the multifunctionality of antigen-specific T cells. Gene Ther, 21(3), 262-271. doi: 10.1038/gt.2013.80

Pender, M. P., Csurhes, P. A., Houghten, R. A., McCombe, P. A., \& Good, M. F. (1996). A study of human T-cell lines generated from multiple sclerosis patients and controls by stimulation with peptides of myelin basic protein. J Neuroimmunol, 70(1), 65-74.

Peterson, L. K., \& Fujinami, R. S. (2007). Inflammation, Demyelination, Neurodegeneration and Neuroprotection in the Pathogenesis of Multiple Sclerosis. Journal of Neuroimmunology, 184(1-2), 37-44. doi: 10.1016/j.jneuroim.2006.11.015

Pette, M., Fujita, K., Kitze, B., Whitaker, J. N., Albert, E., Kappos, L., \& Wekerle, H. (1990). Myelin basic protein-specific $\mathrm{T}$ lymphocyte lines from MS patients and healthy individuals. Neurology, 40(11), 1770-1776.

Peyrin-Biroulet, L., Vignal, C., Dessein, R., Simonet, M., Desreumaux, P., \& Chamaillard, M. (2006). NODs in defence: from vulnerable antimicrobial peptides to chronic 
inflammation. Trends in Microbiology, 14(10), 432-438. doi: http://dx.doi.org/10.1016/j.tim.2006.08.008

Pierrot-Deseilligny, C., \& Souberbielle, J.-C. (2013). Contribution of vitamin D insufficiency to the pathogenesis of multiple sclerosis. Therapeutic Advances in Neurological Disorders, 6(2), 81-116. doi: 10.1177/1756285612473513

Prinz, M., Garbe, F., Schmidt, H., Mildner, A., Gutcher, I., Wolter, K., . . Becher, B. (2006). Innate immunity mediated by TLR9 modulates pathogenicity in an animal model of multiple sclerosis. The Journal of clinical investigation, 116(2), 456-464. doi: 10.1172/JCI26078

Prinz, M., Garbe, F., Schmidt, H., Mildner, A., Gutcher, I., Wolter, K., . . Becher, B. (2006). Innate immunity mediated by TLR9 modulates pathogenicity in an animal model of multiple sclerosis. Journal of Clinical Investigation, 116(2), 456-464. doi: $10.1172 / \mathrm{JCI} 26078$

Prinz, M., \& Priller, J. (2010). Tickets to the brain: role of CCR2 and CX3CR1 in myeloid cell entry in the CNS. $J$ Neuroimmunol, 224(1-2), 80-84. doi: 10.1016/j.jneuroim.2010.05.015

Probst, H. C., McCoy, K., Okazaki, T., Honjo, T., \& van den Broek, M. (2005). Resting dendritic cells induce peripheral CD8+ T cell tolerance through PD-1 and CTLA-4. Nat Immunol, 6(3), 280-286. doi: 10.1038/ni1165

Pucci, E., Giuliani, G., Solari, A., Simi, S., Minozzi, S., Di Pietrantonj, C., \& Galea, I. (2011). Natalizumab for relapsing remitting multiple sclerosis. Cochrane Database Syst $\operatorname{Rev}(10)$, CD007621. doi: 10.1002/14651858.CD007621.pub2

Puddu, P., Fantuzzi, L., Borghi, P., Varano, B., Rainaldi, G., Guillemard, E., . . . Gessani, S. (1997). IL-12 induces IFN-gamma expression and secretion in mouse peritoneal macrophages. J Immunol, 159(7), 3490-3497.

Quah, B. J. C., \& Parish, C. R. (2010). The Use of Carboxyfluorescein Diacetate Succinimidyl Ester (CFSE) to Monitor Lymphocyte Proliferation. (44), e2259. doi: doi:10.3791/2259

Raber, P. L., Thevenot, P., Sierra, R., Wyczechowska, D., Halle, D., Ramirez, M. E., . . . Rodriguez, P. C. (2014). Subpopulations of myeloid-derived suppressor cells impair T cell responses through independent nitric oxide-related pathways. International journal of cancer. Journal international $d u$ cancer, 134(12), 2853-2864. doi: $10.1002 / \mathrm{ijc} .28622$

Racanelli, V., \& Rehermann, B. (2006). The liver as an immunological organ. Hepatology, 43(S1), S54-S62. doi: 10.1002/hep.21060

Racanelli, V., \& Rehermann, B. (2006). The liver as an immunological organ. Hepatology, 43(2 Suppl 1), S54-62. doi: 10.1002/hep.21060

Rachmilewitz, D., Karmeli, F., Takabayashi, K., Hayashi, T., Leider-Trejo, L., Lee, J., . . . Raz, E. (2002). Immunostimulatory DNA ameliorates experimental and spontaneous murine colitis. Gastroenterology, 122(5), 1428-1441. doi: http://dx.doi.org/10.1053/gast.2002.32994

Rachmilewitz, D., Katakura, K., Karmeli, F., Hayashi, T., Reinus, C., Rudensky, B., . . Raz, E. (2004). Toll-like receptor 9 signaling mediates the anti-inflammatory effects of probiotics in murine experimental colitis. Gastroenterology, 126(2), 520-528. doi: http://dx.doi.org/10.1053/j.gastro.2003.11.019

Radu, M., \& Chernoff, J. (2013). An in vivo assay to test blood vessel permeability. J Vis $\operatorname{Exp}(73)$, e50062. doi: 10.3791/50062

Ransohoff, R. M. (2005). Natalizumab and PML. Nat Neurosci, 8(10), 1275-1275.

Reiff, C., \& Kelly, D. (2010). Inflammatory bowel disease, gut bacteria and probiotic therapy. International Journal of Medical Microbiology, 300(1), 25-33. doi: http://dx.doi.org/10.1016/j.ijmm.2009.08.004 
Rizvi, S. A., Kim, E., \& Moodie, J. (2006). Glatiramer in the treatment of multiple sclerosis. Int J Nanomedicine, 1(3), 283-289.

Rostami, A., \& Ciric, B. (2013). Role of Th17 cells in the pathogenesis of CNS inflammatory demyelination. Journal of the neurological sciences, 333(1-2), 76-87. doi: 10.1016/j.jns.2013.03.002

Rutschman, R., Lang, R., Hesse, M., Ihle, J. N., Wynn, T. A., \& Murray, P. J. (2001). Cutting edge: Stat6-dependent substrate depletion regulates nitric oxide production. J Immunol, 166(4), 2173-2177.

Sabatino, J. J., Jr., Shires, J., Altman, J. D., Ford, M. L., \& Evavold, B. D. (2008). Loss of IFN-gamma enables the expansion of autoreactive CD4+ $\mathrm{T}$ cells to induce experimental autoimmune encephalomyelitis by a nonencephalitogenic myelin variant antigen. J Immunol, 180(7), 4451-4457.

Saibil, S. D., Deenick, E. K., \& Ohashi, P. S. (2007). The sound of silence: modulating anergy in $\mathrm{T}$ lymphocytes. Curr Opin Immunol, 19(6), 658-664. doi: http://dx.doi.org/10.1016/j.coi.2007.08.005

Sakaguchi, S., Wing, K., Onishi, Y., Prieto-Martin, P., \& Yamaguchi, T. (2009). Regulatory T cells: how do they suppress immune responses? International Immunology, 21(10), 1105-1111. doi: 10.1093/intimm/dxp095

Sawcer, S., Hellenthal, G., Pirinen, M., Spencer, C. C. A., Patsopoulos, N. A., Moutsianas, L., . . Compston, A. (2011). Genetic risk and a primary role for cell-mediated immune mechanisms in multiple sclerosis. Nature, 476(7359), 214-219. doi: $10.1038 /$ nature 10251

Schmidt, H., Williamson, D., \& Ashley-Koch, A. (2007). HLA-DR15 haplotype and multiple sclerosis: a HuGE review. Am J Epidemiol, 165(10), 1097-1109. doi: 10.1093/aje/kwk118

Schmitt, E., Klein, M., \& Bopp, T. (2014). Th9 cells, new players in adaptive immunity. Trends Immunol, 35(2), 61-68. doi: http://dx.doi.org/10.1016/j.it.2013.10.004

Schreiner, B., Mitsdoerffer, M., Kieseier, B. C., Chen, L., Hartung, H. P., Weller, M., \& Wiendl, H. (2004). Interferon-beta enhances monocyte and dendritic cell expression of B7-H1 (PD-L1), a strong inhibitor of autologous T-cell activation: relevance for the immune modulatory effect in multiple sclerosis. J Neuroimmunol, 155(1-2), 172-182. doi: 10.1016/j.jneuroim.2004.06.013

Schrijver, I. A., van Meurs, M., Melief, M. J., Wim Ang, C., Buljevac, D., Ravid, R., . . . Laman, J. D. (2001). Bacterial peptidoglycan and immune reactivity in the central nervous system in multiple sclerosis. Brain, 124(Pt 8), 1544-1554.

Schroder, K., Hertzog, P. J., Ravasi, T., \& Hume, D. A. (2004). Interferon- $\gamma$ : an overview of signals, mechanisms and functions. Journal of leukocyte biology, 75(2), 163-189. doi: 10.1189/jlb.0603252

Sedgwick, J. D., Schwender, S., Imrich, H., Dorries, R., Butcher, G. W., \& ter Meulen, V. (1991). Isolation and direct characterization of resident microglial cells from the normal and inflamed central nervous system. Proceedings of the National Academy of Sciences of the United States of America, 88(16), 7438-7442.

Shamri, R., Xenakis, J. J., \& Spencer, L. A. (2011). Eosinophils in innate immunity: an evolving story. Cell and tissue research, 343(1), 57-83. doi: 10.1007/s00441-0101049-6

Sharief, M. K., Semra, Y. K., Seidi, O. A., \& Zoukos, Y. (2001). Interferon-beta therapy downregulates the anti-apoptosis protein FLIP in T cells from patients with multiple sclerosis. J Neuroimmunol, 120(1-2), 199-207.

Shaw, P. J., Barr, M. J., Lukens, J. R., McGargill, M. A., Chi, H., Mak, T. W., \& Kanneganti, T. D. (2011). Signaling via the RIP2 adaptor protein in central nervous system- 
infiltrating dendritic cells promotes inflammation and autoimmunity. Immunity, 34(1), 75-84. doi: 10.1016/j.immuni.2010.12.015

Shechter, R., London, A., Varol, C., Raposo, C., Cusimano, M., Yovel, G., . . Schwartz, M. (2009). Infiltrating Blood-Derived Macrophages Are Vital Cells Playing an Antiinflammatory Role in Recovery from Spinal Cord Injury in Mice. PLoS Med, 6(7), e1000113. doi: 10.1371/journal.pmed.1000113

Shechter, R., Miller, O., Yovel, G., Rosenzweig, N., London, A., Ruckh, J., . . Schwartz, M. (2013). Recruitment of beneficial M2 macrophages to injured spinal cord is orchestrated by remote brain choroid plexus. Immunity, 38(3), 555-569. doi: 10.1016/j.immuni.2013.02.012

Shechter, R., \& Schwartz, M. (2013). Harnessing monocyte-derived macrophages to control central nervous system pathologies: no longer 'if' but 'how'. The Journal of pathology, 229(2), 332-346. doi: 10.1002/path.4106

Shen, H., Ackerman, A. L., Cody, V., Giodini, A., Hinson, E. R., Cresswell, P., . . Hanlon, D. J. (2006). Enhanced and prolonged cross-presentation following endosomal escape of exogenous antigens encapsulated in biodegradable nanoparticles. Immunology, 117(1), 78-88. doi: 10.1111/j.1365-2567.2005.02268.x

Shen, L. (2009). Functional Morphology of the Gastrointestinal Tract. In C. Sasakawa (Ed.), Molecular Mechanisms of Bacterial Infection via the Gut (Vol. 337, pp. 1-35): Springer Berlin Heidelberg.

Shi, L., Chen, S., Yang, L., \& Li, Y. (2013). The role of PD-1 and PD-L1 in T-cell immune suppression in patients with hematological malignancies. Journal of hematology \& oncology, 6(1), 74. doi: 10.1186/1756-8722-6-74

Silva, M. T. (2010). Neutrophils and macrophages work in concert as inducers and effectors of adaptive immunity against extracellular and intracellular microbial pathogens. Journal of leukocyte biology, 87(5), 805-813. doi: 10.1189/jlb.1109767

Simpson, S., Jr., Blizzard, L., Otahal, P., Van der Mei, I., \& Taylor, B. (2011). Latitude is significantly associated with the prevalence of multiple sclerosis: a meta-analysis. Journal of neurology, neurosurgery, and psychiatry, 82(10), 1132-1141. doi: 10.1136/jnnp.2011.240432

Slaney, C. Y., Toker, A., La Flamme, A., Backstrom, B. T., \& Harper, J. L. (2011). Naive blood monocytes suppress T-cell function. A possible mechanism for protection from autoimmunity. Immunol Cell Biol, 89(1), 7-13. doi: 10.1038/icb.2010.110

Smith-Garvin, J. E., Koretzky, G. A., \& Jordan, M. S. (2009). T Cell Activation. Annu Rev Immunol, 27, 591-619. doi: 10.1146/annurev.immunol.021908.132706

Smith, P. D., Smythies, L. E., Shen, R., Greenwell-Wild, T., Gliozzi, M., \& Wahl, S. M. (2011). Intestinal macrophages and response to microbial encroachment. Mucosal Immunol, 4(1), 31-42.

Sospedra, M., \& Martin, R. (2005). IMMUNOLOGY OF MULTIPLE SCLEROSIS*. Annu Rev Immunol, 23(1), 683-747. doi: doi:10.1146/annurev.immunol.23.021704.115707

Sriram, S., \& Steiner, I. (2005). Experimental allergic encephalomyelitis: A misleading model of multiple sclerosis. Ann Neurol, 58(6), 939-945. doi: 10.1002/ana.20743

Starr, T. K., Jameson, S. C., \& Hogquist, K. A. (2003). POSITIVE AND NEGATIVE SELECTION OF T CELLS. Annu Rev Immunol, 21(1), 139-176. doi: doi:10.1146/annurev.immunol.21.120601.141107

Steffen, B. J., Butcher, E. C., \& Engelhardt, B. (1994). Evidence for involvement of ICAM-1 and VCAM-1 in lymphocyte interaction with endothelium in experimental autoimmune encephalomyelitis in the central nervous system in the SJL/J mouse. The American journal of pathology, 145(1), 189-201. 
Steinman, L. (1996). Multiple Sclerosis: A Coordinated Immunological Attack against Myelin in the Central Nervous System. Cell, 85(3), 299-302. doi: http://dx.doi.org/10.1016/S0092-8674(00)81107-1

Steinman, L. (2001). Multiple sclerosis: a two-stage disease. Nat Immunol, 2(9), 762-764. doi: $10.1038 /$ ni0901-762

Steinman, L. (2007). A brief history of TH17, the first major revision in the TH1/TH2 hypothesis of T cell-mediated tissue damage. Nature medicine, 13(2), 139-145.

Steinman, L., Merrill, J. T., McInnes, I. B., \& Peakman, M. (2012). Optimization of current and future therapy for autoimmune diseases. Nature medicine, 18(1), 59-65.

Steinman, L., \& Zamvil, S. S. (2005). Virtues and pitfalls of EAE for the development of therapies for multiple sclerosis. Trends Immunol, 26(11), 565-571. doi: 10.1016/j.it.2005.08.014

Steinman, R. M., \& Hemmi, H. (2006). Dendritic cells: translating innate to adaptive immunity. Current topics in microbiology and immunology, 311, 17-58.

Stout, R. D., \& Suttles, J. (2004). Functional plasticity of macrophages: reversible adaptation to changing microenvironments. Journal of leukocyte biology, 76(3), 509-513. doi: 10.1189/jlb.0504272

Streit, W. J., Graeber, M. B., \& Kreutzberg, G. W. (1988). Functional plasticity of microglia: a review. Glia, 1(5), 301-307. doi: 10.1002/glia.440010502

Strober, W., Murray, P. J., Kitani, A., \& Watanabe, T. (2006). Signalling pathways and molecular interactions of NOD1 and NOD2. Nat Rev Immunol, 6(1), 9-20. doi: 10.1038/nri1747

Stromnes, I. M., \& Goverman, J. M. (2006). Active induction of experimental allergic encephalomyelitis. Nat. Protocols, 1(4), 1810-1819.

Stuve, O., Dooley, N. P., Uhm, J. H., Antel, J. P., Francis, G. S., Williams, G., \& Yong, V. W. (1996). Interferon beta-1b decreases the migration of T lymphocytes in vitro: effects on matrix metalloproteinase-9. Ann Neurol, 40(6), 853-863. doi: 10.1002/ana.410400607

Su, G. L. (2002). Lipopolysaccharides in liver injury: molecular mechanisms of Kupffer cell activation (Vol. 283).

Su, Y., Zhang, Z., Trautmann, K., Xu, S., \& Schluesener, H. J. (2005). TLR and NOD2 ligands induce cell proliferation in the rat intact spinal cord. J Neuropathol Exp Neurol, 64(11), 991-997.

Sun, J., Zhang, X., Broderick, M., \& Fein, H. (2003). Measurement of Nitric Oxide Production in Biological Systems by Using Griess Reaction Assay. Sensors, 3(8), 276-284.

Suttles, J., \& Stout, R. D. (2009). Macrophage CD40 signaling: A pivotal regulator of disease protection and pathogenesis. Seminars in Immunology, 21(5), 257-264. doi: http://dx.doi.org/10.1016/j.smim.2009.05.011

Swain, S. L., Weinberg, A. D., English, M., \& Huston, G. (1990). IL-4 directs the development of Th2-like helper effectors. J Immunol, 145(11), 3796-3806.

Szabo, S. J., Kim, S. T., Costa, G. L., Zhang, X., Fathman, C. G., \& Glimcher, L. H. (2000). A novel transcription factor, T-bet, directs Th1 lineage commitment. Cell, 100(6), 655669.

Szmydynger-Chodobska, J., Strazielle, N., Gandy, J. R., Keefe, T. H., Zink, B. J., GhersiEgea, J.-F., \& Chodobski, A. (2012). Posttraumatic invasion of monocytes across the blood-cerebrospinal fluid barrier. Journal of Cerebral Blood Flow \& Metabolism, 32(1), 93-104. doi: 10.1038/jcbfm.2011.111

Takeuchi, O., \& Akira, S. (2010). Pattern recognition receptors and inflammation. Cell, 140(6), 805-820. doi: 10.1016/j.cell.2010.01.022

Talmadge, J. E., \& Gabrilovich, D. I. (2013). History of myeloid-derived suppressor cells. Nat Rev Cancer, 13(10), 739-752. doi: 10.1038/nrc3581 
Taylor, B. V., Pearson, J. F., Clarke, G., Mason, D. F., Abernethy, D. A., Willoughby, E., \& Sabel, C. (2010). MS prevalence in New Zealand, an ethnically and latitudinally diverse country. Multiple sclerosis, 16(12), 1422-1431. doi: $10.1177 / 1352458510379614$

Thacker, E. L., Mirzaei, F., \& Ascherio, A. (2006). Infectious mononucleosis and risk for multiple sclerosis: A meta-analysis. Ann Neurol, 59(3), 499-503. doi: 10.1002/ana.20820

Thomson, A. W., \& Knolle, P. A. (2010). Antigen-presenting cell function in the tolerogenic liver environment. Nature reviews. Immunology, 10(11), 753-766. doi: $10.1038 /$ nri2858

Tiegs, G., \& Lohse, A. W. (2010). Immune tolerance: what is unique about the liver. $J$ Autoimmun, 34(1), 1-6. doi: 10.1016/j.jaut.2009.08.008

Tierney, J. B., Kharkrang, M., \& La Flamme, A. C. (2009). Type II-activated macrophages suppress the development of experimental autoimmune encephalomyelitis. Immunol Cell Biol, 87(3), 235-240. doi: 10.1038/icb.2008.99

Toft-Hansen, H., Buist, R., Sun, X.-J., Schellenberg, A., Peeling, J., \& Owens, T. (2006). Metalloproteinases Control Brain Inflammation Induced by Pertussis Toxin in Mice Overexpressing the Chemokine CCL2 in the Central Nervous System. The Journal of Immunology, 177(10), 7242-7249. doi: 10.4049/jimmunol.177.10.7242

Touil, T., Fitzgerald, D., Zhang, G. X., Rostami, A., \& Gran, B. (2006). Cutting Edge: TLR3 stimulation suppresses experimental autoimmune encephalomyelitis by inducing endogenous IFN-beta. J Immunol, 177(11), 7505-7509.

Trivedi, A., Olivas, A. D., \& Noble-Haeusslein, L. J. (2006). Inflammation and Spinal Cord Injury: Infiltrating Leukocytes as Determinants of Injury and Repair Processes. Clin Neurosci Res, 6(5), 283-292. doi: 10.1016/j.cnr.2006.09.007

Tsunoda, I., Tolley, N. D., Theil, D. J., Whitton, J. L., Kobayashi, H., \& Fujinami, R. S. (1999). Exacerbation of viral and autoimmune animal models for multiple sclerosis by bacterial DNA. Brain Pathol, 9(3), 481-493.

Turvey, S. E., \& Broide, D. H. (2010). Chapter 2: Innate Immunity. J Allergy Clin Immunol, 125(2 Suppl 2), S24-S32. doi: 10.1016/j.jaci.2009.07.016

van der Veen, R. C., Dietlin, T. A., \& Hofman, F. M. (2003). Tissue expression of inducible nitric oxide synthase requires IFN-gamma production by infiltrating splenic $\mathrm{T}$ cells: more evidence for immunosuppression by nitric oxide. J Neuroimmunol, 145(1-2), 8690.

van der Veen, R. C., Dietlin, T. A., Pen, L., Gray, J. D., \& Hofman, F. M. (2000). Antigen presentation to Th1 but not Th2 cells by macrophages results in nitric oxide production and inhibition of $\mathrm{T}$ cell proliferation: interferon-gamma is essential but insufficient. Cell Immunol, 206(2), 125-135. doi: 10.1006/cimm.2000.1741

Vieira, P. L., Christensen, J. R., Minaee, S., O’Neill, E. J., Barrat, F. J., Boonstra, A., . . . O’Garra, A. (2004). IL-10-Secreting Regulatory T Cells Do Not Express Foxp3 but Have Comparable Regulatory Function to Naturally Occurring CD4+CD25+ Regulatory $\mathrm{T}$ Cells. The Journal of Immunology, 172(10), 5986-5993. doi: 10.4049/jimmunol.172.10.5986

Vivier, E., Tomasello, E., Baratin, M., Walzer, T., \& Ugolini, S. (2008). Functions of natural killer cells. Nat Immunol, 9(5), 503-510. doi: http://www.nature.com/ni/journal/v9/n5/suppinfo/ni1582 S1.html

Voskoboinik, I., \& Trapani, J. A. (2006). Addressing the mysteries of perforin function. Immunol Cell Biol, 84(1), 66-71. 
Vyas, J. M., Van der Veen, A. G., \& Ploegh, H. L. (2008). The known unknowns of antigen processing and presentation. Nature reviews. Immunology, 8(8), 607-618. doi: $10.1038 /$ nri2368

Waldner, H. (2009). The role of innate immune responses in autoimmune disease development. Autoimmun Rev, 8(5), 400-404. doi: 10.1016/j.autrev.2008.12.019

Walker, L. S. K., \& Abbas, A. K. (2002). The enemy within: keeping self-reactive T cells at bay in the periphery. Nat Rev Immunol, 2(1), 11-19.

Wang, Z., Hong, J., Sun, W., Xu, G., Li, N., Chen, X., . . Zhang, J. Z. (2006). Role of IFN- $\gamma$ in induction of Foxp3 and conversion of CD4+ CD25- T cells to CD4+ Tregs. The Journal of clinical investigation, 116(9), 2434-2441. doi: 10.1172/jci25826

Weber, M. S., Prod'homme, T., Youssef, S., Dunn, S. E., Rundle, C. D., Lee, L., . . Zamvil, S. S. (2007). Type II monocytes modulate T cell-mediated central nervous system autoimmune disease. Nature medicine, 13(8), 935-943. doi: 10.1038/nm1620

Weber, M. S., Starck, M., Wagenpfeil, S., Meinl, E., Hohlfeld, R., \& Farina, C. (2004). Multiple sclerosis: glatiramer acetate inhibits monocyte reactivity in vitro and in vivo. Brain, 127(Pt 6), 1370-1378. doi: 10.1093/brain/awh163

Weiner, H. L. (2000). Oral tolerance, an active immunologic process mediated by multiple mechanisms. The Journal of clinical investigation, 106(8), 935-937. doi: 10.1172/JCI11348

Weiner, H. L. (2008). A shift from adaptive to innate immunity: a potential mechanism of disease progression in multiple sclerosis. Journal of neurology, 255 Suppl 1, 3-11. doi: $10.1007 / \mathrm{s} 00415-008-1002-8$

Weiner, H. L. (2009). The challenge of multiple sclerosis: how do we cure a chronic heterogeneous disease? Ann Neurol, 65(3), 239-248. doi: 10.1002/ana.21640

Weiner, H. L., da Cunha, A. P., Quintana, F., \& Wu, H. (2011). Oral tolerance. Immunol Rev, 241(1), 241-259. doi: 10.1111/j.1600-065X.2011.01017.x

White, M., Webster, G., O'Sullivan, D., Stone, S., \& La Flamme, A. C. (2014). Targeting innate receptors with MIS416 reshapes Th responses and suppresses CNS disease in a mouse model of multiple sclerosis. PloS one, 9(1), e87712. doi: 10.1371/journal.pone.0087712

Willenborg, D. O., Staykova, M. A., \& Cowden, W. B. (1999). Our shifting understanding of the role of nitric oxide in autoimmune encephalomyelitis: a review. Journal of Neuroimmunology, 100(1-2), 21-35. doi: http://dx.doi.org/10.1016/S01655728(99)00212-X

Willer, C. J., Dyment, D. A., Risch, N. J., Sadovnick, A. D., \& Ebers, G. C. (2003). Twin concordance and sibling recurrence rates in multiple sclerosis. Proceedings of the National Academy of Sciences of the United States of America, 100(22), 12877-12882. doi: 10.1073/pnas.1932604100

Wing, J. B., \& Sakaguchi, S. (2012). Multiple treg suppressive modules and their adaptability. Frontiers in Immunology, 3, 178. doi: 10.3389/fimmu.2012.00178

Wolff, D. J., \& Lubeskie, A. (1995). Aminoguanidine Is an Isoform-Selective, MechanismBased Inactivator of Nitric Oxide Synthase. Archives of Biochemistry and Biophysics, 316(1), 290-301. doi: http://dx.doi.org/10.1006/abbi.1995.1040

Xiao, B. G., Wu, X. C., Yang, J. S., Xu, L. Y., Liu, X., Huang, Y. M., . . Link, H. (2004). Therapeutic potential of IFN-gamma-modified dendritic cells in acute and chronic experimental allergic encephalomyelitis. Int Immunol, 16(1), 13-22.

Xing, Y., \& Hogquist, K. A. (2012). T-Cell Tolerance: Central and Peripheral. Cold Spring Harbor Perspectives in Biology, 4(6). doi: 10.1101/cshperspect.a006957 
Yamazaki, T., Akiba, H., Iwai, H., Matsuda, H., Aoki, M., Tanno, Y., . . . Yagita, H. (2002). Expression of programmed death 1 ligands by murine T cells and APC. J Immunol, 169(10), 5538-5545.

Yong, V. W. (2002). Differential mechanisms of action of interferon-beta and glatiramer aetate in MS. Neurology, 59(6), 802-808.

You, Q., Cheng, L., Kedl, R. M., \& Ju, C. (2008). Mechanism of T Cell Tolerance Induction by Murine Hepatic Kupffer Cells. Hepatology (Baltimore, Md.), 48(3), 978-990. doi: 10.1002/hep. 22395

Youn, J.-I., Nagaraj, S., Collazo, M., \& Gabrilovich, D. I. (2008). Subsets of Myeloid-Derived Suppressor Cells in Tumor-Bearing Mice. The Journal of Immunology, 181(8), 57915802. doi: 10.4049/jimmunol.181.8.5791

Yousry, T. A., Major, E. O., Ryschkewitsch, C., Fahle, G., Fischer, S., Hou, J., . . Clifford, D. B. (2006). Evaluation of Patients Treated with Natalizumab for Progressive Multifocal Leukoencephalopathy. New England Journal of Medicine, 354(9), 924-933. doi: doi:10.1056/NEJMoa054693

Zang, Y. C. Q., Halder, J. B., Samanta, A. K., Hong, J., Rivera, V. M., \& Zhang, J. Z. (2001). Regulation of chemokine receptor CCR5 and production of RANTES and MIP-1 $\alpha$ by interferon- $\beta$. Journal of Neuroimmunology, 112(1-2), 174-180. doi: http://dx.doi.org/10.1016/S0165-5728(00)00397-0

Zehntner, S. P., Brickman, C., Bourbonnière, L., Remington, L., Caruso, M., \& Owens, T. (2005). Neutrophils That Infiltrate the Central Nervous System Regulate T Cell Responses. The Journal of Immunology, 174(8), 5124-5131. doi: 10.4049/jimmunol.174.8.5124

Zhang, N., Pan, H.-F., \& Ye, D.-Q. (2011). Th22 in inflammatory and autoimmune disease: prospects for therapeutic intervention. Molecular and Cellular Biochemistry, 353(1-2), 41-46. doi: 10.1007/s11010-011-0772-y

Zhang, X., Koldzic, D. N., Izikson, L., Reddy, J., Nazareno, R. F., Sakaguchi, S., . . Weiner, H. L. (2004). IL-10 is involved in the suppression of experimental autoimmune encephalomyelitis by $\mathrm{CD} 25+\mathrm{CD} 4+$ regulatory $\mathrm{T}$ cells. International Immunology, 16(2), 249-256. doi: 10.1093/intimm/dxh029

Zhang, Z., Zhong, W., Spencer, D., Chen, H., Lu, H., Kawaguchi, T., \& Rosenbaum, J. T. (2009). Interleukin-17 causes neutrophil mediated inflammation in ovalbumin-induced uveitis in DO11.10 mice. Cytokine, 46(1), 79-91. doi: 10.1016/j.cyto.2008.12.019

Zhu, B., Bando, Y., Xiao, S., Yang, K., Anderson, A. C., Kuchroo, V. K., \& Khoury, S. J. (2007). CD11b+Ly-6C(hi) suppressive monocytes in experimental autoimmune encephalomyelitis. J Immunol, 179(8), 5228-5237.

Zhu, B., Bando, Y., Xiao, S., Yang, K., Anderson, A. C., Kuchroo, V. K., \& Khoury, S. J. (2007). CD11b+Ly-6Chi Suppressive Monocytes in Experimental Autoimmune Encephalomyelitis. The Journal of Immunology, 179(8), 5228-5237. doi: 10.4049/jimmunol.179.8.5228

Ziemssen, T., \& Schrempf, W. (2007). Glatiramer Acetate: Mechanisms of Action in Multiple Sclerosis. In M. Alireza (Ed.), International Review of Neurobiology (Vol. Volume 79, pp. 537-570): Academic Press. 


\section{Chapter 9: Appendices}

\section{Appendix 1: Flow gating strategy and systemic cytokines}

\section{Appendix 1.1 Flow gating strategies for identifying cell populations}
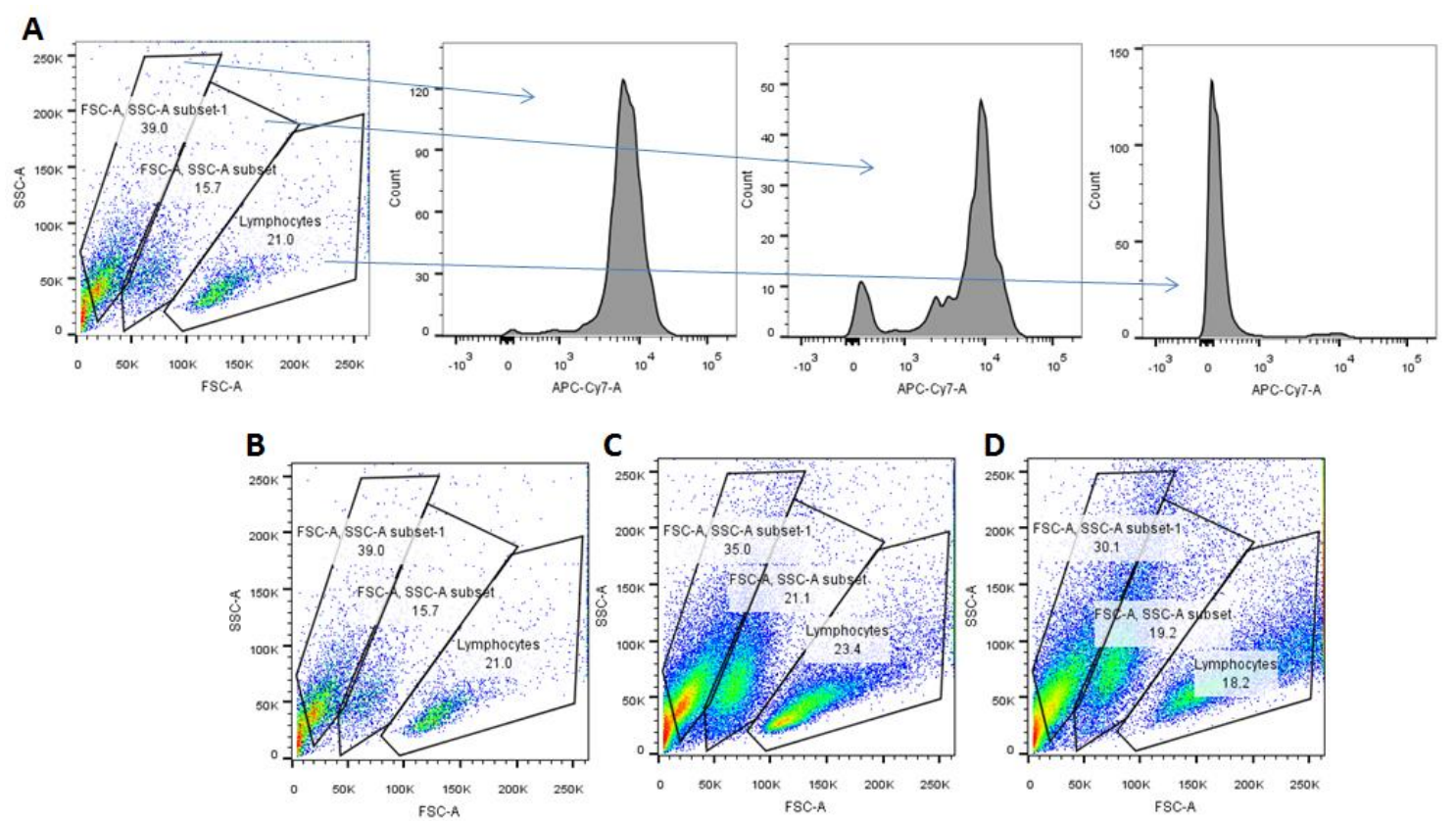

Figure A1-1 Live cell gating strategy for liver and spleen

Due to the complexity of some of the panels and having a limited number of channels for flow cytometry we often were unable to use a live/dead stain. However, using the Zombie NIR dye (Biolegend, CA, USA) we can accurately gate on the live population and exclude dead cells without the need to a viability stain (NB gate states "lymphocytes" which should read "leukocytes". A) Gating on 3 distinct populations in the FSC/SSC and looking at the APC/Cy7 expression we can see that the far left and middle populations are mostly positive for the viability dye, and are therefore dead cells, whereas the cells within the leukocyte gate are negative for the dye and therefore live cells we gate on for further analysis such as in appendix 1.2. B) Gating on media control after 48 hours. C) Gating on MIS416-treated splenocytes for 48 hours. C) Gating on ConA-treated splenocytes for 48 hours. 

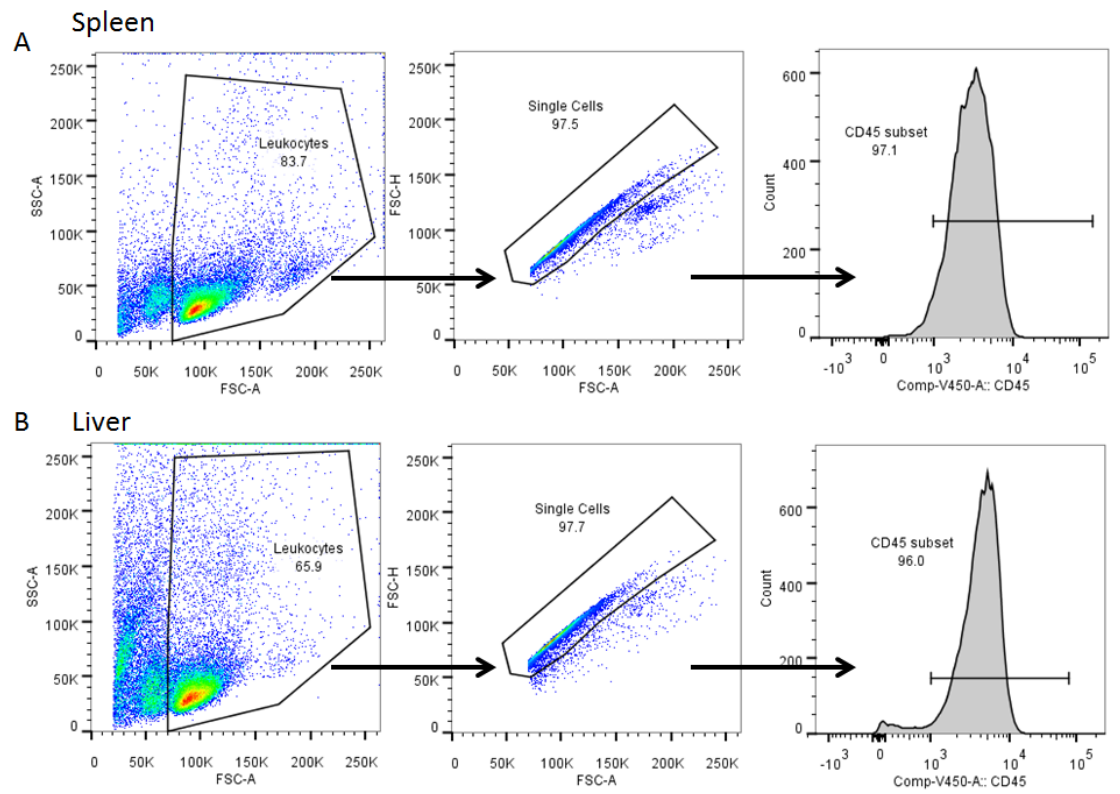

Figure A1-2 Isolating live-single-CD45+ cells from the spleen and liver

After we have identified the live cells in appendix 1.1 we wanted to gate on these cells then remove the doublets using FSC-A versus FSC-H and gating on single cells only. Finally we needed to only include CD45+ cells for further analysis which was typically $\sim 95 \%$ or higher after preliminary gating was performed, then immunophenotyping was done on this live-single-CD45+ population.

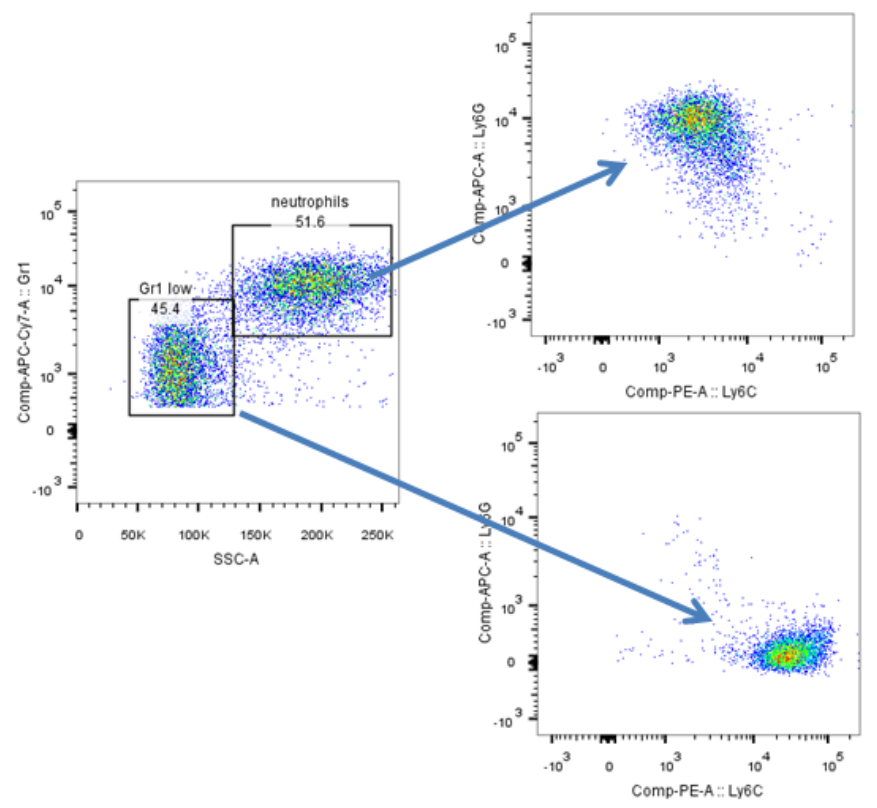

Figure A1-3 Liver and spleen cell populations and differential Gr1+ populations

The Gr-1 positive population is made up of Gr-1 high, which is a granular neutrophil population that expresses high levels of Ly6G and some Ly6C, whereas Gr-1 low population is Ly6G-Ly6C high and most likely monocytic. This figure represents liver Gr1 populations. 
a.
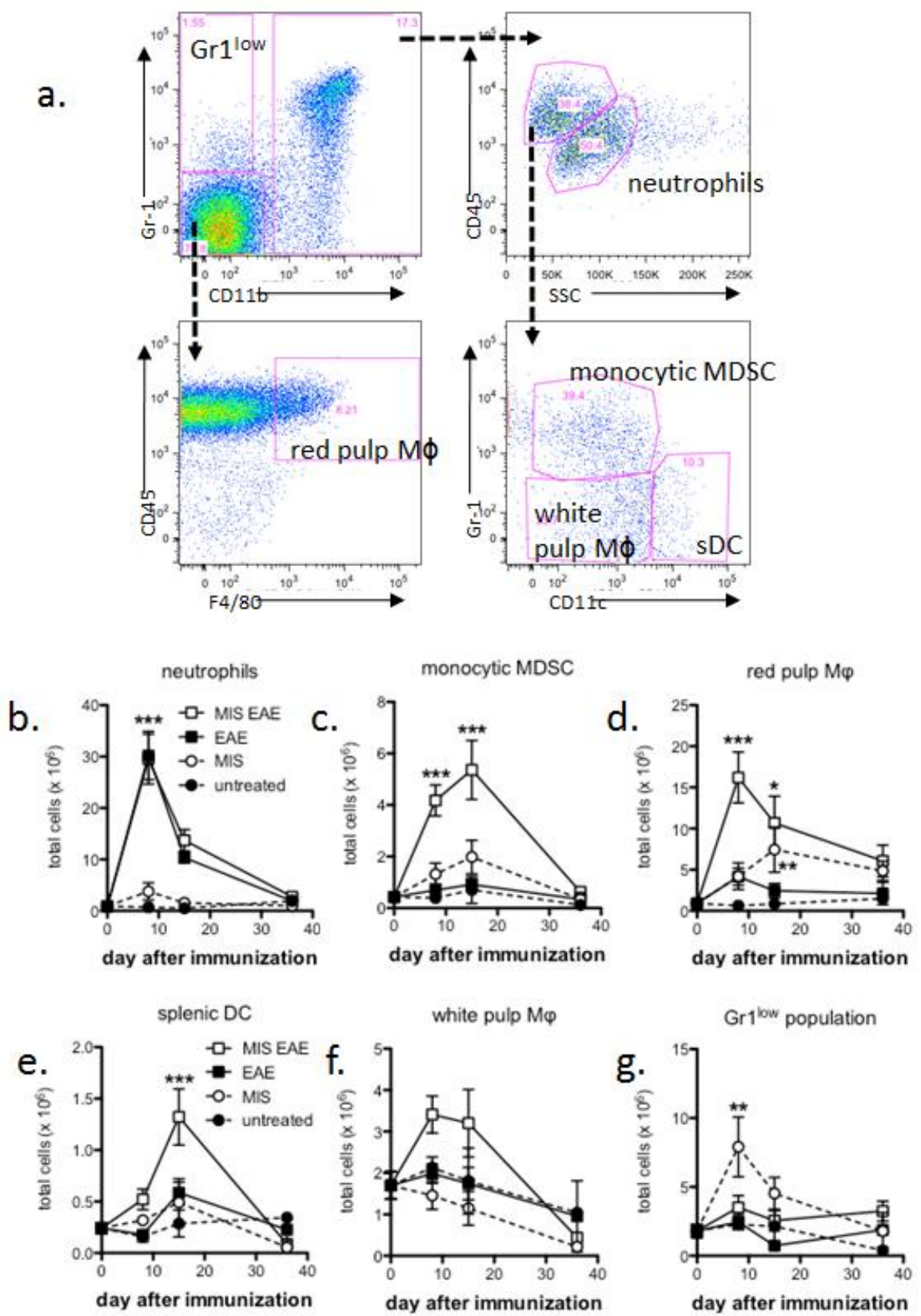

Figure A1-4 The splenic myeloid compartment was significantly altered by MIS416 administration and immunization (as published in White et al, 2014).

C57BL/6 mice were immunized to induce EAE and treated weekly by i.v. administration of $100 \mu \mathrm{g} / \mathrm{mouse}$ MIS416 starting on the day of immunization, and splenocytes were isolated at 8,15 , and 36 days postimmunization and assessed by flow cytometric analysis. (a) Myeloid population gating strategy using CD11b and, Gr-1, CD45, side scatter (SSC), CD11c, and F4/80 expression. Shown are representative plots of live, single cells from a MIS416-treated, immunized mouse. (b-g) MIS416 treatment led to an expansion in the MDSC (c) and red pulp macrophage (d) populations in both immunized and unimmunized mice ( $\mathrm{p}<0.001$ by 2-way ANOVA; MIS EAE compared to EAE and $\mathrm{p}<0.05$; MIS compared to untreated) while MIS416treated, immunized mice had significantly expanded splenic DC (e) but not white pulp macrophages (f) ( $\mathrm{p}<$ 0.05 by 2 -way ANOVA; MIS EAE compared to EAE). In contrast, no significant differences were found between EAE treatment groups in neutrophil numbers (b). The $\mathrm{Gr} 1^{\text {low }}$ population $(\mathbf{g})$ was significantly enhanced in the MIS416-treated, unimmunized animals compared to the untreated animals $(p<0.01$ by 2way ANOVA; MIS compared to untreated), and modestly altered in MIS416-treated immunized animals ( $\mathrm{p}<$ 0.05 by 2 -way ANOVA; MIS EAE compared to EAE). Shown are the means \pm SEM of values from individual mice ( $\mathrm{n}=6-10$ per group) from 2 experiments. $* \mathrm{p}<0.05, * * \mathrm{p}<0.01$, and $* * * \mathrm{p}<0.001$ by Bonferroni's multiple comparison post-test; MIS EAE compared to EAE or MIS compared to untreated. 


\section{Appendix 1.2 Systemic cytokines in MIS416-treated mice}

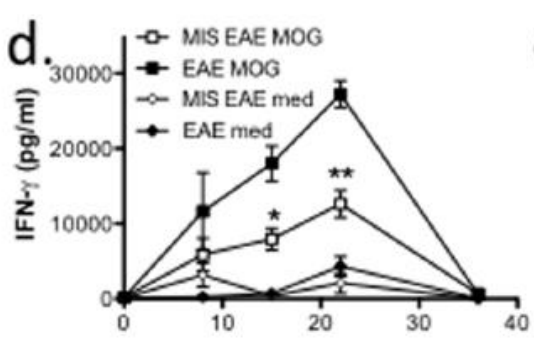

day after immunization

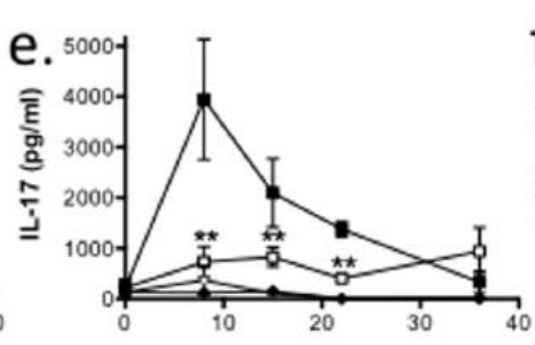

day after immunization

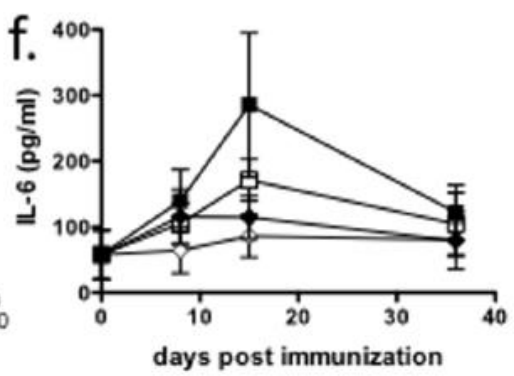

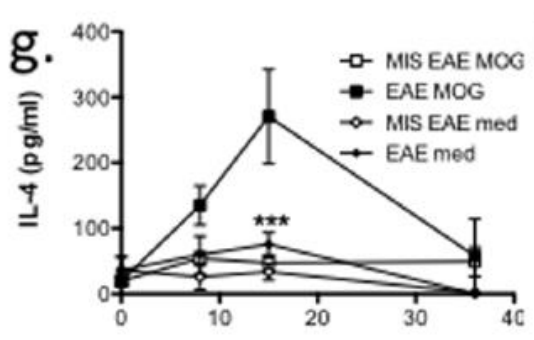

day after immunization

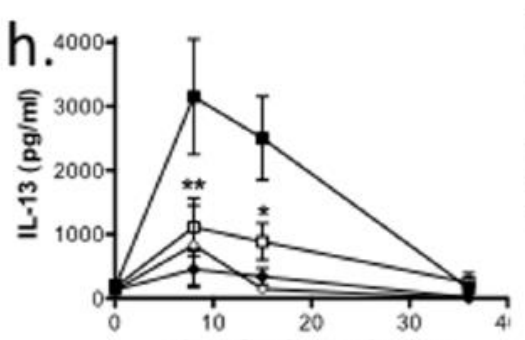

day after immunization

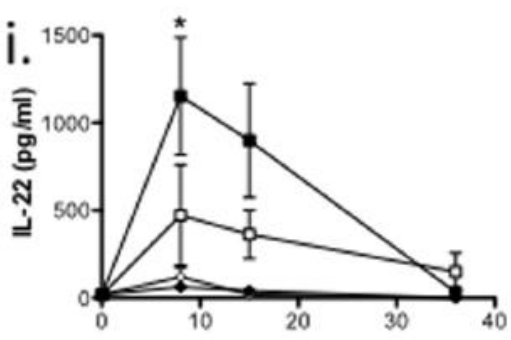

day after immunization

Figure A1-5 MIS416 treatment suppressed systemic antigen-specific responses (as published in White et al, 2014).

C57BL/6 mice were immunised to induce EAE and treated weekly by i.v administration of $100 \mu \mathrm{g} / \mathrm{mouse}$ MIS416 starting on the day of immunisation. Splenocytes were isolated from MIS416-treated or untreated EAE mice and cultured $\left(10^{6}\right.$ cells/well $)$ with MOG peptide $(27 \mu \mathrm{g} / \mathrm{ml})$ or media for 72 hours. Cytokine were assayed by cytokine bead assay and ELISA. Shown are the means \pm SEM of values from individual mice $(\mathrm{n}=3$ 15/timepoint) from 5 experiments. $* \mathrm{p}<0.05$, $* * \mathrm{p}<0.01$, and $* * * \mathrm{p}<0.001$ MOG-stimulated EAE compared to MOG-stimulated MIS EAE by 2-way ANOVA with Bonferronis multiple comparison post-test. 


\section{Appendix 1.3 FMO with isotype gating for activation markers}

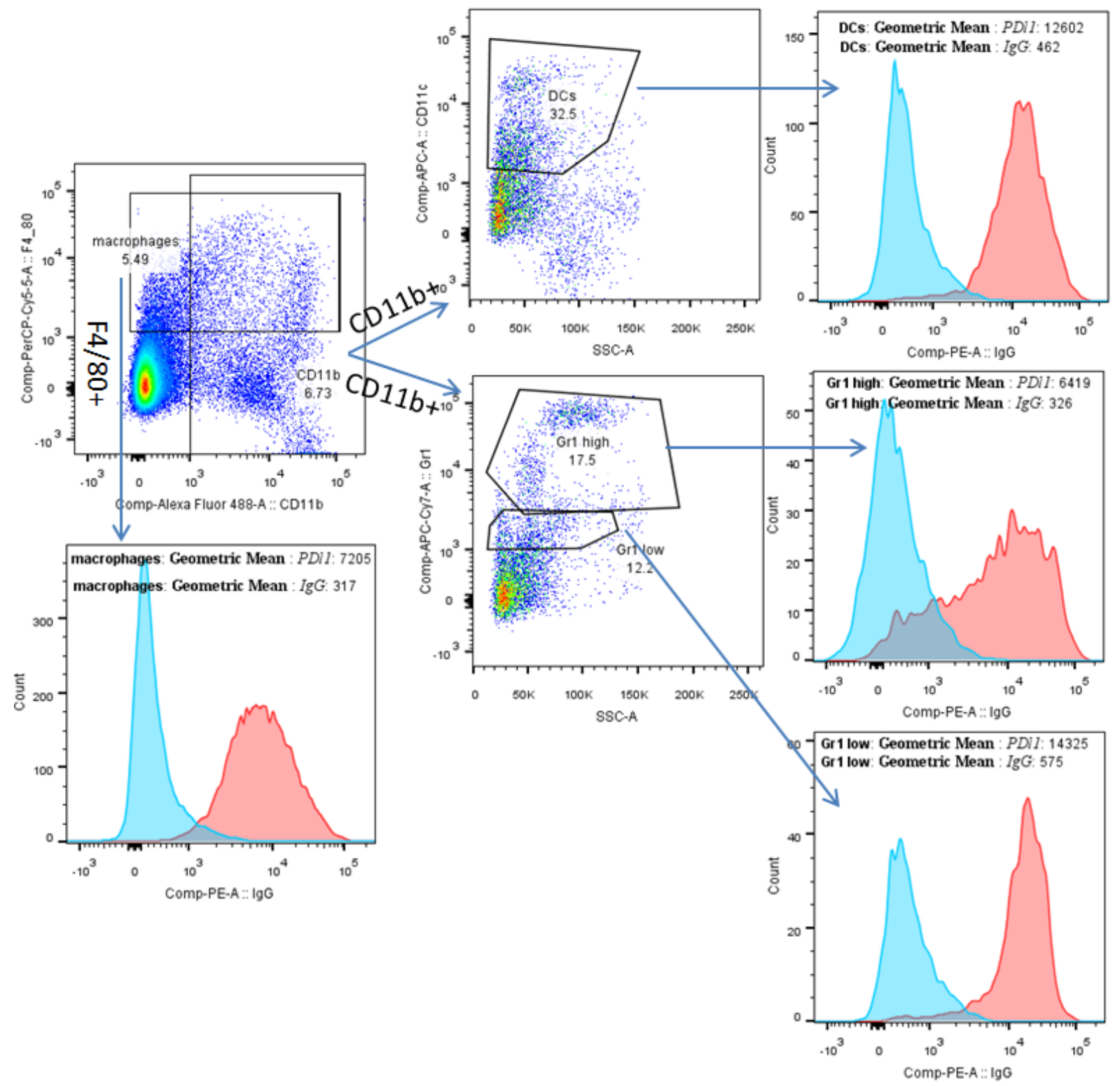

Figure A1-6 Example of FMO gating for activation marker PD-L1.

Firstly leukocytes are gated on using FSC vs SSC then single cells are selected for comparing the FSC-A over FSC-H as events in which there is no linear relationship between FSC-A and FSC-H represent doublets and are excluded from analysis. Using the gating strategy for myeloid populations the expression of PD-L1 was assessed for macrophages (F4/80+), DCs (CD11b+CD11 c+SSC $\left.{ }^{\text {low }}\right)$, Gr-1 high (CD11b+Gr $\left.1^{\text {hi }}\right)$, Gr-1 low (CD11b+Gr- $\left.1^{\text {low }}\right)$ by subtracting the geometric mean of the isotype control (IgG-PE, in blue) from the geometric mean of PD-L1PE (in red). Using the same method, expression of other activation markers were also assessed including MHCII and CD14 expression. 


\section{Appendix 1.4 Intracellular FMO with isotype and gating examples}

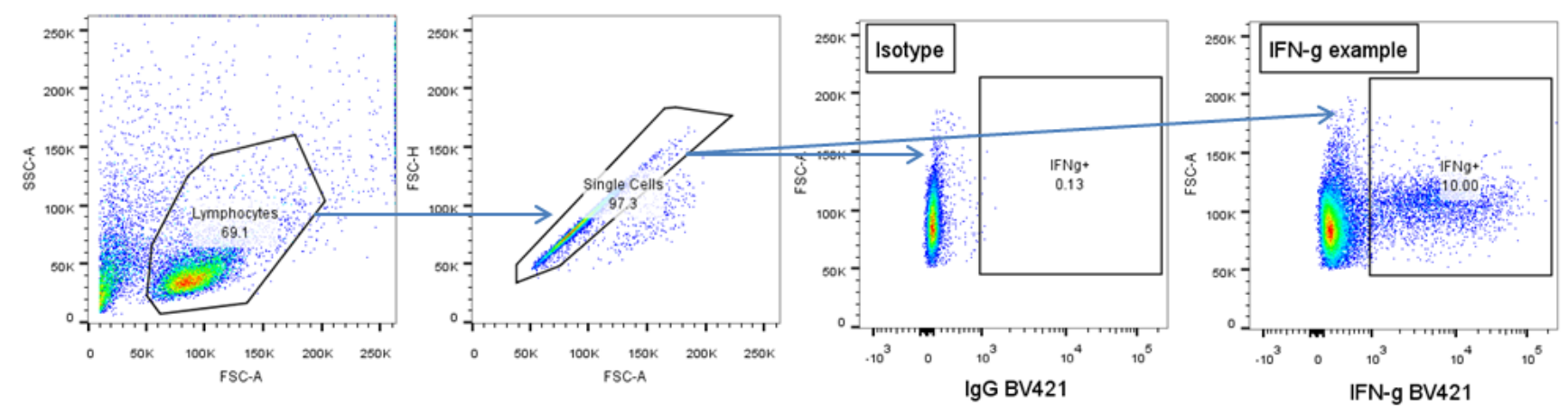

Figure A1-7 Intracellular IFN- $\gamma$ gating strategy.

Firstly leukocytes are gated on using FSC vs SSC then single cells are selected for comparing the FSC-A over FSC-H as events in which there is no linear relationship between FSC-A and FSC-H represent doublets and are excluded from analysis. Using the isotype (contains IgG BV421 control for the IFN- $\gamma$ antibody) set up under the same experimental conditions a negative gate is drawn and applied to the IFN $-\gamma+$ sample, which then identifies cells which are IFN- $\gamma+$ and not cells which non-specifically bind the antibody. Example from a MIS416-treated mouse.

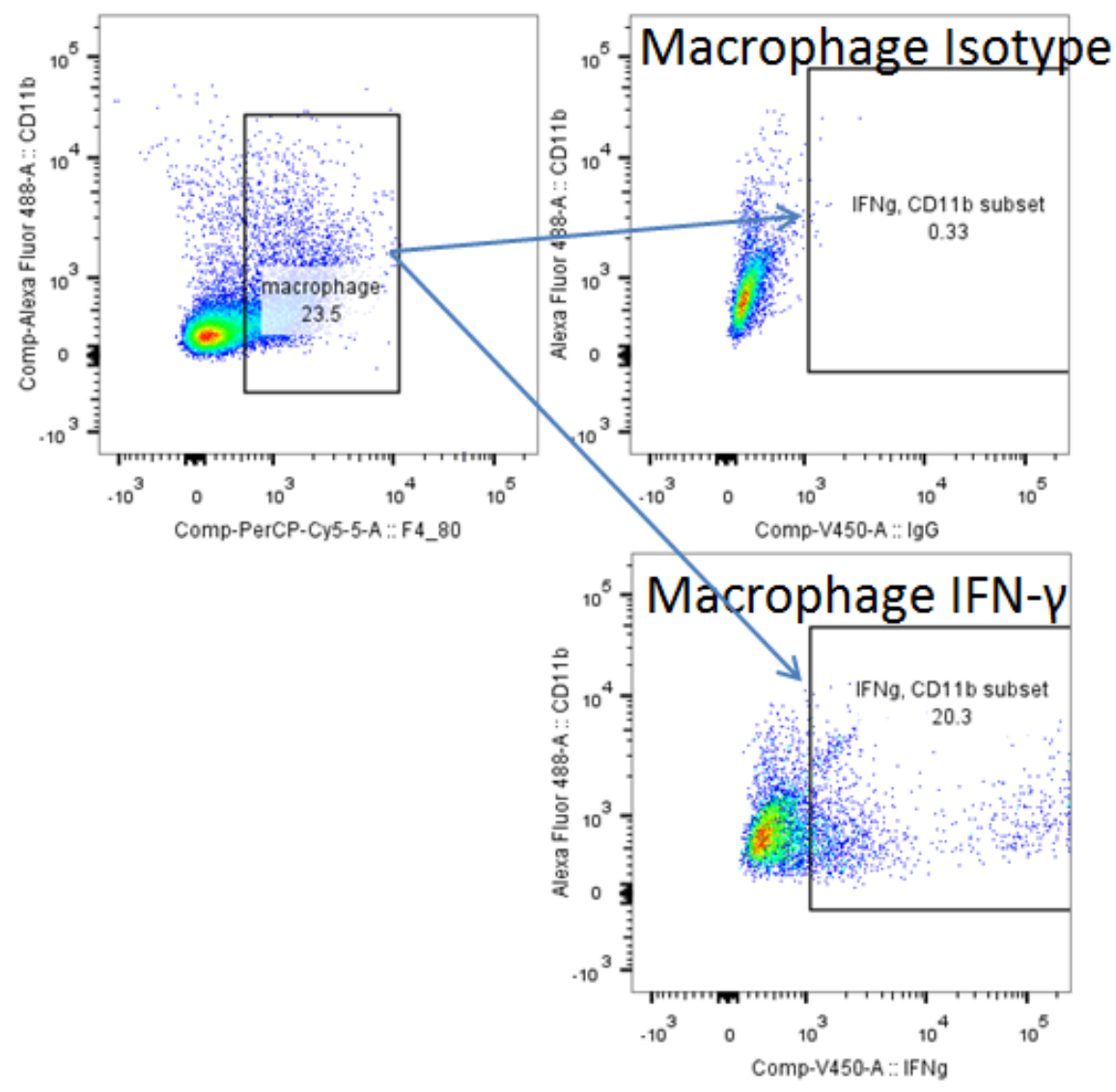

Figure A1-8 Macrophage intracellular IFN- $\gamma$ gating strategy.

Leukocytes were gated on as above in Figure A1-7, and all F4/80+ cells were gated on regardless of CD11b expression, these cells are referred to as macrophages. As done previously, IgG isotype was used to create a negative gate and applied to the IFN- $\gamma+$ sample. Example from a MIS416-treated mouse. 


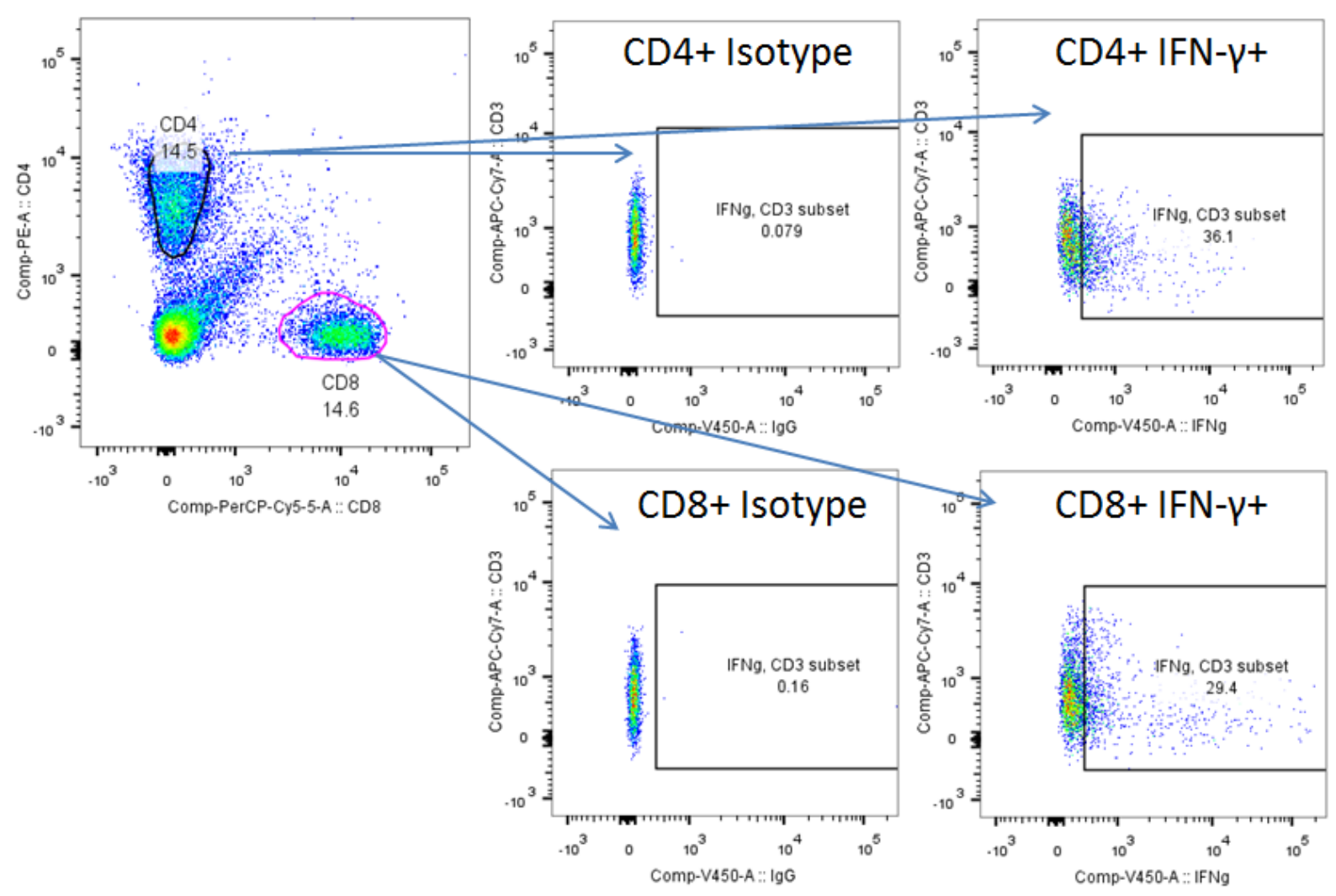

Figure A1-9 Lymphocyte intracellular IFN- $\gamma$ gating strategy.

Leukocytes were gated on as above in Figure A1-6, and cell populations were divided into CD4+ or CD8+. As done previously, IgG isotype was used to create a negative gate and applied to the IFN- $\gamma+$ sample. Example from a MIS416-treated mouse.
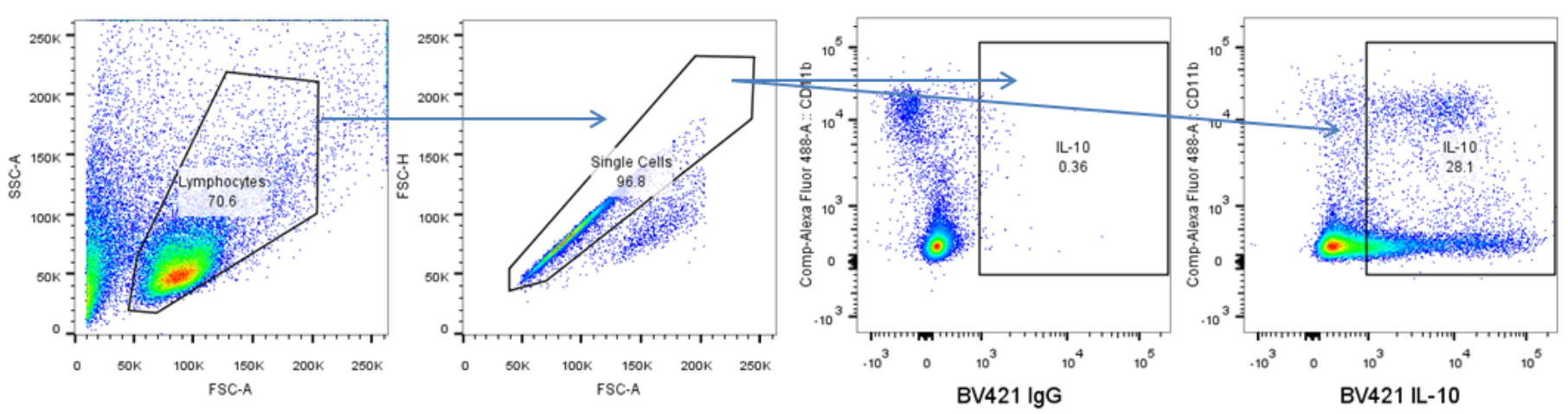

Figure A1-10 Intracellular IL-10 gating strategy.

Firstly leukocytes are gated on using FSC vs SSC then single cells are selected for comparing the FSC-A over FSC-H as events in which there is no linear relationship between FSC-A and FSC-H represent doublets and are excluded from analysis. Using the isotype (contains IgG BV421 control for the IL-10 antibody) set up under the same experimental conditions a negative gate is drawn and applied to the IL-10+ sample, which then identifies cells which are IL-10+ and not cells which non-specifically bind the antibody. Example from a MIS416-treated mouse. 


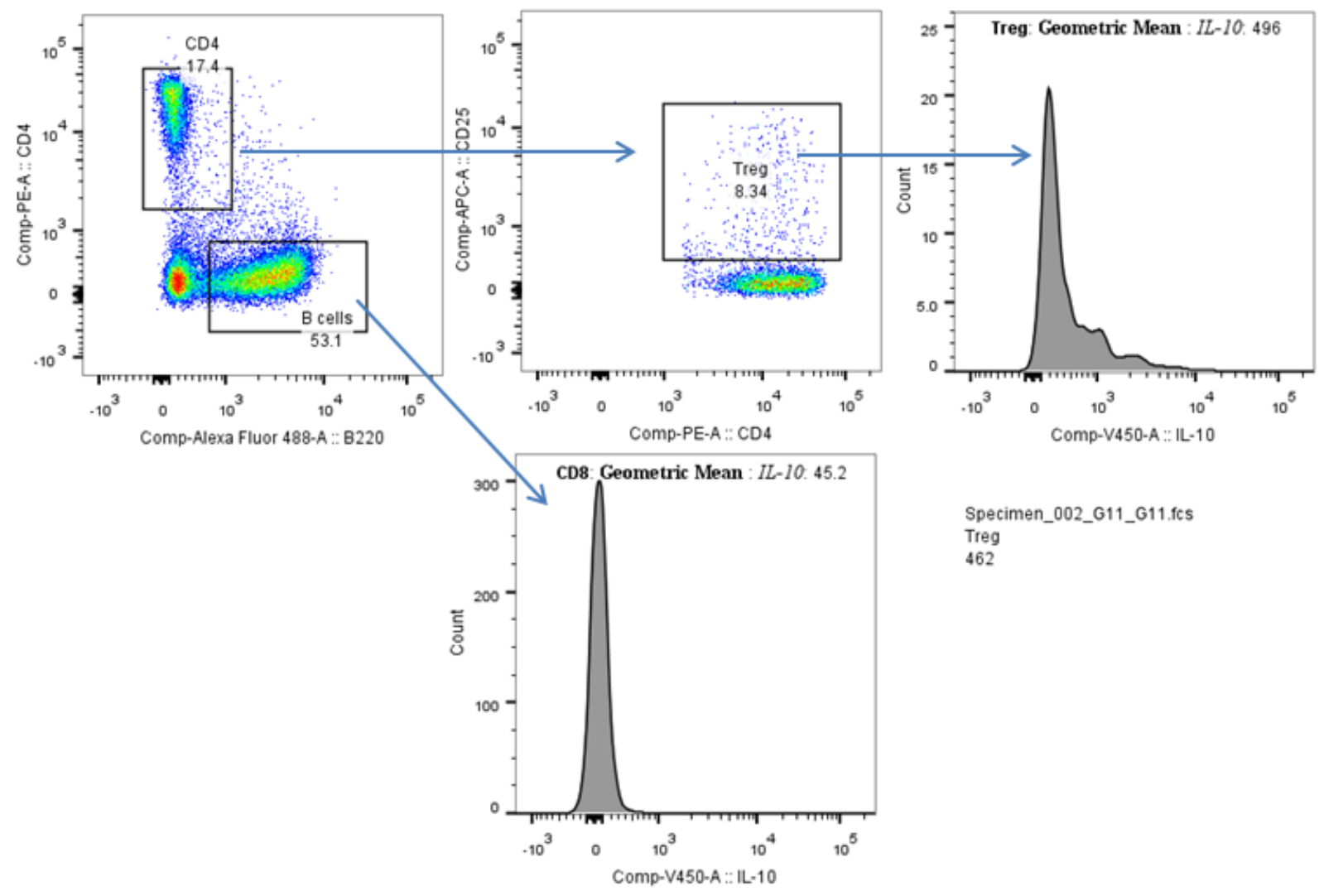

Figure A1-11 Lymphocytes intracellular IL-10 gating strategy.

Leukocytes were gated on as above in Figure A1-9, and cell populations were divided into CD4+ or CD8+. CD4+ cells were then gated on for CD25+ cells (Tregs). As done previously, IgG isotype was used to create a negative gate and applied to the IFN- $\gamma+$ sample, however IL-10 data was expressed as the level of IL-10 produced by each type therefore the geometric mean of BV421-IL-10 was used as indicated above. Example from a MIS416-treated mouse. 


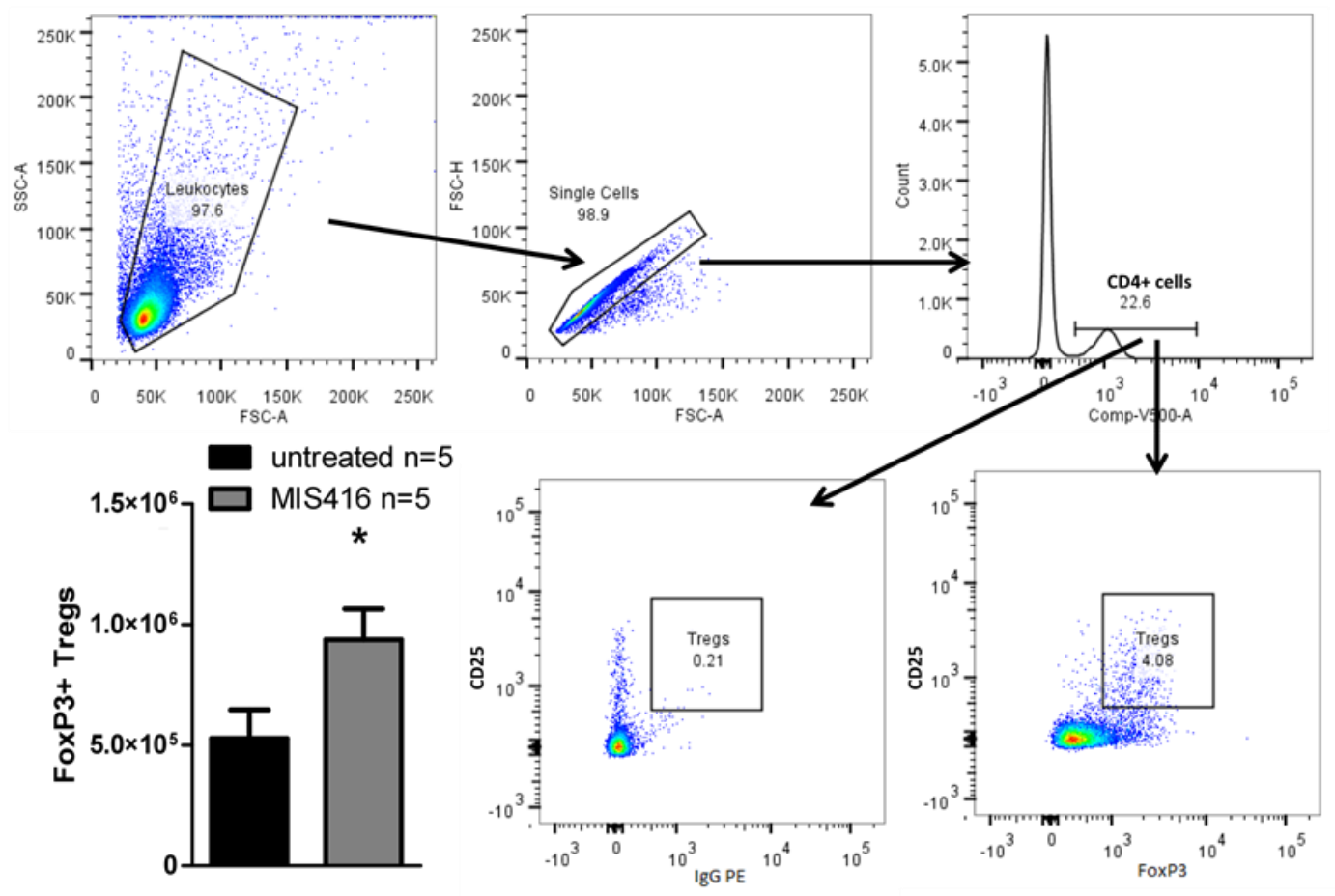

Figure A1-12 Lymphocyte intracellular FoxP3 gating strategy.

Shown are the gating strategy confirming FoxP3 expression in $\mathrm{CD}^{+} \mathrm{CD} 25^{+}$cells and the means and SEM of the total number of FoxP3 ${ }^{+} \mathrm{CD} 4{ }^{+} \mathrm{CD} 25^{+}$cells in MIS or untreated, unimmunized mice ( $\mathrm{n}=5$ per group) at day $15 .{ }^{*} \mathrm{p}$ $<0.05$ by Student's $t$ test; MIS compared to untreated (as shown in supplementary Figure-S1 from White et al 2014). 
Appendix 2: Assessing CFSE labelling and concentration of CD3/CD28

\section{activator beads}
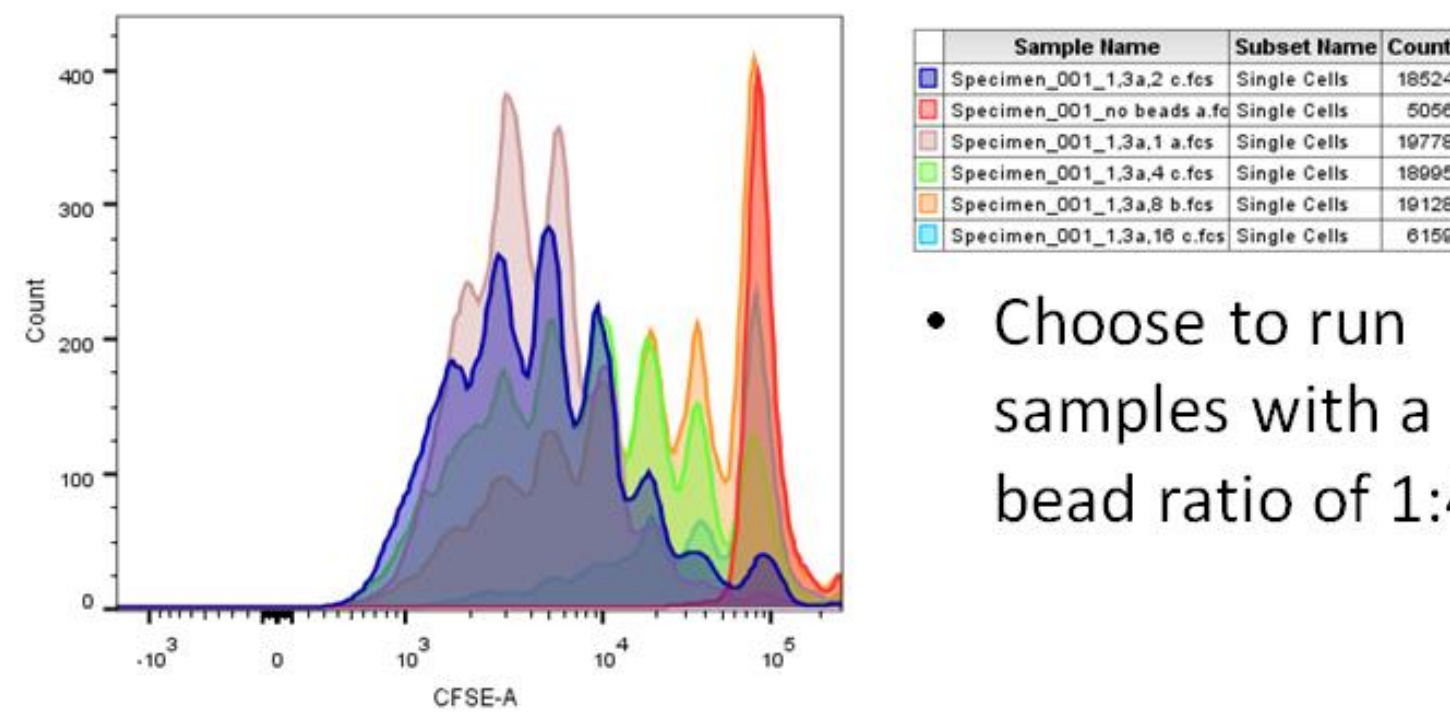

- Choose to run samples with a bead ratio of 1:4

Figure A2-13 CD3/CD28 activator beads in culture with isolated CD4+ $\mathrm{T}$ cells.

CD4+ cells were isolated from splenocytes using the untouched CD4+ dynabead kit and stained with $2 \mu \mathrm{M}$ CFSE and stimulated with CD3/CD28 activator beads at various concentrations. We choose to use the bead ratio of 1:4 for future experiments as the proliferation is consistent but not too high, as shown in the colour green. Unstimulated cells, shown in red, have a clear peak with narrow width. 


\section{Appendix 3: Ly6G+ and Gr-1dim MDSC have different expression profiles}
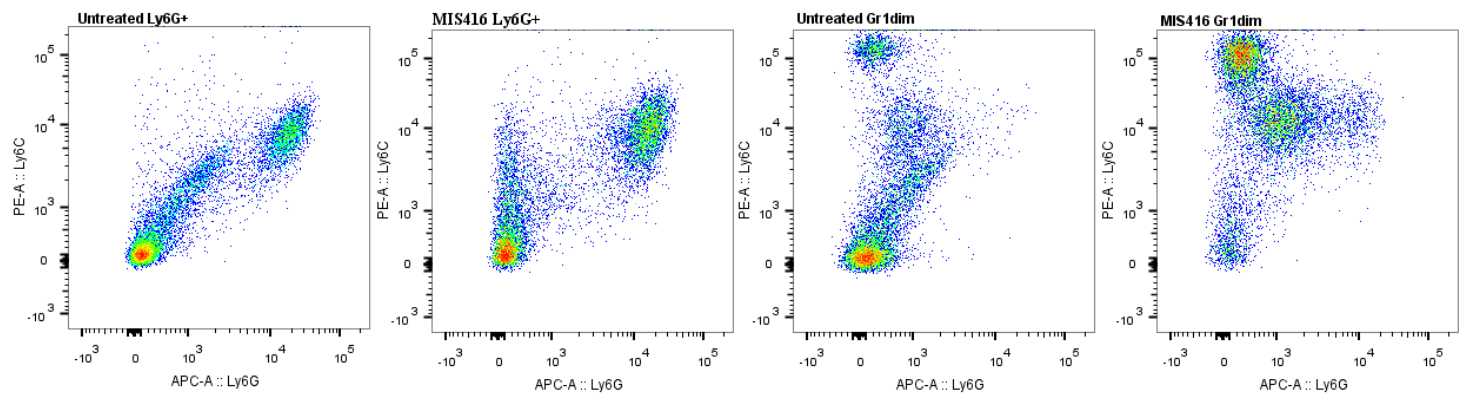

Figure A3-14 MDSC were either monocytic or granulocytic and express different levels of Ly6C and Ly6G.

C57BL/6 mice treated with a weekly dose of MIS416 (100 $\mu \mathrm{g} / \mathrm{mouse})$ and splenocytes were harvested at day 15, 1 day post MIS416 treatment. MDSC were isolated from splenocytes using MACs myeloid-derived suppressor cell kit (Miltenyi Biotec, Germany) and stained for Ly6G and Ly6C expression and analysed using flow cytometry. Both the untreated and MIS416-treated Ly6G+ separated MDSC express high levels of Ly6G and moderate levels of Ly6C, whereas the Gr-1dim MDSC for both MIS416-treated and untreated express very little Ly6G and high levels of Ly6C. 


\section{Appendix 4: In vivo proliferation assay gating strategy}

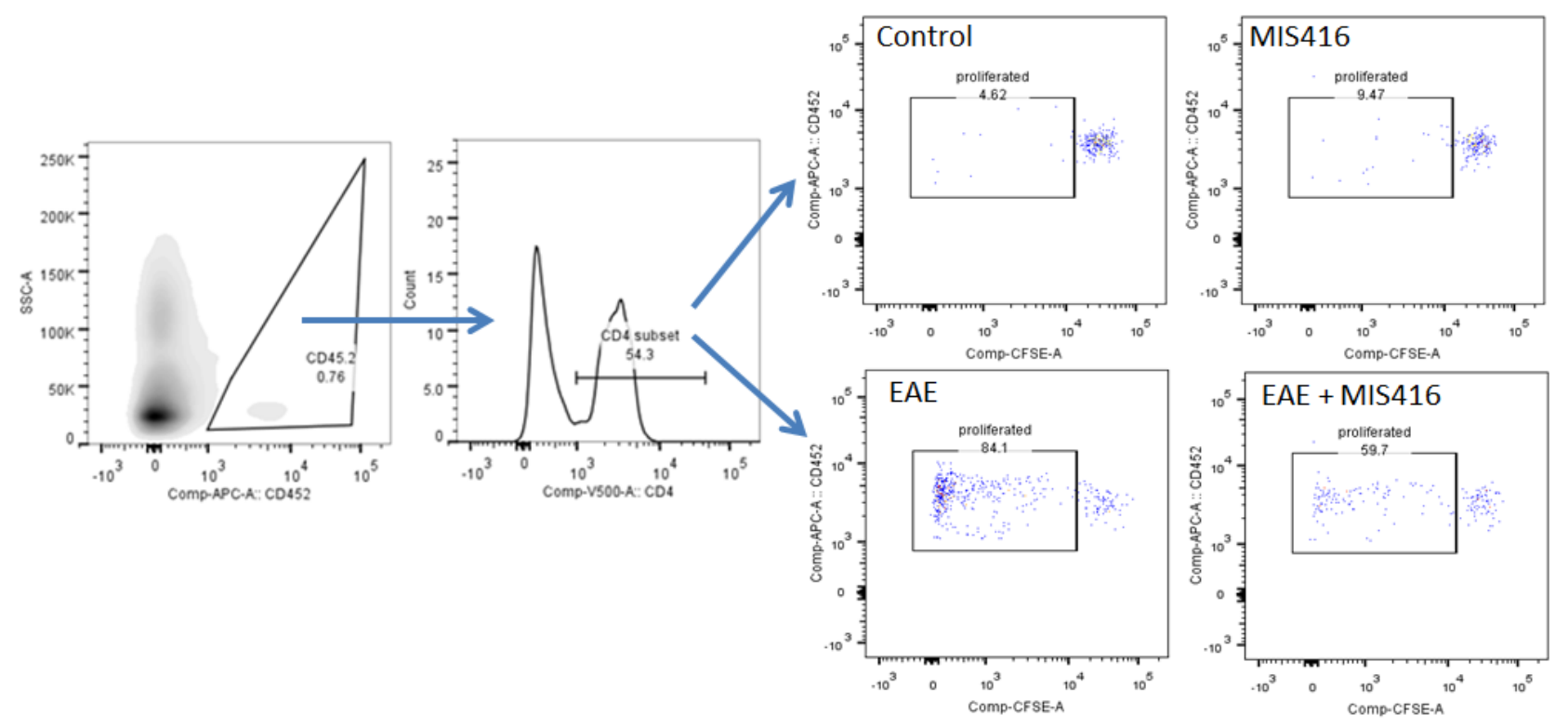

Figure A4-15 gating for in vivo proliferation experiments and examples of each treatment.

B6.SJL-ptprca were injected i.v. with $20 \times 10^{6}$ 2D2 cells at day -1 after which mice received EAE immunisation on day 0 and half were treated with $100 \mu \mathrm{g}$ MIS416. Mice were culled at day 5 and proliferation of CFSE labelled 2D2 cells were assessed using CD45.2 to distinguish injected cells. Firstly, cells were gated on CD45.2 expression after single/live cells were gated on, followed CD4+ expression. The percentage of proliferated cells was gated on based on control samples. Very little proliferation was seen in mice which were not EAE immunised, whereas EAE only mice had significant proliferation, while EAE + MIS416 had a smaller percentage of proliferating cells. 


\section{$\underline{\text { Appendix } 5 \text { Oral dosing of MIS416 }}$}

\section{A5.1 Introduction}

MIS416 treatment has been shown to induce a range of peripheral immune effects which may contribute to the MIS416-induced disease protection seen in EAE mice. These effects include cellular changes such as increased numbers of Tregs, red pulp macrophages and mMDSCs within the spleen and liver of MIS416-treated mice. Furthermore, there were changes in the cytokine profile of splenocyte cultures and serum, as indicated by MIS416-induced elevations of IFN- $\gamma$ and NO within both EAE and non-EAE mice. All together we have shown that these peripheral immune changes result in reduced $\mathrm{Ag}$-specific and non-specific $\mathrm{T}$ cell proliferation both in vitro and in vivo in MIS416-treated mice, which ultimately resulted in reduced EAE disease in treated mice. However, in these studies MIS416 was given in a systemic manner through intravenous injection (i.v.), and considering that MIS416 treatment was tested for the purpose of use in MS patients, we chose to determine the effect of oral MIS416 treatment.

The gastrointestinal tract (GI tract) functions to transport and aid the adsorption of water, electrolytes and nutrients. Because of its function, the GI tract comes into contact with a high bacterial and antigen load compared to any other organ in the body, and is able to defend against pathogens through a series of complex of selective barriers and gut-associated lymphoid tissues (GALT) (L. Shen, 2009). Interestingly, TLR signalling within the GI tract under normal conditions does not appear to result in inflammation, which indicates that immunological tolerance may occur to oral antigens (Nagler-Anderson, 2001). Furthermore, the liver is a major immune organ which comes into contact with antigens and bacterial products from the gut through the portal vein, and yet the liver does not appear to become inflamed in response to these signals (V. Racanelli \& B. Rehermann, 2006).

As we have shown that systemic treatment of MIS416 has immune mediating effects within the liver, we propose that oral treatment of MIS416 may also have effects within the liver and that these effects could be disease altering in EAE.

\subsection{Aims}

To determine if oral administration of MIS416 could induce peripheral or local immune changes and to characterise any changes induced by MIS416. 


\section{A5.3 Results}

\section{A5.3.1 Oral dosing of $100 \mu \mathrm{g}$ MIS416 daily did not induce peripheral immune changes}

Given that MIS416 treatment i.v. induced peripheral cellular alterations within the spleen and liver, we determined whether MIS416 administered orally could alter these sites. In particular, as the liver is a major drainage site for gut-derived bacterial antigens, we were particularly interested in oral MIS416-induced effects at this site. Using a $100 \mu \mathrm{g}$ dose of MIS416 given either weekly i.v. or orally daily in feed, we found very little effect on the weight of the mice (Figure A5-1). Furthermore, of the peripheral immune changes measured it was clear that the elevated spleen and liver cell counts as well as numbers of Tregs and macrophages seen with i.v. MIS416 treatment were not induced with oral treatment (Figure A5-1B,C,D\&E). Furthermore, there was no change in the activation state of macrophages within the spleen of orally treated mice as indicated by the expression of PD-L1 and MHC-II (Figure A5-1F).

In response to re-stimulation ex vivo, we have previously found that i.v. administration of MIS416 resulted in significantly higher amounts of IFN- $\gamma$ and IL-10 being produced in response to MIS416, than untreated mice. While in our experiments we saw this response with MIS416 i.v., we also found that oral administration did not significantly alter splenocyte or liver IFN- $\gamma$ or splenocyte IL-10 in response to either MIS416 or LPS (Figure A5-2). Together these results suggested that MIS416 did not have any effect when given orally although, it is possible that the dose required for MIS416 to act orally is much higher than when given systemically. 

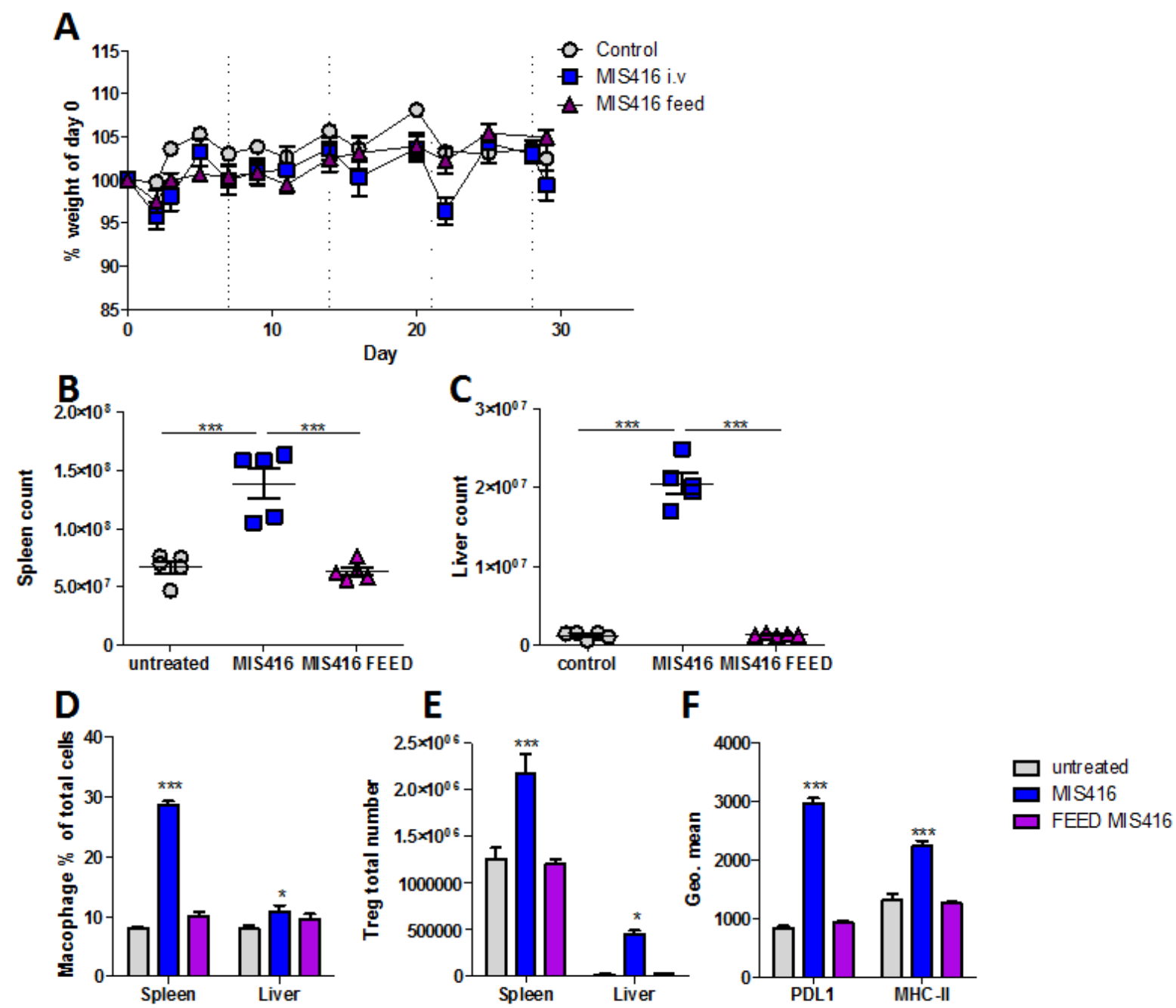

Figure A5-16 MIS416 given in feed at 100 ug daily did not alter the peripheral immune changes unlike i.v. MIS416 treatment.

Mice received daily doses of $100 \mu \mathrm{g}$ MIS416 in diet (MIS416 feed) or weekly doses of $100 \mu \mathrm{g}$ MIS416 i.v. Mice were weighed daily and culled at day 29, spleen and liver were collected and assessed for immunological changes. A) Weight of mice was not significantly altered with either i.v. or feed MIS416. B) Spleen counts were only increased in i.v mice. C) Liver counts were only increased in i.v mice. D) F4/80+ macrophages were only increased in i.v mice. E) Treg numbers were also only increased in i.v. mice. F) The expression of PD-L1 and MHC-II on macrophages was also only increased in i.v. treated mice. Results from 1 experiment, where $\mathrm{n}=5$ /group. Shown are the mean \pm SEM. $* \mathrm{p}<0.05$ and $* * * \mathrm{p}<0.001$, MIS416-treated compared to untreated and MIS416-feed treated by 1-way ANOVA (B \& C) or 2-way ANOVA (D, E \& F) with Bonferonnis post-test. 
A

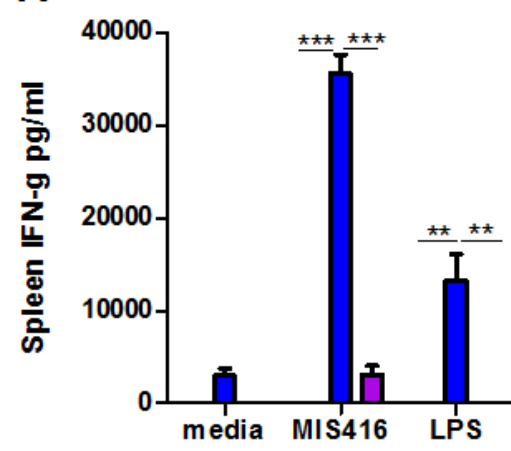

B

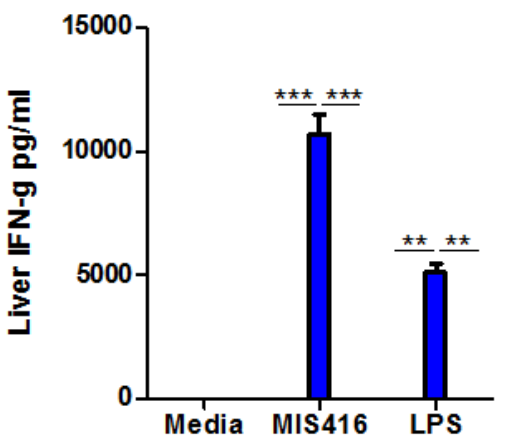

C

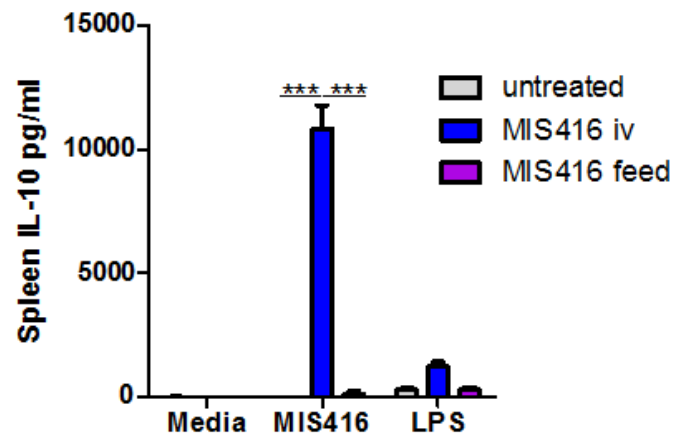

Figure A5-2 Cytokine re-call was not significantly enhanced in diet-treated MIS416 mice.

Mice received daily doses of $100 \mu \mathrm{g}$ MIS416 in diet (MIS416 feed) or weekly doses of $100 \mu \mathrm{g}$ MIS416 i.v. Mice were weighed daily and culled at day 29 , and splenocytes were cultured at $1 \times 10^{6} /$ well and livers cultured at $5 \times 10^{6} /$ well, and re-stimulated with media, MIS416 $(20 \mu \mathrm{g} / \mathrm{ml})$ or LPS $(200 \mathrm{ng} / \mathrm{ml})$. A) Splenocyte IFN- $\gamma$ production was significantly elevated in i.v. treated mice but not diet-treated mice. B) Liver IFN- $\gamma$ production was significantly elevated in i.v. treated mice but not diet-treated mice. C) IL-10 production was significantly elevated in i.v. treated mice but not diet-treated mice. Results from 1 experiment, where n=5/group. Shown are the mean \pm SEM. $* * \mathrm{p}<0.01$ and $* * * \mathrm{p}<0.001$, by 2 -way ANOVA with Bonferonni's post-test. 


\section{A5.3.2 Oral dosing of $2 \mathrm{mg}$ MIS416 daily induced minor immunological changes}

Given that we did not see any effect when MIS416 was given at $100 \mu \mathrm{g}$ dose we chose to use a much higher dose of $2 \mathrm{mg}$ to determine if there will be any effect on gut inflammation or peripheral immune changes. It was found that within the spleen there was an increased proportion of B220+ cells in oral MIS416-treated mice compared to untreated mice (Figure A5-3A). Furthermore, in the mesenteric LN (MLN) and the blood there was a decrease in the percentage of CD4+ $\mathrm{T}$ cells compared to untreated mice (Figure A5-3C\&D). These results indicate that at a higher dose of $2 \mathrm{mg}$, MIS416 administered orally is able to induce moderate changes to the peripheral immune system.

Further assessment of the activation state of splenocytes from orally administered MIS416 showed slight elevations of PD-L1 expression on macrophages and myeloid cells, while no change in the expression of MHCII was detected (Figure A5-4A). Additionally there were also changes in the cytokine production by splenocytes in response to MIS416 and ConA, with oral-treated mice producing less nitric oxide and IFN- $\gamma$ than untreated mice in response to re-stimulation (Figure A5-4B\&C). These results are in contrast with what is seen with i.v. administered MIS416, in which there were consistent increases in NO and IFN- $\gamma$ production in response to MIS416 restimulation, and increases in cellular populations such as CD4+ T cells. 

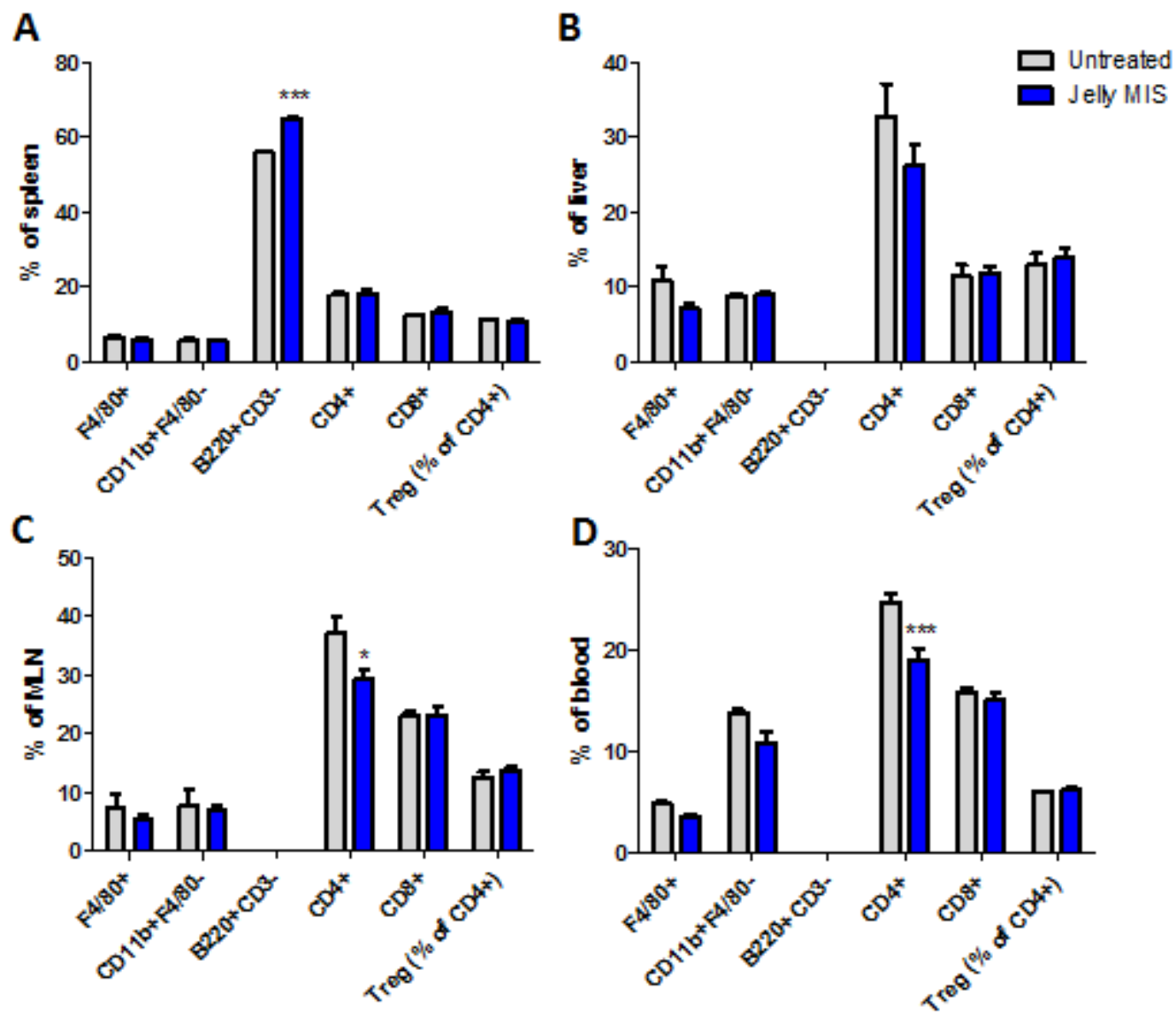

Figure A5-3 Oral MIS416-treated mice had minor cellular changes

Mice received 2mg MIS416 given daily in jelly for 14 days. The mice were culled at day 14 and spleen, liver, blood and LN were harvested for immunological assessment. A) Cellular proportions within the spleen. B) Cellular proportions within the liver (note B220 was not assessed in this organ). C) Cellular proportions within the mesenteric LN (note B220 was not assessed in this organ). D) Cellular proportions within the blood (note B220 was not assessed in this organ). Shown are means \pm SEM of 1-3 experiments where $n=3-9 /$ group. ${ }^{*} \mathrm{p}<0.05$ and $* * * \mathrm{p}<0.001$, by unpaired t-test with Welches correction.
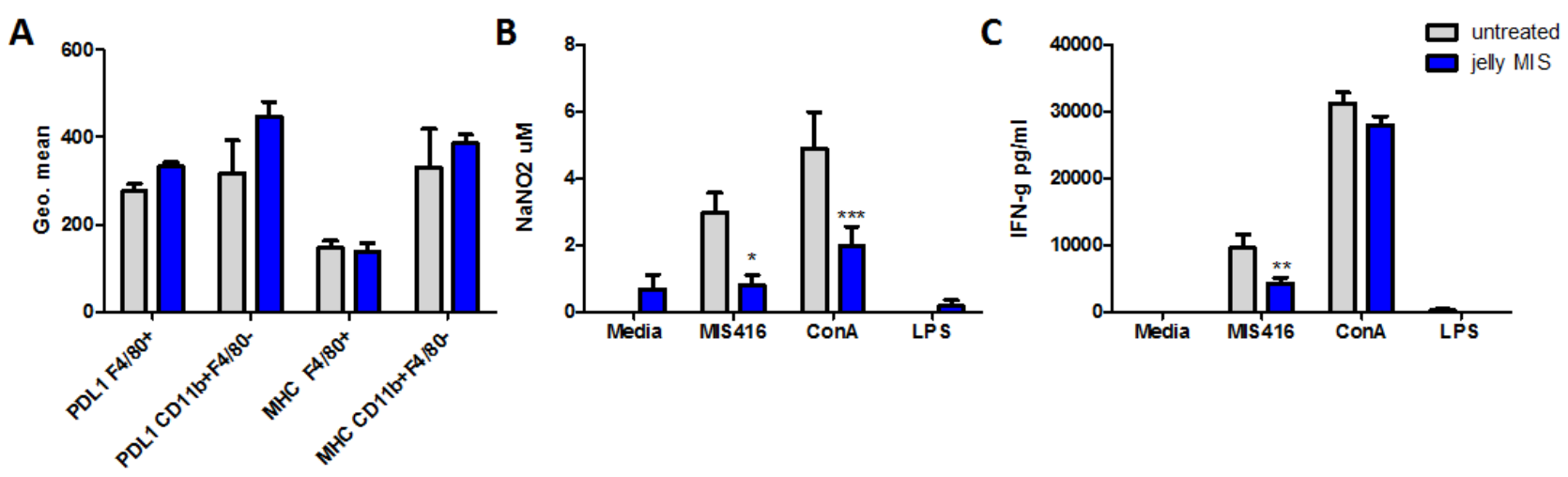

Figure A5-4 Oral MIS416 administration altered activation state within the spleen

Mice received 2mg MIS416 given daily in jelly for 14 days. The mice were culled at day 14 and splenocytes were assessed for activation using flow cytometry and cytokine analysis. A) Expression of PD-L1 and MHC-II on macrophage and myeloid cells in the spleen. B) Nitric oxide production reduced in jelly MIS416-treated mice. C) IFN- $\gamma$ production reduced in jelly MIS416-treated mice. Shown are means \pm SEM of 1-3 experiments where $n=3-$ 6/group. * $\mathrm{p}<0.05, * * \mathrm{p}<0.01$ and $* * * \mathrm{p}<0.001$, by unpaired $\mathrm{t}$-test with Welches correction. 


\section{A5.3.3 Oral dosing of $2 \mathrm{mg}$ MIS416 daily resulted in minor inflammatory gut changes}

As we saw slight changes within the peripheral immune organs assessed (i.e. liver, MLN, spleen and blood) we also determined the effect of oral MIS416 administration on gut inflammation. To do this H\&E sectioning of the small intestine and colon were used to assess immune infiltration and crypt integrity. It was found that oral MIS416 treatment did not significantly alter the crypt integrity as determined by scoring H\&E sections of the small intestine and colon (Figure A5-5). Additionally, oral MIS416 did not affect infiltration of the mucosa, submucosa or muscle of either the colon or small intestine when compared to untreated mice (Figure A5-6). In contrast to the general cellular infiltration measured with $\mathrm{H} \& \mathrm{E}$ staining, when the sections were IHC stained for $\mathrm{F} 4 / 80+$ cells there was a significant decrease in the number of F4/80+ macrophages found within the second half of the small intestine with oral MIS416 treatment (Figure A5-7). Together the gut sections indicate that oral administration of MIS416 does not appear to have a significant effect on local inflammation and cellular infiltration into the gut.

a.

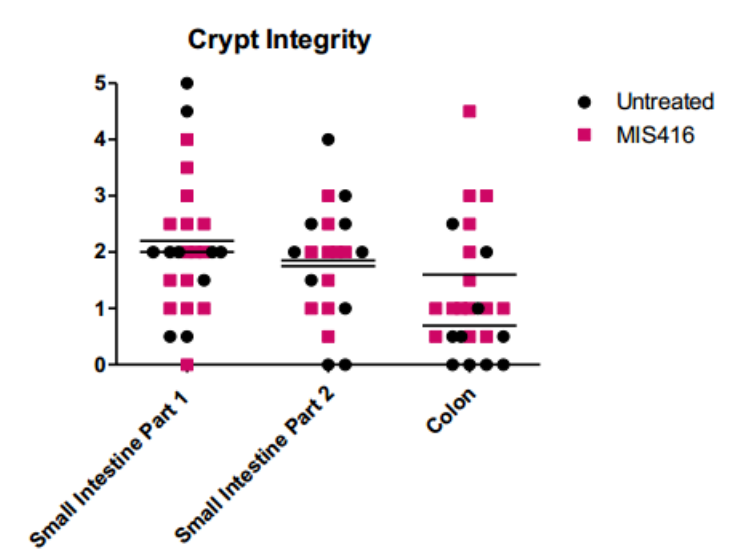

b.
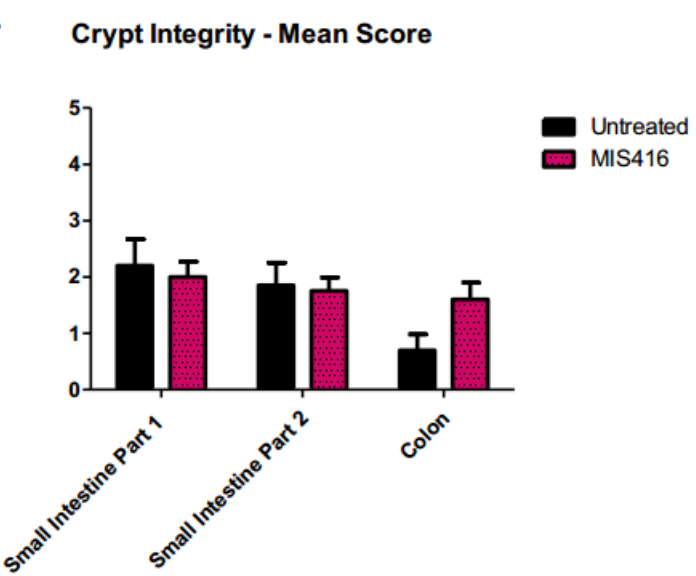

Figure A5-5 Oral MIS416 administration did not alter crypt integrity

Mice received 2mg MIS416 given daily in jelly and culled at day 14. Small intestine (in 2 parts) and the colon were assessed for crypt integrity using $\mathrm{H} \& \mathrm{E}$ staining and independent assessors were used to evaluate crypt scores. A) Crypt integrity showing spread of data for each sample. B) Mean score \pm SEM of crypt integrity. This data was gathered in conjunction with Louise Rooney. 
a. Infiltration of Inflammatory Cells Colon

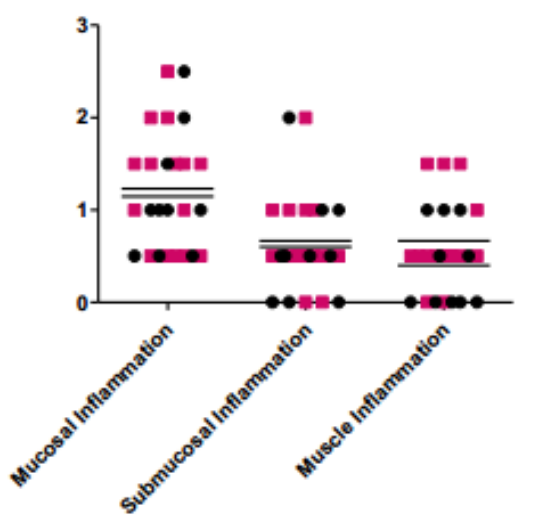

C. Infiltration of Inflammatory Cells Small Intestine Part 1

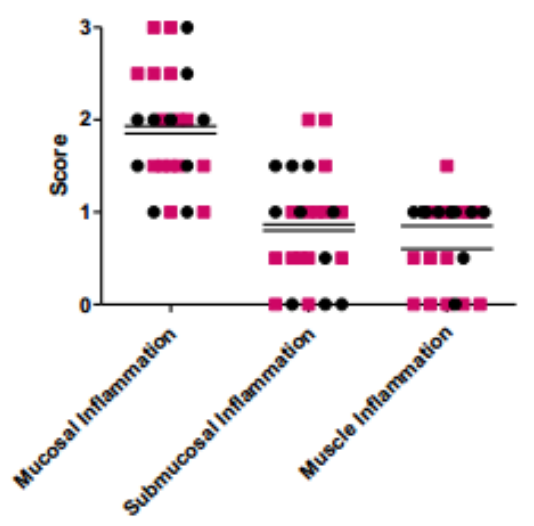

e. Infiltration of Inflammatory Cells Small Intestine Part 2

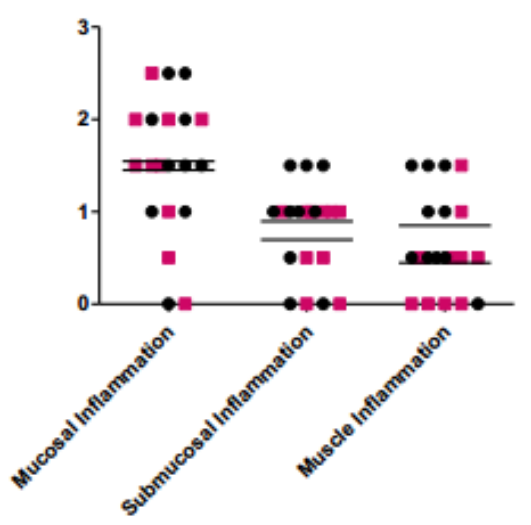

b.

- Untreated

- MIS416

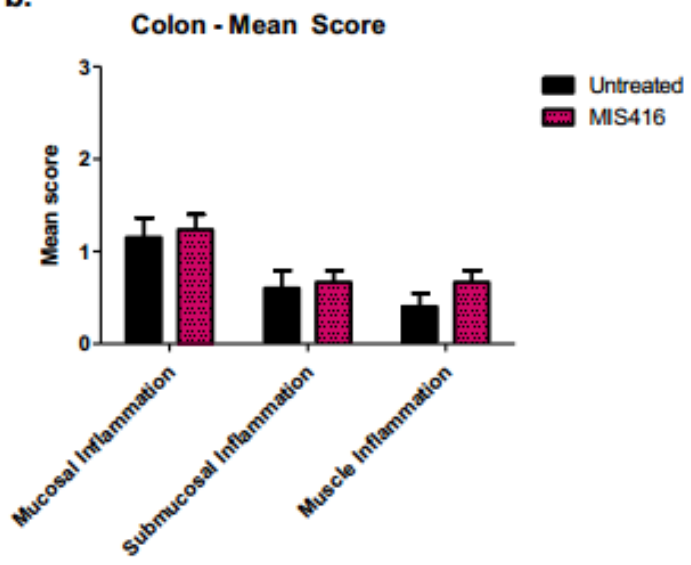

d.

Small Intestine Part 1 - Mean Score
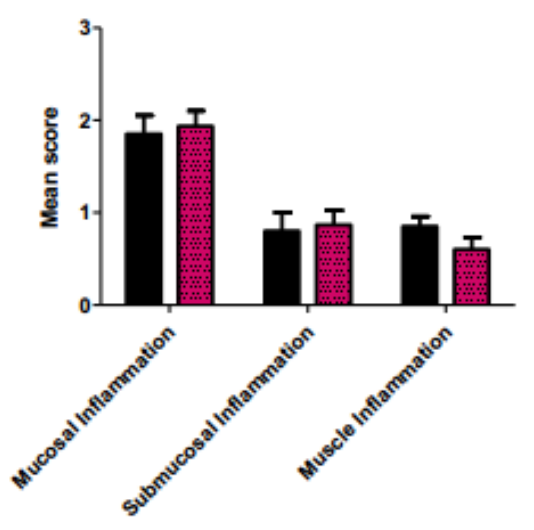

f.

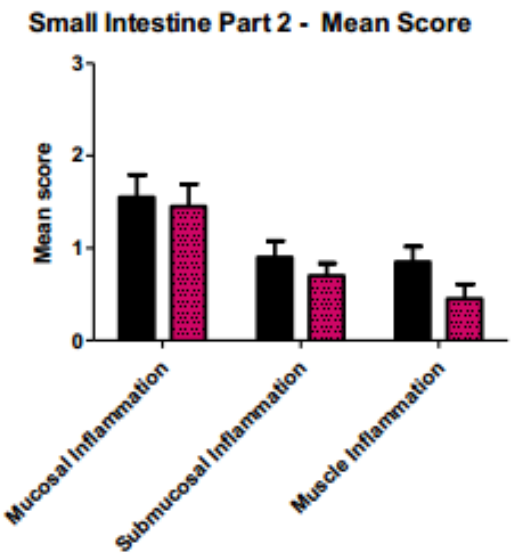

Figure A5-6 Oral MIS416 administration did not induce gut immune infiltration

Mice received 2mg MIS416 given daily in jelly and culled at day 14. Small intestine (in 2 parts) and the colon were assessed for immune infiltration using H\&E staining and independent assessors were used to determine infiltration scores. A\&B) Infiltration of cells into the muscosa, submucosa or muscle of the colon. C\&D) Infiltration of cells into the muscosa, submucosa or muscle of part 1 of the small intestine. E\&F) Infiltration of cells into the muscosa, submucosa or muscle of part 2 of the small intestine. This data was gathered in conjunction with Louise Rooney. 
a.

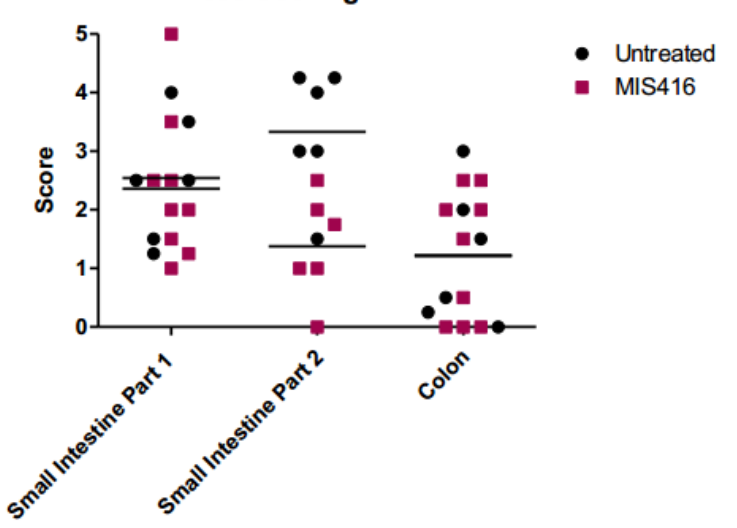

b. F4/80 - Mean Score

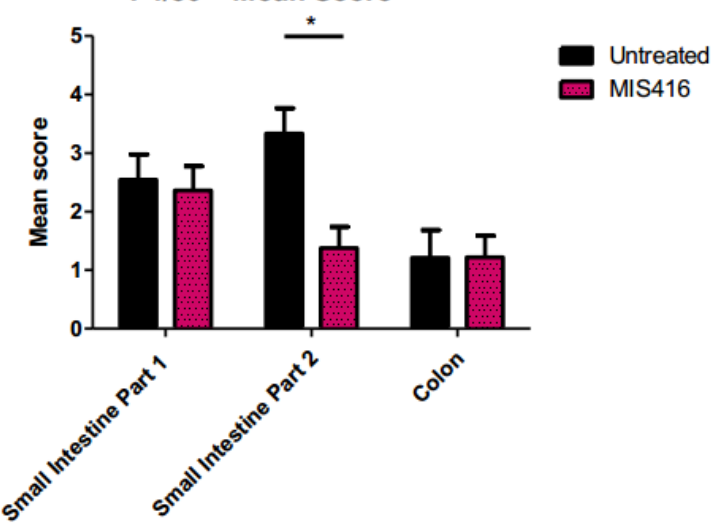

Figure A5-7 Oral MIS416 administration reduced macrophage infiltration in the small intestine

Mice received 2mg MIS416 given daily in jelly and culled at day 14. Small intestine (in 2 parts) and the colon were assessed for F4/80+ infiltration using immunohistochemistry and independent assessors were used to determine infiltration scores. Shown in B) are the means \pm SEM of 2 experiments, $n=6-9 /$ group $* \mathrm{p}<0.05$ by 2 way ANOVA with Bonferroni post-test. This data was gathered in conjunction with Louise Rooney. 


\section{A5.4 Discussion}

The aim of these experiments was to determine if oral administration of MIS416 could induce local or peripheral immune effects. We found that a high dose of MIS416 administered orally induced small changes in the proportion of CD4+ cells within the blood and mesenteric LN compared to untreated mice. Furthermore, that the high dose of oral MIS416 also reduced the splenocyte response to ConA and MIS416 re-stimulation, as seen by reduced NO and IFN- $\gamma$ production. Although MIS416-induced some peripheral changes, there was no significant increase in local inflammation within the gut suggesting that MIS416 could induce a suppressive phenotype locally.

We used cellular infiltration into the gut as a measure of inflammation as it has been suggested that in response to an inflammatory stimuli monocytes from the blood are recruited to the gut (Smith et al., 2011). However, our results showed the opposite effect in response to MIS416 administration, where MIS416-treated mice had a reduced macrophage signal in the second part of the small intestine, suggesting that MIS416-induced TLR9 and NOD2 signalling may reduce inflammation in the gut. These results are further supported by the lack of total cellular infiltration the small intestine and colon of MIS416-treated mice compared to untreated.

A study has assessed the anti-inflammatory effects of probiotics in murine colitis, and determined that TLR9 signalling was responsible for the reduce severity of colitis disease by probiotic treatment (Rachmilewitz et al., 2004). Another study has also shown that continuous exposure to bacterial DNA (i.e. TLR9 agonist), resulted in anti-inflammatory effects within a mouse model of colitis (Rachmilewitz et al., 2002). Additionally, NOD2 signalling has also been shown to have protective effects in inflammatory bowel disease, as it has been shown that Crohns disease is associated with mutations in NOD2 or its downstream pathways; therefore indicating that NOD2 plays a protective role (Peyrin-Biroulet et al., 2006). In addition to the effect of TLR9 and NOD2 signalling on their own, our studies have indicated that when used together as with MIS416 treatment, we can induce an immunoregulatory state which is capable of reducing EAE disease.

An interesting result from the oral administration of MIS416 was the reduced IFN- $\gamma$ and NO production by splenocytes in response to ConA or MIS416 re-stimulation. These results are surprising as they indicate that oral MIS416 treatment may be able to have effects on the immune system outside of the gut. Given that the gut microbiota has been implicated in EAE disease pathogenicity (Lee, Menezes, Umesaki, \& Mazmanian, 2011) and that oral MIS416 can have effects outside of the gut, it would be important to consider the treatment of EAE mice with orally administered MIS416. 
Finally, it has been determined that DCs play a crucial role of the generation of Tregs within the gut (Matteoli et al., 2010), and as we have shown in previous chapters that the addition of TLR9 and NOD2 signalling can induce the formation of Tregs (i.e. with MIS416), this may be a way in which MIS416 has anti-inflammatory actions within the gut. These results altogether suggest that oral MIS416 treatment might be protective in an inflammatory state and whether the protection induced is able to alter a systemic autoimmune disease like EAE is yet to be determined. However, future assessment should include whether oral MIS416 treatment is protective in inflammatory bowel disease (IBD) or Crohns disease. 


\section{Appendix 6: Buffers and solutions}

Phosphate buffered saline (PBS)

10xPBS

$\mathrm{NaCl} 170 \mathrm{~g}$

$\mathrm{Na} 2 \mathrm{HPO} 4.12 \mathrm{H} 2 \mathrm{O} 62.32 \mathrm{~g}$

$\mathrm{NaH} 2 \mathrm{PO} 4.2 \mathrm{H} 2 \mathrm{O} 4.04 \mathrm{~g}$

2 litres ddH2O

$1 x P B S$

Dilute 10:1 ddH2O for working solution of 1X PBS.

\section{Percoll Diluent (v)}

10X PBS $45 \mathrm{ml}$

$0.6 \mathrm{M} \mathrm{HCl} 3 \mathrm{ml}$

$\mathrm{ddH} 2 \mathrm{O} 132 \mathrm{ml}$

Sterilise with a syringe filter

$37 \%$ Percoll (v/v) for CNS

Percoll (33.4\%)

Percoll diluent (19.6\%)

$1 \mathrm{X}$ PBS $(47 \%)$

\section{$33 \%$ Percoll (v) for liver}

$15 \mathrm{ml}$ Percoll,

$30 \mathrm{ml} 1 \mathrm{xPBS}$

$1 \mathrm{ml}$ Alsevers solution (Sigma, USA)

FACs buffer (v/v)

FCS 2\%

Sodium azide (1 M) $0.1 \%$

1xPBS $97.9 \%$

\section{Pertussis toxin buffer}

Tris $15 \mathrm{mM}$

Sodium chloride $0.5 \mathrm{M}$

Triton X-100 0.017\% (v/v)

In $\mathrm{ddH} 2 \mathrm{O}$, filter sterilised using $0.22 \mu \mathrm{m}$ syringe filter

\section{Complete T cell media (CTCM) or culture media (v/v)}

Dulbecco's Modified Eagle Medium 85.9\%

FCS $10 \%$

$\beta$-Mecaptoethanol (55 mM) $0.1 \%$

Non-essential amino acids (10nM) $1 \%$

L-glutamate (200 mM) 1\%

HEPES buffer (1 M) $1 \%$

Penicillin/Streptomycin (100 U/ml/10 mg/ml) 1\% 
Collagenase type II

$2.4 \mathrm{mg}$

$1 \mathrm{ml} 1 \mathrm{xPBS}$

Wash buffer (v/v)

Dulbecco's Modified Eagle Medium 96\%

HEPES buffer (1M) 3\%

Penicillin/Streptomycin (100 U/ml/10 mg/ml) $1 \%$

ELISA capture buffer

$\mathrm{Na} 2 \mathrm{HPO} 40.1 \mathrm{M}$

In ddH2O, adjusted to $\mathrm{pH} 9$.

ELISA stop solution

$\mathrm{H} 2 \mathrm{SO} 40.18 \mathrm{M}$

In ddH2O

Isolation buffer $(\mathbf{v} / \mathrm{v})$

FCS $2 \%$

EDTA $(0.5 \mathrm{mM}) 0.004 \%$

$1 \mathrm{xdPBS} 47.006 \%$

Griess solution A (w/v)

Sulphanilamide $1 \%$

In $2.5 \%$ Phosphoric acid

Griess solution B (w/v)

$\mathrm{N}$-(1-napthyl) ethylenediamine $1 \%$

In $2.5 \%$ Phosphoric acid

Acid alcohol (v/v)

$\mathrm{HCl} 0.3 \%$

Ethanol 70\%

ddH2O $29.7 \%$

0.01\% Lithium Carbonate (w/v)

Lithium Carbonate $0.01 \%$

ddH2O 99.99

4\% Paraformaldehyde (w/v)

Paraformaldehyde 4\%

1xPBS 96\%

ph adjusted to 7.4

$30 \%$ Sucrose

Sucrose (w/v) $30 \%$

Sodium Azide (1 M) (v/v) $0.1 \%$

ddH2O 69.9\% 
Appendix 7: Antibodies and Proteins

\begin{tabular}{|c|c|c|c|c|c|}
\hline Specificity & Label & $\begin{array}{l}\text { Species and } \\
\text { Isotype }\end{array}$ & Manufacturer & $\begin{array}{l}\text { Optimal } \\
\text { dilution }\end{array}$ & Clone \\
\hline \multirow[t]{2}{*}{$\mathrm{B} 220$} & FITC & Rat IgG2a & $\begin{array}{l}\mathrm{BD} \\
\text { pharmingen }\end{array}$ & $1: 400$ & RA3-6B2 \\
\hline & Biotin & Rat IgG2a & $\begin{array}{l}\mathrm{BD} \\
\text { pharmingen }\end{array}$ & $1: 1000$ & RA3-6B2 \\
\hline CD3 & APC/Cy7 & Rat IgG2b, k & biolegend & $1: 200$ & $17 \mathrm{~A} 2$ \\
\hline \multirow[t]{2}{*}{ CD4 } & $\mathrm{PE}$ & Rat IgG2a & $\begin{array}{l}\mathrm{BD} \\
\text { pharmingen }\end{array}$ & $1: 1000$ & RM4-5 \\
\hline & V500 & Rat IgG2a & $\begin{array}{l}\mathrm{BD} \\
\text { pharmingen }\end{array}$ & $1: 400$ & RM4-5 \\
\hline CD8 & PerCP-Cy5.5 & Rat IgG2a & eBioscience & $1: 400$ & $53-6.7$ \\
\hline \multirow[t]{2}{*}{$\begin{array}{l}\text { CD11b (MAC- } \\
1)\end{array}$} & AF488 & Rat IgG2b, k & $\begin{array}{l}\text { BD } \\
\text { pharmingen }\end{array}$ & $1: 1000$ & M1/70 \\
\hline & $\mathrm{PE}$ & Rat IgG2b & eBioscience & $1: 2000$ & $\mathrm{M} 1 / 70$ \\
\hline CD11c & APC & Hamster IgG & Biolegend & $1: 400$ & N418 \\
\hline CD14 & $\mathrm{PE} / \mathrm{Cy} 7$ & Rat IgG2a & Biolegend & $1: 400$ & RTK2758 \\
\hline CD25 & APC & Rat IgG1 & $\begin{array}{l}\mathrm{BD} \\
\text { pharmingen }\end{array}$ & $1: 600$ & PC61 \\
\hline $\mathrm{CD} 45$ & V450 & Rat IgG2b & $\begin{array}{l}\mathrm{BD} \\
\text { pharmingen }\end{array}$ & $1: 400$ & $30-\mathrm{F} 11$ \\
\hline CD45.1 & $\mathrm{PE}$ & Mouse IgG2a & Biolegend & $1: 1000$ & A-20 \\
\hline$\overline{C D} 45.2$ & $\overline{\mathrm{APC}}$ & $\begin{array}{ll}\text { Mouse } & \operatorname{IgG} 2, \\
\mathrm{k} & \\
\end{array}$ & $\begin{array}{l}\mathrm{BD} \\
\text { pharmingen }\end{array}$ & $1: 400$ & 104 \\
\hline $\mathrm{F} 4 / 80$ & PerCP-Cy5.5 & Rat IgG2a & eBioscience & $1: 400$ & BM8 \\
\hline Gr-1 & APC-Cy7 & Rat IgG2b & $\begin{array}{l}\mathrm{BD} \\
\text { pharmingen }\end{array}$ & $1: 400$ & RB6-8C5 \\
\hline $\mathrm{I}-\mathrm{Ab}$ (MHCII) & $\mathrm{PE}$ & Rat IgG2a & $\begin{array}{l}\mathrm{BD} \\
\text { pharmingen }\end{array}$ & $1: 800$ & AF6-120.1 \\
\hline Ly-6C & $\mathrm{PE}$ & Rat IgG2c & Biolegend & $1: 800$ & HK1.4 \\
\hline Ly-6G & $\overline{\mathrm{APC}}$ & Rat IgG2a,k & Biolegend & $1: 400$ & $1 \mathrm{~A} 8$ \\
\hline PD-1 & BV421 & Rat IgG2a & Biolegend & $1: 400$ & 29F.1A12 \\
\hline PDL-1 & PE & Rat IgG2a & eBioscience & $1: 1500$ & MIH5 \\
\hline PDL-2 & $\mathrm{PE}$ & Rat IgG2a & eBioscience & $1: 200$ & TY25 \\
\hline NK1.1 & PE/Cy7 & $\begin{array}{l}\text { Mouse } \operatorname{IgG} 2 \mathrm{a}, \\
\mathrm{k}\end{array}$ & Biolegend & $1: 250$ & PK136 \\
\hline $\begin{array}{lrr}\mathrm{V} \beta & 11 & \mathrm{~T} \text {-cell } \\
\text { receptor } & \\
\end{array}$ & $\mathrm{PE}$ & Rat IgG2b & $\begin{array}{l}\text { BD } \\
\text { pharmingen }\end{array}$ & $1: 400$ & RR3-15 \\
\hline $\mathrm{V} \beta 8.1$ & Biotin & $\begin{array}{l}\text { Mouse IgG2a, } \\
\mathrm{k}\end{array}$ & $\begin{array}{l}\mathrm{BD} \\
\text { pharmingen }\end{array}$ & $1: 400$ & MR5-2 \\
\hline \multicolumn{6}{|l|}{$\begin{array}{l}\text { Isotype } \\
\text { Controls }\end{array}$} \\
\hline Isotype Control & $\overline{\text { FITC }}$ & Rat IgG2a & $\begin{array}{l}\mathrm{BD} \\
\text { pharmingen }\end{array}$ & $1: 400$ & R35-95 \\
\hline
\end{tabular}




\begin{tabular}{|c|c|c|c|c|c|}
\hline \multirow[t]{2}{*}{ Isotype Control } & \multirow[t]{2}{*}{ PE } & Rat IgG1 & $\begin{array}{l}\mathrm{BD} \\
\text { pharmingen }\end{array}$ & $1: 400$ & R3-34 \\
\hline & & Rat IgG2a & $\begin{array}{l}\text { BD } \\
\text { pharmingen }\end{array}$ & $1: 400$ & R35-95 \\
\hline \multirow[t]{3}{*}{ Isotype Control } & \multirow[t]{3}{*}{ APC } & Rat IgG2a & $\begin{array}{l}\text { BD } \\
\text { pharmingen }\end{array}$ & $1: 800$ & R35-95 \\
\hline & & Rat IgG1 & Biolegend & $1: 400$ & RTK2071 \\
\hline & & $\begin{array}{l}\text { Armenian } \\
\text { Hamster IgG }\end{array}$ & Biolegend & $1: 400$ & HTK888 \\
\hline Isotype Control & APC-Cy7 & Rat IgG2b & $\begin{array}{l}\mathrm{BD} \\
\text { pharmingen }\end{array}$ & $1: 800$ & R35-38 \\
\hline Isotype Control & V450 & Rat IgG2b & $\begin{array}{l}\mathrm{BD} \\
\text { pharmingen }\end{array}$ & $1: 800$ & R35-38 \\
\hline Isotype Control & V500 & Rat IgG2a & $\begin{array}{l}\text { BD } \\
\text { pharmingen }\end{array}$ & $1: 800$ & R35-95 \\
\hline Isotype control & BV421 & Rat IgG2b, k & Biolegend & $1: 400$ & RTK4530 \\
\hline \multirow[t]{2}{*}{ Isotype Control } & \multirow[t]{2}{*}{$\mathrm{PE} / \mathrm{Cy} 7$} & Rat IgG2a, k & Biolegend & $1: 250$ & RTK2758 \\
\hline & & $\begin{array}{l}\text { Mouse IgG2a, } \\
\mathrm{k}\end{array}$ & Biolegend & $1: 250$ & MOPC-173 \\
\hline Isotype Control & PerCP/Cy5.5 & Rat IgG2a, k & Biolegend & $1: 400$ & R35-95 \\
\hline Isotype Control & AF488 & Rat IgG1, k & Biolegend & $1: 400$ & RTK2071 \\
\hline Isotype Control & Biotin & Rat IgG2a & $\begin{array}{l}\mathrm{BD} \\
\text { pharmingen }\end{array}$ & $1: 800$ & R35-95 \\
\hline \multicolumn{6}{|l|}{$\begin{array}{l}\text { Intracellular } \\
\text { Molecules }\end{array}$} \\
\hline Fox P3 & $\mathrm{PE}$ & Rat IgG2b & $\begin{array}{l}\text { BD } \\
\text { pharmingen }\end{array}$ & $1: 200$ & $259 \mathrm{D} / \mathrm{C} 7$ \\
\hline \multirow[t]{2}{*}{$\mathrm{IFN}-\gamma$} & $\mathrm{PE}$ & Rat IgG1 & $\begin{array}{l}\text { BD } \\
\text { pharmingen }\end{array}$ & $1: 200$ & XMG1.2 \\
\hline & BV421 & Rat IgG1 & Biolegend & $1: 200$ & XMG1.2 \\
\hline \multirow[t]{2}{*}{$\mathrm{IL}-10$} & $\mathrm{PE}$ & Rat IgG2b & $\begin{array}{l}\mathrm{BD} \\
\text { pharmingen }\end{array}$ & $1: 200$ & JES5-16E3 \\
\hline & BV421 & Rat IgG2b, k & Biolegend & $1: 200$ & JES5-16E3 \\
\hline
\end{tabular}




\section{Appendix 8: ELISA Protocols}

All standard curves were generated by 2 -fold serial dilutions and contained 2 blank wells.

IL-12p40 (BD Biosciences)

\begin{tabular}{|l|l|l|}
\hline Reagent & Concentration/dilution & Diluent \\
\hline Capture & $1: 1000$ & $0.1 \mathrm{M} \mathrm{Na} 2 \mathrm{HPO} 4, \mathrm{pH}=9.0$ \\
\hline Blocking solution & $10 \%$ FCS & 1x PBS pH=7.4 \\
\hline Top standard & $4 \mathrm{ng} / \mathrm{ml}$ & $5 \%$ FCS in 1x PBS pH=7.4 \\
\hline Detection & $1: 1000$ & $5 \%$ FCS in 1x PBS pH=7.4 \\
\hline Streptavidin horseradish peroxidase & $1: 2000$ & $5 \%$ FCS in 1x PBS pH=7.4 \\
\hline
\end{tabular}

IL-10 (BD Biosciences)

\begin{tabular}{|l|l|l|}
\hline Reagent & Concentration/dilution & Diluent \\
\hline Capture & $1: 500$ & $0.1 \mathrm{M} \mathrm{Na} 2 \mathrm{HPO} 4, \mathrm{pH}=9.0$ \\
\hline Blocking solution & $10 \% \mathrm{FCS}$ & $1 \mathrm{x} \mathrm{PBS} \mathrm{pH}=7.4$ \\
\hline Top standard & $25 \mathrm{ng} / \mathrm{ml}$ & $10 \% \mathrm{FCS}$ in $1 \mathrm{x}$ PBS pH=7.4 \\
\hline Detection & $1: 2500$ & $10 \%$ FCS in $1 \mathrm{x}$ PBS pH=7.4 \\
\hline Streptavidin horseradish peroxidase & $1: 1000$ & $10 \%$ FCS in $1 \mathrm{x}$ PBS pH=7.4 \\
\hline
\end{tabular}

IFN- $\gamma$ (BD Biosciences)

\begin{tabular}{|l|l|l|}
\hline Reagent & Concentration/dilution & Diluent \\
\hline Capture & $1: 1000$ & $0.1 \mathrm{M} \mathrm{Na} 2 \mathrm{HPO} 4, \mathrm{pH}=9.0$ \\
\hline Blocking solution & $5 \%$ FCS & 1x PBS pH=7.4 \\
\hline Top standard & $500 \mathrm{pg} / \mathrm{ml}$ & $5 \%$ FCS in 1x PBS pH=7.4 \\
\hline Detection & $1: 1000$ & $5 \%$ FCS in $1 \mathrm{x}$ PBS pH=7.4 \\
\hline Streptavidin horseradish peroxidase & $1: 2000$ & $5 \%$ FCS in $1 x \mathrm{PBS} \mathrm{pH}=7.4$ \\
\hline
\end{tabular}

\section{IL-17A (BD Biosciences)}

\begin{tabular}{|l|l|l|}
\hline Reagent & Concentration/dilution & Diluent \\
\hline Capture & $1: 500$ & $0.1 \mathrm{M} \mathrm{Na} 2 \mathrm{HPO} 4, \mathrm{pH}=9.0$ \\
\hline Blocking solution & $10 \% \mathrm{FCS}$ & $1 \mathrm{x} \mathrm{PBS} \mathrm{pH}=7.4$ \\
\hline Top standard & $1 \mathrm{ng} / \mathrm{ml}$ & $5 \%$ FCS in 1x PBS pH=7.4 \\
\hline Detection & $1: 500$ & $5 \%$ FCS in 1x PBS pH=7.4 \\
\hline Streptavidin horseradish peroxidase & $1: 1000$ & $5 \%$ FCS in $1 \mathrm{x}$ PBS pH=7.4 \\
\hline
\end{tabular}

\section{IL-4 (BD Biosciences)}

\begin{tabular}{|c|c|c|}
\hline Reagent & Concentration/dilution & Diluent \\
\hline Capture & $1: 2000$ & $1 \times \mathrm{PBS} p H=7.4$ \\
\hline Blocking solution & $5 \% \mathrm{FCS}$ & $1 \mathrm{x}$ PBS $\mathrm{pH}=7.4$ \\
\hline Top standard & $2350 \mathrm{pg} / \mathrm{ml}$ & $5 \%$ FCS in $1 x$ PBS $p H=7.4$ \\
\hline Detection & $1: 4000$ & $5 \%$ FCS in $1 x$ PBS $\mathrm{pH}=7.4$ \\
\hline Streptavidin horseradish peroxidase & $1: 2000$ & $5 \%$ FCS in $1 x$ PBS $p H=7.4$ \\
\hline
\end{tabular}




\section{Appendix 9: Alterations of the MIS416 compound}

\section{A9.1 Fluorescently labelled MIS416}

FITC or AF488 was bound via its isothiocyanate group to amine groups in MIS416s amino-sugar backbone (peptidoglycan). MIS416 at $10 \mathrm{mg} / \mathrm{ml}$ was washed three times with excess $0.1 \mathrm{M}$ sodium carbonate/biocarbonate buffer. Then $5 \mathrm{mg} / \mathrm{ml}$ of FITC (fluorescein isothiocyanate; Sigma, USA) in DMSO was added at a ratio of $20 \mu \mathrm{l}$ to each mg of MIS416 to be labelled. The sample was protected from light and left at room temperature overnight on a rotating tube holder. The unbound FITC was removed by washing the MIS416-FITC reaction four times with excess $150 \mathrm{mM} \mathrm{NaCl}$ at $7,000 \times \mathrm{g}$ and resuspending in $\mathrm{NaCl}$ at the original starting volume to obtain a $10 \mathrm{mg} / \mathrm{ml}$ concentration. The sample was then vortexed and to ensure there were no clumps in the suspension, the sample was passed through a $27 \mathrm{G}$ needle and syringe set multiple times. MIS416FITC was then protected from light and stored at $4{ }^{\circ} \mathrm{C}$.

\section{A9.2 Heat treatment of MIS416 removes TLR9 activity only}

MIS416 was heat-treated to remove the TLR9 by denaturing the bacterial DNA at $121^{\circ} \mathrm{C}$. The removal of TLR9 activity was confirmed by the lack of TLR9 dependent cytokine IFN- $\alpha$, whilst the microparticle retained the ability to induce NOD2 cytokines IL-10, IL-1 $\beta$, TNF and IFN- $\gamma$ (Figure A9-1). Furthermore, the microparticle appeared to have not been degraded by heat treatment as the washing steps involve centrifugation and if the particle had degraded, it would not have pelleted at the same centrifuge speed as non-heat-treated MIS416. 
A

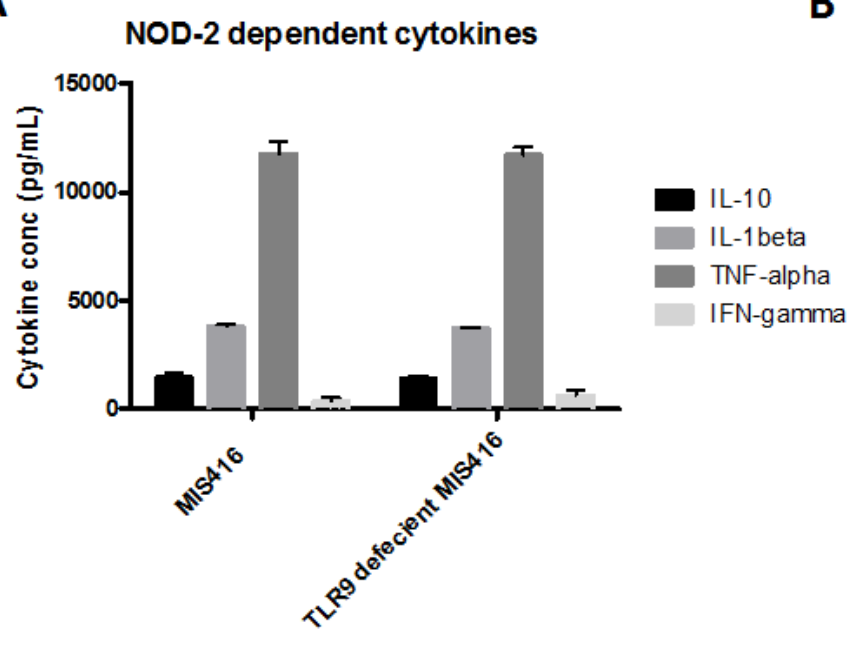

B

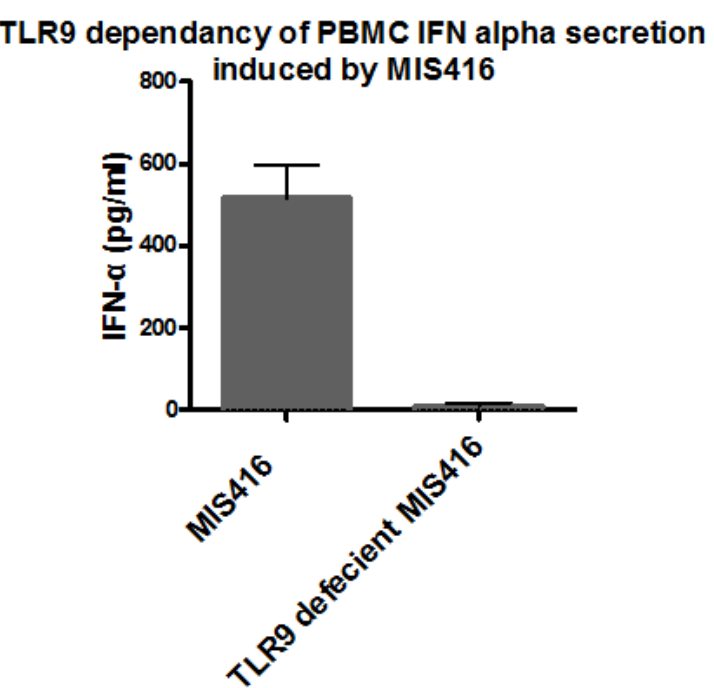

Figure A9-1 Heat treatment of MIS416 retains NOD2 cytokines and TLR9 cytokines are suppressed. Human PBMCs were cultured with $1 \mathrm{mg} / \mathrm{ml}$ of either MIS416 or heat-treated MIS416 and supernatants were assessed for NOD2 dependent cytokines in A) IL-10, IL-1 $\beta$, TNF and IFN- $\gamma$. B) PMBC secretion of IFN- $\alpha$ was dependent on TLR9 activity (data provided by Gill Webster, Innate Immunotherapeutics). 


\section{Appendix 10: Spinal cord sections}

Spinal cord sections were stained with haematoxylin and eosin and images were taken so the infiltration could be scored by independent assessors. The assessors were given information about the scoring system (see below) and scored sections blindly from each mouse. Note: the assessors used for lesion scoring had previous experience analysing CNS sections.

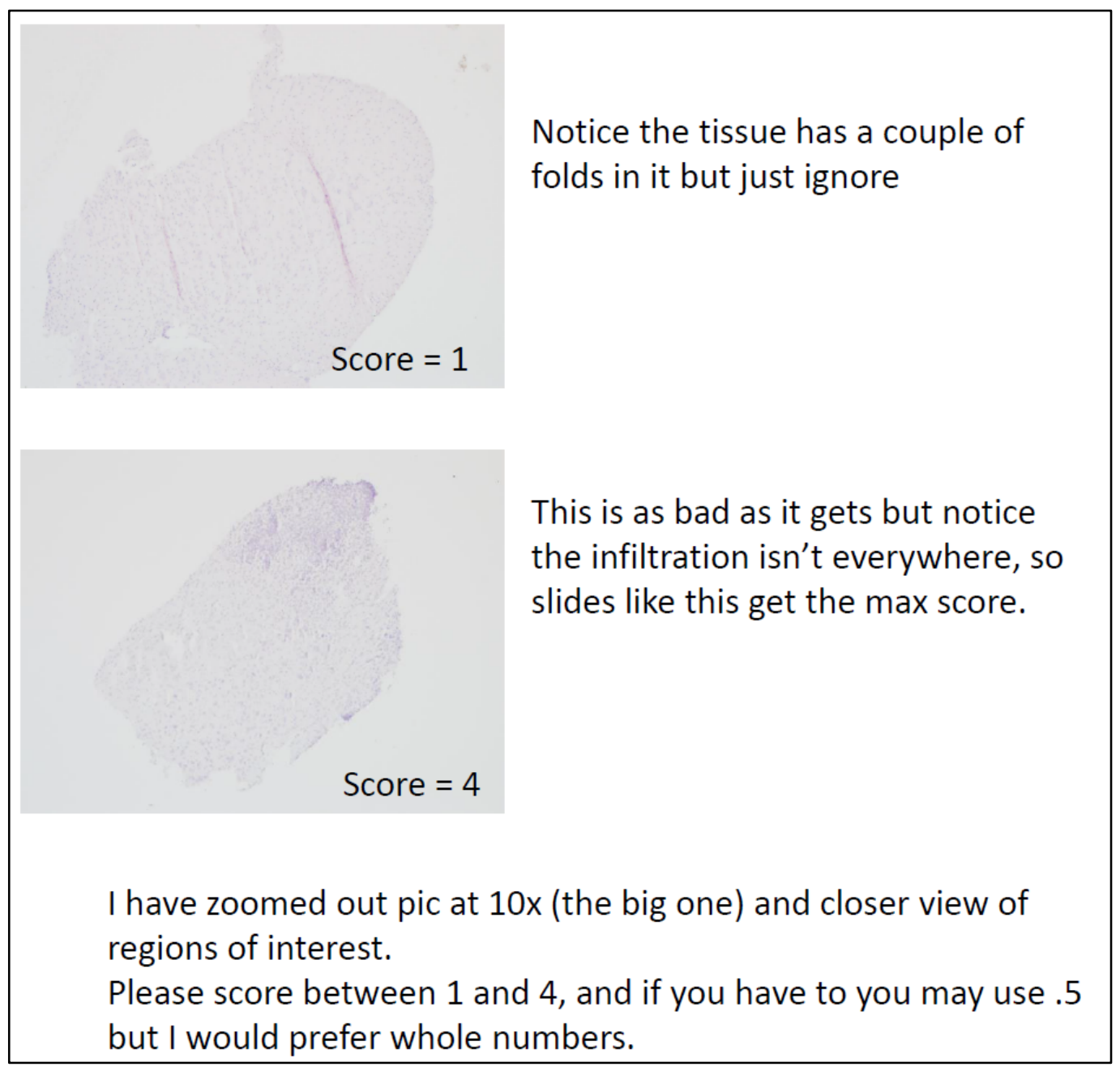

